\title{
ANNOTATED SELECTED BIBLIOGRAPHY
}

· page

1. General botanical handbooks ....... v

2. General and local botanical bibliographies .

3. Interpretation of early botanical works.

4. Keys for identifying Malaysian plants. . VII

5. Floras and botanical enumerations of neighbouring countries ....... VIII page

6. Flora of Malaysia proper . . . . . XIII

a. General works .. . . . . . . XIII

b. Local works . . . . . . . . . Xv

c. Proper taxonomic bibliography, alphabetically arranged according to families $\mathrm{XXV}$

In the absence of a complete bibliography of the botanical literature of Malaysia, comparable to those on Eastern Asia and the Pacific by Merrill \& WALKer, and as the 'Flora Malesiana' will not to be completed within the near future, the need was felt to have at hand a concise, selected bibliography of existing revisions and other phytographical publications temporarily providing taxonomists with a reference to what is roughly available for the identification of Malaysian collections. When the 'Flora Malesiana' is completed, after some decades, this bibliography should no longer be required, as the references contained in it will all have been accounted for in the Flora itself in one way or another.

In the meantime, however, a list arranged by families seems to serve a very practical purpose, as it gives access to the main body of accumulated knowledge precursory to the final revisions in the Flora.

This need is apparently felt in other regions, as during the course of this work, which was started about 1943, similar projects were undertaken for East Asia and the Pacific by MERRILL \& WALKRR and for India by SANTAPAU.

I agree with Dr WALKER that the use of such bibliographies is essentially facilitated by annotations indicating the contents. I have specially paid attention to the presence or absence of keys for identification. In some cases keys lead only to groups of species; I have called these 'partial keys'.

MERRILL \& WALKER's bibliographies aimed at completeness. My object has been more limited and from the beginning I wanted to make it selective for two reasons. On the one hand an exhaustive bibliography would mean years of bibliographical research on which I cannot afford to spend part of the little time left for my scientific work. On the other hand my aim was more limited in that $I$ felt that the need alluded to was focussed rather on a bibliographic guide, enabling rapid orientation in some family or genus, than on a complete bibliographic account. The desired concise data would be swamped in an ocean of detailed references in a really exhaustive work.

The large detailed MS bibliography, which I have gradually compiled during the past twenty five years, is arranged by families and is not suitable for publication; but it will always be at the disposal of revisors working at the Flora Malesiana.

The selection has proved very difficult and was often arbitrary in order to obtain a satisfactory uniform bibliographical level, as the scope and quality of the various papers and their importance for our aim varies enormously. It cannot be denied that, through this selection, a subjective element inevitably enters in.

Many papers are, unfortunately, not provided with keys; still, they have a certain value in efforts towards naming collections. Many papers are not exhaustive, treating representatives of a family or genus from a local region or even part of an island. Other papers were prepared without aiming at a thorough revision. A taxonomist will, however, be glad to have some literature at hand for naming current collections. It is for that aim that the present tool has been forged.

It was, of course, not necessary to give extracts from the great complete Floras and taxonomical handbooks, as these are commonly the recognized tools of the systematist. 
The bibliography tends to go from the large and wide to the small and narrow, as can be observed from the table of contents.

In chapter $6 \mathrm{c}$ families have been arranged alphabetically. It should be borne in mind that the choice of the delimitation of these families is arbitrary. Newly proposed changes have been followed with some conservatism. It has not been found of very great advantage to take up all the segregates which have been proposed in recent times.

Lately there has been a distinct fad towards splitting families, genera, species, in many instances resulting into raising taxa to the next higher rank, a tendency against which the Makers of Botany of the last century were always warning us. On the other hand, if it appears that a family contains entirely discordant elements which, in accordance with additional detailed research into pollen structure, embryology, phytochemistry, anatomy, etc., fit in different places in the system of affinities, such a splitting seems entirely justified. If, however, there is only a difference of opinion about the rank of certain groups, which after segregation merely remain in a different rank (level), but with about the same mutual relationship, there seems to be very little scientific advance gained by this splitting. It seems to me that the recent splitting of Magnoliaceae into several families and raising the complex to the order Magnoliales belongs to this category, as well as similar cases in Olacaceae, Sapotaceae, Icacinaceae, Monimiaceae, Cornaceae, Annocaceae, etc. The case of the anomalous genera Sphenoclea and Pentaphragma now removed from the Campanulaceae seems to me to be of another category, since these genera are removed from the order to which the family Campanulaceae belongs. I am perfectly aware that some families contain definitely anomalous genera and that in such cases the situation is really clarified by the removal of such genera. If the Siphonodontaceae still remain in position next to the Celastraceae there is little scientific gain in raising it to family rank, as the rank of a subfamily would be sufficient to stress its distinction within the Celastraceae. If in Oleaceae, AIRY SHAw has found good arguments in favour of removing Nyctanthes from this family to the Verbenaceae, 1 assume this to be of significant value.

Purely for technical reasons Bambusaceae have, in this bibliography, not been merged with Gramineae proper but are separately treated on a family level immediately after the Gramineae.

Amaryllidaceae, Liliaceae, etc. have only for convenience been accepted in their old delimitation which is, as Hutchinson has demonstrated, certainly artificial in its simplicity and taxonomically untenable.

For the same aim Conifers are treated as an entity, though by general consent they consist of many families. In the Moraceae the genus Ficus has been treated separately on account of its colossal size. Saurauia has also been treated separately within the Actinidiaceae.

For the convenience of those using this bibliography cross-references have been made for family names and for generic names in so far as they have been referred to more than one family.

The choice of the references is not wholly uniform. In general the larger works and Floras have not been classified under families, but in several cases the user will find that $I$ have for instance cited one of BACKER's works on the Java flora. This means that either this treatment is significant for the group in Malaysia or perhaps even comprehensive; from a large experience in using literature for rapid orientation I have intentionally inserted such entries, which at first sight seem superfluous, since they belong to general books dealt with in chapters 5 and $6 \mathrm{a}$.

I was in doubt whether to insert entries for those families which have already been treated in this Flora and would seem, for this reason, now superfluous; it is merely for completeness and uniformity that $I$ have treated such families in the same way as the others.

Families of which only cultivated or introduced species are known to occur in Malaysia, as e.g. Betulaceae, Bromeliaceae, Caricaceae, Tropaeolaceae, etc. are treated in less detail as compared with families possessing mainly native representatives.

Although the 'Flora Malesiana' is principally concerned with native and wild plants, it appeared useful to insert references to revisions of ornamental plants or those cultivated for other reasons.

Concerning the Philippines and New Guinea I have been rather lavish with references and I hope this will be a help for those who have to identify specimens from these islands. 
Those who consult chapter $6 \mathrm{c}$ should bear in mind that it embraces only books and papers which I assume to be of interest to Malaysian botany! When genera are of worldwide distribution, like many in the Scrophulariaceae, I found it necessary to insert also major works on the New World representatives. In the Araceae, Lauraceae, Loranthaceae, etc., however, I have omitted several important papers revising only African or New World representatives.

In families like the Podostemonaceae in which the number of Malaysian representatives is negligeable with respect to the total size of the family, only some of the main works have been mentioned just as far as necessary to understand the taxonomy of the Malaysian members.

Papers in which only a few genera have been revised are not mentioned under the family but have been entered under the respective genera. Comprehensive family monographs as DE Candolle's Monographia Phanerogamarum and the Pflanzenreich have not been cited under the various genera. In some large families treated in the latter work, which consist of a number of parts, names of Malaysian genera have been mentioned as an annotation.

No reference has been made in chapter $6 c$ to the first edition of the 'Pflanzenfamilien', since that work is complete and is provided with indices, but reference has been made to the families treated in the incomplete 2 nd edition. No annotations seemed necessary for the 'Pflanzenfamilien' and the 'Pflanzenreich' entries as the scope of these works is well known.

There are very many articles, reports, and even books, in which plant names are mentioned in the text but do not occur as official lists (with or without references). Although they sometimes contain valuable information, they do not belong to proper phytography and have, for this reason, been omitted.

Papers mainly dealing with anatomy, morphology or geographical distribution are only mentioned if they may be of help for orientation or appear to be of important taxonomic value.

For the convenience of those who can read Dutch, and to whom journals or books written in Dutch are available, references to such works have been cited.

I am perfectly aware that, from the purely bibliographical standpoint of citation, this bibliography leaves much to be desired; sometimes citation of figures has been omitted and there is no absolute uniformity in abbreviations. This is partly due to the fact that the work was done over a considerable period with many interruptions. It should also be considered that no bibliographical aims were pursued, but the purely practical goal of temporarily providing our readers with a tolerable basis for current work. It is not accurate enough to depend on for copying citations without consulting the originals. But $I$ hope that during the typing, retyping, and printing, only few errors will have crept into the tens of thousands of figures it contains.

It should be borne in mind that in titles I have intentionally not kept the original orthography of certain words as regards capitals, the use of which differs in the Latin, German, French, English, etc. languages. I have preferred to capitalize (hence, emphasize) all those words which appear in a title as important words. I have therefore written 'Hortus Indicus Malabaricus' and not 'Hortus indicus malabaricus' which is the correct way in Latin. The names of towns in which works in Latin were issued I have not kept under their Latin equivalents; thus a work issued at 'Leipzig' is not mentioned as having been printed at 'Lipsiae'.

Under each family, country (island) or genus references have been enumerated alphabetically under author's names, except in a very few cases.

Synonyms of several genera have been added in brackets after the accepted generic name. As the references under each genus have been enumerated alphabetically under authors' names, it does not follow that the reduction has been made by the author of the first reference to a generic name.

It is intended to compile, in subsequent volumes, supplementary lists in order to furnish subscribers with up to date references and corrections, and enable them to take full advantage of progress in our field.

In compiling this bibliography I enjoyed the co-operation of Mrs M. J. VAN STEENIS-KRUSEMAN and acknowledge the facilities given by the librarian of the Rijksherbarium who assisted in procuring a great number of books for checking. Dr H. SLEUMER, during a visit to the British 
Museum (Nat. Hist.) and the Royal Botanic Gardens, Kew, was kind enough to verify a number of items not available in Holland in which he was assisted by the Librarian of the Museum and by Mr H. S. MARSHALl. There remained a small number of books which could not be located within my immediate reach and the pertaining references could therefore not be annotated; they are marked 'Non vidi'. 


\section{GENERAL BOTANICAL HANDBOOKS}

Bentham, G. \& J. D. HoOKer, Genera Plantarum, etc. 3 vols (1862-1883).-Generic descriptions; in Latin.

Candolle, A. \& C. DE, Monographia Phanerogamarum prodromi nunc continuatio, nunc revisio, 1-9 (1878-1896).-The families monographed herein are inserted in chapter $6 c$.

Candolle, A. P., A. \& C. DE, c.s., Prodromus systematis naturalis regni vegetabilis sive enume. ratio contracta ordinum generum specierumque plantarum, etc. 17 vols (1824-1873), index 1-4 (1842-1874).-Complete descriptive account of genera and spp. in Latin; no keys.

DAlla TORRE, C. G. DE \& H. HARMs, Genera Siphonogamarum, etc. (1900-1907).-Index of generic and infrageneric names.

ENGler, A. (ed.), c.s., Das Pflanzenreich, etc. Heft 1-107 (1900-1953).-Families of which monographs have appeared in this work have been referred to in chapter 6c. References to these have been made with the separate parts
(Hefte) to avoid the intricate way of numbering of these parts. Keys and descriptions in Latin, other matter in German.

- \& K. Prantl (ed.), Die natürlichen Pflanzenfamilien (1st ed.), 4 Teile (1887-1908), 4 Nachträge (1897-1915).-This widely used work has not been extracted in chapter 6c. Families treated in the 2nd edition of the work which is still far from complete have been referred to in chapter 6c. In German.

Index Kewensis, etc. 2 vols (1893-1895), 11 Suppl. (1901-1953).-References to descriptions of new genera and species.

Index Londinensis, etc. 6 vols (1929-1931), Suppl. 1-2 (1941).-Index to figures of plants.

LEMEE, A., Dictionnaire descriptif et synonymique des genres de plantes phanérogames. Brest. 1-7, 8a-b (1929-1943).-Descriptions of all genera alphabetically arranged; vol. 8 contains keys to the genera of each family.

\section{GENERAL AND LOCAL BOTANICAL BIBLIOGRAPHIES}

with special reference to the tropics of the Old World except Africa

ANonYmous, Tooa Kyoo ei ken-Sigenkagaku-bunkenmokuroku. Bibliographic index for the study of the natural resources of the Great Asia coprosperity sphere. Department of Education, Dai Nippon. Except for the references wholly in Japanese.

1. New Guinea (1942) $259+81$ pp. Botany p. 20-61.

2. French Indo-China \& Thailand (1942) $253+$ 81 pp. Botany p. 19-48.

3. Philippines (1942) $391+61$ pp. Botany p. 35-155.

4. Malay Peninsula ('Malay') (1943) $241+11$ pp. Botany p. 38-108.

5. Micronesia (1944) (by Huzio Utinomi). Bibliographia Micronesica scientiae naturalis et cultus. Kokoryukan. Tokyo (1945) $\mathrm{i}-\mathrm{iii}$, 1-3, 1-208; Botany 1-21. Non vidi.-A 2nd edition in English was edited by the Univ, of Hawaii Press (1952), the botany section of which was translated by $K$. SAKImura and edited by H. ST. JoHN; repr. p. 3-16.

6. East Indies (1944) $689+19$ pp. Non vidi.

- An annotated bibliography of the Southwest Pacific and adjacent areas. Issued by the Allied Forces. 1944. 3 vols. - Non vidi.

1. The Netherlands and British East Indies and Philippine Islands.

2. Malaya. Thailand, Indo-China.

3. The China Coast and the Japanese Empire.

Blake, S. F. \& A. C. ATwood, Geographical guide to Floras of the World. Part 1 (U.S. Dep. Agric. Wash. Misc. Publ. 101, 1942, 1-336).Useful annotated list of Floras and plantgeographical works in the wide sense, geographically arranged. Asia has not yet been treated.
BlATtER, E., A bibliography of the Botany of British India and Ceylon (Journ. Bombay Nat. Hist. Soc. 20, 1911, Ixxix-clxxvi).

Boerlage, J. G., Literatuur Botanie (in F. S. A. DE Clerce, Ethnogr. Beschrijving v. d. Westen Noordkust van Ned. Nieuw-Guinea. Leiden, 1893, 272-273).-Bibl. New Guin. 1884 onwards.

Field, H., Bibliography on Southwestern Asia. Univ. Miami Press (1953) xvi + 106 pp.Anthropogeography, botany and zoology; total 3000 titles. Covering Near East as far as and including Afghanistan.

JACKsoN, B. D., Guide to the literature of botany; being a classified selection of botanical works, including nearly 6000 titles not given in Pritzel's 'Thesaurus'. London (1881) i-xl, 1-626.

JunghuHN, F., Opgaaf betreffende de literatuur over de Flora van Java (in JUNGHUHN, Java, ed. 2, Dutch ed., 1, 1853, 179-198).-Annotated bibliography of Java; in Dutch.

Kuntze, O., Notizen zu Pritzel's Thesaurus Literaturae Botanicae (Rev. Gen. Pl. 1891, cxxii-cxlvi; ibid. pars III, 1898, 153-162).These corrections contain unfortunately many errors.

LAM, H. J., Materials towards a study of the Flora of the island of New Guinea (Blumea 1, 1934, 115-159).-Contains lists of families revised in Bot. Jahrb. and Nova Guinea and a list of books and papers.

Merrill, E. D., Index to Philippine literature 1-6 (Philip. J. Sc. 2, 1907, Bot. 241-250, 345-349, $437-439$; l.c. 3, 1908, 87-94; l.c. 4, 1909, $677-685 ;$ l.c. 5, 1910, 259-266).-Lists of annotated references. 
-, A contribution to the bibliography of the botany of Borneo (Sarawak Mus. J. 2, 1915, 99-136), supplemented in Merrill, E. D., A bibliographic enumeration of Bornean plants (J. Str. R. As. Soc. Special no, 1921, 2-6).-Extensive Bornean bibliography:

-, An enumeration of Philippine flowering plants vol. 4 (1926) 155-239.-Extensive bibliography on Philippine botany.

-, Polynesian botanical bibliography, 1773-1935 (Bull. Bern. P. Bish. Mus. no 144, 1937, 1-194).

-, A botanical bibliography of the islands of the Pacific with $A$ subject index by $\mathrm{E}$. H.' WALKER (Contr. U.S. Nat. Herb. 30, pt 1, 1947, 1-404).Amplification of that of 1937. Annotated!

- \& E. H. WALKER, A bibliography of Eastern Asiatic Botany (Arn. Arb. Harv. Univ. 1938, i-xlii, 1-719).-Comprehensive account.

Ретсн, T., Bibliography of books and papers relating to Agriculture and Botany to the end of the year 1915. Peradeniya Manuals no 3 (1925) 1-256.-Bibliogr. of Ceylon arranged under headings; over 5000 titles. References 3737-4368 deal with taxonomy of Phanerogams and ferns; most references arranged under families.

Pritzel, G. A., Thesaurus Literaturae Botanicae, etc. (ed. JESSEN pp.). Leipzig (1872-1877) 1-577. -Covers all botanical literature with admirable accuracy, specially regarding books and pamphlets.

REHDER, A., The Bradley Bibliography. Arnold Arboretum, Jamaica Plain, Mass. (1911-1918) 5 vols.-Comprehensive bibliography of mainly woody plants described before 1900 .

-, Bibliography of cultivated Trees and Shrubs, etc. Arnold Arboretum, Jamaica Plain, Mass. (1949) i-xl, 1-825.-With many references to infraspecific taxa. Non vidi.
Santapau, H., Contributions to the bibliography of Indian Botany (Journ. Bombay Nat. Hist. Soc. 50, 1952, 520-548; ibid. 51, 1952, 205-259). -Intended as a complement to BLATTER's work (see above). Besides the list of titles, the greater part of the work consists of extracted references arranged in families.

Steenis, C. G. G. J. VAN, Annotated list of literature for the use of botanists and explorers in Malaysia (Fl. Mal. I, 1, 1950, xxxiiixli).

SteENIS-KrusemaN, M. J. VAN, Malaysian plant collectors and collections, etc. (Fl. Mal. I, 1, 1950, i-clii, 1-639).-Bibliographic references to the collections arranged under the collectors.

- \& W. T. Stearn, Dates of Publication (Fl. Mal. I, 4, 1954, clxiii-ccxix).-Extremely useful account of exact dates of publication of certain botanical works; supplementary to PRITZEL's, JACKSON's and other's bibliographies.

TuCKer, E. M., Catalogue of the Library of the Arnold Arboretum of Harvard University. Cambridge, Mass. 3 vols (1914-1933).Valuable catalogue.

WALKER, E. H., Contribution toward a Bibliography of Thai Botany (Nat. Hist. Bull. Siam Soc. 15, 1952, 27-88).-Annotated bibliogr. of Siamese taxonomic botany.

WrT, H. C. D. DE, Short history of the phytography of Malaysian vascular plants (Fl. Mal. I, 4, 1949 , lxx-clxi).-Each chapter has been provided with numerous references relating to the history of Malaysian phytography.

WoODWARD, B. B., Catalogue of the books, manuscripts, maps, and drawings in the British Museum (Natural History). 8 vols, London (1903-1940).-A very useful reference work for botany and zoology.

\section{INTERPRETATION OF EARLY BOTANICAL WORKS}

\section{of importance for Malaysian Botany}

Blanco, M., Flora de Filipinas (1837), ed. 2 (1845), ed. 3 (1877-1883).

cf. Merrill, E. D., A review of the identifications of the species described in Blanco's Flora de Filipinas (Govt Lab. Publ. (Philip.) 27, 1905, 1-132).

-; Species Blancoanae. Manila (1918) 1-423, map.-Evaluation . of species described by Blanco and Llanos.

Burman, N. L,, Flora Indica. Amsterdam (1768). cf. Merrill, E. D., Philip. J. Sc. 19 (1921) 329-388.-This review of the new species of plants proposed by Burmán has been made without actually examining BuRMAN's herbarium at Geneva which has, unfortunately, been merged into the general Herbier DeLESSERT.

HoutTuYn, M., New genera and species published in various works.

cf. Merrill, E. D., J. Arn. Arb. 19 (1938) 291-375; ibid. 20 (1939) 264-268.-Critical consideration of new genera and species proposed by HouTtuYN.

JACK, W., Descriptions of Malayan Plants (Mal. Misc. 1 (1), 1820,1-27; 1 (5), 1821, 1-41; 2 (7), 1822, i-iii, 1-96).

cf. Merrill, E. D., J. Arn. Arb. 33 (1952) 199-250, t. 11.-Evaluation of JACk's new genera and species.

Linnaeus, C., Species Plantarum, etc. ed. 1 (1753-1754) 2 vols.

cf. JACKSON, B. D:, Index to the Linnean Herbarium, with indication of the types of species marked by Carl von Linné (Proc. Linn. Soc. Lond. 124, 1912, Suppl. 1-152).

-, Notes on a catalogue of the Linnean Herbarium (ibid. sess. 134, 1922, Suppl. 1-38, 1 fig.). -Supplementary to the latter entry.

Savage, Sp., A Catalogue of the Linnean Herbarium. Ed. by the Linn. Soc. London (1945) i-xv, 1-225.-A valuable account of the con- 
tents of the Linnean Herbarium in the possession of the Linnean Society of. London indicating the details of the annotations on the sheets.

LOUREIRO, J. DE, Flora Cochinchinensis, etc. (1790) i-xx, 1-744. New verbatim ed. by C. L. WiLLDENOW (1793) i-xxiv, 1-882.

cf. Merrill, E. D., Trans. Amer. Philos. Soc. Philad. new ser. 24, 2 (1935) 1-445.-A critical commentary; as far as necessary and possible the types in the British Museum have been examined.

Pennant, T., Flora Indica (in Outlines of the Globe 4, 1800, 237-317, 1-8).

cf. Merrill, E. D., J. Arn. Arb. 29 (1948) 186-192.-Evaluation of the new accidental combinations proposed in PenNant's compilation.

RAFINESQUE, C. S., Various works.

cf. Merrill, Index Rafinesquianus. Arnold Arboretum, Jamaica Plain, Mass. (1949) i-ix, 1-296.-Complete account and evaluation of the new genera, species, and combinations proposed by RAFINESQUE.

RHEEDE TOT DRAAKESTEIN, H. A. VAN, ${ }^{\prime}$ Hortus Indicus Malabaricus etc. 12 vols (1678-1703). cf. ${ }^{2}$ Burman, J., Flora Malabarica, sive index in omnes tomos horti malabarici, quem juxta normam a botanicis hujus aevi receptam conscripsit, et ordine alphabetico digessit (1769) 1-10. DENNstedt, A. W., Schlüssel zum Hortus Indicus Malabaricus (1818) 1-40.

Buchanan-Hamilton, F., Commentary on the Hortus Malabaricus (1822-1835) 1-410; repr. from Trans. Linn. Soc. 13 (1822) 474-560; 14 (1824) 171-312; 15 (1826) 78-152; 17 (1835) 147-252.

[DILLWYN, L. W.] A review of the references to the Hortus Malabaricus of Henry van Rheede van Draakenstein (1839) i-viii, 1-69.

Rumphius, G. E., Herbarium Amboinense (17411750) 6 vols; (1755), with index universalis, vol. 7 (Auctuarium).-See next entry. $c f^{2}$ Burman, J., Index universalis in sex tomos et auctuarium Herbarii Amboinensis cl. Georgii Everhardi Rumphii (Herb. Amb. Auct. 1755,1-20). -, Index alter in omnes tomos Herbarii Amboinensis cl. G. Everhardi Rumphii, quem de novo recensuit, auxit et emendavit Joannes Burmannus (1769) 1-22.-This was issued in connection with Burman's index to RHEEdE's Hortus Malabaricus and is sometimes bound in the last volume of that work.

LINNAEus, C., Herbarium Amboinense, quod consens. experient. Facult. Medicae in Regia Academia Upsalensi, sub praesidio Linnaei... publico examini : submittit, Alumnus Regius Olavus Stickman (1754) i-iv, 1-28.-Republished with slight alterations under the title: Herbarium Amboinense, sub praesidio $D$. $D$. Car. Linnaei, proposuit Olavus : Stickman (Amoen. Acad. 4, 1759, 112-143).

Buchanan-Hamiton, F., Commentary on the Herbarium Amboinense. Liber Primus (Mem. Wern. Soc. 5, 1826. 307-383).

- A commentary on the second book of the Herbarium Amboinense (ibid. 6, 1832, 268-333). HensChel, A. G. E. T., Clavis Herbarii Amboinensis: in his Vita G. E. Rumphii (1833) 139-202.

HASsKaRL, J. K., Neuer Schlüssel zu Rumph's Herbarium Amboinense (Abh. Naturf. Gesellsch. Halle 9, 1866, 145-389; reprint 1866, 1-247). MERRILL, E. D., An interpretation of Rumphius's Herbarium Amboinense. Manila (1917) 1-595, 2 maps.-The latest evaluation of the Rumphian work, which does not rest on actual herbarium specimens, but on the descriptions and plates on which many genera and species have been based.

ThunberG, C. P. (ed. L. Winberg \& F. 0. WIDMARK), Florula Javanica (1825).

cf. BACKer, C. A., R. C. BAKHUIzen VAN DEN BrINk Jr \& C. G. G. J. VAN Steenis, Blumea 6 (1950) 358-362.-Result of an examination of the types preserved at Uppsala as far as new species are concerned.

\section{KEYS FOR IDENTIFYING MALAYSIAN PLANTS}

BACKER, C. A., Schoolfiora voor Java. Weltevreden (1911) vii-cii.-Contains an original key to Javanese plants which has often proved to be' useful for regions outside Java, though based on characters strictly belonging to the Javanese species. In Dutch.

-, Onkruidflora der Javasche Suikerrietgronden: Handboek ten dienste van de Suikerriet-cultuur en de rietsuikerfabricage op Java. Vol. 7. Soerabaja (1934) Ixxiii-lxxxvii.-Key to c. 750 weeds of sugar-cane fields in Java. In Dutch.

ENDERT, F. H., Geslachtstabellen voor Nederlandsch-Indische boomsoorten naar vegetatieve kenmerken. Thesis, Wageningen (1928).-

(1) The name is spelt in different ways, in the 1 st vol. as $H$. van RHEEDE VAN DRAaKenstein, in the last vol. as H. VAN RHEDE TOT DRAKestein. (2) Chronologically.
Appeared also as “Mededelingen van het Bosbouwproefstation te Buitenzorg' 20 (1928) 242 pp. (Commun. For. Res. Institute Buitenzorg).-Valuable key to the genera containing trees in Indonesia based on vegetative characters with the aid of a hand-lens. In Dutch.

GAGnePaIN, F., Clef analytique et synoptique des familles de plantes vasculaires décrites dans la Flore Générale de l'Indo-Chine. Supplément à la Flore Générale de l'Indo-Chine.-Private issue, Paris (1922) 34 pp. Published in a slightly revised edition in the said Flora, tome préliminaire, Paris (1943) 50-89.

Hutchinson, J., Families of flowering plants. 1. Dicotyledones. London (1926) 9-80; ibid. 2. Monocotyledones.London(1934) 9-25.-Exceedingly useful, general keys covering all genera.

Merrill, E. D., A Flora of Manila. Manila (1912) 33-45.-A small key of a local Flora, still origin- 
al and not without merits for identifying specimens outside the area it covers.

ST. JOHN, H. \& F. R. FOSBERG, Identification of $\mathrm{Ha}$ wailan plants. Part 1, Dicotyledons. University of Hawaii, Occas. Pap. Bern. P. Bish. Mus. no 36 (1938) 4-25; part 2, Gymnosperms \& Monocotyledons, op. cit. no 41 (1942) 5-13.-
Intended only for Hawaii; the second part gives keys as far as the genera.

THONNER, F., Anleitung zum Bestimmen der Familien der Blütenpflanzen. 2nd edition, Berlin (1917) 280 pp.-Originally based on a general key to African plants but extended to all flowering plants.

\section{FLORAS AND BOTANICAL ENUMERATIONS OF NEIGHBOURING COUNTRIES}

\section{India and Pakistan (Nepal, Bhotan) ${ }^{1}$}

Anonymous, Bibliographies of East Indies. See Chapter 2.

BEDDOME, R. H., Icones plantarum Indiae Orientalis, etc. 1 vol., Madras (1868-1874).-300 pl. with descr.

-, The Flora Sylvativa for Southern India, etc. Madras (1869-1874).-Plates of principal timber trees of the Madras Presidency with descr.; 2 vols with 325 pl.; vol. 3 with text of a Flora with keys to genera; species descr. \& listed.

Benthall, A. P., The trees of Calcutta and its neighbourhood. Calcutta (1946) i-cii, 1-513, fig. 1-274.-Keys to spp. mentioned.

BisCOE, W. F., A list of trees and shrubs of Indore State. Bombay (1910) 1-108.-Non vidi.

Blatter, E., $A$ Bibliography of the Botany of British India \& Ceylon. See Chapter 2.

-, Beautiful flowers of Kashmir. London. 2 vols: 1 (1927) i-xv, 1-198, t. 1-33; 2 (1929) i-xv, 1-204, t. 34-62.-Descr.; short key to spp.; plates mostly coloured.

- \& F. HAllberg, The Flora of the Indian desert (Jodhpur and Jaisalmer) (Journ.Bombay Nat. Hist. Soc. 26, 1918-1919, 218-246, 525-551, 811-818, t. 1-31; 968-987; ibid. 27, 1920-1921, $40-47,270-279,506-512$, t. 32-37).-Plant list with some new spp.

- \& C. MCCANN, Revision of the Flora of the Bombay Presidency (ibid. 31-38, 1926-1935, in all 27 parts; unfinished).-Keys.

-, 一, \& T. S. SABNIs, The Flora of the Indus Delta (Journ. Ind. Bot. Soc. 6, 1927, 31-47, 57-78, 115-132; ibid. 7, 1928, 22-43, 70-96, 168-175; ibid. 8, 1929, 19-77; 50 pl., 140 fig.).Lists of spp. and localities; no keys; composition of the flora; ecological notes.

- \& W. S. Millard, Some beautiful Indian Trees. London (1937) $i-x, 1-110$, t. 1-91, fig. $1-60$ (repr. from articles in Journ. Bomb. Nat. Hist. Soc.).

Boissier, E., Flora Orientalis, sive Enumeratio plantarum in Oriente, a Graecia et Aegypto ad Indiae fines hucusque observatarum. Geneva/ Bazel (1867-1884) 5 vols + Suppl. vol. (ed. BUSER) 1888.-Enum. of flora of the Near East.

Bor, N. L., Manual of Indian Forest Botany. Oxford Univ. Pr. (1953) xv + 441, t. 1-31.-

(1) Assam, Andamans \& Nicobars have for convenience been entered separately at the end.
Families generally; key to families \& genera, occasionally to spp.

BRANDIS, D., Indian Trees. London (1906) 767 pp., 201 fig.; 3rd ed. (1911),-Flora for foresters; with keys.

Calder, C. C., V. Narayanaswami \& M. S. RAMASWAMI, List of species and genera of Indian Phanerogams not included in Sir J. D. Hooker's Flora of British India arranged in alphabetical order (Rec. Bot. Surv. Ind. 11, 1926, i-ii, 1-157).-List of plant names with reference arranged alphabetically under specific names.

Collett, H., Flora Simlensis. Calcutta \& Simla (1902); 2nd ed. (1921).-Illustrated Flora with keys.

CoOKe, TH., The Flora of the Presidency of Bombay. 3 vols, London (1901-1909).-Not illustrated; keys.

DuTHIE, J. F., Flora of the Upper Gangetic Plain, etc. Calcutta (1903-1922) 3 vols.-Non-illustrated flora with keys.

Fischer, C. E. C., A survey of the flora of the Anaimalai Hills in the Coimbatore District, Madras Presidency (Rec. Bot. Surv. Ind. 9, 1921, xxi + 218 pp., 5 pl.).-List.

-, New or little-known plants from Southern India I-XIII (Kew Bull. 1932-1940).-Ditto; records and new spp.

FysON, P. F., The Flora of the Nilgiri and Pulney Hill-tops (above $6500 \mathrm{ft}$ ). Madras (1915-1920). 1 (1915) i-xxvi, 1-475 (keys and descr.); 2 (1915) Atlas with illustr. on 286 pp.; 3 (1920) i-xviii, 1-136, illustr. on pp. 287-581 (Supplement to the original edition and including the country round Coonoor and down to $5000 \mathrm{ft}$ ).

- The Flora of the South Indian Hill Stations. Madras (1932) i-xxix, 1-697, Atlas t. 1-611.Flora with keys \& descr.

Gamble, J. S. \& C. E. C. Fischer, Flora of the Presidency of Madras. 11 parts, London (19151936).-Critical non-illustrated Flora with keys.

GUPTA, B. L., Forest Flora of the Chakrata, Dehra Dun and Saharanpur Forest Divisions, United Provinces. Ed. 3. Calcutta (1928) i-xviii, 1-558. -Keys.

HaInes, H. H., The Botany of Bihar and Orissa, etc. 2 vols, London (1921-1925) i-x, 1-1350. Supplement by H. MooNey, Ranchi (1950) i-iii, 1-294.-Important Flora with keys and descr.

HOOKER, J. D. \& T. ThOMSON, Flora Indica vol. 
1 (1855) i-xv, 1-280, 1-285.-Valuable introductory essay; Ranunculaceae to Fumariaceae; as in Fl. Br. Ind. keys to genera, not to spp.

-, c.s., Flora of British India. 7 vols (1872-1897).

Mayuranathan, P. V., The flowering plants of Madras City and its immediate neighbourhood (Bull. Madras Govt Mus. n.s. (Nat. Hist. Sect.) 2, 1929, 1-345, t. 1-38).-Very short descr.; keys.

Supplement by E. BARNES (ibid. 4, no 2, 1938 , i-ii, 1-46, t. 1-7).

MCCANN, C., Trees of India. A popular handbook. Bombay (1947) t. 1-78, 17 fig.-Non vidi.

Osmaston, A. E., A Forest Flora of Kumaon. Allahabad (1927) i-xxxiv, 1-605.-Keys.

PARKer, R. N., A Forest Flora of the Punjab with Hazara and Delhi. Lahore (1918) i-xxxv, 1-577.-Keys. A 2nd ed. in 1924.

Prain, D., Bengal Plants. Calcutta (1903) 2 vols, 1-1319.-Flora with keys; Lower Provinces and Chittagong.

-, Flora of the Sundribuns (Rec. Bot. Surv. Ind. 2, 1903, 231-370).-Key to genera; spp. listed.

RAO, M. R., Flowering Plants of Travancore. Trivandrum (1914).-Non vidi.

Rheede tot Draakestein, H. A. van, Hortus Malabaricus. See Chapter 3.

Roxburgh, W., Plants of the Coast of Coromandel, etc. 3 vols (1795-1820).-Containing 300 tab.

-, Hortus Bengalensis, etc. (1814) i-v, i-xii, 1-76. -Catalogue. For a list of validly published names (based on pre-Linnean descriptions) cf. Robinson, C. B., in Philip. J. Sc. 7 (1912) Bot. 411-419.

-, Flora Indica (ed. Carey) 1 (1820) 1-7, 1-493; 2 (1824) 1-5, i-v, 1-588.

-, ditto ed. 2 (1832) 3 vols, resp. 741, 691, and 875 pp. Repr. by ClARKe literatim (1874).

SANTAPAU, H., Bibliography of Indian Botany. See Chapter 2.

SteWART, J. L. \& D. BRANDIS, The Forest Flora of North-West and Central India. London (1874) 2 vols, text i-xxxi, 1-608; Atlas ('Illustrations') t. 1-70.

Talbot, W. A., The Trees, Shrubs, and Woody Climbers of the Bombay Presidency. Ed. 2, Bombay (1902) i-xi, 1-385.-Keys.

- Forest Flora of the Bombay Presidency and Sind. Poona (1909, 1911) 2 vols: 1 (1909) i-vi, 1-508, i-xxvi, fig. 1-288; 2 (1911) i-xxxvii, 1-574, fig. 289-541.-Descr. illustr., keys.

Wight, R., Icones Plantarum Indiae Orientalis. 6 vols (1838-1853) t. 1-2101.

- \& G. A. Walker-Arnot, Prodromus Florae Peninsulae Indiae Orientalis 1 (1834) i-xxxvii, 1-480.-Partial keys; most of the Choripetalae; also Rubiaceae.

Assam:

BURKILL, I. H., The Botany of the Abor Expedition (Rec. Bot. Surv. India 10, 1924-25, 1-420, t. 1-10).-List; ecology and vegetation.

Fischer, C. E. C., Plants new to Assam I-XIII (Kew Bull. 1929-1940).-Lists of records and new spp.
Kanjllal \& Das, c.s., Flora of Assam. 5 vols (in 6), Calcutta (1935-1940).-Flora with keys; unfinished.

\section{Andaman Islands:}

Gamble, J. S., A preliminary List of the Plants of the Andaman Islands. Port Blair (1903) 54 pp.List.

KURZ, S., Report on the Vegetation of the Andaman Islands, etc. Calcutta (1870) 75 pp.-List of spp. observed.

Parkinson, C. E., A Foriest Flora of the Andaman Islands. Simla (1923) i-v, i-v, i-xiii, 1-325, t. 1-6.-Annotated descr. Flora with keys.

\section{Nicobar Islands :}

KURz, S., A Sketch of the Vegetation of the Nicobar Islands (J. As. Soc. Bengal 45, ii, 1876, 105-164, t. 12-13).-Spp. listed; some new; sketch of vegetation.

PraIN, D., On a botanical visit to Little Andaman and the Nicobars (Proc. As. Soc. Beng. 1891, 156-175, 1892).-Lists of spp. observed.

\section{Ceylon}

Abeyesundere, L. A. J. \& R. A. de Rosayro, Draft of first check-list for Ceylon. Oxford (1939). Mimeogr. 1-115.-Tree catalogue.

Alston, A. H. G., The Kandy Flora. Colombo (1938) i-xvii, 1-109, fig. 1-404.-For the use of schools; keys.

Petch, T., Bibliography of Botany. See Chapter 2.

TRIMEN, H. \& J. D. Hooker, A Handbook to the Flora of Ceylon. 5 vols +1 vol. plates, London (1893-1900).-An important suppl. vol. 6 (1931) by A. H. G. Alston.

WiLlis, J. C. \& M., A revised Catalogue of the Flowering Plants and Ferns of Ceylon (Perad. Manuals of Bot., Entom., Agric. \& Hort. no 2, 1911, 1-188).-Name list.

\section{Burma}

Fischer, C. E. C., Contributions to the Flora of Burma I-XVIII (Kew Bull. 1926-1941).Records and descr. new spp.

KURZ, S., Report on the Forests and Vegetation of Pegu. Calcutta (1875) 1-97, i-cxxxviii, 1-95, i-xiv, 1-34, maps, t. 1-2.-Extract journey; vegetation; vernacular names and data of forest trees; flora with keys.

- Contributions towards a knowledge of the Burmese Flora I-IV (J. As. Soc. Bengal 43-46, 1874-1877).-Keys!

-, Forest Flora of British Burma. 2 vols. Calcutta (1877).-Keys.

Merrilx, E. D., The Upper Burma Plants collected by Capt. F. Kingdon Ward on the Vernay-Cutting Expedition, 1938-1939 (Brittonia 4, 1941, 20188).-List; descr. new spp.

\section{Siam}

ANonymous, Bibliographies of Thailand. See Chapter 2.

Craib, W. G., A. F. G. Kerr, J. B. Imlay, c.s., Contributions to the Flora of Siam I-LIV (Kew 
Bull. 1911-1941).-Lists of records and new spp.

Craib, W. G., A. F. G. KerR, H. R. Fletcher, c.s., Flora Siamensis Enumeratio. Vols 1-32, Bangkok (1925-1954).-Critical synonymy; list; no keys.

Hosseus, C. C., Die botanischen Ergebnisse meiner Expedition nach Siam (Beih. Bot. Centralbl. 28, ii, 1911, 357-457).-List.

RIDLEY, H. N., The Flora of Lower Siam (J. Str. Br. As. Soc. no 59, 1911, 15-234).-List.

-, On a collection of plants from Peninsular Siam

(J. Fed. Mal. Stat. Mus. 10, 1920, 65-126).-List.

W ALKer, E. H., Bibliography of Botany. See Chapter 2.

\section{Indo-China}

Anonymous, Bibliographies of Indo-China. See Chapter 2.

Lecomte, H., F. Gagnepain, c.s., Flore générale de l'Indo-Chine. 7 vols, 1 vol. Suppl. and tome préliminaire. Paris (1907-1951).-Flora with keys and figures.

LOUREIRO, J. DE, Flora Cochinchinensis, etc. (1790) $\mathrm{i}-\mathrm{xx}, 1-744$. For an evaluation $c f$. Merrill, Trans. Amer. Philos. Soc. Philad. new ser. 24, 2 (1935) 1-445.

Pierre, L., Flore Forestière de la Cochinchine. Paris (1879-1907) 26 parts (5 vols) t. 1-400.Plates accompanied by text; quite some Malaysian plants are mentioned or are critically considered.

\section{Hainan}

Masamune, G., Flora Kainantensis. Taiwan (1943) xv + 443 pp.-Enum.; except names and references in Japanese.

Merrill, E. D., An Enumeration of Hainan plants (Lingn. Sc. J. 5, 1927, 1-186).-List; no keys. Many supplements in later vols of same journal and in Sunyatsenia.

- \& Walker, Bibliography of Eastern Asia. See Chapter 2.

\section{Formosa (Taiwan)}

Hayata, B., Flora Montana Formosae (J. Coll. Sc. Imp. Univ. Tokyo 25 (art. 19), 1908, 1-260, t. 1-41).-Enum. plants above $900 \mathrm{~m}$.

-, Materials for a Flora of Formosa (ibid. 30, art. 1, 1911, 1-471).-Descr.

-, Icones Plantarum Formosanarum. (1911-1921) vol. 1-10; Suppl. 5 parts (1925-1932) by Y. Yамамото:-Revisions \& descr.; sometimes keys; beautiful illustrations.

Kanehira, R., Formosan Trees indigenous to the island. Dep. Forestry, Govt Res. Inst. Taihoku. Revised ed. (1936) i-x, 1-754, fig. 1-664, t. 1-50.-Descr.; synon.; no keys.

Kudo, Y. \& G. MASAmune, Genera Plantarum Formosanarum I (Ann. Rep. Taihoku Bot. Gard. 2, 1932, 1-141).-Saururaceae-Rosaceae. Keys to and descr. of genera.

Matsumura, J. \& B. Hayata, Enumeratio Plantarum in Insula Formosa, etc. (J. Coll. Sc. Tokyo 22, 1906, 1-704, t. 1-18).-Enum.; t. 18 is a map.
MerRiLl \& Walker, Bibliography of Eastern Asia. See Chapter 2.

SASAKI, S., A Catalogue of the Government Herbarium. Taihoku (1930) vii $+592 \mathrm{pp}$.

\section{Micronesia}

Glassmann, S. F., The Flora of Ponape (Bern. P. Bish. Mus. Bull. 209, 1952, 1-152, fig. 1-21).List; no keys.

Hosokawa, T., Materials of the botanical research towards the Flora of Micronesia I-XXI (Trans. Nat. Hist. Soc. Formosa 24-31, 1934-1941, only XIV-XVI in J. Jap. Bot. 13, 1937, 53-203, 274-284, 608-617).-A well-illustrated series of papers with records and new spp.; for some genera keys.

KANEHIRA, R., New or noteworthy trees from $\mathrm{Mi}$ cronesia 1-20 (Tokyo Bot. Mag. 46-52, 19321938).-Series of illustrated papers with records and descr. new spp.

-, Flora Micronesica. South Seas Bureau (1933) viii, 468, 37 pp., fig. 1-211, t. 1-21.-Wellillustrated Flora with keys but the text except the Latin names in Japanese.

-, Plantae novae Micronesicae (Trans. Nat. Hist. Soc. Formosa 25, 1935, 136-139).-Records and some new spp in a few families.

-, An Enumeration of Micronesian plants (J. Dept Agric. Kyushu Imp. Univ. 4, 1935, $237-$ 464).

Merrill, E. D., An Enumeration of the plants of Guam (Philip. J. Sc: 9, 1914, Bot. 14-155; ibid. 15, 1919, 539-544).-Enum.; descr. new spp.

-, Bibliography of the Pacific. See Chapter 2.

UTINom, H., Bibliography of Micronesia. See Chapter 2.

Volkens, G., L. Diels, c.s., Beiträge zur Flora von Mikronesien (und Polynesien) I (Bot. Jahrb. $52,1915,1-18$ ) ; ditto II ( ibid. 56, 1921, 429577); ditto III (ibid. 59, 1924, 1-29); ditto IV (ibid. 63, 1930, 272-323); ditto V (ibid. 69, 1938, 395-400).-Revisions; sometimes with keys; incomplete.

WALKER, E. H. \& R. Todin, Additional phanerogams in the Flora of Guam, with notes on unverified records (Contr. U. S. Nat. Herb. 30, 1949 , 449-468, t. 8-9).-List of records; no novelties.

\section{Melanesia}

Bentham, G., Enumeration of the plants collected by $R$. B. Hinds Esq. and Mr Barclay, etc. (in Hook. J. Bot. 2, 1843, 211-240).-List of plants coll. in Fiji, Tanna, New Ireland, and New Guinea, and a few from Ambon (Moluccas).

BURKILL, I. H., c.s., On a collection of Plants from New Britain (Neu Pommern) (Proc. Cambr. Phil. Soc. 9, 1896, 91-98).-List; some new spp.; bibliogr. New Britain Botany.

BURTT, B. L., Melanesian Plants (Kew Bull. 1935, 298-306; ibid. 1936, 459-466).-Descr. some new spp. from the Solomons, a single one from Fiji. 
Diels, L., c.s., Plantae Peekelianae Papuanae (Notizbl. Berl.-Dahlem 10, 1930, 273-282).Descr. some new spp. and records.

ENGLER, A., c.s., Uebersicht über die botanischen Ergebnisse der Gazelle-Expedition (Bot. Jahrb. 7, 1886, 444-480).-Cyperaceae, Gramineae, Orchidaceae were published in op. cit. 5 (1884) 89-94; 6 (1885) 233-248; 7 (1885) 435-443.List of records; also new spp.

FosBerg, F. R.; Melanesian vascular plants (Lloydia 3, 1940, 109-124, 1 pl., 5 fig.).-List of $60 \mathrm{spp}$. from the Templeton Crocker Exp. in the Solomon \& Santa Cruz Isl.; some new.

Hemsley, W. B., C.s., Flora of the Solomon Islands (Kew Bull. 1894, 211-215).-List of new spp.

-, ditto (ibid. 1895, 132-139).-Coll. ComINs; list; new spp.

—, Ann. Bot. 5 (1891) 501-508, t. 27.-Misc. descr. new spp.; Cominsia n.g.

ibid. 6 (1892) 203-210, t. 11-14.-Descr. 2 new gen. of Sapotaceae.

-, J. Linn. Soc. Lond. Bot. 30 (1894) 163-165, 211-217, t. 9-11.-Misc. descr. new spp.; Sararanga n.g.

LAUTERBACH, C., Beiträge zur Flora von NeuMecklenburg (Bot. Jahrb. 45, 1911, 354-365).Records from New Ireland coll. Peekel; some new spp.

-, Beiträge zur Flora Papuasiens.-See under New Guinea. Occasionally Melanesian collections are mentioned.

MERRILL, Bibliography of the Pacific. See Chapter 2.

- \& Perry, Plantae Archboldianae.-See under New Guinea. In this series, as well as in other papers on the New Guinean flora by $A$. C. SmIтh, C. T. White, etc. Melanesian spp. have been recorded or described.

ReChINGER, K., c.s., Botanische Ergebnisse der wissenschaftlichen Forschungsreise nach den Samoainseln, dem Neuguinea-Archipel und den Salomonsinseln (Denkschr. Kais. Ak. Wiss. Wien 84, 1910, 408-455 (Pteridophytes and Cyperaceae); ibid. 89, 1914, 468-621).-List of records and new spp. coll. RECHINGER.

-, Plantae Papuanae (in FedDE, Rep. 11, 1912, 179-187).-Descr. misc. new spp.; some from New Guinea.

RECORD, M., A collection of woody plants from Melanesia (Trop. Woods 81, 1945, 9-45).List of records of $400 \mathrm{spp}$. coll. WATERHOUSE 1932-1936 from Bougainville, New Britain, etc.; no new spp. or new comb.

Schumann, K., Die Flora des Deutschen Ostasiatischen Schutzgebietes (Bot. Jahrb. 9, 1887, 189-223).-List - of plants coll. HóllrunG, NaumanN, etc. from New Guinea, New Britain, Solomons, etc. with occasional new spp.

- c.s., Die Flora von Neu-Pommern (Notizbl. Berl.-Dahlem 2, 1898, 59-158, map).-List records and new spp.

WhItE, C. T., Ligneous plants from the Solomon Islands (and New Guinea) (J. Arn. Arb. 31, 1950, 81-116).-List records and new spp.

\section{New Hebrides}

Guillaumin, A., Contributions to the Flora of the New Hebrides. Plants collected by S. F. Kajewski in 1928 and 1929 (J. Arn. Arb. 12, 1931, 221-264, fig. 1-3; ibid. 13, 1932, 1-29, fig. 1, 81-126, fig. 1-2, t. 43).-Records and new spp.

- Compendium de la Flore Phanérogamique des Nouvelles Hébrides (Ann. Mus. Col. Marseille 55/56, 1948, 1-56).-List of plants known.

Merrill, Bibliography of the Pacific. See Chapter 2.

\section{New Caledonia}

DÄNIKer, A. U., c.s., Katalog der Pteridophyta und Embryophyta Siphonogama (Beibl. Vierteljahrschr. Naturf. Ges. Zürich 77-78, 1932-43, 1-507).

Guillaumin, A., Matériaux pour la Flore de la Nouvelle-Calédonie.-A series of 85 revisions and lists, mostly published in Bull. Soc. Bot. Fr. and Not. Syst. 1914-1945. Indispensable for consulting the next work.

-, Flore analytique et synoptique de la NouvelleCalédonie. Phanérogames. Paris (1948) 1-369.Merely keys; no descr. of genera \& spp.; no author names; no references.

MerrILl, Bibliography of the Pacific. See Chapter 2.

Rendle, A. B., E. G. Baker \& S. Moore, A Systematic Account of the Plants collected in New Caledonia and the Isle of Pines by Prof. R. H. Compton, M. A. in 1914 (J. Linn. Soc. Lond. Bot. 45, 1921, 245-417, t. 13-24).List; many new spp. not considered by GuILLAUMIN.

SCHLECHTER, R., Beiträge zur Kenntnis der Flora von Neu-Kaledonien (Bot. Jahrb. 39, 1907, 1-274, fig. 1-23).-Account of his collections; records and descr.; no keys.

General:

Australia

Bentham, G., assisted by F. von Mueller, Flora Australiensis. 7 vols. London (1863-1878).Standard work; keys.

DoмIN, K., Beiträge zur Flora und Pflanzengeographie Australiens (Bibl. Bot. 20 and 22, 1915-1929, 1317 pp.).-Refers primarily to Queensland.

FrancIs, W. D., Australian Rain-forest Trees. 2nd ed. For. \& Timber Bur. Commonwealth Australia (1951) i-xv, 1-469, 24 fig., 260 tab., 1 map.-Descr.; key to genera; artificial key to spp.

Mueller, F. von, Second systematic census of Australian plants. Melbourne (1889) 244 pp.Enumeration of spp. and their distr. in Australia.

\section{North Australia :}

Ewart, A. J. \& O. B. DAvies, The Flora of the Northern Territory, etc. Melbourne (1917) viii + 387 pp., t. 1-27.-Keys.

Queensland:

BaIley, F. M., The Queensland Flora. 6 parts, 
index. Brisbane (1899-1902) xxxii + 2015 pp., index (1905) 1-66.-Keys.

- Comprehensive Catalogue of Queensland Plants, etc. Brisbane (1913) 879 pp., 976 fig., 16 tab.

Domin, K., See above under Australia. Domm's work deals mostly with Queensland plants.

WhITE, C. T., Ligneous plants collected for the Arnold Arboretum in North Queensland by S. F. Kajewski in 1929 (Contr. Arn. Arb. 4, 1933, 113 pp., t. 1-9).-Enum. \& descr.

- \& W. D. Francis, Contributions to the Queensland Flora 1-8 (Proc. R. Soc. Queensland 1921-1944).-Ditto.

\section{West Australia:}

Diels, L. \& E. Pritzel, Fragmenta phytographiae Australiae occidentalis (Bot. Jahrb. 35, 1904-05, 55-662, fig. 1-70).-Enum.; no keys.

GARDNER, C. A., Enumeratio plantarum Australiae occidentalis. Perth (1931) iv + 150 pp.-Name list.

-, Flora of West Australia. vol. 1, part 1 (Gramineae) (1953); other volumes in course of preparation.

\section{New South Wales:}

Dixon, W. A., The plants of New South Wales, etc. Sydney (1906) xxiv +322 pp.-Analytical keys. Non vidi.

Maiden, J. H. \& E. Betche, A census of New South Wales plants. Sydney (1916) xx + 216 pp.Non vidi.

Moore, Ch. \& E. Betche, Handbook of the Flora of New South Wales. Sydney (1893) xxxix + 582 pp.-Non vidi.

\section{Victoria :}

EWART, A. J., Flora of Victoria. Melbourne (1930) 1257 pp., 349 fig.

\section{South Australia :}

Black, J. M., Flora of South Australia. 4 parts (1922-1929).-Parts 1-3 have already been revised in a 2 nd edition; pt 4 is in the press.

\section{Tasmania}

Rodway, L., The Tasmanian Flora. Hobart (1903) xix +320 pp., tab.-Non vidi.

\section{New Zealand}

Cheeseman, T. F., Manual of the New Zealand Flora. 2nd ed. Wellington (1925) xliv, $1163 \mathrm{pp}$. -Keys.

Hooker, J. D., Handbook of the New Zealand Flora, etc. London (1864-67).-Standard Flora with keys.

\section{Fiji}

GibBs, L. S., c.s., A contribution to the montane Flora of Fiji (including Cryptogams), with ecological notes (J. Linn. Soc. Lond. Bot. 39, 1909, 130212, t. 11-16, fig. 1-2).-Listed records with new spp.

GillesPIE, J. W., New plants from Fiji I-III
(Bern. P. Bish. Mus. Bull. 74, 1930, 1-41, 43-99 (t. 1-57); ibid. 83, 1931, 1-32, 33-72 (t. 1-40); ibid. 91, 1932, 1-38, 39-81 (t. 1-43)).Mainly miscellaneous new spp.

Gray, A., Botany, Phanerogamia (in U.S. Exploring Expedition during the years 1838, 1839, $1840,1841,1842$, under the command of Charles Wilkes, U.S.N., 1854, 1-777, atlas, 1857 , t. 1-100).-Listed records and descr.

MerriLl, Bibliography of the Pacific. See Chapter 2.

SeEmanN, B., Flora Vitiensis: etc. London (18651868) 1-324, t. (col.) 1-90.-Descr.

Smith, A. C., Fijian Plant Studies (Bern. P. Bish. Mus. Bull. 141, 1936, 1-166, fig. 1-83).Account of 1933-1934 expedition; records and new spp. of pteridophytes and phanerogams.

- c.s., Ditto II (Sargentia 1, 1942, i-iv, 1-148, fig. 1-5).-Results of 1940-1941 expeditions by DEGENER; keys.

-, Studies of Pacific Island plants I-XVI (Bull. Torrey Bot. Club 68, 1941, 397-406; J. Arn. Arb. 24, 1943, 347-361; Bull. Torrey Bot. Club 70, 1943, 533-549; J. Arn. Arb. 26, 1945, 97-110, 319-322; ibid. 31, 1950, 137-171, 288-319; ibid. 32, 1951, 27-58, 226-255; Contr. U.S. Nat. Herb. 30, 1952, 469-522; J. Arn. Arb. 33, 1952, 97-149, 367-402; ibid. 34, 1953, 37-51; Contr. U. S. Nat. Herb. 30, 1953, 523-575; J. Arn. Arb. 34, 1953, 97-124).Mostly dealing with Fijian plants, partly as revisions with keys of separate families.

\section{Samoa}

Christophersen, E., c.s., Flowering Plants of Samoa (Bern. P. Bish. Mus. Bull. 128, 1935, 1-221, fig. 1-32).-Records and descr. new spp.

ENGLER, A., c.s., Uebersicht über die botanischen Ergebnisse der Gazelle-Expedition (Bot. Jahrb. 7, 1886, 444-480).-Cyperaceae, Gramineae, Orchidaceae were published in op. cit. 5 (1884) 89-94; 6 (1885) 233-248; 7 (1885) 435-443.List of records; also new spp.

GraY, A., Botany, Phanerogamia (in U.S. Exploring Expedition during the years 1838, 1839, $1840,1841,1842$, under the command of Charles Wilkes, U.S.N., 1854, 1-777, atlas, 1857, t. 1-100).-Listed records and descr.

Merrill, Bibliography of the Pacific. See Chapter 2.

ReCHINGER, K., c.s., Botanische Ergebnisse der wissenschaftlichen Forschungsreise nach den Samoainseln, dem Neuguinea-Archipel und den Salomonsinseln (Denkschr. Kais. Ak. Wiss. Wien 84, 1910, 408-455 (Pteridophytes and Cyperaceae); ibid. 89, 1914, 468-621).-List of records and new spp. coll. RECHINGER.

ReINeCKe, F., Die Flora der Samoa Inseln. II. Siphonogamen (Bot. Jahrb. 25, 1898, 578-708, t. 8-13, fig. 1).-Enum.

\section{Southeastern Polynesia}

Brown, F. B. H., c.s.,Flora of Southeastern Polynesia I-III (Bern. P. Bish. Mus. Bull.; Monocotyle- 
dons: 84, 1931, 1-194, t. 1-35, fig. 1-18; Pteridophytes: 89, 1931, 1-123, t. 1-21, fig. 1-19; Dicotyledons: $130,1935,1-386$, fig. 1-70, t. 1-9).-Flora with keys of the Marquesas, Society Isl., Tuamotu Arch., Austral Isl., Rapa \& Pitcairn.

Drake del Castillo, E., Flore de la Polynésie Francaise. Paris (1893) i-xxiv, 1-352, map (of Tahiti).-Flora with keys of the Marquesas, Society Isl., Pomotou, Gambier, and Wallis Isl. -, Illustrationes Florae Insularum Maris Pacifici. Paris (1886) 1-458, t. 1-50.-Enum. spp. from Fiji, Samoa, Tahiti, Tonga, and SE. Polynesia.
ENGLeR, A., c.s., Uebersicht über die botanischen Ergebnisse der Gazelle-Expedition (Bot. Jahrb. 7, 1886, 444-480).-Cyperaceae, Gramineae, Orchidaceae were published in op. cit. 5 (1884) 89-94; 6 (1885) 233-248; 7 (1885) 435-443.List of records; also new spp.

Gray, A., Botany, Phanerogamia (in U.S. Exploring Expedition during the years 1838, 1839, $1840,1841,1842$, under the command of Charles Wilkes, U.S.N., 1854, 1-777, atlas, 1857 , t. 1-100).-Listed records and descr.

Merrill, Bibliography of the Pacific. See Chapter 2.

\section{FLORA OF MALAYSIA PROPER}

\section{a. GENERAL WORKS}

BACKer, C. A., Contributiones ad cognitionem Florae Indiae Batavae (Bull. Jard. Bot. Btzg III, 2, 1920, 315-330).-Descr. miscellaneous new spp.

BeCCARI, O., c.s.s., Malesia. 3 vols. Firenze (18771890).-Beautifully illustrated series of semimonogr. revisions of various groups mostly on the basis of Beccari's own collections. Other than descr. text in Ital.

Blume, C. L., Bijdragen tot de Flora van Nederlandsch Indië, parts 1-17 (1825-1827).-Descr. of many new genera and over 1000 new spp. from various collections; in Latin.

- Enumeratio Plantarum Javae et insularum adjacentium minus cognitarum vel novarum, etc. Fasc. 1 (1827) i-vi, 1-98; fasc. 2 (1827) i-x, 99-274; Verbatim repr. 1830.-Enum.; descr. (in Latin). Besides plants from Java this work contains also some descr. from other Mal. islands, e.g. from Celebes coll. ReINWARDT; some spp. based on Rumphian references.

-, Rumphia, etc. 4 vols (1836-1849).-Illustrated with col. plates of selected plant groups and genera; in Latin.

- Museum botanicum Lugduno-Batavum, etc. 2 vols (1849-1856) $396+256$ pp.-Account of Leyden collections; mainly descr. text; not confined to Malaysian plants; many new spp., some new genera; in Latin; a few plates with analyses.

Boerlage, J. G., Handleiding tot de kennis der Flora van Nederlandsch Indië. 3 vols (1890-1900). -Incomplete; descriptions of genera, appr. following BenthaM \& HookeR's Genera Plantarum; sometimes keys to the genera; in Dutch.

- Catalogus plantarum phanerogamarum quae in horto botanico Bogoriensi coluntur herbaceis exceptis. 2 parts (1899-1901).-Enum. spp. cultivated in Hort. Bog.; some new.

, Th. Valeton, J. J. SMIth, etc., Icones Bogorienses. 4 vols, Leiden (1897-1914).-Planned like Hooker's Ic. Plant.; with many valuable contributions, several new genera and many new spp.

BURKILL, I. H., Some changes in plant names (Kew Bull. 1935, 316-319).-List of transfers from his Dictionary of Econ. Prod. of the Malay Peninsula.
BuRman, N. L., Flora Indica, etc. Leyden/ Amsterdam (1768) i-iv, 1-242, t. 1-67, indices 1-15.-Enum.; no keys; a bibliographic evaluation is given by Merrill, Philip. J. Sc. 19 (1921) 328-388.

Cholsy, J. D., Plantae Javanicae nec non ex insulis finitimis et etiam e Japonia. Geneve (1858) i-iv, 5-30.-Records and descr. of ZoLLINGER's second collection and a continuation of his 'Syst. Verz.'; contains accounts of Hypericaceae, Guttiferae, Theaceae, Saurauia, and Convolvulaceae; in Latin.

ENGLER, A., c.s., Uebersicht über die botanischen Ergebnisse der Gazelle-Expedition (Bot. Jahrb. 7, 1886, 444-480).-Cyperaceae, Gramineae, Orchidaceae were published in op. cit. 5 (1884) 89-94; 6 (1885) 233-248; 7 (1885) 435-443.List of records; also new spp.; from Timor, Solomon Isl., New Britain, New Guinea, Tonga Isl. and Samoa.

HALlier $f$, H., Neue und bemerkenswerte Pflanzen aus dem Malaiisch-Papuanischen Inselmeer I (Ann. Jard. Bot. Btzg 13, 1896, 276-327); ditto II (Bull. Herb. Boiss. 6, 1898, 213-220, 283-288, 348-360, 604-622); ditto III (ibid. II, 1, 1901, 667-676); ditto IV (Med. Rijksherb. Leiden 26, 1915, 1-8).-Miscellaneous contrib.; records; descr. new spp.

-, Neue Indonesische Dikotyledonen (in FEDDE, Rep. 2, 1906, 59-64).-New spp. and miscellaneous records.

HASSKARL, J. K., Adnotationes de plantis quibusdam Javanicis nonnullisque Japonicis, haud rite cognitis e Catalogo Horti Bogoriensis excerptae. Accedunt nonnullae novae species (in HOEVEN \& DE VRIEse, Tijd. Nat. Geschied. \& Phys. 10, $1843,115-150)$.-List of new records and descr. new spp. occurring in the Catalogue of 1844 , see next entry.

-, Catalogus plantarum in Horto Botanico Bogoriensi cultarum alter. Batavia (1844) 1-391.Contains descr. of new gen. \& spp.; also new comb. and new names.

- Adnotationes de plantis horti Bogoriensis (in HoEven \& DE VRIESE, Tijd. Nat. Geschied. \& Phys. 12, 1845, 77-139).-Continuation of the article of 1843 . 
-, Retzia, sive observationes botanicae, quas in primis in Horto Botanico Bogoriensi, etc. Pugillus I (Nat. Tijd. Ned. Ind. 10, 1855, 1-252, 1856).-Descr. mostly of Javanese plants, partly from the Botanic Gardens at Bogor; in Latin.

-, ditto. Pugillus II (Acta Soc. Sc. Indo-Neerl. 1, 1856, 1-54).-Ditto.

- Hortus Bogoriensis descriptus, sive Retzia editio nova, valde aucta et emendata. Amsterdam \& Bonn (1858) xx +376 pp.-A revised edition of the former work.

-, ditto, pars II (Bonplandia 7, 1859, 170-183, 254-274; ibid. 8, 1860, 90-100, 118-146).Miscellaneous descr., mostly of plants from the Botanic Gardens, Bogor; hardly any new spp.

HeYNe, K., De Nuttige Planten van NederlandschIndië ('The useful plants of the. Netherlands East Indies'). 2nd ed. Weltevreden (1927), 3 vols, ccxi +1662 pp. Repr. literatim (1950) under the title: 'De Nuttige Planten van Indonesië'.Critical enum. of useful plants; in Dutch.

Hochreutiner, B. P. G., Catalogus Bogoriensis novus I-II (Bull. Inst. Bot. Btzg no 19, 1904, 1-48; ibid. no 22,. 1905, 1-132; index 1908, 1-22).-Catalogue of the Botanic Gardens, Bogor; contains descr. of new taxa.

- Plantae Bogorienses exsiccatae novae vel minus cognitae quae in Horto Botanico coluntur. Bogor (1904) 1-75, index i-ix.-Contains descr. of miscellaneous new spp.

-, Descriptiones plantarum Bogoriensium exsiccatarum novarum (Ann. Jard. Bot. Btzg, Suppl. 3, 1910, 815-870).-Miscellaneous descr. of plants cultivated in the Botanic Gardens, Bogor.

KoRthals, P. W., Kruidkunde ('Botany') in Temminck, Verh. Nat. Geschied. 1 vol. (1840 1844) 1-259, t. 1-70.-Treatments of various groups of his collections.

Miquel, F. A. W., Flora Indiae Batavae $1^{1}$ (18551858) xxiv + 1116 pp.; $1^{2}(1858-1859) \times i i+$ 704 pp.; 2 (1856-1859) ix + 1103 pp.; 3 (18551859) $\mathrm{x}+773$ pp.-Enum.; no keys.

-, Choix des plantes rares ou nouvelles, etc. The Hague (-1864) 1-30, t. 1-26, folio.

-, c.s., Annales Musei botanici Lugduno-Batavi. 4 vols, Leyden (1863-1869).-Contains many revisions additional to $\mathrm{Fl}$. Ind. Bat.

-, Illustrations de la Flore de l'Archipel Indien. 3 parts. Leyden (1870-1871) $x+114$ pp., $t$. 1-37.-Semi-monogr. revisions of various families in Malaysia; continuation of the latter work.

OCHSE, J. J. \& R. C. BAKHUIZEN VAN DEN BRINK, Indische Groenten ('Vegetables of the Dutch East Indies'). Batavia-C. (1931) xxxv +1003 pp., 463 fig.-In Dutch; there is also an English edition (1931).-Descriptions.

RoXbURGH, W., Flora Indica. 2 vols. Serampore (1820-1824); ed. CAREY, 3 vols. London (1832). -The latter edition reprinted literatim (1872). -Many types of Malaysian plants are among those described by ROXBURGH, specially from the Malay Peninsula.

SCHEFFER, R. H. C. C., Observationes phytographicae I-III (Nat. Tijd. Ned. Ind. 31, 1870,
1-23, 338-390; ibid. 32, 1873, 387-426).Records and descriptions of new genera \& spp., mostly based on the collections of TEYsmanN.

SMITH, J. J., Plantae novae vel criticae ex herbario et horto bogoriensi I-III (Bull. Jard. Bot. Btzg III, 1, 1918, 399-411; ibid. 4, 1922, 230-240; ibid. 6, 1924, 73-107).-Misc. descr. of Mal. plants with plates.

Steenis, C. G. G. J. VAN, On the origin of the Malaysian mountain flora I (Bull. Jard. Bot. Btzg III, 13, 1934, 174-260).-Semi-critical enum. of spp. belonging to microtherm genera. -, Annotated list of literature. See Chapter 2.

-, Miscellaneous botanical notes I-VI (Bull. Bot. Gard. Btzg III, 17, 1948, 383-411; Blumea 6, 1948, 242-263; Bull. Bot. Gard. Btzg III, 18, 1950, 457-461; Reinwardtia 1, 1952, 467-481; Act. Bot. Neerl. 2, 1953, 298-307; Blumea 7, 1954, 595-598).-Misc. notes, records \& descr. new genera \& spp.

Steenis-Kruseman, M. J. van, Malaysian Plant Collectors. See Chapter 2.

- \&.W. T. Stearn, Dates of Publication. See Chapter 2.

TeysmanN, J. E. \& S. BnNnendiuk, Nieuwe plantensoorten in 's Lands Plantentuin te Buitenzorg (Nat. Tijd. Ned. Ind. 2, 1851, 303-310; ibid. 3, 1852, 326-332; ibid. 4, 1853, 393-398).-Descr. miscellaneous new Malaysian spp.

- Plantae novae in . Horto Bogoriensi cultae (Nat. Tijd. Ned. Ind. 24, 1862, 305-332; ibid. $25,1863,399-428$; ibid. 27, 1864, 15-58).Important series of descr. of miscellaneous new spp. from Malaysia.

Turczaninow, N., Animadversiones, etc. I-III (Bull. Soc. Nat. Moscou 272, 1854, 271bis-372; ibid. 31', 1858, 185-250, 379-476; ibid. 361, 1863, 545-615).-Miscellaneous Latin diagnoses of new genera and $s p p$. from the author's herb. in Charkow; several based on ZoLLINGER numbers.

VRIESE, W. H. DE, Plantae Indiae Batavae Orientalis, etc. Leyden (1856-1857) 1-160, t. 1-8.Revisions of some groups; misc. records and descr. of plants coll. ReINWARDT in Java, Celebes, Ambon, Ternate, etc.

Warburg, O., c.s., Monsunia. Beiträge zur Kenntnis der Vegetation des Süd- und Ostasiatischen Monsungebietes. Leipzig (1900) i-vii, 1-201, t. 1-11.-Descr. various groups of Bryophytes, Fungi, Pteridophytes, and Gymnosperms.

WIT, H. C. D. DE, History of Phytography. See Chapter 2.

ZOLLINGER, H., Observationes phytographicae, etc. Nat. \& Geneesk. Arch. Ned. Ind. 1, 1844 372-405, 599-616; ibid. 2, 1845, 1-19, 200-273, 563-587).-Lists; records \& descr. of new genera \& spp.

-, Systematisches Verzeichnis der im Indischen Archipel in den Jahren 1842-1848 gesammelten sowie der aus Japan empfangenen Pfanzen. Zürich (1854-1855). pt 1 (1854) i-xii,. 1-80, tab.; pt 2 (1854) 81-160; pt 3 (1855) 1-67.Enum. of spp. from his collections made during his second stay in Java and his trips to Bali, 
Lombok, S. Celebes, Saleyer, Flores, Bima, and Sumbawa. Includes also species from Japan received by him. For a continuation see CHOISY and the next entry.

-, Observationes botanicae novae (Nat. Tijd. Ned. Ind. 14, 1857, 145-176, repr. 1-32).-Misc. records and descr. of novelties mostly from Java, partly based on his own collections, partly on those of the Botanic Gardens, Bogor, partly from the heritage of KUHL and VAN HASSELT; continuation and correction of the latter book. In Dutch; descr. in Latin.

\section{b. LOCAL WORKS ${ }^{1}$}

\section{Sumatra}

BARTLETT, H. H., Sumatran plants collected in Asahan and Karoland with notes on their vernacular names (Pap. Mich. Acad. Sc. Arts \& Lett. $6,1926,1-66)$.

Boerlage, J. G., Flora; Systematische lijst, etc. (in VeTh, Midden-Sumatra 4, pt 2, 1884, 16-50). -List; some new spp. from Central Sumatra. In Dutch.

Hildebrand, F. H., Report For. Res. Institute, Bogor, no 8 (1949) 14 pp., mimeogr.-List of tree spp. from Djambi Division, South Sumatra.

-, ditto, no 9 (1949) 50 pp.-Ditto, from Sumatra's East Coast Division.

-, ditto no 56 (1952) 64 pp.-Revised edition of the latter.

一, ditto, no 11 (1949) 67 pp.-Ditto, from Riouw, Bengkalis \& Indragiri.

-, ditto no 36 (1953) 26 pp.-Ditto, from Bengkalis (Riouw).

-, ditto no 61 (1953) 67 pp.-Ditto, from Riouw \& Indragiri.

_, ditto, no 12 (1949) 29 pp.-Ditto, from Banka \& Billiton Islands.

-, ditto, no 57 (1952) 46 pp.-Revised edition of the latter.

-, ditto, no 19 (1949) 78 pp.-Ditto, from Palembang, South Sumatra.

-, ditto, no 20 (1949) 19 pp.-Ditto, from Lampung Districts, South Sumatra.

-, ditto, no 65 (1954) 27 pp.-Revised edition of the latter.

—, ditto, no 22 (1949) 47 pp.-Ditto, from Bencoolen Division.

-, ditto, no 26 (1950) 60 pp.-Ditto, from Sumatra's West Coast Division.

-, ditto, no 64 (1953) 67 pp.-Revised edition of the latter.

-, ditto, no 29 (1950) 43 pp.-Ditto, from Tapanuli Division, Northwest Sumatra.

-, ditto, no 67 (1954) 50 pp.-Revised edition of the latter.

-, ditto, no 32 (1950) 70 pp.-Ditto, from Atjeh Division and neighbouring Simalur Island, N. Sumatra.

JACK, W., Descriptions of Malayan plants (in Mal. Miscell. 1 (1), 1820,1-27; 1 (5), 1821, 1-41; 2 (7), 1822, i-iii, 1-96). Repr. by Hooker (in his J. Bot. 1, 1834, 358-380; Comp. Bot. Mag. 1, 1835, 147-157, 219-224, 253-272). Again reprinted at Calcutta (with addition of data in Calc. J. Nat. Hist. 4, 1843, 1-62, 159-213, 305-374, t. 14-16; repr. 1-230).-Descr. of new genera and spp.-For an evaluation $c f$. Merrill, J. Arn. Arb. 33 (1952) 199-250.

KOORDERS-SCHUMACHER, A., Systematisches Verzeichnis der zum Herbar Koorders, etc. II. Abt. Sumatra. Buitenzorg (1910-1914) 1-62.-Account of his coll. in the islands P. Weh \& P. Bras (extreme N. extremity of Sumatra) and in Central Sumatra; some new spp.

KURZ, S., Korte schets der vegetatie van het eiland Bangka (Nat. Tijd. Ned. Ind. 27, 1864, 142258).-List of his collection from Banka Island; few new spp.; in Dutch.

MERrill, E. D., Notes on the Flora of Sumatra (Philip. J. Sc. 14, 1919, 239-250).-Misc. new records and spp. from the BARTLETT \& LARUE coll.

-, c.s., An enumeration of plants collected in Sumatra by $W$. N. \& C. M. Bangham (Contr. Arn. Arb. 8, 1934, 1-178, t. 1-13).-Records and new spp. listed.

-, New Sumatran plants I-IV (Mich. Acad. Sc. Arts \& Lett. 19, 1933, 149-203, t. 16-35, 1934; ibid. 20, 1934, 95-112, 1935; ibid. 23, 1937, 177-202, 1938; ibid. 24, 1938, 63-92, 1939).Lists of records and new spp. based on collections of Bartlett, Hamel, Rahmat si Toroes and YATES from the northern part of Central Sumatra.

-, Botanical Results of the George Vanderbilt Sumatran Expedition 1939 (Not. Nat. Acad. Sc. Philad. no 47, 1940, 9 pp.).-New spp. from N. Sumatra.

MiQuel, F. A. W., c.s., Plantae Junghuhnianae, etc. 6 parts (1851-1856) 1-570.-Lists, records, $\&$ descr.

-, Sumatra (FI. Ind. Bat. Suppl. 1, 1860-1861; repr. 1862).-Complete enum. of the Sumatran flora with descr. of new genera and spp.

ReNDle, A. B., c.s., Dr H. O. Forbes's Malayan plants (J. Bot. 62-64, 1924-1926, Suppl. 1-149). -Account of Forbes's coll. from S. Sumatra; list with descr. of new gen. \& spp.

Ridley, H. N., c.s., Results of an Expedition to Korinchi Peak, Sumatra. Botany (Fed. Mal. St. Mus. 8, pt 4, 1917, 1-145).-List Boden KLoss coll. W. Central Sumatra.

-, A Botanical Excursion to North Sumatra (J. Mal. Br. R. As. Soc. 1, 1923, 46-113).-List of

(1) The smaller islands are inserted with adjacent large islands or island groups, e.g. those W of Sumatra with Sumatra; Riouw, Banka, Billiton, etc. with Sumatra; Anambas \& Natuna Islands with Borneo; Krakatau \& Madura with Java, etc. In general the map in vol. 1, facing p. cxii has been followed. 
his collection N. of Toba Lake; various new spp. -, Plants from Bencoolen, Sumatra (Kew Bull. 1925, 76-94).-List coll. C. J. Brooks; records and new spp.

-, The Flora of the Mentawi Islands (Kew Bull. 1926, 57-94).-List of BODEN KLOSS coll.

Steenis, C. G. G. J. Van, Die Pteridophyten und Phanerogamen der Deutschen Limnologischen Sunda Expedition (Arch. f. Hydrobiol. Suppl. vol. 11, 1932, 231-387).-Account of coll. by RUTTNER, mostly waterplants from $S$. to Central Sumatra; some new records and new spp.

-, Report of a Botanical Trip to the Ranau Region, S. Sumatra (Bull. Jard. Bot. Btzg III, 13, 1933, 1-56, fig. 1-11).-Records of his own coll.; mostly plant-geographical.

VRIESE, W. H. DE \& F. JUNGHUHN, Bijdragen tot de kennis der Flora van Sumatra (Ned. Kruidk. Arch. 1, 1846, 1-19).-Misc. records and new spp.

\section{Malay Peninsula}

ANONYmous, Bibliographies of the Malay Peninsula. See Chapter 2.

BURKILL, I. H., Some changes in plant names (Kew Bull. 1935, 316-319).-List of new comb. made in the following work.

-, A Dictionary of the Economic Products of the Malay Peninsula. London (1935) 2 vols, i-xi, 1-2402.-Alphabetic standard account; the new comb. are accounted for in Kew Bull. (1935).

- \& M. R. Henderson, The flowering plants of Taiping in the Malay Peninsula (Gard. Bull. Str. Settl. 3, 1925, 303-458).-List.

- \& R. E. HoltTum, A Botanical Reconnaissance upon the Main Range of the Peninsula at Fraser Hill (ibid. 3, 1923, 19-110).-Ditto.

CORNER, E. J. H., Notes on the systematy and distribution of Malayan Phanerogams I-IV (Gard. Bull. Str. Settl. 10, 1939, 1-81; ibid. 239-329; ibid. 11, 1941, 177-235).-Critical consideration of various genera.

-, Wayside Trees of Malaya. Singapore (1940) 2 vols; text i-vii, 1-770, fig. 1-259; Atlas t. 1-228; 2nd ed. (1952) (very slightly revised).Descr. and keys!

CURTIS, C., A catalogue of the flowering plants and ferns found growing wild in the Island of Penang (J. Str. Br. Roy. As .Soc. no 25, 1894, 67-169).Pages 163-169 are occupied by an appendix by RIDLEY on the Botanists of Penang. Non vidi.

Foxworthy, F. W., Commercial timber trees of the Malay Peninsula (Mal. For. Records 3, 1927, 1-195, map, many plates).-Key to principal trees by bark \& wood characters; to each sp. a descr. and one or more plates (photographs).

GRAY, A., Botany, Phanerogamia (in U.S. Exploring Expedition during the years 1838, 1839, 1840, 1841,1842 , under the command of Charles Wilkes, U.S.N., 1854, 1-777, atlas, 1857, t. 1-100).-Listed records and descr.
Griffith, W., Notulae ad Plantas Asiaticas (Posthumous Papers), arranged by J. M.'Clelland, 1 (1847) 1-256; 2 (1849) 257-628, i-viii; 3 (1851) 1-436, i-xii; 4 (1854) i-xli, 1-764.-Contains numerous records, notes and descr. of new genera and spp. from the Malay Peninsula. To this pertains the Atlas of next item.

-, Icones Plantarum Asiaticarum 1 (1847) i-iji, t. 1-62; 2 (1849) i-viii, t. 1-138; 3 (1851) i-v, t. $139-359 ; 4$ (1854) i-xii, t. $360-561$.

-, Some account of the Botanical Collection, brought from the eastward, in 1841 (J. As. Soc. Bengal 23, 1855, 623-650, t. 1-4). Also account of Mal. Pen. coll. p. 631.-Non vidi.

HENDERSON, M. R., On a collection of plants from Gunong Benom, Pahang (J. Fed. Mal. Stat. Mus. 13, 1927, 217-227).-Listed records and new spp.

-, A list of plants from Cameron's Highlands, Pahang (J. Mal. Br. R. As. Soc. 5, 1927, 237277).-Ditto.

-, Additions to the Flora of the Malay Peninsula (Gard. Bull. Str. Settl. 4, 1927, 48-56; ibid. (1929) 411-414; ibid. 5, 1930, 72-80, 92-98;ibid. 7, 1933, 87-128).-Records and descr. of new spp.

-, The Flowering Plants of Kuala Lumpur (Gard. Bull. Str. Settl. 4, 1928, 211-373).-List of spp. with notes on their distribution.

-, New species from the Malay Peninsula (ibid. 5, 1930, 72-80, 5 fig.).-Descr. some new spp.

-, Notes on the Flora of $P$. Tioman and neighbouring islands (ibid. 5, 1930, 80-93).-List.

-, List of additions to the flora of the Malay Peninsula (ibid. 5, 1930, 93-98).-List of additions published since 1925 (last vol. of RIDLEY's Flora).

-, The Flora of the Limestone Hills of the Malay Peninsula (J. Mal. Br. R. As. Soc. 17, 1939, 13-87).-List; distr.

-, Malayan wild flowers. 1. Dicotyledons (Malayan Nature Journ. 4, 1949, 1-181; ibid. 6, 1950, 182-399; ibid. 1952, 400-472, fig. 1-424); 2. Monocotyledons. Kuala Lumpur (1954) 1-357, fig. 1-201.-Descr. \& keys of selected herbaceous plants from the Malay Peninsula.

Holrtum, R. E., New species of vascular plants from the Malay Peninsula (Gard. Bull. Sing. 11, 1947, 267-298).-Descr. of some Pteridophytes \& Monocotyledonous spp.

HOOKER, J. D., Illustrations of the Floras of the Malayan Archipelago and of tropical Africa (Trans. Linn. Soc. Lond. 23, 1860, 155-172, t. 20-28).-Descr. various new genera and spp.

-, Catalogue of the plants distributed at the Roy. Gard. Kew... from the Herbaria of Griffith, Falconer and Helfer. London (1865) iii-iv + $58 \mathrm{pp}$.-List of plant names.

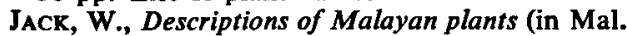
Miscell. 1 (1), 1820, 1-27; 1 (5), 1821, 1-41; 2 (7), 1822, i-iii, 1-96). Repr. by HoOKer (in his J. Bot. 1, 1834, 358-380; Comp. Bot. Mag. 1, 1835, 147-157, 219-224, 253-272). Again reprinted at Calcutta (with addition of data in Calc. J. Nat. Hist. 4, 1843, 1-62, 159-231, 
$305-374$, t. 14-16; repr. 1-230).-Descr. of new genera and spp.-For an evaluation $c f$. MERRILL, J. Arn. Arb. 33 (1952) 199-250.

King, G. \& J. S. GAMble, Materials for a Flora of the Malay Peninsula (J. As. Soc. Beng. 18891936, repr. 5 vols).-Standard Flora with keys and excellent descr. not wholly complete for all families.-For Monocotyledonous plants . $\boldsymbol{c f}$. RIDLEY (1907).

Merrill, E. D., Additions and corrections of Ridley's Flora of the Malay Peninsula (Gard. Bull. Str. Settl. 8, 1935, 131-134).-Additional records, mostly alien weeds; some nomenclatorial notes.

Narayanaswam, V., Provenance of early Malayan plant collections (J. \& Proc. As. Soc. Beng. n.s. $27,1931,327-477,1933)$.-Exact localities of the sheets collected by KUNSTLER, WRAY, and SCORTECHINI.

Planchon, J. E., Catalogue of Malayan plants collected by Thom. Lobb, sets of which have been announced for sale, etc. (in Hook. Lond. J. Bot. $6,1847,469-473)$.-Merely a list of preliminary names on p. $472-473$.

RiDley, H. N., in Kelsall, Notes on a trip to Bukit Etam, Selangor (J. Str. Br. Roy. As. Soc. no 23, 1891, 67-75).-List of plant names; no novelties.

-, On the Flora of the Eastern Coast of the Malay Peninsula (Trans. Linn. Soc. Lond. II, 3, 1893, 267-408, t. 61-66).-Account of his collections from E. Pahang, Kelantan, Trengganu, and $P$. Tioman. Records and descr. of new spp.

-, A botanical excursion to Gunong Jerai (Kedah Peak) (J. Str. Br. Roy. As. Soc. no 34, 1900, 23-30).-Non vidi.

-, The Flora of Singapore (J. Str. Br. R. As. Soc. no 33, 1900, 27-196; ibid. no 35, 1901, 84-90). -List.

-, The Flora of Mt Ophir (ibid. no 35, 1901, 1-28). -Ditto.

- New or rare Malayan plants' I-XII (ibid. nos 41, $44,49,50,54,61,68,73,75,79,82,86,1904$ 1922).-Important series of new records, new genera and spp.

-, Materials for a Flora of the Malay Peninsula. Monocotyledons. 3 vols, Singapore (1907).Revision of the Monocotyledons; descr.; keys.

-, On a collection of plants made by $C$. H. Robinson and $L$. Wray from G. Tahan, Pahang (J. Linn. Soc. Lond. Bot. 38, 1908, 301-336).Records and new spp.

An extract of this appeared under the same title in J. Fed. Mal. St. Mus. $2^{3}, 1907,107-142$. This contains also the descr. of new spp. with reference to their pages in the latter paper (which may have been accomplished from proof sheets); at least the reprints are dated 1907.

- The Flora of the Telom and Batang Padang Valleys (J. Fed. Mal. St. Mus. 4, 1903, 1-98).Records and descr. of new spp.

-, A Scientific Expedition to Temengoh, Upper Perak (J. Str. Br. R. As. Soc. no 57, 1910, 5-122).-Ditto.
-, A. Botanical Excursion to Pulau Adang (ibid. 61, 1912, 45-65).-Ditto.

- An Expedition to Mount Menuang Gasing, Selangor, etc. (J. Linn. Soc. Lond. Bot. 41, 1913, 285-304).-Ditto.

_, On a collection of plants from G. Mengkuang Lebah, Selangor (J. Fed. Mal. St. Mus. 5, 1914, 28-50).-Ditto.

-, An Expedition to Mt Menuang Gasing, Selangor (ibid. 6, 1915, 1-21).-Ditto.

-, Plants from G. Kerbau, Perak (ibid. 6, 1915, 43-62).-Ditto.

-, The Botany of G. Tahan (ibid. 6, 1915, 127-202). -Ditto.

-, The natural history of Kedah Peak. Botany (ibid. 7, 1916, 37-58).-Non vidi.

-, New and rare plants from the Malay Peninsula (ibid. 10, 1920, 128-156).-Ditto.

-, New Malayan plants (J. Bot. 58, 1920, 147 149).-Ditto.

- The Flora of Klang Gates, Selangor (J. Fed. Mal. St. Mus. 10, 1922, 247-251),-Records and descr. new spp.

-, The Flora of the Malay Peninsula. 5 vols, London (1922-1925).-Complete Flora with keys; illustrated.

-, New Malayan plants (J. Bot. 62, 1924, 294 301).-New records \& spp.

-, Additions to the Flora of Malaya (Kew Bull. 1926, 469-479).-New spp. from the Malay Peninsula and Borneo.

\section{Java}

BACKer, C. A., Flora van Batavia. Batavia (1907) $25+x v i+405$ pp.-The first vol. of a planned work; contains only a small part of the Choripetalae: Ranunculaceae-Moringaceae. Keys to genera and spp.; no new spp.; in Dutch.

- Voorlooper eener Schoolflora voor Java. Batavia (1908) $1 x x x+112+8$ pp.-Concise Flora with keys to genera and spp.; contains part of the Choripetalae: Ranunculaceae-Leguminosae; in Dutch.

- Plantes exotiques naturalisées dans Java (Ann. Jard. Bot. Btzg Suppl. 3, 1909, 393-420).List of introduced plants either cultivated or aliens; in French.

- Schoolflora voor Java. Weltevreden (1911) clxv + 676 pp.-Concise Flora with keys to genera and spp.; contains part of the Choripetalae: Ranunculaceae-Myrtaceae; in Dutch.

-, On some results of the botanical investigation of Java (1911-1913) (Bull. Jard. Bot. Btzg II, no 12, 1913, 1-40).-List of records.

- Handboek voor de Flora van Java. 3 parts. Batavia (1924-1928) $66+291+141$ pp.Unfinished ample Flora, containing only revisions of some families of the Gymnosperms and Fern-allies but mostly treating monocotyledonous families (Liliiflorae, Gramineae, etc.); keys; in Dutch.

-, Onkruidflora der Javasche suikerrietgronden (Weed Flora of Javan sugar-cane fields). Soerabaja (1928-1934) 2 vols, text lxxxvii + 
907 pp.; Atlas t. 1-480 (unfinished).-Ample descr.; keys; in Dutch.

-, A revision of Kuntze's types of new Javan species (Brittonia 3, 1938, 75-90).-Evaluation of new spp. described by O. KunTzE from Java. -, c.s., Notes on the Flora of Java I (Bull. Jard. Bot. Btzg III, 16, 1939, 107-110); ditto II (Blumea 5, 1945, 490-524); ditto III (ibid. 6, 1948, 302-309); ditto IV (ibid. 310-336); ditto V (ibid. 1950, 358-362); ditto VI (ibid. 363-406). -Precursory papers to the book of next entry containing new records, synon., new comb., genera and spp.

-, c.s., Beknopte Flora van Java (nooduitgave). Parts 1-13, Leyden (1940-1953).-Mimeographed emergency edition of a complete concise Flora of Java; complete except for the Gramineae, Zingiberaceae, Araceae, Marantaceae; Rubiaceae in print; in Dutch. An English translation is in preparation.

- \& D. F. VAN SLooten, Geillustreerd Handboek der Javaansche Theeonkruiden (Illustrated Handbook of the Weed Flora of Javan tea plantations). Batavia (1924) $47+247$ pp.-Descr.; keys; in Dutch.

BenNetT, J. J. \& R. BRowN, Plantae Javanicae Rariores descriptae iconibus illustratae, etc. (1838-1852) viii, xvi, 1-259, t. 1-50.-Elaboration of HoRSFIELD's coll. in Java 1802-1818; in Latin; comments in English.

Blume, C. L., Enumeratio Plantarum Javae et insularum adjacentium, etc. Leyden 2 fasc. (1827-1828) i-vi, i-x, 1-274; 2nd literatim ed. (1830).-List.

_, Flora Javae. 3 vols (1828-1851); ser. 2 vol. 1 (1858) Orchidaceae; c. 1863, Suppl. only consisting of plates ( $p l$. ined.).-Monogr. treatments; in Latin.

Boldingh, I., Zakflora voor de Landbouwstreken op Java. Batavia (1916) i-xvii, 1-204.-Flora of weeds of agricultural fields in Java, the whole book consisting of one large key. It contains some new comb. which have been considered by Merrill (Brittonia 5, 1948, 25-32).

Cholsy, J. D., $c f$. Chapter 6a.

DoCters VAN LeEUWEN, W. M., Krakatau 1883 1893 (Ann. Jard. Bot. Btzg 46/47, 1936, xii + 506, t. 1-37).-Listed records; in English.

HASSKARL, J. K., Plantarum genera et species novae aut reformatae Javenses (Flora 252, 1842 , Beibl. no 1, 1-114).-Misc. records and descr.; in Latin.

-, Adnotationes de plantis quibusdam Javanicis nonnullisque Jqponicis, haud rite cognitis $e$ Catalogo Horti Bogoriensis excerptae. Accedunt nonnullae novae species (in HOEVEN \& DE VRIESE, Tijd. Nat. Geschied. \& Phys. 10, 1843, 115150).-List of new records and descr. new spp. occurring in the Catalogue of 1844 (see Chapter 6a).

-, Adnotationes de plantis horti Bogoriensis (ibid. 12, 1845, 77-139).-Continuation of the latter item.

-, Plantarum Javanicarum aut novarum aut minus cognitarum adumbrationes (Flora 272, 1844,
583-595, 599-609, 615-626; ibid. 28, 1845, 225-237, 241-251, 292-302, sphalm. 260-269).Ditto; in Latin.

-, Plantae Javanicae Rariores adjectis nonnullis exoticis, in Javae horti cultis. Berlin (1848) i-viii, 1-555 pp.-Ditto; including a number of non-Malaysian species cultivated in the Botanic Gardens at Bogor; in Latin.

Hildebrand, F. H., Report For. Res. Institute, Bogor, no 35 (1950) 171 pp.-List of tree spp. of the islands of Java and Madura. Revised edition, ibid. no 50 (1951) 183 pp.

Hochreutiner, B. P. G., Plantae Hochreutineranae (Ann. Cons. \& Jard. Bot. Genève 15/16, 1912, 145-247; Candollea 2, 1925, 317-513; ibid. 5, 1934, 175-341; ibid. 6, 1934-1936, 399-488; ibid. 8, 1940, 47-60; ibid. 9, 1943, 481-493).-Account of his collections; besides Javanese plants also those from other parts of the world.

JUNGHUhN, F., Bibliography on the Flora of Java. See Chapter 2.

KOORDERS, S. H., Exkursionsflora von Java. 3 vols, Jena (1911-1912) + 1 vol. atlas (19131937).-Atlas not yet completed; keys; in German.

- Atlas der Baumarten von Java. 4 vols, Leyden (1913-1918) t. 1-800.-An illustrated account of most spp. treated in the 'Bijdragen' by KOORDERS \& VALETON, see below.

-, Flora von Tjibodas. Batavia-Buitenzorg. 3 vols (1918-1923) $123+246+139+87+31$ pp.Complete local Flora of Mt Gedeh, W. Java; keys; in German.

- \& Th. Valeton, Bijdragen tot de kennis der Boomsoorten van Java. Batavia (1894-1914) 13 vols.-Issued in the series 'Mededeelingen van 's-Lands Plantentuin te Buitenzorg'; descr. ligneous Jav. spp. in Latin and Dutch; some family revisions are provided with keys in Dutch.

KUNTZE, O., Revisio Generum Plantarum. vol. 1-2 (1891) 1011 pp.; vol. 31 (1893) cccexii pp.; vol. $3^{2}(1898) \mathrm{vi}+201+576$ pp.-Contains descr. of new spp. from his Java tour.

MORITZI, A., Systematisches Verzeichnis der von H. Zollinger in den Jahren 1842-1844 auf Java gesammelten etc. Solothurn (1845-1846) xii + 144 pp.-List ZoLLINGER coll. with occasional new spp.

NoRoÑA, F., Relatio plantarum Javanensium iterfactione usque in Bandong recognitarum (Verh. Bat. Genootsch. K.\& W. 5, 1827, 64-86).-List of Latin and vernacular plant names. $A$ great number of new genera and new species, all nomina nuda. An evaluation on the basis of the vernacular names is given by J. K. HassKarL (in Hoeven \& de Vriese, Tijd. Nat. Geschied. \& Phys. 11, 1844, 209-228).

Planchon, J. E., Catalogue of the first series of plants of Java collected by Thom. Lobb, etc. (in Hook. Lond. J. Bot. 5, 1846, 246-250; 6 (1847) 469-471.-Merely a list of names of preliminary identifications.

RENDle, A. B., c.s., Dr H. O. Forbes's Malayan Plants (J. Bot. Suppl. 1924-1926, 149 pp.).- 
List ForBes's coll. with occasional new genera and species.

Steenis, C. G. G. J. Van, Die Pteridophyten und Phanerogamen der Deutschen Limnologischen Sunda Expedition (Arch. f. Hydrobiol. Suppl. vol. 11, 1932, 231-387).-Account of coll. RUTTNER, mostly water- and marsh-plants; list. - Account of Javan plants collected by $C$. F. Hornstedt (Act. Hort. Berg. 15: 2, 1949, 39-43, fig. 1).-List of coll. HoRnSTEDT; no novelties or new comb.

-, C. A. Backer \& R. C. Bakhuizen van den BRINK Jr, Identification of the new species and combinations proposed by $C$. P. Thunberg in the Florula Javanica by L. Winberg \& F. O. Widmark (1825) (Blumea 6, 1950, 358-362).Nomencl. evaluation; 3 new comb.

-, G. den Hoed, S. Bloembergen \& P. J. EyMA, Flora voor de Scholen in Indonesië. Batavia (1949) 407 pp. 46 fig.; 2nd ed. (1951).-Flora containing only well-known, large-flowered lowland plants, native as well as cultivated, intended only for secondary schools; keys; in Dutch.

-, M. J. van Steenis-Kruseman \& C. A. Backer, Louis Auguste Deschamps (Bull. Brit. Mus. (Nat. Hist.), hist. ser. 1, 1954, 51-68, t. 13).List of 270 spp. from Java.

Teysmann, J. E. \& S. BinnendiJk, Plantae novae Horti Bogoriensis in insula Java (Ned. Kruidk. Arch. 3, 1855, 391-413).-Descr. miscellaneous new spp. from Malaysia.

Thunberg, C. (ed. L. Winberg \& F. O. Widmark) Florula Javanica. Upsala (1825) 23 pp. (cf. also Blumea 6, 1950, 358-362).-List THUNBERG coll.; list with two dozen new spp.

VRIESE, W. H. DE, Plantarum javanicarum minus cognitarum vel novarum, etc. (Tijd. Nat. Geschied. \& Phys. 11, 1844, 336-347).-Descr. of some Jav. plants introduced in the Amsterdam Botanic Garden; a few new spp.

ZollnNGer, H., $c f$. under Moritzi and Chapter 6 .

\section{Christmas Island}

BAKER, E. G., in ANDREWS, $A$ monograph of Christmas Island. London (1900) 171-200, t. 17-18.-Plant list.

HeMSLEY, W. B., Report on the botanical collections from Christmas Island, Indian Ocean, etc. (J. Linn. Soc. Lond. Bot. 25, 1890, 351-362).List; a few new spp.

RIDley, H. N., An Expedition to Christmas Island (J. Str. R. As. Soc. no 45, 1906, 170-271; addit. note l.c. 48, 1907, 107-108).-Contains a plant list with descr. new spp.

\section{Borneo}

Diels, L. \& $\dot{\mathbf{K}}$. G. HaCkenberG, Beiträge zur Vegetationskunde und Floristik von Süd-Borneo (Bot. Jahrb. 60, 1926, 291-316).-Vegetation; records and new spp. coll. HACKENBERG.

(1) Including also the Anambas \& Natuna Islands, S. China Sea.
FISCHER, C. E. C., Contributions towards a Flora of British North Borneo I-IV (Kew Bull. 19321934).-Lists of records \& new spp.

GibBs, L. S., c.s., $A$ contribution to the flora and plant formations of $M t$ Kinabalu, etc. (J. Linn. Soc. Bot. 42, 1914, 1-240, t. 1-8).-Records and new spp. coll. GibBs.

HaLlier $f$., H., Beiträge zur Flora von Borneo (Beih. Bot. Centralbl. 34, ii, 1916, 19-53).Records and descr.

HeINe, H., Diagnoses novae plantarum in Borneo septentrionali a J. et $M$. S. Clemens lectarum (Mitt. Bot. Staatssamml. München 12, 1950, 5761).-Descr. some new spp. from Mt Kinabalu. -, ditto II (ibid. 16, 1953, 208-230).-Ditto.

-, Pflanzen der Sammlung J. \& M. S. Clemens von $M t$ Kinabalu in Britisch Nord-Borneo (in FEDDE, Rep. 54, 1951, 223-248).

- Pflanzen der Sammlung J. \& M. S. Clemens vom Mount Kinabalu in Britisch Nord-Borneo. Thesis, München (1953), mimeographed 1-118. Identifications of an incomplete set of the Clemens Kinabalu coll. 1931-1933; many erroneous identifications.

Henderson, M. R., The 'Padang' fiora of Jemaja, in the Anamba Islands, N. E. I. (Gard. Bull. Str. Settl. 5, 1931, 234-240).-Plant list of a sandy heath.

Hildebrand, F. H., List of tree names issued by For. Res. Institute, Bogor, no 7 (1941).-Mimeographed list of tree spp. from Sampit Subdivision, South Borneo.

-, ditto, no 9 (1941).-Ditto from Bulungan \& Berau, East Indonesian Borneo.

-, ditto, no 55 (1952).-Revised edition of the latter.

-, ditto, no 10 (1941).-Ditto from West Indonesian Borneo.

_, ditto, no 54 (1952) 80 pp.-Revised edition of the latter.

-, Report For. Res. Institute, Bogor, no 2 (1949) 76 pp.-Ditto from Samarinda Division, East Borneo.

-, ditto, no 58 (1952) 105 pp.-Revised edition of the latter.

-, ditto, no 3 (1949) 61 pp.-Ditto from KapuasBarito Division, South Borneo.

-, ditto, no 62 (1953) 77 pp.-Revised edition of the latter.

-, ditto, no 5 (1949) 48 pp.-Ditto from Bandjermasin \& Hulu Sungei Divisions, Southeast Borneo.

-, ditto, no 63 (1953) 62 pp.-Revised edition of the latter.

HoOKER, J. D., Illustrations of the Floras of the Malayan Archipelago and of tropical Africa (Trans. Linn. Soc. Lond. 23, 1860, 155172, t. 20-28).-Descr. various new genera and spp.

IRMSCHER, E., c.s., Beiträge zur Kenntnis der Flora von Borneo (Mitt. Inst. Allg. Bot. Hamburg 7, 1927-1937, 1-310, 10 tab.).-Listed account of HANS WINKLER collection 1925; unfinished.

KeITH, H. G., A preliminary list of North Borneo plant names (North Borneo For. Rec. no 2, 
1938, 256 pp.; 2nd ed. Hong Kong 1952, 528 pp.).-Check list of vernacular names.

Masamune, G., Enumeratio Phanerogamarum Bornearum. Taihoku (1942) 1-739.-Bibliographic enumeration.

MerRILL, E. D., Bibliography of Botany. See Chapter 2.

-, Notes on the Flora of Borneo (Philip. J. Sc. 11, 1916, Bot. 49-100).-Records and descr. new spp.

-, Alabastra Borneensia (J. Str. Br. R. As. Soc. no $77,1917,189-247)$.-Descr. new spp. from a number of families; material from various sources.

-, Contributions to our knowledge of the Flora of Borneo (J. Str. Br. R. As. Soc. no 76, 1917, 75117).-Ditto.

-, New species of Bornean Plants (Philip. J. Sc. 13, 1918, Bot. 67-122).-Ditto.

- A Bibliographic Enumeration of Bornean Plants (J. Str. Br. R. As. Soc., special no, 1921, 1-637).-Accurate bibliographic list of spp. known from the island, with coll. numbers.

-, Additions to our knowledge of the Bornean Flora I-II (Philip. J. Sc. 21, 1922, 515-534; ibid. 30, 1926, 79-87).-Records and descr. new spp.

- New or noteworthy Bornean plants I-III (J. Str. Br. R. As. Soc. no 85, 1922, 151-201; ibid. no 86, 1922, 312-342; ibid. no 87, 1923, 22-45).1-Descr. new spp.

-, The Flora of Banguey Island (Philip. J. Sc. $29,1926,341-427$; cf. also ibid. 24, 1924, $113-$ 116).-List with occasional new spp.

- A collection of plants from Sarawak (Sarawak Mus. J. 3, 1928, 513-557).-Records \& new spp.; middle part of p. 537 belongs on p. 534; $R u b$. from p. 534-537 belong halfway p. 555 .

-, Plantae Elmerianae Borneenses (Univ. Cal. Publ. Bot. 15, 1929, 1-316).-Records \& descr. new spp. coll. ELMER.

Miquel, F. A. W., Analecta Botanica Indica. Pars 1: Stirpes quaedam borneenses (Verh. le K1. Kon.-Ned. Inst. Amsterdam ser. 3, vol. $3,1850,1-30$, t. 1-10).-List of misc. records and new spp. from various sources.

Ridley, H. N., ${ }^{2}$ Plants collected at Penrissen (J. Str. Br. R. As. Soc. no. 33, 1900, 22-24).Small list of records; 2 new spp.

- Some plants collected on Mr. Moulton's Expedition to Batu Lawi (J. Str. Br. R. As. Soc. no 63, 1912, 59-62).-List; descr. some new spp.

- New Bornean plants (Sarawak Mus. J. 1, 1912, 30-38).-Ditto.

-, Contributions to a Flora of Borneo (ibid. 1, 1913, 67-98).-Miscellaneous descr. of new genera \& spp. from various sources.

-, Additions to the Flora of Malaya (Kew Bull. 1926, 469-479).-Descr. new spp.

(1) The third contribution is simultaneously cited: 'J. Roy. As. Soc. Mal. Br. vol. 1'.

(2) Numerous papers by RIDLEY on special groups in Borneo have been inserted in Chapter 6c.
- New species from the Malay Peninsula and Borneo (Kew、Bull. 1929, 254-262).-Descr. new spp.

-, New plants from Sarawak (Sarawak Mus. J. $1,1931,31-52)$.

- \& H. K. AIRY Shaw, ${ }^{1}$ c.s., Additions to the Flora of Borneo and other Malay Islands I-XXII (Kew Bull. 1930-1950).-Valuable series of contributions; records and descr. of new gen. \& spp.

Smith, W. W., Species Borneenses (Not. R. Bot. Gard. Edinb. 8, 1915, 313-348).-Descr. new spp.

StaPf, O., On the flora of Mount Kinabalu, in North Borneo (Trans. Linn. Soc. Lond. II, Bot. 4, 1894, 69-263, t. 11-20).-Basic records; descr. new genera \& spp.

SteEnis, C. G. G. J. VAN, Botanical results of a trip to the Anambas and Natoena Islands (Bull. Jard. Bot. Btzg III, 12, 1932, 151-211, fig. 1-11). -Lists with records; a few new spp.

-, Some remarks on the Kinabalu collection of Chaplain J. \& M. S. Clemens (J. Bot. 72, 1934, 1-12).-List of records \& new spp.

Warburg, O., Plantae novae borneensis (in Fedde, Rep. 18, 1922, 327-330).-Descr. new spp.

Winkler, Hub., c.s., Beiträge zur Kenntnis der Flora und Pflanzengeographie von Borneo (Bot. Jahrb. 44, 1910, 497-571; ibid. 48, 1912, 87-118; ibid 49, 1913, 349-380; ibid. 50, Suppl. 1914, 188-208, t. 3-5).-Records \& new spp. coll. HUb. WINKLER in SE. Borneo.

\section{Lesser Sunda Islands}

Bloembergen, S., Verslag van een exploratie-tocht naar de eilanden Timor en Wetar (Tectona 33, 1940, 101-196).-Report of exploration trip to Timor and Wetar, incl. plant lists; Engl. summary.

Britten, J., W. Fawcett \& H. N. Ridley, c.s., Prodromus Florae Timorensis; etc., in $\mathrm{H}$. $\mathbf{O}$. FORBEs, A Naturalist's Wanderings, etc. London (1885) App. VI, 497-523.-List of names, sometimes with reference to numbers; some new spp.

Decaisne, J., Description d'un herbier de l'tle de Timor (Nouv. Ann. Mus. Paris 3, 1834, 333-501). Also separately issued, under the title 'Herbarii Timoriensis descriptio' Paris (1835) 1-173.

ENGler, A., c.s., Uebersicht über die botanischen Ergebnisse der Gazelle-Expedition (Bot. Jahrb. 7, 1886, 444-480).-Cyperaceae, Gramineae, Orchidaceae were published in op. cit. 5 (1884) 89-94; 6 (1885) 233-248; 7 (1885) 435-443.List of records; also new spp.; Timor.

Hallier, H., Botanische Ergebnisse der Elbert' sche Sunda-Expedition (Meded. Rijksherb. Leiden no 14, 1912, 1-42; ibid. 22, 1914, 1-20; ibid. 37, 1918, 1-92).-Lists of plants collected by Elbert \& Gründler; not finished.

Hildebrand, F. H., List of tree names, ed. by the For. Res. Inst. Bogor, no 2 (1940).-List of tree spp. from the islands of Bali and Lombok.

(1) The later contributions are by Mr SHAw only. 
-, ditto, no 3 (1940).-Ditto of Lesser Sunda Islands, excl. Bali \& Lombok.

-, ditto, no 60 (1953) 51 pp.-Ditto of Timor.

MALM, J. voN, Die Phanerogamenflora der Kleinen Sunda-Inseln und ihre Beziehungen (in FEDDE, Rep. 34, 1934, 255-307).-List of records from the RENSCH coll.

-, Einige bemerkenswerte Arten der Steinschen Sammlung aus Timor (in FEDDE, Rep. 41, 1937, 295-296).-Some records and a new $s p$.

MeIjer Drees, E., Distribution, ecology and silvicultural possibilities of the trees and shrubs from the savanna-forest region in Eastern Sumbawa and Timor (Report For. Res. Inst. Bogor, no 33, 1950, 1-145).-List of tree \& shrub spp. from the islands of Timor and $\mathbf{E}$. Sumbawa, with short descr.; ecol. and distributional data.

-, List of tree and shrub names from Timor (Report For. Res. Inst. Bogor no 33, 1950, mimeogr. 1-61).-Name index coll. For. Res. Inst.

MoORE, S., E. G. BAKER, H. N. RidleY, c.s., Dr H. O. Forbes's Malayan Plants (J. Bot. 62-64, Suppl. 1924-1926, 1-149).-Contains some data on spp. from Timor.

Spanoghe, J. B., Catalogue of plants found on Timor and the neighbouring islands (in Hook. Comp. Bot. Mag. 1, 1835, 344-351).-List of plant names.

-Prodromus Florae Timorensis (Linnaea 15, 1841, 161-208, 314-350, 476-480).-Records and many new spp.

Steenis, C. G. G. J. Van, On the Origin of the Malaysian Mountain Flora III (Bull. Jard. Bot. Btzg III, 14, 1936, 58-62).-List of temperate mountain plants from the Lesser Sunda Islands and their distribution.

Zollinger, H., Opgave der planten gezien ... op het eiland Balie (Nat. \& Geneesk. Arch. Ned. Ind. 2, 1845, 588-595).-List of names only; no numbers or references.

\section{Celebes}

Bloembergen, S., Verslag van een exploratie-tocht naar Midden-Celebes (Tectona 33, 1940, 377418).-Lake Lindu \& Mt Ngilalaki; incl. lists of trees collected.

DOCTERS VAN LEEUWEN, W. M., Botanical results of a trip to the Salajar Islands (Blumea 2, 1937, 239-277; ibid. 3, 1939, 236-237).-List of plant names.

Hemsley, W. B., c.s., Decades Kewenses XXVI$X X V I I$ (Kew Bull. 1896, 36-42).-Plants of Mt Bonthain, SW. Celebes, coll. EVERETT.

Hildebrand, F. H., Report For. Res. Inst. Bogor, no 43 (1950) 105 pp.-List of tree spp. from South Celebes.

-, ditto, no 44 (1951) 53 pp.-Ditto of North Celebes.

KawaKami, T., On some Celebes plants (Bot. Mag. Tokyo, 26, 1912, 49-50).-A few records listed.

KOORDERS, S. H., Enumeratio specierum phanerogamarum Minahassae, in 'Verslag eener botanische dienstreis door de Minahassa' (Meded. 's-Lands Plantentuin, Buitenzorg, no 19, 1898,
253-645).-List of records and new spp. from NE. Celebes from the KoORDERs coll.

Some minor corrections on this book were incorporated in 3 articles by its author (in Natuurk. Tijd. Ned. Ind. 61, 1902, 250-261; ibid. 63, $1903,76-89,90-99)$, the 1st in Dutch, the others in German.

-, Supplement op het eerste overzicht der Flora van N. O. Celebes, vol. 1, \$ 1, Batavia (1918) 1-30; $\$$ 2, Bull. Jard. Bot. Btzg III, 2 (1920) 245-260; vol. $2, \S 1$ (1922) $9+127$ pl.; vol. 3 (1922) 1-60. -Descr. and plates of new records and spp.

KOORDERS-SCHUMACHER, A., Systematisches Verzeichnis der zum Herbar Koorders, etc. III. Abt., Celebes (1914) 7-141.-NE. Celebes; list KOORDERs numbers.

LAM, H. J., c.s., Contributions to our knowledge of the Flora of Celebes (coll. C. Monod de Froideville) and of some other Malaysian islands (Blumea 5, 1945, 554-599).

Oliver, D., Note on a collection of plants made by Mr Riedel of Gorontalo (J. Linn. Soc. Lond. Bot. 15, 1877, 97-100).-N. Celebes, coll. A. B. MEYER; descr. miscellaneous new spp.

Richard, A., in Dumont d'URVille, Voyage de découvertes de L'Astrolabe, etc. vol. 2. Sertum Astrolabianum. Paris (1834) i-lvi, 1-167; At las (1833) t. 1-39.-Records and descr. new spp. Non vidi.

\section{Philippines}

Ahern, G. P., Compilation of notes on the most important timber tree species of the Philippine Islands (1901) 1-112, t. (col.) 1-43.-Descr. plates.

ANONYMOUS, Bibliographies of the Philippines. See Chapter 2.

Blanco, M., Flora de Filipinas, etc. (1837) i-lxxviii, 1-887; ed. 2 (1845) i-lix, 1-619; ed. 3, directed by Naves \& FERnANDEZ-VILLAR, 1 (1877) i-xxx, 1-350, index, 2 (1878-79) 1-419, index i-viii, 3 (1879) 1-271, index i-vi, $4^{1}$ (1880) i-xvii, $1-108,4^{2}$ (1880) i-vi, 1-63, $4^{3}$ (1880-83) i-ix, 1-375, t. 1-473:

For dates of publication $c f$. Merrill, E. D., Philip. J. Sc. 12 (1917) Bot. 113-116.

For an evaluation of the works, see Chapter 3.

Brown, W. H., Minor products of Philippine forests (Philip. Forestry Bull. 22; 3 vols, Manila 1920-1921).-Illustrated account of useful plants with descr. arranged according to uses; not a phytographic work.

-, Useful Plants of the Philippines (Commonwealth of the Philippines, Dep. Agric. \& Commerce, Manila, Techn. Bull. no 10, 3 vols, 1950: 1-590, fig. 1-253; 1-513, fig. 1-239; 1-507, fig. 1-170).-Useful plants systematically arranged in families; descr. of spp.; in some genera also keys. Photo-lithographed in Australia.

Elmer, A. D. E., c.s., Leaftets of Philippine Botany, vol. 1-10 (1906-1939).-Accounts of records and new spp.; extracted for Chapter 6c.

Gray, A., Botany, Phanerogamia (in U.S. Exploring Expedition during the years 1838, 1839, 
$1840,1841,1842$, under the command of Charles Wilkes, U.S.N., 1854, 1-777, Atlas, 1857, t. 1-100).-Listed records and descr.

KANEHIRA, R., A list of plants collected at Davao, P.I. (Bult. Sci. Fak. Terkult. Kjuŝu Imp. Univ. 7, 1936, 228-238).-List of vascular plants coll. KaneHIRA in Mindanao; text in Japanese. Non vidi.

Merrill, E. D., Plantae Ahernianae, in Report on investigations made in Java in the year 1902 (Philip. Bur. For. Bull. 1, 1903, 15-55).-List of AHERN coll.; records and some new spp.

-, Notes on Cuming's Philippine plants in the Herbarium of the Bureau of Government Laboratories (Govt Lab. Publ. (Philip.) no 35, 1905, 69-77).-Evaluation of a number of spp.; descr. of some new spp.

-, New or noteworthy Philippine plants I-IV. (Govt Lab. Publ. Philip. 6, 17, 29, 35, 1904 1905); ditto $V-X V I I$ (Philip. J. Sc. 1-20, 19061922).-Records; descr. new genera and spp.

-, The flora of the Lamao Forest Reserve (Philip. J. Sc. 1, 1906, Suppl. 1-141).-List; also new spp.

-, Index to Philippine literature. See Chapter 2.

-, Some genera and species new to the Philippine Flora (ibid. 2, 1907, Bot. 421-428).-Misc. records and new spp.

-, The Flora of Mt Halcon, Mindoro (ibid. 2, 1907, Bot. 251-309).-List of records and new spp.

-,The Philippine plants collected by the Wilkes United States Exploring Expedition (ibid. 3, 1908, Bot. 73-84, t. 1-4).-Evaluation.

- New Philippine plants from the collections of M.S. Clemens I (ibid. 3, 1908, Bot. 129-165). -Descr. of new genera and spp.

-, On a collection of plants from the Batanes and Babuyanes Islands (ibid. 3, 1908, Bot. 385-442).-List of records and new spp.

A Flora of Manila. Manila (1912) 1-490.Flora with keys.

- Nomenclatural and systematic notes on the Flora of Manila (Philip. J. Sc. 7, 1912, Bot. 227-251).-Records; some new names, new spp. and comb.

-. Sertulum Bontocense: new or interesting plants collected in Bontoc Subprovince, Luzon, by Father Morice Vanoverbergh I (ibid. 7, 1912, Bot. 71-107); ditto II (ibid. 9, 1914, Bot. 443459).-Lists of records and new spp.

-, Plantae Wenzelianae (ibid. 8, 1913, Bot. 363390); ditto II (ibid. 9, 1914, Bot. 353-389); ditto III (ibid. 10, 1915, Bot. 265-285).-List of records and new spp.

-, Genera and species erroneously credited to the Philippine flora (ibid. 10, 1915, Bot. 171-194).

-, New plants from Sorsogon Province, Luzon (ibid. 11, 1916, Bot. 1-35).-Descr. of miscellaneous new spp.

-, New plants from Samar (ibid. 11, 1916, Bot. 175-206).-Descr. new spp.

-, New Philippine shrubs and trees (ibid. 12, 1917, Bot. 263-303).-Ditto.

-, Species Blancoanae. Manila (1918) 1-423.
-, An Enumeration of Philippine Flowering Plants. Manila, 4 vols (1923-1926).-Indispensable reference work; general chapters; bibliography. -, New species of Philippine plants collected by $A$. Loher (Philip. J. Sc. 27, 1925, 21-59).-Descr. new spp.

-, Additions to our knowledge of the Philippine Flora (ibid. 26, 1925, 447-496); ditto II (ibid. 29, 1926, 475-497, t. 1); ditto III (ibid. 30, 1926, 389-430).-Ditto.

-, Nomenclatural notes on Philippine plants (ibid. $35,1928,1-9$ ).

-, Miscellaneous notes on Philippine botany (ibid. 60, 1936, 27-35).-Records and new spp.

- \& M. L. MerRTt, The Flora of Mt Pulog (ibid. 5, 1910, Bot. 287-401).-Collecting list of records and new spp.

- \& R. A. Rolfe, Notes on Philippine Botany (ibid. 3, 1908, Bot. 95-127).-List records and new spp.

Naves, A. \& C. Fernandez-Villar, Novissima Appendix ad Floram Philippinarum R. P. Fr. Emmanuelis Blanco, seu enumeratio contracta plantarum philippinensium hucusque cognitarum. Cum synonymis P. P. Blanco, Llanos, Mercado et aliorum auctorum (in BlANCO, Fl. FiliP. ed. $\left.3,4^{3}, 1880-83, \mathrm{i}-\mathrm{ix}, 1-375\right)$.-List of records, sometimes descr.; some new spp.

Perkins, J., c.s., Fragmenta Florae Philippinae (1904-1905) pt 1-3, 1-212, t. 1-4.

Presl, C. B., Reliquiae Haenkeanae, seu descriptiones et icones plantarum, quas in America meridionali et boreali, in insulis Philippinis et Marianis collegit Thaddeus Haenke. 1 (1825-30) i-xv, 1-356, t. 1-48; 2 (1835-36) 1-152, t. 49-72. Vol. 1 (1825) 1-84, (1827) 85-148, (1828) 149. 206, (1830) 207-356. Vol. 2 (1831) 1-56, (1835) 57-152.-List; descr. of gen. and spp.

Quisumbing, E., Medicinal plants of the Philippines. Manila (1951) 1234 pp.-Synonymy; popular descr.; not a phytographic work.

-, New or interesting Philippine plants I (Philip. J. Sc. 41, 1930, 315-371, fig. 1-28,t. 1-3); ditto II (ibid. 76, 1944, 37-56).-New records and descr.

- \& E. D. Merrill, New Philippine plants I-II (ibid. 37, 1928, 133-213, t. 1-4; ibid. 82, 1953, 323-339, t. 1-5).-Ditto.

Robnson, C. B., Alabastra philippinensia I (Bull. Torrey Bot. Club 35, 1908, 63-75); ditto II (Philip. J. Sc. 3, 1908, Bot. 175-218); ditto III (ibid. 6, 1911, Bot. 319-358).-Ditto.

-, Botanical notes upon the island of Polillo (ibid. 6, 1911, Bot. 185-228).-Records and descr. new spp.

Rolfe, R. A., Supplementary list of Philippine plants (J. Bot. 23, 1885, 209-216).-List of names of CuMING numbers; some new comb.

VIDAL Y SOLER, S., Sinopsis de familias y géneros de plantas leñosas de Filipinas. Introdución a la flora forestal del archipiélago filipino (1883) i-xviii, 1-411, Atlas t. 1-100.-Non vidi.

-, Phanerogamae Cumingianae Philipinarum $b$ fndice numèrico y catálogo sistemático de las plantas fanerogamas coleccionadas en Filipinas por Hugh Cuming, con caracteristicas de algunas 
especies no descritas y del género Cumingia (Malváceas) (1885) i-xv, 1-217, t. 1.-Name list with descr. new spp. in Latin.

-, Revision de plantas vasculares Filipinas (1886) i-vi, 1-454, t. 1-2.-List; descr. new spp. in Latin; text in Span.

\section{Moluccas}

BeNTHAM, G., Enumeration of the plants collected by $R$. B. Hinds Esq. and Mr Barclay ...; to which are added a few species gathered in Amboyna by Mr Barclay (in Hook. Lond. J. Bot. 2, 1843, 211-240).-Contains also miscellaneous records from the Philippines, Moluccas, New Guinea, Tanna, and Fiji.

BritTEN, J., c.s., List of plants from Timor Laut (in: FoRBes's Wanderings, etc. 1885, App. 354-355).-List of plants from the Tenimber Islands (= Timor Laut); coll. Forbes and RIEDEL.

Hemsley, W. B., c.s., The Southeastern Moluccas (in Rep. Scient. Results H. M. S. Challenger, Botany, vol. 1, pt 3, 1885, 101-226).-Records.

HiLdebrand, F. H., Report For. Res. Inst. Bogor, no 49 (1951) $59 \mathrm{pp}$.-List of tree spp. of Southern Moluccas.

-, ditto, no 45 (1951) 53 pp.-Ditto of Northern Moluccas.

Holthuis, L. B. \& H. J. LAM, A first contribution to our knowledge of the flora of the Talaud Islands and Morotai (Blumea 5, 1942, 93-256).List records \& new spp.

LAM, H. J., Mianghas (Palmas). Batavia (1932) 1-66.-List of plants of this N. Moluccan islet in App. 2, p. 57-62.

Merrill, E. D., Reliquiae Robinsonianae (Philip. J. Sc. 11, 1916, Bot. 243-249).-Records and new spp. from Ambon.

-, An interpretation of Rumphius's Herbarium Amboinense. Manila (1917) 1-595, 2 maps.Evaluation of names based on Rumphian plants.

RANT, A., Twee botanische reizen naar Ambon (Nat. Tijd. Ned. Ind. 94, 1934, 100-133).List of spp. from Ambon \& Ceram.

RichaRd, A., in Dumont D'URville, Voyage de découvertes de L'Astrolabe, etc. vol. 2. Sertum Astrolabianum. Paris (1834) i-lvi, 1-167; Atlas (1833) t. 1-39.-Records and descr. new spp. from Buru \& Ceram.-Non vidi.

Rumphius, G. E., Herbarium Amboinense. See Chapter 3.

THISELTON-DYER, W. T., Report on the botany of Mr H. O. Forbes's expedition to Timor Laut (J. Linn. Soc. Lond. Bot. 21, 1885, 370-374).Merely listed names without references or numbers compiled by OLIVER.

WARBurg, O., Beiträge zur Papuanischen Flora (Bot. Jahrb. 13, 1891, 230-455).-Records and new descr. of his coll.; incl. also those of the S. Moluccas (Aru \& Key Islands).

-, Vegetationsschilderungen aus Südost Asien (Bot. Jahrb. 17, 1893, 169-176).-Plants of Ceram Laut (= Gebeh), an islet off SE. Ceram; only names in text; no proper records.

\section{New Guinea}

ANonymous, Bibliography of New Guinean botany. See Chapter 2.

BaIley, F. M., Contributions to the Flora of (British) New Guinea (or: to the Flora of Queensland and (British) New Guinea) (Queensl. Agric. J. 1897-1914).-A number of small contributions; records and descr. new spp.

-, etc., Botanical reports in the Appendices of the Annual Reports of British New Guinea (18971909).-Ditto.

-, Contributions to the New Guinea Flora (Proc. R. Soc. Queensl. 18, 1903, repr. p. 1-5).List; some new spp.

BAKer, E. G., S. MOORE, c.s., Dr H. O. Forbes's New Guinea plants (J. Bot. 61, 1923, 53-55 and Suppl. 1-64).-Listed account of the Forbes coll.; records and new genera and spp.

BecCari, O., Malesia (1877-1890).-Contains a great number of records and new spp. from New Guinea.

-, Catalogo delle piante del fiume Fly (in d'Albertis, Nuova Guinea 1880, 575-577; transl. d'Albertis, New Guinea: What I did and what I saw, 2, 1880, 396-400).-List Fly River plants; no new spp.

BENTHAM, G., Enumeration of the plants collected by $R$. B. Hinds Esq. and Mr Barclay... in New Guinea; to which are added a few species gathered in Amboyna by Mr Barclay (in Hook. Lond. J. Bot. 2, 1843, 211-240).-Miscellaneous records mixed with those of surrounding islands.

BoERLAGE, J. G., Bibliography of New Guinea. See Chapter 2.

Diels, L., c.s., Beiträge zur Flora des Saruwaged Gebirges (Bot. Jahrb. 62, 1929, 452-501).Records and descr. of coll. KEYsser.

-, Descriptions of new species collected in British Papua by L. J. Brass (J. Arn. Arb. 10, 1929, 75-84).-New spp. of some families.

DIJK, L. J. vAN, Boschbedrijf en Boschbeheer in de Residentie Molukken, in het bijzonder NoordNieuw-Guinea (eilanden der Geelvinkbaai). For. Bur., Buitenzorg (1939) mimeogr., 92 pp., many app.-In app. B, p. 1-26, a list of tree $s p p$. collected in the islands of Japen and Meos Noem.

ENGLER, A., c.s., Uebersicht über die botanischen Ergebnisse der Gazelle-Expedition (Bot. Jahrb. 7, 1886, 444-480).-Cyperaceae, Gramineae, Orchidaceae were published in op. cit. 5 (1884) 89-94; 6 (1885) 233-248; 7 (1885) 435-443.List of records; also new spp.

Grbes, L. S., c.s., A Contribution to the Phytogeography and Flora of the Arfak Mountains, etc. London (1917) i-iv, 1-226, fig. 1-15, t. 1-4. -Records and descr. new gen. \& spp.

Hemsley, W. B., c.s., The Admiralty Islands (in Report Scient. Results H. M. S. Challenger, Botany, vol. 1, pt 3, 1885, 227-275).-Records listed.

-, c.s., Flora of British New Guinea (Kew Bull. 1899, 95-126).-Records and descr. new spp. coll. ENGlish, Giulianetti, etc. 
-, Report on Botanical Collections (Ann. Report Brit. New Guinea 1897/1898, 1899, 147-150).List records.

Hildebrand, F. H., List of tree names issued by For. Res. Institute, Bogor, no 6 (1941).-List of tree spp. from West New Guinea.

-, ditto (1953) 46 pp.-Revised edition of the latter.

KANEHIRA, R., An enumeration of plants collected in the Mandated Territory of New Guinea I-III (Bot. Mag. Tokyo 52, 1938, 349-357, 409-416; ibid. 53, 1939, 8-16).-Records; few new spp.

- \& Hatusima, S., c.s., The Kanehira-Hatusima 1940 collection of New Guinea plants I-XXI (Bot. Mag. Tokyo 55-57, 1941-1943).-Mostly on the flora of Mt Arfak; records and descr. new spp.

Kostermans, A. J. G. H., Notes on New Guinea plants I-III (Bull. Bot. Gard. Btzg III, 18, $1950,435-448$, fig. 1-3).-Descr. new gen. \& spp.

LAM, H. J., Bibliography of New Guinea. See Chapter 2.

LANE-Poole, C. E., The Forest Resources of the Territories of Papua and New Guinea. Parliam. Commonw. Australia (1925) iv +209 pp., figs.-Contains important field-notes of L.$P$.'s coll.

Lauterbach, C., L. Diels \& R. Schlechter, c.s., Beiträge zur Flora von Papuasien (Bot. Jahrb. onwards of vol. 49, 1912).-Important series of revisions, mostly dealing with the former area, but sometimes covering the whole of New Guinea and incl. data on Melanesia; often keys.

Merrill, E. D. \& L. M. Perry, c.s., Plantae Papuanae Archboldianae I-XVIII (J. Arn. Arb. 20-30, 1939-1949).-Important series of records and descr. mainly of the BRAss collections, continued by Miss PERry in the shape of revisions of families and genera which have been included in Chapter $6 \mathrm{c}$.

-, Note on some Papuan collections of $M$. $S$. Clemens (ibid. 29, 1948, 152-168).-Records and descr. new spp.

Miklouho-Maclay, N. de (\& F. Von Muelier), List of plants in use by the natives of the Maclaycoast, New Guinea (Proc. Linn. Soc. N.S.W. 10, $1885,346-358)$.-List useful spp. with notes.

MOORE, S., New species of Papuan plants (J. Bot. 67, 1929, 49-51).-Records and some new spp. coll. Brass.

Mueller, F. VoN, Descriptive notes on Papuan plants. Parts 1-9 (2 vols): 1 (1875-1877) 1-94; 2 (1877-1890) 1-70.-Records and descr. new spp.

-, Numerous small papers in 'Melbourne Chemist and Druggist', 'Australasian Journal of Pharmacy', 'Victorian Naturalist', 'Wing's Southern Science Record', 'Proc. Linnean Soc. New South Wales', 'Trans. Roy. Soc. Victoria', 'Journal of Botany', 'Ann. Rep. Br. N. G.', etc. (18841896).-Inserted in Chapter $6 \mathrm{c}$ as far as suitable.

-, Report on a small collection of plants from the Aird-River, obtained by $\mathrm{Mr}$ Theodore Bevan during his recent expedition (Proc. Linn. Soc. N.S.W. II, 2, 1887, 419-422, t. 6-7).-List of names; descr. some new spp.

-, Records of observations on Sir William MacGregor's highland plants from New Guinea 1889 (Trans. R. Soc. Victoria $\left.1^{2}, 1889,1-45\right)$.Records; descr. new spp.

OlIVER, D., List of plants collected in New Guinea by Dr. A. B. Meyer . . . (J. Linn. Soc. Bot. 15, 1877, 29-30).-Small list with a few new spp.

Pulle, A. A., c.s., Nova Guinea, botanical volumes 8, 12, 14, 18 (1911-1936), many plates; new ser. 4 (1940) 109-112, t. 4-11; 133-150, t. 14.Records and descr. new spp.

Rechinger, K., Plantae novae Papuanae (in Fedde, Rep. 11, 1912, 179-187).-Descr. new spp. from New Guinea (\& the Solomons).

-, Botanische und zoologische Ergebnisse einer wissenschaftlichen Forschungsreise nach den Samoainseln, dem Neuguinea-Archipel und den Salomonsinseln (Denkschr. Math.-Naturw. Kl. K. Ak. Wiss. Wien 89, 1914, 443-672; 707, t. 1-8).-List of records with new spp.; few from New Guinea.

Rehder, A., c.s., A Supplement to C. T. White, 'Ligneous plants collected in the Territory of Papua (British New Guinea) in 1925-26 by L. J. Brass' (J. Arn. Arb. 14, 1933, 62-67).Listed records; no new spp.

RIDLEY, H. N., On the Monocotyledonous plants of New Guinea collected by $M r H$. O. Forbes (J. Bot. 24, 1886, 321-327, 353-360).-Records with new spp.

-, Plantae novae papuanae (ibid. 52, 1914, 289 296). On Forbes's coll.; records \& new spp.

-, c.s., Report on the botany of the Wollaston Expedition to Dutch New'Guinea 1912-1913 (Trans. Linn. Soc. Lond. II, Bot. 9, 1916, 1-269, t. 1-6).-Descr. \& records of the BoDEN KLoss coll. from Mt Carstensz.

SAlVerda, Z., Rapport van een ...exploratie in $Z W$. Nieuw Guinea. For. Rep. mimeogr., Buitenzorg (1938) 122 pp. + many app.-In App. I, p. 1-12, a list of tree spp. collected in West New Guinea, MacCluer Gulf area; in Dutch.

SChefFer, R. H. C. C., Enumeration des plantes de la Nouvelle-Guinée (Ann. Jard. Bot. Btzg 1, 1876, 1-60, 178-181).-List; descr. new spp.

SchumanN, K., Die Flora des Deutschen Ostasiatischen Schutzgebietes (Bot. Jahrb. 9, 1888, 189-223).-List of records and new spp. from New Guinea incl. also Micronesia and Melanesia.

-, Plantae Bammlerianae (Notizbl. Berl.-Dahlem 1, 1895, 44-57).-List of plants from Tami Isl., near SE. Huon Peninsula; a few new spp.

- \& M. Hollrung, Die Flora won Kaiser Wilhelms Land (Beih. zu den Nachr. Kais. Wilh. Land 1889, 1-137).-Enum. with records and descr. of new genera \& spp.

- \& C. LAuterbach, Die Flora der deutschen Schutzgebiete in der Südsee (1900) i-xvi, 1-613, t. 1-22, map.-Enum.; descr. new spp., incl. also those of Micronesia. 
-, ditto, Nachträge (1905).-Ditto.

SмIтH, A. C., Studies of Papuasian plants I-VI (J. Arn. Arb. 22, 1941, 60-80, 231-252, 343-374, 497-528; ibid. 23, 1942, 417-443; ibid. 25, 1944, 104-298).-Revisions of several families; records and descr. new spp.; no keys.

STEENIS, C. G. G. J. VAN, Notes on a number of New Guinean species (J. Arn. Arb. 28; 1947, 419-423).-Listed records.

Valeton, Th., Plantae papuanae (Bull. Dép. Agr. Ind. Néerl. no 10, 1907, 1-72).-Records and new spp.

WARBURG, O., Beiträge zur Kenntnis der papuanischen Flora (Bot. Jahrb. 13, 1891, 230-455).Account Warburg coll.; records; new gen. \& spp.

-, Bergpflanzen aus Kaiser Wilhelmsland (ibid. 16, 1892, 1-32).-Records and descr. new spp.
-, Plantae Hellwigianae (ibid. 18, 1894, 184-212). -Ditto.

WHITE, C. T., A Contribution to our Knowledge of the Flora of Papua (Proc. R. Soc. Queensl. 34, 1922, 5-65).-Listed records and new spp.

-, On a small collection of plants from the Rigo District, Papua (Proc. Linn. Soc. N. S. W. 51, 1926, 296-298, t. 17).-Ditto.

-, c.s., Ligneous plants collected in the Territory of Papua in 1925-1926 by L. J. Brass (J. Arn. Arb. 10, 1929, 197-274).-Ditto.

-, Ligneous plants from the Solomon Islands (and New Guinea) (ibid. 31, 1950, 81-116).-Ditto.

- \& W. B. Francis, Plants collected in Papua by C. E. Lane-Poole (Proc. R. Soc. Queensl. 38, 1927, 225-261, fig. 1-19).-Ditto.

-, Plants collected in the Mandated Territory of New Guinea by C. E. Lane-Poole (ibid. 39, $1928,61-70$, t. 4-5).

\section{PROPER TAXONOMIC BIBLIOGRAPHY}

\section{ACANTHACEAE}

ANDERSON, T., On the identification of the Acanthaceae of the Linnean Herbarium, etc. (J. Linn. Soc. Bot. 7, 1863, 111-118).-List.

BremeKamp, C. E. B., Materials for a monograph of the Strobilanthinae (Verh. Kon. Ned. Akad. Wet. A'dam sect. 2, 41, no 1, 1944, 1-306, t.1-6). -Mainly dealing with Hemigraphis and Strobilanthes; 43 new genera are segregated! In all genera keys to the Mal. spp.

- \& N. E. NanNenga-Bremekamp, A premiliminary survey of the Ruelliinae of the Malay Archipelago and New Guinea (Verh. Kon. Ned. Akad. Wet. A'dam sect. 2, 45, no 1, 1948, 1-39). -Key to subtribes, genera and spp. comprising e.g. revisions of Dipteracanthus and Eranthemum. Two new genera: Nothoruellia and Pararuellia (Aporuellia).

Hallier $f$., H., Indonesische Acanthaceen (Nova Acta, Abh. Kais. Leop.-Car. Deut. Akad. Naturf. 70, 1897, 195-224, t. 9-16).-New spp. mainly in Hemigraphis; revision of Ptyssiglossis.

-, Ueber Pseuderanthemum metallicum n. sp. und das System der Acanthaceae (Ann. Jard. Bot. Btzg 15, 1898, 26-37, t. 9).-Descr. new sp.; 2 new sections; discussion evaluation of characters.

LINDAU, G., Beiträge zur Systematik der Acanthaceen (Bot. Jahrb. 18, 1893. 36-64, fig. 1-2, t. 1-2). -Tribal and generic delimitation with pollen structure; key to genera by pollen characters.

RADLKOFER, L., Ueber den systematischen Werth der Pollenbeschaffenheit bei den Acanthaceen (Sitz.-Ber. Math. Phys. Kl. Bayer. Akad. Wiss. $13,1883,256-314)$.-Significance of pollen structure in subdividing the family.

Valeton, TH., Ic. Bog. 3 (1908) t. 251-257.-New spp. of Ptyssiglottis, Ruellia and Rungia.

\section{Local}

Africa: Lindau, Bot. Jahrb. 17 (1892) 89-113; ibid. 20 (1894) 1-76.-List; descr. new spp. \& gen.

SE. Asia: Anderson, J. Linn. Soc. Lond. Bot. 9 (1867) 425-526.-Conspectus generum; enum. of spp. in India; no keys.

Bombay: SantaPaU, Univ. Bombay, Bot. Mem. 2 (1952) i-iii, 1-104, t. 1-6.-Revision Bombay Presid. spp.

-, J. Bombay Nat. Hist. Soc. 51 (1953) 349-368. -Addition to latter paper; 'some new keys to spp. of various genera.

Siam: Clarke (ed. Hosseus), Bot. Jahrb. 41 (1907) 62-73.-Enum.

ImLAY, Kew Bull. (1939) 109-150.-Critical notes and descr. of new spp. \& genera from Siam.

Sumatra: Merrill, Contr. Arn. Arb. 8 (1934) 154-155.-Small list; some new comb.

RidLeY, J. Fed. Mal. Stat. Mus. 8, 4 (1917) 7174.-List; several new spp.

-, J. Mal Br. R. As. Soc. no 87 (1923) 81-83. -List; several new spp.

-, Kew Bull. (1925) 87.-New Sphinctacainthus.

Malay Peninsula: AIrY Shaw, Kew Bull. 1951 (1952) 370.-Limnophila viscida RIDL. reduced to Staurogyne.

Java: BACKER, Onkruidfl. Jav. Suiker: (1931) 637685.

BremeKamp, Verh. Kon. Ned. Akad. Wet. A'dam sect. 2, 45, no 2, 1948, 1-78.-Extensive critical notes; many new spp.; no keys.

- \& BACKer, in BACKer, Bekn. Fl. Java (em. ed.) 9 (1949) fam. 198, p. 1-101.-In Dutch; keys!

LINDAU, in FEDDE, Rep. 13 (1915) 550-554.Descr. 5 new spp. from Java, 1 from Sumbawa, 1 from Celebes.

VALETON, Ic. Bog. 4 (1912) t. 326.-Lamiacanthus viscosus.

Borneo: Van Steenis, Bull. Bot. Gard. 'Btzg III, 17 (1948) 398.-New rheophytic Hygrophila.

Philippines: Bremekamp, Philip. J. Sc. 80 (1951) 11-21.-Descr. new spp. 
-, ibid. 81 (1952) 133-134.-2 new spp.

Clarke, Govt Lab. Publ. Philip. no 35 (1905) 89-93.-Misc. descr.

—, Philip. J. Sc. 1 (1906) Suppl. 247-249.-Descr. some new spp.

Elmer, Leafi. Philip. Bot. 5 (1913) 1685-1704. -Misc. new spp. from Palawan.

一, ibid. 10 (1939) 3673-3679.-Misc. new spp. Lindau, in Perkins, Fragm. Fl. Philip. (1904) 38-41.-List; no new spp.

MerRILL, Philip. J. Sc. 26 (1925) 490-491.-New

Hypoestes.

—, ibid. 27 (1925) 54-55.-2 new spp.

—, ibid. 29 (1926) 486-489.-5 new spp.

-, ibid. 30 (1926) 426-428.-2 new spp.

-, ibid. 41 (1930) 358-361, fig. 23-24.-Ditto.

Quisumbing \& MerRILl, Philip. J. Sc. 37 (1928)

199-200.-New Hallieracantha.

Celebes: BremeKaMP, Svensk Bot. Tidskr. 42 (1948) 372-403.-List of KAUDERN \& KJELLBERG coll.; new spp.

-, ibid. 43 (1949) 63-64.-New Hypoestes from Saleier Isl.

Hemsley, Kew Bull. (1896) 39.-New Strobilanthes.

Moluccas: Merrill, Philip. J. Sc. 11(1916) Bot. 313.-New Lepidagathis and Pseuderanthemum from Ambon.

New Guinea: LAUTERbach, Nova Guinea 8 (1910) 333-334.-New Staurogyne.

LindaU, Bot. Jahrb. 19 (1894) Beibl. 48, 3-7.List; some new spp.

$\longrightarrow$, ibid. 50 (1913) 166-170.-Descr. Strobilanthes n. sp.; Ancylacanthus and Jadunia n. .

一, ibid. 55 (1918) 135-136.-Descr. 2 new spp.; Gymnophragma n. $\mathrm{g}$.

MOORE, J. Bot. 58 (1920) 190-195.-List; descr. new spp.; Hulemacanthus n.g.

Ridley, J. Bot. 52 (1914) 294-296.-Descr. 2 new spp.

\section{Genera}

Acanthus: VAN SteEnIs, Trop. Natuur 26 (1937) 202-207.-Revision Mal. spp.; in Dutch; key.

Eranthemum (Daedalacanthus): BREMEKAMP \& NanNenga-Bremekamp, Verh. Kon. Ned. Akad. Wet. A'dam sect. 2,45, no 1 (1948) 33-39.-Key to endemic spp. and var. of Mal.

Hallieracantha: STAPF, J. Linn. Soc. Bot. 38 (1907) 6-17.-Revision; now very incomplete.

Hemiadelphys: Merrill, Philip. J. Sc. 12 (1917) Bot. 109-110.-Record.

Lepidagathis: NeEs AB EsEnBECK, Monographia generis Lepidagathis (Nov. Act. Ac. Caes. Leop.Car. Nat. Cur. 19, Suppl. 2, 1841, xxi-liv; repr. separately under the title: 'Lepidagathis illustratio monographica' 1841, 1-40).-Descr. \& synopsis of spp., some new; key to groups.

Pseuderanthemum: Hallier $f$., Ann. Jard. Bot. Btzg 15 (1898) 26-37.-Descr. new Sumatran sp.; 2 new sections.

Ptyssiglottis: Hallier $f$., Nova Acta, Abh. Kais. Leop.-Car. Deut. Akad. Naturf. 70 (1897) 212224, t. 14-16.-Revision; key.

MOORE, J. Bot. 60 (1922) 355-358.-Ditto.
Pycnostachys: BruCE, Kew Bull. (1939) 563-593, 5 maps.-Complete revision; key; Afr. genus, some spp. sometimes cultivated.

Sanchesia: LeONARD, J. Wash. Acad. Sc. 16 (1926) 484-492.-Revision; key.

Sautiera: VALETON, Ic. Bog. 4 (1912) t. 327.-Native of Timor.

Sciaphyllum: Bremekamp, Rec. Trav. Bot. Néerl. 37 (1940) 293-300, 1 fig.-Monotypic.

Strobilanthes: Benoist, Bull. Soc. Bot. Fr. 80 (1933) 730-732.-New spp. from Siam and Indo-China.

Strophacanthus: Bremeramp, Verh. Kon. Ned. Ak. Wet. A'dam sect. 2, 45, no 2 (1948) 43-45.-List.

Thunbergia: BACKer, Trop. Natuur 5 (1916) 136137.-Native and cultivated Jav. spp.; in Dutch; key!

LINDAU, Bot. Jahrb. 17 (1893) Beibl. 41, 31-44.

-Revision; no descr.; key!

\section{ACERACEAE}

Bloembergen, S., Fl. Mal. I, 4 (1948) 3-4, 1 fig. PAX, F., Pflanzenreich Heft 8 (1902) 1-89.

\section{Genera}

Acer: FANG, Contr. Biol. Lab. Sc. Soc. China, bot. ser. 11 (1939) ix + 346 pp.-Revision Chin. spp. LI, Pacif. Science 6 (1952) 288-294.-Spp. of Formosa and Riukiu Isl.; descr.; key!

Van Steenis, Bull. Bot. Gard. Btzg III, 18 (1950) 458-459.-Records from Malaya and Timor. -, Fl. Mal. 1, 4 (1954) 592.-Proper name of Mal. Acer is A. laurinum HAssk.

\section{ACTINIDIACEAE \\ (incl. also Saurauiaceae ${ }^{1}$ )}

Gilo, E. \& E. WerdermanN, Pflanzenfamilien ed. 2, 21 (1925) 36-45.

\section{Genera}

Actinidia: P'er \& LAw, Bot. Bull. Acad. Sinica 2 (1948) 25-33.-Spp. of Szechuan \& Sikang; key! DunN, J. Linn. Soc. Bot. 39 (1911) 394-410.Revision; key!

LI, J. Arn. Arb. 33 (1952) 1-61.-Revision; key!

Van Steenis, Fl. Mal. I, 4 (1948) 37-39, fig. 1.

\section{Saurauia \\ (Trematanthera)}

Burtr, B. L., in Hook. Ic. PI. 34 (1936) t. 3316.Descr. of a new sp. of the Solomons. Constructive remarks on the structure of the subdivisional affinities (series of DIELs).

Buscalion, L. \& Muscatello, G., Malpighia 24-30.-Revision New World spp.

Gilg, C. \& E. WerdermanN, Pflanzenfamilien ed. 2, 21 (1925) 42-45.

Korthals, P. W., in Temminck, Verh. Nat. Gesch. Bot. (1841-42) 120-134, t. 19.-Descr. 6 spp. Miquel, F. A. W., Ann. Mus. Bot. Lugd.-Bat. 4 (1869) 106-112.-List; many new spp.

(1) Saurauia has been included in several other families (Dilleniaceae, Theaceae, etc.). 
Schnarf, K., Sitz. Ber. Ak. Wiss. Wien, Math.Naturw. K1. Abt. 1, 122 (1924) 17-28, 22 fig.Ontogeny and seed structure point to affinity with Theaceae.

VRIESE, W. H. DE, P1. Ind. Bat. Orient. (1856) 3157.-List Mal. spp.; new spp.; no key.

\section{Local}

Sumatra: BAKeR, J. Bot. 56 (1918) 161-167.-Important list; some new spp.; key.

Merrill, Contr. Arn. Arb. 8 (1934) 104-106.List records.

Miquel, Fl. Ind. Bat. Suppl. (1860) 480-482.New spp.

Ridley, J. Mal Br. R. As. Soc. no 87 (1923) 5354.-Records and new $s p$.

-, Kew Bull. (1926) 60.-New sp.

Borneo: Merrill, Philip. J. Sc. 13 (1918) Bot. 8594.-Descr. 11 new spp.

-, J. Str. Br. R. As. Soc. no 79 (1919) 31-35.Records and 4 new spp.

—, ibid. no 86 (1922) 330-331.-New sp. and a new comb. in Actinidia.

-, Philip. J. Sc. 21 (1922) 529-530.-New sp.

—, ibid. 29 (1926) 396-397.-New sp.

—, Plant. Elm. Born. (1929) 196-197.-Records and new $s p$.

Ridley, Kew Bull. (1929) 262.-New sp.

Alry SHAw, Kew Bull. (1940) 248-249.-List records.

Philippines: Elmer, Leafl. Philip. Bot. 10 (1939) 3726-3728.-2 new spp.

Merrill, Philip. J. Sc. 9 (1914) Bot. 521-530.12 new spp.

一, ibid. 26 (1925) 471.-New sp.

-, ibid. 27 (1925) 36.-New sp.

—, ibid. 29 (1926) 396-397.-New sp.

Quisumbing, Philip. J. Sc. 41 (1930) 332-337,

fig. 9-11.-3 new spp.

一, ibid. 76 (1944) 48-52.-4 new spp.

Celebes: KoORDERs, Suppl. Flora N. O. Celebes 2 (1922) t. 69-80; ibid. 3 (1922) 34-40.-Descr. \& plates.

New Guinea: Diels, Bot. Jahrb. 57 (1922) 441-459. -Key to groups and spp. of New Guinea.

-, Nova Guinea 14 (1924) 82-84.-Records and new spp.

Kanehira \& Hatusima, Bot. Mag. Tokyo 57 (1943) 63-74.-Records; 8 new spp.

LAUterbach, Nova Guinea 8 (1910) 305-307. -List; 3 new spp.

一, ibid. 8 (1912) 835-839.-List; 8 new spp. Von Mueller, Vict. Nat. 2 (1886) 134-135.Descr. Trematanthera.

Ridley, Trans. Linn. Soc. Lond. II. Bot. 9 (1916) 14-15.-5 new spp.

Smith, A. C., J. Arn. Arb. 22 (1941) 502-524. -List; 18 new spp.

White \& Francis, Proc. R. Soc. Queensl. 38

(1927) 244-247, fig. 10-11.-List; 3 new spp.

\section{AEGINETIA CEAE}

See Orobanchaceae

AGAVACEAE

See Amaryllidaceae

\section{AIZOACEAE}

(incl. Molluginaceae \& Ficoidaceae)

Backer, C. A., Fl. Mal. I, 4 (1951) 267-275, fig. $1-2$.

Pax, F. \& K. Hoffmann, Pflanzenfamilien ed. 2, $16 \mathrm{c}$ (1934) 179-233.

\section{Local}

Java: BACKER, Onkruidfl. Jav. Suiker. (1930) $237-$ 242.-In Dutch; key!

New Guinea: Merrill \& Perry, J. Arn. Arb. 23 (1942) 386. -2 records.

Pulle, Nova Guinea 8 (1910) 355. - List.

\section{Genera}

Mesembryanthemum: VoN PoellnItz, in Fedde, Rep. 32 (1933) 1-73.-Segregation of the genus; descr. \& keys to segregate genera.

Trianthema: Melville, Kew Bull. (1952) 261-269. -Some critical spp. allied to $T$. pentandra.

\section{ALANGIACEAE}

Wangerin, W., Pflanzenreich Heft $41^{3}$ (1910) 1-25, fig. 1-6.

\section{Local}

China: Melchior, Notizbl. Berl.-Dahlem 10 (1929) 822-830.-Revision; key! —, \& MANSFELD, Bot. Jahrb. 60 (1925) 162-166. -Revision; key.

\section{Genera}

Alangium: BloEmbergen, Blumea 1 (1935) 241294, fig. 1-5.-Revision Neth. Ind. spp. -, Bull. Jard. Bot. Btzg III, 16 (1939) 139-235, 10 fig.-Revision of the genus; key!

\section{ALISMATACEAE}

Buchenau, F., Pflanzenreich Heft $16^{2}$ (1903) 166, fig. 1-19.

Micheli, M., in DC. Mon. Phan. 3 (1881) 29-83. Miquel, F. A. W., Ill. Fl. Arch. Ind. (1870) 49-51. -Census Sagittaria and Lophotocarpus.

\section{Local}

Java: BACKer, Handb. Fl. Java pt 1 (1925) 52-55. -A practically complete Mal. survey; in Dutch; keys!

KERN, Trop. Natuur 32 (1952) 127-129, 1 fig.Record of a Sagittaria newly naturalized in Java; in Dutch.

\section{Genera}

Alisma: SAmuelsson, Ark. Bot. 24A, no 7 (1932) 1-46, t. 1-6.-Revision; key to spp. and sspp.

Echinodorus (Ranalisma): GAGNEPAIN, Bull. Soc. Bot. Fr. 76 (1929) 274-276.-Reduction of $R a-$ nalisma.

STAPF, in Hook. Ic. PI. IV, 7 (1901) t. 2652.Ranalisma from Mal. Pen.

VAN SteEnis, Arch. Hydrobiol. Suppl. 11 (1932) 240.-Recomb.

Elisma: VAN Steenis, Trop. Natuur 24 (1935) 54 56.-Record of this European plant in Java. 
VAN DER WERFF, Blumea 7 (1954) 599-601.Elisma record from Java is based on a wrongly localized plant.

Lophotocarpus: Smith, J. G., 11th Ann. Rep. Missouri Bot. Gard. (1900) 145-150.-Key to U.S. Am. spp.

Sagittaria: Smith, J. G., 6 th Ann. Rep. Missourri Bot. Gard. (1895) 27-64, t. 1-29.-Revision N. Amer. spp.; descr.; key!

\section{AMARANTHACEAE}

BACKer, C. A., Fl. Mal. I, 4 (1949) 69-98, 593594,8 fig.

LOPRIORE, G., Ueber die geographische Verbreitung der Amaranthaceae in Beziehung zu ihren Verwandtschaftsverhältnisse (Bot. Jahrb. 30, 1901, 1-38, t. 1).-General discussion.

Moquin-Tandon, A., in DC. Prod. 13, 2 (1849) $231-424,462-463$.

Schinz, H., Pflanzenfamilien ed. 2, 16c (1934) 7-85, fig. 1-46.

Suessenguth, K., Amaranthaceen-Studien (in FedDE, Rep. 44, 1938, 36-48).

\section{Local}

Malay Peninsula: Suessenguth, Mitt. Bot. Staatssamml. München, Heft 6 (1953) 194.-Psilotrichum malaccense $n$. sp.; this appears to be Alternanthera brasiliana, cf. FI. Mal. I, 4, 594b.

Java: Van Steenis, Buil. Bot. Gard. Btzg III, 17 (1948) 402.-New record of Gomphrena celosioides.

Philippines: Merrill, Philip. J. Sc. 29 (1926) 478.Descr. new var. of Cyathula.

New Guinea: Pulle, Nova Guinea 8 (1910) 351352.-Small list of records.

\section{Genera}

Achyranthes: Boerlage, Ned. Kruidk. Arch. II, $5^{3}$ (1889) $420-430$, t. 6.-Account specim. at Leyden.

Merrill, Trans. Amer. Phil. Soc. Philad. n.s. 24, ii (1935) 148-149.-Typification.

Amaranthus: MerriLl, Amer. J. Bot. 23 (1936) 609-612, 1 fig.-Applic. of the name A. viridis. WILLDENow, Historia Amaranthorum (1798) 1-38, t. 1-12.

\section{AMAR YLLIDACEAE}

(incl. also Agavaceae \& Hypoxidaceae)

BAKER, J. G., A new key to the genera of Amaryllidaceae (J. Bot. 16, 1878, 161-169).-Synopsis; in Latin.

-, A synopsis of Hypoxidaceae (J. Linn. Soc. Bot. 17, 1878, 93-126).-Descr.; keys!

- Handbook of the Amaryllideae. London (1888) xii +216 pp.-Non vidi.

Pax F. \& G. F. Hoffmann, Pflanzenfamilien ed. 2, 15a (1930) 391-430, fig. 163-187.

Traub, H. P. \& H. N. Moldenke, Amaryllidaceae: tribe Amarylleae. The Amer. Plant Life Soc. Stanford Cal. 1949, 194 pp., 15 pl.-Elaborate systematic treatment; key to genera.

\section{Local}

Malay Peninsula: Ridley, Mat. Fl. Mal. Pen. (Monocot.) 2 (1907) 65-69.-Revision; keys.

Java: BACKER, Hand. Fl. Java pt 3 (1928) 81-105. -Practically complete for Malaysia, many cultivated spp. included; in Dutch; keys!

KoshimizU, Jap. J. Bot. 27 (1952) 180.-Crinum gigas NakaI reduced to $C$. asiaticum.

Philippines: Elmer, Leafl. Philip. Bot. 5 (1913) 1645-1649.-List Curculigo, 3 new spp.

New Guinea: Hallier, Nova Guinea 8 (1913) 899902.-Records; 2 new Crinums.

LAUTERBACH, Bot. Jahrb. 50 (1913) 301-305.List; no new spp.; keys!

\section{Genera}

Agave: Berger, Die Agaven. Beiträge zu einer Monographie. Jena 1915, vi +288 pp., 79 fig., 2 pl.-Contr. towards monogr.

Amaryllis: Traub, Hamilton \& UPHOF, Herbertia 5 (1938) 114-131.-Tentative revision.

Crinum: BaKer, Gard. Chron. II, 15 (1881) 763, 786 ; ibid. 16 (1881) 39, 40, 72, 180, 398, 495-496, 588-589, 760, 784-785.-Enum.; spp. geographically under subgen. \& sect.

UPHOF, Herbertia 9 (1942) 63-84 (1943).Complete list.

Hymenocallis: Sealy, Kew Bull. (1954) 201-240. -Revision; key.

Polianthes: Rose, Contr. U. S. Nat. Herb. 8 (1903) 8-14, fig. 1-4.-Revision; new delimitation; key.

Zephyranthes: Hume, Nat. Hort. Mag. 14 (1935) 258-275.-Non vidi.

SEALY, J. Roy. Hort. Soc. 62 (1937) 195-209.Delimitation against allied genera; lists of spp.; no references, syn. or keys.

\section{AMPELIDACEAE See Vitaceae}

\section{ANACARDIACEAE}

BARKLEY, F. A., A key to the genera of the Anacardiaceae (Amer. Midl. Nat. 28, 1942, 465474).

ENGLER, A., Ueber die morphologischen Verhältnisse und die geographische Verbreitung der Gattung Rhus, etc. (Bot. Jahrb. 1, 1881, 365427).-Generic delimitation and descr. new gen. \& spp.

-, in DC. Mon. Phan. 4 (1883) 171-500, t. 4 15.-Now incomplete.

MrQuel, F. A. W., De quibusdam Burseraceis et Anacardiaceis Archipelagi Indici (Ann. Mus. Lugd.-Bat 4, 1869, 117-118).-Enum. spp.; some new Buchananias.

\section{Local}

Indo-China: Evrard, Bull. Soc. Bot. Fr. 99 (1952) 131-132.-Some new Melanorrhoea \& Bouea.

Malay Peninsula: Burkill, Gard. Bull. Str. Settl. 5 (1931) 224-230.-Critical list of Gluta.

CORNER, Gard. Bull. Str. Settl. 10 (1939) 253256.-Key to the 3 Campnospermas. Henderson, Gard. Bull. Str. Settl. 3 (1924) 290291.-Notes on some Semecarpus. 
-, ibid. 7. (1933) 97-99, t. 19.-The first Pistacia in Malaysia!

Sumatra: Merrill, Contr. Arn. Arb. 8 (1934) 9192.-Critical note on Rhus chinensis MiLL.

Java: Adelbert, Blumea 6 (1948) 325-327.Some notes.

Borneo: Merrill, J. Str. Br. R. As. Soc. no 86 (1922) 322-324.-New spp. of Melanochyla and Semecarpus.

_, Plant. Elm. Born. (1929) 166-170.-Several new spp.

RIDLEY, Kew Bull. (1933) 193-202.-Numerous new spp. and records; Nothopegia new for Mal.; Melanocommia n.g.; also a Buchanania from Aru Isl. (Moluccas).

Philippines: ELMER, Leafl. Philip. Bot. 9 (1934) 3179-3180.-New Semecarpus.

—, ibid. 10 (1939) 3679-3684.-Several new spp. Merrill, Philip. J. Sc. 30 (1926) 407-408.New Semecarpus from Samar.

QuisumbING, Philip. J. Sc. 76 (1944) 43-44.New Semecarpus.

New Guinea: Kanehira \& Hatusima, Bot. Mag. Tokyo 56 (1942) 166-171, fig. 9.-Records; new spp. in Buchanania and Koordersiodendron.

Lauterbach, Nova Guinea 8 (1910) 297-299.

-Records; 2 new spp. in Rhus.

一, ibid. 8 (1912) 829-830.-Records; new spp. in Semecarpus and Buchanania.

-, Bot. Jahrb. 56 (1921) 345-373, fig. 1-5.-

Descr. new sp. and 2 new genera; keys!

—, ibid. 59 (1924) 536.-New Semecarpus.

Merrill \& Perry, J. Arn. Arb. 22 (1941) 529

541.-Records; many new spp. of BRAss coll.

-, ibid. 29 (1948) 158-160.-New spp. of Euroschinus and Rhus.

SuIS, Nova Guinea 14 (1924) 97-99, t. 8.-List records; new Rhus and 2 Semecarpus.

White, Proc. R. Soc. Queensl. 34 (1922) 40-42. -New records; new sp. in Buchanania and Semecarpus.

-, J. Arn. Arb. 31 (1950) 95-96.-New Mangifera; record of Pleiogynium.

\section{Genera}

Bouea (Tropidopetalum): FEDTsChENKo, Svensk Bot. Tidskr. 19 (1926) 493.-Reduction of Tro. pidopetalum.

Campnosperma (Coelopyrum): Van STEens, F1. Mal. Bull. 3 (1948) 74.-Campnosperma prop. to be conserved.

Mangifera: MukHerJeE, S., Lloydia 12 (1949) 73136.-Revision; key.

Pegia (Phlebochiton): Colebrooke, Trans. Linn. Soc. Lond. 15 (1827) 364-365.-Type descr.

Diels, Sunyatsenia 1 (1933) 123.-New sp. from China.

Handel-Mazzetti, Sinensia 3 (1933) 186-187. -New sp. and recomb.

Lecomte, Bull. Soc. Bot. Fr. 54 (1907) 525-529. -Revision; no key!

VAN STEENIS, J. Bot. 72 (1934) 10-12.-Revision; key.

Pentaspadon (Nothoprotium); Hooker f., Fl. Br. Ind. 2 (1876) 28-29.
Pleiogynium: LeENhouts, Blumea 7 (1952) 159.New comb. for $P$. solandri.

White, Proc. R. Soc. Queensl. 45 (1933) 27-28, t. 3 (1934).-Descr. new sp. Papua.

Rhus: Barkley, Ann. Missouri Bot. Gard. 24 (1937) 265-466.-N. \& Centr. Am. spp.; keys of Rhus and allied genera.

Merrill, J. Arn. Arb. 9 (1928) 1-4, t. 10-11.Type study Linnean herbarium.

Schinus: BARKLEY, Brittonia 5 (1944) 160-198, fig. 1-20.-Revision; key.

Sorindeja: Van SteenIS, Bull. Bot. Gard. Btzg III, 17 (1948) 462-463.-The record for Mal. is based on a cult. plant.

Swintonia (Anauxanopetalum): MrQueL, J. Bot. Néerl. 1 (1861) 368-369.

\section{ANCISTROCLADACEAE}

GiLG, E., Pflanzenfamilien ed. 2, 21 (1925) 589592.

Steenis, C. G. G. J. vaN, Fl. Mal. I, 4 (1948) 8-10, 2 fig.

\section{ANNONACEAE}

(excl. Eupomatiaceae \& Himantandraceae)

Baillon, H., Mémoire sur la famille des Anonacées (Adansonia 8, 1868, 162-184, 295-344).General discussion and synopsis of genera.

BoerlaGe, J. G., Notes sur les Anonacées du Jardin Botanique de Buitenzorg (Ic. Bog. 1, 1899, 79-208, t. 26-75).-Key to Mal. genera; spp. enumerated, no. new genera, few new spp.

Candollae, A. De, Mémoire sur la famille des Anonacées (Mém. Soc. Phys. \& Hist. Nat. Genève $5,1832,177-221$, t. 1-5). Enum.; descr. new gen. and spp.; no keys.

Corner, E. J. H., The Annonaceous seed and its four integuments (New Phytologist 38, 1949, 332-364, 31 fig.).-Anat. of seed coat and its interpretation.

Diels, L., Die Gliederung der Annonaceen und ihre Phylogenie (Sitz.-Ber. Preuss. Akad. Wiss. Phys.-Math. KI. 1932, 77-85).-Generic alliances.

Engler, A. \& L. Diels, Ubersicht über die bekannten Gattungen der Anonaceen und Beschreibung einiger neuen Gattungen dieser Familie aus dem tropischen Afrika (Notizbl. Berl.Dahlem 3, 1900, 45-59, 1 tab.).-Key to the genera.

FrIEs, R. E., Revision der Arten einiger Anonaceen-Gattungen I-V (Acta Hort. Berg. 10, 1930$1931,1-341$, t. 1-27; ibid. 12, 1934-1939, 1-577, 66 fig., 68 pl.)-Rev. of many genera, mostly neotropical; keys.

- Einige Gesichtspunkte zur systematischen Gruppierung der Amerikanischen AnnonaceenGattungen (Ark. f. Bot. 30A, no 8, 1943, 1-31, 11 fig.).

Hutchinson, J., The genera of Anonaceae (Kew Bull. 1923, 241-261).-Key to the genera.

KINO, G., The Anonaceae of British India (Ann. R. Bot. Gard. Calc. 4, 1893, 1-169, t. 1-220).Magnificent monograph; keys!

MrQuel, F. A. W., Anonaceae Archipelagi indici 
(Ann. Mus. Bot. Lugd.-Bat. 2, 1865, 1-45).Complete enum. with descr. of new spp.

Scheffer, R. H. C. C. ., Nat. Tijd. Ned. Ind. 31 (1869) 2-15; ibid. (1870) 338-344; ibid. 32 (1873) 387-392.-Misc. new spp. and records; some new genera.

-, Sur quelques plantes nouvelles ou peu connues de l'Archipel Indien (Ann. Jard. Bot. Btzg 2, 1885, 1-31).-Important list of records and new spp.; new genera: Ararocarpus, Rauwenhoffia, mostly based on BECCARI and TEYSMANN coll.

Sprague, T. A. \& J. Hutchinson, African Anonaceae (Kew Bull. 1916, 145-161).-Key to genera; list; descr. new spp.

\section{Local}

Siam: SinClaIR, Gard. Bull. Sing. 14 (1953) 40-44. -List; corrections and notes; recomb.

India-Burma: SINCLAIR, ibid. 14 (1953) 45-48.Ditto.

Sumatra: BAKER, J. Bot. (1924) Suppl. p. 2-5.Records Forbes coll.; some new spp.

Merrill, Contr. Arn. Arb. 8 (1934) 58-60.Records; new sp. in Disepalum and Orophea. RIdLey, J. Mal. Br. R. As. Soc. no 87 (1923) 51-52.-New Melodorum.

-, Kew Bull. (1926) 59.-New Popowia.

Malay Peninsula: Corner, Gard. Bull. Str. Settl. 10 (1939) 11-15.-Critical records; some new var.

Henderson, Gard. Bull. Str. Settl. 7 (1933) 8890, t. 15-16.-2 new spp.

Java: BACKER, Schoolfl. Java (1911) 24-37. -, Blumea 5 (1945) 491-493.-Critical notes; monotypic Polyaulax n.g.

Borneo: BeccarI, Nuovo Giorn. Bot. Ital. 3 (1871) 177-193, t. 2-7.-List; descr. new spp. and Eburopetalum, Enicosanthum, Marcuccia, and Mezzettia n.g.

Diels, Mitt. Inst. Alig. Bot. Hamburg 7 (1927) 77-80.-List coll. WINKLER; some new spp.

$\rightarrow$, Notizbl. Berl.-Dahlem 11 (1931) 80-82, 8586.-Descr. new spp. coll. BECCARI.

Merrill, J. Str. Br. R. As. Soc. no 85 (1922) 174-189.-Many new records and new spp.; monotypic Woodiella n.g.

RIDLEY, Kew Bull. (1912) 382-390.-Many new spp.

-, Sarawak Mus. J. 1 (1913) 73-98.-Important list of records; many new spp.; Mezzettiopsis n.e.

AIRY Shaw, Kew Bull. (1939) 275-290.-Enum. coll. Richards! Critical notes on Enicosanthum, Neouvaria n. g., and Uvariella.

SinClaIR, Sarawak Mus. J. no 18 (n.s. vol. 5, no 3) (1951) 597-609.-Valuable critical notes.

Philippines: Elmer, Leafl. Philip. Bot. 5 (1913) 1705-1750.-Descr. new spp.

-, ibid. 10 (1939) 3684-3692.-Descr. new spp. Merrill, Philip. J. Sc. 10 (1915) Bot. 227264.-Important study of Philip. and other Annon.

—, ibid. 26 (1925) 453-457.-Descr. 4 add. new spp.
-, ibid. 29 (1926) 479-480.-New Orophea and Papualthia.

-, ibid. 30 (1926) 392-394.-2 new spp.

Quisumbing, Philip. J. Sc. 41 (1930) 323-324, fig. 4.-New Papualthia.

—, ibid. 76 (1944) 39-40.-2 new spp.

New Guinea: BuRck, Nova Guinea 8 (1910) 427433 , t. 69-70.-List records \& new spp.

DiELs, Nova Guinea 8 (1912) 871-873.-List of records; some new spp.

一, Bot. Jahrb. 49 (1913) 113-167, 5 fig.-Keys to gen. \& spp.; new genera Papualthia, Oncodostigma, Oreomitra, Schefferomitra.

—, ibid. 52 (1914) 177-186.-Addit. new spp. and records.

—, Notizbl. Berl.-Dahlem 11 (1931) 82-83.-

Descr. new spp.

Kostermans, Reinwardtia 1 (1952) 459-461, fig. 1.-New Saccopetalum.

Scheffer, Ann. Jard. Bot. Btzg 1 (1876) 2-5.List; some new spp.

White, J. Arn. Arb. 10 (1929) 212-213.-Small name list.

\section{Genera}

Anaxagorea (Spermabolus): FrIES, Svensk Bot. Tidskr. 34 (1940) 400-408.-Discussion affinity of the genus.

SCHEFFER, Nat. Tijd. Ned. Ind. 31 (1869) 9.Reduction Spermabolus.

Annona: Frues, Act. Hort. Berg. 10 (1931) 197315.-Revision; key.

SAFford, J. Wash. Acad. Sc. 1 (1911) 118-120.Sectional division.

-, Contr. U. S. Nat. Herb. 18 (1914) 1-68, t. 1-41.-Key to subdivisions; descr. new spp.; no key to $s p p$.

Dasymaschalon (Unona §): MerrILL, Philip. J. Sc. 10 (1915) Bot. 235-239.-Segregation; list of spp.; new comb.

Desmos (Unona auct. pro reg. paleotrop.):MERRILL, Philip. J. Sc. 10 (1915) 234-236.-Delimitation; complete list; new comb.

SAFFORD, Bull. Torr. Bot. Club 39 (1912) 501508.-Generic delimitation; Desmos retained for Old World spp. of Unona; key to sect.; 16 spp. examined; no key to spp.

Disepalum: Van Steenis, Bull. Bot. Gard. Btzg III, 17 (1948) 405-406, fig. 2.-Revision; key!

Enicosanthum (Griffithia KING non al., Griffithianthus, Marcuccia): AIRY SHAw, Kew Bull. (1939) 275-278.-List spp.

Eupomatia. See Eupomatiaceae.

Fissistigma (Melodorum auctt. non LoUR., Pyramidanthe, Mitrella): Merrill, Philip. J. Sc. 15 (1919) 130-137.-Recomb. of all spp.

Friesodielsia (Oxymitra Hook. f. \& TH. non BISCHOFf): Van Steenis, Bull. Bot. Gard. Btzg III, 17 (1948) 458.-A new generic name proposed.

Himantandra. See Himantandraceae.

Pseuduvaria: Merrill, Philip. J. Sc. 10 (1915) Bot. 254-256.-Delimitation; new comb.

Sageraea: ChatterJee, Proc. Linn. Soc. Lond. sess. 154 (1943) 263-269.-Revision Ind. \& Burm. spp.; short key; enum. of specimens. 


\section{APOCYNACEAE}

Blume, C. L., De quibusdam Apocyneis, etc. (Rumphia 4, 1848, 25-29, t. 179-181).

Boerlage, J. G., Apocynacées (Bull. Inst. Bot. Btzg 5, 1900, 4-20).-List caoutchouc furnishing spp., some new, coll. VAN ROMBURGH in W. Malaysia.

Hallier $f$., H., Ueber Kautschuklianen und andere Apocynaceen, nebst Bemerkungen über Hevea (Jahrb. Hamb. Wiss. Anst. 17, 1900, 1-216, t. 1-3).-Contains a census of spp. of Willughbeia, Chilocarpus, Otopetalum reduced to Micrechites, Melodinus, Leuconotis, Hunteria.

Hasskarl, J. K., Plantarum nonnularum Javanicarum e familiis Asclepiadearum et Apocynearum adumbrationes (Flora 40, 1857, 103-106). -Enum.; descr.

MARKGRAF, F., Die Gliederung der Asiatischen Tabernaemontanoideen (Notizbl. Berl.-Dahlem 12, 1935, 540-552).-Segregation of some genera; distinction of Rejoua, Pagiantha, Oistanthera, Ervatamia (with sections), Eucorymbia, and Voacanga; key to the genera; enum. spp.; descr. new gen. \& spp.

-, Neue Diagnosen (Mitt. Bot. Staatssamml. München 1, 1950, 26-31).-New comb. \& spp.

Merrill \& Quisumbing, Philip. J. Sc. 82 (1953) 334.-3 new Alyxias.

MrQuel, F. A. W., Nova genera Apocynearum indicarum (Versl. Kon. Akad. Wet. A'dam 6, 1857, 191-194).-Descr. new genera.

-, in Siebold \& DE VRIEse, Flor. Jard. 2 (1859) 142-144.-Same as above.

-, Ann. Mus. Bot. Lugd.-Bat. 4 (1869) 137141, t. 5.-Descr.; some new spp.

Monachino, J., A resumé of the American Carisseae (Lloydia 9, 1946, 293-309).-Key to the genera.

Pichon, M., Classification des Apocynacées I. Carissées et Ambélaniées (Mém. Mus. Hist. Nat. Paris n.s. 24, 1948, 111-181, t. 1-4).-Keys to tribes, genera \& sections.

-, ditto V. Cerbéroidées (Not. Syst. 13, 1948, 212-229, t. 1-2).-Treated as a new subfamily; key to genera; generic descr. \& syn.

-, ditto IX. Rauvolfiées. Alstoniées, Allamandées, et Tabernaemontanoidées (Mém. Mus. Hist. Nat. Paris n.s. 27, 1948, 153-251, t. 10-20). -Keys to tribes and genera.

-, ditto XVI. Clef des genres d'Ecdysanthérées (Bull. Mus. Hist. Nat. Paris II, 20, 1948, 296-303). -Key; Parameriopsis n.g.

-, ditto XIX. Le rétinacle des Echitoidées (Bull. Soc. Bot. Fr. 95, 1948, 211-216, fig. 1).-Structure of stamens.

-, ditto XXV. Echitoidées (Mém. Mus. Hist. Nat. Paris. n.s. B. Bot. 1, 1950, 1-143, t. 1-10). -Keys to genera, generic descr. \& syn.; sometimes keys to spp.

-, ditto XXVI. Détermination des échantillons fleuris des Plumérioidées (Bull. Mus. Hist. Nat. Paris II, 21, 1949, 140-146).-Key to groups and genera by aid of floral characters.

-, ditto XXVII. Détermination des graines des
Plumérioidées et des Cerbéroidées (Bull. Mus. Hist. Nat. Paris II, 21, 1949, 226-269).-Keys based on seed characters.

-, ditto XXVIII. Supplément aux Plumérioidées (Mém. Mus. Hist. Nat. Paris. n.s. Bot. 1, 1950, $145-172$, t. 11).

-, ditto XXXIII. Les sous-tribus des Carissées (Not. Syst. 14, 1952, 310-315).-Key to subtribes.

-, ditto XXXV. Monographie des Landolphiées (Mém. Inst. Fr. Afr. Noire no 35, 1953, 1-437). -None native in Malaysia.

-, Les glandes nodales des Apocynacées et leurs modifications (Bull. Mus. Hist. Nat. Paris II, 21, 1949, 467-473).

Woonson, R. E., Observations on the inflorescence of Apocynaceae (Ann. Missouri Bot. Gard. 22, 1935, 1-48).-Special reference to Amer. genera of Echitoideae.

- The American genera of Echitoideae (ibid. 22, 1935, 153-306).-Keys!

\section{Local}

Siam: KERR, Kew Bull. (1937) 40-44, 87-94.Descr. new spp.

China \& Malaysia: Tsiang, Notes on Asiatic Apocynales I (Sinensia 3, 1932, 157-160).-Various notes, with occasional mention of Malaysian specimens!

二, ditto II (Sunyatsenia 2, 1934, 89-174).Ditto.

-, ditto III (ibid. 3, 1936, 121-160).-Ditto.

-, ditto IV (ibid. 4, 1939, 31-54).-Ditto.

-, ditto V (ibid. 6, 1941, 109-120).-Ditto.

Sumatra: LÜTJEHARMS \& VAN OOSTSTROOM, Blumea 3 (1938) 102-105.-2 new spp. of Tabernaemontana ( = Ervatamia).

Merrill, Contr. Arn. Arb. 8 (1934) 139-144.Critical notes; new $s p$.

一, Pap. Mich. Acad. Sc. Arts \& Lett. 19 (1934) 188-189.-New Chilocarpus \& Urceola.

MOORE, J. Bot. (1923) Suppl. 31-34.-Descr. Clitandropsis n.g.; new spp. coll. ForBes.

RidLey, Kew Bull. (1925) 84-85.-New Lyonsia. -, ibid. (1926) 73-74.-List and new Chilocarpus.

Malay Peninsula: Henderson, Gard. Bull. Str. Settl. 5 (1930) 78-79.-New Kopsia. 一, ibid. 7 (1933) 108-109.-New Urceola.

Java: BAKHUIZEN VAN DEN BRINK Jr, in BACKER, Bekn. Flora Java (em. ed.) 7 (1948) fam. 172, 1-50.

-, Blumea 6 (1950) 384-393.-Critical notes; some new spp., one new genus.

Borneo: Hallier $f$., Bot. Jahrb. 49 (1913) 371-375. -List of critical notes.

Markgraf, Mitt. Bot. Staatssamml. München 1 (1950) 26-30.-New spp.

MerRill, J. Str. Br. R. As. Soc. no 77 (1917) 237-238.-New Alyxia.

_, Philip. J. Sc. 21 (1922) 531-532.-New Tabernaemontana.

-, J. Mal. Br. R. As. Soc. 1 (1923) 27-29.New Epigynum; notes and new sp. of Willughbeia. 
_, Philip. J. Sc. 29 (1926) 411-413.-List; new Kopsia.

RIDLEY, Kew Bull. (1934) 123-124.-Records; 2 new spp.

SchWARTz, Mitt. Inst. Allg. Bot. Hamb. 7(1931) 258.-New Alyxia.

VAN STEENIS, Bull. Bot. Gard. Btzg III, 17 (1948) 407-409.-Descr. Neokeithia n.g.; distr. Eucorymbia; record of Spirolobium.

Philippines: Elmer, Leafl. Philip. Bot. 4 (1912) 1445-1467.-Descr. new spp.

-, ibid. 10 (1939) 3692-3699.-Descr. new spp.

MerrILL, Philip. J. Sc. 7 (1912) Bot. 242-243.-

New Tabernaemontana.

一, ibid. 13 (1919) 52-55.-Some new spp.

-, ibid. 27 (1925) 50-53.-Some new spp.

-, ibid. 29 (1926) 483-484.-2 new spp.

-, ibid.-30 (1926) 423-424.-New Kibatalia.

-, \& Quisumbing, ibid. 82 (1953) 334-336, t. 4 5.-3 new Alyxias.

Quisumbing \& Merrill, Philip. J. Sc. 37 (1928) 191-195.-4 new spp.

New Guinea: Kanehira \& Hatusima, Bot. Mag. Tokyo 55 (1941) 489-504.-List; many new spp. specially in Alyxia and Parsonsia.

MARKGRAF, Bot.. Jahrb. 61 (1927) 164-222, t. 10.-Revision; keys!

-, Nova Guinea 14 (1927) 278-291, t. 31-32. List; many new spp.; 3 new genera Discalyxia, Lamechites, and Papuechites.

-, Notizbl. Berl.-Dahlem 10 (1930) 282.-

Record of an Excavatia.

-, Brittonia 2 (1936) 139-140.-New Parsonsia and Clitandropsis.

-, Notizbl. Berl.-Dahlem 15 (1940) 130-131.-

New Alyxia \& Clitandropsis.

-, J. Arn. Arb. 21 (1940) 199-200.-New Excavatia.

Merrill \& Perry, J. Arn. Arb. 24 (1943) 210217.-List records; some new spp.; reinstatement of Bleekeria, a segregate of Ochrosia.

-, ibid. 29 (1948) 163-164.-Some records \& a new sp.

SCHLECHTER, Die Guttapercha- und KautschukExp., etc. (1911) 13, 126, 127.-Some new spp.

\section{Genera}

Aganosma: Tsiang, Sunyatsenia 4 (1939) 31-42.Critical list of spp.

Allamanda: HolTTUM, M. A. H. A. Mag. 11 (1941) 54-58, fig. 1.-Key to 3 spp. cult. in Malaya.

Alstonia (Amblyocalyx): Monachino, Pac. Sc. 3 (1949) 133-182.-Complete revision with keys to spp.; complete for Mal.

PICHON, Bull. Mus. Hist. Nat. Paris II, 19 (1947) 294-298.-Subdivision; no key to spp.; Blaberopus treated as a separate genus (cf. l.c. p. 300-301).

Carissa: Haines, Ind. For. 45 (1919) 375-388, t. 17-20.-Revision Indian spp.; key. 一, ibid. 47 (1921) 377-379.-Some addit. notes.

Cerbera: Blake, Proc. R. Soc. Queensl. 59 (1948) 161-162.-New sp. closely allied to C. floribunda. CORNER, Wayside Trees Malaya (1940) 143-144. Valeton, Ann. Jard. Bot. Btzg 12 (1895) 238248 , t. $26-27$.
Chonemorpha: Chatrerjee, Kew Bull. (1947) 4752; ibid (1948) 68.-Revision; key; 1 new sp. FurTaDo, Gard. Bull. Str. Settl. 9 (1935) 113117.-Revision; key!

SeshagIRI, J. Ind. Bot. Soc. 32 (1953) 34-45, 4 fig.-Revision; new sp.; key.

Corymbia: Pichon, Bull. Mus. Hist. Nat. Paris II, 21 (1949) 270-271, fig. 1.-Belongs to the Echitoideae of trib. Ichnocarpeae, allied to Epigynum.

Dyera: Hooker $f$., J. Linn. Soc. Bot. 19 (1882) 291-293.-Type descr.; 2 spp.

MONACHINO, Lloydia 9 (1946) 174-202.-Revision; 2 spp.; key!

Pichon, Bull. Mus. Hist. Nat. Paris II, 21 (1949) 600-602.-Descr. fruit structure.

Symington, Mal. Forester 4 (1935) 82-85, 1 pl. $-D$. costulata.

Ervatamia (segr. of Tabernaemontana sens. lat.): Hallier $f$, Bot. Jahrb. 49 (1913) 372-375. Critical remarks on generic segregation of the genus.

Markgraf, Nova Guinea 14 (1927) 278-281.

-, Notizbl. Berl.-Dahlem 12 (1935) 547-548.

-Sect. \& spp. enum.

Merrill, Contr. Arn. Arb. 8 (1934) 139-144.Author expresses doubt about the generic distinction from Tabernaemontana!

Holarrhena: Chevalier, Rev. Int. Bot. Appl. \& Agr. Col. 29 (1948) 115-120.

Hunteria: PICHON, Bol. Soc. Brot. II, 27 (1953) 88-112, 149-150.-Revision; key!

Hymenolophus: TsIang, Sunyatsenia 4 (1939) 2829.-Monotypic; Sumatra.

Kentochrosia: Merrill \& Perry, Philip. J. Sc. 76 (1941) 19-21.-Discussion of the genus; no descr.; enum. of material present in Arn. Arb. Herb.

Kibatalia (Kickxia, Vallaris auct., Paravallaris): Pichon, Mém. Mus. Hist. Nat. Paris n.s. 1 (1950) 70-73.-Key to spp.

Woodson, Philip. J. Sc. 60 (1936) 209-227, t. 1. -Revision; key!

Lepinia: Hosokawa, Tokyo Bot. Mag. 48 (1934) 528-530, 1 fig.-Revision; key!

Lepiniopsis: Valeton, Ann. Jard. Bot. Btzg 12 (1895) 249-253, t. 28.-Monotypic.

Mixantum: PichoN, Bull. Mus. Hist. Nat. Paris II, 20 (1948) 382.-Monotypic, aff. Beaumontia; from Puket.

Neokeithia: Pichon, Bull. Mus. Hist. Nat. Paris II, 21 (1949) 375-377.-Descr. seed structure. VAN SteEnIS, Bull. Bot. Gard. Btzg III, 17 (1948) 407-408.-Type descr.; Borneo.

Neuburgia $=$ Crateriphytum $=$ Couthovia $($ Loganiaceae).

Ochrosia: Merrill \& Perry, J. Arn. Arb. 24 (1943) 213-214.-Raising Bleekeria, a subgenus of Ochrosia, to generic rank.

Pichon, Bull. Mus. Hist. Nat. Paris II, 19 (1947) 205-212.-Sections \& series; keys to spp. studied.

VALETON, Ann. Jard. Bot. Btzg 12 (1895) 233237, t. 22-25.-Revision; no key.

Parameria: Moldenke, Revista Sudamer. Bot. 6 (1940) 176-178.-P. barbata should be named $P$. laevigata (Juss.) MoLD. 
Parsonsia (Helygia, Lyonsia): Blake, Proc. R. Soc. Queensl. 59 (1948) 164-167.-Nomencl. not. Austral. spp.

MERRILL, Brittonia 1 (1933) 233-237.-Nomencl. PichoN, Not. Syst. 14 (1950) 4-21.-Partly with keys.

Plumeria: Van Steenis, Trop. Natuur 26 (1937) 63-66.-Key Mal. spp.

Woodson, Ann. Missouri Bot. Gard. 25 (1938) 202-224.-Revision; key!

Rauwolfia (Huntera); Hallier $f$., Meded. Rijksherb. Leiden 1 (1910) 26.-Huntera reduced.

Pichon, Bull. Soc. Bot. Fr. 94 (1947) 31-39.-14 sections; descr.; syn. of listed species.

Rendle, Proc. Linn. Soc. Lond. 149th session, pt 3 (1937) 106-109, 1 fig.-Identity Linnean spp.; syn.

VALETON, Ic. Bog. 4 (1913) 207-210, t. 367.Critical notes on $R$. densiflora BTH.

Strophanthus: Franchet, Nouv. Ann. Mus. Hist. Nat. Paris IV, 5 (1893) 221-294, t. 7-17.

Monachino, Phytologia 3 (1951) 366-373, pl. 1-3.-New sect.

Pax, Bot. Jahrb. 15 (1893) 362-386, t. 10-11.Revision; key!

Staner \& Michotte, Bull. Jard. Bot. Brux. 13 (1934) 23-56.-African spp.

Toxopeus, Meded. Alg. Proefst. Landb. Btzg no 74 (1948) 1-22.-Biology of fruit setting in S. gratus.

WIT, Landbouw 17 (1941) 98-105.-Ditto.

Tabernaemontana: HaLlier $f$., Bot. Jahrb. 49 (1913) 372-375.-Critical remarks on generic segregation of the genus.

Markgraf, Nova Guinea 14 (1927) 278-281. -, Notizbl. Berl. Dahlem 12 (1935) 550.

Merrill, Contr. Arn. Arb. 8 (1934) 139-144.Author expresses doubt about the generic distinction of Ervatamia.

Pichon, Not. Syst. 13 (1948) 230-254.-Genus subdivided into 8 subgen., each with mostly several sect.

Thevetia: Pichon, Bull. Mus. Hist. Nat. Paris II, 22 (1950) 291-294.-Descr. fruit structure.

Trachelospermum: PICHON, Bull. Mus. Hist. Nat. Paris II, 20 (1948) 190-192.-Subdiv.; no key.

TsIANG, Sunyatsenia 4 (1939) 29-30.-New sp. from Java.

Woodson, Sunyatsenia 3 (1936) 65-105.-Revision Asiat. spp.; key.

Vallariopsis: WoODson, Philip. J. Sc. 60 (1938) 228-229.-Monotypic; Mal. Pen.

Vinca: Pichon, Bull. Mus. Nat. Hist. Nat. Paris II, 23 (1951) 439-444.-Revision; key.

Voacanga (Orchipeda, Pootia): Mrquel, Ann. Mus. Bot. Lugd.-Bat. 1 (1864) 316-317.

Pichon, Bull. Mus. Hist. Nat. Paris II, 19 (1947) 409-416.-Key to African spp.

ROLFE, J. Bot. 21 (1883) 201-202.-Nomencl. spp.

Wrightia: PIchon, Not. Syst. 14 (1951) 77-87.With keys to spp.

TsIANG, Sunyatsenia 4 (1939) 42-54.-Critical list of spp.
APONOGETONACEAE

Local

Asia: CAMus, Not. Syst. 2 (1912) 202-204.-Key to Asiat. spp.

New Guinea: Martelli, Nuovo Giorn. Bot. Ital. II, 3 (1897) 472-473, t. 8.-Descr.

Van Steenis, J. Arn. Arb. 28 (1947) 419.

\section{Genera}

Aponogeton: Camus, Bull. Soc. Bot. Fr. 70 (1923) 670-676.-Revision; key!

ENGLer, Bot. Jahrb. 8 (1887) 261-274, f. A, t. 6.-Revision; key!

Krause, Pflanzenreich Heft 24 (1906) 1-24, fig. 1-9.

Van Steenis, Fl. Mal. I, 4 (1948) 11-12, fig. 1.

\section{APOSTASIACEAE}

Blume, C. L., Apostasieae (in Hoeven \& De VResE, Tijd. Nat. Geschied. \& Phys. 1, 1834, 137142).-Census; in Latin; no keys.

Pfitzer, E., Orchidaceae-Pleonandrae (Pflanzenreich Heft 12, 1903, 1-9).

Rolfe, R. A., A morphological and systematic review of the Apostasiaceae (J. Linn. Soc. Bot. 25, 1889, 211-243, t. 48).-Revision; ssp. enumerated.

\section{Local}

Indo-China: Gagnepann, Bull. Soc. Bot. Fr. 80 (1933) 348-353.-A new genus; some new spp.

Sumatra: Holtrum, Orchids of Malaya (1953) 59-65.-Keys!

Smith, in Fedde, Rep. 32 (1933) 129-131.-List.

Malay Peninsula: Ridley, J. Linn. Soc. Bot. 32 (1896) 415-416.-List. -, Mat. Fl. Mal. Pen. (Monoc.) 1 (1907) 231233.-Keys.

Java: BACKer, Bekn. Fl. Java (em. ed.) 10 (1949) fam. 240 , p. $1-3$.

Borneo: SmITH, J. J., Mitt. Inst. Allg. Bot. Hamburg 7 (1927) 10-11.-New sp. and var. of Apostasia.

Celebes: SmiтH, J. J., Bot. Jahrb. 65 (1933) 449450.-New Apostasia.

New Guinea: RidLeY, J. Bot. 24 (1886) 355-356, t. 271-New Neuwiedia.

SmITH, J. J., Nova Guinea 8 (1911) 522-523, t. 80.-New Neuwiedia.

\section{Genera}

Adactylus: Cretziou, J. Jap. Bot. 17 (1941) 407408, map 2.-Two new comb.

Neuwiedia: SMiTH, J. J., Bull. Jard. Bot. Btzg II, no 4 (1914) 1-8.-Critical new descr. spp.; subdiv. genus.

Schoenomorphus $=$ Tropidia (Orchidaceae) .

\section{AQUIFOLIACEAE}

LOesener, TH., Vorstudien zu einer Monographie der Aquifoliaceen (Verh. Ver. Prov. Brandenburg 33, 1892, 1-45, fig. 1-12).-General morphol.; biol.; anat.; key to subdiv. of Ilex.

-, Monographia Aquifoliacearum (Nova Acta 
Acad Caes. Leop.-Car. Nat. Cur. 78, 1901, viii +570 pp.; ibid 89, 1908, 313 pp.).

—, Pflanzenfamilien ed. 2, 20b (1942) 36-86.

\section{Local}

Sumatra: Merrill, Pap. Mich. Acad. Sc. Arts \& Lett. 19 (1934) 162-163.-3 new spp.

Borneo: MERRILL, Sarawak Mus. J. 3 (1928) 526527.-New Ilex.

—, J. Arn. Arb. 20 (1939) 222-224.-2 new spp. of Ilex with opposite leaves.

RIDLeY, Kew Bull. (1931) 34.-New Ilex.

-, ibid. (1939) 409-411 (sphalm. 509-511).List; new Ilex spp.

Philippines: Elmer, Leafl. Philip. Bot. 5 (1913) 1663-1669.-List Ilex; 4 new spp.

Merrull, Philip. J. Sc. 26 (1925) 468-469.New Ilex.

-, ibid. 27 (1925) 31.-New Ilex.

Celebes: Capitaine, Bull. Soc. Bot. Fr. 57 (1910) 234-236, t. 10.-New Ilex.

New Guinea: LOESENER, Bot. Jahrb. 59 (1924) 80 83.-Key.

Merrill \& Perry, J. Arn. Arb. 20 (1939) 333-

334.-Descr. new spp.; records.

-, ibid. 22 (1941) 258-260.-Ditto.

—, ibid. 30 (1949) 40.-Ditto.

\section{Genera}

Ilex: Comber, Not. R. Bot. Gard. Edinb. 18 (1933)

37-62.-List Chin. \& Himal. spp.; several new.

Hallier $f$., Rec. Trav. Bot. Néerl. 15 (1918) 66. -Reduction Octas.

Hu, J. Arn. Arb. 30 (1949) 233-344, 348-387.Revision of Chin. spp.

—, ibid. 31 (1950) 39-80, 214-263.-Ditto.

-, ibid. 34 (1953) 138-162.-Spp. from Formosa \& Riu Kiu; key!

LOESENER, Mitt. Deut. Dendr. Ges. 28 (1919) 1-66.

-Subdiv. of Aquifoliaceae; key to genera; in-

frageneric key to Ilex; key to spp. cult. in Europe.

Maximowicz, Mém. Acad. Imp. St Pétersb. VII, $29^{3}$ (1881) 14-53.-Annot. in Ilex.

VAN SteEnIS, Bull. Bot. Gard. Btzg III, 17 (1948)

389-390.-Occurrence of stipules.

Sphenostemon (Idenburgia, Nouhuysia): GibBs, Contr. Fl. Phytogr. Arfak Mts (1917) 136-140,

fig. 10-11.-Descr. Idenburgia.

LAuTERbach, Nova Guinea 8 (1912) 843-844.-

Descr. Nouhuysia.

Van Steens, Acta Bot. Neerl. 1 (1952) 94-98, fig. 2.-Reduction Idenburgia.

-, Svensk Bot. Tidskr. (in the press).-Reduction.

\section{ARACEAE}

Alderwerelt van Rosenburgh, C. $R$. W. $K$. VAN, New or noteworthy Malayan Araceae I (Bull. Jard. Bot. Btzg III, 1, 1918, 359-389); ditto II (ibid. 4, 1922, 163-229); ditto III (ibid. 4, 1922, 320-347).-Practical key to Mal. genera; addit. descr. of new spp.

Brown, N. E., On some new Aroideae; with observations on other known forms. Part 1 (J.
Linn. Soc. Bot. 18, 1880, 242-263, t. 4-6).Misc. notes and records.

Engler, A., in DC. Mon. Phan. 2 (1879) 1-681. -, Neue Araceen vom Indischen Archipel (Bot. Jahrb. 1, 1881, 179-186).-Notes and descr.

- Aracee della Malesia e della Papuasia raccolte da O. Beccari (Malesia 1, 1885, 259-304, t. 16-28).-Important treatments; descr. and some keys. In Latin.

- Araceae novae Asiae tropicae et subtropicae (Bot. Jahrb. 25, 1898, 1-28).-List records \& descr.

-, Beiträge zur Kenntniss der Araceae X (ibid. 37, 1906, 110-143).-Ditto.

- Pothoideae (Pflanzenreich Heft 21, 1905. 1-330, fig. 1-88); additamentum (ibid. Heft 37, 1908, 2-3, 138-139).-Acorus, Anadendron, Anthurium, Pothoideum, Pothos.

-, Lasioideae (ibid. Heft 48, 1911, 1-130, fig. 1-44).-Amorphophallus, Cyrtosperma, Lasia, Podolasia.

-, Philodendroideae - Anubiadeae, Aglaonemateae, Dieffenbachieae, Zantedeschieae, Typhonodoreae, Peltandreae (ibid. Heft. 64.1915 1-78, fig. 1-34).-Aglaodorum, Aglaonema, Dieffenbachia, Zantedeschia.

-, Aroideae - Pistioideae (ibid. Heft 73, 1920, 1-274, fig. 1-64).-Arisaema, Arum, Pistia, Sauromatum, Typhonium.

-, \& K. KRAUSE, Monsteroideae (ibid. Heft 37, 1908, 4-138, fig. 1-56).-Amydrium, Epipremnum, Holochlamys, Monstera, Raphidophora, Scindapsus, Spathiphyllum.

-, Philodendroideae - Philodendreae - Homalomeninae, Schismatoglottidinae (ibid. Heft 55 , 1912, 1-134, fig. 1-77).-Bucephalandra, Diandriella, Gamogyne, Homalomena, Microcasia, Piptospatha, Schismatoglottis.

-, Colocasioideae (ibid. Heft 71, 1920, 1-139, fig. 1-29).-Alocasia, Aridarum, Caladium, Colocasia, Remusatia, Schizocasia, Syngonium, Xanthosoma.

-, Araceae, pars generalis et index familiae generalis (ibid. Heft 74, 1920, 1-71).-General considerations; system of the family.

Krause, K., Philodendroideae-Philodendreae-Philodendrinae (ibid. Heft 60, 1913, 1-143, fig. 145).-Philodendron.

Miquel, F. A. W., in de Vriese, Pl. Ind. Bat. Or. (1856) 148-153.-List; descr. new spp.

-, Mantissa Aroidearum indicarum (Ann. Mus. Bot. Lugd.-Bat. 3, 1867, 79-82).

Schотт, H. W., Synopsis Aroidearum, etc. (1856) 1-140.-Enum.; many descr.

-, Genera Aroidearum exposita. Vienna (1858) 1-101, t. 1-98.-Plates and descr. of genera; no key to these.

-, Aroideenskizzen (Bonplandia 7, 1859, 30-31, 164).-Descr. new spp. of Homalomena and Chamaecladon.

-, Prodromus systematis Aroidearum (1860) 1-602.-Monograph; no keys!

-, in Miquel, Ann. Mus. Bot. Lugd.-Bat. 1 (1863-64) 122-131, 221, 278-286.-Records; list; new $s p p$. 


\section{Local}

Sumatra: Merrill, Pap. Mich. Acad. Sc. Arts \& Lett. 19 (1934) 152.-New Homalomena.

-, J. Mal. Br. R. As. Soc. no 87 (1923) 104-106. -List; new Pothos and Homalomenas.

-, Kew Bull. (1925) 92-93.-List; 2 new spp. -, ibid. (1926) 92-93.-New Pothos.

De Vriese, in Miquel, Pl. Jungh. (1852) 99106.-List; several new Pothos.

Malay Peninsula: Furtado, Gard. Bull. Str. Settl. 8 (1935) 145-158. - New descr. and reductions in Cryptocoryne, Pothos, Rhaphidophora, Scindapsus.

一, M. A. H. A. Mag. 10 (1941) 11-17.-Key to edible aroids.

Ridley, Mat. Fl. Mal. Pen. (Monocot.) pt 3 (1908) 1-52.-Keys.

Java: BACKER, Trop. Natuur 9 (1920) 1-4, 19-32. -Key to Jav. Amorphophallus.

KoORDers, Bull. Jard. Bot. Btzg III, 1 (1919) 159-161.-Note on Cyrtosperma merkusii.

-, ibid. III, 1 (1919) 162-167, t. 5-7.-Descr. new Alocasia.

MiQuel, Berl. Bot. Zeit. 14 (1856) 561-565.Descr.

VAN SteEnIs, Bull. Jard. Bot. Btzg III, 17 (1948) 403.-New comb. in Typhonium.

—, ibid. III, 17 (1948) 447-456.-Key to Jav. Arisaema.

-, c.s., Blumea 6 (1950) 359.-New comb. in Homalomena.

Borneo: Brown, J. Bot. 20 (1882) 195-196.Descr. Gamogyne.

ENGLer, Bull. Soc. Tosc. Ort. (1879) 265-271; 295-302.-Misc. descr. coll. BecCARI.

— \& Krause, Bot. Jahrb. 48 (1912) 93-95.-List; some new Homalomenas and Schismatoglottis. Furtado, Gard. Bull. Str. Settl. 8 (1935) 145158.-New spp. and critical remarks in Pothos, Rhaphidophora, Schismatoglottis, and Scindapsus. MERrILl, J. Str. Br. R. As. Soc. no 85 (1922) 159-160.-New Schismatoglottis.

—, Philip. J. Sc. 29 (1926) 353-354.-List; new Scindapsus.

Ridley, J. Str. Br. R. As. Soc. no 44 (1905) 169188.-List; many new spp.

-, J. Bot. 51 (1913) 201-202, t. 527.-Aridarum n.g.; critical remarks on Piptospatha and $\mathrm{Ga}$ mogyne.

DE WrT, Webbia 9 (1954) 455-458, fig.1.-Descr. neotype Cryptocoryne longicauda BECC. \& ENGL.

Celebes: BoK \& LAM, Blumea 2 (1936) 33, 1 fig.New Amorphophallus.

KRaUSE, Notizbl. Berl.-Dahlem 11 (1932) 331332.-New Rhaphidophora.

Philippines: Elmer, Leafi. Philip. Bot. 10 (1938) 3611-3635; ibid. 10 (1939) 3699-3704.-New spp.

Krause, Bot. Jahrb. 44 (1910) Beibl. 101, p. 11-12.-Some new Rhaphidophora.

-, ibid. 45 (1911) 657-660.-Descr. new spp.

—, Notizbl. Berl.-Dahlem 5 (1912). 266-267.-

Descr. 2 new spp.

一, in Elmer, Leafl. Philip. Bot. 6 (1914) 2284

2285.-Descr. 2 new Rhaphidophoras.
MerrilL, Philip. J. Sc. 21 (1922) 516.-New Pothos.

-, ibid. 26 (1925) 451-452.-New Rhaphidophora \& Aglaonema.

Quisumbing \& Merrill, Philip. J. Sc. 37 (1928) 136.-New Schismatoglottis.

SCHOTT, Bonplandia 10 (1862) 148.-Descr. Alocasia.

New Guinea: Engler, Bull. Soc. Tosc. Ort. (1879) 265-271, 295-302.-Misc. descr. coll. BECCARI. -\& Krause, Nova Guinea 8 (1910) 247-252. -, ibid. 8 (1912) 805-809, t. 147-148.

-, Bot. Jahrb. 49 (1912) 90-99.

一, ibid. 54 (1916) 74-91.-New spp.; no keys.

KraUSE, Bot. Jahrb. 45 (1911) Beibl. 104, p. 84. -New Epipremnum.

- \& Van Alderwerelt van Rosenburgh, Nova Guinea 14 (1924) 210-220.-List; new spp.

\section{Genera}

Alocasia: Furtado, Gard. Bull. Str. Settl. 11 (1941) 244-257.-A. macrorrhiza and its varieties.

Amorphophallus: Blume ex Decaisne, Nouv. Ann. Mus. Paris 3 (1834) 366.'

WINKLER, Ber. Deut. Bot. Ges. 49 (1931) 91-101, t. 6-11.-Details on A. titanum.

Arisaema: BUCHET, Not. Syst. 1 (1911) 366-375.New spp.

Colocasia: HiLl, Bot. Mus. Leafl. Harv. 7 (1939) 113-118.-Nomencl. C. esculenta; varieties.

Cryptocoryne: Engler, in BecCari, Malesia 1 (1883) 296-300.-Key!

Petch, Ann. R. Bot. Gard. Peradeniya 11 (1928) $11-26$, t. 2-5.-Notes on some Ceylon spp.

Epipremnum: EnGLER, in BeCCARI, Malesia 1 (1885) 272-275.-Key!

Homalomena: Furtado, Gard. Bull. Str. Settl. 10 (1939) 183-238.-Synopsis Indo-Mal. spp.; no key.

Piptospatha (Gamogyne): EnGLer, Pflanzenreich Heft 71 (1920) 2.-Reduction.

Rhaphidophora: ENGLER, in BECCARI, Malesia 1 (1885) 266-272.-Key!

Schismatoglottis: ENGLER, in BeccARI, Malesia 1 (1885) 284-288.-Key!

Scindapsus: ENGLER, in BeCCARI, Malesia 1 (1885) 275-278.-Key!

\section{ARALIACEAE}

Miquel, F. A. W., in DE VRIESE, Pl. Ind. Bat. Or. (1856) 81-92.

-, Ann. Mus. Bot. Lugd.-Bat. 1 (1864) 1-27,

(1) The first legitimate description of Amorphophallus has been referred bij BLume to 'Bat. Courant 1825', and this source has always been copied. However, the instalment of this Government issue of Nov. 23rd, 1825, contained only a short, clear description in Dutch of an aroid which BLUME claims to represent a new genus and which he expects to describe and name in a future paper. As far as we can trace the name Amorphophallus was first mentioned in 1834. 
219-220.-Descr. new gen. \& spp.; list; no keys!

SeEmanN, B., Revision of the natural order Hederaceae (J. Bot. 2, 1864, 235-250, 289-309; ibid. $3,1865,73-81$, t. $27,173-181,265-276,361-$ 363 , t. 41; ibid. 4, 1866, 293-299, 352-353; ibid. $5,1867,236-239,285-286$; ibid. 6, 1868, 52-58, 129-142, 161-165).

-, A revised reprint of the same work (1868) 107 pp.

Viguier, R., Sur les Araliacées du groupe de Polyscias (Bull. Soc. Bot. Fr. 52, 1905, 285-314).

-, Recherches anatomiques sur la classification des Araliacées (Ann. Sc. Nat. Bot. IX, 4, 1906, 1-210).-New system of the family; see critics by Harms, Bot. Jahrb. 40 (1907) Lit.-Ber. 2227.

-, Nouvelles recherches sur les Araliacées (ibid. IX, 9, 1909, 305-405).

\section{Local}

North America: SmITH, A. C., Brittonia 2 (1936) 247-261.-List.

China: LI, Sargentia 2 (1942) 1-134, fig. 1-14.Survey of Chin. Aral.; keys.

- Proc. Acad. Nat. Sc. Philad. 96 (1944) 249277, map.-Phytogeogr. div. of China based on distr. of Aral.

Sumatra: Merrill, Contr. Arn. Arb. 8 (1934) 116-121.-New Trevesia and Scheffleras.

-, Pap. Mich. Acad. Sc. Arts \& Lett. 19 (1934) 177-179.-3 new Scheffleras.

-, ibid. 23, 1937 (1938) 186-187.-New Schefflera.

RidLey, J. Mal. Br. R. As. Soc. no 87 (1923) 64. -List; new Aralia.

De VRIEsE, Ned. Kruidk. Arch. 1 (1846) 15-19. -Some descr. of Aralia and Arthrophyllum.

Malay Peninsula: Henderson, Gard. Bull. Str. Settl. 7 (1933) 105-106, t. 22-23.-New Schefflera and Acanthopanax.

Ridley, Kew Bull. (1929) 124.-Second sp. of Hederopsis.

Java: BAKHUIZEN VAN DEN BRINK \& VAN OOSTSTROOM, in BACKER, Bekn. Fl. Java (em. ed.) afl. 7 (1948) fam. 159, p. 1-19.-In Dutch; keys. Koorders, Bull. Jard. Bot. Btzg III, 1 (1919) 181-186, t. 16-17.-New Pentapanax.

MiQuel, Plant. Jungh. (1855) 420-426.-Many new spp. and new gen.

Borneo: Merrill, Philip. J. Sc. 13 (1918) Bot. 99-103.-4 new Scheffleras.

-, J. Str. Br. R. As. Soc. no 79 (1919) 27-31.New spp. of Schefflera; no key.

—, Sarawak Mus. J. 3 (1928) 537-538.-Two new Schefferas.

Philipson, J. Bot. 78 (1940) 116-119.-List Clemens records; new sp. in Polyscias and Gilibertia.

Philippines: Elmer, Leafl. Philip. Bot. 6 (1914) 2325-2341.-New spp. Mindanao.

一, ibid. 10 (1938) 3637-3648, 3704-3705.New spp. of Schefflera; no key.

Merrill, Philip. J. Sc. 10 (1915) Bot. 195-205.New Scheffleras.
-, ibid. 26 (1925)

Schefflera.

Celebes: Harms, Ann. Jard. Bot. Btzg 19 (1904)

12, 17-18.-New Tetraplasandra, 2 Scheffleras. -, in FEDDE, Rep. 3 (1907) 23-24.-New Schefflera.

New Guinea: Harms, Nova Guinea 8 (1910) 271277, t. 62-64.

-, Bot. Jahrb. 56 (1920) 374-414, f. 1-4.-

Revision; keys!

—, ibid. 69 (1938) 277-283.-Descr.

-, J. Arn. Arb. 20 (1939) 321-323.

-, Bot. Jahrb. 72 (1942) 205-207.-3 new Scheffleras.

Philipson, Bull. Br. Mus. Nat. Hist. Bot. 1 (1951) 9-18.-New spp. from New Guinea \& Solomons.

RIDLEY, J. Bot. 52 (1914) 290-291.-2 new Scheffleras.

Solomon Islands: HaRms, Notizbl. Berl.-Dahlem 15 (1942) 677-680.-List coll. WATERHOUSE; 3 new spp.

\section{Genera}

Acanthopanax: Harms, Mitt. Deut. Dendr. Ges. 27 (1918) 1-39, t. 1-8b.-Keys.

Acanthophora: Van Steenis, Bull. Bot. Gard. Btzg III, 17 (1948) 390-391.-Distr.

Aralia: Harms, Bot. Jahrb. 23 (1896) 1-20.Revision; list; no key.

Van STeEnIS, Bull. Bot. Gard. Btzg III, 17 (1948) 391-395, fig. 1.-Critical notes.

Boerlagiodendron (Eschweileria, Osmoxylon): BECCARI, Malesia 1 (1878) 193-198.-Census.

Boerlage, Ned. Kruidk. Arch. 4 (1886) 1-15.Remarks.

-, Ann. Jard. Bot. Btzg 6 (1887) 112-125, t. 13-16.-Key!

HaRMs, Bot. Jahrb. 56 (1921) 377-384.-Nearly a revision; key.

Dendropanax (Gilibertia R. \& P. non al.): MERRILL, Brittonia 4 (1941) 129-134.-List of Old World spp.

Philipson, Bull. Br. Mus. Nat. Hist. Bot. 1 (1951) 18-19.-Critical remarks.

Smith, A. C., Trop. Woods no 66 (1941) 1-3.-Dendropanax should be conserved; new comb.

Harmsiopanax (Horsfieldia non WILLD., BL.): Harms, Bot. Jahrb. 56 (1920) 413.

Macklinlaya (incl. Anomopanax): Harms, Ann. Jard. Bot. Btzg 19 (1904) 13-16.-Enum.

Philipson, Bull. Br. Mus. Nat. Hist. Bot. 1 (1951) 3-9.-Revision; key!

Nothopanax: MerrilL, Philip. J. Sc. 7 (1912) Bot. 241-243.-List.

Sprague \& Green, Kew Bull. (1933) 154-155.Nomencl.

Panax: Harms, Bot. Jahrb. 23 (1896) 1-23.Delimitation against Aralia.

Peekeliopanax: HARMS, Notizbl. Berl.-Dahlem 9 (1929) 478-484.-Spp. from New Britain.

Pentapanax: Harms, Bot. Jahrb. 23 (1896) 20-22. -Some critical remarks.

Koorders, Bull. Jard. Bot. Btzg III, 1 (1919) 181-186, t. 16-17.-New sp. from Java. 
Schefflera (Sciodaphyllum): SмIтн, A. C., Trop. Woods no 66 (1941) 3-6.-Nomencl.

Tetraplasandra: HARMs, Ann. Jard. Bot. Btzg 19 (1904) 12.-New sp. from Celebes.

SherfF, in SherfF, Bot. Leafl. no 6 (1952) 1941.-Descr. Pac. spp.

-, ibid. no 8 (1953) 1-13.-Further notes.

Trevesia: BoERlage, Ned. Kruidk. Arch. 4 (1886) 1-15.-Remarks; new sp.

-, Ann. Jard. Bot. Btzg 6 (1887) 106-112, t. 11-12.-Key!

\section{ARAUCARIACEAE \\ See Coniferae}

\section{ARISTOLOCHIACEAE}

Schmidt, O. C., Pflanzenfamilien ed. 2, $16 \mathrm{~b}$ (1935) 204-242, fig. 103-123.

\section{Local}

Sumatra: Merrill, Pap. Mich. Acad. Sc. Arts \& Lett. 23, 1937 (1938) 178.-New Apama.

Java: BACKER, Trop. Natuur 7 (1918) 177-183; ibid. 8 (1919) 133-138, 150-155, 161-168.-Key to wild \& cult. spp. in last ref.

-, Bull. Jard. Bot. Btzg III, 2 (1920) 320-322.2 new Aristolochias.

Philippines: MerrILL, Philip. J. Sc. 29 (1926) 478. -New Aristolochia.

Quisumbing, Philip. J. Sc. 41 (1930) 322-323.New Thottea.

Warburg, in Perkins, Fragm. Fl. Philip. (1905) 169-170.-2 new Aristolochias.

New Guinea: LAUTERBACH, Bot. Jahrb. 52 (1914) 104-107.-Key.

Merrill \& Perry, J. Arn. Arb. 23 (1942) 383384.-Descr.

一, ibid. 29 (1948) 152-154.-Descr.

SchmidT, Bot. Jahrb. 58 (1923) 488-491.-New spp.

-, in FEDdE, Rep. 23 (1927) 298-299.-New comb. \& record.

\section{Genera}

Apama (Bragantia): VAleton, Ic. Bog. 3 (1908) 153-154, t. 160.-New sp. from Sumatra.

Aristolochia: MASTERS, J. Linn. Soc. Bot. 14 (1875) 387-495.

SchmidT, in Fedde, Rep. 30 (1932) 72-75.Austral. spp.; key!

Thottea: BECCARI, Nuovo Giorn. Bot. Ital. 2 (1870) 5-8, t. 1.-2 new sp. from Borneo.

VAN Steenis, Bull. Jard. Bot. Btzg III, 12 (1932) 204-207.-New sp. from Natuna Isl.

VAleton, Ic. Bog. 3 (1908) 155-156, t. 161.New sp. from Borneo.

\section{ASCLEPIADACEAE}

Blume, C. L., Mus. Bot. Lugd.-Bat. 1 (1849) 43$46,57-60,125-126$; ibid. (1850) 146-150.Descr.

GoOD, R., An atlas of the Asclepiadaceae (New Phytol. 51, 1952, 198-209, maps 1-17).-Distr. of tribes and some genera.

LUtckнof, C. A., The Stapelieae of South Africa (1952) 283 pp., t. 1-5, photographs.
SChlechter, R., Beiträge zur Kenntnis der Asclepiadaceen des Monsun-Gebietes (Bot. Jahrb. 40 , 1908, Beibl. 92, 1-19).-Misc. new spp.; Oistonema, Anatropanthus n.g.

-, Beih. Bot. Centralbl. 34 Abt. 2 (1917) 1-18. -Misc. new gen. \& spp.

- \& WarbURG, O., Asclepiadaceae novae Asiae australis et orientalis (in FEDDE, Rep. 3, 1907, 305-315, 339-347).-New spp.

White, A. \& B. L. Sloane, c.s., The Stapelieae. 2nd ed. Pasadena, Cal. (1937) 3 vols, 1233 fig. 39 col. pl.-Revision; keys.

\section{Local}

North America: Woodson, Ann. Missouri Bot. Gard. 28 (1941) 193-244.-Keys to genera \& sections.

Asia: Tsiang, Sunyatsenia 2 (1934) 174-202; ibid. 3 (1936) 160-240; ibid. 4 (1939) 54-94; ibid. 6 (1941) 169.-Various notes.

India: WIGHT, Contr. Bot. India (1834) 29-67.Revision; partial keys.

Siam: KerR, Kew Bull. (1938) 445-454.-Descr. new spp.

-, ibid. (1939) 456-465.-Ditto.

-, Fl. Siam. En. 3 (1951) 1-51.-List.

Sumatra: Ridley, J. Fed. Mal. Stat. Mus. 8, 4 (1917) 62-63.-New Hoya, Dischidia, and Hoyella.n.g.

一, J. Mal. Br. R. As. Soc. no 87 (1923) 77-78.New Hoya and Dischidia.

-, Kew Bull. (1925) 85-86.-New Hoya. -, ibid. (1926) 74.-List; new Hoya.

Malay Peninsula: Henderson, Gard. Bull. Str. Settl. 7 (1933) 109-113, t. 26-27.-New Tylophora and 2 Toxocarpus.

Java: BACKER, Ic. Bog. 3 (1908) 157-159, t. 262.Critical notes on Hoya densifolia.

BAKhuIzen VAN DEN BRINK, in BACKer, Bekn. Fl. Java (em. ed.) 8 (1949) 1-65.-In Dutch; keys! -, Blumea 6 (1950) 368-382.-Critical notes; new spp. in Cynanchum, Toxocarpus, Tylophora, Dischidia, Hoya, and Heynella n.g.

Borneo: Merrill, Philip. J. Sc. 13 (1918) Bot. 120-121.-New Ceropegia.

Schwarz, Mitt. Inst. Allg. Bot. Hamburg 7 (1931) 259-262.-List; new spp. in Hoya and Dischidia.

Philippines: Elmer, Leafl. Philip. Bot. 10 (1938) 3543-3599.-List; descr.; no keys.

Koorders, Philip. J. Sc. 15 (1919) 263-265.Some remarks on a Hoya.

Merrill, Philip. J. Sc. 7 (1912) Bot. 243.-New comb. in Telosma (Pergularia)

一, ibid. 27 (1925) 53-54.-New Tylophora. -, ibid. 60 (1936) 33.-New gen. name.

Schlechter, Philip. J. Sc. 1 (1906) Suppl. 295303, 453-472.

—, in FEDDE, Rep. 13 (1915) 537-544; ibid. 554 566.

Celebes: Koorders, Bull. Jard. Bot. Btzg III, 2 (1920) $245-254$, t. 4-6.-Critical note on Hoya maxima.

New Guinea: Markgraf, J. Arn. Arb. 10 (1929) 84.-1 n. sp. from Papua. 
Norman, Brittonia 2 (1937) 328.-2 new Hoyas. RIDLEY, J. Bot. 52 (1914) 293-294.-3 new Hoyas.

SCHLECHTER, Bot. Jahrb. 50 (1913) 81-164.Enum. NE. New Guinea; many new spp.; Astelma n.g.

Van SteEnIs, Blumea 7 (1954) 596.-Records of both Phyllantheras.

\section{Genera}

Asclepias: Woodson, Ann. Missouri Bot. Gard. 41 (1954) 1-211, 261, 114 fig.-Monogr.; key!

Ceropegia: Tsiang, Sunyatsenia 4 (1940) 290.Nomencl. note.

Dischidia (Conchophyllum): BecCARI, Malesia 2 (1878) 248-274, t. 60-63.-Synopsis; descr.; no key.

Gomphocarpus: Bulzocx, Kew Bull. (1952) 405410.-Critical list Afr. spp.; no key.

Hoya: BLume, Rumphia 4 (1849) 29-33, t. 182188.-Some nice plates; enum. spp.

Parsonsia: Merrill, Brittonia 1 (1933) 233-237.Syn. of $P$. helicandra.

Pentasacme (Spiladocorys): Schlechter, J. Bot. 34 (1896) 15-16.-Reduction; revision; no key.

Pergularia-Telosma: Brown, Kew Bull. (1907) 323-325.-Nomencl.

Phyllanthera: Van Steenis, Bull. Bot. Gard. Btzg III, 18 (1950) 459.-Key.

Quisumbingia (Schlechterianthus, Petalonema): Merrill, Philip. J. Sc. 60(1936) 33.-Nomencl.

Sarcostemma (incl. Oxystelma): Woodson, Ann. Missouri Bot. Gard. 37 (1950) 477-560.

Wattakaka (Dregea \& Wattakaka): STAPF, Bot. Mag. (1922) 4 pp., t. 8976.-Java.

\section{AVICENNIACEAE \\ See Verbenaceae}

\section{BALANOPHORACEAE}

Harms, H., Pflanzenfamilien ed. 2, 16b (1935) 282-296.

HOOKER, J. D., On the structure and affinities of Balanophoraceae (Trans. Linn. Soc. Lond. 22, 1859 , 1-68, t. 1-16).-Synopsis of the family; no keys.

JunGHUHN, F., Uber Javan'sche Balanophoreen (Nova Acta Acad. Caes. Leop.-Car. Nat. Cur. 18, Suppl. 1841, 201-228, t. 1-2).-Survey Jav. spp. and descr. Rhopalocnemis.

Setchell, W. A., Balania harlandii (Hook. f.) Tiegh. of the Hong Kong region and its relatives (Hong Kong Naturalist Suppl. 1, 1932, 2-14, t. 1-9).-Discussion subdiv. in Balanophora, creating a new genus; E. Asiat. sp.; no Mal. spp.

-, Acroblastum vs. Polyplethia: a complex of the Balanophoraceae (Univ. Calif. Publ. Bot. 19, 1935, 141-158, t. 16-19).-Segregation of Acroblastum from Balanophora; incl. several Mal. spp.

\section{Local}

Java: Junghunn, Nova Acta Acad. Caes. Leop.Car. Nat. Cur. 18, Suppl. 1841, 201-228, t. 1-2.Descr.
Van Steenis, Arch. Hydrobiol. Suppl. 10 (1932) 301-302.-Reduction Balanophora alutacea; descr. B. abbreviata.

Borneo: BeCCARI, Atti Soc. Ital. Sc. Nat. 11 (1868) repr. p. 2-3.-Descr. Balanophora reflexa.

-, Nuovo Giorn. Bot. Ital. 1, 1869, repr. 1-20, t. 2-4.-Descr. new sp.; general considerations on Balanophoraceae; bibliogr.; enum. Balanophora spp.

HOOKER f., Trans. Linn. Soc. 22 (1859) 426-427, t. 75.-New Balanophora.

Philippines: Elmer, Leafl. Philip. Bot. 5 (1913) 1659-1662.-List; 3 new Balanophoras.

Warburg, in Perkins, Fragm. Fl. Philip. (1905) 169.-New Balanophora.

Moluccas: Ridley, J. Str. Br. R. As. Soc. no 39 (1903) 207.-New sp. from Tenimber Isl.

New Guinea: Merrill \& Perry, J. Arn. Arb. 23 (1942) 383.-Record.

-, ibid. 29 (1948) 152.-Record.

SCHLECHTER, Bot. Jahrb. 50 (1913) 68-69, fig. 1. -Descr. new sp.

Valeton, Nova Guinea 8 (1913) 919-921, t. 161.-Descr. new sp.

Micronesia: Hosokawa, J. Soc. Trop. Agric. Taihoku Imp. Univ. 6 (1934) 572.-List; one new sp.

\section{Genera}

Balanophora: FAwCETT, Trans. Linn. Soc. II, 2, Bot. (1886) 233-238, t. 33-36.-Synopsis; new spp. from Sumatra, Java, Saleier; key.

GöPPERT, Nova Acta Acad. Caes. Leop.-Car. Nat. Cur. 18 (1842) Suppl. 231-272, t. 1-3.Anat.

Lotsy, Ann. Jard. Bot. Btzg 16 (1899) 174-185, t. 16-19.-Embryol. B. globosa.

Exorhopala: VAN STEENIS, Handel. 6e N.1. Natuurwet. Congr. (1931) 464-473 (1932).

Rhopalocnemis: GöPPERT, Nova Acta Acad. Caes. Leop.-Car. Nat. Cur. 22 (1847) 119-158, t. 11-15.-Structure; anat.

Lorsy, Ann. Jard. Bot. Btzg 17 (1901) 73-101, t. 3-14.-Morph.; embryology.

Van SteEnIS, Handel. 6e N. I. Natuurwet. Congr. (1931) 464-473 (1932).-Descr.; ecol.

\section{BALSAMINACEAE}

Miquel, F. A. W., Ill. Fl. Arch. Ind. (1871) 92104.-Census; descr.

\section{Local}

Malay Peninsula: Henderson, Gard. Bull. Str. Settl. 5 (1930) 72-73, fig.-New Impatiens.

HOOKER $f$., KeW Bull. (1909) 1-12; ibid. (1911) 249-250.-Mal. Pen. \& Indo-Chin. spp.

Sumatra: Hooker $f$., Kew Bull. (1920) 246.-New Impatiens.

-, J. Bot. (1924) Suppl. 27-29.-Descr. new spp.

MIQUEL, Fl. Ind. Bat. Suppl. (1861) 161-162, 395-398.-Descr.

-, Choix (1864) t. 5.-Descr. of a sp.

Ridley, J. Fed. Mal. St. Mus. 8, pt 4 (1917) 2325.-Several new Impatiens. 
Ridley, Kew Bull. (1925) 78.-New sp.

Java: BACKER, Bull. Jard. Bot. Btzg III, 16 (1939) 109-110.-New sp.

Van Steenis, Bull. Bot. Gard. Btzg III, 17 (1948) 384-387.-Key.

Borneo: Hooker f., Kew Bull. (1910) 74-76.Descr. 3 new Impatiens.

Philippines: HoOKer $f$., Kew Bull. (1909) 281-289. -Revision; key to Philip. Impatiens.

New Guinea: Hooker $f$., Bot. Mag. (1909) t. 8247; (1911) t. 8396.

RIDLEY, Trans. Linn. Soc. Lond. II, Bot. 9 (1916) 23-24.-New sp.

SCHLECHTER, Bot. Jahrb. 55 (1918) 114-120, fig. 1.-Key.

\section{Genera}

Impatiens: Hooker $f$, J. Linn. Soc. Lond. Bot. 37 (1904) 22-32.-Interpretation of the spp. in the Wallichian herb. of the Linn. Soc. London. HoOKer $f$., Rec. Bot. Surv. Ind. 4 (1904-06) 110, 11-36, 37-59.-Epitome of Indian spp.

Semeiocardium: BACKER, Gard. Bull. Str. Settl. 9 (1935) $70-73$, t. 2.

\section{BARRINGTONIACEAE See Lecythidaceae}

\section{BASELLACEAE}

BACKer, C. A., Bekn. Fl. Java (em. ed.) 4A (1942) fam. 64, 1-2.-In Dutch; key.

Ulbrich, E., Pflanzenfamilien ed. 2, 16c (1934) 263-291.

\section{Genera}

Boussingaultia: Hauman, Anal. Mus. Nac. Hist. Nat. Buenos-Aires Bot. 33 (1925) 347-359.Revision of the genus; key.

\section{BEGONIACEAE}

Benecke, F., Beitrag zur Kenntnis der Begoniaceen (Bot. Jahrb. 3, 1882, 288-318).

Candolle, A. De, Mémoire sur la famille des Bégoniacées (Ann. Sc. Nat. Bot. IV, 11, 1859, 93-149).-Census; subdiv. of family; descr. sections and new spp.

IRMSCHER, E., Die Verteilung der Geschlechter in den Infloreszenzen der Begoniaceen unter Berücksichtigung der morphologischen Verhältnisse (Bot. Jahrb. 50, Suppl. 1914, 556-577).

-, Pflanzenfamilien ed. 2, 21 (1925) 548-588, fig. 251-268.

_, Ủber eine Abänderung des Zahlenverhältnis zwischen männlichen und weiblichen Blüten bei der monoecischen Begonia wallichiana (Mitt. Inst. Alg. Bot. Hamburg 6, 1927, 149-158).Variability in floral numbers.

- Neue Begoniaceen von O. Beccari in Malesien gesammelt (Webbia 9, 1954, 469-509, fig: 1-8).-Descr. new spp. mainly from Borneo and New Guinea.

KLotzsch, J. F., Begoniaceengattungen und Arten (Abh. Akad. Wiss. Berlin 1854, 121-255, t. 1-12).

\section{Local}

East Asia: IrMSChER, Mitt. Inst. Allg. Bot. Hamburg 6 (1927) 343-360.-Descr. new spp.

Sumatra: Ridley, J. Fed. Mal. Stat. Mus. 8 (4) (1917) 37-41.-Descr. new spp.

-, J. Mal. Br. R. As. Soc. 87 (1923) 62-63.Records; 2 new spp.

一, Kew Bull. (1925) 83.-Records; new sp.

Malay Peninsula: BurkiLL, J. Str. Br. R. As. Soc. 79 (1918) 103.-New sp. Langkawi Isl.

IrMSCHER, Mitt. Inst. Alg. Bot. Hamburg 8 (1929) 86-160.--Revision; key.

RiDley, Kew Bull. (1929) 258.-New sp.

Borneo: IRMSCHER, Webbia 9 (1954) 469-508, fig. 1-8.-Descr: new spp. coll. BECCARI.

Merrill, J. Str. Br. R. As. Soc. no 86 (1922) 334.-New sp.

—, Sarawak Mus. J. 3 (1928) 529-532.-Records; 3 new spp.

Ridley, Sarawak Mus. J. 2 (1916) 177-178.New sp.

WARBURG, in FedDE, Rep. 18 (1922) 329.New sp.

Celebes: Hemsley, Kew Bull. (1896) 37.-New sp. IRMSCHER, Bot. Jahrb. 50 (1913) 335-383.Descr. new spp.

Philippines: Elmer, Leafl. Philip. Bot. 10 (1939) 3706-3709.-New spp.

Merrill, Philip. J. Sc. 6 (1911) Bot. 369-406.Revision; key.

一, ibid. 26 (1925) 477-483.-Addit. new spp.

一, ibid. 30 (1926) 413-414.-New sp.

-\& Quisumbing, Addisonia 17 (1932) 57-58, t. 573.-New sp.

Quisumbing \& Merrill, Philip. J. Sc. 37 (1928) 172-175.-New $s p$.

New Guinea : IRMSCHER, Bot. Jahrb. 50 (1913) 335383, fig. 1-5.-Descr. new spp.; no key.

-, Notizbl. Berl.-Dahlem 7 (1917) 102-103.New $s p$.

—, Webbia 9 (1954) 469-509, fig. 1-8.-Descr. new spp. coll. BeCCARI.

Merrill \& Perry, J. Arn. Arb. 24 (1943) 4159, 7 fig.

- ibid. 29 (1948) 160.

RidLey, J. Bot. 52 (1914) 289-290.-New sp.

-, Trans. Linn. Soc. Lond. II, Bot. 9 (1916) 58-62.-Descr. new spp.

Smith, J. J., Bull. Dép. Agr. Ind. Néerl. no 2 (1906) 47-48.-New $s p$.

\section{Genera}

Begonia: Dryander, Trans. Linn. Soc. 1 (1791) 155-173.

Fotsch, K. A., Die Begonien, ihre Beschreibung, Kultur, Zuchtung und Geschichte. Stuttgart 1933, 254 pp., 87 fig., t. 1-5.-A sort of key to cult. spp. not complete and not scientific; descr. of spp. mentioned; some of Malaysia.

\section{BERBERIDACEAE}

Koch, K., in MiQ. Ann. Mus. Bot. Lugd.-Bat. 1 (1864) 252.-List Mal. spp.

Tischler, G., Die Berberidaceen und Podophyl- 
laceen. Versuch einer morphologisch-biologischen Monographie (Bot. Jahrb. 1 1902, 596727, fig. 1-30).-Morph.; key to genera.

\section{Local}

India: ChatterJee, Rec. Bot. Surv. India 16 (1953) 1-86.-Keys!

Sumatra: Merrill, Contr. Arn. Arb. 8 (1934) 5556, t. 1.-New sp.

\section{Genera}

Berberis: AHRENDT, J. Bot. 79 (1941) Suppl. 1-80; (1942) 81-104; (1944) 105-112; (1945) 113-116. -Revision of the genus in Asia; keys.

SCHNEIDER, Bull. Herb. Boiss. II, 5 (1905) 33-48, 133-148, 391-403, 449-464, 655-670, 800-831; ibid. 8 (1908) 192-204, 258-266.-Precursory to monograph; key to spp.

-, Mitt. Deut. Dendrol. Ges. 55 (1942) 1-60 (1943).-Revision sect. Wallichianae; key.

Epimedium: StEARN, J. Linn. Soc. Lond. Bot. 51 (1938) 409-536, t. 24-31.-Monogr.; key.

Mahonia: FedDe, Bot. Jahrb. 31 (1901) 30-133.Revision; key.

TAKEDA, Not. R. Bot. Gard. Edinb. 6 (1917) 209-245, t. 1-37.-Old World spp. with descr.; no keys.

\section{BETULACEAE}

Winkler, Hub., Pflanzenreich Heft 19 (1904) 1-149, fig. 1-28, t. 1-2 (maps).

\section{Genera}

Alnus: O. Kuntze, Rev. Gen. Pl. (1891) 639-640. -Key.

Carpinus: Hub. WINKLER, Bot. Jahrb. 50 (1914) 488-508.

Corylus: Beijerinck, Jaarb. Ned. Dendr. Ver. 1948-1949 (1951) 67-107.-Revision; in Dutch.

\section{BIGNONIACEAE}

Bureau, E., Monographie des Bignoniacées, etc. Thèse, Paris (1864) 1-215, t. 1-31.

Dop, P., Contribution à l'étude des Bignoniacées (Bull. Soc. Bot. Fr. 62, 1926, 887-891, fig. 1-4). -Descr. Haplophragma n.g.; key to some As.Afr. genera of Tecomeae.

Miquel, F. A. W., Animadversiones in nonnullas Bignoniaceas (Ann. Mus. Bot. Lugd.-Bat. 1, 1864, 197-202).-Survey; descr.; no key.

SeEmanN, B., Synopsis Crescentiacearum: an enumeration of all the Crescentiaceous plants at present known (Trans. Linn. Soc. 23, 1860, 1-22).-Keys to genera, not to spp.

-, Revision of the natural order Bignoniaceae (Ann. Mag. Nat. Hist. III, 10 1862, 29-33).Subdiv. of family; enum. of genera; descr. of Aistanthus \& Campsidium.

- Revision of the natural order Bignoniaceae (J. Bot. 1, 1863, 18-23, 87-91, 225-228, 257-258; ibid. 3, 1865, 329-333; ibid. 5, 1867, 371-375; ibid. 8, 1870, 145-149, 337-341, 379-383).Precursory to revisions of some genera.

Seibert, R. J., The use of glands in a taxonomic consideration of the family Bignoniaceae (Ann. Missouri Bot. Gard. 35, 1948, 123-136, t. 3-7).
SteenIS, C. G. G. J. VAN, Malayan Bignoniaceae, their taxonomy, origin, and geographical distribution (Rec. Trav. Bot. Néerl. 1927, 787-1049, fig. 1-16, map).-Rev. Mal. spp.; keys.

-, The Bignoniaceae of the Netherlands Indies (Bull. Jard. Bot. Btzg III, 10, 1928, 173-290, fig. 1-13).-Revision Neth. Ind. spp.; keys.

\section{Local}

India \& Burma: ChatTerjee, Bull. Bot. Soc. Beng. 2 (1948) 62-79.

Indo-China: DoP, Bull. Mus. Hist. Nat. Paris (1926) 182-185, 233-235.-Descr. new spp.

Malay Peninsula: Holtrum, M. A. H. A. Mag. 11 (1941) 3-11.-Cultivated spp.; key.

Java: Merrill, Philip. J. Sc. 19 (1921) 381.Discussion ident. Crescentia ovata BuRM.

Borneo: VAN STEENIS, J. Bot. 72 (1934) 5-6.-New Radermachera.

Philippines: Elmer, Leafl. Philip. Bot. 10 (1939) 3709-3711.-New Radermachera.

Merrill, Philip. J. Sc. 3 (1908) Bot. 331-338.Rev. Radermachera; key. —, ibid. 26 (1925) 489.-New Radermachera.

New Guinea: DiELs, Bot. Jahrb. 57 (1922) 496-500. -Descr. Neosepicaea n.g.; new spp.; key to Tecomanthe.

GibBs, Fl. Phyt. Arfak Mts (1917) 179-180.New sp.

LAM \& Meeuse, Blumea 3 (1938) 201.-Reduction.

Moore, J. Bot. (1923) Suppl. 38-39.-2 new Tecomanthes.

Van Steenis, Nova Guinea 14 (1927) 293-303, t. 33-34.-Revision; keys to spp.

-, J. Arn. Arb. 28 (1947) 423.-Records Neosepicaea.

Qeensland: Van Steenis, Proc. R. Soc. Queensl. 41 (1929) 39-58, fig. 1-2.-Revision; keys.

\section{Genera}

Bignonia: Blake \& Sprague, J. Bot. 61 (1923) 191-193.-Further notes on generic typification.

Sprague, ibid. 60 (1922) 236-238, 363-364.Generic typification.

Campsis: SeemanN, J. Bot. 5 (1867) 371-374.Enum. : no key.

Catalpa: PaclT, Candollea 13 (1952) 241-285.Revision; key.

Deplanchea (Diplanthera F. v. M. non al.): VoN MUELleR, J. Bot. 5 (1867) 212-213.

SCHEFFER, Nat. Tijd. Ned. Ind. 31 (1870) 332337.-Revision; no key.

Dolichandrone: Sprague, Kew Bull. (1919) 302314.-Revision; key.

Nyctocalos: MiQuel, Ann. Mus. Bot. Lugd.-Bat. 3 (1867) 249, t. 8.-Revision.

SeemanN, J. Bot. 8 (1870) 147-149.-Incl. Haussmannia; key.

Radermachera: MiQuel, Ann. Mus. Bot. Lugd.Bat. 3 (1867) 250.-Revision.

SeEmann, J. Bot. 8 (1870) 145-147.-Enum.

Spathodea: SeemanN, J. Bot. 1 (1863) 225; ibid. 3 (1865) 332; ibid. 8 (1870) 339-341.-New defin.; list epithets. 
Tecoma (Stenolobium): Melchior, Ber. Deut. Bot. Ges. 59 (1941) 18-31.-Critical remarks on some spp.

ReHDer, Mitt. Deut. Dendr. Ges. 22 (1913) 262. -Generic typification.

Seemann, J. Bot. 1 (1863) 87-91.-List; key.

Sprague, J. Bot. 60 (1922) 364.-Generic typification.

Tecomanthe: Blume, Rumphia 4 (1849) 34-36, t. 190.-Enum. (under Tecoma).

Tecomaria: SeEmanN, J. Bot. 1 (1863) 19-23.Enum.

Wightia. See Scrophulariaceae.

\section{BIXACEAE \\ in the restricted sense}

BACKer, C. A., Fl. Mal. I, 4 (1951) 239-241, 2 fig.

PILGER, R., Pflanzenfamilien ed. 2, 21 (1925) 313315, fig. 139.

\section{BOERLA GELLACEAE}

LAM, H. J., Bull. Jard. Bot. Btzg III, 7 (1925) 250252.

\section{BOMBACACEAE}

Bakhuizen van DeN Brink, R. C., Revisio Bombacacearum' (Bull. Jard. Bot. Btzg III, 6, 1924, 161-240, t. 26-38).-Family revision; keys.

-, ibid. 241-254.-Rev. Indonesian spp.; keys.

BecCari, O., Le Bombaceae Malesi descritte ed illustrate (Malesia 3, 1889, .201-280, t. 12-36).Revision Mal. spp.; keys.

Masters, M. T., Monographic sketch of the Durioneae (J. Linn. Soc. Bot. 14, 1875, 495-508, t. 14-16).-Synopsis; descr. gen. \& spp.; no key.

\section{Local}

Malay Peninsula: Corner, Gard. Bull. Str. Settl. 10 (1939) 302-308.-Revision Mal. Pen. Durio; key.

Furtado, Gard. Bull. Str. Settl. 4 (1929) 421425.-Key to spp. of Neesia.

WyatT-Smith. See under Durio.

Borneo: Kostermans, Trop. Natuur 33 (1953) 31-35, 3 fig.-Biol. notes; new comb. in Durio. Merrill, Philip. J. Sc. 29 (1926) 393-394.-New Durio from Banguey Isl.

RIDleY, Kew Bull. (1938) 221.-New sp.

WyatT-Smith. See under Durio.

Philippines: MendozA, Philip. J. For. 4 (1941) 27-35, 2 pl.-Notes on Durio in Palawan.

New Guinea: Van Steenis, J. Arn. Arb. 28 (1947) 422.-Record of Papuodendron.

WhItE. See under Papuodendron.

\section{Genera}

Adansonia: HochreutnNer, Ann. Cons. Jard. Bot. Genève 11-12 (1908) 136-143, t.-New sp.; revision of the genus; key.

Bombax, Gossampinus, Pseudobombax, Salmalia (typification, chronol.): BAKHUIZEN VAN DE Brink, Bull. Jard. Bot. Btzg III, 6 (1924) 161. Alston, Handb. Fl. Ceylon 6 (Suppl.) (1931) 30. Furtado, Gard. Bull. Str. Settl. 10 (1939) 173181.

Dugand, Caldasia 6 (1943) 47-68.
Chatterjee \& RaIzada, Ind. For. 76 (1950) 139, 154-155.-Non vidi. -, Taxon 3 (1954) 102

Camptostemon (Cumingia): Troll, Flora 128 (1933) 348-360.-Key; distr.

Ceiba: Toxopeus, Meded. Alg. Proefst. Landb. Btzg no 56 (1948) 1-19, map.-Discussion origin Ceiba in Afr., Asia \& Mal.

Durio (Boschia, Heteropyxis, Lahia): WyATTSmItH, Kew Bull. (1953) 513-532 (1954).-Some new comb.; valuable notes on Mal. Pen. \& Bornean spp.; key to Malayan spp.

Neesia (Boschia): Korthals, in Temminck, Verh. Nat. Gesch. Bot. (1844) 257-258, t. 69. Merrill, J. Arn. Arb. 29 (1948) 201.-Glabraria L. (1771) is the oldest name for Boschia KORTH. (1842).

Ochroma: Pierce, Trop. Woods no 69 (1942) 1-2. -O. pyramidale.

-, ibid. no 70 (1942) 20-23.-Critical study of RowleE's types; syn. of $O$. lagopus.

RowleE, J. Wash. Acad. Sc. 9 (1919) 157-167. -Synopsis.

Papuodendron: White, J. Arn. Arb. 27 (1946) $272-274$, t. 1.-Placed by C. T. Whrte in Malvaceae.

\section{BORRAGINACEAE}

Brand, A., Cynoglosseae (Pflanzenreich 78, 1921, 1-183, fig. 1-22).

Johnston, I. M., Studies in the Boraginaceae III (Contr. Gray Herb. n. s. 73, 1924, 42-78).Genera; specially p. 68 Plagiobothrys.

-, ditto XI (J. Arn. Atb. 16, 1935, 145-205).Key to Tournefortia, Messerschmidia and Havilandia.

-, ditto XX. Representatives of 3 subfamilies in Eastern Asia (J. Arn. Arb. 32, 1951, 1-26, 99122).-Cordioideae and Heliotropoideae from China, Northern Indo-China, Hainan, Formosa, Riukius and Japan. Full keys \& elaborate notes on Cordia, Coldenia, Rotula, Ehretia, Heliotropium, Tournefortia, Messerschmidia.

\section{Local}

Formosa: Ding Hou, Taiwania 1 (1950) 197-223. -Revision; keys.

Siam: Fletcher \& Kerr, Flora Siam. Enum. 3 (1951) 77-88.-Enum.

Java: Hochreutiner, C. R. Soc. Phys. Hist. Nat. Genève 44 (1927) 74.-Record of Cynoglossum australe in E. Java.

Philippines: RobInson, Philip. J. Sc. 4 (1909) Bot. 687-698.-Revision; keys.

New Guinea: Johnston, J. Arn. Arb. 33 (1952) 68-69.-New Trigonotis.

Pulle, Nova Guinea 8 (1910) 399.-Records. 一, ibid. 8 (1912) 683.-Ditto.

\section{Genera}

Cordia: Hallier $f$., Meded. Rijksherb. Leiden no 36 (1918) 3.-Some critical notes on synonymy of some spp.

Hutchinson, Kew Bull. (1918) 217-222, fig. 1-2.-C. myxa \& 2 allied spp.; key. 
Johnston, Contr. Gray Herb. no 92 (1930) 1-65. -Amer. spp.; keys.

-, J. Arn. Arb. 21 (1940) 336-355.-Key to Centr. Amer. spp. of sect. Pilicordia.

Ehretia: Nakal, J. Arn. Arb. 5 (1924) 36-41.Rev. some spp. allied to E. acuminata; key.

Lithospermum: Johnston, J. Arn. Arb. 33 (1952) 299-363, t. 1-3.-Revision; key.

Mertensia: Williams, Ann. Missouri Bot. Gard. 24 (1937) 17-159.-Monogr.; keys to N. Amer. spp.

Myosotis: Stroh, Beih. Bot. Centralbl. 61 B (1941) 317-345.-Enum.; synon.; no keys; no descr.

Pteleocarpa: BecCari, Malesia 1 (1877) 130-131. -Under Icacin.

Sleumer, Pflanzenfamilien ed. 2, 20 b (1942) 400. -Comment.

Williams, Bull. Herb. Boiss. II, 5 (1905) 225.Places it close to Peripterygium (Icacin.)

Rotula (Rhabdia): BuNTING, J. Bot. 47 (1909) 269270.-Reduction; revision; monotypic.

Johnston, J. Arn. Arb. 32 (1951) 14-16.-Revision; 2 spp.

KuhlmanN, Arq. Inst. Biol. Veg. Rio de Janeiro 1 (1934) 113-114, fig. 1-14.-Descr. new Braz. Rhabdia.

Tournefortia: Johnston, Contr. Gray Herb. no 92 (1930) 66-89.-Sect.; key to Amer. spp.

Trigonotis (Havilandia, Zoelleria): Johnston, J. Arn. Arb. 21 (1940) 57-63.-Descr. new Pap. spp.; key to part of Mal. Trigonotis; reduction 2 genera and discussion of generic delimitation.

\section{BROMELIACEAE}

HaRms, H., Pflanzenfamilien ed. 2, 15a (1930) 65159, fig. 31-54.

Mez, C., in DC. Mon. Phan. 9 (1896) 1-990.

-, Pflanzenreich Heft 100 (1932) 1-437.

\section{Local}

Java: BACKER, Handb. Fl. Java 3 (1924) 8-18.Only cultivated.

\section{BUDDLEIACEAE \\ See Loganiaceae}

\section{BURMANNIACEAE}

BeCCARI, O., Malesia 1 (1878) 240-254, t. 10-15.Synopsis Indo-Austr. spp.; no key.

JONKER, F., A monograph of the Burmanniaceae. Thesis, Utrecht (1938) 1-279, fig. 1-20.-Keys! -, Fl. Mal. I, 4 (1948) 13-26, fig. 1-11.

SChLeChTER, R., Die Thismieae (Notizbl. Berl.Dahlem 8, 1921, 31-45).-Revision; new genera and spp.; keys.

\section{Local}

Sumatra: JoNKER, Blumea 3 (1938) 108-110, fig.New Burmannia from Enggano Isl.

SmITH, J. J., Bull. Jard. Bot. Btzg III, 9 (1927) 220-222, 1 fig.-New Thismia.

Malay Peninsula: RidLeY, J. Str. Br. R. As. Soc. 22 (1890) 331-339.

- , Ann. Bot. 9 (1895) 323-326, t. 12.-2 new Thismias.
-, Mat. Fl. Mal. Peninsula (Monocot.) 2 (1907) 69-75.-Keys.

Borneo: Warburg, in Fedde, Rep. 18 (1922) 330. -New Burmannia.

Philippines: Schlechter, Philip. J. Sc. 1 (1906) Suppl. 305.-New Burmannia.

New Guinea: Schlechter, Bot. Jahrb. 49 (1912) 100-108, fig. 1.-List descr.; no key.

一, ibid. 55 (1918) 202-203, fig. 1.-New Thismia. Smith, J. J., Nova Guinea 8 (1909) 193-195, t. 49.-List some new spp.

—, ibid. 8 (1912) 895-896, t. 159.-New Burmannia.

\section{Genera}

Gymnosiphon: Schlechter, in Fedde, Rep. 17 (1921) 253-258.-Distinction against Ptychomeria; enum.

\section{BURSERACEAE}

Cretziou, P., Burseraceae malayanae (in Fedde, Rep. 36, 1934, 265-267).-Ident. Vienna sheets; listed records.

ENGler, A., in DC. Mon. Phan. 4 (1883) 1-169, t. 1-3.

-, Pflanzenfamilien ed. 2, 19a (1931) 405-456, fig. 191-220.

Husson, A. M. \& H. J. LAM, Scandent Burseraceae (Dacryodes and Canarium) (Blumea 7, 1952, 163-167, fig. 1).-New Dacryodes from Borneo.

LAM, H. J., Bull. Jard. Bot. Btzg III, 12 (1933) 282-561.-Revision; keys.

Miquel, F. A. W., Ann. Mus. Bot. Lugd.-Bat. 4 (1869) 116-117.-List Garuga, Canarium.

\section{Local}

Indo-China: Guillaumin, Bull. Soc. Bot. Fr. 55 (1908) 611-618.-New Canariums from IndoChina.

Sumatra: Ridley, Kew Bull. (1925) 79.-New Santiria.

Borneo: Merrill, Philip. J. Sc. 30 (1926) 81-82.New Canarium.

Ridley, Kew Bull. (1930) 80-87.-New Canarium \& Santiria. -, ibid. (1931) 493.-A new Canarium.

Philippines: ElmEr, Leafi. Philip. Bot. 3 (1911) 1077-1088.-Canarium of Mt Apo. -, ibid. 10 (1939) 3711-3714.-Two new Canariums.

Quisumbing \& Merrill, Philip. J. Sc. 37 (1928) 155.-New Canarium.

Celebes: LAM, Blumea 3 (1938) 111-113.-New Haplolobus.

Moluccas: LAM, Blumea 5 (1942) 197-198.-List Talaud \& Morotai spp.

New Guinea: Husson \& LAM, Blumea 7 (1952) 167-170, fig. 1.-New Dacryodes.

LAUTERBACH, Bot. Jahrb. 56 (1920) 317-340.Keys.

-, Nova Guinea 8 (1910) 295.-Records.

一, ibid. 8 (1912) 827.-Ditto.

-, ibid. 14 (1924) 135.-Ditto.

Solomons: White, J. Arn. Arb. 31 (1950) 91-93.New Haplolobus. 
Melanesia: Rechinger, in Fedde, Rep. 11 (1912) 184.-New Canarium.

\section{Genera}

Canarium: Krng, J. As. Soc. Bengal 62, ii (1893) 184-188, t. 10-13.-List descr. some Ind. Canariums.

Dacryodes: Kalkman, Blumea 7 (1954) 500-522. -Revision; key.

Garuga: KalKMAN, Blumea 7 (1953) 459-472, fig. 1-3.-Revision; key. -, ibid. 7 (1954) 498-499.-Additional note.

Haplolobus: Husson \& LAM, Blumea 7 (1953) 413-458, fig. 1-15.-Revision; key.

Protium: Leenhouts, Blumea 7 (1952) 154-160, fig. 1.-Rev. Mal. spp.; key.

Swart, Rec. Trav. Bot. Néerl. 39 (1942) 211-395. -Monogr.; key.

Santiria: KALKMAN, Blumea 7 (1954) 522-552.Revision; key.

Scutinanthe: LeEnhouts, Blumea 7 (1952) 160163, fig. 1.-Rev. Mal. spp.; key.

Triomma: KalkmaN, Blumea 7 (1954) 499-500.Revision.

\section{BUTOMACEAE}

Buchenau, F. G. Ph., Beiträge zur Kenntnis der Butomaceen, Alismaceen und Juncaginaceen (Bot. Jahrb. 2, 1882, 465-510.)-Notes and additions to Michelr's monograph.

-, Pfianzenreich Heft 16 (1903) 1-12.

Micheli, M., in DC. Mon. Phan. 3 (1881) 84-93.

Steenis, C. G. G. J. van, Fl. Mal. I, 5 (1954) 118120 , fig. 1.

\section{Local}

Java: BACKer, Handb. F1. Java 1 (1925) 56-57.

\section{BUXACEAE \\ Local}

Java: SMith, J. J., in KoORders \& Valeton, Bijdr. Booms. Java 12 (1910) 3-8.

Malay Peninsula: Rideey, Kew Bull. (1926) 475476.-New Buxus.

\section{Genera}

Buxus: Hatusima, J. Dep. Agr. Kyushu Imp. Univ. 6 (1942) 261-342.-Revision; key to Asiatic spp.

Sarcococca: SEALY, in Hook. Ic. Pl. 35 (1947) t. 3469-3471, p. 1-10.-Descr. and discussion of several spp.

-, J. R. Hort. Soc. 74 (1949) 301-306.-Key to cultivated spp.

\section{CACTACEAE}

Britton, N. L. \& J. N. Rose, Descriptions and illustrations of the Cactus family (Carnegie Inst. Washington Publ. 248, 1, 1919, vii + 236; 2,1920 , vii $+239 ; 3,1922$, vii $+255 ; 4,1923$, viii +318$)$.

VAuPEL, F., Pflanzenfamilien ed. 2, 21 (1925) 594-651.

\section{Local}

Java: BACKer, Bekn. Fl. Java (em. ed.) 4B (1944) fam. 92, p. 1-9.-In Dutch; key.

\section{Genera}

Cereus: BERGer, Missouri Bot. Gard. Report 16 (1905) 57-86, t. 1-12.-Key to and descr. of subgenera; specific names listed.

Opuntia: BuRKILL, Rec. Bot. Surv. Ind. 4 (1911) 287-322.-Opuntia in India; key.

\section{CAESALPINIACEAE \\ See Leguminosae}

\section{CALLITRICHACEAE}

BACKer, C. A., Fl. Mal. I, 4 (1950) 251-252, fig. 1. PaX, F. \& G. F. HoffmanN, Pflanzenfamilien ed. 2, 19c (1931) 236-240.

\section{Local}

New Guinea: Merrill \& Perry, J. Arn. Arb. 22 (1941) 258.-New sp.

-, ibid. 29 (1948) 158.-Record of C. verna.

\section{Genera}

Callitriche: Samuelsson, Veröff. Geob. Inst. Rübel 3 (1925) 603-628.-Critical study Swiss spp.

\section{CAMPANULACEAE (incl. Lobeliaceae)}

\section{(excl. Pentaphragmataceae \& Sphenocleaceae)}

Candolle, A. DE, Monographie des Campanulées. Paris (1830) i-viii, 1-384, t. 1-20.-Partial keys.

Prest, C. B., Prodromus monographiae Lobeliacearum (Abh. Böhm. Ges. Wiss. n.s. 4, 1836, 1-52).-Enum. genera and ssp.; subdivisions of genera, hardly partial keys.

WiMmer, F., Campanulaceae-Lobelioideae (Pfian'zenreich Heft 106, 1943, 206 pp.).-For Malaysia only containing Pratia.

-, Vorarbeiten zur Monographie des Campanulaceae-Lobelioideae II. Trib. Lobelieae (Ann. Naturh. Mus. Wien 56, 1948, 317-374).

\section{Local}

Sumatra: Merrill, Not. Nat. Acad. Nat. Sc. Philadelphia no 47 (1940) 9.-New Lobelia.

Celebes: Fischer, Kew Bull. (1928) 141.-New Lobelia.

SkotTSBERG. See under Lobelia.

WIMMER, in FEDDE, Rep. 38 (1935) 78-79.New Lobelia.

Philippines: Elmer, Leafl. Philip. Bot. 9 (1934) 3180.-New variety.

New Guinea: Diels, Bot. Jahrb. 55 (1918) 121-125, fig. 1.-Records; Phyllocharis n.g. -, 62 (1929) 493.-Record of Pratia. Gibis, Contr. Fl. Phytog. Arfak Mts (1917) 183.-New Lobelia.

Merrill \& Perry, J. Arn. Arb. 22 (1941) 385387.-New sp. in Lobelia \& Phyllocharis; new records in Lobelia.

—, ibid. 30 (1949) 59-60.-New sp. in Pratia and Lobelia.

Moore, Trans. Linn. Soc. Lond. Bot. II, 9 (1916) 88-89.-Two n. spp. of Pratia. 


\section{Genera}

Campanumaea: CHIP, J. Linn. Soc. Bot. 38 (1908) 391.-Generic synonyms.

Merrill, Pap. Mich. Acad. Sc. 20 (1934) 111 (1935).-C. lancifolia.

Lobelia: KURz, Flora 30 (1872) 302.-L. dopatrioides from Lower Burma.

SkotTsberG, Acta Hort. Gothob. 4 (1928) 1-26. -Arborescent Asiatic spp.

Wahlenbergia (Campanulopsis): Brown, Gard. Chron. 54 (1913) 316-317.-Austral.-N. Caled. spp.

Lothian, Proc. Linn. Soc. N.S.W. for 1946, 71 (1947) 201-236.-Prov. rev. Old World spp.

Van Steenis, Bull. Bot. Gard. Btzg III, 17 (1948) 463.-Reduction Campanulopsis.

\section{CANNACEAE}

KRÄnzlin, F., Pflanzenreich Heft 56 (1912) 1-77, fig. 1-16.

WINkLer, Hub., Pflanzenfamilien ed. 2, 15a (1930) 640-654, fig. 290-295.

\section{Genera}

Canna (Java): BACker, Onkruidfl. Jav. Suiker. (1928) 194-197.-Key to 3 Jav. spp.

\section{CANNABINACEAE}

BACKer, C. A., Fl. Mal. I, 4 (1951) 222-223, fig. 1.

PraIN, D., On the morphology, teratology and diclinism of the flowers of Cannabis (Sc. Mem. Off. Med. \& Sanit. Dep. Govt India, Calcutta n.s., no 12, 1904, 1-32, t. 1-5).-Monographical; morph.

\section{CAPPARIDACEAE}

Miquel, F. A. W., Ill. Fl. Arch. Ind. (1870) 19-36, t. 11-19.-List Mal. repres.

Pax, F. \& G. F. HoffmanN, Pflanzenfamilien ed. 2, $17 \mathrm{~b}$ (1936) 146-223.

Woodson, R. E., Gynandropsis, Cleome, and Podandrogyne (Ann. Missouri Bot. Gard. 35, 1948, 139-146, t. 8).-Relation of these genera; key to Podandrogyne.

\section{Local}

Argentine: GómEz, Lilloa 26 (1953) 279-341, fig. 1-12, map 1-4, 1 pl.-Revision; keys; in Spanish.

Sumatra: Jochems, Trop. Natuur 17 (1928) 80-82, fig. 1-2.-Record of Cleome ciliata.

Malay Peninsula: Burkill, Gard. Bull. Str. Settl. 3 (1924) 280-281.-New record of Cleome. MERRILL, Gard. Bull. Str. Settl. 8 (1935) 132.New record of Cleome.

Java: BACKER, Bekn. Fl. Java (em. ed.) 4 (1942) fam. 45, p. 1-11.-In Dutch; keys.

Borneo: Hallier $f$., Bull. Herb. Boiss. 6(1898) 216.

New Guinea: LAUTERBACH, Bot. Jahrb. 52 (1915) 108-114, fig. 1.-Rev.; keys.

\section{Genera}

Capparis: Gagnepain, in MOROT, J. de Bot. 21 (1908) 53-65. Essay in classification; key to Asiat. spp.
-, Bull. Soc. Bot. Fr. 55 (1908) 209-215, 269-270, 322-325.-Descr. new spp. and new gen.; mainly Indo-China.

Radlkofer, Sitz. Ber. K. Bayer. Akad. Wiss. Math.-Phys. Cl. 14 (1884) 101-182; ibid. 17 (1887) 365-422.-Critical taxon. notes; syn.; anat.; etc.; no keys.

Crataeva: Corner, Gard. Bull. Str. Settl. 10 (1939) 15-21.-Critical notes.

Gagneparn, Not. Syst. 8 (1939) 211-213.Indo-Chin. spp.; key.

KuRz, J. Bot. 12 (1874) 193-196, t. 147-148.Indian spp.; key.

Stixis: Hallier $f$, Beih. Bot. Centralbl. 39, ii (1921) 34.-Syn. Covilhamia.

Pierre, Bull. Soc. Linn. Paris 1 (1887) 652-656.

\section{CAPRIFOLIACEAE}

KeRn, J. H. \& C. G. G. J. van Steents, Fl. Mal. I, 4 (1951) 175-194, fig. 1-5.

\section{Local}

Sumatra: Merrill, Pap. Mich. Acad. Sc. Arts \& Lett. 19 (1934) 199.-New sp. of Lonicera. KIDleY, J. MaI. Br. R. As. Soc. no 87 (1923) 64-65.-New Lonicera.

Malay Peninsula: Henderson, J. Fed. Mal. Stat. Mus. 11 (1924) 187-188.-New Lonicera.

Philippines: Elmer, Leafl. Philip. Bot. 9 (1934) 3181-3182.-New Viburnum.

Merrill, Philip. J. Sc. 26 (1925) 491-492.New Viburnum.

\section{Genera}

Carlemannia: Bremekamp, Rec. Trav. Bot. Néerl. 36 (1939) 372.-Discussion taxon. position.

Lonicera: ReHDER, Rep. Missouri Bot. Gard. 14 (1903) 27-232, t. 1-20.-Synopsis of genus; keys.

Van Steenis, J. Arn. Arb. 27 (1946) 442.Preliminary revision Mal. spp.; key.

-, Blumea 6 (1948) 243-244.-Critical notes on distr. \& synonymy of Mal. spp.

Sambucus: Hutchinson, Kew Bull. (1909) 191193.-Notes on Indian spp.

Von Schwerin, Mitt. Deut. Dendr. Ges. (1920) 194-231.-Revision.

Symphoricarpus: JonEs, J. Arn. Arb. 21 (1940) 201252.-Revision; key.

Viburnum: KERN, Reinwardtia 1 (1951) 107-170, 10 fig.-Revision Mal. spp.; keys.

-, ibid. 2 (1952) 131-132, 1 fig.-Add. note 0.1 V. clemensae.

Rehder, in Sargent, Trees and Shrubs 2 (1908) 81-116, t. 138-150 (new spp. republ. in Fedde, Rep. 9, 1911, 179-186).-Descr. \& pl. of mostly Chin. spp.

\section{CARICACEAE}

Harms, H., Pflanzenfamilien ed. 2, 21 (1925) 510-522, fig. 235-241.

Mello, J. C. \& R. SPruce, Notes on Papayaceae (J. Linn. Soc. Bot. 10, 1869, 1-15, t. 1).Discussion and notes.

Solms-Laubach, H., in Mart. Fl. Bras. 13, 3 (1889) 174-195, t. 49-52.-Key. 


\section{Genera}

Carica: Heilborn, O., Ark. Bot. 17, no 12 (1922) 1-16, t. 1.-Tax. \& Cytol. of Ecuador spp.

\section{CARYOPHYLLACEAE}

Mattfeld, J., Biologische und morphologische Blütenformen bei den Caryophyllaceen (Notizbl. Berl.-Dahl. 14, 1939, 470-482, fig. 1-15).Interpr. floral morph.

Pax, F. \& G. F. Hoffmann, Pflanzenfamilien ed. 2, 16c (1934) 275-367.

SteEnIS, C. G. G. J. vaN, Bull. Jard. Bot. Btzg III, 13 (1934) 180.-List Mal. mountain spp.

\section{Local}

Java: BACKER, Bekn. Fl. Java (em.ed.) 4A (1942) no 56, p. 1-16.

FenZl, in Zol.LnNGer, Syst. Verz. Heft 2 (1854) 141-142.-List.

New Guinea: MatTreld, Bot. Jahrb. 62 (1929) 475-479.-New Sagina and 2 Cerastiums. —, ibid. 69 (1938) 267-273.-Revi:ion; key to Cerastiums and Saginas; new comb. in Scleranthus.

-, ibid. 70 (1940) 468.-New Sagina.

Merrill \& Perry, J. Arn. Arb. 23 (1942) 386-388.-Records.

—, ibid. 30 (1949) 39-40.-New Sagina.

WARBURG, Bot. Jahrb. 16 (1892) 21.-New Sagina.

\section{Genera}

Arenaria: Williams, Bull. Herb. Boiss. 3 (1895) 593-603.-Sections.

-, J. Linn. Soc. Bot. 33 (1898) 326-437.Revision; no keys.

Cerastium: Gartner, in Fedde, Rep. Beih. 113 (1939) 1-96, fig. 1-101, t. 1-19.-Monograph. Möschl, Mem. Soc. Brot. 7 (1951) 15-119, maps, fig.-Revision Afr. spp. S. of the Sahara; map of $C$. indicum.

Williams, Bull. Herb. Boiss. 6 (1898) 893-904. Provisional enum.; spp. in key.

Dianthus: Williams, J. Linn. Soc. Lond. Bot. 29 (1893) 346-478.-Monograph.

Drymaria: Wiggins, Proc. Calif. Ac. Sc. IV, 25 (1944) 189-214, t. 20-22.-Key to Sonora desert spp.; incl. also some spp. introd. into Malaysia.

Gypsophila: LAWRENCE, Baileya 1 (1953) 16-18, fig. 5-12.-Key to cult. spp.

Williams, J. Bot. 27 (1889) 321-329.-Revision; no keys.

Lychnis: LAWRENCE, Baileya 1 (1953) 105-111, fig. 52-56.-Cult. spp.; key.

Polycarpaea: Gagnepain, in Morot, J. de Bot. 21 (1908) 275-280.-Revision; key.

Saponaria: Simmler, Denkschr. Akad. Wiss. Wien Math.-Naturw. K1. 85 (1910) 433-510, t. 1-2.-Monograph; partial keys.

Silene: Williams, J. Linn. Soc. Lond. Bot. 32 (1896) 1-196.-Revision; partial keys.

Stellaria: Briquet, Ann. Cons. Jard. Bot. Geneve 13-14 (1909-11) 378-380.-Critical notes.

SpRaGUE, Kew Bull. (1920) 308-318.-Nomenclature Stellaria versus Alsine.
Tunica: Williams, J. Bot. 28 (1890) 193-199.Revision; partial keys.

\section{CASTANEACEAE See Fagaceae}

\section{CASUARINACEAE}

Diels, L. \& E. Pritzel, Bot. Jahrb. 35 (1905) 122-130.-Revision W. Austral. spp.; key.

MACKLIN, E. D., The Casuarina distyla complex (Kew Bull. 1931, 145-151).-Key to the Austral. spp. of the $C$. distyla complex.

Miquel, F. A. W., Revisio critica Casuarinarum (Verh. Ned. Inst. 1. Kl. 13, 1848, 1-84).

-, Synopsis specierum Casuarinae (Flora 48, 1865, 17-24).

-, in DC. Prod. $16^{2}$ (1868) 332-344.

-, Ill. Fl. Arch. Ind. (1870) 8-11, t. 7-8.-Descr.

VRIESE, W. H. DE, Sur les Casuarinacées des Iles de la Sonde et des Moluques (in DE VRIESE, Pl. Nov. Ind. Bat. Or. 1845, 1-4, t. 1).-Descr.

\section{Local}

Sumatra: DE VRIESE, Tijd. Nat. Gesch. \& Phys. 11 (1844) 113-117.-New sp.

New Guinea: Moore, J. Bot. 61 (1923) Suppl. 53.New sp.

Pulle, Nova Guinea 8 (1910) 347.-Records. -, ibid. (1911) 621.-Records.

Solomons: Van Steenis, J. Arn. Arb. 20 (1939) 220-221.-Distr. C. rumphiana; reduced to C. nodiflora.

\section{CELASTRACEAE}

(incl. Siphonodontaceae)

LOESENER, TH., Ưber die geographische Verbreitung einiger Celastraceen (Bot. Jahrb. 24, 1897, 197-201).-First record of Microtropis in New World; reduction of a Chin. Ilex to Perrottetia and discussion of the distr. of the latter genus. -, Celastraceae novae, etc. (Notizbl. Berl.Dahlem 13, 1936, 220-225).-New tribe Lophopetaleae; key to its genera; census of Solenospermum; new sp. in Lophopetalum and Solenospermum from Borneo and New Guinea.

-, ibid. 13 (1937) 563-564.-Discuss. affinity Celastraceae and Hippocrateaceae.

—, Pflanzenfamilien ed. 2, $20 \mathrm{~b}$ (1942) 87-197.

\section{Local}

China: Loesener, Bot. Jahrb. 30 (1901-02) 446474.-Survey Chin. spp.; key to genera; partial keys to spp.

Formosa: Hou, Taiwania 1 (1950) 169-195.-Key.

Indo-China: Tardieu-Blot, Fl. Gén. I.-C. Suppl. 1 (1948) 781-812, fig. 94-99.-Keys!

-, Not. Syst. 14 (1950) 43-49.-Misc. descr. new or noteworthy spp.

Sumatra: Merrill, Contr. Arn. Arb. 8 (1934) 9293.-List; new Evonymus.

Ridley, J. Mal. Br. R. As. Soc. no 87 (1923) 56-57.-New Celastrus.

Java: Amshoff, Blumea 5 (1945) 517.-New record of a Celastrus.

VAN STEENIS, Trop. Natuur 22 (1933) 175-176, 
fig. 1.-Remarks on a climbing form of Evonymus japonicus.

-, Bull. Bot. Gard. Btzg III, 17 (1948) 400.Ditto, addit. remarks.

Borneo: Merrill, J. Str. Br. R. As. Soc. no 76 (1917) 93.-Record of an Elaeodendron.

Ridley, Kew Bull. (1931) 36-39.-Descr. new spp. in Evonymus, Microtropis and Lophopetalum.

一, ibid. (1938) 235-237.-Descr. new spp.

Rolfe, Kew Bull. (1918) 47-48.-Critical note on a Lophopetalum.

AIRY Shaw, Kew Bull. (1949) 162.-2 records.

Philippines: LOHER, Ic. Bog. 1 (1897) 55-56, t. 16.New Lophopetalum.

MerRILl, Philip. J. Sc. 16 (1920) 449-451, t. 1.-New comb. in Elaeodendron and record of an Evonymus.

—, ibid. 26 (1925) 466-467.-New Glyptopetalum from Palawan.

-, ibid. 27 (1925) 32-33.-New Kokoona and new var. in Siphonodon.

New Guinea: Loesener, Nova Guinea 8 (1910) 279-280.-New Celastrus and Lophopetalum.

-, Notizbl. Berl.-Dahlem 12 (1934) 36-38.2 new Perrottetias.

Merrill \& Perry, J. Arn. Arb. 20 (1939) 335-337.-New sp. in Gymnosporia, Kurrimia and Elaeodendron.

-, ibid. 22 (1941) 260-262.-New sp. in Celastrus \& Perrottetia.

New Caledonia: Guillaumin, Bull. Soc. Bot. Fr. 73 (1926) 429-433.-Key to genera and to spp. of Elaeodendron and Gymnosporia.

LOESENER, Bot. Jahrb. 39 (1907) 158-171.Revision; keys.

\section{Genera}

Alcineanthus = Neoscortechinia (Euphorbiaceae) Cremnobates = Schizomeria (Cunoniaceae).

Evonymus (Conocarpus): Blakelock, Kew Bull. (1948) 237-244.-E. frigidus and its allies. -, ibid. (1951) 210-290.-Revision with keys.

Glyptopetalum: Prain, J. As. Soc. Bengal 60, ii (1891) 206-210 (repr. in Nov. Ind.).-Revision; key.

Microtropis: Merrill \& Freeman, Proc. Amer. Acad. Arts \& Sc. 73 (1940) 271-310.-Revision Old World spp.; key.

Perrottetia (Caryospermum): KoORders \& VAlE'ron, Ic. Bog. 2 (1904) 137-138, t. 127.-Jav. sp.; reduction Caryospermum.

Siphonodon (Capusia): Crolzat, Lilloa 13 (1947) 31-43, 8 fig--Raises Siphonodon to subfamily rank.

Hooker $f$., Trans. Linn. Soc. Lond. 22 (1857) 133-139, t. 26.-Morph. struct.

Merrill, J. Arn. Arb. 21 (1940) 108-109.Reduction Capusia from Indo-China.

TARDieU-Blot, Not. Syst. 14 (1951) 101-104.Raises Siphonodon to family rank.

Solenospermum (Lophopetalum p.p.): LOESENER, Notizbl. Berl.-Dahlem 13 (1936) 220-226.List.

VAleton, Ic. Bog. 1 (1901) t. 90, p. 43-46.-
Descr. Jav. sp. with discussion on generic delimitation of the genus from Kokoona.

\section{CENTROLEPIDACEAE}

Gilg, E. \& C. Benedict, Pflanzenfamilien ed. 2, 15a (1930) 27-32.

Hieronymus, G., Beiträge zur Kenntnis der Centrolepidaceen (Abh. Naturf. Ges. Halle 12, 1873, 115-222, t. 1-4).-Monogr.; no key.

-, Úber Blüte und Blütenstand der Centrolepidaceen (Bot. Jahrb. 7, 1886, 319-330).-Monogr.; diagr.

Steudel, E. G., Enumeratio plantarum Glumacearum pars II. Stuttgart (1855) 266-267.-Enum.; descr. in Latin.

\section{Genera}

Centrolepis: Van Steenis, Bull. Jard. Bot. Btzg III, 13 (1934) 181.-List Mal. spp.

\section{CERATOPHYLLACEAE}

Steenis, C. G. G. J. van, Bull. Jard. Bot. Btzg III, 13 (1933) 102-103.-Revision Mal. spp.

一, Fl. Mal. I, 4 (1949) 41-42, fig. 1.

\section{Local}

New Guinea: Merrill \& Perry, J. Arn. Arb. 23 (1942) 390.-Record.

\section{Genera}

Ceratophyllum: Muenscher, Amer. J. Bot. 27 (1940) 231-233, 2 fig.-Descr. fruit \& seedlings.

\section{CHAILLETIACEAE \\ See Dichapetalaceae}

\section{CHENOPODIACEAE}

BACKer, C. A., Fl. Mal. I, 4 (1949) 99-106, fig. 1.

Ularich, E., Pflanzenfamilien ed. 2, 16c (1934) 379-584, 587.

Ungern-Sternberg, F., Salicorniearum synopsis (Atti Congr. Int. Bot. Firenze 1874 (1876) 259-343, synopsis).-Monograph of tribe Salicornieae; incl. are e.g. revisions of Salicornia and Arthrocnemum; keys to genera and spp.; in Latin.

\section{Local}

Java: BaCker, Onkruidfl. Jav. Suiker. (1930) 214-215.-Descr. Suaeda maritima.

New Guinea: Merrill \& Perry, J. Aro. Arb. 29 (1948) 154.-Record Chenopodium carinatum. Pulle, Nova Guinea 8 (1910) 349.-Records. Valeton, Bull. Dép. Agric. Ind. Néerl. 10 (1907) 9.-Records i.a. of Tecticornia.

Australia \& New Zealand: Aellen, Bot. Jahrb. 68 (1938) 347-434; ibid. 71 (1940) 228-232.Revision; keys.

—, Candollea 12 (1949) 153-155.-Ditto.

\section{Genera}

Arthrocnemum: Moss, J. S. Afr. Bot. 20 (1954) 1-22.-Revision S. Afr. spp.; keys; one new comb.

Chenopodium: Aellen \& Just, Amer. Midl. Nat. 30 (1943) 47-76, 1 pl.-Key to Amer. spp. 


\section{CHLORANTHACEAE}

Cordemoy, C. J. DE, Monographie du groupe des Chloranthacées (Adansonia 3, 1863, 280-310).Key to genera; descr. of spp.

Steenis, C. G. G. J. van, Bull. Jard. Bot. Btzg III, 13 (1934) 182-184.-Enum. Mal. spp. of Ascarina \& Hedyosmum.

\section{Local}

China: Groff, Lingn. Sc. J. 11 (1932) 94-97:Kwantung spp.; key.

P'El, Bot. Bull. Acad. Sin. 1, no 2 (1947) 111-117.-Keys to genera and spp. of Eastern China.

Sumatra: Ridley, J. Mal. Br. R. As. Soc. no 87 (1923) 89.-New variety.

Borneo: HeIne, in Fedde, Rep. 54 (1951) 226.Record.

Merrill, Philip. J. Sc. 13 (1918) Bot. 103-105.Two new spp.

Philippines: Merrill \& Quisumbing, Philip. J. Sc. 82 (1953) 324.-Nomencl. notes; reduction of a Chloranthus.

Robinson, Philip. J. Sc. 4 (1909) Bot. 69-70.Revision; key.

New Guinea: Kanehira \& Hatusima, Bot. Mag. Tokyo 55 (1941) 388-389.-Record.

Pulle, Nova Guinea 8 (1911) 623.-Record.

\section{Genera}

Chloranthus: P'eI, Sinensia 6 (1935) 665-688.Descr. \& key to Chinese Chloranthus.

- \& Renhwa San, Contr. Biol. Lab. Sc. Soc. China 10 (1938) 207-212.-Study of Chloranthus in China.

Hedyosmum: Merrill, Lingn. J. Sc. 5 (1927) 59.Record of Jamaican $s p$. in Hainan.

ViCTORIN, Contr. Inst. Bot. Univ. Montreal 63 (1948) 5-10.-Cuban spp.; key!

\section{CLETHRA CEAE}

Turczaninow, N., Quelques observations sur les espèces du genre Clethra (Bull. Soc. Nat. Moscou 36², 1863, 228-235).

\section{Local}

Asia: Dop, Bull. Soc. Bot. Fr. 75 (1928) 729-733.Key to 8 spp.

Sumatra: Merrill, Contr. Arn. Arb. 8 (1934) 121-122.-Record.

-, Pap. Mich. Acad. Sc. Arts \& Lett. 19 (1934) 179-181.-Two new spp.

Ridley, J. Mal. Br. R. As. Soc. no 87 (1923) 75.-New $s p$.

SмITH, J. J., Ic. Bog. 4 (1910) 65-66, t. 319.New sp.

Java: Koorders, Bull. Jard. Bot. Btzg III, 1 (1918) 148-152, t. 10.-Note on Clethra javanica.

Celebes: Koorders, Bull. Jard. Bot. Btzg III, 2 (1920) 255-257.-Note on Clethra canescens. MiQuel, Ann. Mus. Lugd.-Bat. 1 (1863) 41.Record.

Smith, J.J., Bull. Jard. Bot. Btzg III, 1 (1920) 398-399, t. 47.-New sp.
-, ibid. 4 (1922) 240.-New name for the above.

Philippines: Elmer, Leafl. Philip. Bot. 9 (1934) 3182-3184.-New sp.

New Guinea: Schlechter, Bot. Jahrb. 52 (1915) 219-220.-Enum.; new sp.

一, ibid. 55 (1918) 194.-New comb.

SḾrth, J. J., Nova Guinea 12 (1914) 169-170.Descr. new sp.

\section{COCHLOSPERMACEAE}

Pilger, R., Pflanzenfamilien ed. 2, 21 (1925) 316-320.

STeEnIS, C. G. G. J. van, Bull. Jard. Bot. Btzg III, 13 (1936) 519-524, fig. 1.-Rev. Mal. spp.

-, Fl. Mal. I, 4 (1949) 61-63, fig. 1.

\section{Local}

New Guinea: Balley, Queensl. Agric. J. 24 (1910) 20.-Record.

BAKER, J. Bot. (1923) Suppl. p. 4.-New var.

White, Proc. R. Soc. Queensl. 34 (1922) 45.Record.

— \& Francis, Proc. R. Soc. Queensl. 38 (1927) 248.-Record.

\section{COMBRETACEAE}

Candolle, A. de, Mémoire sur la famille des Combrétacées (Mém. Soc. Phys. Hist. Nat. Genève 4, 1828, 1-41).-Discussion family affinitities; descr. some new spp., mostly Indian.

EXELL, A. W., The genera of Combretaceae (J. Bot. $69,1931,113-128)$.-Key to and syn. of genera.

-, Fl. Mal. I, 4 (1954) 533-589, fig. 1-33.

Slooten, D. F. van, Bijdrage tot de kennis der Combretaceeën en Flacourtiaceeën van Nederlandsch-Indië. Thesis, Utrecht (1919) 5-54.Rev. Indonesian spp.; keys.

-, The Combretaceae of the Dutch East Indies (Bull. Jard. Bot. Btzg III, 6, 1924, 11-64).Rev. Indonesian spp.

\section{Local}

Malay Peninsula: RidLeY, Kew Bull. (1931) 449.New Terminalia.

Java: MrQuel, Ann. Mus. Bot. Lugd.-Bat. 4 (1869) 115.-Descr. new Combretum.

Borneo: Exell, Blumea 7 (1954) 557.-New Combretum.

Philippines: Merrill, Philip. J. Sc. 30 (1926) 414-415.-New Terminalia.

New Guinea: Ditss, Bot. Jahrb. 57 (1922) 427-430. -List; key for Terminalias.

Exell, Brittonia 2 (1936) 137-138.-New Terminalias.

一, J. Arn. Arb. 20 (1939) 317-320.-List

BRASS coll.; one new $s p$.

LAUTERBACH, Nova Guinea 8 (1910) 317.Record.

一, ibid. 8 (1912) 847.

VAN Slooten, Nova Guinea 14 (1927) 196-198.

-List Dutch New Guinean spp.; no key.

WhITE, Proc. R. Soc. Queensl. 34 (1922) 46-47. -List. 
-, J. Arn. Arb. 31 (1950) 100.-Reduction of a Terminalia.

\section{Genera}

Combretum: Don, G., Trans. Linn. Soc. Lond. 15 (1827) 412-441.-Review of the genus; descr. new spp.; partial keys.

Lumnitzera: VAN SLOOTEN, Trop. Natuur 11 (1922) 51-56, 65-70, 4 fig.-Discussion distr. -, Blumea Suppl. 1 (1937) 163-175.-Ditto.

Quisqualis: ExelL, J. Bot. 69 (1931) 119-120.Key to all spp.

Terminalia: BlatTer, J. Ind. Bot. Soc. 8 (1929) 245-262.-Revision of Indian spp.; complete synon.; descr.; key.

EXELL, Blumea 7 (1953) 322-328.-Descr. new spp.

PARkinson, Indian For. Rec. n.s., Bot. 1 (1937)

1-28, t. 1-3.-Indian spp. of § Pentaptera; key.

\section{COMMELINACEAE}

BRÜCKNeR, G., Zur speziellen Systematik der Commelinaceae (Notizbl. Berl.-Dahlem 10, 1927, 55-61).-For Mal. only important nomenclaturally as many new comb. are proposed in passing through splitting of the genera Aneilema and Pollia.

—, Pflanzenfamilien ed. 2, 15a (1930) 159-181, fig. 55-66.

Clarke, C.B., in DC. Mon. Phan. 3 (1881) $113-324$, t. 1-8.

Hasskarl, J. K., Commelinaceae Indicae, imprimis Archipelagi indici adjectis nonnullis hisce terris alienis (1870) 1-182.-Monograph; no keys.

Pichon, M., Sur les Commélinacées (Not. Syst. 12, 1946, 217-242).-Cartonema is segregated as a separate famuly; segregation of Tradescantia into various other, partly new, genera; key to all genera of the family, arranged according to a new system.

Woodson, R. E., Commentary on the North American genera of Commelinaceae (Ann. Missouri Bot. Gard. 29, 1942, 141-154).-Key to genera.

\section{Local}

India: Clarke, Commelinaceae et Cyrtandraceae Bengalenses. Calcutta (1874) 1-62, t. 1-42.Descr. \& pl.

Sumatra: RidLey, J. Mal. Br. R. As. Soc. no 87 (1923) 102-103.-List; 2 new Forrestias.

Malay Peninsula: RIDLey, Mat. Fl. Mal. Pen. (Monocot.) 2 (1907) 113-125.-Keys.

Java: BACKER, Handb. Fl. Java 3 (1924) 18-39.In Dutch; keys.

BAKHUIZEN VAN DEN BRINK, Blumea 6 (1950) 398-399.-List; various new comb.

Philippines: ElmER, Leafl. Philip. Bot. 9 (1934) 3184-3185.-New Commelina.

-, ibid. 10 (1939) 3714-3715.-New Cyanotis.

Moluccas: VAN SteEnIs, Blumea 7 (1954) 598.First record of a Cartonema.

New Guinea: Hallier $f$., Nova Guinea 8 (1913) 905-908.-List; one new Pollia.
LAUTERBACH, Bot. Jahrb. 50 (1913) 54-65.Rev.; keys; some new spp.

\section{Genera}

Belosynapsis (Dalzellia HAssk. non WighT): SPRAGUe \& Fischer, Kew Bull. (1928) 252-254. -Census; new comb.; segregate of Cyanotis.

Cyanotis: Livera, Ceylon J. Sc. A. Bot. (Ann. Bot. Gard. Perad.) 9 (1924) 185-190.-Revision Ceylon spp.; key.

Forrestia: HassKarl, Flora (Bot. Zeit.) 47 (1864) 625-631.-Revision; key.

Spatholirion: DUNN, Kew Bull. (1911) 161-162.Revision; key.

RIDLEY, J. Bot. (1896) 329-330, t. 360.-Type description.

Tradescantia: ANDERson \& WoOdson, Contr. Arn. Arb. 9 (1935) 1-132, t. 1-12.-Account of indig. spp. of U.S.A.; key.

\section{COMPOSITAE}

Bentham, G., Notes on the classification, history, and geographic distribution of the Compositae (J. Linn. Soc. Lond. Bot. 13, 1873, 335-577, t. 8-11).-General; interrelations within the family; distrib. notes; no descr.; no keys.

Boerlage, J. G., Handl. Fl. Ned. Ind. $2^{1}$ (1891) 165-170.-Key to genera.

Clarke, C. B., Compositae Indicae descriptae et secus genera Benthamii ordinatae. Calcutta (1876) xxiv $+347+$ xlv pp.-Synopsis; no keys.

Koster, J. TH., The Compositae of the Malay Archipelago. Vernonieae-Eupatorieae (Blumea 1, 1935, 351-536).-Rev.; keys. Spp. from New Guinea, the Philippines, and the Malay Peninsula are not treated.

Martelli, U., Le Composite raccolte dalla Dottore O. Beccari nell' Archipelago Malese e nelle Papuasia (Nuovo Giorn. Ital. 15, 1883, 281-305).-List.

RoBINSON, B. L., Records preliminary to a general treatment of the Eupatorieae I (Contr. Gray Herb. Harv. Univ. 64, 1921, 1-21); ditto II (ibid. 65, 1922, 46-54); ditto III (ibid. 68, 1923, 3-43); ditto IV (ibid. 73, 1924, 3-31); ditto V (ibid. 75, 1925, 3-15); ditto VI (ibid. 77, 1926, 1-62); ditto VII (ibid. 80, 1928, . . ditto VIII (ibid. 90, 1930, 3-36); ditto IX (ibid. 96, 1931, 1-27); ditto X (ibid. 100, 1932, 1-69); ditto XI (ibid. 104, 1934, 1-71).

Stebins Jr, G. L., A new classification of the tribe Cichorieae (Madroño 12, 1953, 65-89).

Steenis, C. G. G. J. van, Bull. Jard. Bot. Btzg III, 13 (1934) 184.-List of spp. of Mal. mountain genera.

\section{Local}

India: De CANDolle, in Wight, Contr. Bot. India (1834) 1-27.-Revision; partial keys.

Japan: Kitamura, Mem. Coll. Sc. Kyoto Imp. Univ. ser. B 13 (1937) 1-421, t. 1-35.-Monograph Cynareae, Inuleae, Eupatorieae, Astereae; keys.

-, ibid. ser. B $15^{3}$ (1940) 285-446, t. 1-11.- 
Ditto; this second part of the monogr. contains the Vernonieae, Mutisieae, and Anthemideae. -, ibid. ser. B 16 (1942) 155-292, t. .1-8.Tribes Senecioneae, Heliantheae and Ambrosieae.

Formosa: Kitamura, Acta Phytotax. \& Geobot. 10 (1941) 15-37.-Revision; no descr.; keys.

Sumatra: BoErlage, in Veth, Midden-Sumatra $4^{2}$ (1884) 20-21.-New Senecio.

KOSTER, Blumea 7 (1953) 362.-Reduction of Erechthites petiolata BTH.

Merrill, Pap. Mich. Acad. Sc. Arts \& Lett. 24 (1939) 90-92.-List; new Ainsliaea.

Ridley, J. Mal. Br. R. As. Soc. no 87 (1923)

72-74.-List; new sp. in Blumea \& Gynura.

Van Steenis, Bull. Bot. Gard. Btzg III, 17 (1948) 403.-Record of Blumeopsis.

Java: Backer, Bull. Jard. Bot. Btzg II, no 12 (1913) 39-40.-New Chrysanthellum; key.

-, Trop. Natuur 7 (1918) 58-59, fig. 11.Launaea pinnatifida var. javanica.

$\longrightarrow$ Rec. Trav. Bot. Néerl. 36 (1939) 449-459.-

Rev. of Gynura \& Crassocephalum.

Koster, in BACKer, Bekn. Fl. Java (em. ed.) 13A (1953) fam. 178, p. 1-145.-Revision; in Dutch; keys.

Van Steenis, Blumea 6 (1948) 245.-Note on Carpesium.

Lesser Sunda Islands: GagnepaIN, Bull. Soc. Bot. Fr. 67 (1920) 87-88.--Record of Pterocaulon redolens from Timor.

KOSTER, in Fedde, Rep. 34 (1933) 1-29.-List ElBERT coll.; various $n$. spp.

-, Blumea 7 (1952) 288-289.-Various notes; a new Vernonia.

VAN Steenis, Blumea 6 (1948) 246.-Gerbera from Bali.

Borneo: Koster, Blumea 7 (1953) 362.-Record of Spilanthes chamaecaula; reduction of a Lactuca. MerRILl, J. Str. Br. R. As. Soc. no 77 (1917) 245-247.-New sp. in Lactuca and Lagenophora. VAN SteENIS, Blumea 6 (1948) 246.-Record Rhynchospermum.

Celebes: Hemsley, Kew Bull. (1896) 38.-New Senecio from Mt Bonthain.

Philippines: Elmer, Leafl. Philip. Bot. 1 (1906) 83-186.-Manual of Philip. Compos.; keys. -, ibid. 10 (1939) 3715-3716.-New Gynura. KLATT, Flora (Bot. Zeit.) 68 (1885) 203-204.A new Vernonia.

Merrill, Philip. J. Sc. 12 (1917) Bot. 117-123, t. 2-3.-Fenixia, Guerreroia n.g.; 2 new Gynuras and some records.

—, ibid. 29 (1926) 495-496.-New Blumea.

—, ibid. 30 (1926) 429-430.-New Wedelia.

-, ibid. 60 (1936) 35.-Acanthospermum.

— \& Quisumbing, Philip. J. Sc. 82 (1953) 336.Record 3 weeds.

Quisumbing, Philip. J. Sc. 41 (1930) 368.Record of Galinsoga.

Moluccas: Merrill, Philip. J. Sc. 11 (1916) Bot. 319-320.-List weeds, mostly from Ambon.

New Guinea: Lauterbach, Nova Guinea 8 (1910) 335-338.

—, ibid. (1912) 863-866.
MatTfeld, Bot. Jahrb. 62 (1929) 386-451.-

Full important treatment; keys.

-, ibid. 494-501.-Saruwaged spp.; new spp., records \& comb.

一, ibid. 67 (1937) 248-268,-Many new spp.; revisions Keysseria and Olearia in Papua.

—, ibid. 69 (1938) 283-294.-New records \& spp.; Arrhenechthites n.g.

一, ibid. 70 (1940) 470-482.-New spp.; first record of Raoulia.

Moore, in Gibss, Contr. Fl. Phytog. Arfak Mts (1917) 183-184.-List; new Lactuca.

WhITE, Proc. R. Soc. Queensl. 34 (1922) 59-60. -List; mostly weeds.

-, ibid. 38 (1927) 260-261, fig. 19.-New Olzaria.

Micronesia: Kiramura, Acta Phytotax. \& Geobot. 10 (1941) 70-74.-List; no new spp.

\section{Genera}

Acanthospermum: Blake, Contr. U.S. Nat. Herb. 20 (1921) 383-393.-Revision.

Ageratum: Robinson, Proc. Am. Ac. Arts \& Sc. 49 (1913) 454-483.-Monograph; key.

Ainsliaea: Beauverd, Bull. Soc. Bot. Genève II, 1 (1900) 364-388, ibid. II, 2 (1910) 36-51.Monograph.

Anaphalis-Gnaphalium: GAMBLE, Kew Bull. (1921) 315-316.-Generic distinction.

KOSTER, Blumea 4 (1941) 483-484.-Various notes.

Arrhenechthites: MATTFELD, Bot. Jahrb. 69 (1938) 288-292.-Rev. of this Pap. end.; key.

Artemisia: KeCk, Proc. Cal. Ac. Sc. IV, 25 (1946) 421-468.-Revision $A$. vulgaris complex in N. Am.

Athroisma: MatTfeld, Notizbl. Berl.-Dahlem 13 (1936) 300-303.-Revision.

Bidens: Sherff, Field Mus. Nat. Hist. (Chicago) bot. ser. 16 (1937) 1-709, t. 1-189.

Blainvillea: Burkıl, Gard. Bull. Str. Settl. 5 (1930) 123.

Koster \& Philipson, Blumea 6 (1950) 349-354.

Blumea: Koster, Blumea 4 (1941) 485-490.Various notes; new var. \& forms. -, ibid. 6 (1948) 264-265.-Study of some types.

Blumeopsis: Gagnepain, Bull. Mus. Hist. Nat. Paris 26 (1920) 75-76.-Monotypic.

Merrill, J. Arn. Arb. 19 (1938) 70.-Record from Sumatra.

Brachycome: Davis, Proc. Linn. Soc. N.S.W. 73 (1948) 142-241.-Rev. Austr. spp.

Calotis (Tolbonia); DAvis, J. Linn. Soc. N.S.W. 76 (1951) 146-188.-Revision.

Merrill, Bull. Soc. Bot. Fr. 77 (1930) 340-341.

Chrysanthellum: KOSTER, in BACKER, Bekn. Fl. Java (em. ed.) 13A (1953) 92-93.-Key to 2 spp. Milne-Redhead, Kew Bull. (1948) 466.-Chr. indicum DC. = Chr. americanum (L.) VATKE; syn. \& distr.

Coreopsis: Sherff, Field Mus. Nat. Hist. (Chicago) bot. ser. 11 (1936) 279-475.-Revision.

Cosmos: Burkill, Gard. Bull. Str. Settl. 5 (1930) 118-120.-Some notes on spp. cult. in Malaya. SherfF, Field Mus. Nat. Hist. (Chicago) bot. ser. 7 (1932) 401-447.-Revision. 
Crepis: Babcock, Univ. Calif. Publ. Bot. 14 (1929) 323-333.-New spp. from contin. SE. Asia. -, Univ. Calif. Publ. Bot. 21 (1947) xii +197. -Distr.; cytol.

一, ibid. 22 (1947) 199-1030, fig. 1-304, t. 1-36. -Monogr.; keys.

Elephantopus: Baker, Trans. Acad. Sc. St Louis 12 (1902) 43-56.-Key to allied gen. \& ssp.

Philipson, J. Bot. 76 (1938) 299-305.-African spp.; key.

Emilia: Garabedian, Kew Bull. (1924) 137-144.With key.

Eschenbachia (Conyza): Koster, Blumea 7 (1952) 290.-Recomb. Mal. spp.

Eupatorium: Koster, Blumea 1 (1935) 492-503.Key.

-, ibid. 4 (1941) 491-492.-Two records.

VAN STEENIS, Reinwardtia 1 (1952) 478-479.Notes on $E$. odoratum.

Glossogyne: Van Steenis, Bull. Jard. Bot. Btzg III, 12 (1932) 168-169.-New record from Natuna Isl.

Gynura (incl. Crassocephalum); BACKER, Rec. Trav. Bot. Néerl. 36 (1939) 449-459.-Only Jav. spp.

Helianthus: Watson, Pap. Mich. Acad. Sc. Arts \& Lett. 9 (1928) 305-475 (1929).-Contr. to monograph.

Ixeris: MatTfELD, Bot. Jahrb. 62 (1929) 447-450. -Key to 6 spp. under Lactuca \$ Ixeris.

StebBins, J. Bot. 75 (1937) 43-51.-Critical notes; enum. of spp.

Keysseria (Hecatactis, Myriactis \& Hecatactis): Mattfeld, Bot. Jahrb. 67 (1937) 249-253.-Papuan genus; revision; key.

Lactuca: Stebrins, J. Bot. 75 (1937) 12-18.Critical notes on Lactuca and related genera.

Lagenophora: Davis, Proc. Linn. Soc. N.S.W. 75 (1950) 122-132.-Revision Austral. spp.

Launaea: MerRILl \& ChUN, Sunyatsenia 2 (1935) 328.

Melampodium: Robinson, Proc. Amer. Ac. Arts \& Sc. 36 (1901) 455-466.-Revision; key.

Mikania: RobINson, Contr. Gray Herb. Harv. Univ. 104 (1934) 55-71.

Montanoa: Robinson \& Greenman, Proc. Am. Ac. Arts \& Sc. 34 (1899) 508-521 (repr. in Contr. Gray Herb. new ser. 16).-Revision; key.

Moonia: Brown, Bern. P. Bish. Mus. Bull. 130 (1935) $340-345$, fig. 61-62.-Moonia is merged with Chrysogonum L. and emended.

Olearia: MatTfeld, Bot. Jahrb. 67 (1937) 253262.-Revision; key Pap. spp. 一, ibid. 70 (1940) 470-472.-New spp.

Porophyllum: Robinson \& Greenman, Proc. Am. Ac. Arts Sc. \& Lett. 32 (1896) 31-33 (repr, in Contr. Gray Herb. new ser. 10).-Provis. partial key to spp. N. of Panama.

Prenanthes: Stebins, Bull. Jard. Bot. Brux. 14 (1937) 333-352, fig.-List scandent spp. of Prenanthes and Lactuca in Africa.

Pterocaulon: GagnepanN, Bull. Soc. Bot. Fr. 67 (1920) 87-88.-Timor.

Merrill \& Chun, Sunyatsenia 2 (1935) 329330.
Soliva: Cabrera, Not. Mus. La Plata 14 (1949) Bot. no 70, p. 123-139, fig. 1-2.-Revision; key. Healy, Trans. R. Soc. New Zeal. 81 (1953) 23-26.-Key to 4 introd. spp. in New Zealand; key.

Sphaeranthus: RoBuns, Kew Bull. (1924) 177-199. -Revision.

Spilanthes: Koster \& PhILIPson, Blumea 6 (1950) 349-354.-Preliminary key to Mal. spp. Moore, Proc. Amer. Acad. Arts \& Sc. 42 (1907) 521-569.-Revision.

Tithonia: Blake, Contr. U.S. Herb. 20 (1921) 423-436.-Revision.

Tridax: Robinson \& Greenman, Proc. Am. Ac. Arts Sc. \& Lett. 32 (1896) 3-10 (repr. in Contr. Gray Herb. new ser. 10).-Revision; keys.

Verbesina: Robinson \& Greenman, Proc. Am. Ac. Arts \& Sc. 34 (1899) 534-566 (repr. in Contr. Gray Herb. new ser. 16).-Revision; key.

Vernonia: Koster, Blumea 1 (1935) 380-455.Keys.

-, Blumea 4 (1941) 490-491.-Various additions.

Xanthium: Widder, in Fedde, Rep. Beih. 20 (1923) 1-221.-Revision.

Youngia (segregate from Crepis): BABCock \& STEBBINS, Carn. Inst. Washington no 484 (1937) 1-106.-Revision.

Zinnia: Robinson \& Greenman, Proc. Am. Ac. Arts Sc. \& Lett. 32 (1896) 14-20 (repr. in Contr. Gray Herb. new ser. 10).-Revision; key.

\section{CONIFERAE}

(incl. Araucariaceae, Cupressaceae, Pinaceae, Podocarpaceae, and Taxaceae)

Boer, P. DE, De Coniferis Archipelagi Indici. Groningen (1866) 1-54, t. 1-3.-Revision; no keys.

-, in MiQ. Ann. Mus. Bot. Lugd.-Bat. 3 (1867) 255.-Some additions; no new spp.

Dallimore, W. \& A. B. Jackson, A handbook of Coniferae, etc. (1923) i-xi, 1-570, t. 1-32, fig. 1-120.-Keys to genera and sometimes to spp.; of little importance for Malaysia.

FitzPatrick, H. M., Coniferae; keys to the genera and species, with economic notes (Proc. Dublin Soc. 19, 1929, 189-260, t. 9-15).-Partial keys to genera and spp.

Hutchinson, J., Kew Bull. (1924) 49-66, maps.Key to the tribes \& genera of Cycadaceae, Taxaceae and Pinaceae with maps of distr.

MAsters, M. T., The genera of Taxaceae and Coniferae (J. Linn. Soc. Lond. Bot. 30, 1893, 1-42).-Distr.; keys to genera.

Pilger, R., Pflanzenreich Heft 18 (1903) 1-124, fig. 1-24.-Revision of Dacrydium, Podocarpus, Phyllocladus, and Taxus.

-, Kritische Úbersicht über die neuere Literatur betreffend die Familie der Taxaceae (Bot. Jahrb. 54, 1916, 1-43).-Taxac. in the old wide sense.

—, Pflanzenfamilien ed. 2, 13 (1926) 121-407, fig. 109-215.

Steenis, C. G. G. J. van, Bull. Jard. Bot. Btzg III, 
13 (1934) 184-193.-List Mal. spp. of mountain genera.

Warburg, O., Monsunia 1 (1900) 182-194.Synopsis of Agathis, Araucaria, Libocedrus, Podocarpus, Dacrydium, Phyllocladus, with new spp.

\section{Local}

Formosa: LI, Taiwania 1 (1950) 285-310.-Key to Formosan spp.

Sumatra: Merrill, Contr. Arn. Arb. 8 (1934) 14-15.-List.

MiQueL, Pl. Jungh. (1851) 1-6.-List; 2 new spp. De VrIEse, Pl. Nov. Ind. Bat. Or. (1845) 5-8, t. 2.-Descr. Pinus merkusii.

Malay Peninsula: Corner, Gard. Bull. Str. Settl. 10 (1939) 239-247, t. 5-10.-Dacrydium; one new sp.; key; notes on Agathis.

-, ibid. 10 (1939) 251-252.-Notes on some Podocarpus.

-, Wayside trees of Malaya (1940) 713-725.

Ridley, J. Str. Br. R. As. Soc. no 60 (1911) 53-68.-Complete account; no key.

Java: BACKer, DANSER \& WASSCHER, Bekn. Fl. Java (em. ed.) 2 (1940) fam. 17-21.-Also cult. spp.; in Dutch; keys.

Borneo: STAPF, in GibBs, J. Linn. Soc. Lond. Bot. 42 (1914) 191-195.-List; new Dacrydium from Mt Kinabalu.

Philippines: Foxworthy, Philip. J. Sc. 6 (1911) Bot. 132-172, t. 28-31.-Complete account; keys.

New Guinea: BecCARI, Malesia 1 (1877) 178-181. -List.

GibBs, Contr. Fl. Phytog. Arfak Mts (1917) 78-87, fig. 3-6b.-List \& new Dacrydium and Libocedrus.

Kanehira \& Hatusima, Bot. Mag. Tokyo 55 (1941) 385-387.-List.

KOORDERS, Nova Guinea 8 (1909) 177-182.Taxaceae.

-, ibid. (1912) 613-616.-Pinaceae, Taxaceae.

LAUTERBACH, Bot. Jahrb. 50 (1913) 46-53, fig. 1-2.-Revision; keys to Libocedrus and Araucaria.

Merrill \& Perry, J. Arn. Arb. 30 (1949) 39.Dacrydium sp.

Pilger, Bot. Jahrb. 54 (1917) 207-211.Taxaceae; keys to Podocarpus and Phyllocladus spp.

-, ibid. 68 (1937) 244-247.-Podocarpaceae; list; new Podocarpus.

一, ibid. 69 (1938) 252-253.-New Dacrydium and Podocarpus.

RIDleY, Trans. Linn. Soc. Lond. II, Bot. 9 (1916) 158-159.-New Podocarpus.

White, J. Arn. Arb. 28 (1947) 259-260.Critical notes; a new section of Araucaria.

\section{Genera}

Agathis: Foxworthy, Philip. J. Sc. Sect. A, 5 (1910) 173-175.-A. alba.

MeiJer Drees, Bull. Jard. Bot. Btzg III, 16 (1940) 455-474.-Revision; various new spp.; key.
Araucaria: Barsali, Atti Soc. Toscana Sc. Nat. Pisa 25 (1909) 145-185, t. 1 (fig. 1-15).-Complete revision; syn.; keys.

White, J. Arn. Arb. 28 (1947) 259-260.-New sect.; critical notes Pap. spp.

Cupressus: Masters, J. Linn. Soc. Lond. Bot. 31 (1896) 312-363.-Revision.

Libocedrus: FloRIN, Svensk Bot. Tidskr. 24 (1930) 117-131, t. 1-2.-E. Asiat. spp. — \& Boutelje, Acta Hort. Berg. 17: 2 (1954) 1-37, t. 1-10.-The genus segregated into 5 genera; key to the spp. by external morph. \& epiderm. characters.

LI, J. Arn. Arb. 34 (1953) 17-34, t. 1-2.Splitting Libocedrus into 4 segregate genera; the New Guinean species belong to Papuacedrus.

Pinus: Engelmann, Trans. St Louis Acad. Sc. 4 (1880) 161-190, t. 1-3.-Revision; key.

Masters, J. Linn. Soc. Lond. Bot. 35 (1904) 560-659.-Revision.

Shaw, Arn. Arb. Publ. 5 (1914) iv + 96 pp.Revision.

Podocarpus: Gray \& BuchHolz, J. Arn. Arb. 31 (1950) 82-92, t. 1-4, 93-97.-Taxon. revision; S. Pac.spp. of $\S$ Stachycarpus and $\S$ Sundacarpus; notes on $P$. amara.

WASSCHER, Blumea 4 (1941) 359-482.-Revision Indonesian spp.; keys.

\section{CONNARACEAE}

Planchon, J. E., Prodromus monographiae ordinis Connaracearum (Linnaea 23, 1850, 409-442).

Radlkofer, L., Ủber die durchsichtigen Punkte und andere anatomische Charactere der Connaraceae (Sitz. Ber. Bayer. Akad. Wiss. 16, 1886, 345-378).

SChEllenberG, G., Beiträge zur vergleichenden Anatomie und zur Systematik der Connaraceen. Thesis, Zürich (1910) 1-158.

-, Bot. Jahrb. 60 (1925) 207-251.-Sections.

-, Pflanzenreich Heft 103 (1938) 1-326.

\section{Local}

Sumatra: Merrill, Pap. Mich. Acad. Sc. Arts \& Lett. 23 (1938) 178-179.-Records; a new Rourea.

Borneo: Merrill, J. Str. Br. R. As. Soc. no 76 (1917) 84.-Record.

-, Philip. J. Sc. 13 (1918) Bot. 68-73.-5 new Connarus spp.

—, J. Str. Br. R. As. Soc. no 85 (1922) 198-201. -3 new Agelaeas and a Connarus; recomb. in Cnestis.

Ridley, Kew Bull. (1938) 275-276.-List records.

SchellenBerG, Bot. Jahrb. 59 (1924) Beibl. 131, p. 22-41.-Important survey of Born. spp.; several new spp. and comb.

Java: BAKHUIZEN VAN DEN BRINK, Blumea 6 (1950) 365.-New name in Connarus.

Philippines: Elmer, Leafl. Philip. Bot. 10 (1939) 3717-3719.-2 new spp. 
MerriLl, Philip. J. Sc. 4 (1909) Bot. 117-128.Revision; keys.

New Guinea: Merrill \& Perry, J. Arn. Arb. 23 (1942) 391.-A new Rourea.

SCHELLENBERG, Bot. Jahrb. 58 (1923) 178-181.-

Revision; no key; 2 new spp.

\section{Genera}

Cnestis: Andreas \& Prop, Blumea 7 (1954) 602-616, fig. 1-7.-Revision, SE. Asiat. \& Mal. spp.

Ellipanthus: PraIN, J. As. Soc. Beng. 59, ii (1890) 208-210, t. 8.-Key to Ind. spp.

Pseudellipanthus: Schellenderg, in Mez, Bot. Arch. 1 (1922) 314-315.-Type descr.; Borneo; key.

Rourea: Schellenderg, Bot. Jahrb. 56 (1920) 21-29.-Critical remarks on various neotrop. spp.

\section{CONVOLVULACEAE}

Chorsy, J. D., Convolvulaceae orientales, etc. (Mém. Soc. Phys. Hist. Nat. Genève 6, 1833, 383-502, t. 1-6).-Conspectus of genera and spp.; key only to genera; descr. Indo-Mal.Austr. spp.-Also reprinted (1834) with independent pagination.

-, De Convolvulaceis dissertatio secunda, etc. (ibid. 8, 1838, 43-86, t. 1-4).-Continuation of the above; ditto.

- De Convolvulaceis dissertatio tertia, etc. (ibid. 9, 1841, 261-288, t. 1-5).-Monogr. Cuscuta; no key.

Hallier $f$., H., Versuch einer natürlichen Gliederung der Convolvulaceen, etc. (Bot. Jahrb. 16, 1893, 453-594).-Evaluation morph. \& anat. characters; keys to tribes and genera.

-, Bausteine zu einer Monographie der Convolvulaceen (Bull. Herb. Boiss. 5, 1897, 366-387, 736-754, 804-820, 996-1013, 1021-1052; ibid. 6, 1898, 714-724; ibid.7, 1899, 408-418).-No keys.

Ooststroom, S. J. VAN, The Convolvulaceae of Malaysia I-V (Blumea 3, 1938, 62-94; ibid. 1939, 267-371; ibid. 1940, 481-582; ibid. 5, 1943, 339-411; ibid. 1945, 689-691; ibid. 6, 1950, 337-348; ibid. 7, 1952, 171-178).-Revision, not complete for Argyreia; Erycibe not treated; keys!

- \& R. D. Hoogland, Fl. Mal. I, 4 (1952) 388-512, fig. 1-62.

\section{Local}

Africa: Hallier $f$., Bot. Jahrb. 18 (1893) 81-160. -Enum.; no keys.

India: Prain, J. As. Soc. Beng. 63, ii (1894) 83115.-Additions to Fl. Br. Ind.; many new spp.; no keys.

-, ibid. 65, ii (1896) 536-538.-Ditto.

SantapaU, J. Bombay Nat. Hist. Soc. 47 (1947) 337-354.-Revision spp. of Bombay Pres.; keys.

Siam: KeRR, Kew Bull. (1941) 10-21.-Descr. new spp.

Sumatra: Merrill, Pap. Mich. Acad. Sc. Arts \& Lett. 19 (1934) 189-191.-2 new Erycibes.
Van Ooststroom, Blumea 5 (1945) 686-688, fig. 1.-New Argyreia.

-, ibid. 7 (1952) 171-178.-New Argyreias.

RidLeY, J. Fed. Mal. St. Mus. 8, 4 (1917) 64-65. -List; new Lettsomia.

Malay Peninsula: Hoogland, Blumea 7 (1952) 179-192, fig. 1.-Revision Argyreia; key.

Borneo: VAN OOSTSTROOM, Kew Bull. (1938) 175176.-Records RICHARDS coll.; one new Merremia.

-, Blumea 7 (1952) 175-177, fig. 1.-New Argyreias.

Philippines: Elmer, Leafl. Philip. Bot. 10 (1939) 3720-3722.-2 new Riveas.

MerRILL, Philip. J. Sc. 7 (1912) Bot. 244-245.Note on Ipomoea reptans; new comb. in Merremia.

-, ibid. 26 (1925) 488-489.-New Lettsomia.

-, ibid. 29 (1926) 485.-Ditto.

New Guinea: van Ooststroom, J. Arn. Arb. 29 (1948) 413-418, t. 1.-Misc. notes; new Erycibe; key to Pap. Erycibe.

-, Nova Guinea n.s. 6 (1955) (in the press).Complete survey.

Pilger, Bot. Jahrb. 59 (1924) 84-86.-Descr. new spp.

\section{Genera}

Argyreia (Rivea, Lettsomia): Hoogland, Blumea 7 (1952) 179-192, fig. 1.-Rev. Mal. spp.; key. Van Ooststroom, Blumea 6 (1950) 337-348.Kèy \& descr.

-, ibid. 7 (1952) 171-178, fig. 1-2.-Additions.

Cuscuta: EngelmanN, Trans. Acad. Sc. St Louis 1 , no 3 (1859) 453-523.-Syst. arrangement of $s p p . ;$ key to groups, but no complete key to spp. - , Generis Cuscutae species, etc. Berlin (1860) viii $+88 \mathrm{pp}$.-Latin ed. by Ascherson of the above.

YUNCKeR, Illinois Biol. Monogr. 6 (1921) 91241, t. 1-13.-Revision N. Amer. \& W. Indian spp.; keys.

-, Mem. Torrey Bot. Club 18 (1932) 113-331, fig. 1-158.-Monogr.; keys.

Decalobanthus: van OosTstroom, Blumea 2 (1936) 98-100.-Endemic in Sumatra.

Erycibe (Fissipetalum): Hoogland, Blumea 7 (1953) 310-319.-Descr. new spp.

-, ibid. 7 (1953) 342-361.-Critical review of all spp.; enum.

Evolvulus: van OOSTSTROOM, A monograph of the genus Evolvulus (1934) 1-267.-Keys.

Ipomoea: House, Ann. N.Y. Ac. Sc. 18 (1908) 181-263.-Rev. N. Amer. spp.

Hewittia (Shutereia): ChOdAT \& Roulet, Bull. Herb. Boiss. 1 (1893) 191-196, 5 fig.-Critical remarks.

Neuropeltis: vaN Ooststroom, Blumea 5 (1942) 268-273, fig. 1-2.-Rev. Indo-Mal. spp.; key.

\section{CORIARIACEAE}

Good, R. D'O, New Phytologist 29 (1930) 170198.-Geogr. distr.; groups; no key.

Maximowicz, K. J., De Coriaria, etc. (Mém. Acad. Imp. Sc. St Pétersb. VII, 29, no 3, 1881, 
1-13).-Survey of spp. and morph. \& anat. differences of spp. of $\mathrm{N}$. and $\mathrm{S}$. hemisphere; key (copied in Bot. Jahrb. 3, 1882, 186-187).

\section{Local}

Philippines: Merrild, En. Philip. Fl. Pl. 2 (1923) 465.-Record.

New Guinea: WARBURG, Bot. Jahrb. 16 (1892) 22. -New spp.

New Zealand: Oliver, Rec. Dominion Mus. 1 (1942) 21-43, 6 pl.-Revision; key.

\section{CORNACEAE \\ (excl. Alangiaceae \& Nyssaceae)}

Danser, B. H., The Cornaceae, sensu stricto, of the Netherlands Indies (Blumea 1, 1934, 46-74). -Full revision of Mal. Mastixia.

Hutchinson, J., Neglected generic characters in the family Cornaceae (Ann. Bot. n.s. 6, 1942, 83-93, fig. 1-5).-Splitting of the genus Cornus.

WANGERIN, Pflanzenreich Heft $41^{4}$ (1910) 1-110, fig. 1-24.

\section{Local}

Banka: ValeTon, Ic. Bog. 2 (1906) 267-268, t. 179.-New sp.

Java: Miquel, Pl. Junghuhn. (1855) 426.-New Mastixia.

Philippines: Merrill, Philip. J. Sc. 26 (1925) 486-487.-New Mastixia.

WANGERIN, in FEDDE, Rep. 10 (1912) 273.New Mastixia.

Solomons: Merrill \& Perry, J. Arn. Arb. 21 (1940) 527.-Record.

\section{Genera}

Alangium. See Alangiaceae.

Mastixiodendron. See Rubiaceae.

Nyssa. See Nyssaceae.

\section{CORSIACEAE} Local

New Guinea: BecCARI, Malesia 1 (1878) 238-240, t. 9.-Type descr.

Gibis, Contr. Fl. Phytogr. Arfak Mts (1917) 103-105, fig. 7.-Record \& new sp.

SCHLEChTER, Bot. Jahrb. 49 (1913) 109-112, fig. 1.-Descr. 2 new spp.

SMITH, J. J., Nova Guinea 8 (1909) 197. 一, ibid. 8 (1912) 893.

一, ibid. 12 (1914) 171-172, t. 54.-New sp.

Williams, Bot. Mus. Leafl. Harv. Univ. 12 (1946) 179-182.-New sp.

\section{CORYNOCARPACEAE}

Hemsley, W. Botting \& Fritsch, On the genus Corynocarpus Forst. (Ann. Bot. 17, 1903, 743760, t. 36; ibid. 18, 1904, 179-180).-Anat.; descr. gen. and 2 spp.

Krause, K., Pflanzenfamilien ed. 2, 20 b (1942) 22-35.

Steenis, C. G. G. J. Van, Fl. Mal. I, 4 (1951) 262-264, fig. 1-2.

Tieghem, Ph. van, Sur le genre Corynocarpe, considéré comme type d'une famille distincte, les Corynocarpacées (in Morot, J. de Bot. 14, 1900, 193-197).-Anat.; morph.; taxon. position.

\section{Local}

New Guinea: Merrill \& Perry, J. Arn. Arb. 22 (1941) 541-542.-Record.

Van Steenis, J. Arn. Arb. 28 (1947) 421.Record.

Queensland: White, Contr. Arn. Arb. 4 (1933) 57, t. 5.-Descr. new sp.

\section{Genera}

Corynocarpus: van Steens, Bull. Jard. Bot. Btzg III, 13 (1933) 99-101, fig. 1.-Rev. Mal. sp.

\section{CRASSULACEAE}

BACKer, C. A., Fl. Mal. I, 4 (1951) 197-202, 2 fig.

Berger, A., Pflanzenfamilien ed. 2, 18a (1930) 352-483, fig. 183-212.

Candolle, A. P. DE, Mémoire sur la famille des Crassulacées. Paris (1828) 1-47, t. 1-13.Discussion of genera \& descr. new spp.; no key; not dealing with Mal. spp.

\section{Local}

Java: Koorders, Bull. Jard. Bot. Btzg III, 1 (1919) 169-180, t. 14-15.-Descr. new Kalanchoe from $E$. Java.

\section{Genera}

Kalanchoe: HaMET, Bull. Herb. Boiss. II, 7 (1907) 869-900; ibid. 8 (1908) 17-48.-Monograph.

Sedum: Fröderström, Act. Hort. Gothob. 5 (1930) App. 1-75; ibid. 6 (1931) App. 1-111.

Praeger, J. R. Hort. Soc. Lond. 46 (1921) 1-314.-Only cult. spp.

\section{CRUCIFERAE}

Miquel, F. A. W., Ill. Fl. Arch. Ind. (1870) 14-19, t. 9-10.-Synopsis; no keys.

Schulz, O. E., Brassiceae, pars prima (Pflanzenreich Heft 70, 1919, 1-290, fig. 1-35).-Brassica, Rapistrum, Sinapis.

-, ditta, pars secunda (ibid. Heft 84, 1923, 1-100, fig. 1-26).

-, Sisymbrieae (ibid. Heft 86, 1924, 1-388, fig. 1-74).-Sisymbrium.

-, Draba et Erophila (ibid. Heft 89, 1927, 396, fig. 1-35).

-, Pflanzenfamilien ed. 2, 17 b (1936) 227-658, fig. 121-426.

\section{Local}

Malay Peninsula: Burkill, Gard. Bull. Str. Settl. 5 (1930) 99-117,-Key to cultivated Brassicas.

Furtado \& Holttum, M. A. H. A. Mag. 10 (1940) 47-51.-Cult. Brassicas; with key to Chin. vernac. names.

Java: HoCHREUTINER, Candollea 2 (1925) 367-372. -Records; Nasturtium under Rorippa.

New Guinea: Merrill \& Perry, J. Arn. Arb. 24 (1943) 207.-Records, also of Papuzilla. 
RIDLEY, Trans. Linn. Soc. Bot. II, 9 (1916) 16-17, t. 1, fig. 7-14.-Descr. Papuzilla.

ScHulz, Bot. Jahrb. 55 (1918) 266-272, fig. 1.Key.

\section{Genera}

Brassica: BAILEY, Gentes Herb. 1 (1922) 53-108, fig. 18-49; ibid. 2 (1930) 209-267, fig. 108-146; ibid. 4 (1940) 319-330, fig. 199-210.-Cult. spp.; keys.

Lathouwers, Bull. Inst. Agron. \& Sta. Rech. Gembloux 2 (1933) 3-16.-Cult. spp.

Pearson, Proc. Amer. Soc. Hort. Sc. 25 (1928) 105-110.-Classification of genus.

Prain, Dept Land Rec. \& Agric. Bengal, Agric. ser. no 3, Bull. no 4 (1898); repr. in Prain, Bot. Not. \& Pap. (1901) 145-222, t. 1-10.-Key, descr. \& fig. cult. Bengal spp.

SabNIS, Ind. J. Agric. Sc. 5 (1935) 559-578.Revision Indian spp., var. \& strains (types); key.

Cardamine: Schulz, Bot. Jahrb. 32 (1903) 280623.-Monograph; keys.

Nasturtium: SPRAGUE, J. Bot. 62 (1924) 225-228.Nomencl. of $N$. officinale.

\section{CRYPTERONIACEAE}

Niedenzu, F., Zur Kenntnis der Gattung Crypteronia Bl. (Bot. Jahrb. 15, 1892, 161-179, 1 fig.).-Taxon. position; revision; key.

\section{Local}

New Guinea: Merrill \& Perry, J. Arn. Arb. 22 (1941) 270.-Record.

\section{CUCURBITACEAE}

Cogniaux, A., in DC. Mon. Phan. 3 (1881) 325-954.

-, Fevilleae et Melothrieae (Pflanzenreich Heft 66, 1916, 1-277, fig. 1-65).-Alsomitra (= Neoalsomitra), Cerasiocarpum, Hemsleya, Macrozanonia (= Alsomitra), Melothria, Muellerargia, Thladiantha, Zanonia.

- \& H. HARMs, Cucurbiteae-Cucumerineae (Pflanzenreich Heft 88, 1924, 1-246, fig. 1-26).Citrullus, Cucumus, Gymnopetalum, Lagenaria, Luffa.

ZimmermanN, A., Die Cucurbitaceae. Jena (1922). Pt I. Beiträge zur Anatomie und Physiologie, i-viii, 1-205, fig. 1-95; pt 1I. Beiträge zur Morphologie, Anatomie, Biologie, Pathologie und Systematik, i-iv, 1-86, fig. 1-99.-General chapters; taxon. consisting of descr. of some new spp. from Africa.

\section{Local}

India: Chakravarty, Ind. J. Agric. Sc. 16 (1946) 1-89.-Studies Ind. spp.; special remarks on distr. \& uses of economic spp.

Sumatra: Merrill, Pap. Mich. Acad. Sc. Arts \& Lett. 19 (1934) 199-201.-New Melothria; Siraitia n.g.

-, Contr. Arn. Arb. 8 (1934) 165.-2 records. Ridley, J. Mal. Br. R. As. Soc. no 87 (1923) 62.-Records.
Borneo: Merrill, J. Mal. Br. R. As. Soc. 1 (1923) 44-45.-New Melothria and Momordica.

Lesser Sunda Islands: JACOBs, Blumea 7 (1954) 617.-Distr. Gynostemma hederifolia.

VAN Woerden, Trop. Natuur 29 (1940) 6, fig. 7.-Fig. of 2 podagric spp.

Philippines: Elmer, Leafl. Philip. Bot. 10 (1939) 3722-3723.-New Alsomitra.

Merrill, Philip. J. Sc. 29 (1926) 495.-New Melothria.

-, ibid. 30 (1926) 429.-Record Zanonia (Juppia).

New Guinea: Cogniaux, Bull. Acad. Roy. Belg. III, 14 (1887) 355, 363.-New Melothria and Alsomitra.

HaRms, Bot. Jahrb. 60 (1926) 150-161.-Synopsis; keys in Alsomitra and Trichosanthes.

JACOBS, Blumea 7 (1954) 617-621, fig. 1-2.New Melothria.

Merrill \& Perry, J. Arn. Arb. 29 (1948) 167-168.-3 new Melothrias; note on a Gynostemma.

-, ibid. 30 (1949) 56-59.-Notes on Neoalsomitra; 3 new Melothrias; 2 new Trichosanthes.

Pulle, Nova Guinea 8 (1910) 405-406.-List; new Trichosanthes.

-, ibid. (1912) 689.-Records.

\section{Genera}

Alsomitra (Macrozanonia); Cogniaux, Bull. Soc. Bot. Belg. 43 (1906) 357-360.-Descr.; tax. position.

DE WIT, Bull. Bot. Gard. Btzg III, 18 (1949) 193-200.-Revision.

Citrullus: Fosberg, Taxon 2 (1953) 99-101.Prop. to conserve Citrullus.

Cucumis: Kitamura, Act. Phytotax. \& Geobot. 14 (1950) 41-44, 2 fig.-East Asiat. spp.; $C$. melo and $C$. sativus with varieties.

NaUdIN, Ann. Sc. Nat. IV, 11 (1859) 1-87.Revision of spp. and var.; no key.

Cucurbita: BaIley, Gent. Herb. 2 (1929) 63-115, fig. 29-64.-Domesticated spp.; key.

-, ibid. 6 (1943) 267-322.-American spp.

NAUdIN, Ann. Sc. Nat. IV, 6 (1856) 1-73.Revision; no key.

Russell, J. Wash. Acad. Sc. 14 (1924) 265.Ident. of seeds.

Whitaker, Ann. Missouri Bot. Gard. 34 (1947) 101-111, t. 11-12.-Amer. origin of cultiv. spp.

WhitAKer \& BoHN, Econ. Bot. 4 (1950) 52-81. -Taxonomy, genetics, etc.; key to 5 spp. distinguished.

Hodgsonia: Kundu, J. Bot. 76 (1938) 364-366.Revision; key.

—, J. Bomb. Nat. Hist. Soc. 43 (1942) 362-388. -Indian spp.

Luffa: ExeLL, J. Bot. 71 (1934) 352.-Synon. of $L$. aegyptica.

Neoalsomitra (Alsomitra auct.): Hutchinson, Ann Bot. n.s. 6 (1942) 95-102.-Monogr.; key.

JACOBS, Blumea 7 (1954) 621-622, fig. 3.-Distr. N. integrifoliola.

Thladiantha: GaGnepanN, Bull. Mus. Hist. Nat. 
Paris 24 (1918) 287-296.-Revision Asiat. spp. in Paris herb.

Trichosanthes: Kundu, J. Bot. 77 (1939) 9-14.New spp. from India.

—, J. Bomb. Nat. Hist. Soc. 43 (1942) 362-388. -Revision Indian spp.

Zanonia (Juppia): HARMs, Notizbl. Berl.-Dahlem 8 (1924) 717-719.-Reduction.

MERRILl, Philip. J. Sc. 30 (1926) 429.-Record from Philippines.

\section{CUNONIACEAE}

Engler, A., Pflanzenfamilien ed. 2, 18a (1930) 229-262.

Steenis, C. G. G. J. van, Bull. Jard. Bot. Btzg III, 13 (1934) 196.-List spp. of Mal. mountain genera.

\section{Local}

Borneo: AIRY SHAw, Kew Bull. (1940) 259-261.New Weinmannia.

Van Steenis, J. Bot. 70 (1934) 3-5.-New Weinmannia.

Moluccas: Valeton, Ic. Bog. 3 (1907) 69-70, t. 228.-New Schizomeria from Ambon.

New Guinea: Gibss, Contr. Fl. Phytog. Arfak Mts (1917) 141-143.-New spp. of Pullea and Spiraeanthemum.

Kanehira \& Hatusima, Bot. Mag. Tokyo 56 (1942) 105-109.-New spp. in Aistopetalum, Betchea, and Opocunonia.

Mattfeld, J. Arn. Arb. 20 (1939) 432-436.New spp. in Ceratopetalum (1) and Schizomeria (4).

Perry, J. Arn. Arb. 30 (1949) 139-165.-Keys to spp. represented at the Arn. Arb., Weinmannia excepted; various new spp.

Pulle, Nova Guinea 8 (1911) 645-646.-New Ackama and Spiraeanthemum.

Ridley, Trans. Linn. Soc. Bot. II, 9 (1916) $56-63$, t. 4 , fig. 55 .

Schlechter, Nova Guinea 12 (1914) 491-493.New spp. in Spiraeanthemum, Pullea, and Weinmannia.

-, Bot. Jahrb. 52 (1915) 139-166, fig. 1-9.Synopsis; keys to spp.; various new genera.

一, ibid. 55 (1918) 194.-Reduction Cremnobates to Schizomeria.

SchmidT, Nova Guinea 14 (1924) 150.-New Schizomeria.

Solomons: BURTT, Kew Bull. (1936) 462.-Record of Spiraeopsis celebica.

Fiji-Samoa: Smrth, A. C., J. Arn. Arb. 33 (1952) 119-149.-Revision; ' keys. First record of Pullea.

\section{Genera}

Ackama: White \& Francis, Proc. R. Soc. Queensl. 47 (1936) 59.-Discussions generic delimit. with Betchea in Australia.

\section{CUPRESSA CEAE} See Coniferae

\section{CUPULIFERAE}

See Fagaceae

\section{CYANASTRACEAE}

Clausen, R. T., A review of the Cyanastraceae (Gent. Herb. 4, 1940, 293-304).-Revision Cyanastrum; key.

Engler, A., Pflanzenfamilien ed. 2, 15a (1930) 188-190, fig. 72.

\section{Local}

Java: BACKER, Handb. Fl. Java 3 (1924) 42-43.Only cult. spp.

\section{CYCADACEAE}

Blume, C. L., Rumphia 4 (1848) 11-18, t. 176B-C. MiQuel, F. A. W., Monographia Cycadearum. Utrecht, folio (1842) 1-82, t. 1-8.-Monograph; keys.

- Prodromus systematis Cycadearum (1861) 1-36.-Survey; partial keys.

- Nouveaux matériaux pour servir à la connaissance des Cycadées (Adansonia 9, 1868, 29-73).-Revision.

Pilger, R., Pflanzenfamilien ed. 2, 13 (1926) 44-82, fig. 48-82.

Schuster, J., Pflanzenreich Heft 99 (1932) 1-168.

\section{Local}

Malay Peninsula: Ridley, J. Str. Br. R. As. Soc. no 60 (1911) 67-68.-Enum.

Java: BACKER, Handb. Fl. Java 1 (1924) 25-27.In Dutch; key.

Philippines: Brown \& Kienholz, Philip. J. Sc. 26 (1925) 47-50, t. 1-2.-New sp. from Luzon hills.

Foxworthy, Philip. J. Sc. 6 (1911) Bot. 150152, t. 26-27.-Revision; key.

Merrill, Philip. J. Sc. 60 (1936) 233-241, t. 1-4.-New sp. from Culion.

New Guinea: BECCARI, Malesia 1 (1877) 183.Record.

Kanehira \& Hatusima, Bot. Mag. Tokyo 55 (1941) 385.-Record.

Pulle, Nova Guinea 8 (1910) 343.-Ditto.

Micronesia: Kanehira, J. Jap. Bot. 14 (1938) 579-588, fig. 1-7.-Rev.; no key.

\section{Genera}

Cycas: STapf, Kew Bull. (1916) 1-8.-Distinction between $C$. circinalis, thouarsii and rumphii tabulated.

TANDY, J. Bot. 65 (1927) . 281.-Interpr. of Cycas inermis LoUR.

Warburg, O., Monsunia 1 (1900) 178-181.Revision; key.

\section{CYCLANTHACEAE}

Gleason, H. A., New or noteworthy Monocotyledons from British Guiana (Bull. Torr. Bot. Club 56, 1929, 1-8).-Revision of Carludovica.

Harling, G., Studien über den Blütenbau und die Embryologie der Familie Cyclanthaceae (Svensk Bot. Tidskr. 40, 1946, 257-272, fig. 1-4).

-, Contribution to the knowlegde of the $\mathrm{Cy}$ clanthaceae in Ecuador (Acta Hort. Berg. 15, 9, 1950, 185-206). 
Kuntze, O., Cyclanthaceae (Rev. Gen. Pl. 1891, 737-738).-Revision of Carludovica; key.

SuRANGe, K. R., A contribution to the morphology $\&$ anatomy of the Cyclanthaceae (Trans. Nat. Inst. Sc. India III, 4, 1949, 159-209).

\section{CYPERACEAE}

BoeCKeler, O., Die Cyperaceen des Königlichen Herbariums zu Berlin (Linnaea 35, 1868, 397612; ibid. 36, 1870, 271-512, 691-768; ibid. 37, $1871,1-144,1873,520-647$; ibid. 38, 1874, 223 544 ; ibid. 39, 1875, 1-152; ibid. 40, 1876, 327452; ibid. 41, 1877, 145-356).-Also as a reprint in 2 vols. Berlin (1879).

-, Bot. Jahrb. 5 (1884) 497-521.-New Cyp., some Mal.

Clarke, C. B., Reductions of the Wallich Herbarium (Kew Bull. 1907, 264-281).-Modern names of the Cyperaceae in the Wallichian numerical list.

-, New genera and species of Cyperaceae (Kew Bull. add. ser. 8, 1908, 1-196).-Extract from MS. monogr.; new genera; many new spp.; partial keys.

-, Illustrations of Cyperaceae (1909) i-vi, 1-6, t. 1-144.

Holtrum, R. E., The spikelet in Cyperaceae (Bot. Rev. 14, 1948, 525-541).-Discussion morph. value.

Kükenthal, G., Caricoideae (Pflanzenreich Heft 38, 1909, 1-824, fig. 1-128).-Carex, Schoenoxiphium, Uncinia.

-, Scirpoideae-Cypereae (Pflanzenreich Heft 101, 1935-1936, 1-671, fig. 1-65).-Cyperus.

-, Vorarbeiten zu einer Monographie der Rhynchosporoideae (in FedDE, Rep. 44, 1938, 1-32, 65-101, 161-195; ibid. 46, 1939, 13-32, 65-76; ibid. 47, 1939, 101-119, 209-216; ibid. 48, 1940, 49-72, 195-250; ibid. 50, 1941, 19-50, 112128 ; ibid. 51, 1942, 1-17, 139-193; ibid. 52, 1943, 52-111; ibid. 53, 1944, 85-100, 187-219).Monograph; keys; extracted for genera in Malaysia.

-, ditto (Bot. Jahrb. 74, 1949, 375-509; ibid. 75, 1950, 90-195; ibid. 75, 1951, 273-314; ibid. $75,1952,451-497$ ).-Ditto.

- Neue Cyperaceen aus dem Malayischen und Papuanischen Gebiet (Bull. Jard. Bot. Btzg III, 16, 1940, 300-323).-Also printed in FeDDE, Rep. 53 (1944) 100-111.-Various important critical notes, records and new spp.

-, Neue oder nicht genügend bekannte Cyperaceen (Mitt. Thür. Bot. Ver., N. F., Heft 50, 1943, repr. 1-13).-Misc. notes on Cyperus, Fimbristylis, and Scirpus, mostly Papuan; some from the Philippines and Java; some new spp.

KUNTH, C. S., Enumeratio plantarum II. Cyperaceae. Stuttgart (1837) 1-592.-Critical enumeration; partial keys.

KURz, S., Uber Pandanophyllum and verwandte Gattungen, insbesondere solche, welche im Indischen Archipel vorkommen (J. As. Soc. Beng. 38, ii, 1869, 70-85).-Prelim. revision of Mapanieae; partial key to genera.
Mrquel, F. A. W., Sur quelques genres des Cypéracées de la tribu des Hypolytrées (IIl. FI. Arch. Ind. 1870, 57-66, t. 20-28).-Descr.

NeLMEs, E., Facts and speculations on phylogeny in the tribe Cariceae of the Cyperaceae (Kew Bull. $1951^{3}$, 427-436, 1952).-Phylog. considerations.

PfFiffer, H., Vorarbeiten zur systematischen Monographie der Cyperaceae-Mapanieae (in MEz, Bot. Arch. 12, 1925, 446-472).-Survey of genera \& spp. and their synonymy; key to genera.

Steudel, E. G., Synopsis plantarum Glumacearum pars II. Cyperaceae. Stuttgart (1855) 1-246, 314-319.-Enum. descr. in Latin; contains also spp. based on specimens of CUMING, ZoLLINGER, etc.

UITTIEN, H., Studies in Cyperaceae-Mapanieae IVIII (Rec. Trav. Bot. Néerl. 33, 1936, 133-155, 277-291, fig. 1-3).-Precursory to revision of Mal. spp.

\section{Local}

India: Clarke, J. Linn. Soc. Lond. Bot. 34 (1898) 1-146.-Cyperaceae used for phytogeogr. subdivisions of India; list of material used in preparing the account in Fl. Br. Ind.

Nees von Esenbeck, in Wight, Contr. Bot. India (1834) 69-129.-Revision; partial keys.

SeDGWICK, J. Bombay Nat. Hist. Soc. 25 (1918) 682-700; ibid. 26 (1918) 192-209.-Revision and keys to Cyp. of Bombay Presid.

Sumatra: Backer, Bull. Jard. Bot. Btzg III, 2 (1920) 328.-New Mapania.

KüKenthal, Bull. Jard. Bot. Btzg III, 16 (1940) 300-323.-Many new records.

Nelmes, Kew Bull. (1952) 83-84.-New Carex. RidLey, J. Bot. 23 (1885) 35-36.-New Carex. -, J. Mal. Br. R. As. Soc. no 87 (1923) 107-108. -List.

Malay Peninsula: Clarke, J. Linn. Soc. Lond. Bot. 37 (1904) 1-16.-Carices listed.

Holtrum, Gard. Bull. Str. Settl. 11 (1947) 293296, fig. 6.-New comb. and spp. in Mapania, Scleria, and Diplacrum.

Nelmes, Kew Bull. (1937) 353-355, 1 fig.-New Carex.

-, ibid. (1950) 209.-Ditto.

RIDLEY, J. Str. Br. R. As. Soc. no 23 (1891) 1-19. -Listed records; no new spp.; no key.

-, Mat. Fl. Mal. Pen. (Monocot.) 3 (1907) 55118.-Keys.

UrTTIEN, Gard. Bull. Str. Settl. 10 (1939) 182.New Mapania.

Java: KüKenthal, Bull. Jard. Bot. Btzg III, 16 (1940) 300-323.-Various records.

VAN STEENIS, Bull. Bot. Gard. Btzg III, 17 (1948) 399.-Record of Scleria oryzoides.

Borneo: KüKenthal, Bull. Jard. Bot. Btzg III, 14 (1936) 47-49, fig. 1.-New Oreobolus.

- , ibid. 16 (1940) 300-323.-Various records. MERRILL, Philip. J. Sc. 11 (1916) Bot. 53-54.2 new Mapanias.

-, J. Str. Br. R. As. Soc. no 76 (1917) 78-80.New Mapania; further listed records.

-, ibid. no 85 (1922) 156-159.-4 new Mapanias. 
Pfeiffer, Mitt. Inst. Allg. Bot. Hamburg 7 (1928) 166-176.-Listed records WINKLER coll.; a new Scirpus.

Ridley, J. Str. Br. R. As. Soc. no 46 (1906) 221228.-Enum.; new spp. in the Mapanieae.

StapF, Trans. Linn. Soc. Bot. II, 4 (1894) 244246.-Kinabalu records; new spp. in Scirpus and Cladium.

- \& Turrill, J. Linn. Soc. Lond. Bot. 42 (1914) 173-185, fig. 7.-List of Kinabalu records; new spp. in Scirpus, Schoenus, Vincentia, and Carex; Lophoschoenus n.g.

UitTIEN, Rec. Trav. Bot. Néerl. 32 (1935) 193202.-List RichaRDs's coll.

Celebes: Kükenthal, Bull. Jard. Bot. Btzg III, 16 (1940) 300-323.-Various records.

Philippines: Clarke, Philip. J. Sc. 2 (1907) Bot. 77-110.-List of spp. represented at Kew.

Elmer, Leafl. Philip. Bot. 3 (1910) 853-855.New spp. in Carex, Cladium and Fimbristylis. -, ibid. 10 (1938) 3525-3542.-List of Irosin Valley spp.; new spp. in Mapania, Carex, and Scleria; no keys.

GANDoger, Bull. Soc. Bot. Fr. 66 (1919) 296.New Fuirena and Fimbristylis from Philippines and Java.

Kükenthal, in Elmer, Leafl. Philip. Bot. 4 (1911) 1169-1170.-New Carex.

-, Philip. J. Sc. 6 (1911) Bot. 57-64.-Conspectus of Caricoideae; keys.

Merrill, Philip. J. Sc. 7 (1912) Bot. 231.-New Fimbristylis.

-, ibid. 35 (1928) 4-6.-Nomencl. notes.

-, ibid. 60 (1936) 27-28.-Identif. of an erroneously localized Amer. Scleria.

Palla, Allg. Bot. Zeitschr. 13 (1907) 49-50.New Scleria.

StAPF, Philip. J. Sc. 19 (1921) 65-66.-Descr. new Vincentia.

Moluccas: Merrill, Philip. J. Sc. 11 (1916) Bot. 256-259.-Ambon records; new Vincentia.

New Guinea: Blake, J. Arn. Arb. 28 (1947) 99-116, fig. 1-4; 207-229, fig. 1, t. 1-2; ibid. 29 (1948) 90-102, fig. 1; ibid. 35 (1954) 203-238, fig. 1-2, t. 1.-Listed records coll. BRAss; new spp. \& comb. in Carex, Mapania, Paramapania, Cyperus, Schoenus, Fimbristylis; key to Papuan Sclerias! KükeNTHAL, Bot. Jahrb. 59 (1924) 41-60.Listed records; new spp. in Fimbristylis, Mapania, Thoracostachyum.

一, ibid. 69 (1938) 255-265.-List; new spp. in Cyperus and Carex.

一, ibid. 70 (1940) 463-468.-List Clemens coll., 3 new Carices.

—, Bull. Jard. Bot. Btzg III, 16 (1940) 300-323. -Various records and new spp.

一, Mitt. Thür. Bot. Ver., N. F., Heft 50 (1943) repr. 1-13.-Various critical notes on Cyperus, 2 new Fimbristylis and a new Scirpus.

Nelmes, Kew Bull. (1949) 378-386.-Brass coll.; no key.

-, ibid. (1949) 387-392.-Clemens coll.; no key.

OHwi, Bot. Mag. Tokyo 56 (1942) 199-215.Listed Kanehira-Hatusima records; new spp. in Carex, Mapania, Thoracostachyum, Cladium, Schoenus, Scirpus, and Fimbristylis.

RIDLEY, Trans. Linn. Soc. Lond. Bot. II, 9 (1916) 241-247.-Listed records; new spp. in Cyperus, Lipocarpha, Cladium, Hypolytrum, Thoracostachyum, Mapania, and Carex.

UitTIEN, J. Arn. Arb. 20 (1939) 213-215.-List Brass coll.; only Mapanieae.

VAlCKenier Suringar, Nova Guinea 8 (1912) 695-713, t. 113-118.-List; many new spp.

Micronesia: Kükenthal, Bot. Jahrb. 59 (1924) 2-10.-List of $48 s p p$.

Australia: Blake, Notes on Australian Cyperaceae I (Proc. R. Soc. Queensl. 48, 1937, 89-94, t. 2-3); ditto II (ibid. 49, 1938, 154-155); ditto III (ibid. 51, 1940, 32-50); ditto IV (ibid. 177-182); ditto V (ibid. 52, 1941, 55-61); ditto VI (ibid. 54, 1943, 69-74); ditto VII (ibid. 58, 1947, 35-50); ditto VIII (ibid. 60, 1949, 45-53).-Include occasionally Mal. spp.

-, Transact. R. Soc. South Australia 67 (1943) 52-61.-Critical notes on S. Austr. spp.; key to the Cladium-Mariscus-alliance.

\section{Genera}

Arthrostylis: Kükenthal, in Fedde, Rep. 53 (1944) 192-200.-Revision.

Capitularia: RidLEY, Trans. Linn. Soc. Lond. Bot. II, 9 (1916) 244-245.-Reduces Capitularia to Chorisandra!

UitTIEN, Rec. Trav. Bot. Néerl. 33 (1936) 289291.-New Solomon Isl. $s p$.

Valckenier SurINGAR, Nova Guinea 8 (1912) 711, t. 118.-Type descr.

Carex: Bootr, Illustrations of the genus Carex. London 4 parts. I (1858) 1-74, t. 1-200; II (1860) $75-103$, t. $201-310$; III (1862) 105-126, t. 311411 ; IV (1867) 127-233, t. 412-600.-Monogr. descr, and plates.

Kükenthal, Pflanzenreich Heft 38 (1909) 67824, fig. 1-128.

Nelmes, Kew Bull. (1938) 106-110.-Critical notes on some spp. with key.

—, Proc. Linn. Soc. Lond. sess. 155 (1944) 277285.-Rev. Austral. spp.; key.

—, Kew Bull. (1946) 5-29.-Key to Mal. \& Polyn. spp.

—, ibid (1950) 198-208.-Descr. new or noteworthy spp.

—, ibid. (1951) 121.-Descr. 3 new sect.

-, Reinwardtia 1 (1951) 221-450.-Complete revision; keys.

—, ibid. 2 (1954) 373-383.-Addit. notes listed.

Carpha: Kükenthal, in Fedde, Rep. 47 (1939) 101-119, 209-211.-Revision.

Cladium: Kükenthal, in Fedde, Rep. 51 (1942) 1-17, 139-193.-Revision; key.

VAN SteEnIs, Arch. Hydrobiol. Suppl. 3 (1932) 281-282, fig. 3.-Records of 2 spp.

Costularia (Lophoschoenus): KÜKENTHAL, in FEDDE, Rep. 46 (1939) 13-32, 65-76.-Revision; key.

Cyperus (Kyllinga, Juncellus, Pycreus, Mariscus): Blake, Univ. Queensl. Pap. Dep. Biol. 2, no 2 (1942) 1-14, t. 1-8.-C. rotundus \& allied spp. in Australia; synon.; key. 
Clarke, J. Linn. Soc. Lond. Bot. 21 (1884) i-xi, 1-202, t. 1-4.-Revision Indian spp.; partial keys. KERN, Reinwardtia 1 (1952) 463-466, fig. 1.C. alulatus $n$. $s p$., hitherto often confused with C. iria; India.

一, ibid. 2 (1952) 97-130. fig. 1-14.-Critical notes; some new spp.; precursory to revision. -, ibid. 3 (1954) 27-66, fig. 1-12.-Ditto, 2nd part. Kükenthal, Pflanzenreich Heft 101 (1936) 1671.-Monograph; keys.

—, Mitt. Thür. Bot. Ver., N. F., Heft 50 (1943) repr. 1-8.-Critical addit. notes; new key for $\S$ Graciles.

Valckenier Suringar, Het geslacht Cyperus (sensu amplo) in den Maleischen Archipel benevens een overzicht van de geschiedenis der systematiek van de familie der Cyperaceën. Thesis, Leeuwarden (1898) i-xv, 1-192, t. 1-6.Revision Indon. spp.; no keys; in Dutch; descr. in Latin.

Eleocharis: Blake, Proc. R. Soc. Queensl. 50 (1939) 88-132, t. 7-10.-Monograph of Austr. \& N. Zeal. spp.; key.

Nelmes, Kew Bull. (1952) 289-290.-Submerged Afr. 1-flowered spp.

SVENSON, Rhodora 31 (1929) 121-135, 152-163, 167-191, 199-219, 224-242; ibid. 34 (1932) 193203, 215-227; ibid. 36 (1934) 377-389; ibid. 39 (1937) 210-231, 236-272; ibid. 41 (1939) 1-19, 43-77, 90-110.-Monographic studies, with plates and text maps.

Fimbristylis (Abildgaardia): BLAKE, Univ. Queensl. Pap. Dept of Biol. 1, no 13 (1940) 1-14.-New Queensl. spp.

BOECKELER, Linnaea 37 (1871) 2-56.-Revision Berlin specim.; partial keys.

Fuirena: Bush, Rep. Missouri Bot. Gard. 16 (1905) 87-99.-North Amer. spp.; key.

Gahnia: BenL, Flora 131 (1937) 369-386, fig. 1-11. -Dispersal mechanisms.

-, in FEDDE, Rep. 44 (1938) 196-199.-New spp. —, ibid. 49 (1940) 30-34.-Nomenclator.

-, Bot. Arch. 40 (1940) 151-257, fig. 1-30.Compl. monogr.; keys.

-, Bot. Jahrb. 75 (1950) 82-89.-Critical remarks on following paper.

KüKENTHAL, in FEDDE, Rep. 52 (1943) 52-111.Revision; key.

Hypolytrum p.p.; UITTIEN, Rec. Trav. Bot. Néerl. 33 (1936) 153-155.-Key.

Isolepis: BeETLE, Amer. Midl. Nat. 34 (1945) 723734.-Evaluation of 470 combinations published under Isolepis with their correct name (belonging to 18 genera of $C y p$. presently accepted).

Lepidosperma: Kükenthal, in Fedde, Rep. 50 (1941) 19-50, 112-128.-Revision; key.

Lipocarpha: DANDY, J. Bot. 70 (1932) 331-332.Synon. L. senegalensis.

Mapania \$ Halostemma: UITTIEN, Rec. Trav. Bot. Néerl. 33 (1936) 151-153, fig. 3, 284-289.Revision; key.

Mapania \& Pandanophyllum: UITTIEN, Rec. Trav. Bot. Néerl. 33 (1936) 145-151.-Key.

Mapania \$ Pandanoscirpus: UrrTIEN, Rec. Trav Bot. Néerl. 33 (1936) 277-284.-Revision; key.
Oreobolus: KüKenthaL, in Fedde, Rep. 48 (1940) 60-72.-Revision; key.

Pfeiffer, in Fedde, Rep. 23 (1927) 350-352.Key.

Paramapania: UrtTIEN, Rec. Trav. Bot. Néerl. 32 (1935) 184-192; ibid. 33 (1936) 141-145.-Revision; key.

Remirea: Kükenthal, in Fedde, Rep. 53 (1944) 200-209.-Revision.

Rhynchospora: BLAKe, Proc. R. Soc. Queensl. 51 (1940) 47.-New sp. from Philippines, Madura \& Queensl.

KükenthaL, Bot. Jahrb. 74 (1949) 375-509; ibid. 75 (1950) 90-195; ibid. 75 (1951) 273-314.Revision; keys.

Schoenoxiphium: Kükenthal, Pflanzenreich Heft 38 (1909) 28-33.

—, Bull. Jard. Bot. Btzg III, 16 (1940) 312-313. -New Sumatran sp.

Schoenus: KüKenthal, in Fedde, Rep. 44 (1938) 5-32, 65-101, 161-195; corrections ibid. 48 (1940) 195-250.

Scirpodendron: Kurz, J. As. Soc. Beng. 38, ii (1869) 70-85; cf. Flora 52 (1869) 441.

Scirpus: BaCkER, Onkruid. Jav. Suiker. (1928) 147-151.-In Dutch; key to Jav. spp.

Beetle, Amer. J. Bot. 27 (1940) 63-64.-Delimitation of subgenera.

—, ibid. 29 (1942) 82-88.-Key for $\S$ Bolboschoenus.

一, ibid. 30 (1943) 395-401.-Key for $\S$ Schoenoplectus.

Blake, Proc. R. Soc. Queensl. 58 (1947) 38-42. -Some critical notes; key for Sc. merrillii and allies in Australia.

-, ibid. 62 (1952) 83-88.-Critical notes and key to Sc. supinus, lateriflorus, and juncoides.

Palla, Bot. Jahrb. 10 (1889) 293-301, t. 11.Key to allied genera, partly segregates.

Scleria: Blake, Proc. R. Soc. Queensl. 62 (1952) 88-89.-Critical notes; $S$. oryzoides reduced. CORE, Brittonia 2 (1936) 1-105, t. 1-3.-Revision Amer. spp.; key.

Pí́rart, Lejeunea 13 (1951) 1-70.-Rev. spp. Congo Belge.

Thoracostachyum: UITTIEN, Rec. Trav. Bot. Néerl. 33 (1936) 133-140.

Uncinia: Kükenthal, Pflanzenreich Heft 38 (1909) 50-67.

NeLMEs, Kew Bull. (1949) 140-145.-Three new spp., 2 from New Guinea.

\section{CYRTANDRACEAE}

See Gesneriaceae

\section{DAPHNIPHYLLACEAE}

Rosenthal, K., Pflanzenreich Heft $68^{5}$ (1919) 1-16, fig. 1.

-, Pflanzenfamilien ed. 2, 19c (1931) 233-235, fig. 124.

\section{Local}

Philippines: Quisumbing \& Merrill, Philip. J. Sc. 37 (1928) 161-162.-New sp. 


\section{Genera}

Daphniphyllum: CroIzat, Lingn. Sc. J. 20 (1941) 79-103.-Taxon. position.

—\& MetCalfF, Lingn. Sc. J. 20 (1941) 105-127. -Chin. \& Jap. spp.

HARRISON, Kew Bull. (1951) 369-370, fig. 1-2 (1952).-Note on pseudohermaphrodism in $D$. macropodum MiQ. cult. at Kew.

P'EI, Contr. Biol. Lab. Sc. Soc. China (bot. ser.) 8 (1933) 233-243.-Chin. spp.

\section{DATISCACEAE}

GilG, E., Pflanzenfamilien ed. 2, 21 (1925) 543547, fig. 249-250.

Steenis, C. G. G. J. vaN, F1. Mal. I, 4 (1953) 382387, fig. $1-6$.

\section{Genera}

Octomeles: Miquel, Fl. Ind. Bat. Suppl. (1861) 336.-Type descr.

WARBURG, Bot. Jahrb. 13 (1891) 385-386.Record New Guinea; new sp.

Tetrameles: KoORders \& Valeton, Bijdr. Booms. Java 9 (1903) 36-40.

Merrill \& Perry, J. Arn. Arb. 23 (1942) 407. -New Guinea.

\section{DICHAPETALACEAE \\ (Chailletiaceae)}

BeCCARI, O., Malesia 1 (1877) 175-177.-Affinity genus; list 3 spp. of which one Papuan new.

Engler, A., Pfianzenfamilien ed. 2, 19 c (1931) 1-11, fig. 1-5.

\section{Local}

Borneo: Merrill, J. Str. Br. R. As. Soc. no 86 (1922) 319.--Record of a Philip. sp.

RIDLEY, Kew Bull. (1930) 372-373.-Descr. new spp.; records.

-, ibid. (1938) 234.-Descr. of new sp. with 4merous flowers and entire petals.

Philippines: Merrill, Philip. J. Sc. 17 (1920) 271272.-Descr. 3 new spp., one with entire petals. —, ibid. 30 (1926) 401-402.-New sp. from Sulu.

New Guinea: KRAUSE, Bot. Jahrb. 49 (1912) 168169.-Descr. 2 new spp.

一, ibid. 62 (1929) 341-346.-Survey; key.

Queensland: White, Proc. R. Soc. Queensl. 53 (1942) 211-212.-First (new) sp. from Australia.

\section{DILLENIACEAE}

(for Saurauia see Actinidiaceae)

Gilg, E. \& E. WerdermanN, Pflanzenfamilien ed. 2, 21 (1925) 7-36, fig. 1-25.

Hoogland, R. D., Fl. Mal. I, 4 (1951) 141-174, fig. 1-13.

Martelli, U., Le Dilleniaceae Malesi e Papuane delle collezioni Beccari (in BeCCARI, Malesia 3, 1886, 150-167).-Census \& descr. BecCARI coll.; no keys; compl. en. of Dillenia and Wormia.

Miquel, F. A. W., Ann. Mus. Bot. Lugd.-Bat. 4 (1868) 73-80, t. 1.-Revision; no keys.

VRIESE, W. H. DE, Pl. Ind. Bat. Orient. (1856) 7981, t. 6-7.-Descr.

\section{Local}

India: Parkinson, Ind. For. 61 (1935) 447-453, t. 28-29.-Key to 3 allied spp. of Dillenia, one new (Andamans).

Sumatra: Miquel, Ann. Mus. Bot. Lugd.-Bat. 1 (1864) 315, t. 9.-New Wormia from Banka.

Malay Peninsula: CoRNer, Gard. Bull. Str. Settl. 10 (1939) 3-11.-Critical remarks on some spp.

Borneo: Merrill, Plant. Elm. Born. (1929) 194196.-List; new sp. in Tetracera and Dillenia.

RIDley, Sarawak Mus. J. 1 (1913) 68-71.-List; 2 new Tetraceras.

Java: DE WIT, Bull. Bot. Gard. Btzg III, 18 (1949) 208-209.-Disc. whether Dillenia suffruticosa is native.

Philippines: Merrill, Philip. J. Sc. 9 (1914) Bot. 517-521.-4 new Dillenias.

New Guinea: DiELs, Bot. Jahrb. 57 (1922) 436-441. -Revision; keys.

-, Nova Guinea 14 (1923) 81.-Records.

Gibis, Contr. Fl. Phytog. Arfak Mts (1917) 148-149.-New Hibbertia.

Kanehira \& Hatusima, Bot. Mag. Tokyo 57 (1943) 63.-Records.

LAUTERBach, Nova Guinea 8 (1910) 305.-Some records.

RIDLEY, Trans. Linn. Soc. Lond. II, Bot. 9 (1916) 13-14.-New Wormia.

Smith, A. C., J. Arn. Arb. 22 (1941) 497-502.List; 3 new Wormias.

White \& Francis, Proc. R. Soc. Queensl. 38 (1927) 242-243, fig. 9.-New Wormia.

\section{Genera}

Dillenia (Wormia): Corner, Gard. Bull. Str. Settl. 10 (1939) 3-11.-Critical remarks.

Hoogland, Blumea 7 (1952) 1-145, fig. 1-11.Complete revision of the genus; key.

Tetracera: Hoogland, Reinwardtia 2 (1953) 185224, pl. 1.-Revision Indo-Austr. spp.; key.

Trematanthera $=$ Saurauia (Actinidiaceae).

\section{DIOSCOREACEAE}

Burkill, I. H., Fl. Mal. I, 4 (1951) 293-335, fig. 1-14.

KNUTH, R., Pflanzenreich Heft 87 (1924) 1-387, fig. 1-69.

-, Pflanzenfamilien ed. 2, 15a (1930) 438-462, fig. 192-206.

Prain, D. \& I. H. Burkill, Kew Bull. (1926) 118120.-Corrections on KNUTH's monograph.

Uline, E. B., Eine Monographie der Dioscoreaceen (Bot. Jahrb. 25, 1881, 126-165).-Key to genera and sections.

\section{Local}

Malay Peninsula: BurkilL, Gard. Bull. Str. Settl. 3 (1925) 289-290.-Observations on Stenomeris. Prain \& Burkill, ibid. 4 (1927) 86-88, 2 fig.Observ. on Diosc. tamarisciflora.

Java: BACKER, Handb. Fl. Java 3 (1924) 108-116. -In Dutch; key.

Borneo: KNUTH, in Fedde, Rep. 36 (1934) 127.New sp. 
Lesser Sunda Islands: KNUTH, in Fedde, Rep. 38 (1935) 119-120.-New Dioscorea from Sumba.

Philippines: Prann \& Burkill, in Elmer, Leaf Philip. Bot. 5 (1913) 1589-1599.-List; descr new spp.

Celebes: Burkill, Kew Bull. (1950) 259-260. fig -New sp.

KNUTH, in FeDDE, Rep. 36 (1934) 127-128.new spp. coll. KJELLBERG.

New Guinea: Knuth, in Fedde, Rep. 42 (1937 163-164.-Descr. 3 new spp.

\section{Genera}

Dioscorea (Peripetasma) : BuRKILL, Gard. Bull. Str Settl. 3 (1924) 258-259, t.-Varieties of $D$. pentaphylla in Mal.; key to var.

-, J. S. Afr. Bot. (1952) 177-191.-Reduction of Testudinaria as an Afr. sect.; key.

- \& Holtrum, Gard. Bull. Str. Settl. 3 (1924) 260.-D. piscatorum as a fish poison.

- \& PraIn, Ann. R. Bot. Gard. Calcutta 14, part 1 (1936) x + 210 pp.; part 2 (1938) ii + $211-$ $528+x x$, t. 1-150.-Complete revision of IndoAustr. spp.

Prann \& Burkill, J, As. Soc. Beng. II, 4 (1908) 447-457.-Misc. descr. new spp.

-, ibid. II, 10 (1914) 5-41.-Revision IndoAustr. spp.; key.

-, Kew Bull. (1919) 339-375.-Key to Linnean spp. of Dioscorea; nomencl. D. sativa.

一, ibid. (1925) 58-66.-List misc. new spp.; reduction Peripetasma p. 66.

-, ibid. (1926) 118-120.-Reduction of KNUTH's and ULINE's spp.

-, ibid. (1931) 88-91, map.-Key to spp. of $\S$ Stenocorea.

-, Gard. Bull. Str. Settl. 5 (1932) 51-57, fig. 1-13.-Observ. on D. gibbiflora; new key to Dioscorea series of $D$. alata.

-, Kew Bull. (1933) 240-246.-Descr. new spp. from SE. Asia, incl. one from Sumatra \& Malaya.

Stenomeris: Beccari, Nuovo Giorn. Bot. Ital. 2. (1870) 8-12, t. 2.-New sp. from the Philippines. TAubert, Bot. Jahrb. 15 (1893) Beibl. 38, p. 1-2. -Revision; key.

Trichopus: Beccari, Nuovo Giorn. Bot. Ital. 2 (1870) 13-19, t. 3 .

\section{DIPSACACEAE}

Alvarado, S., Die morphologische Aufbau des Hüllkelches der Dipsacaceen (Bot. Jahrb. 61, 1927, Beibl. 138, p. 10-21, t. 9).-Morph. of epicalyx.

Steenis, C. G. G. J. vaN, Fl. Mal. I, 4 (1951) 290 292, fig. 1.

\section{Genera}

Triplostegia (Hoeckia): VAN STEENIS, Fl. Mal. I, 4 (1951) 290-292, fig. 1.-Complete revision.

\section{DIPTEROCARPACEAE}

BERGER, L. G. DEN, Unterscheidungsmerkmale von rezenten und fossilen Dipterocarpaceengattungen (Bull. Jard. Bot. Btzg III, 8, 1927, 495-498). -Anat.
Brandis, D., An enumeration of the Dipterocarpaceae, etc. (J. Linn. Soc. Bot. 31, 1895, 1-148, t. 1-3).-Monograph chiefly based on specimens at Kew and London; partial geogr. keys.

Burck, W., Sur les Diptérocarpacées des Indes néerlandaises (Ann. Jard. Bot. Btzg 6, 1887, 145-249).-Anat.; key to genera; crit. enum. of spp.; descr. of spp.

FoxworthY, F. W., Distribution of the Dipterocarpaceae (J. Arn. Arb. 27, 1946, 347-354).General figures.

GILG, E., Pflanzenfamilien ed. 2, 21 (1925) 237269, fig. 108-118.

HeIM, F., Recherches sur les Diptérocarpacées. Introduction à la monographie générale de la famille (1892) i-v, 1-186, t. 1-11.-Anat.; no exact key to the genera, but several keys on basis of different organs; no spp. treated.

Merrill, E. D., Distribution of the Dipterocarpaceae (Philip. J. Sc. 23, 1923, 1-33, 2 maps, $t$. 3-8).-Distr. in the light of geol. hist. of Malaysia.

MrQuel, F. A. W., Dipterocarpaceae novae vel minus cognitae (Ann. Mus. Bot. Lugd.-Bat. 1, 1864, 213-215).-Descr. new spp.

-, Annotationes de Dipterocarpeis (Ann. Mus. Bot. Lugd.-Bat. 3, 1867, 83-85).-Complete list of Mal. spp.; records.

Parius, J. P., Bijdrage tot de kennis van de OostIndische damarhars. Thesis, Leiden (1933) 1135, fig. 1-15, t.-Descr. some resinous Dipteroc.; anat.; phytochem.; some new spp.; in Dutch.

Scheffer, R. H. C. C., Nat. Tijd. Ned. Ind. 31 (1870) 346-351.-Misc. descr. many new spp.

, ibid. 32 (1873) 407-409.-Misc. descr. from Banka and Borneo.

Slooten, D. F. van, Sertulum Dipterocarpacearum malayensium I (Bull. Jard. Bot. Btzg III, $16,1940,430-454$, fig. 1-10).-New or noteworthy spp. in Anisoptera, Dipterocarpus, Vatica, and Dryobalanops.

_, ditto II (ibid. 17, 1941, 96-138, fig. 11-20).Ditto, Dipterocarpus, Shorea (key to \$ Isoptera), Hopea, and Vatica.

-, ditto III (ibid. 17, 1942, 220-255, fig. 21-36). -Ditto, one Shorea; new spp. of Vatica subg. Isauxis; key to spp. of subg. Synaptea.

-, ditto IV (ibid. 18, 1949, 229-269, fig. 1-14). -Ditto, Shorea.

-, ditto V (Reinwardtia 2, 1952, 1-68, fig. 1-22). -The Dipterocarpaceae of Eastern Malaysia (Celebes, Moluccas, and New Guinea); Anisoptera, Hopea, Shorea.

\section{Local}

Siam: RYAN \& KerR, J. Siam Soc. $8^{1}$ (1911) 1-24, t. 1-5.-Revision spp. of N. Siam; key; econ. \& sylvic. possibilities; S. Siam. spp. listed.

Sumatra: PARus, in Fedde, Rep. 33 (1933) 243-244. -Descr. some new spp.

Malay Peninsula: Foxworthy, Mal. For. Rec. 10 (1932) 1-289, t. 1-23, 1 map.-Descr.; distr.; uses; keys.

Herm, Bull. Mens. Soc. Linn. Paris no 127 (1892) 
1009-1011.-Descr. Duvalliella problematica $n$. g. $n$. sp. from Penang (= Dipterocarpus). $C f$. SYMINGTON (1937) below.

Symington, Notes on Malayan Dipterocarpaceae (Gard. Bull. Str. Settl. 7, 1933, 129-159, t. 33-47).-Notes on Shoreas.

-, ditto II (ibid. 8, 1934, 1-40, t. 1-10).-Anisoptera, Balanocarpus, Hopea, Vatica, Cotylelobium.

-, ditto III (ibid. 8, 1935, 265-292, t. 16-28).Notes on Shoreas and Hopeas.

-, ditto IV (ibid. 9, 1938, 319-354, t. 17-27).Various notes on Dipterocarpus, Hopea, Shorea, and Parashorea.

- , ditto V (ibid. 10, 1939, 336-386, t. 11-27).Cont. Hopea, Shorea, Parashorea, Dryobalanops. -, ditto VI (J. Mal. Br. R. As. Soc. 19, 1941, 139-168, t. 1-7).-Descr. new spp. in Hopea (6), Vatica (1), Shorea (1); discussion of special groups of Shorea and Vatica.

-, Kew Bull. (1937) 318-319.-Reduction Duvalliella to Dipterocarpus.

-, Mal. For. Rec. no 16 (1943) i-xliii, 1-244, fig. 1-114.-Complete revision of Mal. Pen. spp. Thiselton-DYER, J. Bot. 12 (1874) 154.-New Vatica.

Borneo: HeIm, Bull. Mens. Soc. Linn. Paris no 120 (1891) 954-958; ibid. 122 (1891) 970-976.Descr. new spp. coll. BECCARI; Richetia n.g.

—, ibid. no 120 (1891) 958-960.-Descr. Pierrea (coll. BecCari).

Merrill, Philip. J. Sc. 29 (1926) 398-400.-2 new Dipterocarpus.

PARIJS, in FEDde, Rep. 33 (1933) 243-244.Misc. new spp.

Van Slooten, in Merrill, Pl. EIm. Born. (1929) 200-206.-List; one new comb.

Vesque, C. R. Ac. Sc. Paris 70 (1874) 625-627.Descr. of new spp. of Dipterocarpus from the BeCCARI collection.

Philippines: Elmer, Leafl. Philip. Bot. 4 (1912) 1469-1474.-4 new spp.

FoxworThy, Philippine Dipterocarpaceae (Philip. J. Sc. 6, 1911, Bot. 231-285, t. 34-44; ibid. 13, 1919, 163-199, t. 1-2).-Revision; keys.

一, ditto III (ibid. 67, 1938, 241-331, t. 1-9).Complete revision; keys!

—, in Elmer, Leafl. Philip. Bot. 6 (1913) 19491958.-Records \& new spp. from Agusan region (Mindanao).

Gil., Bot. Jahrb. 18 (1894) Beibl. 45, p. 38-39. -New Philip. Shorea.

Merrill, Philip. J. Sc. 26 (1925) 475-477.New Shorea.

-, ibid. 30 (1926) 411.-Record of Vatica papuana.

Thiselton-Dyer, J. Bot. 16(1878) 100.-New sp.

Celebes: van Slooten, Reinwardtia 2 (1952) 1-68, fig. 1-21.-Survey of spp.

Moluccas: van Slooten, Reinwardtia 2 (1952) 1-68, fig. 1-21.-Survey of spp.

New Guinea: Diels, Bot. Jahrb. 57 (1922) 460463.-Revision; keys.

GılG, Bot. Jahrb. 18 (1894) Beibl. 45, p. 38-39. -New Vatica.
Van Slooten, Nova Guinea 14 (1927) 222-227. -, Reinwardtia 2 (1952) 1-68, fig. 1-21.-Survey; no keys.

Thiselton-Dyer, J. Bot. 16 (1878) 98-103.-2 new spp.

White, Proc. R. Soc. Queensl. 43 (1932) 49.New Hopea.

\section{Genera}

Anisoptera: van Slooten, Bull. Jard. Bot. Btzg III, 8 (1926) 1-17, fig. 1-2.-Revision; key.

Cotylelobium: van Slooten, Bull. Jard. Bot. Btzg III, 10 (1929) 393-406, fig. 1-3; ibid. 12 (1932) 43-44.-Revision; keys in both papers.

Dipterocarpus: van Slooten, Bull. Jard. Bot. Btzg III, 8 (1927) 263-352, fig. 1-15; ibid. 16 (1940) 443-448; ibid. 17 (1941) 97-110.-Revision with additions; key in the first paper.

Thiselton-DYeR, J. Bot. 12 (1874) 101-108, $t$. 143-145.-Revision; partial key.

-, ibid. 12 (1874) 152-154.-Critical notes on VESQUE's spp. (see below).

Dryobalanops: van Slooten, Bull. Jard. Bot. Btzg III, 12 (1932) 1-45, fig. 1-5; ibid. 16 (1940) 448451.-Revision; key.

Thiselton-Dyer, J. Bot. 12 (1874) 98-101, t. 142.-Revision; no key.

Parashorea: van Slooten, Bull. Jard. Bot. Btzg III, 8 (1927) 370-380, fig. 1-3.-Revision; key.

Shorea \& Isoptera: van SLOOTEN, Bull. Bot. Gard. Btzg III, 17 (1941) 115-130.

Shorea: van Slooten, Bull. Bot. Gard. Btzg III, 18 (1949) 230-269.-New spp.

Upuna: Symington, Bull. Jard. Bot. Btzg III, 17 (1941) 88-95, t. 1-2, fig. I-II.-New Bornean genus.

Vatica: van Slooten, Bull. Jard. Bot. Btzg III, 9 (1927) 67-137, fig. 1-13.-Revision; key. -, ibid. 17 (1941) 133-137.-Additions.

Vatica \& Isauxis: vaN SLooren, Bull. Jard. Bot. Btzg III, 17 (1942) 223-242.

Vatica \& Synaptea: vaN Slooten, Bull. Jard. Bot. Btzg III, 17 (1942) 243-254.-Key.

\section{DROSERACEAE}

Drels, L., Pflanzenreich Heft 26 (1906) 1-136, fig. $1-40$.

-, Pflanzenfamilien ed. 2, $17 \mathrm{~b}$ (1936) 766-784, fig. 473-486.

Steenis, C. G. G. J. van, Bull. Jard. Bot. Btzg III, 13 (1933) 106-109, 202.

-, Fl. Mal. I, 4 (1953) 377-381, fig. 1-7.

\section{Local}

Timor: von MaLm, in Fedde, Rep. 41 (1937) 295. -Record Aldrovanda.

New Guinea: van Steenis, J. Arn. Arb. 28 (1947) 420-421.-List BRAss coll.

\section{EBENACEAE}

Bakhuizen van den Brink, R. C., Revisio Ebenacearum Malayensium (Bull. Jard. Bot. Btzg III, 15, 1936, 1-49; ibid. 1937, 59-178; ibid. 1938, 179-368; ibid. 1941, 369-515).-Plates and final part in the press.-Complete revision; keys. 
Hiern, W. P., A monograph of Ebenaceae (Trans. Cambr. Philos. Soc. 12, 1873, 27-300, 1 fig., t. 111.-Complete monograph; keys to genera and spp.

\section{Local}

Siam: Bakhuizen van den Brink, Kew Bull. (1937) 382-392. 一, ibid. (1938) 24-26, t.-New sp.

Ceylon: Wright, Ann. R. Bot. Gard. Perad. 2 (1904) 1-106, 133-210, t. 1-20.-Revision; key.

Malay Peninsula: BAKhUIZEN VAN DEN BRINK, Gard. Bull. Str. Setl. 7 (1933) 161-190, t. 48-50.

Borneo: Diels \& Hackenberg, Bot. Jahrb. 60 (1926) 313-314.-2 new Diospyros.

MerRILL, J. Str. Br. R. As. Soc. no 87 (1923) 2526.-New Diospyros.

RIDLEY, J. Bot. 63 (1925) 51-52.-New sp.

Philippines: MerriLl, Philip. J. Sc. 26 (1925) 487-488.-New sp.

-, ibid. 29 (1926) 483.-New sp.

-, ibid. 30 (1926) 421-423.-2 new spp.

Moluccas: Merrill, Philip. J. Sc. 11 (1916) Bot. 303-304.-New Maba from Ambon.

New Guinea: Hiern, Nova Guinea 8 (1909) 199200.-List; 2 new spp.

Solomon Islands: BAKHUIZEN VAN DEN BRINK, J. Arn. Arb. 16 (1935) 68-69, t. 120-122,-Records.

\section{ELAEAGNACEAE}

Nelson, Aven, The Elaeagnaceae. A mono-generic family (Am. J. Bot. 22, 1935, 681-683).Shepherdia and Hippophae are reduced to Elaeagnus. Key to Rocky Mt spp.

Schlechtendal, D. F. L. von, Elaeagnacearum in Candollei prodromo (Vol. XIV) expositarum adumbratis (Linnaea 30, 1859-60, 304-386).Comments; descr.; no keys; in Latin.

Servetraz, C., Note préliminaire sur la systématique des Elaeagnacées (Bull. Herb. Boiss. II, 8, 1908, 381-394).-Prelim. revision; keys.

-, Monographie des Eléagnacées (Beih. Bot. Centralbl. 25, ii, 1909, 1-420, fig. 1-145).-Monogr.; keys.

\section{Local}

Formosa: LI, Lloydia 15 (1952) 156-160.-Key to 8 spp. of Elaeagnus; 2 new.

Java: MiQueL, Pl. Jungh. (1852) 173,-List.

New Guinea: Merrill \& Perry, J. Arn. Arb. 22 (1941) 267-268.-Record.

\section{ELA EOCARPACEAE}

\section{Local}

Sumatra: Merrill, Pap. Mich. Acad. Sc. Arts \& Lett. 19 (1934) 168.-New Elaeocarpus.

Malay Peninsula: BurkiLl, J. Str. Br. R. As. Soc. no 75 (1917) 41.-New Elaeocarpus.

Corner, Gard. Bull. Str. Settl. 10 (1939) 308329.-Revision; 2 new spp.; key.

Henderson, Gard. Bull. Str. Settl. 5 (1930) 74 76, 2 fig.-New Elaeocarpus.

Java: Adelbert, Blumea 6 (1948) 310-313.Critical notes.
Koorders, Bull. Jard. Bot. Btzg III, 1 (1919) 140-144, t. 7-8.-Record of Elaeocarpus littoralis.

Borneo: de Candolle, Bull. Herb. Boiss. II, 3 (1902) 367-368.-2 new Elaeocarpus.

KNUTH, in FEDde, Rep. 44 (1938) 124-132.20 new spp. of Elaeocarpus.

Merrill, J. Str. Br. R. As. Soc. no 76 (1917) 96-97.-New Elaeocarpus.

-, ibid. no 77 (1917) 191-203.-Critical notes, a dozen new spp. of Elaeocarpus.

一, ibid. no 86 (1922) 327.-New Elaeocarpus.

-, Sarawak Mus. J. 3 (1928) 527-528.-New

Elaeocarpus.

RIDLEy, Kew Bull. (1938) 229-234.-List records; 9 new Elaeocarpus.

AIRY SHaw, Kew Bull. (1949) 163-165.-Records; new Elaeocarpus.

WARBURG, in Fedde, Rep. 18 (1922) 327-329. 5 new Elaeocarpus.

Philippines: de Candolle, in Elmer, Leafl. Philip. Bot. 2 (1909) 633-638.-Revision \& key to Elaeocarpus.

Elmer, Leafl. Philip. Bot. 4 (1911) 1171-1190. -New spp.

一, ibid. 9 (1934) 3185-3186.-New sp.

Merrill, Philip. J. Sc. 26 (1925) 470.-New Elaeocarpus.

— \& QUISUMBING, ibid. 82 (1953) 328.-Record Sloanea sigun.

Van Steenis, Bull. Bot. Gard. Btzg III, 17 (1948) 409.-Syn. Elaeocarpus sphaericus.

Celebes: Koorders, Bull. Jard. Bot. Btzg III, 2 (1920) 258-260, t. 7A-B.-Descr. new Elaeocarpus.

DE WIT, Bull. Bot. Gard. Btzg III, 18 (1949) 210.-Reduction of an Elaeocarpus.

New Guinea: Gibss, Contr. Fl. Phytog. Arfak Mts (1917) 148.-One Elaeocarpus and 2 Sericoleas.

Kanehira-Hatusima, Bot. Mag. Tokyo 56 (1942) 308-322, fig. 4-14.-New spp. in Aceratium, Echinocarpus, Elaeocarpus, and Sericolea.

KOORDERS, Nova Guinea 8 (1909) 173-174.Record and new Elaeocarpus.

Pulle, Nova Guinea 8 (1912) 661-662.-2 new Elaeocarpus.

RIDLEY, Trans. Linn. Soc. Lond. II, Bot. 9 (1916) 21-22.-New Elaeocarpus spp. and a Sloanea.

SCHLeChter, Bot. Jahrb. 54 (1916) 92-155.Extensive revision; keys.

Schmidt, Nova Guinea 14 (1924) 151-157.New spp. and records.

-, J. Arn. Arb. 10 (1929) 79-80.

Smirh, A. C., J. Arn. Arb. 25 (1944) 104-121, 222-270, 271-298.-Many novelties.

VAN StEenIS, Nova Guinea 14 (1927) 304-305.Sericolea records.

\section{Genera}

Aceratium: Schlechrer, Bot. Jahrb. 54 (1916) 100-107.-Key.

Smith, A. C., J. Arn. Arb. 25 (1944) 110-121.Records and 11 new Pap. spp. 
Whrte, Kew Bull. (1932) 42-43.-Aceratium in Queensland.

Dubouzetia: Brongniart \& Gris, Bull. Soc. Bot.

Fr. 8 (1881) 199-200.-Type descr.

Smith, A. C., J. Arn. Arb. 25 (1944) 272-273.-

2 new spp.

Sprague, Kew Bull. (1907) 11, 125-128.Revision; key to New Caled. spp.

Elaeocarpus: KNUTH, in FEDDE, Rep. 48 (1940) 7279.-20 new spp. from New Guinea, Celebes, Bismarcks \& Timor.

一, ibid. 49 (1940) 66-73.-New spp. from various regions.

一, ibid. 50 (1941) 81-88.-New spp. from New Guinea, Philippines, Celebes \& Java.

Merrill, J. Arn. Arb. 32 (1951) 157-200.New spp. and critical notes, specially on KNUTH's spp.; many reductions; no keys.

-, Proc. R. Soc. Queensl. 62 (1952) 49-56.Further critical notes and reductions; no keys. SmIth, A. C., J. Arn. Arb. 25 (1944) 222-270.Key to sect. in Papua; records and c. 30 new spp. Turczaninow, Bull. Soc. Nat. Moscou $19^{2}$ (1846) 489-496.-Key to 3 sect.; no keys to spp.; 11 spp. treated from CUMING's and ZoLliNGER's Mal. coll.

Sericolea (Hormopetalum, Mischopleura, Pyrsonota): SChlechter, Bot. Jahrb. 55 (1918) 194.Reduction of Pyrsonota.

-, in FEDDE, Rep. 16 (1919) 29-32.-Census spp.; nomencl.

Schmidt, Nova Guinea 14 (1919) 151-153.2 new spp.

Smith, A. C., J. Arn. Arb. 25 (1944) 104-110.Records and 5 new spp.

WERNHAM, in HoOK. Ic. Pl. 31 (1916) t. 3059, pp. 1-2.-2 spp. under Mischopleura.

Sloanea (Antholoma, Anoniodes, Echinocarpus, Phoenicosperma): Miquel, Ann. Mus. Bot. Lugd.-Bat. 2 (1865) 68.-Descr. Jav. sp.

Von Mueller, Vict. Nat. 8 (1892) 164; ibid. 9 (1892) 111.-2 new spp. from New Guinea.

Smith, A. C., J. Arn. Arb. 25 (1944) 273-297.Rev. Pap. spp.; sect. in Papua; records and 11 new spp.

Smith, C. E., Contr. Gray Herb. 175 (1954) 1114.-Revision New World spp.; key.

\section{ELATINACEAE}

BaCker, C. A., Fl. Mal. I, 4 (1951) 203-206, 1 fig. NiedenzU, F., Pflanzenfamilien ed. 2, 21 (1925) 270-276.

\section{Genera}

Bergia: Milne-Redhead, Kew Bull. (1948) 450.Synon. B. capensis.

\section{EPACRIDACEAE}

Copeland, H. F., Observations on certain Epacridaceae (Am. J. Bot. 41, 1954, 215-222, fig. 1-44). -General discussion; main subdiv.

LAM, H. J., Blumea 5 (1945) 571-574.-Distinction of Styphelia and Trochocarpa by means of venation, referring a number of Mal. spp. to the latter genus.
SmITH, J. J., Nova Guinea 12 (1912) 797-798.List of Mal. spp.

\section{Local}

Java: De Vriese, in Miquel, Plant. Jungh. (1851) 84-86.-Redescr. Jav, spp. and remarks on occurrence of Styphelia in Mal.

Lesser Sunda Islands: FAWCETT, in Forbes, Wand. Nat. (1885) 509.-New sp. from Timor.

SMITH, J. J., Ic. Bog. 4 (1913) 171-173, t. 352.New sp.

Borneo: Gibrs, J. Linn. Soc. Lond. Bot. 42 (1914) 105-107, fig. 7.-List \& new sp. from Mt Kinabalu.

Celebes: LAM, Blumea 5 (1945) 571-574.-Records; referring a number of Mal. spp to Trochocarpa. Smith, J. J., Ic. Bog. 4 (1910) 81-82, t. 325.

Philippines: Merrill, Philip. J. Sc. 20 (1922) 419. -New $s p$.

Moluccas: Scheffer, Nat. Tijd. Ned. Ind. 32 (1871) 419.-New sp. from Gebeh Isl.

New Guinea: Diels, Bot. Jahrb. 62 (1929) 488.2 records.

Kanehira \& Hatusima, Tokyo Bot. Mag. 56 (1942) 483-485.-Records; 1 new sp.

RIDLEY, Trans. Linn. Soc. Lond. II, Bot. 9 (1916) 100-102.-4 new spp.

SmIrH, J. J., Nova Guinea 8 (1912) 797-803, t. 143-146.-List Mal. spp.; 5 new spp.

-, ibid. 12 (1917) 539-541, t. 224-225.-Records; 2 new spp.

-, in Gibss, Contr. Fl. Phytog. Arfak Mts (1917) 167-168.-Some records.

\section{ERICACEAE}

(incl. Monotropaceae, Pirolaceae, Vacciniaceae)

Copeland, H. F., A study, anatomical, and taxonomic, of the genera of Rhododendroideae (Am. Midl. Natural. 30, 1943, 533-625, fig. 1229).-Morph.

Cox, H. T., Studies in the comparative anatomy of the Ericales, Ericaceae subfamily Rhododendroideae (Amer. Midl. Nat. 39, 1948, 220-245). -Morphology and phylogeny.

Miquel, F. A. W., Ericaceae Archipelagi Indici (Ann. Mus. Bot. Lugd.-Bat. 1, 1864, 36-45; 1866, 220-221, t. 2).-Descr.; records.

Sleumer, H., Vaccinioideen-Studien (Bot. Jahrb. $71,1941,375-510)$.-Subdiv. of the subfamily; keys to tribes and genera and subdivision of genera (e. g. Vaccinium); many new comb.

SteENIS, C. G. G. J. vaN, Bull. Jard. Bot. Btzg III, 13 (1934) 203-207.-List of a number of mountain spp.

\section{Local}

Sumatra: Merrill, Pap. Mich. Acad. Sc. Arts \& Lett. 19 (1934) 181-184.-New spp.

-, Not. Nat. Acad. Nat. Sc. Philadelphia no 47 (1940) 3-6.-3 new Rhododendrons from Atjeh. MOORE, J. Bot. (1925) Suppl. 55-57, 147.-List; 3 new Vacciniums.

Ridley, J. Fed. Mal. Stat. Mus. 8, 4 (1917) 56-58.-List; 2 new Vacciniums. 
AIRY SHaw, Kew Bull. (1948) 158-161.-Pictures of 2 spp.

SMITH, J. J., Med. Rijksherb. Leiden no 30 (1916)

1-10.-Some Vacciniums.

-, Contr. Arn. Arb. 8 (1934) 122-129.-

Records and new spp. from N. Sumatra.

一, in Fedde, Rep. 35 (1934) 292-297.-New spp.

-, Bull. Jard. Bot. Btzg III, 13 (1935) 443-464. -Some new spp. \& var.

Malay Peninsula: AIRY SHAw, Kew Bull. (1940) 304-305.-New Gaultheria.

SMITH, J. J., Gard. Bull. Str. Settl. 8 (1935) 262263.-New Rhododendron.

Java: SMITH, J. J., Ic. Bog. 4 (1910) 73-74, t. 322.New Rhododendron.

一, ibid. (1912) 99-101, t. 331.-New Vaccinium. 一, in Koord. \& VAl., Bijdr. Booms. Java 13 (1914) 88-169.

VAN STEENIS, Bull. Bot. Gard. Btzg III, 17 (1948) 387-389.-Critical notes on Rhododendron and Vaccinium.

-, Act. Hort. Berg. 15 (2) (1949) 41, fig. 1.Distr. Vaccinium bracteatum in Mal.

Lesser Sunda Islands: SLeUMer, Bot. Jahrb. 71 (1940) 146-147.-New Rhododendron from Flores.

Borneo: Beccari, Malesia 1 (1878) 199-213.Descr. new spp.

Fischer, Kew Bull. (1932) 293.-New Vaccinium.

GibBs, J. Linn. Soc. Lond. Bot. 42 (1914) 101-105.-List Kinabalu; a new Rhododendron. MERRILL, J. Str. Br. R. As. Soc. no 76 (1917) 102-110.-New spp.

-, J. Mal. Br. R. As. Soc. 1 (1923) 22-23.New Vaccinium.

-, Sarawak Mus. J. 3 (1928) 541-543.-List; 2 new Rhododendrons.

RidLey, Kew Bull. (1914) 209-210.-New Rhododendron.

-, ibid. (1922) 106-108.-Descr. new Vacciniums, partly under Rigiolepis.

SLeUMer, Bot. Jahrb. 71 (1940) 138-168.-

Descr. new spp. mainly from Mt Kinabalu.

Sмгтн, J. J., Ic. Bog. 4 (1910) 67-72, 75-79, t. 320-321, 323-324.-2 new Vacciniums, a Rhododendron and Costera n.g.

-, Bull. Jard. Bot. Btzg III, 1 (1920) 399-410.-

Few new spp.

-, ibid. III, 13 (1935) 443-464.-Various new spp. \& var.

STAPF, Trans. Linn. Soc. Lond. II, Bot. 4 (1894) 189-198, t. 14-15.-Kinabalu coll.; new spp. in all genera.

Philippines: Copeland, Philip. J. Sc. 40 (1929) 133-179, t. 1-16.-Rhododendron; key.

-, ibid. 42 (1930) 537-607, t. 1-7.-Vaccinium; key.

一, ibid. 47 (1932) 57-118, t. 1-8.-Other ericaceous genera; keys.

Elmer, Leafl. Philip. Bot. 3 (1911) 1089-1107.Account Eric. Mt Apo, Mindanao; many new spp.

-, ibid. 10 (1939) 3730-3731.-New Vaccinium.
Merrill, Philip. J. Sc. 3 (1908) Bot. 369-382.-

Revision; keys.

-, ibid. 27 (1925) 43-45.-2 new spp.

-, ibid. 30 (1926) 419.-New Rhododendron.

- \& QuisumBing, ibid. 82 (1953) 333-334.-2

new spp. of Rhododendron from Palawan.

RENDLE, J. Bot. 34 (1896) 355-357.-Some new spp.

Warburg, in Perkins, Fragm. Fl. Philip. (1905) 172-175.-4 new spp.

Celebes: Sleumer, Bot. Jahrb. 71 (1940) 138-168. -Descr. new spp. from coll. Warburg, HeINRICH, and SARASIN.

Smith, J. J., Bull. Jard. Bot. Btzg II, no 8 (1912) 48-49.-New var. of a Vaccinium.

-, Med. Rijksherb. Leiden no 30 (1916) 7-9.New Vaccinium var.

-, Bull. Jard. Bot. Btzg III, 1 (1920) 399-410.Descr. new spp.

-, ibid. 4 (1922) 240.-New Rhododendron.

-, in FeDDE, Rep. 30 (1932) 162-178.-Descr. new spp. Mt Bonthain.

-, Bull. Jard. Bot. Btzg III, 13 (1935) 443-464. -Some new spp. and var.

-, Bot. Jahrb. 68 (1937) 199-215.-Novelties coll. KJellbero.

Moluccas: Smith, J. J., in Fedde, Rep. 30 (1932) 162-178.-Descr. new spp.

New Guinea: BecCarI, Malesia 1 (1878) 199-213. -Descr. new spp.

Diels, Bot. Jahrb. 62 (1929) 486-488.-List; 3 new spp.

FörSTER, in FEDDE, Rep. 13 (1914) 221-225.6 new Rhododendrons, leg. KEYsSER.

Kanehira \& Hatusima, Bot. Mag. Tokyo 56 (1942) 476-483.-List; 2 new spp.

KoORders, Nova Guinea 8 (1909) 183-191, t. 48.-New spp.

一, ibid. 8 (1912) 875-887, t. 150-157.-New spp. VoN MUeller, Vict. Nat. 1 (1884) 101-102.New Rhododendron.

-, J. Bot. 24 (1886) 289-291.-Agapetes n. sp., Catanthera n. g. (= Medinilla!)

-, in WING's South. Sc. Record new ser. 2 (1886) 2 pp.-Type descr. Dimorphanthera under Agapetes.

-, Vict. Nat. 4 (1887) 110-112.-New Rhododendron.

SCHLECHTER, Bot.Jahrb. 55(1918) 137-194.-Keys. SLEUMER, Bot. Jahrb. 70 (1939) 95-124.-Revision Agapetes \& Dimorphanthera; keys.

一, ibid. 72 (1942) 207-269.-Revision of Diplycosia, Gaultheria, Vaccinium; many new spp.; keys.

SMITH, J. J., Bull. Jard. Bot. Btzg II, no 8 (1912) 50-55.-List prelim. descr. new spp.

- , in FEDdE, Rep. 12 (1913) 209.-New Rhododendron.

一, Ic. Bog. 4 (1913) 175-178, t. 353.-New Dimorphanthera.

-, Nova Guinea 12 (1914) 129-168, t. 29-52.Records; new spp.

-, Meded. Rijksherb. Leiden no 25 (1915) 1-14.-Descr. new spp.

, Nova Guinea 12 (1917) 495-537, t. 193-223; 
correct. vol. 18 , p. $89 .-$ Key to Rhododendron \& Vaccinium; records and new spp.

-, in Gibss, Contr. Fl. Phytog. Arfak Mts (1917) 168-174.-List; records \& new spp.

WernhaM, Trans. Linn. Soc. Lond. II, Bot. 9 (1916) 89-99.-Many new spp. also Mischopleura n.g. (= Elaeoc.).

Solomon Isl.: SLeumer, Notizbl. Berl.-Dahlem 12 (1935) 485-486.-3 Rhododendrons from Bougainville.

\section{Genera}

Agapetes (Pentapterygium, Paphia, Corallobotrys, Desmogyne): Alry Shaw, Kew Bull. (1935) 2453.-Critical notes; keys.

-, ibid. (1948) 77-104.

Sleumer, Bot. Jahrb. 70 (1939) 97-106.-Revision; key.

Catanthera = Medinilla (Melastomataceae).

Cheilotheca: RIDLEY, F1. Mal. Pen. 2 (1923) 222223, fig. 93.-Descr. sp. from Mal. Pen.

Costera (Cymothoë, Iaera): AIRY SHAw, in Hook. Ic. Pl. 33 (1935) t. 3281, pp. 1-3.-New sp. 一, Kew Bull. (1935) 150-156.-Cymothoë. Smith, J. J., Bull. Jard. Bot. Btzg III, 13 (1935) 418-424, fig. 1.-Revision; key.

Dimorphanthera: SLEUMER, Bot. Jahrb. 70 (1939) 106-124.-Revision; key.

Gaultheria: AIRY SHAw, Kew Bull. (1940) 306330.-Classif. Asiat. spp.; keys; some Sumatran spp. included.

-, ibid. (1952) 171-174.-Key to Sino-Himalayan spp. of $\S$ Leucothoides.

Mischopleura $=$ Sericolea (Elaeocarpaceae).

Pirola: Andres, Bull. Jard. Bot. Btzg III, 14 (1936) 4-7, fig. 1.-Descr. new sp. from N. Sumatra.

Rhododendron: Cowan, J. M., The Rhododendron leaf. A study of the epidermal appendages. Edinburgh \& London (1950) 1-120, t. 1-15, fig. 1-8.-Structure of indument as a taxonomic character.

Hayes, Keenan \& Cowan, Not. R. Bot. Gard. Edinb. 21 (1951) 1-33, t. 1-11.-Anat. of leaf in relation to taxon.

Von HofF, Ber. Deut. Bot. Ges. 63 (1950) 3135.-Significance of hair structures for allianceships and their interrelation.

-, Jahrbuch Rhododendron Gesellschaft Bremen (1953) 58-71, 81 fig.-Ontogeny of indumentum and importance of taxonomy.

-, ibid. (1954) 42-55, 11 fig.-Considerations on phylogeny on the basis of geogr. and morph.

Hutchinson, Rhododendron Yearbook (1946) 42-47.-Evolution and classification of the genus.

KINGDON WARD, J. Bot. 73 (1935) 241-247, fig. 1-3.-Seed types in Rhododendron.

-, Rhododendron Yearbook (1947) 99-114.Observ. on classification.

Sinclair, Not. R. Bot. Gard. Edinb. 19 (1937) 267-271.-Structure of bud as a taxonomic character.

Sleumer, Bot. Jahrb. 74 (1949) 511-553.Attempt of a subdivision of the genus.
Vaccinium (Disiphon, Neojunghuhnia, Rigiolepis): Sleumer, Bot. Jahrb. 71 (1941) 408-493.Many new comb.; subdivision of the genus; keys to Indian \& SE.-E. Asiat. spp.; occasionally a Mal. sp. included.

SMrTH, J. J., Blumea 1 (1935) 323-342, fig. 1.Revision Mal. spp. of § Rigiolepis; no key.

\section{ERIOCAULACEAE}

KoERniCKe, F., Monographia scripta de Eriocaulaceis. Berlin (1856) i-ii, 1-36.-Non vidi.

- Eriocaulacearum monographiae supplementum (Linnaea 27, 1856, 561-692).-List; descr.; partial keys.

-, in Miquel, Ann. Mus. Bot. Lugd.-Bat. 3 (1867) 238-241.-Some records and one new sp.

Moldenke, H. N., The known geographic distribution of the members of the ... and Eriocaulaceae. New York (1949) mimeogr. 1-215.Checklist of names, geogr. arranged.

Ruhland, W., Pflanzenreich Heft 13 (1903) 1-294, fig. $1-40$.

- Zur geographischen Verbreitung der Eriocaulaceen (Bot. Jahrb. 50, Suppl. 1914, 363374).-Geogr. distr.

-, Pflanzenfamilien ed. 2, $15 a$ (1930) 39-57, fig. 16-25.

Steudel, E. G., Enumeratio plantarum Glumacearum pars II. Stuttgart (1855) 267-283.Enum. descr. in Latin.

\section{Local}

India: Fyson, J. Ind. Bot. 1 (1919) 49-53; ibid. 2 (1921) 133-150, 192-207, 259-266, 307-320; ibid. 3 (1922) 12-18, 91-115, 51 t. \& fig.-Non vidi.

-, The Indian species of Eriocaulon (1923) 188, t. 1-55.-Reprint of the above. Non vidi.

Raizada, Sc. \& Cult. 14 (1949) 387-388.Floral proliferation.

Indo-China: Lecomte, in Morot, J. de Bot. 21 (1908) 86-94, 101-109, fig. 1-3, 129-136, fig. 1-3.-Key to I.-C. spp.; morphol.; ecol.; dispersal.

Malay Peninsula: Ridley, Mat. Fl. Mal. Pen. (Monocot.) 3 (1907) 53-55.-Key.

Java: BACKER, Handb. Fl. Java 3 (1924) 5-8.Key; in Dutch.

Borneo: GIB BS, J. Linn. Soc. Bot. 42 (1914) 172.List records.

STAPF, Trans. Linn. Soc. Lond. II, Bot. 4 (1894) 243-244.-New sp.

Suessenguth \& Heine, Mitt. Bot. Staatssamml. München Heft 2 (1950) 57-58.-New sp.

Philippines: Ruhland, in Perkins, Fragm. Fl. Philip. (1904) 136.-New sp.

New Guinea: RIDLEY, Trans. Linn. Soc. Lond. II, Bot. 9 (1916) 240-241.-New sp.

\section{Genera}

Eriocaulon: van SteEnis, Bull. Bot. Gard. Btzg III, 18 (1950) 460-461.-Reduction of the forgotten genus Busseuillia Less. from Australia to Eriocaulon. 


\section{ERYTHROPALACEAE See Olacaceae}

\section{ERYTHROXYLACEAE}

Burck, W., Les Erythroxyleae des Indes Néerlandaises (Ann. Jard. Bot. Btzg 11, 1893, 190-194, t. 15-16).-Survey; no key; 4 new spp.

Schulz, O. E., Pflanzenreich Heft 29 (1907) 1-176. -, Pflanzenfamilien ed. 2, 19a (1931) 130-143, fig. 60-67.

\section{Local}

New Guinea: Schulz, Bot. Jahrb. 58 (1924) 249.Records; new var.

Solomon Islands: WhrTe, J. Arn. Arb. 31 (1950) 89-90.-New sp.

\section{EUPHORBIACEAE (excl. Daphniphyllaceae)}

Baillon, H., Etude générale du groupe des Euph or biacées. Paris (1858) 1-684, Atlas 1-52, t. 1-27.Classification of genera.

-, Nouvelles observations sur les Euphorbiacées (Adansonia 11, 1873-74, 72-138).-Mostly relating to New Caled. spp.; some of Africa \& Malaysia; Cephalomappa n.g.

Croizat, L. C. M., The tribe Plukenetiinae of the Euphorbiaceae in eastern tropical Asia (J. Arn. Arb. 22, 1941, 417-431).-Plukenetia, Tragia, Megistostigma (Clavistylus), Cnesmone; no keys to spp.

GRÚNING, G., Porantheroideae, Ricinocarpoideae (Pflanzenreich Heft 58, 1913, 1-97, fig. 1-16).Contains only some Australian genera, some of which might occur in New Guinea.

JABlONsZKY, E., Euphorbiaceae-Bridelieae (Pflanzenreich Heft 65, 1915, 1-98, fig. 1-15).-Bridelia, Cleistanthus.

Mueller(-Arg.), J., Euphorbiaceae. Vorlăufige Mittheilungen aus dem für De Candolle's Prodromus bestimmten Manuscript über diese Familie (Linnaea 32, 1863, 1-126; ibid. 34, 1865, 1-224).-Descr.; partial keys.

- \& E. Boissier, in DC. Prodr. $15^{2}$ (1866) 11286.

PAX, F., Euphorbiaceae-Jatropheae (Pflanzenreich Heft 42, 1910, 1-148, fig. 1-45).-Aleurites, Elateriospermum, Hevea, Jatropha, Tritaxis.

-, Euphorbiaceae-Adrianeae (ibid. Heft 44. 1910, 1-111, fig. 1-35).-Cephalomappa, Manihot.

- \& K. Hoffmann, Euphorbiaceae-Cluytieae (ibid. Heft 47, 1911, 1-124, fig. 1-35).-Codiaeum, Dimorphocalyx, Erismanthus, Galearia, Microdesmis, Paracroton, Strophioblachia, Syndyophyllum, Trigonopleura, Trigonostemon.

- Euphorbiaceae-Gelonieae (ibid. Heft 52', 1912, 1-41, fig. 1-11).-Baliospermum, Chaetocarpus, Cheilosa, Endospermum, and Gelonium.

-, Euphorbiaceae-Hippomaneae (ibid. Heft $52^{2}$, 1912, 1-319, fig. 1-58).-Excoecaria, Homalanthus, Hura, Omphalea, Pimeleodendron, Sapium, Sebastiania.
-, Euphorbiaceae - Acalypheae - Chrozophorinae (ibid. Heft 57, 1912, 1-142, fig. 1-45).-Agrostistachys, Chrozophora, Sumbavia, Sumbaviopsis.

- Euphorbiaceae - Acalypheae - Mercurialinae (ibid. Heft 63, 1914, 1-473, fig. 1-67).-Alchornea, Alcinaeanthus, Blumeodendron, Calpigyne, Cladogynos, Claoxylon, Clarorivinia, Cleidion, Coccoceras, Coelodepas, Macaranga, Melanolepis, Micrococca, Neotrewia, Podadenia, Trewia, Wetria.

- Additamentum V (ibid. Heft 63, 1914, 397427).

- Acalypheae - Plukenetiinae, A.-Epiprininae, A.-Ricininae, Pereae (ibid. 68 1 , 1919, 1-134, fig. 1-29).-Clavistylus, Cnesmone, Epiprinus, Homonoia, Pachystylidium, Plukenetia, Pterococcus, Ricinus, Sphaerostylis, Tragia.

-, ibid. Heft $68^{2}$ (1919) 1-59, fig. 1-9.-Dalechampia.

-, Additamentum VI (ibid. Heft 684, 1919, 181).-System of the Crotonoideae; Annesijoa n. g. (Papua); Blumeodendron n. spp.; Claoxylon n. spp. (Papua), Mallotus n. spp. (Malaysia), Coelodepas n. sp. (Borneo), Cleidion n. spp. (Malaysia), Macaranga (ditto; many of Papua), Neomphalea n.g. (Papua), Homalanthus n. sp. (Papua).

-, Euphorbiaceae - Phyllanthoideae - Phyllantheae (ibid. Heft 81, 1922, 1-349, fig. 1-26).Actephila, Agyneia, Andrachne, Antidesma, Aporosa, Baccaurea, Bischofia, Dicoelia, Drypetes, Hymenocardia, Longetia, Richeriella, Sauropus.

- Euphorbiaceae - Crotonoideae - Acalyphinae (ibid. Heft. 85, 1924, 1-178, fig. 1-3).-Acalypha, Acalyphopsis n. g. (Celebes).

-, Additamentum VII (ibid. Heft 85, 1924, 179 204).-New Mal. spp. in Claoxylon, Macaranga, and Pimeleodendron.

-, Pflanzenfamilien ed. 2, 19c (1931) 1-233, fig. 6-123.

SChefFer, R. H. C. C., Observationes de quibusdam Euphorbiaceis Archipelagi indici (in MIQuel, Ann. Mus. Bot. Lugd.-Bat. 4, 1869, 119127).-List; based on MüLLER's monogr.; few new spp.

SмIтн, J. J., Ic. Bog. 4 (1910) 16-47, t. 306-316.New spp. of Antidesma, Baccaurea, Croton, Cyclostemon and Glochidion.

-, Bull. Jard. Bot. Btzg III, 1 (1920) 390-396, t. 38-44.-Misc. new spp.

一, ibid. 4 (1922) 235-237, t. 9.-Ditto.

一, ibid. 6 (1924) 82-107.-Ditto.

Whebler, L. C., The genera of the living Euphorbiaceae (Amer. Midl. Nat. 30, 1943, 456-503). -List of genera and their types.

ZOLLINGer, H., Over de soorten van Rottlera ..., alsmede over eenige verwante geslachten uit de familie der Euphorbiaceae (Acta Soc. Sc. IndoNeerl. 1, 1856, 1-32).-Redefinition of Rottlera (= Mallotus); enum. spp. based on coll. in Herb. Hort. Bog.; descr. some new genera under joint authorship with REICHENBACH.

-, Nachtrag zu dem Aufsatze über Rottlera und verwandte Euphorbiaceen (Linnaea 29, 1857, 462-469).-List of spp. of Mappa; Doryxylon n.g. 


\section{Local}

Asia: Croizat, J. Arn. Arb. 21 (1940) 490-510.Various misc. notes and descr. also incl. Mal. spp.

-, ibid. 23 (1942) 29-54.-Ditto.

Sumatra: CroizAT, J. Arn. Arb. 23 (1942) 54.New Trigonostemon.

Merrill, Contr. Arn. Arb. 8 (1934) 85-91.List; new Endospermum.

-, Pap. Mich. Acad. Sc. Arts \& Lett. 19 (1934) 161-162.-New Macaranga.

MoORE, J. Bot. (1925) Suppl. p. 92-105.-List FORBES coll.; new spp. in Cleistanthus, Glochidion, Drypetes, Neoscortechinia, Gelonium, Blumeodendron; Clonostylis n. $\mathrm{g}$.

RIDLEY, J. Fed. Mal. Stat. Mus. 8, 4 (1917) 8284.-List; new Homalanthus.

-, Kew Bull. (1925) 88-90.-New spp. in Glochidion, Macaranga \& Trigonostemon.

- , ibid. 1 (1926) 80-81.-List; new Gelonium and Trigonostemon.

Malay Peninsula: CoRner, Gard. Bull. Str. Settl. 10 (1939) 288-299.-Critical notes on species of various genera.

Croizat, Gard. Bull. Str. Settl. 9 (1937) 145-151. -New name for a Euphorbia.

GAGE, Kew Bull. (1914) 239-241.-New spp. of Cleistanthus \& Phyllanthus.

- , Rec. Bot. Surv. Ind. 9 (1920) 219-249.New spp. and records.

-, J. \& Proc. As. Soc. Bengal 75 (1936) 469527.-Good general keys to genera; revisions of Bridelia, Cleistanthus, Actephila, Andrachne, Agyneia, Flueggea.-Continuation of KING \& GAMBLE's 'Materials', but not complete.

Henderson, Gard. Bull. Str. Settl. 7 (1933) $121-$ 125, t. 32.-List; new Sauropus \& Richeriella. RidLeY, Kew Bull. (1923) 360-369.-New spp., Actephiliopsis n. $\mathrm{g}$.

Java: KOORDERs, Bull. Jard. Bot. Btzg III, 1 (1919) 145-147, t. 9.-Descr. new Glochidion.

Meeuse \& Adelbert, in BaCker, Bekn. Fl. Java (em. ed.) 4C (1943) fam. 112, p. 1-134.-Revision, in Dutch; keys.

Pax \& Hoffmann, Blumea 3 (1938) 60-61.New $s p$. from E. Java.

SmIth, J. J., in Koord. \& Val. Bijdr. Booms. Java 12 (1910) 1-782.-Very important contribution.

Van SteenIs, Bull. Bot. Gard. Btzg III, 17 (1948) 399.-Record of Chrozophora.

Borneo: Diels \& HaCKEnBerg, Bot. Jahrb. 60 (1926) 309.-New Macaranga.

GagnePain, Bull. Soc. Bot. Fr. 71 (1924) 621.New Dimorphocalyx.

Hutchinson, J. Linn. Soc. Lond. Bot. 42 (1914) 133-186.-List coll. GibBs; new Antidesma.

Merrill, Philip. J. Sc. 11 (1916) Bot. 54-77.Many new spp. in various genera; Moultonianthus n. $g$.

—, J. Str. Br. R. As. Soc. no 76 (1917) 89-93.3 Antidesmas, 1 Ostodes.

-, Philip. J. Sc. 13 (1918) Bot. 80-84.-3 new

Mallotus, 1 new Cleistanthus.
一, J. Str. Br. R. As. Soc. no 86 (1922) 319-322.

-List; new spp. in Galeria, Glochidion.

-, Philip. J. Sc. 21 (1922) 521-523.-List; new Aporosa \& Cyclostemon.

-, ibid. 30 (1926) 80-81.-New Coelodepas.

-, Sarawak Mus. J. 3 (1928) 525-526.-List; new Mallotus and Trigonostemon.

-, Pl. Elm. Born. (1929) 139-166.-List; new spp. in many genera.

Pax \& HofrmanN, Pflanzenreich Heft 63 (1914) 418, 422-423.-New spp. of Endospermum \& Excoecaria.

-, Mitt. Allg. Inst. Bot. Hamburg 7 (1931) 222 230.-List; new sp. in Antidesma, Glochidion, Alchornea, Macaranga and Gelonium.

STAPF, Trans. Linn. Soc. Lond. II, 4 Bot. (1894) 223-226.-New sp. in Claoxylon, Glochidion.

Philippines: Elmer, Leafl. Philip. Bot. 2 (1908) 427-434.-6 Macarangas.

-, ibid. 3 (1910) 903-931.-List \& new spp. from Sibuyan.

-, ibid. 4 (1911) 1271-1306.-List; new spp. from Palawan.

-, ibid. 9 (1934) 3186-3187.-New Gelonium.

-, ibid. 10 (1939) 3731-3736.-4 new spp.

Merrill, Philip. J. Sc. 7 (1912) Bot. 379-410; ibid. 9 (1914) 461-493; ibid. 11 (1916) Bot. 189191; ibid. 16 (1920) 539-597.-Misc. new spp.; no keys.

-, ibid. 26 (1925) 462-466.-New spp. in Glochidion and Phyllanthus.

-, ibid. 27 (1925) 30-31.-New Gelonium and Dimorphocalyx.

-, ibid. 30 (1926) 402-407.-New sp. in Cleidion, Croton, and 2 in Phyllanthus.

Quisumbino, Philip. J. Sc. 41 (1930) 328-332, fig. 7-8.-2 new Trigonostemons.

-, ibid. 76 (1944) 42-43.-New Claoxylon.

- \& Merrill, ibid. 37 (1928) 158-161.-New sp. in Actephila, Antidesma and Phyllanthus.

Robinson, Philip. J. Sc. 4 (1909) Bot. 71-105.Philippine Phyllanthinae; keys.

STAPF, in Elmer, Leafl. Philip. Bot. 1 (1907) 206-207.-New Trigonostemon.

Moluccas: Merrill, Philip. J. Sc. 11 (1916) Bot. 281-285.-Records; new spp. in Breynia and Macaranga.

New Guinea: Bailey, Proc. R. Soc. Queensl. 18 (1903) repr. p. 3-4.-New Baccaureas.

Croizat, Bull. Bot. Gard. Btzg III, 16 (1940) 351-357.-New Euphorbia.

-, J. Arn. Arb. 23 (1942) 369-376.-New Crotons.

GAGE, Nova Guinea 12 (1914) 479-486, t. 182 187.-List; new spp. in Phyllanthus, Macaranga, Homalanthus, and Omphalea.

Hutchinson, in Gibis, Contr. Fl. Phytog. Arfak Mts (1917) 145, 213-214.-New Homalanthus and some records.

Mansfeld, J. Arn. Arb. 10 (1929) 77-79.-New sp. of Macaranga \& Aporosa.

MOORE, J. Bot. (1923) Suppl. 44-49.-List; new Glochidion, 2 Phyllanthus, Antidesmas, Acalyphas, Macarangas. 
Pax, in Fedde, Rep. 8 (1910) 325.-New Phyllanthus.

- \& Hoffmann, Pflanzenreich Heft 63 (1914) 419.-New Omphalea.

Perry, J. Arn. Arb. 34 (1953) 191-257.-Revision Macaranga; keys.

RIDLEY, Trans. Linn. Soc. Lond. II, Bot. 9 (1916)

147-148.-List; 2 new Macarangas.

SMITH, J. J., Nova Guinea 8 (1910) 221-245, t. 52-60.-Several new spp.

-, ibid. 8 (1912) 779-795, t. 130-142.-Ditto.

-, ibid. 12 (1917) 543-548, t. 226-229.-Ditto.

Smith, L. S., Proc. R. Soc. Queensl. 58 (1946) 51-60, t. 1-2.-Revision Endospermum; key.

-, North Queensl. Natur. 15 (no 84) (1947) 1-2.

-Notes on Phyllanthus $\$$ Nymania; one new name.

WARBURG, Bot. Jahrb. 13 (1891) 347-361.List; new spp. in many genera.

-, ibid. 18 (1893) 196-199.-Descr. of a new genus and of new spp. in several genera.

Fiji: Smith, A. C., J. Arn. Arb. 33 (1952) 367-402. -Revision of Antidesma, Acalypha \& Macaranga.

\section{Genera}

Acalypha: NrTschKe, in Mez, Bot. Arch. 4 (1923) 277-317, map.-Geogr. distr. \& system. of the genus.

Agyneia: van Steenis, Bull. Bot. Gard. Btzg III, 17 (1948) 410-411.-Distr. A. bacciformis in Malaysia.

Aleurites: Hemsley, Kew Bull. (1906) 119-121; ibid. (1908) 93; ibid. (1914) 1-4.-Revisions; new spp.; synon.; no key.

一, in Hook. Ic. Pl. 29 (1906) t. 2801-2802.-A. cordata \& trisperma.

LANGERON, M., Le genre Aleurites Forst. Systématique-Anatomie-Pharmacologie. Paris (1902) 1-160, fig. 1-52.-Key to 3 spp.

Antidesma: Mrquel, Ann. Mus. Bot. Lugd.-Bat. 1 (1864) 218.-3 new spp.

Botryophora: BEUMÉ, Hoe het groeide. Wageningen (1947) 10-12.-Reduction of a Sumatran Sterculia to B.; o floral morphol.; in Dutch; no new comb.

AIry Shaw, Kew Bull. (1948) 484.-Syn. \& distr. of the genus and $s p$.

Breynia: Croizat, Bull. Bot. Gard. Btzg III, 17 (1942) 211-212.-Nomencl. generic name.

Bridelia: Gehrmann, Bot. Jahrb. 41 (1908) Beibl. 95, 1-42, fig. 1-3.-Precursor to monogr.; special attention to African spp.; general remarks; key to spp.

HAINES, J. Bot. 59 (1921) 188-193.-Critical notes on some Indian spp.

Chondrostylis: BoERLAGE, Ic. Bog. 1 (1897) 71-72, t. 23.

Koorders, Ann. Jard. Bot. Btzg 19 (1904) 4556, t. 4-5.-Descr.; morph.; anat.; taxon. position.

Chrozophora: Prann, Kew Bull. (1918) 49-120.Monogr.; key.

Claoxylon (Erythrococca, Micrococca): PranN, Ann. Bot. $25^{2}$ (1911) 575-638.-Erythrococca
\& Micrococca segregated as separate genera; keys.

Cleistanthus (Paracleisthus): Croizar, J. Arn. Arb. 23 (1942) 38-41.-Reduction; new spp. and recomb. from SE. Asia.

GaGnePain, Bull. Soc. Bot. Fr. 70 (1923) 497502.-Disc. of Paracleisthus; delimit.; 3 new sp.

Coelodepas: Crolzat, J. Arn. Arb. 23 (1942) 29.Calpigyne auct. $=$ Coelodepas HAssK. $($ Nephrostylus GAGN.)

Coelodiscus: PAX, in FedDE, Rep. 8 (1910) 481482.-Two new Pap. spp.

Daphniphyllum. See Daphniphyllaceae.

Dicoelia: Crolzat, J. Arn. Arb. 23 (1942) 38.Some critical notes.

Dimorphocalyx: WHITE, Proc. R. Soc. Queensl. 47 (1935) 80 (1936).-Descr. 1st Austr.

Elaeogene = Vatica (Dipterocarpaceae), of. HALLIER $f$., Med. Rijksherb. Leiden no 36 (1918) 4.

Endospermum (Capellenia T. \& B.): KURZ, J. Bot. 5 (1867) 23.-Reduction Capellenia; enum. spp. VAN SteEnis, Bull. Bot. Gard. Btzg III, 17 (1948) 457-458; Fl. Mal. Bull. 3 (1948) 74.-Prop. conserv.

Epiprinus (Symphyllia): Croizat, J. Arn. Arb. 23 (1942) 52-54.-Critical notes; some new comb.; new sp. from SE. Asia.

Euphorbia: Keng, Quart. J. Taiw. Mus. 4 (1951) 253-260, fig. 1-2.-Revision; key.

SEDGWICK, J. Bombay Nat. Hist. Soc. 26 (1919) 599-600.-E. rothiana reduced to $E$. laeta; $E$. oreophila restored for the mountain sp.

SherfF, Ann. Missouri Bot. Gard. 25 (1937) 1-94, t. 2-11.-Revision Hawaiian spp.; keys.

White, A., R. A. Dyer \& B. L. SlonNe, The succulent Euphorbieae (Southern Africa). Pasadena, Cal. (1941) 2 vol., xiv $+990+11$ pp., 1102 fig., 26 col. pl.-Descr.; key to the spp.

White, C. T., Proc. R. Soc. Queensl. 53 (1942) 227.-Record of Euph. plumerioides from Thursday Isl.

Glochidion: Alston, Ann. R. Bot. Gard. Peradeniya 11 (1928) 1-10, t. 1.-Revision \& key to Ceylon spp.

MUELLER-ARG., Flora 48 (1865) 369-380, 385391.-Brief revision; partial keys.

Hevea: DuckE, Arch. Jard. Bot. Rio de Janeiro 5 (1930) 147-157.-Key; synopsis.

-, ibid. 6 (1933) 49-57.-List.

-, Arq. Inst. Biol. Veget. (Rio de Janeiro) 2 (1935) 217-246, t. 1-3.-Revision of mainly Brazilian spp.; key.

-, Arq. Serv. Florestal, Rio de Jan. $2^{1}$ (1943)

25-49.-Discussion of some spp.; hybridization; no key.

HUBER, Bol. Mus. Goeld. 4 (1905) 620-651.Synopsis; partial key; in Portuguese.

- , ibid. 7 (1913?) 199-281, map.-New contrib.; new spp.; partly in German, partly in Portug.; descr. in Latin.

Jatropha: MCVAugh, Bull. Torr. Bot. Club 72 (1945) 271-294, fig. 1-24.-Rev. Amer. sp.; keys.

WILBUR, J. Elisha Mitchell Sc. Soc. 70 (1954) 92-101.-Synopsis \& Eucurcas; key to spp. 
Lophopyxis: Holthuis \& LAM, Blumea 5 (1942) 205-208, fig. 7.-Synon. \& distr.

Macaranga: BAKER, Gard. Bull. Str. Settl. 8 (1934) 63-68, t. 11-15.-Biol. of Macaranga spp. in relation to ants.

Perry, J. Arn. Arb. 34 (1953) 191-257.-Full rev. of 60 Pap. spp. incl. some of adjacent islands; keys!

Mallotus (Aconceveibum): MERRILl \& VAN STEENIS, Webbia 8 (1952) 405-406.-Reduction.

Megistostigma (Clavistylus): VAN STEENIS, Bull. Jard. Bot. Btzg III, 12 (1932) 174, 201-204, fig. 6, 10.-Full descr. of $C$. peltatus.

Melanolepis: Gagnepain, Bull. Soc. Bot. Fr. 71 (1924) 1026-1027.-2nd sp. of the genus.

Moultonianthus: Merrill, Philip. J. Sc. 11 (1916) Bot. 70-71.-Type descr.

Van Stzenis, Bull. Bot. Gard. Btzg III, 17 (1948) 405.-New comb.

Neoscortechinia (Alcinaeanthus, Scortechinia Hook. f. non SACc.): Merrill, Philip. J. Sc. 11 (1916) Bot. 75-76.-Census of the genus.

-, Pl. Elm. Born. (1929) 164-165.-Critical note on identity of Alcinaeanthus.

Phyllanthodendron (Phyllanthus \$, etc.): Croizat, J. Arn. Arb. 23 (1942) 32-38.-Revision; key to subdivision; no key to spp.; recomb.

Phyllanthus \& Nymania (Leichhardtia, Nymania, Phyllanthus \& Leichhardtia): Diels, Notizbl. Berl.-Dahlem 11 (1931) 308-311.-Revision; key.

Smith, L. B., North Queensl. Natur. 15 (1947) 1-2.-Nomencl.; addit. descr.

Van SteEnis, Bull. Bot. Gard. Btzg III, 17 (1948) 460.-Nomencl.

Pimeleodendron: Smith, J. J., Bull. Jard. Bot. Btzg III, 6 (1924) 100-107.-Census; descr. new spp.; no key.

Ptychopyxis (Calpigyne, Podadenia): Crolzat, J. Arn. Arb. 23 (1942) 47-50.-Critical notes; Calpigyne is regarded as a nomen confusum; new subdiv.; no key.

Sauropus (Breyniopsis): Crolzat, J. Arn. Arb. 21 (1940) 494-496.-Breyniopsis is reduced. -, Bull. Bot. Gard. Btzg III, 17 (1941) 209-211. -Proposed conserv. against Arachne.

Sebastiana: van STEenIS, Bull. Bot. Gard. Btzg III, 17 (1948) 409-410.-Key to some W. Mal. spp.

Suregada (Gelonium RoXB. non GAERTN.): CrolzAT, Bull. Jard. Bot. Btzg III, 17 (1942) 212-217. -Reduction; all recombinations.

MerRiLL, J. Arn. Arb. 32 (1951) 79-81.-Addit. critical remarks on the above; some spp. removed to other families.

Trigonopleura (Peniculifera): Symngron, Kew Bull. (1937) 319.-Reduction.

Trigonostemon: van StEenIs, Fl. Mal. Bull. 3 (1948) 74-75.-Proposed conserv, against Enchidium.

Trisyngyne $=$ Nothofagus (Fagaceae)

\section{EUPOMATIACEAE}

Diels, L., Die verwandtschaftlichen Beziehungen von Eupomatia (Bot. Jahrb. 48, Beibl. 107, 1912, 11-13).
Local

New Guinea: Diels, Bot. Jahrb. 49 (1912) 165.Under Annonaceae.

White, J. Arn. Arb. 10 (1929) 213.

\section{FAGACEAE \\ (Castaneaceae, Cupuliferae)}

BarnetT, E. C., Keys to the groups of Quercus, Lithocarpus, and Castanopsis of Eastern Asia with notes to their distribution (Trans. \& Proc. Bot. Soc. Edinb. 34, 1944, 159-204).-List; keys to genera, sections, and species groups.

HJelmQVIST, H., Studies on the floral morphology and phylogeny of the Amentiferae (Bot. Notiser 1948, Suppl. vol. 2, no 1, p. 1-171, fig. 1-58).

KoIDzumi, G., On the classification of Castaneaceae (Bot. Mag. Tokyo 30, 1916, 92-103, 185215).-Complete enum.; for Lithocarpus the name Synaedrys is introduced; otherwise no new comb.

LendNer, A., Sur la cupule des Fagacées (Bull. Soc. Bot. Genève II, 8, 1916, 161-166, 2 fig.).

Miquel, F. A. W., Plant. Jungh. (1851) 8-14.List Quercus; descr. Callaeocarpus (= Castanopsis).

- Adnotationes de Cupuliferis (Ann. Mus. Bot. Lugd.-Bat. 1, 1863, 102-121, 221).-Enum.; descr.

PrantL, K., Beiträge zur Kenntnis der Cupuliferen (Bot. Jahrb. 8, 1887, 321-336).-General discussion morph.

SCHWARZ, O., Entwurf zu einem natürlichen System der Cupuliferen und der Gattung Quercus L. (Notizbl. Berl.-Dahlem 13, 1936, 1-22).System of the family; subdiv. Quercus; keys to these subdivisions and segregates!

Seemen, A. von, Cupuliferen in dem Herbar zu Buitenzorg (Bull. Dép. Agric. Ind. Néerl. no 1, 1906, 1-14).-List spp. in Herb. Bog., some new.

\section{Local}

Formosa: LI, Bull. Torr. Bot. Club 80 (1953) 317324.-Taxon. notes.

Siam: BarNETT, Kew Bull. (1938) 98-106.-Descr. new spp. Quercus and Castanopsis.

-, Trans. \& Proc. Bot. Soc. Edinb. 33 (1942) 327-343.-Revision; no descr.; keys to genera \& spp.!

Sumatra: Merrill, Pap. Mich. Acad. Sc. Arts \& Lett. 19 (1934) 152-153.-New Castanopsis.

-, Contr. Arn. Arb. 8 (1934) 42-44.-List Quercus.

-, J. Arn. Arb. 33 (1952) 241-242.-Interpr. 2 spp. of JACK.

SCHEFFER, Nat. Tijd. Ned. Ind. 31 (1870) 359363; ibid. 32 (1873) 415-417.-New records and spp. of Quercus and Castanopsis from Banka.

Malay Peninsula: Camus, Bull. Mus. Hist. Nat. Paris II, 4 (1932) 912-914.-New Quercus spp. from the Mal. Pen.

-, Bull. Soc. Bot. Fr. 80 (1933) 353-355.New Quercus; also from SE. Asia.

-, Bull. Mus. Hist. Nat. Paris II, 5 (1933) 8889.-New Quercus. 
-, ibid. II, 6 (1934) 92-94.-New Quercus \& Castanopsis.

-, Bull. Soc. Bot. Fr. 94 (1947) 4-5.-New spp. of Castanopsis and Quercus.

CORNER, Gard. Bull. Str. Settl. 10 (1939) 279280.-Synon. Quercus lappacea; critics on subdivision of Quercus.

GamBLE, Kew Bull. (1914) 177-181.-Descr. new spp. Quercus \& Castanopsis.

Henderson, Gard. Bull. Str. Settl. 5 (1930) 7678, 1 fig.-New Pasania.

Borneo: Camus, Bull. Soc. Bot. Fr. 75 (1928) 698. -New Castanopsis.

SchOTTKY, Bot. Jahrb. 49 (1913) 356-359.Records coll. WINKLER; 2 new Pasanias and 2 new Castanopsis.

WARBurg, Kew Bull. (1936) 19-21.-3 new Castanopsis from Sarawak.

Java: Blume, Verh. Bat. Gen. K. \& W. 9 (1823) 205-221, 6 pl.-Descr. Quercus.

JunghuHN, Nat. Tijd. Ned. Ind. 15 (1858) 119123.-Descr. Quercus.

Koorders, Teysmannia 11 (1900) 133-138.In Dutch; key to Castanopsis.

- \& Valeton, Bijdr. Booms. Java 10 (1904) 1-65. Miquel, Plant. Jungh. (1851) 8-13,-List; some new spp.

Oudemans, in Miquel, J. de Bot. (1861) 244 249.-Prelim. note Quercus coll. JUNGHUHN.

Celebes: Koorders, Suppl. Flora N. O. Celebes 2 (1922) t. 2; ibid. 3 (1922) 1.-New Quercus.

Philippines: Camus, Bull. Soc. Bot. Fr. 81 (1934) 816.-New Quercus.

Elmer, Leafl. Philip. Bot. 3 (1910) 933-946.Account of Quercus \& Castanopsis on Mt Apo; some new spp.

-, ibid. 6 (1913) 1981-1986.-Quercus from Mt Urdaneta; some new spp.

_, ibid. 9 (1934) 3188-3189.-New Quercus from Mt Pinatubo.

-, ibid. 10 (1939) 3736-3738.-New Quercus from Mt Bulusan.

MerRILL, Philip. J. Sc. 3 (1908) Bot. 317-330.Revision of Fagac.; keys!

-, ibid. 21 (1922) 516-517.-A Quercus $\$ P a-$ sania.

-, ibid. 29 (1926) 362-363.-New Castanopsis. -, ibid. 29 (1926) 476-477.-New Quercus § Pasania.

-, ibid. 30 (1926) 79-80.-New Castanopsis. -, ibid. 35 (1928) 6.-New name in Quercus.

Von Seemen, in Fedde, Rep. 5 (1908) 21.-New Quercus.

New Guinea: BaILEY, Queensl. Agric J. 22 (1908) 149.-New Castanopsis.

MARKGRAF, Bot. Jahrb. 59 (1924) 61-79, 4 fig.; ibid. (1925) 538-539.-Revision.

VAN SteenIS, Blumea 7 (1952) 146-147, 306.Diagn. new Nothofagus.

-, J. Arn. Arb. 34 (1953) 301-374, fig. 1-23.Revision Nothofagus; keys!

-, ibid. 35 (1954) 266-267, 1 fig.-Add. note Nothofagus.

White \& Francis, Proc. R. Soc. Queensl. 38 (1927) 227.-List of records coll. LANE-PooLE.

\section{Genera}

Castanopsis: den Berger, Hand. 4e Ned. Ind. Natuurw. Congr. (Weltevreden) (1926) 402.Quercus acuminatissima is a Castanopsis as to wood-anat.

Blume, Mus. Bot. Lugd.-Bat. 1 (1850) 282-286. -List.

Camus, A., Les châtaigniers. Monographie des genres Castanea et Castanopsis (Encycl. Écon. Sylv. 3, 1929, 1-604, t. 1-24, maps 1-4; Atlas (folio) (1929) t. 1-76, I-XXXIV).-Monogr.; keys.

KING, Ann. R. Bot. Gard. Calc. 2 (1889) 17-18. 93-107, t. 83-103.-Revision Indo-Mal. spp.; key.

LitTle, Phytologia 3 (1949) 81-82.-Prop. conserv. Castanopsis.

Miquel, Pl. Jungh. (1851) 13-14.

ReHDER, J. Arn. Arb. 1 (1919) 121-122.-New comb. Malaysian spp.

Nothofagus: Camus, Rev. Int. Bot. Appl. \& Agric. Col. 31 (1951) 71-84, pl. 4 (1952).-Survey; (incomplete and wrong) key!

LANGDON, Bot. Gazette 108 (1947) 350-371, 37 fig-Compar. morphol.

Du RIETZ, Svensk Bot. Tidskr. 24 (1930) 504 510.-Domatia in New Zeal. spp.

VAN STeEnis, Taxon 2 (1953) 177.-Proposal for conservation.

-, J. Arn. Arb. 34 (1953) 301-374, fig. 1-23.Revision of the genus; keys!

-, ibid. 35 (1954) 266-267, fig. 1.-Reduction of Trisyngyne BallL. (New Caledonia); notes on some S. Amer. \& Papuan spp.

Quercus s. l. (Lithocarpus, Pasania, etc.): Blume, Mus. Bot. Lugd.-Bat. 1 (1850) 287-288.-List of some spp.

Camus, A., Les chênes. Monographie du genre Quercus sous-genre Cyclobalanopsis et Euquercus (sect. Cerris \& Mesobalanus) (Encycl. Écon. Sylv. 7, 1936-1938, 1-686, fig. 1-35; Atlas (1934) 1-93, t. 1-178, 1-XXX).-Monograph; keys. -, ditto, sous-genre Euquercus sect. Lepidobalanus et Macrobalanus (ibid. 7, 1938-1939, 1-830, fig. 1-59; Atlas (folio) 1935-1936, 1-177, t. 79236, XXXI-LVIII).-Ditto. Not more seen.

De Candolle, Ann. Sc. Nat. Bot. IV, 18 (1862) 49-58.-New subdiv. Quercus. Cf. also J. Bot. 1 (1863) 134-142.

HANCE, J. Bot. 1 (1863) 173-183.-On generic delimitation in Quercus and other Fagaceae.

-, ibid. 12 (1874) 240-243.-Some new Mal. spp. of Quercus.

King, Ann. R. Bot. Gard. Calc. 2 (1889) 17-92, t. 15-82, 104.-Revision Indo-Mal. spp.; keys! Korthals, in Temminck, Verh. Nat. Gesch., Botanie (1842-44) 195-217, t. 43-47.-Descr.

Muller, U. S. Dep. Agric. Misc. Publ. 477 (1942) 1-216, t. 1-124.-Revision Central Amer. spp.; key!

-, Chron. Bot. 7 (1942) 12-14.-Discussion infrageneric subdiv.

Oudemans, Annotationes criticae in Cupuliferas nonnullas javanicas (Nat. Verh. Kon. Akad. 11, 
1865, repr. 1-24).-Descr. some new spp.; revision; key to Mal. spp:

ReHDer, J. Arn. Arb. 1 (1919) 122-136.-New comb. in Lithocarpus and Quercus, several from Malaysia.

-, ibid. 10 (1929) 132-134.-A number of transfers of Mal. Quercus to Lithocarpus.

Schwarz, Notizbl. Berl.-Dahlem 13 (1936) 1-22.-Segregates of Quercus s. l. and other subdivisions; keys to these.

Von Seemen, Bot. Jahrb. 27 (1900) Beibl. 64, p. 11-18.-Various descr. of spp. from Java, Borneo, Celebes, etc. herb. KoORdERs and cult. in Hort. Bog.

Troll, Ber. Deut. Bot. Ges. 44 (1926) 290-295. -Morph. 9 inflor. in Quercus.

Trelease, Mem. Nat. Acad. Sc. 20 (1924) 1-255, t. 1-420.-Revision American Quercus; keys! Wenzig, Jahrb. Bot. Gart. Berlin 3 (1884) 175219.-List American spp.; no key.

一, ibid. 4 (1886) 179-213.-Quercus in Europe, N. Africa \& Orient.

-, ibid. 4 (1886) 214-240.-Enum. Asiat. \& Mal. spp.; no keys!

\section{FICOIDACEAE See Aizoaceae}

\section{FLAC O URTIA CEA E}

(Samydaceae, Bixaceae pro parte)

Bentham, G., Notes on Bixaceae and Samydaceae (J. \& Proc. Linn. Soc. Lond. 5, 1861, 2nd Suppl., 75-94).-Struct. of fam.; delimitation of genera and descr. new spp.; no keys.

Clos, D., Révision des genres et des espèces appartenant à la famille des Flacourtiacées (Ann. Sc. Nat. IV, 8, 1857, 209-274).-Revision; keys to spp.; contains also several genera now assigned to different families.

Gilg, E., Pflanzenfamilien ed. 2, 21 (1925) 377457, fig. 163-212.

Sleumer, H., New Malaysian Flacourtiaceae (Blu.mea 7, 1954, 484-497).-Descr. new spp. and a new gen.; precursor to next revision.

-, Fl. Mal. I, 5 (1954) 1-106, fig. 1-37.

Slooten, D. F. VAN, Bijdrage tot de kennis der Combretaceeën en Flacourtiaceeën van Nederlandsch-Indië. Thesis, Utrecht (1919) 57-175.Keys.

-, Bull. Jard. Bot. Btzg III, 7 (1925) 291-421.New revision; keys.

\section{Local}

Sumatra: BAKER $f$., J. Bot. (1924-25) Suppl. 6, 42-43, 142-143.-List Forbes coll.; new var. in Hydnocarpus; $n$. sp. in Casearia.

Merrill, Philip. J. Sc. 14 (1919) 246-247.New Osmelia.

-, Pap. Mich. Acad. Sc. Arts \& Lett. 19 (1934) 174-175.-New Casearia; redescr. of a Hydnocarpus.

Malay Peninsula: Bunting \& Milsum, Kew Bull. (1927) 49-56, 3 fig., t. 8.-Cultivation data on Hydnocarpus \& Taraktogenos in the F.M.S.

Borneo: Merrill, Philip. J. Sc. 11 (1916) Bot. 92100.-New records and new spp.
-, J. Str. Br. R. As. Soc. no 86 (1922) 332-333. -New Taraktogenos and Casearia.

-, Plant. Elm. Born. (1929) 207-210.-List ELMER coll.; 2 new Hydnocarpus.

RIDLEY, in HoOK. Ic. Pl. V, 2 (1932) t. 3167.New Taraktogenos.

一, J. Bot. 74 (1936) 221-225.-Descr. new spp. of Flacourtia, Ryparosa, and Xylosma.

-, Kew Bull. (1938) 110-112.-Descr. new spp. AIRY Shaw, Kew Bull. (1949) 157-158.-2 records and a new Hydnocarpus.

Philippines: De Candolle, Philip. J. Sc. 11 (1916) Bot. 37-38.-New Hydnocarpus.

Elmer, Leafl. Philip. Bot. 9 (1934) 3189-3190. -New Casearia.

-, ibid. 10 (1939) 3738-3739.-New Casearia. Merrill, Philip. J. Sc. 27 (1925) 37-39.-2 new Homaliums.

—, ibid. 30 (1926) 411-413.-New Casearia.

— \& Quisumbing, ibid. 82 (1953) 330.-New Hydnocarpus and Casearia; syn. Flacourtia jangomas.

Quisumbing, Philip. J. Sc. 76 (1944) 54-55.New Casearia.

- \& MERrILL, ibid. 37 (1928) 171-172.Ditto.

New Guinea: Baker $f$., J. Bot. (1923) 22.-New Casearia.

GILG, Bot. Jahrb. 55 (1918) 273-294.-Revision; keys.

Mildbraed, see sub Ryparosa.

Pulle, Nova Guinea 8 (1912) 671-672.-List : new Flacourtia \& Hydnocarpus.

Van Slooten, Nova Guinea 14 (1927) 190-194. -List.

\section{Genera}

Erythrospermum: voN TIEGHEM, in MOROT, J. de Bot. 14 (1900) 125-129.-Proposed as type of a new family.

Flacourtia: GAGNEPAIN, in Morot, J. de Bot. 21 (1908) 168-173.-Revision \& key.

Ridley, J. Bot. 74 (1936) 221-224.-Critical notes on Mal. spp.; new sp. from Borneo and Assam.

Homalium: Bentham, J. Linn. Soc. Lond. Bot. 4 (1860) 31-38.-Revision; no key.

Hydnocarpus (Taraktogenos): SLeumer, Bot. Jahrb. 69 (1938) 1-93.-Monograph; key.

Itoa (Mesaulosperma): SLeuMER, Notizbl. Berl.Dahlem 11 (1934) 1024-1026.

Ryparosa (Gertrudia): Mildbraed, Notizbl. Berl.Dahlem 10 (1928) 338-340.-New Guinea; reduction.

Scolopia: Gagnepain, in Morot, J. de Bot. 21 (1908 )164-168.-Revision; key.

Xylosma: Sleumer, Notizbl. Berl.-Dahlem 14 (1938) 288-297.-Mal.-Pac. spp.

\section{FLA GELLARIACEAE}

BACker, C. A., Fl. Mal. I, 4 (1951) 245-250, fig. 1-2.

ENGler, A., Pflanzenfamilien ed. 2, 15a (1930) 6-8, fig. 1 . 


\section{Local}

Sumatra: Merrill, Contr. Arn. Arb. 8 (1934) 18. -Record of Joinvillea.

Malay Peninsula: Ridley, J. Str. Br. R. As. Soc. no 44 (1905) 199-200.-New Joinvillea.

Java: BACKER, Handb. Fl. Java 3 (1924) 2-3.-Key; in Dutch.

BakHUizen VAN Den Brink Jr, Blumea 6 (1950) 399.-Comb. nov. of a var.

New Guinea: Krause, Bot. Jahrb. 59 (1925) 544 546.-List.

-, Nova Guinea 14 (1927) 180.-Records.

LAUTERBACH, Bot. Jahrb. 50 (1913) 288-289.Key.

\section{Genera}

Hanguana (Susum, Veratronia): ErdtManN, Rapp. \& Commun. 8e Congr. Int. Bot. Sect. 2-6 (1954) 33.-Pollen \& epidermal structure are in favour of removing this genus to Liliaceae.

KURz, Flora 56 (1873) 224.-Revision; reduction of genera.

-, J. As. Soc. Bengal 44, ii (1875) 199.-Addit. note.

Joinvillea: Brongniart \& Gris, Bull. Soc. Bot. Fr. 8 (1861) 268-269.-Survey; no key.

\section{FUMARIACEAE}

Fedde, F., Pflanzenfamilien ed. 2, $17 \mathrm{~b}$ (1936) 140145, fig. 75-76.

Hutchinson, J., The genera of Fumariaceae and their distribution (Kew Bull. 1921, 97-115).Key.

\section{Local}

Java: BACKER, Bekn. Fl. Java (em. ed.) 4 A (1942) fam. 42.-Keys; in Dutch.

\section{Genera}

Fumaria: Pugsley, J. Linn. Soc. Lond. Bot. 44 (1919) 233-355, t. 9-13.-Revision; partial keys.

\section{GENTIANACEAE}

Grisebach, A. H. R., Genera \& species Gentianearum, etc. (1839) 1-364.-Monograph; keys.

KNoblauch, E., Beiträge zur Kenntnis der Gentianaceae (Bot. Centralbl. 60, 1894, 321-334, 353-363, 385-401).-Notes on genera \& spp.

SteEnIs, C. G. G. J. van, Bull. Jard. Bot. Btzg III, 13 (1934) 208-209.-List Mal. Crawfurdia, Gentiana, Swertia.

\section{Local}

China: Franchet, Bull. Soc. Bot. Fr. 46 (1899) 302-324.-List, with new spp.; no key.

India: Clarke, J. Linn. Soc. Lond. Bot. 14 (1875) 423-457.-Revision; concise descr.; partial keys.

Sumatra: BACKER, Bull. Jard. Bot. Btzg III, 2 (1920) 326-328.-New Gentiana.

Merrill, Contr. Arn. Arb. 8 (1934) 138-139.Record of Crawfurdia.

- , Not. Nat. Acad. Nat. Sc. Philad. no 47 (1940) 7.-2 new Gentianas.

MOORE, J. Bot. (1925) Suppl. 70-71.-List; new Gentiana \& Limnanthemum.
Ridley, J. Fed. Mal. Stat. Mus. 8, 4 (1917) 58.New Gentiana.

-, Kew Bull. (1939) 29-31.-New Gentiana.

Malay Peninsula: Henderson, J. Mal. Br. R. As. Soc. no 17 (1939) 58.-List limestone spp.

Java: RideEY, Kew Bull. (1939) 29-31.-Record of 2nd Gentiana from Java.

Borneo: Merrill, Philip. J. Sc. 13 (1918) Bot. 120. -New Gentiana.

STAPF, Trans. Linn. Soc. Lond. II, 4 Bot. (1894) 208-209.-New Gentiana.

Philippines: Elmer, Leafl. Philip. Bot. 10 (1939) 3739-3740.-New Microphium.

Robinson, Philip. J. Sc. 3 (1908) Bot. 214.New Swertia.

Van Steenis, Bull. Bot. Gard. Btzg III, 17 (1948) 409.-New record of a Gentiana.

New Guinea: Diels, Bot. Jahrb. 62 (1929) 488-489. -New Swertia.

HeMSLEY, Kew Bull. (1899) 106-107.-Record and 2 new Gentianas.

KoORDERS, Nova Guinea 8 (1909) 175-176.List; new Gentiana.

一, ibid. 8 (1912) 889-890.-Records.

MANSFELD, Bot. Jahrb. 61 (1927) 28-29.-List. Merrill. \& Perry, J. Arn. Arb. 30 (1949) 45.Records of 2 spp. of Nymphoides.

Wernham, Trans. Linn. Soc. Lond. II, Bot. 9 (1916) 117-118.-4 new Gentianas.

\section{Genera}

Cotylanthera: Figdor, Ann. Jard. Bot. Btzg 14 (1897) 213-240, t. 16-17.-Morph.

Exacum: Hallier $f$., Beih. Bot. Centralbl. 34, ii (1916) 42-43.-Mal. E. 'tetragonum' is a separate sp.

Nauen, M. A. H. A. Mag. 10 (1940) 152.Cult. spp.

VAN STEEnIs, Bull. Bot. Gard. Btzg III, 17 (1948) 462.-Syn. Paracelsea Z. \& M. 1844.

Gentiana (Crawfurdia): KusNezow, Act. Hort. Petrop. 15 (1904) i-iv, 1-507, 4 maps, 1 tab.Monograph of subgen. Eugentiana; key to sect. and spp.; \pm verbatim German translation of a Russian edition dated 1894.

Marquand, Kew Bull. (1931) 68-70.-Crawfurdia p. maj. $p$. is reduced to Gentiana.

-, J. R. Hort. Soc. 57 (1932) 188-211.-Rev. Chin. \& Himal. sect.; descr.; keys to spp.

-, Kew Bull. (1937) 134-180.-Revision of Chin. spp.; keys.

Nymphoides (Limnanthemum): Goesel, Ann. Jard. Bot. Btzg 9 (1891) 120-126, t. 16.-Morph.

MIKI, Tokyo Bot. Mag. 49 (1935) 779-780, fig. 10.-Rev. Japan. spp.; key.

Van Steenis, Arch. Hydrobiol. Suppl. 3 (1932) $320-322$, fig. 6.-Records of Sumatra; details on 3 spp.

\section{GERANIACEAE}

KNUTh, R., Pflanzenreich Heft 53 (1912) 1-640, fig. $1-80$.

-, Pflanzenfamilien ed. 2, 19a (1931) 43-66, fig. 20-34.

SteEnis, C. G. G. J. van, Bull. Jard. Bot. Btzg III, 13 (1934) 209-210.-List Mal. mountain spp. 


\section{Local}

New Guinea: KnUth, in Fedde, Rep. 45 (1938) 61-62.-Two new Geraniums.

\section{GESNERIACEAE \\ (Cyrtandraceae)}

Beddome, R. H., Gesneraceae, with annotated list of the genera and species which have been introduced to cultivation (J. R. Hort. Soc. 33, 1908, 74-100).-List with short descr. of spp.

BURTT, B. L., Studies in the Gesneraceae of the Old World (Not. R. Bot. Gard. Edinb. 21, 1954, 185-218, t. 1-2).-Introd.; types of certain genera and other groups; status of Ceratoscyphus; new Burmese Didymocarpus; notes on Ancylostemon.

Clarke, C. B., in DC. Mon. Phan. 5 (1883) 1-303, t. 1-32.

Craib, W. G., Didissandra and allied genera in China and North India (Not. R. Bot. Gard. Edinb. 11, 1919, 255-268).-Keys.

\section{Local}

SE. Asia: Clarke, Commelinaceae et Cyrtandraceae Bengalenses. Calcutta (1874) 63-133, t. 43-93.-Descr. \& pl.

Indo-China: Pellegrin, Bull. Soc. Bot. Fr. 73 (1926) 412-429.-Subfam. Cyrtandreae; keys.

Sumatra: For BES, J. Linn. Soc. Lond. Bot. 19 (1882) 297.-New Boea.

Merrill, Contr. Arn. Arb. 8 (1934) 151-154, t. 14.-List; 2 new Trichosporums.

MOORE, J. Bot. (1925) Suppl. 72-76.-List FORBES coll.; new spp. in Agalmyla (1), Stauranthera (1), Cyrtandra (5).

Ridley, J. Mal. Br. R. As. Soc. no 87 (1923) 79 81).-List; new spp. in Aeschynanthus (1), Didymocarpus (1), Rhynchothecum (1), Cyrtandra (2).

-. Kew Bull. (1925) 86-87.-List; new Didymocarpus.

-, ibid. (1926) 75-76.-List; new spp. in Didissandra (1), Cyrtandra (2), Didymocarpus (1).

-, Arch. Hydrobiol. Suppl. 11 (1932) 326-330,

fig. 7.-List; new Didissandra.

Malay Peninsula: Henderson, Gard. Bull. Str. Settl. 4 (1929) 412-413.-New Loxocarpus. -, ibid. 7 (1933) 115-118.-3 new Paraboeas.

-, J. Mal. Br. R. As. Soc. 17 (1939) 60-62.List limestone spp.

KRÄnzlin, Notizbl. Berl.-Dahlem 4 (1907) 293-294.-Two new Didymocarpus.

RIDLEY, J. Linn. Soc. Bot. 32 (1896) 497-528.Rev.; Malayan Cyrtandreae listed.

-, Kew Bull. (1929) 258-260.-3 new spp.

Spare, M. A. H. A. Mag. 10 (1940) 131-133.Key to some ornamental spp. of Aeschynanhus.

Java: BACKER, Trop. Natuur 1 (1912) 173-175, 179-183, fig. 1-3.-Key to Jav. Trichosporum; in Dutch.

BAKHUIZEN VAN DEN BRINK Jr, Blumea 6 (1950) 394-398.-Critical list.

Borneo: Bayley Balfour \& W. W. Smith, Not. R. Bot. Gard. Edinb. 8 (1915) 349-356, t. 146.Moultonia n. g.; full discussion.
-, Sarawak Mus. J. 6 (1915) 277-286, t. 2.Copy of the above.

KRäNZLIN, J. Linn. Soc. Lond. Bot. 37 (1906) 275-285.-3 new spp. in each Cyrtandra and Aeschynanthus.

-, Notizbl. Berl.-Dahlem 4 (1907) 292-293.2 Cyrtandras.

-, Mitt. Inst. Allg. Bot. Hamburg 7 (1927) 80113, fig. 1-2.-List HANS WINKLER coll.; new spp. in Aeschynanthus (3), Didissandra (1), Didymocarpus (9), Monophyllaea (1), Stauranthera (1), Hexatheca (2), Cyrtandra (16).

Merrill, J. Mal. Br. R. As. Soc. no 87 (1923) 32-36.-New spp. in Didymocarpus (1) and Cyrtandra (3).

-, Sarawak Mus. J. 3 (1928) 549-552.-Records \& new spp. of Didymocarpus (1), Trichosporum (1), Dichrotrichum (1).

MOORE, J. Linn. Soc. Lond. Bot. 42 (1914) 116118.-List Gibes; new Cyrtandra and Aeschynanthus.

StAPF, Trans. Linn. Soc. Lond. II, 4 Bot. (1894) 212-214.-List; 4 new spp

WinkLeR, Bot. Jahrb. 44 (1910) 537-541.-List Hub. Winkler; 5 new Cyrtandras.

Celebes: Kränzlin, J. Linn. Soc. Lond. Bot. 37 (1906) 275-285.-New Cyrtandra.

Philippines: Elmer, Leafl. Philip. Bot. 2 (1908) 553-567.-List spp. Cuernos Mts; several new; no key.

-, ibid. 3 (1910) 947-970.-Descr. new \& interesting spp.

-, ibid. 9 (1934) 3191-3194.-2 new Cyrtandras. -, ibid. 10 (1939) 3741-3744.-3 spp. ditto.

KRÄNZLIN, J. Linn. Soc. Bot. 37 (1906) 275285.-5 new Cyrtandras.

-, Philip. J. Sc. 8 (1913) Bot. 163-179, 311-333. -List new spp.; no key.

Merrill, Philip. J. Sc. 10 (1915) Bot. 75-84, 340-341.-Descr. new spp.

-, ibid. 26 (1925) 489-490.-New Didymocarpus from Palawan.

Moluccas: Merrill, Philip. J. Sc. 11 (1916) Bot. 313.-Ambon records; new Trichosporum.

New Guinea: Diels, Bot. Jahrb. 62 (1929) 492493.-A Cyrtandra and 2 Dichrotrichums.

FORBES, J. Bot. 25 (1887) 347-348.-New Boea. Kanehira, Hatusima \& Ohwi, Bot. Mag. Tokyo 57 (1943) 111-128.-Many new spp.

LAUTER BACH, Nova Guinea 8 (1910) 325-331, t. 66.-List; new spp. in Dichrotrichum (1), Monophyllaea (1), Cyrtandra (7); Cyrtandropsis n. g. -, ibid. 8(1912) 859-862.-List; 3 new Cyrtandras. MOORE, J. Bot. 37 (1899) 172-175.-Notes on FORBES coll.; listed records; new spp. in Trichosporum and Chirita from Papua.

-, Trans. Linn. Soc. Lond. II, Bot. 9 (1916) 122-132.-List Wollaston Exp.; new spp. in Aeschynanthus (3), Dichrotrichum (5), Monophyllaea (1), Cyrtandra (8).

-, J. Bot. 67 (1929) 50-51.-New Cyrtandra.

VoN MUeller, in Wing's South. Sc. Rec. 2 (1882) 229.-New Didymocarpus.

-, Melbourne Chem. \& Drugg. (June 1884) 14. -New Dichrotrichum. 
SCHLECHTER, Bot. Jahrb. 58 (1923) 255 -379, fig 1-11.-Revision; keys.

-, Nova Guinea 14 (1927) 308-322.-New spp. in Aeschynanthus (4), Dichrotrichum (2), Cyrtandra (16); Oxychlamys n. 8 .

\section{Genera}

Aeschynanthus (Trichosporum): Hallier $f$, Nat. Tijd. Ned. Ind. 56 (1896) 333-346.-Rev. spp. in Herb. Bog.; no key.

Pellegrin, Bull. Soc. Bot. Fr. 72 (1926) 821824.-Key to Indo-Chin. spp.

Anna: Pellegrin, Bull. Soc. Bot. Fr. 77 (1930) 45-46.-Indo-Chin. spp.

Corallodiscus: BuRTT, Gard. Chron. III, 121 (1947) 204, 212.-Segregate of Didissandra.

Cyrtandra: Hosoxawa, Trans. Nat. Hist. Soc. Formosa 25 (1935) 410-473.-Distr.

Sмiтh, A. C., J. Arn. Arb. 34 (1953) 37-51.Revision; descr. many new spp.

Cyrtandropsis: van Steenis, Bull. Bot. Gard. Btzg III, 17 (1948) 459-460.-Nomencl. generic name.

Dichrotrichum: SCHLEChTER, in FedDe, Rep. 16 (1919) 212-213.-D. borneense; definition new infrageneric group.

Didissandra: BaKhUIzEN VAN DEN BRINK Jr, Fl. Mal. Bull. no 3 (1948) 72-73.-Conservation against Ellobum.

CraIB, Not. R. Bot. Gard. Edinb. 11 (1919) 255-268.-Segregation of the genus.

Moultonia: Bayley Balfour \& W. W. SMith, Sarawak Mus. J. 2 (1916) 277-286, t. 2.

Paraboea \& Paraboea: BurTt, Kew Bull. (1948) 55-56.-Survey; no key.

Tetradema: Schlechrer, Notizbl. Berl.-Dahlem 7 (1920) 359-362,-Key.

\section{GNET ACEAE}

BecCARI, O., Malesia 1 (1877) 181-183.-His coll. listed; 2 new spp.

Blume, C. L., De Gnetaceis (Rumphia 4, 1848, 1-10, t. 174-176).-List.

MarKGraf, F., Monographie der Gattung Gnetum (Bull. Jard. Bot. Btzg III, 10, 1930, 407-511, t. 1-14).-Revision; keys.

-, Fl. Mal. I, 4 (1951) 336-347, fig. 1-5.

WARBURG, O., Monsunia 1 (1900) 195-197.Survey; no key.

\section{Local}

Malay Peninsula: Ridley, J. Str. Br. R. As. Soc. no 60 (1911) 59-66.-Key.

Borneo: MARKgraf, Reinwardtia 1 (1952) 462.New variety.

Philippines: Foxworthy, Philip. J. Sc. 6 (1911) Bot. 172-176, t. 32-33.-Revision; key.

New Guinea: Markgraf, Bot. Jahrb. 60 (1925) 144-149.-Key.

-, Nova Guinea 14 (1927) 221.-Record.

Pulle, Nova Guinea 8 (1910) 345.-Record.

\section{GONYSTYLACEAE See Thymelaeaceae}

\section{GOODENIACEAE}

Krause, K., Pflanzenreich 54 (1912) 1-207, fig. 1-34.
VRIESE, W. H. DE, Goodenovieae (Natuurk. Verh. Holl. Mij. Wet. Haarlem, 2e verzam., 10, 1854, i-viii, 1-194, t. 1-38).-Monograph.

\section{Local}

Java: BACKer, Bekn. Fl. Java (em. ed.) 8 (1949) fam. 185.

Philippines: Merrill, Publ. Govt Lab. (Manila) no 35 (1906) 66-68.-Disc. Calogyne record.

-, Philip. J. Sc. 5 (1910) Bot. 249-252.-List; descr. 3 new spp.

MiQuel, Ann. Mus. Bot. Lugd.-Bat. 1 (1864) 210.-2 new spp.

New Guinea: Merrill \& Perry, J. Arn. Arb. 22 (1941) 387-388.-Velleia, Calogyne.

-, ibid. 30 (1949) 60.-Leschenaultia.

VoN Mueller, Vict. Nat. 1 (1884) 122-123.

Pulle, Nova Guinea 8 (1912) 693.

\section{GRAMINEAE!}

Amshoff, A. \& J. Th. Henrard, Gramineae, in Pulle, Flora of Surinam 1, part 1 (1948) 273442.

BLAKE, S. T., Monographic studies in the Australian Andropogoneae I (Queenst. Univ. Pap. Dept Biol. 2, 1944, 1-62).-Revisions of the genera Botriochloa, Capillipedium, Chrysopogon, Vetiveria and Spathia.

Gardner, C. A., Flora of Western Australia vol. 1, pt 1 (1952) 1-400, t. 1-103, 6 diagr., map.Revision; keys.

HACKel, E., Die auf der Expedition S. M. S. 'Gazelle' von Dr NaumanN gesammelten Gramineen (Bot. Jahrb. 6, 1885, 233-248).

-, Andropogoneae in DC. Mon. Phan. 8 (1889) $1-716$, t. $1-2$.

Henrakd, J. Th., A contribution to the knowledge of the Indian Maydeae (Meded. Rijksherb. Leiden no 67, 1931, 17 pp.).-Keys.

-, Identification of some Malaysian grasses (Blumea 1, 1935, 305-311).-Notes on Paspalum, Schizachyrium, Deschampsia, and Cenchrus.

-, Notes on the nomenclature of some grasses (Blumea 3, 1940, 411-480.)

-, Ditto II (ibid. 4, 1941, 496-542).

HiтcHCOCK, A. S., The grasses of Hawaii (Mem. Bern. P. Bish. Mus. 8, no 3, 1922, 132 pp.).

-, Grasses of British Guyana (Contr. U. S. Nat. Herb. 22, 1922, 439-514).

- Manual of the grasses of the United States (U. S. Dept Agric. Misc. Publ. no 200, 1935, 1040 pp.).

- \& A. Chase, Revisions of North American grasses (Contr. U. S. Nat. Herb. 22, 1920, 1-77, 115-234).-Ichnanthus, Brachiaria, Cenchrus, Isachne, Oplismenus, Echinochloa, Setaria, Pennisetum.

Hubbard, C. E., The genera of the tribe Arundinelleae (Kew Bull. 1936, 317-322).-Key to the genera.

-, Apochiton burtti C. E. Hubbard (in Hook.

(1) Bambusaceae have been treated here, for convenience, separately at the end of the Gramineae. 
Ic. Pl. V, 4, 1936, t. 3319, 9 pp.).-Key to genera of Eragrosteae.

-, Key to the genera of the Rottboelliastrae in Australia (in Hook. Ic. Pl. V, 4, 1936, t. 33133314 , p. 5-6).

-, Limnopoa meeboldii (C. E. C. Fischer) C. E. HubBard (in Hook. Ic. Pl. V, 5, 1943, t. 3432, 4 pp.).-Contains a key to the genera of Isachneae. - \& O. STAPF, Sclerandrium truncatiglume (F. Muell.) Stapf \& C. E. Hubbard (in Hook. Ic. Pl. V, 3, 1935, t. 3262, 7 pp.).-Key to genera of Polliniastrae.

JANOWSKI, M., Arundinellearum, Graminum tribus conspectus (Bot. Arch. 1, 1922 21-28).-Keys to spp. of Arundinella \& Thepanolaena.

JANSEN, P., Notes on Malaysian grasses I (Reinwardtia 2, 1953, 225-350, fig. 1-18).-Misc. notes; one new genus, many new spp. and var. and new comb.

-, ditto II (Acta Bot. Neerl. 1, 1953, 468-483, fig. 1-4).-Ditto. ditto III (ibid. 2, 1953, 363-386, fig. 1-10). - Ditto.

KuNTH, K. S., Révision des Graminées, etc. (in Humboldt \& BonPland, Nova genera et species plantarum). 2 vols (issued in 44 parts, $c f$. Fl. Mal. I, 4, cxciv-cxcv, in 1829-1834). The same work issued by KuNTH, under the title: 'Distribution méthodique de la famille des Graminées', Paris (1835), plates and letterpress identical but paging different; I, i-xlv, 1-175, II, 177-579, errata, 220 tab.

- Enumeratio plantarum, etc. I. Gramineae (Agrostographia synoptica sive enumeratio Graminearum). Stuttgart (1833) 1-606.-Monograph; descr. of genera and spp.; no keys.

-, ditto II (Suppl. to vol. I). Stuttgart (1835) 1-436, t. J.-Ditto.

Mez, C. (\& M. JANowski), Graminea novae vel minus cognitae (in FedDE, Rep. 17, 1921, 83-86, 145-153, 291-303).-Mixed descr.

Munro, W., On the identification of the grasses of Linnaeus's Herbarium, etc. (J. Linn. Soc. Lond. $6,1861,35-55)$.

OHw, J., New or noteworthy grasses from Asia (Bull. Tokyo Sc. Mus. no 18, 1947, 1-15).Almost entirely dealing with Mal. grasses.

Pilger, R., Pflanzenfamilien ed. 2, 14e (1940) 1208, fig. 1-106.-Subfam. Panicoideae; tribes Paniceae, Arthropogoneae, Andropogoneae, and Maydeae.

-, Additamenta agrostologica 1-III (Bot. Jahrb. $74,1945,1-27, \quad 199-265,554-567)$.-Sclerodeyeuxia n. g. (Malaysia).

-, Das System der Gramineae (Bot. Jahrb. 76, 1954, 281-384).-New key to all genera.

-, Pflanzenfamilien ed. 2, 14d. In preparation.Subfam. Festucoideae, Micrairoideae, Eragrostoideae, Oryzoideae, Olyroideae.

StapF, O., The oilgrasses of India and Ceylon (Kew Bull. 1906, 297-363).-Cymbopogon, Vetiveria \& Andropogon spp. with key.

- \& C. E. Hubbard, Gramineae. MaydeaePaniceae in Fl. Trop. Afr. 9 (1917-1934) 1-1132; 10 (1937-?) unfinished.
Steudel, E. G., Synopsis plantarum Glumacea- rum pars I. Gramineae. Stuttgart (1854) i-viii, 1-475.-Enum. descr. in Latin; contains also spp. based on specimens of CUMING, ZOLLINGER, etc.

Local

Assam: BoR, N. L., in KanJlal, c.s., F1. Assam 5, $1940,1-480$.

Malay Peninsula: Hackel, Oesterr. Bot. Z. 41 (1891) 5-9, 47-50.-Records and descr.

HoltTuM, Gard. Bull. Sing. 11 (1947) 297-298. -New Spodiopogon and Rottboellia.

Hubbard, Kew Bull. (1941) 24-25.-New Cymbopogon and new comb. in Eustachys.

-, J. Str. Br. R. As. Soc. no 23 (1891) 19-32.Listed records; no new spp.; no keys.

Ridley, Mat. Fl. Mal. Pen. (Monocot.) 3 (1907) 118-182.-Keys.

Sumatra: HenRard, in Fedde, Rep. 22 (1926) 349350.-New Erianthus.

Java: BACKER, Bull. Jard. Bot. Btzg III, 2 (1920) 325-326.-New Diplachne.

-, Handb. Fl. Java 2 (1927) 1-300.-Important revision; keys; in Dutch.

Henrard, Blumea 3 (1939) 238-242.-New Polytoca.

Borneo: Pilger, Mitt. Inst. Allg. Bot. Hamburg 7 (1928) 177-178.-List WINKLER coll.

Ridley, J. Str. Br. R. As. Soc. no 46 (1906) 215221.-Listed records; no new spp.

StaPF, J. Linn. Soc. Lond. Bot. 42 (1914) 185189. -Listed Kinabalu records.

Philippines: Hackel, Govt Lab. Publ. Philip. 35 (1905) 79-82.

-, Philip. J. Sc. 1 (1906) Suppl. 263-269.

一, ibid. 2 (1907) Suppl. 419-420.

一, ibid. 3 (1908) 167-169.

Merrill, Philip. J. Sc. 1 (1906) Suppl. 307-392. -Important enumeration; keys.

—, Philip. J. Sc. 30 (1926) 390.-Koordersiochloa. -, Philip. J. Sc. 35 (1928) 1-4.

Quisumbing \& MerRILL, Philip. J. Sc. 37 (1928) 135-136.-New Panicum.

Santos, J. Wash. Acad. Sc. 33 (1943) 135140.-Two new species.

Moluccas: Henrard, in Fedde, Rep. 22 (1925) 106-107.-New Andropogon.

Merrill, Philip. J. Sc. 11 (1916) Bot 253-255. -List of Ambon Gram.

Lesser Sunda Islands: Henrard, in Fedde, Rep. 18 (1922) 240-241.-New Eragrostis.

New Guinea: BlaKe, Blumea Suppl. 3 (1946) 5662.-Ancistagrostis \& Buergersiochloa n. g. Chase, J. Arn. Arb. 20 (1939) 304-316.-Brass coll.

-, ibid. 24 (1943) 77-89.-Brass coll.

HenRARD, Blumea 4 (1941) 525-526.-New Arthraxon.

Hrtchcock, Proc. Linn. Soc. N. S. W. 54 (1929) 145-146.-See also J. Arn. Arb. 10 (1929) 272273.-List Brass coll.

-, Brittonia 2 (1936) 107-130.-Brass coll. Hubbard, Kew Bull. (1949) 474.-New Poa. Pilger, Bot. Jahrb. 52 (1914) 167-176.-List. 
一, ibid. 62 (1929) 457-461.-New spp.

—, ibid. 69 (1938) 253-255.-New spp. of Digitaria, Deschampsia, Dichelachne.

ReEDer, J. Arn. Arb. 29 (1948) 257-318, 321392.-Full account of Panicoideae; keys.

-, J. Arn. Arb. 31 (1950) 320-328.-New \& noteworthy spp.

STAPF, in Hook. Ic. Pl. IV, 7 (1901) t. 26052608.-Deyeuxia, Poa, and Danthonia spp.

Micronesia: Hosoxawa, Contr. Herb. Taihoku Imp. Univ. no 42, 7 (1935) 305-325.-Enumeration.

\section{Genera}

Acrachne: HenRard, Blumea 3 (1938) 164-167.

Acroceras: HeNRARD, Blumea 3 (1940) 444-452.Critical notes.

Aegilops: Eig in Fedde, Rep. Beih. 55 (1929) 1228.-Monograph.

Agrostis: HenraRd, Blumea 3 (1940) 436-439.Critical notes.

Ancistagrostis: Blake, Blumea Suppl. 3 (1946) 56-59.-From New Guinea.

Ancistrachne: Blake, Univ. Queensl. Pap. Dept Biol. 1, no 19 (1941) 4-7.

Aristida: Blake, Proc. R. Soc. Queensl. 51 (1940) 169-176.-New spp. of Aristida from Queensland.

-, ibid. 56 (1944) 11-20.-A. utilis.

HeNRARD, Meded. Rijksherb. Leiden no 54, 54A, 54B, I (1926) 1-220; II (1927) 221-464; III (1928) 465-701.

一, ibid. no 58 (1929) 1-153; 58A (1932) 157325.-Monograph.

Arundinella: HuBBARD, Kew Bull. (1936) 317-322. JanowsKI, in Mez, Bot. Arch. 1 (1922) 21-28. -Key.

Asthenochloa: Henrard, Ned. Kruidk. Arch. (1929) 579-582.

Avena \& Avenastrum: SAINT-Yves, Candollea 4 (1930) 353-503.-Key.

Aulacolepis: HaCkel, in Fedde, Rep. 3 (1907) 241-245.

HrTchCOCk, J. Wash. Acad. Sc. 24 (1934) 290 292.

Bothriochloa: Camus, Ann. Soc. Linn. Lyon 76 (1930) Mém. 162-165.-Revision Old World spp.; no key.

Henrard, Blumea 3 (1940) 456-457.-New comb. -, Gedenkb. J. Valckenier Suringar (1942) 181-187.-Notes; new comb.

Brachyachne (Cynodon \$ Brachyachne): HuBBARD, Kew Bull. (1934) 448.-List.

Brachypodium: Sannt-Yves, Candollea 5 (1934) 427-493.-Key.

Bromus: STAPF, Kew Bull. (1928) 209-211.Survey of generic concept.

Buergersiochloa: BLAKE, Blumea Suppl. 3 (1946) 59-62.-New Guinea.

LAUTERBaCh, Bot. Jahrb. 52 (1914) 167-169.

Calamagrostis : MATUszkIEwicz, Ann. Univ. CurieSklodowska sect. C. Biol. 3 (1948) 223-256.

Capillipedium: Henrard, Blumea 3 (1940) 457463.-Critical notes; new spp. from the Philippines and Java.
JANSEN, Reinwardtia 2 (1953) 246-252.-Critical notes; key.

Chionachne: Henrard, Meded. Rijksherb. Leiden no 67 (1931) 13-17.

Chloris: Merrill, Bull. Soc. Bot. Fr. 73 (1926) 27-30.-Syn. of C. tenera.

Chrysopogon: Henrard, Blumea 4 (1941) 531535.-Nomencl., some new Mal. spp.

Coelachne (pulchella): HubBard, in HoOK. Ic. Pl. V, 5 (1943) t. 3440.-List of spp.

Coelorhachis: JANSEN, Reinwardtia 2 (1953) 254256.-Rev. Mal. spp.; key.

Coix : Koorders, Bull. Jard. Bot. Btzg III, 1 (1919) 153-158, 190-191.-C. palustris from Java.

Vallaeys, Bull. Agric. Congo Belg. 39 (1948) no 2; repr. 1-70.-Monogr.

Cyrtococcum: Camus, Bull. Mus. Hist. Nat. Paris 27 (1921) 118.-List.

JANSEN, Reinwardtia 2 (1953) 256-258.-Rev. Mal. spp.; key.

Danthonia: JANSEN, Reinwardtia 2 (1953) 258265, fig. 4-6.-Rev. Mal. ssp.; key.

Deyeuxia: JANSEN, Act. Bot. Neerl. 1 (1952) 468472, fig. 1.-Crit. enum.

-, ibid. 2 (1953) 363-365, fig. 1.-New Sum. sp. VICKery, Contr. N. S. W. Nat. Herb. 1 (1940) 43-82.-Delimit. of the genus; rev. Austral. spp.

Dichanthium: JANSEN, Act. Bot. Neerl. 1 (1952) 473-474.-Descr. 2 spp.

Digitaria: Henrard, Meded. Rijksherb. Leiden no 61 (1930) 1-21.-New \& noteworthy spp.; key to $\$$ Solitaria.

-, Blumea 1 (1934) 90-114.-Notes and new spp.

-, Monograph of the genus Digitaria. Leiden (1950) xxi + 999 pp.

Echinochloa: Blake, Proc. R. Soc. Queensl. 62 (1952) 91-92.-Key to Austral. spp.

Echinopogon (intermedius): HuBBARD, in Hook. Ic. P1. V, 3 (1935) t. 3261.-Monograph; key.

Ectrosia: HubBard, in Hook. Ic. Pl. V, 4 (1936) t. 3312, 16 pp.-Revision; key.

Ectrosiopsis: Jansen, Act. Bot. Neerl. 1 (1952) 474-475, fig. 2.-New sp.

—, Reinwardtia 2 (1953) 267-269.-Rev. Mal. spp.; key.

Enneapogon: Burbidge, Proc. Linn. Soc. Lond, 60 (sess. 153) (1941) 52-91, 5 fig.-Revision Austral. spp.; key.

Eragrostis: Blake, Proc. R. Soc. Queensl. 59 (1948) 153-156.-Queensl. spp.

Camus, Not. Syst. 2 (1911-13) 226-229.-List E. Asiat. spp.

JANSEN, Reinwardtia 2 (1953) 269-275.-Critical notes.

JeDwabnick, in Mez, Bot. Arch. 5 (1924) 177 216. List; no key; incl. new spp.

Eremochloa: CAmus, Not. Syst. 3 (1914) 85-88.Asiat. spp.; key.

Erianthus: JANSEN, Reinwardtia 2 (1953) 275-276. -2 new comb. Mal. spp.

Eulalia: JANSEN, Reinwardtia 2 (1953) 277-278.Rev. Mal. spp.; key.

Festuca subg. Eufestuca: JANSEN, Act. Bot. Neerl. 2 (1953) 365-376.-Rev. Mal. spp.; key. 
SAINT-YVES, Candollea 4 (1929) 65-129, 147165, (1930) 293-397.-African, Malaysian, and N. Zealand spp.; key.

Garnotia: JANSEN, Acta Bot. Neerl. 2 (1953) 376382, fig. 9.-Critical notes; key to Mal. spp. Santos, J. Arn. Arb. 25 (1944) 85-95.-Philip., Chin., and Indo-Chinese spp.

—, Nat. \& Appl. Sc. Bull. (Philip.) 10 (1950) 3179.-Compl. revision; key.

Garnotiella: STAPF, Kew Bull. (1910) 301-302.Morph.; syst. position.

Helictotrichon (Avenastrum): HENRARD, Blumea 3 (1940) 425-431.-Nomencl.

Imperata: HubBard, in Joint Publ. no 7 Imp. Agric. Bur. (1944) 5-13.

Isachne: HENRARD, Blumea 3 (1940) 463-473.Critical notes.

JANSEN, Reinwardtia 2 (1953) 279-292.-Critical notes; key Mal. spp.

-, Act. Bot. Neerl. 2 (1953) 382-384, fig. 10.New sp. from Sumba.

Ischaemum: JANSEN, Reinwardtia 2 (1953) 292301.-Critical notes.

Iseilema: Blake, Proc. R. Soc. Queens1. 49 (1938) 82-94.-Queensl. spp.; key.

Camus, Bull. Mus. Hist. Nat. Paris 24 (1918) 539-541.-Key.

Hubbard, in Hook. Ic. Pl. V, 3 (1935) t. 3286, 1-16.-Revision Austral. spp.; key.

JANSEN, Reinwardtia 2 (1953) 302-303, fig. 11. -New sp. from Wetar.

Leptaspis: JANSEN, Reinwardtia 2 (1953) 302-305. -Rev. Mal. spp.; key.

Lophatherum: Camus, Bull. Mus. Hist. Nat. Paris 25 (1919) 494-496.-Key.

Microstegium: Henrard, Blumea 3 (1940) 453455.-Critical notes.

JANSEN, Reinwardtia 2 (1953) 305-307.-Ditto.

Mnesithea: JANSEN, Reinwardtia 2 (1953) 307309.-Rev. \& key to Mal. spp.

Monostachya: Merrill, Philip. J. Sc. 5 (1910) Bot. 330-332.-Orig. descr.

Oryza: BACKeR, Blumea Suppl. 3 (1946) 45-55.Revision wild Mal. spp.; key.

Chatterjee, Ind. J. Agric. Sc. 18 (1948) 185192 (1949).-Enum. spp.; key.

JANSEN, Reinwardtia 2 (1953) 312-313, fig. 13. -New Pap. sp.

Prodoehl, in Mez, Bot. Arch. 1 (1922) 211-224, 231-255.-Monograph.

Roschevicz, Bull. Appl. Bot. Gen. \& Pl. Breed. $27^{2}$ (1931) 3-134.-Monogr.; summary, bibliogr. \& key in Engl. p. 119-134.

Ottochloa: DandY, J. Bot. 69 (1931) 55.-Nomencl. HENRARD, Blumea 4 (1941) 530-531.-Key.

JANSEN, Reinwardtia 2 (1953) 313-314, fig. 14.New Pap. sp.

Panicum: Camus, Not. Syst. 2 (1911-13) 246-253. -List E. Asiat. spp.

Hugres, Kew Bull. (1923) 305-332.-Nomencl. Austr. spp..

JANSEN, Reinwardtia 2 (1953) 314-319.-Critical notes.

Paspalidium: Blake, Proc. R. Soc. Queensl. 62 (1952) 92-99.-List Austral. spp.; key to some spp.
Paspalum: Camus, Not. Syst. 2 (1911-13) 216-226. -Key to E. Asiat. spp.

VAsEY, Bull. Torrey Bot. Club (1886) 162-168. -Synopsis.

Pennisetum: Leeke, Zeitschr. f. Naturw. 79 (1907) 1-108. Also Thesis, Halle, 1907. New spp. copied in FeDDE, Rep. 5 (1908) 66-76.

Poa: Bor, J. Bombay Nat. Hist. Soc. 50 (1952) 787-838; ibid. 51 (1952) 61-103, 7 pl., 38 fig.Extensive revision Indian spp. (49); descr.; key.

JANSEN, Reinwardtia 2 (1953) 322-333, fig. 1517.-Rev. Mal. spp.; key.

Pogonatherum: CHASE, J. Arn. Arb. 31 (1950) 130 132.

JANSEN, Reinwardtia 2 (1953) 333-334.-Rev. Mal. spp.; key.

Polytoca: Henrard, Meded. Rijksherb. Leiden no 67 (1931) 7-13.-Key.

—, Blumea 3 (1939) 238-242.-New Jav. sp.

Pseudoraphis: PILGER, Notizbl. Berl.-Dahlem 10 (1928) 207-210.-No key.

Ramosia: Merrill, Philip. J. Sc. 11 (1916) Bot. $2-4$.

Schizachyrium: JANSEN, Reinwardtia 2 (1953) 336339.-Rev. Mal. spp.; key.

Sclerachne: HENRARD, Meded. Rijksherb. Leiden no 67 (1931) 7 .

Sehima: Camus, Bull. Mus. Hist. Nat. Paris 27 (1921) 372-373.-Key to Asiat. spp.

Setaria (Chaetochloa): HubBaRd, Amer. J. Bot. 2 (1915) 169-198.-S. italica \& allied spp.

JANSEN, Reinwardtia 2 (1953) 339-343.-Rev. Mal. spp.; key.

StaPF, Kew Bull. (1920) 124-127.-Discussion generic concept in relation to Chaetochloa. -, ibid. (1928) 147-149.-S. glauca and $S$. lutescens.

Sorghum: SNOwDEN, Kew Bull. (1935) 221-255.Cult. spp. classified.

-, The cultivated races of Sorghum. London (1936) i-vii, 1-272, t. 1-4, fig. 1-31.

Sporobolus: Blake, Univ. Queensl. Pap. Dept Biol. 1 (1941) no 18, 3-12.-Key Queensl. spp.

JANSEN, Act. Bot. Neerl. 1 (1952) 475-479, fig. 3-4.-Critical notes.

Stylagrostis: MEZ, Bot. Arch. 1 (1922) 20.-Conspectus.

Thaumastochloa: HubBard, in Hook. Ic. Pl. V, 4 (1936) t. 3313-3314, 6 pp.

Themeda: Camus, Bull. Mus. Hist. Nat. Paris 26 (1920) 266-273.-Key.

JANSEN, Act. Bot. Neerl. 1 (1952) 479-483.Critical notes; key to group of $T$. gigantea.

Thysanolaena: JANOWsKI, in Mez, Bot. Arch. 1 (1922) 21-28.-Revision; key.

Triodia: BurbidGe, Austr. J. Bot. 1 (1953) 121184.-Descr.; key.

Tripsacum: Curler \& ANDERson, Ann. Missouri Bot. Gard. 28 (1941) 249-269.-Prelim. survey.

Vulpia: Bor, J. Bombay Nat. Hist. Soc. 50 (1951) 340-343.-Rev. 3 Ind. spp.; key.

Xerochloa: Diels, Bot. Jahrb. 35 (1905) 64-68.

Zoysia: HubBard, in Hook. Ic. Pl. V, 3 (1935) t. 3264, 1-4.-Critical notes. 


\section{BAMBUSACEAE}

Blatter, E. (\& R. N. Parker), The Indian bamboos brought up to date (Ind. For. 55, 1929, 541-562, 586-613).

Buse, L. H., Trib. Bambusaceae (in MiQueL, Plant. Jungh. 1854, 386-394).

Camus, E. G., Les Bambusées. Monographie. Paris (1913) 215 pp.

GAmble, J. S., The Bambusaceae of British India (Ann. R. Bot. Gard. Calc. 7, 1896, xvii +133 pp.).

McClure, F. A., The bamboo genera Dinochloa and Melocalamus (Kew Bull. 1936, 251-254).Generic distinction.

MunRo, W., A monograph of the Bambusaceae, including descriptions of all the species (Trans. Linn. Soc. Lond. 26, 1866, 1-157, t. 1-6).

\section{Local}

Malay Peninsula: Holtrum, J. Arn. Arb. 27 (1946) 340.-Classification Malayan genera; key. —, Gard. Bull. Sing. 11 (1947) 296.-Some new comb.

—, Kew Bull. (1953) 493-496.-New Schizostachyum.

RIDLEY, J. Str. Br. R. As. Soc. no 23 (1891) 32-33.-Listed records; no new spp.; no key. - , Mat. Fl. Mal. Pen. (Monocot.) 3 (1908) 182-197.-Keys.

Java: BACKeR, Handb. Fl. Java pt 2 (1928) 260289.-With valuable keys for sterile and fertile material; cult. spp. are included; in Dutch.

Borneo: Stapf, J. Linn. Soc. Lond. Bot. 42 (1914) 189-191.-2 new spp.

Philippines: Brown \& Fischer, Bull. Bur. For. Philip. Isl. no 22 (1920) 249-310.-Keys.

Gamble, Philip. J. Sc. 5 (1910) Bot. 267-281.Revision; keys.

—, ibid. 6 (1911) Bot. 289.-New sp.

-, ibid. 8 (1913) Bot. 203-206.-Additions; no key.

MerRILl, Amer. J. Bot. 3 (1916) 58-64.-Identity of BLANCO's spp.

New Guinea: Camus, J. Arn. Arb. 9 (1928) 144 145.-New spp..

Chase, J. Arn. Arb. 20 (1939) 304-305.-Records. 一, ibid. 24 (1943) 77-78.-Ditto.

Pilger, Bot. Jahrb. 52 (1914) 172-176.-Descr.

RidLey, J. Bot. 24 (1886) 360.-New Dendrocalamus.

\section{Genera}

Arundinaria: Gamble, Kew Bull. (1912) 135-140. -Key to Sikkim Himal. spp.

NakAI, J. Arn. Arb. 6 (1925) 141-153.-Segregation of the genus.

Bambusa: McClure, Blumea Suppl. 3 (1946) 90-112.

Chloothamnus (Oreiostachys): HenRard, Blumea 2 (1936) 60-73.

Melocanna: Parkinson, Ind. For. 61 (1935) 325327.-New name for $M$. humilis.

Schizostachyum: MCClure, Lingn. Sc. J. 14 (1935) 575-602.-Chin. spp. $\rightarrow$, Lingn. Sc. J. 15 (1936) 301-304.-Revised descr.

-, Blumea 2 (1936) 86-96.-Typif.; new Jav. sp.

\section{GUTTIFERAE \\ (incl. Hypericaceae)}

Chorsy, J. D., Description des Guttifères de l'Inde, etc. (Mém. Soc. Phys. Hist. Nat. Genève 122, 1851 ; 381-440, t. 1-4).-Treats WALLICH coll. and some Amer. spp.; synopsis of genera; no keys to spp.

Engler, A. (\& R. Keller): Pflanzenfamilien ed. 2,21 (1925) 154-237.

Planchon, J. E. \& J. Triana, Mémoire sur la amille des Guttifères (Ann. Sc. Nat. Bot. IV, 13, $1860,306-376 ; 14,1860,226-367 ; 15,1861$, $240-319 ; 16,1862,263-308$; reprint 1862 , iv + 336 pp.).

-, Sur la famille des Guttifères (Bull. Soc. Bot. Fr. 8, 1861, 26-29, 66-73, 96-100).

VESQUE, J., Epharmosis sive materiae ad instruendam anatomiam systematis naturalis II. Genitalia foliaque Garciniearium et Calophyllearum (1889) 1-30, t. 1-162.-Revision of Garcinia \& Calophyllum on an anatomical basis.

-, in DC. Mon. Phan. 8 (1893) 1-669.

\section{Local}

Borneo: Corner, Gard. Bull. Str. Settl. 10 (1939) 36-38.-Notes on some Malay Garcinias.

RidLey, Kew Bull. (1938) 115-123.-New spp.

Philippines: Elmer, Leafl. Philip. Bot. 3 (1911) 1047-1055.-New Garcinia from Sibuyan.

—, Leafl. Philip. Bot. 10 (1939) 3744-3748.New spp.

Merrill, Philip. J. Sc. 3 (1908) Bot. 359-368.Philip. Garcinia; key.

—, Philip. J. Sc. 26 (1925) 472-475; 27 (1925) 37. -New Garcinias.

New Guinea: Diels, Bot. Jahrb. 62 (1929) 482483.-New Hypericum.

LAUterbach, Nova Guinea 8 (1910) 309-312. -Records; new Garcinias.

一, ibid. 8 (1912) 843-844.-Records; Nouhuysia n. $g$.

-, Bot. Jahrb. 58 (1923) 1-49.-Rev.; keys.

-, ibid. 61 (1927) 31.-New Garcinia.

Smith, J. Arn. Arb. 22 (1941) 343-374.-No keys, also some Melanesian spp.

Micronesia: Lauterbach, Bot. Jahrb. 59 (1924) 18-23.

\section{Genera}

Cratoxylon: Corner, Gard. Bull. Str. Settl. 10 (1939) 21-36.-Critical notes.

Gagnepain, Not. Syst. 1 (1909) 14-22.-Attempt to classify the Asiat. spp.

Korthals, in Temminck, Verh. Nat. Gesch. Bot. (1842) 175-180.

Garcinia: PIERRE, Fl. For. Cochinch. 1 (1883) i-xlii, t. 54-92.-Monograph; no keys.

Hypericum: Keller, Bull. Herb. Boiss. II, 8 (1908) 175-191.-Rev, sect. Brathys.

Van Steenis, Bull. Jard. Bot. Btzg III, 13 (1934) 219-220.-List of Mal. spp.

Nouhuysia $=$ Sphenostemon (Aquifoliaceae). 
Ochrocarpus: Merrill, J. Arn. Arb. 26 (1945) 93-96.-Syn. of $O$. odoratus with a new species from Samoa.

Pentaphalangium: SмIтн, J. Arn. Arb. 22 (1941) 370-374.-No key.

Tetrathalamus $=$ Bubbia (Winteraceae)

\section{HAEMODORACEAE}

Pax, F., Pflanzenfamilien ed. 2, 15a (1930) 386390.

Steenis, C. G. G. J. van, Fl. Mal. I, 5 (1954) 111113 , fig. 1.

\section{Local}

New Guinea: Hallier $f$., Nova Guinea 8 (1913) 902.-Record of Haemodorum.

Van Steenis, J. Arn. Arb. 28 (1947) 420.Ditto.

\section{HALORAGACEAE}

Schindler, A. K., Pflanzenreich Heft 23 (1905) 1-133, fig. 1-36.

Steenis, C. G. G. J. van, Bull. Jard. Bot. Btzg III, 13 (1934) 217-218.-List.

\section{Local}

New Guinea: Grbas, Contr. Fl. Phytog. Arfak Mts (1917) 159.

MANSFELD, Bot. Jahrb. 61 (1927) 26-27.-No keys.

MATTFELD, Bot. Jahrb. 69 (1938) 275-277.New sp. of Myriophyllum § Pentapteris §\$ Pelonastes.

Merrill \& Perry, J. Arn. Arb. 23 (1942) 407408.-New records.

- J. Arn. Arb. 29 (1948) 161-162.

WENT, Nova Guinea 14 (1924) 105-109.-New spp. of Haloragis.

\section{Genera}

Laurembergia: VAN SteEnIS, Arch. Hydrobiol. Suppl. 3 (1932) 315-316, fig. 5.-Account of the 2 Mal. spp.

\section{HAMAMELIDACEAE}

HaRms, H., Pflanzenfamilien ed. 2, 18a (1930) 305-345.

LecomTe, H., Hamamelidacées du Haut-Tonkin (Bull. Mus. Hist. Nat. Paris 30, 1924, 390-393).

Steenis, C. G. G. J. van, Bull. Jard. Bot. Btzg III, 13 (1934) 218.-List mountain genera.

\section{Local}

Malay Peninsula: Symington, J. Mal. Br. R. As. Soc. 14, pt 3 (1936) 351.-Records.

Philippines: Hemsley, in Hook. Ic. Pl. IV, 9 (1907) t. 2836.-New Sycopsis.

\section{Genera}

Distylium: Walker, J. Arn. Arb. 25 (1944) 322335.-Revision with key.

Embolanthera: MerriLl, Philip. J. Sc. 4 (1910) Bot. 263-264.-Palawan, monotypic.

Exbucklandia (Bucklandia R. BR. non PR., Symingtonia): vAN STEENIS, Acta Bot. Neerl. 1 (1952) 443-444.-Nomencl. note.
-, Blumea 7 (1954) 595.-Ditto.

Rhodoleia: ExelL, Sunyatsenia 1 (1933) 95-102. -Revision with key.

Sycopsis: WALKER, J. Arn. Arb. 25 (1944) 335341.-Revision with key; first record in New Guinea!

\section{HERNANDIACEAE}

Blume, C. L., Illigereae (in HoeVen \& DE VRIESE, Tijd. Nat. Geschied. \& Phys. 1, 1834, 142-147). -Census of Illigera \& Gyrocarpus; spp. listed; in Latin.

\section{Local}

Malay Peninsula : CoRNer, Wayside trees of Malaya (1940) 323.

New Guinea: Pulle, Nova Guinea 8 (1911) 639.

\section{Genera}

Hernandia: Tuyama, Bull. Sigenkagaku Kenkyusyo 1 , no 1 (1943) 41-44.-H. javanica, $H$. sono$r a$, and a new sp. from the Marianes.

Illigera: DunN, J. Linn. Soc. Lond. Bot. 38 (1908) 290-297.-Revision.

Miquel, Ann. Mus. Bot. Lugd.-Bat. 2 (1866) 213-215.-Revision Mal. spp.; no key.

Wu, Bot. Jahrb. 71 (1941) 177.-Key to $6 \mathrm{~S}$. Chin. spp.

\section{HIMANTANDRACEAE}

Bailey, I. W., Nast, \& A. C. SMith, The family Himantandraceae (J. Arn. Arb. 24, 1943, 190206).-Revision; key to the 2 spp. of Himantandra.

SMith, A. C., A nomenclatural note on the Himantandraceae (J. Arn. Arb. 23, 1942, 366-368). - List of $s p p$.

\section{Local}

New Guinea : Diels, Bot. Jahrb. 49 (1913) 164-165, fig. 6.-Under Annon.

—, ibid. 52 (1914) 185-186.-Emended descr.

\section{Genera}

Himantandra (Galbulimima): DieLs, Bot. Jahrb. 55 (1918) 126-143, 1 fig.

\section{HIPPOCRATEACEAE}

LOESENER, TH., Pflanzenfamilien ed. 2, $20 \mathrm{~b}$ (1942) 198-231.

Mrquel, F. A. W., Hippocrateaceae Archipelagi Indici (Ann. Mus. Bot. Lugd.-Bat. 4, 1869, 148-154).

Smrth, A. C., The American species of Hippocrateaceae (Brittonia 3, 1940, 341-555, fig. 1-12).Revision; key to genera and spp.; notes on Old World genera.

- Notes on Old World Hippocrateaceae (Amer. J. Bot. 28, 1941, 438-443).-Loeseneriella, Pristimera, Salacia, Salacicratea, and Dicarpellum; many new comb.

-, Notes on Hippocrateaceae in Southeastern Asia (J. Arn. Arb. 26, 1945, 169-179).-Asiat. spp. of Pristimera \& Loeseneriella; keys. 
TARDIEU-BLot, Hippocrateaceae nouvelles ou litigieuses d'Indochine (Bull. Soc. Bot. Fr. 95, 1948, 261-265).

\section{Local}

Borneo: RidLEY, Kew Bull. (1938) 237-242.-New spp. in Hippocratea and Salacia.

Banka: SCHEFfER, Nat. Tijd. Ned. Ind. 31 (1870) 16-17.-New Salacia.

New Guinea: Loesener, Nova Guinea 8 (1910) 281-282.

\section{Genera}

Brassiantha: SmITH, A. C., \& Balley, J. Arn. Arb. 22 (1941) 389-394.-New Guinea.

Hippocratea: RoLfe, Kew Bull. (1918) 47-48.Critical note on a few spp.

Loeseneriella: Smith, A. C., J. Arn. Arb. 26 (1945) 169-175.-SE. Asiat. genus; possibly also in Malaysia.

Pristimera: Smith, A. C., J. Arn. Arb. 26 (1945) 175-179.

Salacicratea: HARMS, Notizbl. Berl.-Dahlem 15 (1942) 676-677.-New spp. from N. Guinea \& Celebes.

LOESENER, Bot. Jahrb. 63 (1930) 275.-From Melanesia \& Micronesia.

Smith, A. C., Am. J. Bot. 28 (1941) 441-442.

\section{HYDR OCAR YACEAE}

\section{(Trapaceae)}

Fleroff, A. Th., Genus Trapa L., seine Verbreitung und systematische Uebersicht (Bull. Jard. Bot. Rép. Russ. 24, 1925, 13-45, in Russian). (cf. Bot. Centralbl. 151, 1927, 260.)

Steenis, C. G. G. J. VAN, Fl. Mal. I, 4 (1949) 43-44, fig. 1.

\section{HYDROCHARITACEAE}

Ascherson, P., Vorarbeiten zu einer Ubersicht der phanerogamen Meeresgewächse (Linnaea 35, 1867, 152-208).-Conspectus; no key.

ERNST-SChWARZENBACH, M., Zur Blütenbiologie einiger Hydrocharitaceen (Ber. Schweiz. Bot. Ges. 55, 1945, 33-69, t. 1-2).-On Hydrilla verticillata and some others.

Miquel, F. A. W., Illustr. Fl. Arch. Ind. (1870) 51-56.-No key.

OStenfeld, C. H., Pflanzenareale $1^{3}$ (1927) 35-38, Karten 21-24.-Distr. of marine genera.

\section{Local}

Malay Peninsula: Ridley, Mat. Fl. Mal. Pen. (Monocot.) 1 (1907) 3-6.-Keys.

Java: BACKER, Handb. Fl. Java pt 1 (1925) 57-64. -Keys; in Dutch.

Celebes: Hallier $f$., Meded. Rijksherb. Leiden no 26 (1915) 7-8.-Boottia mesenterium.

New Guinea: Graebner, Bot. Jahrb. 49 (1912) 68-69.-Vallisneria gigantea.

HALliER, Nova Guinea 8 (1913) 915-917.-2 new Hydrocharis, record of Vallisneria.

SOLEREDER, Meded. Rijksherb. Leiden no 21 (1914) 2 pp.-Anat. new Pap. spp.

\section{Genera}

Blyxa: van SteEnis, Trop. Natuur 18 (1929) 197-200, fig. 1-3.-Distr. 2 Mal. spp.

Boottia: Dandy, J. Bot. 70 (1932) 328.-Reduct. of Hydrocharis renifolia.

GürRKE, Festschr. ASCHERSON (1904) 533-547. -Notes on trop. Afr. spp. of Boottia \& Ottelia. Merrill \& MetCalf, Lingn. Sc. J. 17 (1938) 567-570.-New Chin. sp.

Echinodorus (Ranalisma): van SteEnis, Arch. f. Hydrobiol. Suppl. 9 (1932) 240, footnote 3.Nomencl.

En(h)alus: Troll, Planta 125 (1931) 427-456, 15 fig.-Biology.

Halophila: BALfour, Trans. Bot. Soc. Edinb. 13 (1878) 290-343, pl. 8-12.-Mainly morphol. Ostenfeld, Philip. J. Sc. 4 (1909) Bot. 67-68.H. ovata.

SeTChell, Amer. Midl. Nat. 69 (1935) 560-577. -Geogr. distr.

Hydrocharis: Hallier $f$., Nova Guinea 8 (1913) 916-917).-2 new spp.; survey Mal. spp.

Ottelia (Boottia, Oligolobos): DANDY, J. Bot. 72 (1934) 132-139.-Revision; list of spp.; no key; Boottia reduced.

-, ibid. 73 (1935) 209-217, fig. 1.-Ottelia in China; key.

GÜRKE, Festschr. AsChERSON (1904) 533-547.Notes on trop. Afr. spp. of Boottia \& Ottelia.

\section{HYDROPHYLLACEAE}

BACKer, C. A., Fl. Mal. I, 4 (1952) 207-209, 1 fig. Cholsy, J., Description des Hydroléacées (Mém. Soc. Phys. \& Hist. Nat. Genève 6, 1833, 95-122, t. 1-3).-Revision; descr.; no key.

Bentham, G., Review of the Order of Hydrophylleae (Trans. Linn. Soc. 172, 1835, 267-282).Descr. of genera and spp.; no key.

Brand, A., Pflanzenreich Heft 59 (1913) 1-210, fig. 1-39.

\section{Local}

Java: BACKer, Onkruidfl. Jav. Suiker. (1931) $532-534$

\section{Genera}

Hydrolea (Nama): Hirchcock, Amer. J. Bot. 20 (1933) 415-430.-Taxonomy.

Nemophila: Constance, Univ. Calif. Publ. Bot. 19 (1941) 341-398.-Rev.: key.

Phacelia: Howell, Proc. Cal. Ac. Sc. IV, 25( 1944) 357-376.-Revision sect. Miltitzia; key.

\section{HYPERICACEAE See Guttiferae \\ HYPOXIDACEAE \\ See Amaryllidaceae \\ ICACINACEAE \\ (Peripterygiaceae)}

BaIllon, H. E., Deuxième étude sur les Mappiées (Adansonia 10, 1872, 261-282; 11, 1875, 239$273,292-312,366-373 ; 12,1876-79,220-254$, 282-296). 
BecCARI, O., Malesia 1 (1877) 105-134, t. 3-8, (1878) 255-256, (1883) 257.-Complete list of Mal. spp. with descr. of new spp.

Howard, R. A., Studies of the Icacinaceae. Preliminary taxonomic notes (J. Arn. Arb. 21, 1940, 461-488).-Crit. notes; key to genera.

-. Studies of the Icacinaceae II (ibid. 23, 1942, 55-78).-Mostly extra-Mal. genera.

- Studies of the Icacinaceae IV. Consideration of the New World genera (Contr. Gray Herb. $142,1942,3-60$ ).

-; Studies of the Icacinaceae VIII (Lloydia 6, 1943, 144-154).-Descr. new spp.

Sleumer, H., Beiträge zur Kenntnis der Icacinaceen und Peripterygiaceen (Notizbl. Berl.Dahlem 15, 1940, 228-257).

—, Pflanzenfamilien ed. 2, $20 \mathrm{~b}$ (1942) 322-396. '

\section{Local}

Borneo: Merrill, J. Str. Br. R. As. Soc. no 86 (1922) 325-326.-New Phytocrene.

Stapf, Kew Bull. (1906) 71-72.-New spp.

Philippines: Elmer, Leafl. Philip. Bot. 10 (1939) 3748-3750.-New spp.

MerriLl, Philip. J. Sc. 26 (1925) 467-468.New Mappia.

New Guinea: BIRNIE, Nova Guinea 14 (1927) 275277.

ENGLER, Bot. Jahrb. 16 (1893) Beibl. 39, p. 13. -New Polyporandra.

Pulle, Nova Guinea 8 (1912) 657-660.

RidLeY, Trans. Linn. Soc. Lond. II, Bot. 9 (1916) 27-30.-3 New genera.

SCHellenBerg, Bot. Jahrb. 58 (1923) 155-177.Keys to spp.

Micronesia: Schellenderg, Bot. Jahrb. 59 (1924) 17.-New Urandra from Palau.

\section{Genera}

Cantleya: Howard, J. Arn. Arb. 21 (1940) 479 481.

Cardiopteris (Peripterygium): Wall. ex RoYle, Illustr. Bot. Him. Mts (1834) 136.-Short descr. BLume, Rumphia 3 (1849) 206, t. 177.-As a distinct family Cardiopterideae.

Hasskarl, Tijd. Nat. Geschied. \& Phys. 10 (1843) 142; Cat. Hort. Bog. (1844) 234-235; Retzia 1 (1855) 64-65.

BeCCARI, Nuov. Giorn. Bot. Ital. 9 (1877) 100108, t. 8.-Emended descr.

SLeumer, Pflanzenfamilien ed. 2, 20b (1942) 397-400.-As a distinct family Peripterygiaceae.

Citronella (Villaresia, Chariessa, Pleuropetalon): Howard, J. Arn. Arb. 21 (1940) 471-479.

-, Contr. Gray Herb. 142 (1942) 60-92.Revision; keys.

Codiocarpus: Howard, Brittonia 5 (1943) 53-57.

Gonocaryum: Scheffer, Ann. Jard. Bot. Btzg 1 (1876) 96-102.

Lophopyxis: Holthuis \& LAM, Blumea 5 (1942) 205-208.

Medusanthera (Lasianthera, Tylecarpus, Stemonurus p.p.): Howard, J. Arn. Arb. 21 (1940) 469-471.

-, Lloydia 6 (1943) 134-143.-Revision; key.
Merrilliodendron (Peekeliodendron): KANEHIRA, Bot. Mag. Tokyo 48 (1934) 920-921.

SLEUMER, Notizbl. Berl.-Dahlem 13 (1937) 509512.

Nothapodytes: Howard, J. Arn. Arb. 23 (1942) 66-73.-Key.

Phytocrene: Miquel, Ann. Mus. Lugd.-Bat. 3 (1867) 247-248.

Polyporandra: Koorders, Versl. Gew. Verg. Akad. Wet. A'dam 27 Febr. 1909, vol. 17, 780-782, Proc., p. 763-765.

Pseudobotrys: MOESER, in Fedde, Rep. 10 (1912) 310-311.

Urandra (Stemonurus p. p.): HowARd, J. Arn. Arb. 21 (1940) 462-469.

ThwaItes, in Hook. J. Bot. \& Kew Gard. Misc. 7 (1855) 211-212.

\section{ILLICIACEAE}

SmIth, A. C., The families Illiciaceae and Schisandraceae (Sargentia 7, 1947, 1-224, 41 fig.).Revision; key!

\section{IRIDACEAE}

BAKER, J. G., Handbook of the Irideae. London 1892.

Diels, L., Pflanzenfamilien ed. 2, 15a (1930) 463-505.

Steenis, C. G. G. J. van, Bull. Jard. Bot. Btzg III, 13 (1934) 220.-List mountain spp.

\section{Local}

Sumatra: Merrill, Not. Nat. Acad. Nat. Sc. Philad. no 47 (1940) 2.

Java: BACKER, Handb. Fl. Java 3 (1924) 116-130. -Also cult. spp.; keys; in Dutch.

Borneo: STAPF, Trans. Linn. Soc. Lond. II, Bot. 4 (1894) 241-242.

New Guinea: Diels, Bot. Jahrb. 62 (1929) 462. GibBs, Contr. Fl. Phytog. Arfak Mts (1917) 101. VoN Mueller, Trans. R. Soc. Victoria pt 2 (1889) 34.

WENT, Nova Guinea 14 (1924) 114.

\section{Genera}

Crocus: MAw, A monograph of the genus Crocus. London (1886) 1-326, xx, index, t. (col.) 1-67.No keys.

Iris: LAWRENCE, Gentes Herb. 8 (1953) 346-371.Reclassification; sections.

Neomarica (Marica): SpraGUE, Kew Bull. (1928) 278-281.

Sisyrinchium: Bicknell, Bull. Torr. Bot. Club 2628 (1899-1901).-Segregation; keys; no complete monograph.

\section{IRVINGIACEAE \\ See Simaroubaceae}

\section{JUGLANDACEAE}

\section{Local}

Java: Koorders \& VAleton, Bijdr. Booms. Java 5 (1900) 161-177.

Philippines: Elmer, Leafl. Philip. Bot. 9 (1934) 3194-3197.-Two new spp. 
Quisumbrng, Philip. J. Sc. 76 (1944) 37.

New Guinea: SchlechteR, Bot. Jahrb. 50 (1913) 66-67.

\section{JUNCACEAE}

BaCker, C. A., Fl. Mal. I, 4 (1951) 210-215, fig. 1-2.

Buchenau, F. G, PH., Monographia Juncacearum (Bot. Jahrb. 12, 1890, 1-495, t. 1-3, fig. 1-9).-Monogr.; keys.

-, Pflanzenreich Heft 25 (1906) 1-284.

Steenis, C. G. G. J. van, Bull. Jard. Bot. Btzg III, 13 (1934) 220-221.-List.

STEudel, E. G., Enumeratio plantarum Glumacearum pars II. Stuttgart (1855) 290-314.Enum. descr. in Latin.

VIERHAPPER, F., Pflanzenfamilien ed. 2, 15a (1930) 192-224.

\section{Local}

India: BuchenAu, Bot. Jahrb. 6 (1885) 187-232, t. 2-3.-Revision, specially Himal. spp.; keys. New Guinea: Diels, Bot. Jahrb. 62 (1929) 461-462.

\section{Genera}

Juncus: RidLeY, J. Bot. 73 (1935) 342-343.-Spp. of Java \& Sumatra.

\section{JUNCA GINACEAE}

(Scheuchzeriaceae)

Buchenau, F. G. PH., Pflanzenreich Heft 16 (1903) 1-20, fig. 1-9.

Steenis, C. G. G. J. vaN, Fl. Mal. I, 4 (1949) 5657, fig. 1.

\section{Local}

New Guinea: van Steenis, J. Arn. Arb. 28 (1947) 419.

\section{LABIATAE}

Bentham, G., Labiatarum genera et species: etc. (1832-36) lxviii + 783 pp.

BrIQUET, J., Fragmenta Monographiae Labiatarum I (Bull. Soc. Bot. Genève 5, 1888, 20-122); II (Bull. Herb. Boiss. 2, 1894, 119-141); III (ibid. 689-724); IV (ibid. 4, 1896, 676-696, 762808, 847-878); V (Ann. Conserv. Jard. Bot. Genève $2,1898,102-251$ ).

DuNN, S. T., A key to the Labiatae of China (Not. R. Bot. Gard. Edinb. 6, 1915, 127-190),-Keys to genera \& spp.

Eplng, C., Synopsis of the South American Labiatae (in FEDDE, Rep. Beih. 85, 1935-1937, 1935, 1-96, 1936, 97-288, 1937, 289-341).

JuNELL, S., Zur Gynäceummorphologie und Systematik der Verbenaceen und Labiaten, etc. (Symb. Bot. Uppsala 1, no 4, 1934, 1-219, fig. 1-257).-Delimitation of the families on the basis of $\&$ morph.; result strongly deviating from current opinion.

MUKherJee, S. K., A revision of the Labiatae of the Indian Empire (Rec. Bot. Surv. India 14, 1940, viii +228 pp.).

PraIN, D., Noviciae Indicae III. Some additional species of Labiatae (J. As. Soc. Bengal 59, ii, 1890, 294-318).-Add. to Fl. Br. Ind.
Steenis, C. G. G. J. van, Bull. Jard. Bot. Btzg III, 13 (1934) 221-223.-List mountain plants.

\section{Local}

Sumatra: Merrill, Contr. Arn. Arb. 8 (1934) 148-149.

-, Pap. Mich. Ac. Sc. Arts \& Lett. 19 (1934) 191-194.-New spp.

Malay Peninsula: FurTado, Gard. Bull. Str. Settl. 4 (1929) 415.-Key of Hyptis in Mal. Pen.

-, ibid. 4 (1929) 416-419.-Account of Ocimum; key.

PranN, Kew Bull. (1908) 114-115.-Acrymia n.g.; monotypic.

RIDLEY, J. Str. Br. As. Soc. no 50 (1908) 105-108. -Survey.

Philippines: Elmer, Leafl. Philip. Bot. 9 (1934) 3197.-New Pogostemon.

Prain, Kew Bull. (1908) 116.-New Pogostemon.

New Guinea: Mansfeld, Bot. Jahrb. 62 (1921) 376-381.-Full account; keys.

\section{Genera}

Achyrospermum: BruCE, Kew Bull. (1936) 47-60. -Afr. spp.; key.

Ceratanthus: TAYLOR, J. Bot. 74 (1936) 33-41.Survey.

Cymaria (Anthocoma): PraIN, Ann. Bot. 6 (1892) 214-217.-Syn. of C. acuminata.

Gomphostemma: PraIN, Ann. R. Bot. Gard. Calc. 3 (1891) 228-273, t. 75-105.-Revision; key.

Hyptis: EplING, Kew Bull. (1936) 278-280.-List distr. of Old World spp.

_, Rev. Mus. La Plata n. s., 7. Bot. (1949) 153497, fig. 1-42 (maps).-Complete revision; keys; in Span.

Lavandula: ChaYtor, J. Linn. Soc. Lond. Bot. 51 (1937) 153-204.-Revision; keys.

GrEeN, Kew Bull. (1932) 295-297.-Nomencl.

Microtoena: Prain, Bull. Soc. Bot. Fr. 42 (1895) 417-427.-Revision; key.

Pogostemon: PraIN, Kew Bull. (1908) 78-82.$P$. cablin (patchouly).

Salvia \& Calosphace: Epling, in Fedde, Rep. Beih. 110 (1938-1939) 1-380.

\section{LAURACEAE}

Allen, C. K., Studies in the Lauraceae. I. Chinese and Indo-Chinese species of Litsea, Neolitsea, and Actinodaphne (Ann. Missouri Bot. Gard. 25, 1938, 361-434).

-, Ditto. V. Some Eastern Asiatic species of Beilschmiedia and related genera (J. Arn. Arb. 23, 1942, 444-463.)-Lauromerrillia $n$. $g$.

Gamble, J. S., New Lauraceae from the Malayan region (Kew Bull. 1910, 142-153, 253); II (ibid. 218-228); III (ibid. 312-321); IV (ibid. 357-368). -New spp. from the Mal. Pen., also some from New Guinea \& Borneo. No key.

Kostermans, A. J. G. H., Studies in South American Malpighiaceae, Lauraceae and Hernandiaceae, especially of Surinam. Thesis Utrecht, (1936). 70 pp. (Repr. in Med. Bot. Mus. en Herb. 
Univ. Utrecht no 25).-Contains also notes on Mal. Laur.

-, Revision of the Lauraceae I (Rec. Trav. Bot. Néerl. 33, 1936, 719-757).-On American genera.

-, ditto II (ibid. 34, 1937, 500-609).-Ditto.

-, A historical survey of Lauraceae (J. Sc. Res. Indon. 1, 1952, 83-95, 113-127, 141-159).Chronol. review; many new comb.; index to scientific names.

LeComte, H., Lauracées de Chine et d'Indo-Chine (Nouv. Arch. Mus. Hist. Nat. V, 5, 1913, 43120, t. 3-9).-No keys.

Liou, Ho, Contribution à l'étude systématique et phytogéographique des Lauracées de Chine et d'Indochine. Thèse, Paris (1932) 1-207.-Monographic; descr. and keys.

Meissner, K. F., Lauraceae (in DC. Prod. 15, 1, 1864, 1-260).

Mez, C., Lauraceae Americanae monographice descriptae (Jahrb. Kön. Bot. Gart. Berlin 5, $1889,1-556)$.

-, Spicilegium Laureanum (Arb. aus den Kgl. Bot. Gart. Breslau 1, Heft 1, 1892, 71-166).Georg. distr. of genera; additions to his monogr. on the Amer. Laur.

NakAI, T., On the Japanese species of the tribe Litseae of Lauraceae ( J. Jap. Bot. 14, 1938, 117-195).

\section{Local}

Sumatra: KURz, Flora 55 (1872) 171-172.-Disc. Litsea rugosa.

Merrill, Contr. Arn. Arb. 8 (1934) 61-65. RIDLEY, J. Str. Br. R. As. Soc. no 87 (1923) 8990.-New sp.

—, Kew Bull. (1926) 79.

Malay Peninsula: Corner, Gard. Bull. Str. Settl. 10 (1939) 276-278.-Cinnamomum, Lindera \& Phoebe spp.

Henderson, Gard. Bull. Str. Settl. 7 (1933) 120

121, t. 31.-New Endiandra.

Symington, Kew Bull. (1937) 319.-Reduction of Alseodaphne crassipes.

Borneo: Merrill, J. Str. Br. R. As. Soc. no 85 (1922) 191-197.-New spp.

AIRY Shaw, Kew Bull. (1939) 434-438 (sphalm. 534-538).-Also new spp.

Philippines: Elmer, Leafi. Philip. Bot. 2 (1908) 375-384.-Descr. new spp.; no key.

一, ibid. 2 (1910) 703-728.-Ditto.

一, ibid. 10 (1939) 3750-3757.-Ditto.

Merrill, Philip. J. Sc. 1 (1906) Suppl. 56-58.

-, ibid. 12 (1917) Bot. 125-141.-New spp.

一, ibid. 27 (1925) 23.-Two new spp.

Quisumbing, Philip. J. Sc. 76 (1944) 40-41.-

Two new spp.

- \& MERRILL, ibid. 37 (1928) 146-149.-Three new spp.

WhIte, Kew Bull. (1933) 46.-New name in Cryptocarya.

New Guinea: Allen, J. Arn. Arb. 23 (1942) 112131, 133-155.-Many new spp. and a n.g.; no key. Kanehira \& Hatusima, Bot. Mag. Tokyo 57 (1943) 185-192.-New spp.
Kostermans, Bull. Bot. Gard. Btzg III, 18 (1950) 435-443.-New spp. and critical notes. LAUTERBACH, Nova Guinea 8 (1912) 819-820. TesChNer, Bot. Jahrb. 58 (1923) 380-440.Keys.

—, ibid. 58 (1924) 492-495.-New spp. of Cinnamomum; no key.

Solomon Islands: WHITE, J. Arn. Arb. 31 (1950) 83-86.

\section{Genera}

Caryodaphnopsis: AIRY SHaw, Kew Bull. (1940) 74-76.-Segregate of Notaphoebe to which added a Philippine 'Persea'. 一, in Hook. Ic. Pl. 35 (1943) t. 3436.

Cinnamomum (Camphorina): Allen, J. Arn. Arb. 20 (1939) 44-62.-Crit. notes on Asiat. spp. Blume, Rumphia 1 (1835) 25-45.

Cammerloher, Bull. Jard. Bot. Btzg III, 7 (1924) 446-497.-Neth. Ind. spp.; key.

Merrill, Bot. Gaz. 70 (1920) 84-85.-Reduction Camphorina.

Miquel, Ann. Mus. Bot. Lugd.-Bat. 1 (1864) 254-270, 317.

Dehaasia: BLume, Rumphia 1 (1835) 161-164.

Dryadodaphne $=$ Levieria (Monimiaceae), cf. KosTERMANS, Rec. Trav. Bot. Néerl. 34 (1937) 605.

Eusideroxylon: Symington, in Hook. Ic. Pl. 35 (1940) t. 3409.-Second sp. from Borneo.

Teysmann \& BinnendiJK, Nat. Tijd. Ned. Ind. 25 (1863) 289-294.-Orig. descr.

De WIT, Bull. Bot. Gard. Btzg III, 18 (1949) 200-208.-Revision.

Lindera: Allen, J. Arn. Arb. 22 (1941) 1-31.Critical notes; no key.

Neocinnamomum: Allen, J. Arn. Arb. 20 (1939) 62-63.-Chin. spp.

LIoU, in Lauracées de Chine et d'Indochine. 1932.

Merrill, Contr. Arn. Arb. 8 (1934) 64.-Sumatra record.

Neolitsea (Bryantea RAFIN., Tetradenia NEES, non BTh.): Gamble, Kew Bull. (1911) 172.

Merrill, Philip. J. Sc. 1 (1906) Suppl. 56-57. —, J. Arn. Arb. 29 (1948) 198-201.

Persea: Blake, J. Wash. Acad. Sc. 10 (1920) 9. AIRy Shaw, in Hook. Ic. Pl. 35 (1943) t. 3472 (\& 3436).

Pseudocryptocarya: Teschner, Bot. Jahrb. 58 (1923) 411-413.-New Guinea.

Stemmatodaphne: Gamble, in Hook. Ic. Pl. IV, 10 (1913) t. 2984.-Mal. Pen.; aff. Phoebe. AIRY Shaw, Kew Bull. (1940) 76-77.

\section{LECYTHIDACEAE}

\section{(Barringtoniaceae)}

Blume, C. L., Notice sur quelques Barringtoniées de l'Archipel des Indes (Flore des Serres 7, 1851, 21-25).

Knuth, R., Pflanzenreich Heft 105 (1939) fam. 219a, 82 pp.

Korthals, P. W., Bijdrage tot de kennis der Myrtaceae (Ned. Kruidk. Arch. 1, 1846, 205-206).

Miers, J., On the Lecythidaceae (Trans. Linn. Soc. Lond. $30,1874,157-318$ ). 
-, On the Barringtoniaceae (Trans. Linn. Soc. Lond. II, Bot. 1, 1875, 47-118, t. 10-18).Monographic; key to the genera; no keys to spp. WhITE, C. T., A revised account of the Queensland Lecythidaceae (Proc. Linn. Soc. N. S. Wales 44, 1920, 822-825).-No key.

\section{Local}

Java: BACKER, Schoolfl. Java (1911) 527-530.In Dutch; key.

Borneo: Merrill, J. Str. Br. R. As. Soc. no 77 (1917) 204-206.-Two new spp. of Barringtonia. Ridley, Kew Bull. (1938) 284-285.-Three new spp.

AIRY Shaw, Kew Bull. 1 (1950) 137-138.New sp.

New Guinea: Lauterbach, Nova Guinea 8 (1910) 313-315.

-, ibid. 8 (1912) 845.

-, Bot. Jahrb. 57 (1922) 341-353.-Keys.

Merrill \& Perry, J. Arn. Arb. 21 (1940) 292296.-New spp.; no key.

Melanesia: LAUTERBACH, Bot. Jahrb. 45 (1911) 362-363.-Two new Barringtonias.

ReCHINGER, in FedDe, Rep. 11 (1912) 183-184. -New Barringtonia.

\section{Genera}

Barringtonia: LütJEHARMS \& VAN OOSTSTROOM, Blumea 3 (1938) 95-105.-Two new Barringtonias. Chydenanthus: AlrY SHAw, Kew Bull. (1949) 152.

Petersianthus (Petersia WELW., non KLoTzSCH): Merrill, Philip. J. Sc. 11 (1916) Bot. 200-201. -Census of the genus under this new name (Africa, Philippines).

\section{LEEACEAE See Vitaceae}

\section{LEGUMINOSAE}

(incl. Caesalpiniaceae, Mimosaceae, Papilionaceae)

Baker J. G., \& D. Oliver, in Fl. Trop. Afr. 2 (1871) 1-364.

BAKER, E. G., The Leguminosae of tropical Africa. 3 pts. London (1926-30) 953 pp.

Bentham, G., Notes on Mimoseae, with a short synopsis of species (in Hook. J. Bot. 4, 1842, 323-418; Hook. Lond. J. Bot. 1, 1842, 318392 , 494-528; $3,1844,82-112,195-226$; 4, $1845,577-622 ; 5,1846,75-108)$.

- Enumeration of Leguminosae indigenous to Southern Asia, etc. (in Hook. Lond. J. Bot. 2, 1843, 423-481, 559-613; ibid. 3, 1844, 338-365; ibid. $7,1848,580-657)$.

-, Synopsis of the Dalbergiae (J. Linn. Soc. Bot. 4, 1860, Suppl. 1-134).

-, Description of some new genera and species of tropical Leguminosae (Trans. Linn. Soc. 25, 1866 , 297-320, t. 33-43).-Mostly African, some Asiat.

- Revision of the suborder Mimoseae (Trans. Linn. Soc. 30, 1875, 335-664, t. 66-70).

Burkart, A., Las Leguminosas Argentinas, ed. 2. Buenos Aires (1952).

Gagnepain, F., Mimosées nouvelles (Not. Syst. 2, 1911, 56-62).-New Mal. spp. of Entada, Adenanthera \& Parkia.
Pellegrin, F., Les Légumineuses du Gabon (Mém. Inst. Et. Centrafr. 1, 1948, 284 pp.).

PraIN, D., Some additional Leguminosae (J. As. Soc. Beng. 66, ii, 1897, 347-518).-Additions to Fl. Br. India; incl. the Malay Peninsula.

Schindler, A. K., Die Desmodiinen in der botanischen Literatur nach LINNE (in FEDDE, Rep. Beih. 49, 1928, 1-371).--Bibl.; no keys and descr.

Steenis, C. G. G. J. vaN, Bull. Jard. Bot. Btzg III, 13 (1934) 223.-List mountain spp.

WIT, H. C. D. DE, Revision of Malaysian Bauhinieae.-The MS of this large monograph has been in the hands of the Keeper of the Herb. Bog. for about 4 years and was intended to be published in Reinwardtia in 1955.

Local

Sumatra: Merrill, Contr. Am. Arb. 8 (1934) 72-79.

Ridley, J. Mal. Br. R. As. Soc. no 87 (1923) 58. -List.

Malay Peninsula: Henderson, Gard. Bull. Str. Settl. 7 (1933) 99-101.-List; one new Bauhinia. Prain, J. As. Soc. Bengal 66, ii (1897) 21-275.

Java: BACKER, Voorl. Schoolfl. Java (1908) 66112.-In Dutch; keys.

-, Schoolf. Java (1911) 289-443.-In Dutch; keys.

—, Bull. Jard. Bot. Btzg III, 2 (1920) 323-324.New sp. of Bauhinia and Crotalaria.

—, Bull. Jard. Bot. Btzg III, 16 (1939) 107-110. -Teyleria n. 8.; Tephrosia n. comb.

-, Bekn. Fl. Java (em. ed.) pt 5 (1941) fam. 118-120.-In Dutch; keys.

—, Blumea 5 (1945) 508-515.-Crit. notes; new spp.

Bentham, in Miquel, Pl. Junghuhn. (1852) 205-270.

HassKarl, Flora (1842) Beibl. 57-114.-New and emend. spp.

—, Tijd. Nat. Gesch. \& Phys. 11 (1844) 49-112.Descr. of Papil.

—, Nat. \& Geneesk. Arch. 2 (1845) 58-71, 183199, 341-361.-New descr. Jav. Papil.

Zol.linger, Nat. \& Geneesk. Arch. Ned. Ind. 3 (1846) 51-82.-Enum.; descr. new spp.

Borneo: Merrill, J. Str. Br. R. As. Soc. no 76 (1917) 84-86.-Records \& new spp.

-, Philip. J. Sc. 11 (1916) Bot. 77-92.-Many new spp. \& records.

一, ibid. 13 (1918) Bot. 73-74.-New Crudia.

-, ibid. 29 (1926) 372-375.-List Banguey Isl.

Miquel, F. A. W., Anal. Bot. Ind. 1 (1850) 7-12 (repr. from Verh. Kon. Ned. Inst. le kl. 3e reeks, vol. 3 ).

Ridley, Kew Bull. (1929) 254-258.-New spp., some from the Mal. Pen.

- Kew Bull. (1938) 276-280.-Many new records; new spp. in Baphia, Saraca, and Spatholobus.

SMITH, Ic. Bog. 3 (1907) t. 230-231.-New Millettia; $c f$. Whitfordiodendron.

Philippines: DunN, Philip. J. Sc. 6 (1911) Bot. $315-$ 317.-Revision Millettia; key! 
Elmer, Leafl. Philip. Bot. 1 (1907) 220-232.New gen. \& spp.

-, ibid. 2 (1910) 689-701.-New gen. \& spp.

-, ibid. 9 (1934) 3198-3200.-Two new spp.

一, ibid. 10 (1939) 3757-3758.-New Mezoneurum.

Merrill, Philip. J. Sc. 5 (1910) Bot. 1-136.-

Full account; keys.

ibid. 7 (1912) Bot. 235.-Nomencl.

-, ibid. 27 (1925) 25-26.-New Bauhinia.

-, ibid. 30 (1926) 396-399.-New spp. from Sulu.

— \& QuisumbING, ibid. 82 (1953) 326-328.-

New records.

Quisumbing, Philip. J. Sc. 41 (1930) 324-326.-

New Derris.

Quisumbing \& Merrill, Philip. J. Sc. 37 (1928)

151-153.-Three new spp. in Bauhinia, Mucuna, \& Rhynchosia.

Celebes: OlIVER, J. Linn. Soc. Lond. Bot. 15 (1877) 98-99.-New Phanera.

Moluccas: Holthuis \& LAM, Blumea 5 (1942) 186-196.-Morotai; Talaud.

New Guinea: BaIley, Ann. Rep. Br. New Guinea (1901-1902) App. (1902).

-, Queensl. Agric. J. (1901) 410-411.-New Cassia.

-, ibid. 24 (1910) 20-21.-Mucuna var.

BAKER, Brittonia 2 (1937) .318.-New Desmodium.

-, J. Arn. Arb. 20 (1939) 329-330.-New Adenanthera.

HaRms, Bot. Jahrb. 55 (1918) 38-58.-New spp.; no key.

-, Notizbl. Berl.-Dahlem 7 (1920) 28-30.Three new Mucunas.

Kostermans, Bull. Bot. Gard. Btzg III, 18 (1950) 443-448.-New Koompassia and Inocarpus.

Merrill \& Perry, J. Arn. Arb. 23 (1942) 391 407.-Brass coll.

一, ibid. 29 (1948) 155-157.-CLEMENs coll.

Pulle, Nova Guinea 8 (1910) 369-387.-Records. 一, ibid. 8 (1911) 649-653.-Ditto.

SchumanN, Bot. Jahrb. 9 (1887) 201-204.-Ditto.

WARBURG, Bot. Jahrb. 13 (1891) 321-337, 453-

454.-Ditto.

一, ibid. 18 (1893) 193-194.

White, Proc. R. Soc. Queensl. 34 (1922) 32-37. -List.

-, J. Arn. Arb. 8 (1927) 130.-New Maniltoa. —, ibid. 10 (1929) 219-224, 273.-List.

一, ibid. 31 (1950) 88-89.-Two new Albizzias. - \& Francis, Proc. R. Soc. Queensl. 38 (1927) 230-232.-Records and new Albizzia.

\section{Genera}

Adenanthera: BAKER, J. Arn. Arb. 20 (1939) 329330.-New sp.

Afzelia (Pahudia); LEONARD, Reinwardtia 1 (1950) 61-65.-Reduction of Pahudia. List of spp.

MetCalf, Lingn. Sc. J. 19 (1940) 257-258.New record for SE. China of Pahudia martabanica Prain.

Meijer Drees, Bull. Jard. Bot. Btzg III, 16 (1938) 96-102,-Key.
-, Tectona 31 (1938) 851-864.

PranN, Ind. For. 29 (1900) 310-312.-Revision of Asiat. spp.; key.

-, Sc. Mem. Med. Off. Army India 12 (1901) 1-17.

De WrT, Bull. Bot. Gard. Btzg III, 17 (1941)

139-154.-New revision; key.

Albizzia: Fournier, Ann. Sc. Nat. IV, Bot. 15 (1861) 161-178.-No key.

Von Mueller, J. Bot. 10 (1872) 7-11.-Defin. Austr. spp.; relations to allied genera.

Ricker, J. Wash. Acad. Sc. 8 (1918) 242-246.Key to Chin. \& Form. spp.; no descr.; collectors numbers enum.

Wu, Bot. Jahrb. 71 (1941) 180-182.-Key to some Chin. spp.

Alysicarpus: SEDGwick, J. Ind. Bot. 1 (1919) 14 18. - A. rugosus and allies.

Archidendron: DE WrT, Bull. Jard. Bot. Btzg III, 17 (1941) 256-272.-Revision; key.

-, Reinwardtia 2 (1952) 69-96.-New revision. -, Webbia 9 (1954) 458-459.-New sp. from New Guinea.

Atylosia (Cantharospermum): Sprague, . Kew Bull. (1927) 134-135.-Nomencl.

Baphia: Ridley, Kew Bull. (1938) 280.-2nd Mal. sp.

Bauhinia: CHEN, J. Arn. Arb. 19 (1938) 129-133.New spp. from China.

Gagnepain, C. R. Ass. Fr. 43 me sess. Le Hâvre 1914 (1915) 411-426.-Distr. \& classif. E. Asiat. \& Mal. spp.; key; no descr.; coll. numbers.

Korthals; in Temminck, Verh. Nat. Geschied. Bot. (1841) 77-92.-Descr. new spp.

Brownea: PitTier, Contr. U. S. Nat. Herb. 18 (1916) 145-157.-Rev. of spp. of Panama \& Venezuela.

Butea: Anonymous, Kew Bull. (1908) 381.-A new sp. with notes on the genus.

Blatter, J. Ind. Bot. Soc. 8 (1929) 133-138. Revision of the genus. Non vidi.

PraIN, Kew Bull. (1908) 381-387.-New sp. and crit. notes.

Caesalpinia: DANDY \& Exell, J. Bot. 76 (1938) 175-180.-Nomencl. of 3 common spp.

Fischer, Bot. Gaz. 18 (1893) 121-123.-Reduction of Hoffmannseggia and list of North Amer. spp.

Petch, Ann. R. Bot. Gard. Perad. 9 (1925) 299 305.-Nomencl.

Canavalia: Chatterjee, J. Ind. Bot. Soc. 28 (1949) 83-95, 5 pl.-Indian spp.

PIPER, U. S. Dep. Agric. Circ. no 110 (1913) 2936, t. 3.-Data on C. ensifolia \& C. gladiata.

-, Proc. Biol. Soc. Wash. 30 (1917) 174-178. Notes \& descr. of 2 new spp., one from Luzon; C. turgida reduced to $C$. microcarpa (DC.) PIPER comb. nov.

- Contr. U. S. Nat. Herb. 20 (1925) 555-588. -Revision Amer. spp.; key.

- \& DunN, Kew Bull. (1922) 129-145.-Rev.; key to Old World spp.

Cassia: Bentham, Trans. Linn. Soc. 27 (1871) 503-591, t. 60-63.-Revision.

Corner, M. A. H. A. Mag. 5 (1935) 37-50.Account of Mal. Pen. spp.; key. 
Petch, Ann. R. Bot. Gard. Perad. 9 (1924) 229236. $C$. mimosoides \& C. leschenaultiana.

SteyaerT, Bull. Jard. Bot. Brux. 20 (1950) 233-

268. - $\$$ Chamaecrista, Asiat. \& Afr. spp.

DE WrT, Webbia 11 (1954) 197-292.-Rev.; keys.

Cicer: Popov, Bull. Appl. Bot. of Gen. \& PlantBreed. 21 (1929) 1-240, fig. 1-48.-Apparently revision; in Russ. with Latin descr.; summary. Non vidi.

Clianthus (Sarcodum): Merrill, J. Bot. 66 (1928) 264-265.-Gen. nomencl.

Clitoria: Bentham, J. Linn. Soc. Lond. Bot. 2 (1858) 33-44.-Synopsis.

Copaifera (Pseudosindora): DWYER, Brittonia 7 (1951) 143-172.-Revision Central Amer., West Indian \& S. Amer. spp.; key.

DE WrT, Webbia 9 (1954) 459-463.-Bornean tree; first record in Malaysia.

Crotalaria: BAKER, J. Linn. Soc. Lond. Bot. 42 (1914) 241-425.-Rev. Afr. spp.; key.

Gagnepann, Bull. Soc. Bot. Fr. 61 (1914) 282$289,310-315$.-Division of the genus into 2 groups and enum. of their spp.

Santapau, J. Bombay Nat. Hist. Soc. 51 (1953) 960-962.-Key to Deccan spp.

SENN, Contr. Gray Herb. no 125 (1939) 317-370. -Repr. from Rhodora 41.-N. Amer. spp.; key.

Crudia: Prain, J. As. Soc. Beng. 73, ii (1904) 199_ 200.-Born. sp.

DE WIT, Bull. Bot. Gard. Btzg III, 18 (1950) 407-434.-Not incl. Philippines; revision; key.

Cynometra: Harms, Notizbl. Berl.-Dahlem 3 (1902) 186-188.-New spp. from Philip. \& Papua.

Dalbergia: PrTtrer, J. Wash. Acad. Sc. 12 (1922) 54.-Mexican \& Central Amer. spp.; key; descr.; enum. of coll.

Prain, J. As. Soc. Beng. 70, ii (1901) 39-65.List of 74 Asiat. and Mal. spp.; no key.

-, Ann. R. Bot. Gard. Calc. 10 (1905) 1-114, t. 1-91.-Revision; key.

Dansera: van Steenis, Bull. Bot. Gard. Btzg III, 17 (1948) 413-416.-Sumatra.

SteyaERT, Reinwardtia 2 (1953) 351-355.Reduced to subgenus of Dialium; new comb.

Derris: Backer, Blumea 5 (1945) 513-514.-Key to some Jav. spp.

Blake, S. F., J. Wash. Acad. Sc. 19 (1929) 472 475.-Distinction of Deguelia from Derris; transfer of 7 Mal. spp. of Derris to Deguelia.

GaGnePaIn, Not. Syst. 2 (1913) 341-350.Asiat. spp.; key.

WhIte, Kew Bull. (1929) 309-310.-Queensl. sp.

Desmodium s. l.: SchindleR, Bot. Jahrb. 54 (1916) 51-68.

—, in Fedde, Rep. 20 (1924) 136-155.-Amer. spp. of Meibomia, a segregate of Desmodium. -, in Fedde, Rep. 20 (1924) 266-286.-Dicerma, Phyllodium, Pteroloma, Hanslia, Dendrolobium, Codariocalyx, Hegnera and other segregates of Desmodium.

-, in FEDDE, Rep. 21 (1925) 1-21.-New spp. and comb.

-, in Fedde, Rep. Beih. 49 (1928) 1-371.Bibl.; syn.
Dialium: PraIN, Ind. For. 22 (1896) 460-461.Vern. names of $s p p$.

STEYart, Bull. Soc. R. Bot. Belg. 84 (1951) 2943.-Subdivisions.

- Reinwardtia 2 (1953) 351-355.-Dansera and Uittienia are reduced to subgenera of Dialium.

DE WIT, Blumea 7 (1953) 320-321.-Descr. 8 new spp. precursory to revision.

Dolichos: CraIB, Kew Bull. (1914) 76-77.-D. hosei from Borneo.

Milne-Redhead, Kew Bull. (1905) 353-354.D. falcatus in Africa.

Dunbaria: WU, Bot. Jahrb. 71 (1941) 182-185.Key to Chin. spp.

Endertia: vaN STEENIS \& DE WIT, Bull. Bot. Gard. Btzg III, 17 (1947) 323-327.-Borneo.

Entada: RidleY, J. Bot. 58 (1920) 195-196.

Eriosema: Staner \& de Craene, Ann. Mus. Congo Belge, Bot., II, 1 (1934) 41-92.-Congo spp.; key.

Erythrina: BoERlaGe, Teysmannia 3 (1892) 535542.-Indonesian spp.; no key.

KRUKOFF, Brittonia 3 (1939) 205-337.-American spp..

-, J. Arn. Arb. 20 (1939) 225-233.-Prelim. not. Asiat.-Polyn. spp.

McCuntock, Baileya 1 (1953) 53-58, fig. 3840.-Key to cult. spp.

ReiJnvaAn \& Docters van Leeuwen, Trop. Natuur 21 (1932) 193-199.

Erythrophloeum: Planchon, Ann. Mus. Col. Marseille 15 (1907) 161-304, fig. 1-107.-Deals with many extra-Mal. spp.

一, ibid. 19 (1911) 303-329, t. 3-4.-Anat. morph. E. densiflorum.

Fissicalyx: Bentham, Trans. Linn. Soc. 23 (1861) 389.

Flemingia: LI, Amer. J. Bot. 31 (1944) 224-228.Nomencl.

Fordia: DuNN, Kew Bull. (1911) 62-64.-Revision; key.

Glycine: Piper, J. Amer. Soc. Agron. 6 (1914) 75-84.-Nomencl.

$-\&$ W. J. Morse, The Soybean. xv + 329, fig. 1-84.-On G. soja.

Indigofera: BAKER, J. Bot. 41 (1903) 185-194.Trop. Afr. spp.

Burkart, Darwiniana 4 (1942) 145-178, fig. 1-6.-Argentine spp.; revision; key; in Spanish. Gagnepain, Not. Syst. 3 (1915) 111-123.General observations on structure of indument; descr. new spp. of China and Indo-China.

PraIn \& BAKer, J. Bot. 40 (1902) 60-67, 136144.-Critical notes on old spp.; no key.

Inocarpus: FosberG, J. Wash. Acad. Sc. 31 (1941) 93-96.-Nomencl. sp.

Kostermans, Bull. Bot. Gard. Btzg III, 18 (1950) 446-448.-New sp. New Guinea.

Inga: PITtIER, Contr. U. S. Nat. Herb. 18 (1916) 173-223, t. 81-105.-Revision; descr.; list; no key.

Intsia: Meijer Drees, Bull. Jard. Bot. Btzg III, 16 (1938) 87-96.-Key.

-, Tectona 31 (1938) 851-864. 
DE WIT, Bull. Bot. Gard. Btzg III, 17 (1941) 139-154.-Newest revision.

Kalappia: Kostermans, Reinwardtia 1 (1952) 451456.-New gen. from E. Malaysia.

Kingiodendron: BuRTT, Kew Bull. (1936) 460-462. -Melan. spp.

DE WIT, Bull. Bot. Gard. Btzg III, 18 (1949) 211-212.

Koompassia (Abauria): BeCCARI, Malesia 1 (1877) 169-171.

Kostermans, Bull. Bot. Gard. Btzg III, 18 (1950) 443-446.-New sp. from N. Guinea.

TAubert, Ber. Deut. Bot. Ges. 10 (1892) 640 642.

DE WIT, Bull. Bot. Gard. Btzg III, 17 (1947) 309-322.-Revision.

Lespedeza: FranchET, Rev. Hort. 62 (1890) 225227.-W. Chin. spp.

HOCHREUTINER, Rhodora 36 (1934) 390-392.Nomencl. gen. name.

RICKER, Rhodora 36 (1934) 130-132.-Nomencl. gen. name.

Schindler, in Fedde, Rep. 11 (1912) 338-347. - Campylotropis segregated from Lespedeza.

-, Bot. Jahrb. 49 (1913) 570-658.-No key.

Lonchocarpus: PITTIER, Contr. U. S. Nat. Herb. 20 (1917) 37-93.-Centr. Amer. spp.

White, Kew Bull. (1929) 309-310.-Austral. spp.

Lupinus: Zhuкovsкy, Bull. Appl. Bot. Gen. \& Plant-Breed. 21, 1 (1928-29) 241-294.-Descr. Old World spp. and some New World ones; key; in Russian, Engl. summary.

Maniltoa: HaRms, Notizbl. Berl.-Dahlem 3 (1902) 189-191.-Synopsis; new spp.

Whrte, J. Arn. Arb. 8 (1927) 130.-New sp.

Mastersia: HaRMs, in FedDE, Rep. 9 (1911) 367369.-Revision; no key.

Medicago: Urban, Verh. Bot. Ver. Brandenb. 15 (1873) 1-85.-Revision; key.

Melilotus: Schulz, Bot. Jahrb. 29 (1900) 660-735, t. 6-8.-Revision; key.

Mezoneurum: Prain, J. As. Soc. Beng. 61, ii (1892) 130-131.-New sp. Andamans.

Millettia: DunN, Philip. J. Sc. 6 (1911) Bot. 315317.-New spp.

-, J. Linn. Soc. Lond. Bot. 41 (1912) 123-243.Revision.

GAGNEPAIN, Not. Syst. 2 (1911) 350-367.-New spp. Indo-China.

Mimosa: BURkaRT, Darwiniana 8 (1948) 9-231, fig. 1-31, t. 1-26.-Argentine spp.; keys; in Spanish.

Gamble, Kew Bull. (1920) 1-6, fig. 1-7.Indian spp.; key.

Robinson, Proc. Amer. Ac. Arts \& Sc. 33 (1898) 305-331.-Revision North American \& Mexican spp.

Monarthrocarpus (Desmofischera): HoLthuIs, Blumea 5 (1942) 188-192.

Kostermans, Reinwardtia 1 (1952) 456-457. Merrill, Philip. J. Sc. 5 (1910) Bot. 89.

Mucuna (Stizolobium): Burck, Ann. Jard. Bot. Btzg 11 (1893) 183-190.-Revision; no key. DYER, Kew Bull. (1931) 464.-M. gigantea.
HaRms, Notizbl. Berl.-Dahlem 7 (1920) 372374.-Three new Pap. spp.

PIPER, Proc. Biol. Soc. Wash. 30 (1917) 51-62.

Myroxylon: HaRms, Notizbl. Berl.-Dahlem 5 (1908) 85-98.-Revision; no key.

Neptunia: Robinson, Proc. Amer. Ac. Arts \& Sc. 33 (1898) 332-334 (repr. in Contr. Gray Herb. new ser. 13).-Revision Amer. spp.

Ormocarpum: TeYsmanN \& BINNENDIJK, Nat. Tijd. Ned. Ind. 27 (1864) 54-56.-New spp.

Ormosia: Jackson, Trans. Linn. Soc. 10 (1811) 360 , t. 25.

Merrill \& Chen, Sargentia 3 (1943) 77-120.Chin. \& Indo-Chin. spp.; key.

Prain, J. As. Soc. Beng. 69, ii (1900) 175-186. -List of Asiat. spp.; key.

—, J. As. Soc. Beng. 73, ii (1904) 45-46.-Add. to prec. item.

Padbruggea: DunN, Kew Bull. (1911) 197-198.Key.

Pahudia $=$ Afzelia.

Parkia: BURKILL, Dict. (1935) 1668-1671. Chevalier, Bull. Mus. Hist. Nat. Paris (1910) 169-174.-Afr. spp.

Phaseolus: Balley, Gentes Herb. 4 (1940) 336-341. -Ph. lunatus and relatives.

Peekelia: HARMS, Notizbl. Berl.-Dahlem 7 (1920) 370-371.-Segr. from Phaseolus; Papua.

Prosopis: Burkart, Darwiniana 4 (1940) 57-128, fig. 1-15, t. 1-23.-Monogr.; keys; in Span.

Pterocarpus: Prain, Ind. For. 26 (1900) 397-412. -Revision; key.

AIRY SHAW, Kew Bull. (1937) 63-64, 477-479.

Pueraria: Bentham, J. Linn. Soc. Lond. Bot. 9 (1867) 121-125.-Revision.

Orwı, Tokyo Sc. Mus. no 18 (1947) 16.-New comb.

Samanea: Merrill, J. Wash. Acad. Sc. 6 (1916) 42-48.-Segr. of Pithecellobium.

Saraca (Leucostegane): RIDLEY, Kew Bull. (1929) 257.-New sp. Borneo.

Serianthus: GuillaumiN, Not. Syst. 2 (1911) 374 376.-New Caled. spp.

Kostermans, Reinwardtia 2 (1953) 357-359, fig. 1.-New sp.

Sesbania: Sprague \& Milne-Redhead, Kew Bull. (1939) 159-162.-Nomencl. of S. aculeata.

Sindora: Symington, Kew Bull. (1938) 73-79, t. -Notes on Mal. Pen. spp.

DE WIT, Bull. Bot. Gard. Btzg III, 18 (1949) 582.-Revision; key.

Sophora: GaGnePain, Not. Syst. 3 (1914) 12-21.Key to Asiat. spp.; misc. new spp.

VAN SteenIs, Bull. Bot. Gard. Btzg III, 17 (1948) 421-428.-Revision; key.

Stylosanthes: TAubert, Abh. Bot. Ver. Prov. Brandenb. 32 (1890) 1-34.-Monogr.; keys.

Tephrosia: BACKER, Bull. Jard. Bot. Btzg III, 16 (1939) 110.-New name for Salajar $s p$.

Teyleria: BACKER, Bull. Jard. Bot. Btzg III, 16 (1939) 107-109.

Uittienia: van Steenis, Bull. Bot. Gard. Btzg III, 17 (1948) 416-419.-Borneo.

SteyaerT, Reinwardtia 2 (1953) 351-355.Reduced to subgenus of Dialium; new comb. 
Vandasia (Kennedya or Hardenbergia retusa) : BACKER, Ic. Bog. 3 (1908) 167-169, t. 265.-Papua. DomIN, Bibl. Bot. 89 (1926) 220.

Vigna: Piper, Bull. U. S. Dep. Agric. Bur. Pl. Ind. 229 (1912).

Voandzeia: HaRms, Notizbl. Berl.-Dahlem 5 (1912) 253-258.

STAPF, Kew Bull. (1912) 209-213.

Whitfordiodendron (Adinobotrys, Whitfordia): Burkill, Kew Bull. (1935) 319.

DuNN, Kew Bull. (1911) 193-197.-Rev.; key. -, ibid. (1912) 363-364.-Adjustment.

Elmer, Leafl. Philip. Bot. 2 (1910) 689.

Merrill, Philip. J. Sc. 29 (1926) 373.

\section{LEMNACEAE}

Hegelmaier, F., Die Lemnaceen. Eine monographische Untersuchung. Leipzig (1868) 169 pp.

-, Systematische Ubersicht der Lemnaceen (Bot. Jahrb. 21, 1895, 268-305).

KURz, S., Enumeration of Indian Lemnaceae (J. Linn. Soc. Lond. Bot. 9, 1867, 264-268).

-, J. Bot. 5 (1867) 115-116.-Ditto of Austral. spp. and additions to latter paper.

Lawalré, A., La position des Lemnaceae et leur classification (Bull. Soc. Bot. Roy. Belg. 77, 1945, 27-38).-Affinity is with Najadaceae and not with Araceae!

\section{Local}

Java: BACKer, Handb. Fl. Java pt 3 (1924) 1-2.In Dutch; key.

\section{Genera}

Wolffia: Miquel, Ned. Kruidk. Arch. 3 (1853) 425-429.-New sp.

\section{LENTIBULARIACEAE}

Barnhart, J. H., Segregation of genera in Lentibulariaceae (Mem. N. Y. Bot. Gard. 6, 1916, 39-64, fig. 1-25).-Key to segregate genera.

Benjamin, L., Neue Gattungen und Arten der Utricularien, nebst einer neuen Einteilung der Gattung Utricularia (Linnaea 20, 1847, 299-315). -Synopsis; no keys.

Lloyd, F. E., Utricularia (Biol. Rev. 10, 1935, 72-110, fig. 1-35).

-, Notes on Utricularia, with special reference to Australia, with descriptions of four new species (Vict. Naturalist 1936, 91-112).

OLIVER, D., The Indian species of Utricularia (J. Linn. Soc. Lond. Bot. 3, 1859, 170-190).-Incl. notes on Mal. \& Born. spp.; no key.

\section{Local}

Java: BACKER, Bekn. Fl. Java (em. ed.) 8 (1949) fam. 194.

Van Steenis, Arch. f. Hydrobiol. Suppl. 11 (1932) 330-332, fig. 8 (i-v).-Records \& details on 5 spp.

New Guinea: Melchior, Bot. Jahrb. 62 (1929) 382-385.

\section{LILIACEAE}

BAKer, J. G., Revision of the genera and species of Tulipeae (J. Linn. Soc. Bot. 14, 1875, 211-310).
- Revision of the genera and species of the Asparagaceae (ibid. 14, 1875, 508-632).

-, Revision of the genera and species of Anthericeae and Eriospermeac (ibid. 15, 1877, 253363).

- A synopsis of Colchicaceae and the aberrant tribes of Liliaceae (J. Linn. Soc. Bot. 17, 1879, 405-510).-Ditto.

-, A synopsis of Aloineae and Yuccoideae (J. Linn. Soc. Bot. 18, 1880, 148-241).-Ditto.

Berger, A., Liliaceae-Asphodeloideae-Aloineae (Pflanzenreich Heft 33, 1908, 1-347, fig. 1-14).

Brown, N. E., Notes on the genera Cordyline, Dracaena, Pleomele, Sansevieria \& Taetsia (Kew Bull. 1914, 273-279).

Candolle, A. De, Smilacées (in DC. Mon. Phan. $1,1878,1-217)$.-Contains revisions of Smilax, Heterosmilax, and Rhipogonum.

Gagnepain, F., Les Aspidistrées d'Indochine (Bull. Mus. Hist. Nat. Paris II, 6, 1934, 189-192).

Krause, K., Pflanzenfamilien ed. II, 15a (1930) 227-386.

Trelease, W., The Yucceae (Rep. Missouri Bot. Gard. 13, 1902, 27-133, t. 1-99).-Revision; key.

\section{Local}

Sumatra: Ridley, J. Fed. Mal. St. Mus. 8, 4 (1917) 117-118.-Incl. also some new Peliosanthes.

Malay Peninsula: Ridley, J. Bot. 34 (1896) 162168.-Dracaena in Mal. Pen.

—, J. Str. Br. R. As. Soc. no 31 (1898) 91-98.Mal. Pen. Peliosanthes.

—, Mat. Fl. Mal. Pen. (Monocot.) 2 (1907) 86108.-Keys.

Java: BACKER, Handb. Fl. Java pt 3 (1924) 4580.-Important; key; many cultivated species included; in Dutch.

Borneo: Merrill, Philip. J. Sc. 29 (1926) 355-356. -New Pleomele.

Ridley, Sarawak Mus. J. 12 (1912) 38.-New Dracaena.

Philippines: Merrill \& Quisumbing, Philip. J. Sc. 82 (1953) 323.-Syn. of a Peliosanthes.

New Guinea: Hallier $f$, Nova Guinea 8 (1914) 989-1003.

KRAUSE, Bot. Jahrb. 59 (1925) 547-567.

-, Nova Guinea 14 (1927) 173-179.

LAUTERBACH, Bot. Jahrb. 50 (1913) 290-300.

\section{Genera}

Aletris: Franchet, in Morot, J. de Bot. 10 (1896) 178-180, 195-203.-Reduces Metanarthecium; key to Asiat. spp. of Aletris.

Masumune, Bull. Soc. Bot. Fr. 84 (1937) 18.New sp. of Sumatra \& Borneo.

Allium: BAKER, J. Bot. 12 (1874) 289-295.-Asiat. spp.

Regel, Alliorum adhuc cognitarum monographia. Petersburg (1875) 1-266.

STEARN, Herbertia 11 (1944) 11-34, 45-63, fig. 117-120, t. 255-262.-Distr. \& classif. sect. of old World spp.

—, ibid. 226-245, fig. 122, t. 263-267.-Synon. and nomencl. of $A$. odorum \& tuberosum; improved key to allied spp. 
VVEDENSKY, ibid. 65-225.-Key to 225 spp. occurring in the URSS, a translation by AIRY SHAw from the Flora URSS 4 (1935) 112-280.

Aloe: ReYNolds, G. W., The Aloes of South Africa (1950) xxiv + 520 pp., 572 fig., 77 (col.) tab. -Revision with descr. \& syn. of 132 S. Afr. spp.; keys.

Astelia: Skottsberg, Kungl. Svensk. Vetensk. Handl. III, 14, no 2 (1934) 3-106.-Monograph; key.

Chlorophytum: von Poellnitz, Ber. Deut. Bot. Ges. 61 (1943) 126-131.-Afr. spp.; div. of genus.

Dianella: Schlrttler, Mitt. Bot. Mus. Univ. Zürich 163 (1940). Inaug. Diss. 284 pp.-Monograph; keys.

-, Blumea 6 (1948) 200-228.-Rev. of Mal. spp.; key.

Disporum: Don, Trans. Linn. Soc. 18 (1841) 513524.-Monogr.

Dracaena (Cordyline): BAKER, J. Bot. 11 (1873) 261-266.-Synopsis of Indian spp. of Dracaena \& Cordyline.

ROTHERT, Bull. Dép. Agric. Ind. Néerl. 24 (1909) 1-15.-Anat. diff. between Dracaena \& Cordyline.

Haworthia: voN Poellnitz, in Fedde, Rep. 31 (1932) 82-91.

Hemerocallis: BaILEY, Gent. Herb. 2 (1930) 143156, fig. 80-86.-Key.

Hosta: Maekawa, J. Fac. Sc. Imp. Univ. Tokyo sect. iii, Bot. 5 (1940) 317-425.-Monogr.; keys.

Lilium: BAKER, J. R. Hort. Soc. 26 (1901) 335345.-Add. to Elwes's monogr.

ELWES, A monograph of the genus Lilium. London $1880, x v+84$ pp., 49 tab.-Suppl. by Grove, 58 pp., 16 tab. (1933-1936).

WAUGH, Bot. Gaz. 27 (1899) 235-254, 340-360, fig. 1-14.-Conspectus of the genus; key to spp. WILson, The lilies of Eastern Asia. London $1925,124 \mathrm{pp}$.

WOODCock \& Stearn, Lilies of the World, their cultivation and classification (1950) 461 pp.-Non vidi.

Metanarthecium (cf. Aletris): MasumuNe, Bull. Soc. Bot. Fr. 84 (1937) 18-19.-New sp. from the Philippines.

Ophiopogon (Chloopsis, Mondo): BaILeY, Gentes Herb. 2 (1929) 3-37.-Nomencl.; nom. cons. FARWell, Amer. Midl. Nat. 7 (1921) 41-43.Nomencl.

HASSKARL, Flora 9 (1851) 481-486.

Petrosavia (Protolirion): Beccari, Nuovo Giorn. Bot. Ital. 3 (1871) 7-11, t. 1.-Type descr.

Hutchinson, Kew Bull. (1933) 156-157.Reduction of Protolirion.

RIDLEY, Ann. Bot. 9 (1895) 55-57.

VAN SteENIS, Trop. Natuur 23 (1934) 52.Reduction of Protolirion; key.

Pleomele: Ridley, J. Bot. 68 (1930) 177-181.Asiat. spp.

Sansevieria: BrowN, Kew Bull. (1915) 185-261.Monogr.; key!

Tupistra: BAKER, J. Linn. Soc. Bot. 14 (1875) 580 581.

KuRz, J. As. Soc. Beng. 44, ii (1875) 199.New sp. from Tenasserim.

\section{LINACEA E}

Hallier $f$., H., Beiträge zur Kenntnis der Linaceae (DC. 1819) DumorT. (Beih. Bot. Centralbl. 39, ii, 1921, 1-178). -A very wide delimitation of the family, not accepted by present workers.

Miquel, F. A. W., Revue des Linées, indigènes dans l'Archipel Indien (Ill. Fl. Arch. Ind. 1870, 67-71).-Very incomplete treatment.

Planchon, E., Sur la famille des Linées (in Hook. Lond. J. Bot. 6, 1847, 588-603).

WinkLer, Hub., Pflanzenfamilien ed. 2, 19a (1931) 82-130.

\section{Local}

Sumatra: Merrill, Pap. Mich. Acad. Sc. 23 (1938) 180.

Borneo: RidLEY, Kew Bull. 1930, 74-75.-Ixonanthes.

Philippines: Elmer, Leaf. Philip. Bot. 10 (1939) 3758.

Moluccas: Merrill, Philip. J. Sc. 11 (1916) Bot. 277.-New Hugonia from Ambon.

New Guinea: LAUTERBACH, Bot. Jahrb. 52 (1915) 115-117.

Pulle, Nova Guinea 8 (1910) 391.

Went Jr, Nova Guinea 14 (1924) 112.

\section{Genera}

Ctenolophon: BeCCARI, Malesia 1 (1877) 119-120. -Under Icacinaceae.

OLIVER, Trans. Linn. Soc. Lond. 28 (1873) 515516, t. 43.-Under Olacineae.

Indorouchera (Rouchera p. p.): HALLIER $f$, Beih. Bot. Centralbl. 39, ii (1916) 50-53.-Census.

Ixonanthes: Hallier $f$., Beih. Bot. Centralbl. 39, ii (1916) 6-11.-Census.

Philbornea: Hallịer $f$., Arch. Néerl. Sc. Exact. Nat. III B, 1 (1912) 104-111.-Also general notes on affinities.

Schuurmansia \& Schuurmansiella: Haluier $f$., Rec. Trav. Bot. Néerl. 10 (1914) 340-351.Segregates of Durandea.

\section{LOASACEAE}

Ging, E., Pflanzenfamilien ed. 2, 21 (1925) 522543.

Urban, I., Monographia Loasacearum (Nova Act. Acad. Caes. Leop.-Carol. Nat. Cur. 76 ${ }^{1}$, 1900, liv, 1-370, t. 1-8).

\section{LOBELIACEAE See Campanulaceae \\ LOGANIACEAE (incl. Buddleiaceae)}

Bentham, G., Notes on Loganiaceae (J. Linn. Soc. Bot. 1, 1857, 52-114).-Discussion of \& key to the genera; enum. of spp. not mentioned in DC. Prod. or those emended; of small genera all spp. enumerated but no keys; descr. many new spp.

(1) ExELl \& MendonçA have segregated Linaceae into a number of related families among which Ixonanthaceae, Hugoniaceae and Ctenolophonaceae (Consp. FI. Angol. 1, 1951, 242-248). 
Blume, C. L., Museum Botanicum Lugduno-Batavum 1 (1850) 163-174, 238-240.-Enum.

Cammerloher, H. C., Bull. Jard. Bot. Btzg III, 5 (1923) 294-333.-Unsatisfactory revision of Indonesian spp.

KLETT, W., Umfang und Inhalt der Loganiaceen (in Mez, Bot. Arch. 5, 1924, 312-338).-Key and treatment of genera.

\section{Local}

Sumatra: Merrild, Contr. Arn. Arb. 8 (1934) 137-138.

-, Pap. Mich. Acad. Sc. 23 (1937) 188-189 (1938).-Cynoctonum; new Fagraea.

Malay Peninsula: Henderson, Gard. Bull. Str. Settl. 7 (1933) 113-115, t. 28.-2 new Fagraeas.

Java: BAKHUIZEN VAN DEN BRINK Jr, Blumea 6 (1950) 382-383.-Crit. notes.

Borneo: Merrill, J. Str. Br. R. As. Soc. no 77 (1917) 231-237.-New spp. -, J. Mal. Br. R. As. Soc. 1 (1923) 27.

Philippines: Elmer, Leafl. Philip. Bot. 2 (1909) 595-601.-Key \& descr. Fagraea.

-, ibid. 3 (1910) 857-860.-Notes; new Fagraeas.

Merrill, Philip. J. Sc. 27 (1925) 49.-New Fagraea.

—, ibid. 37 (1928) 190.-New Geniostoma.

New Guinea: Cammerloher, Nova Guinea 14 (1924) 115-118.

Gilo, Bot. Jahrb. 54 (1916) 156-197.

Hiern, Nova Guinea 8 (1909) 201-202.

Kanehira \& Hatusima, Bot. Mag. Tokyo 56 (1942) 157-166.-Several new spp. in Buddleia, Couthovia, Fagraea and Geniostoma.

Merrill \& Perry, J. Arn. Arb. 23 (1942) 408416.

MoOre, J. Bot. 66 (1928) 105.-New Fagraea. -, ibid. 67 (1929) 49.-New Fagraea \& Couthovia.

\section{Genera}

Buddleia: Cammerloher, Bull. Jard. Bot. Btzg III, 5 (1923) 334-335.

GagnePaIN, Not. Syst. 2 (1912) 182-194.-

Rev. Asiat. spp.

Marquand, Kew Bull. (1930) 177-208.-Rev. Old World spp.

Couthovia (Crateriphytum, Neuburgia): BLUME, Mus. Bot. 1 (1850) 156-157.

Koorders, Bull. Inst. Bot. Btzg no 16 (1902) 6-12.-Diagn. Crateriphytum.

-, Abbildung und Beschreibung von Crateriphytum moluccanum SCHEFF. Batavia 1919, pp. 1-8, t. 1 a-b.

Markgraf, Bot. Jahrb. 61 (1927) 222.

Fagraea: Blume, Rumphia 2 (1836) 25-36.Descr. \& fig. some spp.

MiQuel, Ann. Mus. Lugd.-Bat. 2 (1866) 216218, t. 5-6.-List.

Geniostoma: Valeton, Bull. Inst. Bot. Btzg no 12 (1902) 1-28.

Strychnos: BLume, Rumphia 1 (1835) 66-72, t. 25-26.

HiLl, Kew Bull. (1911) 281-302.-Various Asiat. \& Philip. spp.
-, ibid. (1917) 121-210.-Rev. Ind. \& Mal. spp.; keys; illustr.

-, ibid. (1919) 238-239.-S. nux-vomica in Cochin-China.

\section{LORANTHACEAE}

Danser, B. H., On the taxonomy and the nomenclature of the Loranthaceae of Asia and Australia (Bull. Jard. Bot. Btzg III, 10, 1929, 291-373). -System; generic key; nomenclator and general notes.

- The Loranthaceae of the Netherlands Indies (ibid. 11, 1930, 233-519).-Complete revision of Mal. Loranth. save the Philippines; keys.

- A new system for the genera of Loranthaceae-Loranthoideae with a nomenclator for the Old World species of this subfamily (Verh. Kon. Akad. Wet. A'dam, afd. Natuurk. 29, 1933, 4-129).

- Miscellaneous notes on Loranthaceae 1-6 (Rec. Trav. Bot. Néerl. 31, 1934, 223-236).

-, ditto 7-8 (ibid. 751-760.)

-, ditto 9-15 (Blumea 2, 1936 34-59).

-, ditto 16-18 (ibid. 3, 1938, 34-59).

-, ditto 19-24 (ibid. 3, 1940, 389-404).

ENGler \& Krause, Pflanzenfamilien ed. 2, 16b (1935) 98-203.

Korthals, P. W., Aanteekeningen over eenige soorten Loranthus (Tijd. Nat. Gesch. \& Phys. 3, 1836, 187-202).-Embryol.

- Verhandeling over de op Java, Sumatra en Borneo verzamelde Loranthaceae (Verh. Bat. Gen. K. \& W. 17, 1839, 199-288, pl. 1-2).Repr. p. 1-92.

\section{Local}

Indo-China \& Siam: DANser, Bull. Jard. Bot. Btzg III, 16 (1938) 1-63; additions ibid. (1940) 235267.-Keys.

Sumatra: Danser, in Merrill, Contr. Arn. Arb. 8 (1934) 53-54.

Malay Peninsula: KING, J. As. Soc. Beng. 56, ii (1887) 89-100.-Perak spp.; no key.

Java: DANSER, Candollea 6 (1936) 457-459,-List.

Borneo: DANSER, Rec. Trav. Bot. Néerl. 31 (1934) 237-747 -Sarawak

—, ibid. 31 (1934) 229-235, 751-758.-Mt Kinabalu.

—, Blumea 2 (1936) 39-42; 3 (1938) 36-37.

-, ibid. 4 (1941) 259-260.-New Macrosolen.

Celebes: DANSER, Blumea 2 (1936) 37-39.

Philippines: Danser, Rec. Trav. Bot. Néerl. 30 (1933) 464-481.-On Thaumasianthes.

-, Philip. J. Sc. 58 (1935) 1-151.-Complete revision.

—, Blumea 3 (1940) 393-394.-New Lepidellas.

Elmer, Leafl. Philip. Bot. 3 (1911) 1067-1075.Various new spp. Mt Apo.

一, ibid. 6 (1913) 1959-1971.-New spp. Mt Urdaneta.

一, ibid. 9 (1934) 3201-3202.-New sp.

Merrill, Philip. J. Sc. 3 (1909) Bot. 129-153.

-Revision.

-, ibid. 30 (1926) 391-392.-New sp. from Samar.

—, ibid. 29 (1926) 477-478.-New Ginalloa. 
New Guinea: DANSER, Brittonia 2 (1936) 131-134. -., Blumea 3 (1938) 37-59; ibid. 3 (1940) 394 401.-Carr, Brass, and Clemens coll. KraUSe, Nova Guinea 14 (1924) 100-104. —, Bot. Jahrb. 57 (1922) 464 495. -, ibid. 62 (1929) 474-475.-New sp. LAUTERBACH, Nova Guinea 8 (1910) 289-290. 一, ibid. 8 (1912) 815-816.

Solomon Islands: DaNSER, J. Arn. Arb. 16 (1935) 206-209.

Melanesia: Danser, Bull. Jard. Bot. Btzg III, 14 (1936) 73-98.

-, Candollea 6 (1936) 457-459.

\section{Genera}

Korthalsella: Danser, Rec. Trav. Bot. Néerl. 31 (1934) 758-760.

_, Bull. Jard. Bot. Btzg III, 14 (1937) 115-159.

-Revision.

一, ibid. 16 (1940) 329-342.-Suppl. to revision. MEKEL, Blumea 1 (1935) 312-319.-Status of K. dacrydii.

WASSCHER, Blumea 4 (1941) 320-321.-Distr. of K. dacrydii.

Thaumasianthes: Danser, Rec. Trav. Bot. Néerl. 30 (1933) 464-481.-New gen. Philip.

Viscum: DANSER, Blumea 4 (1941) 261-319.Rev. Indo-Austr. spp.

\section{LOWIACEAE}

WinkLer, HuB., Pflanzenfamilien ed. 2, 15a (1930) 541.

\section{Local}

Malay Peninsula: Henderson, Gard. Bull. Str. Settl. 7 (1933) 125-126.-New sp.

RIDley, J. Str. Br. Roy. As. Soc. no 32 (1899) 183-184.-Enum.

-, Mat. Fl. Mal. Pen. (Monocot.) 2 (1907) 61-62.-Key.

\section{LYTHRACEAE}

KozhNe, E., Lythraceae monographice describuntur (Bot. Jahrb. 1, 1880, 142-178, 240-266, 305$335,436-458 ; 2,1881,136-176 ; 3,1882,129$ $155,319-352,395-429 ; 4,1883,12-37,386-431$; $5,1884,95-132 ; 6,1885,1-48 ; 7,1885,1-61)$. -, Pflanzenreich 17 (1903) 1-326.

-, Lythraceae, Nachträge (Bot. Jahrb. 41, 1907, 74-110).-Additional to monogr,; enum.

\section{Local}

Java: BACKer, Onkruidfl. Jav. Suiker. (1930) 460-466.-Herbac. genera only.

KoORders \& Valeton, Bijdr. Booms. Java 1 (1894) 186-205.

Philippines: Merrill \& Quisumbing, Philip. J. Sc. 82 (1953) 332.-Record of a Cuphea.

New Guinea: MaNsfeld, Bot. Jahrb. 61 (1927) 23-25.

Merrill \& Perry, J. Arn. Arb. 22 (1941) 268269.

Pulle, Nova Guinea 8 (1911) 675.

\section{Genera}

Ammannia: Blatter \& Hallberg, J. Bomb. Nat. Hist. Soc. 25 (1918) 701-722; ibid. 26 (1918) 210-217.-Rev. Ind. spp.

Rotala: Blatter \& Hallberg, J. Bomb. Nat. Hist. Soc. 25 (1918) 701-722; ibid. 26 (1918) 210-217. -Rev. Ind. spp.; keys.

\section{MAGNOLIACEAE}

(excl. Illiciaceae, Schisandraceae, and Winteraceae)

DANDY, J. E., The genera of Magnolieae (Kew Bull. 1927, 257-264).-Key to genera; descr. of new spp. \& gen.

- Three new Magnolieae (J. Bot. 66, 1928, 46-48).

- \& Good, Pflanzenareale II. Reihe, Heft 5 (1929) Karte 41-43.

GagnePain, F., Quelques synonymes de Magnoliacées (Bull. Soc. Bot. Fr. 76, 1929, 738-739). -Nomencl. correct.

Kng, G., The Magnoliaceae of British India (Ann. R. Bot. Gard. Calc. 3 (2) 1891, 197-226, t. 3874).

Korthals, P. W., Bijdrage tot de kennis der Indische Magnoliaceae (Ned. Kruidk. Arch. 2, 1851, 91-98).-Repr. with other titel \& separ. pagin. 1-8.

Miquel, F. A. W., Ann. Mus. Bot. Lugd.-Bat. 4 (1868) -Talauma, Michelia, Manglietia.

\section{Local}

Sumatra: Merrill, Contr. Arn. Arb. 8 (1934) 57. Malay Peninsula: DANDY, Kew Bull. (1928) 183193.

Java: Blume, Flora Javae fasc. 19-20 (1829).

Philippines: Warburg, in Perkins, Fragm. Fl. Philip. 3 (1905) 171.-New Talauma.

New Guinea: Diels, Bot. Jahrb. 54 (1917) 239245.

-, Nova Guinea 14 (1924) 75-79.

Kanehira \& Hatusima, Bot. Mag. Tokyo 57 (1943) 147-153.-Aromadendron.

Pulle, Nova Guinea 8 (1912) 633-634.

SCHLECHTER, Bot. Jahrb. 50 (1913) 70-72.

White \& Francis, Proc. R. Soc. Queensl. 38 (1927) 228

\section{Genera}

Bubbia \& Drimys. See Winteraceae.

Illicium. See Illiciaceae.

Kadsura. See Schisandraceae.

Magnolia: DANDY, J. R. Hort. Soc. 52 (1927) 260 264.-Key to all spp.

Michelia: DANDY, J. Bot. (1929) 222-224.-New spp. Indo-China.

Pachylarnax: DANDY, J. Bot. 71 (1933) 312.-2nd spp.

Schisandra. See Schisandraceae.

Talauma: DANDY, Kew Bull. (1927) 419-420.Syn. T. gitingensis.

\section{MALPIGHIA CEAE}

Jussieu, A. de, Monographie des Malpighiacées (Arch. Mus. Paris 3, 1843, 5-152, 255-616; repr. 1845, 1-368). 
Niedenzu, F., Ueber eine neue Eintheilung der Malpighiaceae (Ber. Deut. Bot. Ges. 8, 1890, 190-194).-General.

- Malpighiaceae palaeotropicae II (Verz. d. Vorles. Akad. Braunsweig 1924, 1-19).

-, Pflanzenreich Heft 91, 93, 94 (1928) 1-870, fig. 1-48.

\section{Local}

India: BLATTER, E., A proposed revision of the Flora of British India (J. Ind. Bot. Soc. 9, 1930, 140-150).

Indo-China: ARÈnEs, Not. Syst. 11 (1943) 62-80.

Malaysia: ARènEs, Reinwardtia 3 (1954) 67-74. -Descr. new spp.

Philippines: Merrill, E. D., Philip. J. Sc. 5 (1910) 186-191.-Key Philip. Hiptages.

\section{Genera}

Aspidopterys: Hutchnson, Kew Bull. (1917) 91103.-Revision.

Tristellateia: ArÈnEs, Mém. Mus. Hist. Nat. Paris 21 (1947) 275-330.-Monogr.

\section{MALVACEAE}

BAKER, E. G., Synopsis of genera and species of Malveae (J. Bot. 28, 1890, 15-18, 140-145, 207213, 239-243, 339-343, 367-371 ; 29, 1891, 49-53, $164-172,362-366 ; 30,1892,71-78,136-142$, $235-240,290-296,324-332 ; 31,1893,68-76$, 212-217, 267-273, 334-338, 361-368).

BenthaM, G., Notes on Malvaceae and Sterculiaceae (J. Proc. Linn. Soc. Bot. 6, 1862, 97-107). -Generic distinction.

Edlin, H. L., A critical revision of certain taxonomic groups of the Malvales (New Phytol. 34, 1935, 1-20, 122-143).

Gürke, M., Beiträge zur Systematik der Malvaceen (Bot. Jahrb. 16, 1893, 330-385).

Hochreutiner, B. P. G., Malvaceae novae vel minus cognitae (Ann. Cons. Jard. Bot. Genève 6, 1902, 10-59).-Mixed notes; no keys.

-, Notulae in Malvaceas, etc. (Ann. Cons. Jard. Bot. Genève 20, 1917, 107-172).-Mixed notes; no keys.

-, Les relations des Malvacées avec les Sterculiacées (C. R. Soc. Phys. \& Hist. Nat. Genève 42, 1925, 112-113).

Howard, R. A., Atkinsia gen. nov., Thespesia, and related West Indian genera of the Malvaceae (Bull. Torr. Bot. Club 76, 1949, 89-100).

Hutchinson, J. B., Notes on the classification and distribution of genera related to Gossypium (New Phytol. 46, 1947, 123-141).-Key to these genera; incl. also Thespesia; key to spp. of Cienfugosia.

\section{Local}

Sumatra: Merrill, Contr. Arn. Arb. 8 (1934) 101. Borneo: Merrill, Sarawak Mus. J. 3 (1928) 528. RIDLEY, Kew Bull. (1938) 221.-Few records. AIRy Shaw, in Hook. Ic. Pl. V, 4 (1939) t. 3377. -Hibiscus borneensis.

Java: BACKER, Schoolf. Java (1911) 111-130.In Dutch; keys.
HasskarL, Flora 25, ii (1842) Beibl. 38-39. 一, Plant. Jav. Rar. (1848) 300-306.

Van Steenis, Bull. Bot. Gard. Btzg III, 17 (1948) 402.-Hibiscus venustus BL.

Lesser Sunda Islands: WARBURG \& UlBrich, Notizbl. Berl.-Dahlem 7 (1920) 365.-Hibiscus sumbawanus.

New Guinea: Hochreutiner, Nova Guinea 14 (1924) 159-166.

UlBRICH, Notizbl. Berl.-Dahlem 14 (1939) 367368.-2 new Hibiscus.

Whrte, Proc. R. Soc. Queensl. 34 (1922) 43-44.-Listed records.

-, J. Arn. Arb. 10 (1929) 238-239.-Listed records.

\section{Genera}

Abelmoschus: Hochreutiner, Candollea 2 (1924) 83-87.-List.

Abutilon: Gagnepain, Not. Syst. 1 (1909) 67-72.Classif. Asiat. spp.

GARCKE, Bot. Jahrb. 15 (1893) 480-493.Notes; no descr.; no keys.

Cephalohibiscus: UlBrich, Notizbl. Berl.-Dahlem 12 (1935) 494-500, fig. 6.-Melanesia.

Gossypium: Alıotra, Ann. R. Scuola Sup. Agric. Portici 5 (1903) $111 \mathrm{pp}$.

Hutchinson, New Phytologist 46 (1947) 123141.-Classif. \& distr. of the genus and related genera.

-, Silow \& Stephens, The evolution of Gossypium and the differentiation of the cultivated cottons. Oxford Univ. Press. (1947) xi + 160 pp., 10 fig.-Monogr.; keys.

Roberty, Candollea 7 (1938) 297-360; 9 (1942/3) 19-103; 10 (1943-46) 345-398.

WATT, Revision of the genus Gossypium. London 1907.

—, Kew Bull. (1926) 193-210.-Descr.; no key. -, Kew Bull. (1927) 321-356.-Ditto.

Zartsev, Bull. Appl. Bot. Gen. \& Plant-Breed. 18 (1927-1928) 1-65.-Contr. classif.

Hibiscus (Abelmoschus): Hochreutiner, Ann. Cons. Jard. Bot. Genève 4 (1900) 23-191, 9 fig. Tren Hsiang Ho, Bot. Bull. Ac. Sin. 1 (1947) 45-52.-Key to Kwantung spp.

Malachra: GÜRKE, Bot. Jahrb. 16 (1892) 345-361. -Monograph, in Latin; key.

Malvastrum: GrAY, Bonplandia 5 (1857) 292298.

Malvaviscus: Schery, Ann. Missouri Bot. Gard. 29 (1942) 183-244.-Revision.

Papuodendron. See Bombacaceae.

Pavonia: Ulbrich, Bot. Jahrb. 57 (1920) 54-184., -Stress on Afr. spp.

Pseudoabutilon: FrIEs, Kungl. Vetensk. Handl. Stockholm 43 (1908) no 4.

Sida: Gagnepain, Not. Syst. 1 (1909) 27-32.Asiat. spp.

GANDoger, Bull. Soc. Bot. Fr. 71 (1924) 627633.-Kev.

Sidalcea: Eling Roush, Ann. Missouri Bot. Gard. 18 (1931) 117-244.-Monogr.

Sphaeralcea : Kearney, Univ. Calif. Publ. Bot. 19 (1925) 1-128.-N. Am. spp. 
Thespesia: BaKer, J. Bot. 35 (1897) 50-54,Revision; partial key.

Hutchinson, New Phytologist 46 (1947) 134 138.-Revision; key.

Urena: Hochreutiner, Ann. Cons. Jard. Bot. Genève 5 (1901) 131-146.-Key to var. of U. lobata.

Wissadula: Fries, Kungl. Vetensk. Handl. Stockholm 43 (1908) no 4, 114 pp., 10 tab.

\section{MARANTACEAE}

EichleR, A. W., Beiträge zur Morphologie und Systematik der Marantaceen (Abh. K. Preuss. Akad. Wiss. Berl. 1883 (1884) 1-99, t. 1-7).

Gagnepain, F., Zingiberacées et Marantacées nouvelles de l'herbier du Muséum (Bull. Soc. Bot. Fr. 50, 1903, 586-590; ibid. 51, 1904, 164-182).

Loesener, Th., Pfianzenfamilien ed. 2, 15a (1930) 654-693.

Schumann, K., Pflanzenreich Heft 11 (1902) 1-184.

\section{Local}

Malay Peninsula: Ridley, J. Str. Br. Roy. As. Soc. no 32 (1899) 175-182.-Enum.

-, Mat. Fl. Mal. Pen. (Monocot.) pt 2 (1907) 55-61.-Keys.

Philippines: Perkins, Fragm. Fl. Philip. 1 (1904) 68-73.-Revisions; keys.

New Guinea: Schulze, Bot. Jahrb. 69 (1938) 265-267.-New Phryniums; no key.

\section{Genera}

Cominsia: Valeton, Bull. Jard. Bot. Btzg III, 2 (1920) 351-353.-Revision; key.

Donax: Rolfe, J. Bot. 45 (1907) 242-244.Nomencl.; revision.

Schumannianthes: RoLfe, J. Bot. 45 (1907) 244. Revision.

\section{MARTYNIACEAE \\ See Pedaliaceae}

\section{MELASTOMATACEAE}

Bakhuizen van den Brink Jr, R. C., A contribution to the knowledge of the Melastomataceae occurring in the Malay Archipelago especially in the Netherlands Indies (Rec. Trav. Bot. Néerl. 40, 1943, 1-391).-Partial revision; keys. Preissued reprint.

Blume, C. L., Over eenige Oostindische, bijzonder Javaansche Melastomaceae (in VAN HALL, c.s., Bijdr. Nat. Wet. 6, 1831, 211-268).-In German in Flora 15, 2 (1831) 465-479, 481-496, 497-512, 513-528.

-, Mus. Bot. Lugd.-Bat. 1 (1849) 5-13, 17-25, 33-41, 49-56; ibid. (1851) 353-363.-Enum.

Cogniaux, in DC. Mon. Phan. 7 (1891) 1-1256. -Now very incomplete monograph.

Guillaumin, A., Contribution à l'étude des Mélastomatacées d'extrême Orient I (Not. Syst. 2, 1913, 301-329); II (Bull. Soc. Bot. Fr. 60, 1913, 86-92); III (ibid. 273-276, 337-345, 362-371, 401-406).

Korthals, P. W., Bijdrage tot de kennis der
Indische Melastomataceae (in Temminck, Verh. Nat. Gesch. Bot. 1844, 218-255).

LI, HuI-LIN, Studies in the Melastomataceae of China (J. Arn. Arb. 25, 1944, 1-42).-Keys.

Miquel, F. A. W., Melastomataceae Teysmanno et De Vriese in Celebes et insulis Moluccis collectae (Ann. Mus. Bot. Lugd.-Bat. 1, 1864, 216-217).-Small list.

NAUdiN, C., Melastomacearum quae in Musaeo parisiensi continentur Monographicae descriptionis et secundum affinitates distributionis tentamen (Ann. Sc. Nat. Bot. III, 12, 1849, $196-284$; 13, 1849, 25-39, 126-159, 273-303, $347-362 ; 14,1850,53-76,115-165 ; 15,1851$, $43-79,276-345 ; 16,1851,83-246 ; 17,1852$, $305-382 ; 18,1852,85-154,257-294)$.

Triana, J., Les Melastomacées (Trans. Linn. Soc. 28, 1871, 1-188).

\section{Local}

Sumatra: Merrill, Contr. Arn. Arb. 8 (1934) 113-116.-Enum.

Ridley, J. Mal. Br. R. As. Soc. no 87 (1923) 60. -, Kew Bull. (1925) 81-82.-Bencoolen records. -, Arch. Hydrobiol. Suppl. 11 (1932) 312-314, fig. 4.-New Sonerila.

Malay Peninsula: Corner, Gard. Bull. Str. Settl. 10 (1939) 299-301.-Key Mal. Pen. Melastomas. Henderson, J. Fed. Mal. Stat. Mus. 13 (1927) 219.

-, J. Mal. Br. R. As. Soc. 5 (1927) 247-250.List; new Sonerila.

-, Gard. Bull. Str. Settl. 7 (1933) 103-105.Misc. records.

Borneo: Diels \& HaCkenberg, Bot. Jahrb. 60 (1926) 312.

MerRILl, Sarawak Mus. J. 3 (1928) 539-541. RIDLEY, J. Str. Br. Roy. As. Soc. no 63 (1912) 59-60.-New Sonerila.

SchwarTz, Mitt. Inst. Allg. Bot. Hamb. 7 (1931) 237-257.-Many new spp.

Celebes: Bakhuizen VAN Den Brink Jr, Blumea 5 (1945) 582-584. -2 new Sonerilas.

Philippines: Elmer, Leafl. Philip. Bot. 4 (1911) 1191-1230.

-, ibid. 9 (1934) 3202-3205.-3 new spp.

—, ibid. 10 (1939) 3649-3672.-Mt Irosin new spp.

Merrill, Philip. J. Sc. 8 (1913) Bot. 207-250, 335-360.-Revision Philip. spp. and gen.; keys. -, ibid. 12 (1917) Bot. 337-360.-List new spp. - \& Quisumbnng, ibid. 37 (1928) 177-185.New spp.: Eisocreochiton n.g.

Moluccas: Holthuis \& LAM, Blumea 5 (1942) 219-221.-List Talaud \& Morotai.

Merrill, Philip. J. Sc. 11 (1916) Bot. 298-299. -Some Ambon records; one new Dissochaeta.

New Guinea: Mansfeld, Nova Guinea 14 (1927) 199-207.

-, Bot. Jahrb. 60 (1925) 105-143.-Rev.; keys.

MARKGRAF, Brittonia 2 (1936) 141-144.-Some new spp.

Merrill \& Perry, J. Arn. Arb. 24 (1943) 422-439. 
Mueller, in Wing's South. Sc. Rec. N. S. 2 (1886) 8-9.-New Medinilla.

OHwI, Bot. Mag. Tokyo 57 (1943) 1-20.-List

KaNEHIRA coll.; many new spp.

\section{Genera}

Astronidium (Naudiniella): Markgraf, Notizbl. Berl.-Dahlem 12 (1934) 47-50.

Blastus: SPARE, Kew Bull. (1929) 317-319.-Rev.; key.

Creochiton: Wagner, Sitz. Ber. Kais. Akad. Wiss. Wien Math. Naturw. Kl. 115 (1907) 411-427.-Morphol.

Enaulophyton: van SteEnis, Bull. Jard. Bot. Btzg III, 12 (1932) 194-198.-Monotypic; Natuna Isl.

Krassera: Schwartz, Mitt. Inst. Allg. Bot. Hamburg 7 (1931) 247-250.-Borneo.

Melastoma: Guillaumin, Not. Syst. 2 (1913) 311-323.-Key.

Memecylon: Bentham, J. Linn. Soc. Bot. 5 (1861) 77-78.-Cuming's coll.

Osbeckia: Gamble, J. Ind. Bot. Soc. 1 (1919) 60-61.-3 new Ind. spp.

Guillaumin, Not. Syst. 2 (1913) 305-311.-Key.

Phaulanthus: Ridley, J. Str. Br. R. As. Soc. no 57 (1910) 41-44.-6 spp.

Plethiandra: BuRkill, J. Str. Br. R. As. Soc. no 77 (1917) 265-269.-Sarawak.

Pternandra (Kibessia): BurkiLl, Kew Bull. (1935) 319.-Kibessia reduced to Pternandra.

Sonerila: StAPF, Ann. Bot. 6 (1892) 291-323.Revision.

Triuranthera: BACKer, Bull. Jard. Bot. Btzg III, 2 (1920) 319-320.-Borneo, Java, monotypic.

\section{MELIA CEAE}

Candolle, C. De, in DC. Mon. Phan. 1 (1878) 399-752, t. 6-9.-World monograph.

HaRMS, H., Zur Kenntnis einiger Malayischen Meliaceen (Notizbl. Berl.-Dahlem 15, 1941, 472-478).-Listed new spp. and records.

-, in Pflanzenfamilien ed. 2, $19 \mathrm{b1}$ (1940) 1-172.

Miquel, F. A. W., Monographia Meliacearum Archipelagi Indici (Ann. Mus. Lugd.-Bat. 4, 1868, 1-64).

Pellegrin, F., Sur les genres Aglaia, Amoora et Lansium (Not. Syst. 1, 1909-1911, 284-290).Disc. gen. distinction.

\section{Local}

Sumatra: Merrill, Contr. Arn. Arb. 8 (1934) 81-84.-List.

Malay Peninsula: Henderson, Gard. Bull. Str. Settl. 7 (1933) 90-95, t. 17.-3 new spp.

Borneo: Merrill, J. Str. Br. R. As. Soc. no 76 (1917) 87-89.-2 new Aglaias.

-, J. Str. Br. R. As. Soc. no 86 (1922) 315-318. -New spp.

RidLey, Kew Bull. (1930) 364-372.-New spp. Schwartz, Mitt. Inst. Allg. Bot. Hamb. 7 (1931) 235-236.

Airy Shaw, Kew Bull. (1940) 254-258.-Many new records.
Java: ADELBERT, Blumea 6 (1948) 313-322.

Candolle, C. DE, Ann. Cons. Jard. Bot. Genève 15-16 (1911-1913) 245-247.-List coll. HochREUTINER.

KoORders \& Valeton, Ic. Bog. 1 (1897-1901); 2 (1903-1906).-Several spp. fig.

Lesser Sunda Islands (\& Celebes): De Candolle, Med. Rijksherb. Leiden no 22 (1914) 7-10.New records.

Celebes: Koorders, Suppl. vol. 1 Flora N.O. Celebes 1 (1918) 23-27.

-, Suppl. vol. 2 ditto 2 (1922) t. 34-44.

-, Suppl. vol. 3 ditto 3 (1922) 19-24.

Moluccas: Merrill, Philip. J. Sc. 11 (1916) Bot. 278-280.-Records; new Dysoxylum.

Philippines: Elmer, Leafl. Philip. Bot. 9 (1934) 3205-3206.-A new Aphanamixis.

一, ibid. 9 (1937) 3273-3395.-New spp. \& records.

MerrIll, Philip. J. Sc. 9 (1914) Bot. 531-541.New spp.

一, ibid. 26 (1925) 459-462.-New spp.

Perkins, Notizbl. Berl.-Dahlem 4 (1903) 78-79. -2 new spp.

-, Fragm. Fl. Philip. (1904) 30-35.-List new rec. \& spp.

Quisumbing \& Merrill, Philip. J. Sc. 37 (1928) 156-158.-2 new spp.

New Guinea: de CANdolle, Bull. Herb. Boiss. II, 3 (1902) 161-180.-List; also spp. of New Caledonia \& Samoa.

—, Nova Guinea 8 (1910) 423-426.

一, ibid. 8 (1914) 1011-1017.

HARMs, Bot. Jahrb. 72 (1942) 158-205.Many new spp.; no keys.

Kurata, Bull. Tokyo Univ. For. no 35 (1947) art. 6, 198-199.-New Vavaea.

Merrill \& Perry, J. Arn. Arb. 21 (1940) 296327.

一, ibid. 22 (1941) 256-258.

-, ibid. 29 (1948) 157.

Whrte, Proc. R. Soc. Queensl. 34 (1922) 38-39.

-Small list of records.

一, ibid. 38 (1927) 234-237.-Some new spp.

Polynesia: SMITH, A. C., Contr. U.S. Nat. Herb. 30 (1952) 469-522.-Rev. of Xylocarpus, Vavaea, Aglaia, and Dysoxylum.

\section{Genera}

Aglaia (Oncostemma): AIRY SHAw, Kew Bull. (1949) 166.-Some crit. notes.

Amoora: HaINES, Kew Bull. (1920) 238-242.Crit. note A. spectabilis.

Cedrela $=$ Toona .

Chisocheton (Clemensia): AIRY SHAw, in HoOK. Ic. PI. (1937) t. 3333.-Descr. new $s p$. in $\delta$ Clemensia.

Chukrassia (Chickrassia): LIVERA, Ann. R. Bot. Gard. Perad. 9 (1925) 307-308.-Revision; key!

Dysoxylum (Piptosaccos): Fedtschenko, Svensk Bot. Tidskr. 19 (1924) 490-493.-Crit. note syn.

Flindersia. See Rutaceae.

Heynea (Ailanthopsis): GagnePaIn, Not. Syst. 13 (1947) 62-63. 
Munronia: Harms, Ber. Deut. Bot. Ges. 35 (1917) 74-82.

Pseudocarapa: Merrill \& Perry, J. Arn. Arb. 21 (1940) 315-316.

Reinwardtiodendron: Perkins, Fragm. Fl. Philip. 1 (1904) 74-76.

Sandoricum: MerRILL, J. Bot. 61 (1923) 172-174, 200.-S. koetjape.

Swietenia: BLAKE, J. Wash. Acad. Sc. 10 (1920) 286-297.

RolfE, Kew Bull. (1919) 201-207.-Crit. notes.

Toona (Cedrela): BuRkILL, Gard. Bull. Str. Settl. 5 (1930) 120-122.-Mal. Pen. list.

De Candolle, Rec. Bot. Surv. Ind. 3 (1908) 357-376.-Rev. Indo-Mal. spp.

HuI LIN LI, Trop. Woods 79 (1944) 16-24.Chin. spp.

Symington, Mal. For. 4 (1935) 119-126.-Mal. Pen. spp.

Vavaea (Lamiofrutex): BURTT, Kew Bull. (1935) 303-304.-Crit. notes.

Van Steenis, Bull. Bot. Gard. Btzg III, 18 (1950) 457.-Geogr. distr.

-, Act. Bot. Neerl. 1 (1952) 93-94.-Reduction Lamiofrutex.

Walsura (Napaeodendron): Pellegrin, Not. Syst. 1 (1909-1911) 227-229.-New spp. Tonkin. SYMINGTON, Kew Bull. (1937) 319.

Xylocarpus: Parkinson, Ind. For. 60 (1934) 136-143, t. 15-17.-Indian spp.

RIDLEY, Kew Bull. (1938) 288-292.

\section{MENISPERMACEAE}

BeCCARI, O, Malesia 1 (1877) 135-165.

Diels, L., Pflanzenreich Heft 46 (1910) 1-345, fig. 1-93.

Miers, J., A few remarks on the Menispermaceae (Ann. Mag. Nat. Hist. II, 7, 1851, 33-45).Concise revision; annnotated generic list; no descr. or keys to spp.

-, On the Menispermaceae (Ann. Mag. Nat. Hist. III, 13, 1864, 1-15, 122-135, 315-323, $486-491 ; 14,1865,97-103,252-261,363-374)$.

-, A complete monograph of the Menispermaceae (Contr. Bot. 3, 1864-71, 1-402, pl. 88-154).

Miquel, F. A. W., Ann. Mus. Bot. Lugd.-Bat. 4 (1868) 80-88.

\section{Local}

Riu Kiu: Yамamoto, Trans. Nat. Hist. Soc. Formosa 29 (1939) 195-200.

Java: BACKER, Schoolfl. Java (1911) 37-47.

Philippines: Diels in Elmer, Leafl. Philip. Bot. 4 (1911) 1161-1167.-Synopsis of Philip. spp. 一, Philip. J. Sc. 8 (1913) Bot. 157-158.-3 new spp.

ELMER, Leafl. Philip. Bot. 10 (1939) 3760-3762. -New Tinospora.

New Guinea: Diels, Nova Guinea 8 (1910) 283; 8 (1912) 869; 14 (1924) 80.

-, Bot. Jahrb. 52 (1914) 187-190.

Kanehira \& Hatusima, Tokyo Bot. Mag. 56 (1942) 471-474.

\section{Genera}

Juppia $=$ Zanonia (Cucurbitaceae).

Peripetasma $=$ Dioscorea (Dioscoreaceae)

Stephania: Yamamoto, Trans. Nat. Hist. Soc. Formosa 26 (1936) 239-243.-Japan. \& Formosan spp.

\section{MIMOSACEAE}

See Leguminosae

\section{MOLLUGINACEAE}

See Aizoaceae

\section{MONIMIACEAE}

BecCARI, O., Malesia 1 (1877) 186-193.

Money, L. M., I. W. Bailey \& B. G. L. Swamy, The morphology and relationships of the Monimiaceae (J. Arn. Arb. 31, 1950, 372-404).

Perkins, J., Pflanzenreich, Nachtr. Heft 49 (1911) 1-67.

-, Einige neue Asiatische Monimiaceae (Bot. Jahrb. 45, 1911, 422-425).

-, Uebersicht über die Gattungen der Monimiaceae sowie Zusammenstellung der Abbildungen und der Literatur über die Arten dieser Familie bis zum Jahre 1925. Engelmann (Leipzig), 1925,55 pp., 47 fig.

- (\& GILG, E.), Pflanzenreich Heft 4 (1901) $1-122$.

Local

New Guinea: Gilg \& SCHLECHTER, Bot. Jahrb. 55 (1918) 195-201.

一, ibid. 58 (1923) 244-248.

Kanehira \& Hatusima, Bot. Mag. Tokyo 56 (1942) 249-262.

Perkins, Nova Guinea 8 (1912) 867-868.

-, Bot. Jahrb. 52 (1912) 191-218.

Sмith, J. Arn. Arb. 22 (1941) 231-252.-No key. Queensland: FrancIS, Kew Bull. (1931) 457-458.

\section{Genera}

Levieria (Dryadodaphne, Isomerocarpa): KosteRMANS, Rec. Trav. Bot. Néerl. 34 (1937) 605.

Moore, J. Bot. 61 (1923) Suppl. 109.-Descr. Dryadodaphne in Laur.

Smith, A. C., J. Arn. Arb. 23 (1942) 442-443.S. maintains that Dryadodaphne is different from Levieria.

\section{MONOTROPACEAE} See Ericaceae

\section{MORACEAE}

For Ficus see below

Miquel, F. A. W., Ann. Mus. Bot. Lugd.-Bat. 3 (1867) 210-214.

RENNER, O., Beiträge zur Anatomie und Systematik der Artocarpeen und Conocephaleen, insbesondere der Gattung Ficus (Bot. Jahrb. 39, $1907,319-448$ ).

Trécul, A., Mémoire sur la famille des Artocarpées (Ann. Sc. Nat. Bot. III, 8, 1847, 38-157, t. 1-6). 


\section{Local}

Borneo: Merrill, Plant. Elm. Born. (1929) 45-50. - List.

Philippines: Merrill, Philip. J. Sc. 19 (1921) 49-69.-New spp.

Warburg, in Perkins, Fragm. Fl. Philip. (1905) 165-167.

New Guinea: Diels, Bot. Jahrb. 67 (1935) 171-177. WhITE, Proc. R. Soc. Queensl. 34 (1922) 23.List.

\section{Genera}

Antiaris: BLume, Rumphia 1 (1836) 46-59, 171-173. Merrill, Philip. J. Sc. 2 (1907) Bot. 111-112.

Artocarpus: BecCARI, Nelle For. Borneo (1902) 625-636.-Born. \& Pap. spp.

CORNer, Gard. Bull. Str. Settl. 10 (1939) 56-81. Elmer, Leaft. Philip. Bot. 2 (1909) 609-626.Synopsis \& key to Philip. spp.

FOSBERG, J. Wash. Acad. Sc. 31 (1941) 93.Nomencl.

KING, Ann. R. Bot. Gard. Calc. 2 (1889) 1-16, t. 1-14.-Revision Ind. spp.

Quisumbing, Philip. J. Sc. 72 (1940) 331-335.A. camansi BlanCo.

Morus: Horto, Jap. J. Bot. 52 (1938) 363-368.Syst. cult. Jap. Morus.

NaKaI, J. Arn. Arb. 8 (1927) 234-238.

Parartocarpus: SMITH, Bull. Jard. Bot. Btzg III, 4 (1932) 233-234; ibid. 6 (1924) 80.

Paratrophis: vaN SteENIS, J. Bot. 72 (1934) 89.Syn. P. glabra.

Poikilospermum (Conocephalus): Merrill, Contr. Arn. Arb. 8 (1934) 47-52.-Complete list. Miquel, Ann. Mus. Bot. Lugd.-Bat. 1 (1864) 203.-Type descr.

PetrucCI, Nuovo Giorn. Bot. Ital. n.s. 9 (1902) 213-230.-Revision.

Taxotrophis: Hutchinson, Kew Bull. (1918) 147-152.-Revision; key!

\section{Ficus}

Barrett, M. F., Bull. Torr. Bot. Club 73 (1946) 321-325.-Misc. notes.

-, ibid. 76 (1949) 53-64.-Syn. F. retusa.

Corner, E. J. H., A revision of the Malayan species of Ficus: § Covellia and § Neomorphe (J. Mal. Br. R. As. Soc. 11, 1933, 1-65).

-, A revision of Ficus, subg. Synoecia (Gard. Bull. Str. Settl. 10, 1939, 82-161).

Diess, L., Ueber ungewöhnliche Abwandlungen des Ficus-Rezeptaculums (Flora 28, 1933, 2833).-Dammaropsis reduced to Ficus.

KING, G., Observations on the genus Ficus with special reference to the Indo-Malayan and Chinese species (J. Linn. Soc. Bot. 24, 1887, 27-44).

- The species of Ficus of the Indo-Malayan and Chinese countries (Ann. R. Bot. Gard. Calc. 1, 1,1887 , xiii, 1-66; ibid. 1, 2, 1888, 67-185).Almost a revision.

Miquel, F. A. W., Prodromus monographiae Ficuum (Hook. Lond. J. Bot. 6, 1847, 514-588; ibid. 7, 1848, 64-78, 109-116, 221-236, 451-471).
-, Annotationes de Ficus speciebus (Ann. Mus. Lugd.-Bat. 3, 1867, 214-235, 260-300).-No keys.

SATA, N., A monographic study of the genus Ficus from the point of view of economic botany (Contr. Inst. Hort. \& Econ. Bot., Fac. of Agric. Taihoku Imp. Univ. no 32, 1944, 289 pp.).-Non vidi.

\section{Local}

Sumatra: KING, J. As. Soc. Bengal 56, ii (1888) 65-67.-FORBES coll.

Java: BACKER, Blumea 6 (1948) 302-309.Critical list.

Borneo: Merrill, J. Str. Br. R. As. Soc. no 76 (1917) 80-81.

Celebes: Teysmann \& de Vriese, Nat. Tijd. Ned. Ind. 23 (1861) 212-214.-Bosscheria n.g. (=Ficus).

Philippines: Elmer, Leafl. Philip. Bot. 1 (1906) 187-205.-Misc. spp. from Leyte.

- , ibid. 1 (1906) 42-62.-Misc. spp. from Benguet.

-, ibid. 1 (1907) 236-261.-Misc. spp. from Tayabas.

-, ibid. 2 (1908) 531-551.-Misc. spp. from S. Negros.

-, ibid. 4 (1911) 1232-1270.-Misc. spp. from

Davao.

-, ibid. 4 (1911) 1307-1325:-Misc. spp. from Sibuyan.

-, ibid. 4 (1912) 1363-1397.-Misc. spp. from Palawan.

-, ibid. 7 (1914) 2359-2415.-Misc. spp. from

N. Agusan.

-, ibid. 9 (1937) 3397-3487.-Misc. spp. from Sorsogon.

Quisumbing, Philip. J. Sc. 41 (1930) 316-317.3 spp.

Warburg, in Perkins, Fragm. Fl. Philip. (1905) 194-202.-New spp.

Moluccas: Merrill, Philip. J. Sc. 11 (1916) Bot. 264-265.-2 new spp. from Ambon.

New Guinea: DiELs, Bot. Jahrb. 67 (1935) 177235.-Keys.

KING, J. As. Soc. Beng. 55, ii (1886) 394-411.Forbes, Beccari, D'Albertis coll.

-, Ann. R. Bot. Gard. Calc. I, App. 1 (1888) 1-9.

-, J. As. Soc. Beng. 56, ii (1888) 61-65.BeCCARI \& D'Albertis coll.

ReCHINGER, in FedDe, Rep. 11 (1912) 179181.-Some new spp., partly from the Solomon Isl. Summerhayes, J. Arn. Arb. 10 (1929) 142-154, 206-209.-BRASS coll.

- ibid. 14 (1933) 62.-Some corrig.

-, ibid. 22 (1941) 81-109.-List Brass coll.

Solomon Islands: RECHINGER, in FEDDE, Rep. 11 (1912) 179-181.-New spp.

Micronesia: Diels, Bot. Jahrb. 69 (1938) 398-400. - Key.

\section{MORINGACEAE}

Pax, F., Pfianzenfamilien ed. 2, 17b (1936) 693-698. 
Steenis, C. G. G. J. vaN, Fl. Mal. I, 4 (1949) 45-46, 1 fig.

\section{MUSACEAE \\ (excl. Lowiaceae)}

BAKER, J. G., A synopsis of the genera and species of Musaceae (Ann. Bot. 7, 1893, 189222).

LOESENER, TH., Ueber Musaceen, im besonderen uber Heliconia (Verh. Bot. Ver. Prov. Brandenb. 57, 1915-1916, 211-214).

SchumanN, K., Pflanzenreich Heft 1 (1900) 1-45, fig. 1-10.

Winkler, Hub., Pflanzenfamilien ed. 2, 15a (1930) 505-541, fig. 224-235.

-, Pflanzenareale 1. Reihe, Heft 2 (1926) maps 11-12.

\section{Local}

Sumatra: Ridley, Kew Bull. (1926) 90.-Descr. Musa sumatrana.

Malay Peninsula: Ridley, J. Str. Br. Roy. As. Soc. no 32 (1899) 182-183.-Enum.

-, Mat. Fl. Mal. Pen. (Monocot.) pt 2 (1907) 62-64.-Key.

Java: BACKer, Handb. Fl. Java 3 (1924) 130-141.

Philippines: QuisumbIng, Philip. Agric. Rev. 12 (1919) 1-90.

Teodoro, Philip. J. Sc. 10 (1915) Bot. 379-421.

New Guinea: Lauterkach, Bot. Jahrb. 50 (1913) 306-307.

MUELleR, Vict. Nat. 13 (1896) 53-55.

\section{Genera}

Heliconia: Backer, Bull. Jard. Bot. Btzg III, 2 (1920) 315-319.-Key H. indica \& bihai.

Lowia. See Lowiaceae.

Musa: BAKER, Kew Bull. (1894) 229-314.-Repr. as Kew Bull. Addit. Ser. no 6 (1906) 1-88, 9 fig. Cheesman, Kew Bull. 1947 (1948) 97-117; ibid. (1948) 11-28, 145-157; ibid. (1949) 24-28, 133-137, 445-452; ibid. (1950) 27-31, 151-155. -Critical descr. \& notes.

MacDaniels, Bern. P. Bish. Mus. Bull. 190 (1947) 56 pp.-Fe'Banana in connection with migrations in Polynesia. -

Quisumbing, Philip. Agric. Rev. 12 (1919) 1-90. -Cult. var.

RANT, Nat. Tijd. Ned. Ind. 94 (1934) 103-111.$M$. troglodytarum from Moluccas.

Simmonds, Kew Bull (1953) 571-574 (1954).Descr. 2 new spp., one from New Guinea, one from the Solomon Islands.

Ravenala: Winkler, Pflanzenfamilien ed. 2, 15a (1930) 532-533.-Key.

\section{MYOPORACEAE}

Bloembergen, S., The genus Myoporum in the Netherlands Indies (Blumea 3, 1938, 179-182). -, Fl. Mal. I, 4 (1951) 265-266, fig. 1.

KraenzlnN, F., Beiträge zur Kenntnis der Familie der Myoporinae R.BR. (in FEDDE, Rep., Beihefte 54, 1929, 129 pp.).-Revision; keys.

\section{Local}

New Guinea: Kraenzlin, in Fedde, Rep. 22 (1926) 338.

\section{MYRICACEAE}

BACker, C. A., Fl. Mal. I, 4 (1951) 276-279, fig. 1. Chevalier, A., Monographie des Myricacées (1901-1902) 1-257 (Repr. from Mém. Soc. Nat. Sc. Math. \& Nat. Cherbourg 32, 1901, 85-340).

Vloten-van den Berg, O. VAN \& Vloten, $H$. VAN, in KIRCHNER, LOEW \& SCHROETER, Lebensgeschichte der Blütenpflanzen Mitteleuropas 2, 1 (1928) 559-599.

\section{Local}

New Guinea: Markgraf, Bot. Jahrb. 59 (1925) 540.

\section{MYRISTICACEAE}

BuUME, C. L., Rumphia 1 (1836) 174-191.-List. WARBURG, O., Zur Charakterisierung und Gliederung der Myristicaceen (Ber. Deut. Bot. Ges. 13, 1895, 82-95).

-, Monographie der Myristicaceen (Nova Acta Acad. Caes. Leop.-Carol. Nat. Cur. 68, 1897, $1-680$, t. 1-25).

\section{Local}

Malay Peninsula: Henderson, Gard. Bull. Str. Settl. 7 (1933) 120, t. 30.-New Horsfieldia. AIRY SHAW, Kew Bull. (1948) 251-252.-New Myristica.

Borneo: Diels \& Hackenberg, Bot. Jahrb. 60 (1926) 308.-New Myristica.

Alry. Shaw, Kew Bull. (1939) 439-445.-List Sarawak Exp.

Philippines: Elmer, Leafl. Philip. Bot. 3 (1911) 1057-1066.-New spp.

一, ibid. 10 (1939) 3763-3764.-New Horsfieldia. Merrill, Philip. J. Sc. 30 (1926) 394-395.New Sulu Knema.

Quisumbing \& Merrill, Philip. J. Sc. 37 (1928) 144-145.-New Palawan Knema.

Warburg, in Perkins, Fragm. Fl. Philip. (1904) 49-50.-New records.

New Guinea: Markgraf, Bot. Jahrb. 67 (1935) 143-170.-Keys.

一, J. Arn. Arb. 10 (1929) 77.-New var. of Myr. fatua.

Pulle, Nova Guinea 8 (1911) 635-637.

Smith, J. Arn. Arb. 22 (1941) 60-80.-New spp.

Micronesia: KanehiRA, J. Dep. Agr. Kyushu Imp. Univ. 4 (1935) 319-320.-List.

Markgraf, Bot. Jahrb. 69 (1938) 395-397.Keys.

\section{Genera}

Myristica: KING, Ann. R. Bot. Gard. Calc. 3 (1891) 275-331, t. 106-174.-Rev. Ind. spp. MiQuel, Ann. Mus. Bot. Lugd.-Bat. 1 (1864) 205-207.-List.

-, ibid. 2 (1865) 46-51.-Completed list. Smith, Bull. Torr. Bot. Club 68 (1941) 397-406. -Rev. Pac. sp.; key. 


\section{MYRSINACEAE}

Blume, C. L., Aegicereae (in Hoeven \& De VRIEse, Tijd. Nat. Geschied. \& Phys. 1, 1834, 147-153).-Census; descr. listed; in Latin.

Candolle, A. De, Review of the Natural Order Myrsineae (Trans. Linn. Soc. 17, 1834, 95138).-No keys.

Grosze, A., Anatomisch-systematische Untersuchungen der Myrsinaceen (Bot. Jahrb. 41, 1907, Beibl. 96, 1-46, fig. 1-31).-Types of indument, epidermis, etc.

MEz, C., Pflanzenreich Heft 9 (1901) 1-437, fig. 1-61.-See for additions under New Guinea.

NakaI, T., Nova Flora Japonica: 'Ardisiaceae'. Tokyo \& Osaka (1943) 1-170, fig. 1-42.-In Latin and Japanese. Nomenclature deviates widely from the usual one.

SCHefFer, R. H. C. C., De Myrsinaceis Archipelagi Indici. Thesis, Utrecht (1867) 1-113, 1 tab. -In Latin; keys, descr.

-, Nat. Tijd. Ned. Ind. 31 (1870) 363-375.-List of records; some new spp.

-, ibid. 32 (1871) 419-426.

\section{Local}

East Asia: Walker, Philip. J. Sc. 73 (1940) 1-258. -Revision; keys.

-, J. Arn. Arb. 23 (1942) 344-355, 2 fig.Additions.

Siam: Fletcher, Not. R. Bot. Gard. Edinb. 20 (1948) 106-120.-Concise revision; keys; no references. For descr. $c f$. Kew Bull. (1936) 42-47; ibid. (1937) $26-40$ and enum. in Fl. Siam. En. 2 (1938) 325-352.

Sumatra: Merrill, Contr. Arn. Arb. 8 (1934) 129-134.-Important list.

-, Pap. Mich. Acad. Sc. 19 (1934) 184-186. -Embelia, Maesa n. sp.

Ridley, Kew Bull. (1926) 71-72.-2 new Ardisias.

Borneo: Merrill, Philip. J. Sc. 30 (1926) 85.New Ardisia.

_, Sarawak Mus. J. 3 (1928) 543-545.-2 new Ardisias.

- P1. Elm. Born. (1929) 234-237.-3 new

Ardisias listed \& records.

Philipson, J. Bot. 77 (1939) 101-106.-Important list.

RIDLEY, Kew Bull. (1934) 120.-New sp.

AIry Shaw, Kew Bull. (1939) 411-417 (sphalm. 511-517).-List RICHARDS coll.

Philippines: Elmer, Leafl. Philip. Bot. 2 (1908) 439-444.-New spp.

-, ibid. 2 (1910) 659-675.-Ditto.

-, ibid. 10 (1939) 3764-3766.-Ditto.

Merrill, Philip. J. Sc. 5 (1910) Bot. 212-223.New spp.

-, ibid. 12 (1917) Bot. 143-158.-New spp. of Ardisia, Discocalyx, and Maesa.

—, ibid. 26 (1925) 484 485.-Ardisia New sp.

-, ibid. 27 (1925) 45-46. -Embelia n. sp.

-, ibid. 30 (1928) 420-421.-New Discocalyx.

_, ibid. 41 (1930) 341-342.-New Maesa.

Mez, Philip. J. Sc. 1 Suppl. (1906) 271-276.
-, in FEDdE, Rep. 3 (1906) 97-104.-1 new Maesa, 4 Ardisias, 1 Amblyanthopsis, 1 Discocalyx.

-, ibid. 16 (1920) 309-312, 410-425.-3 new Maesa, 13 Ardisias, 3 Discocalyx, 2 Jubilarias, 2 Embelias, 1 Rapanea.

Quisumbing, Philip. J. Sc. 41 (1930) 341-343.Maesa n. sp.

一, ibid. 76 (1944) 52-53.-Loheria n. sp.

— \& MERRILL, ibid. 37 (1928) 185-187.-Maesa \& Discocalyx n. sp.

Moluccas: Merrill, Philip. J. Sc. 11 (1916) Bot. 300-302.-Ambon records; new Maesa.

New Guinea: Kanehira \& Hatusima, Tokyo Bot. Mag. 57 (1943) 215-234.-Many new spp.

MEz, Bot. Arch. 1 (1922) 125-128, 230, 255-256. -New spp. in Maesa, Ardisia, Conandrium. 一, ibid. 2 (1922) 34, 173-176, 210-212, 259-260. -New spp. of Ardisia, Conandrium, Discocalyx, Loheria, Grenacheria, Rapanea.

-, ibid. 6 (1924) 131-132.-New spp. in Maesa \& Rapanea.

WhITE, J. Bot. 65 (1927) 248.-New Ardisia.

-, J. Arn. Arb. 10 (1929) 257-258.-Listed LANE-POOLE coll.

\section{Genera}

Abromeitia: MEz, Bot. Arch. 1 (1922) 100.

Apoia $=$ Sarcosperma (Sarcospermataceae) .

Ardisia: Walker, J. Wash. Acad. Sc. 29 (1939) 256-261.-Key to A. crispa \& crenata.

Embelia: Walker, Lingn. Sc. J. 10 (1931) 475480.-Embelia scandens and allies; key.

Fittingia: Mez, Bot. Arch. 1 (1922) 105-106.

Labisia (Angiopetalum): VAN STEENIS, FI. Mal. Bull. 4 (1948) 109; Bull. Bot. Gard. Btzg III, 17 (1948) 459.-Prop. cons. Labisia.

Rapanea (Hunsteinia), sec. Hatusima, June 1944, unpublished.

Tapeinosperma: Philipson, J. Bot. 77 (1939) 102-103.-Bornean spp.

\section{MYRTACEAE}

Burret, M., Myrtaceen Studien I (Notizbl. Berl.Dahlem 15, 1941, 479-550).-Crit. notes; mostly extra-Malaysian, many New Caledonian; list of Rhodomyrtus; no keys.

-, Myrtaceen Studien II (in Fedde, Rep. 50, 1941, 50-60).-Extra-Malaysian; few New Caled.

Candolle, A. P. DE, Mémoire sur la famille des Myrtacées (Mém. Soc. Phys. \& Hist. Nat. Genève $9,1841,301-361$, t. 1-22).-General discussion various genera.

Korthals, P. W., Bijdrage tot de kennis der Myrtaceae (Ned. Kruidk. Arch. 1, 1846, 185 205).-List with descr. of new spp.

Vriese, W. H. DE, Plant. Ind. Batav. Orient. (1856) 63-79, pl. 5.-Listed records.

\section{Local}

India: Gamble, J. Ind. Bot. Soc. 1 (1919) 60.Short diagnoses of some SE. Ind. spp.

China: Merrill \& Perry, J. Arn. Arb. 19 (1938) 191-247.-Rev. of fam.; keys. 
Indo-China: Merrill \& Perry, J. Arn. Arb. 19 (1938) 99-116.-List Syzygium; no keys.

Mal. Peninsula: Henderson, Gard. Bull. Sing. 11 (1947) 299-338, 18 fig.-New spp. Eugenia. -, ibid. 12 (1949) 1-293, 54 fig.-Important revision of 138 spp. and var. of Eugenia (Syzygium); keys.

—, ibid. 14 (1953) 1-3.-New Tristania.

Java: AmshoFF, Blumea 5 (1945) 495-502.-List Jav. spp. under Syzygium.

BACKER, Schoolfl. Java (1911) 494-532.Key Jav. spp.

Borneo: Merrill, J. Str. Br. R. As. Soc. no 77 (1917) 206-231.-Descr. mainly Eugenia \& Tristania.

-, ibid. no 79 (1919) 19-27.-List with new spp. -, ibid. no 86 (1922) 335-337.-New Eugenia \& Xanthomyrtus.

-, Sarawak Mus. J. 3 (1928) 532-534, 537 (partim).-Listed records.

-, Mitt. Inst. Allg. Bot. Hamburg 7 (1937) 269.-Small list.

Merrill \& Perry, Mem. Am. Acad. Arts \& Sc. 18 (1939) 135-202.-Rev. Born. spp.; keys; descr.

Miquel, Anal. Bot. Ind. 1 (1850) 12-29, t. 1-10 repr. from Verh. Kon. Ned. Inst. 1e kl. $3 e$ reeks, vol. 3.-List \& new spp.

RidLey, J. Bot. 68 (1930) 10-17, 33-39,-List of Eugenias \& Tristanias.

AIRY SHaw, Kew Bull. (1949) 117-125.-In Syzygium 4 new spp. and several new comb.; key to Aphanamyrtus group.

Philippines: MaIden, Proc. U. S. Nat. Mus. 26 (1903) 691-692.-Identification Eucalyptus.

ElMER, Leafl. Philip. Bot. 4 (1912) 1399-1444.Descr. Eugenia spp.

-, ibid. 7 (1914) 2343-2358.-Ditto; various Myrt.

一, ibid. 10 (1939) 3767-3770.

Merrill, Philip. J. Sc. 10 (1915) Bot. 207-225.

-New spp. of Eugenia.

-, ibid. 11 (1916) Bot. 21-25.-Ditto.

-, ibid. 19 (1921) 289-308.

-, ibid. 27 (1925) 39-43.-4 new Eugenias.

-, ibid. 79 (1950) 351-430.-Nomenclatural readjustments under Syzygium.

Quisumbing, Philip. J. Sc. 41 (1930) 337-340.2 new Eugenias.

— \& MERriLl, ibid. 37 (1928) 175-177.-New Eugenia \& Tristania.

Moluccas: Merrill, Philip. J. Sc. 11 (1916) Bot. 296-297.-New Eugenia from Ambon.

New Guinea: Diels, Bot. Jahrb. 57 (1922) 356426, 1 fig.-Keys.

-, Nova Guinea 14 (1924) 85-96.

LAuterbach, Nova Guinea 8 (1910) 319-323. 一, ibid. 8 (1912) 849-855.

Merrill \& Perry, J. Arn. Arb. 23 (1942) 233265, 267-297.-Many new spp.; no keys.

Rechinger, in Fedde, Rep. 11 (1912) 183.-

Some new Syzygiums.

White, Proc. R. Soc. Queensl. 34 (1922) 47-48. -Listed records.

—, J. Arn. Arb. 23 (1942) 79-92, 1 pl.-Mostly capsular-fruited genera; many new spp.; no keys.

—, ibid. 32 (1951) 139-149, pl. 1.-New spp. \& records, incl. Moluccas and Solomon Isl.

N. Australia: CheEL and MAIDEN, in EWART \& DAvies, Fl. North. Terr. (1917) 203-210, 290 317.-Eucalyptus; key to infraspec. taxa in $\mathrm{Me}$ laleuca leucadendron.

New Caledonia: Guillaumin, Bull. Soc. Bot. Fr. 81 (1934) 3-17.

-, ibid. 85 (1939) 626-653.

Fiji: Perry, J. Arn. Arb. 31 (1950) 350-371.

\section{Genera}

Acmena (Xenodendron): Merrill \& Perry, J. Arn. Arb. 19 (1938) 1-20.-Synopsis.

Aphanomyrtus (Pseudoeugenia): KOORDERs \& Valeton, Ann. Jard. Bot. Btzg Suppl. II (1898) 145-151, Taf. 6-8.-A segregate from Eugenia.

Merrill, Blumea Suppl. 1 (1937) 107-111.

Airy Shaw, Kew Bull. (1949) 121.-Key.

VALETON, in FEDDE, Rep. 4 (1907) 234-235.

Basisperma: White, J. Arn. Arb. 23 (1942) 84-85.

Cleistocalyx (Acicalyptus): Merrill \& PERRY, J. Arn. Arb. 18 (1937) 322-344, 1 pl.-Revision; no key. Segregate of Eugenia s.l.

Cloezia: Baker $f$., J. Linn. Soc. Lond. Bot. 45 (1921) 309-310.-New Caled. spp.; key.

Eucalyptopsis: Whrte, J. Arn. Arb. 32 (1951) 139-144, pl. 1 .

Eucalyptus: BLAKE, Austr. J. Bot. 1 (1953) 185352,35 pl.-Principally N. Australian, but also considering Mal. spp.; keys.

BlAKely, A key to the Eucalypts, with descriptions of 500 species and 138 varieties; and a companion to J. H. MAIDEN's Critical Revision of the genus Eucalyptus. Sydney (1934) 339 pp. Burbidge, Trans. R. Soc. South Austr. 71 (1947) 137-167.-Key to S. Austr. spp.

MAIDEN, A critical revision of the genus Eucalyptus. Sydney (1903-1933) 8 vols, 75 pts. -For key see BLAKELY.

Mueller, J. Linn. Soc. Bot. 3 (1859) 81-101.Conspectus; descr. new sp.; no key.

Eugenia (Jambosa): Several authors maintain Eugenia s.l. as a genus. See Henderson in Malaya. Diels and Merrill \& Perry advocate splitting Mal. spp. into Syzygium, Jossinia, Acmena, Cleistocalyx, Tetraeugenia, Aphanomyrtus. Gagnepain, Bull. Soc. Bot. Fr. 64 (1917) 94 103.-Discussion characters for delimitation of spp.

Jossinia: Merrill, J. Arn. Arb. 31 (1950) 329-333. —, Philip. J. Sc. 79 (1950) 356-360.-Reinstatement of Jossinia.

Kjellbergiodendron: BurReT, Notizbl. Berl.-Dahlem 13 (1936) 101-106, fig. 5

MerRill, J. Arn. Arb. 33 (1952) 162-165. VAN Steenis, Act. Bot. Neerl. 1 (1952) 440 442, fig. 2.-Revision.

Melaleuca: VAN SteENIS, Tectona 32 (1939) 863-864.-Some records \& fig.; in Dutch; no keys. 
Octamyrtus: Diels, Bot. Jahrb. 57 (1922) 373-376. White, J. Arn. Arb. 32 (1951) 143-147.-Key.

Osbornia: van SteEnIS, Trop. Natuur 25 (1936) 194-196, fig. 1-2.

Psidium: Rant, Ann. Jard. Bot. Btzg 41 (1930) 27-32, tab. 8-12.

Syncarpia (Nania): Mrquel, J. Bot. Néerl. 1 (1861) 292-297, pl. 3.

Syzygium: Merrill \& Perry, Mem. Am. Acad. Arts \& Sc. 18 (1939) 135-202.-Rev. Born. spp. -, J. Arn. Arb. 19 (1938) 99-116.-Rev. Indo-Chin. spp.

Tetraeugenia (a segregate from Eugenia): MERRILL, J. Str. Br. R. As. Soc. no 77 (1917) 230-231.

AIRY Shaw, Kew Bull. (1949) 123.

Xanthomyrtus: Diels, Bot. Jahrb. 57 (1922) 362-367.

MerrilL, Philip. J. Sc. 30 (1926) 415-416.

-, Sarawak Mus. J. 3 (1928) 533.

Xanthostemon: GUGerLI, in FEDDE, Rep. Beih. 120 (1940) 1-149, t. 1-16. Also Thesis, Zürich. -Keys; descr.; unsatisfactory.

Merrill, J. Arn. Arb. 33 (1952) 150-162.Corrections for latter paper.

Whiteodendron: van SteEnIs, Act. Bot. Neerl. 1 (1952) 436-440, fig. 1.-New genus from Borneo.

\section{NAJADACEAE}

Rendle, A. B., A systematic revision of the genus Najas (Trans. Linn. Soc. Bot. II, 5, 1899, $379-436$; ibid. $1900,437-444$, t. 39-42).Monograph; keys!

-, Pflanzenreich Heft 7 (1901) 1-21, fig. 1-5.

\section{Local}

Java: BACKER, Handb. Fl. Java 1 (1924) 51.-Key; in Dutch.

Kосн, Ber. Schweiz. Bot. Ges. 44 (1935) 339342.-New spp.

Bali: van SteEnIs, Arch. Hydrobiol. Suppl. Bd 11 (1932) 271-272, fig. 2.-Najas marina.

\section{Genera}

Najas: Braun, J. Bot. 2 (1864) 274-279, 2 fig.Revision.

Mikı, Tokyo Bot. Mag. 49 (1935) 773-778, fig. 6-8.-Key to Japan. spp.

\section{NEPENTHACEAE}

Beccari, O., Revista delle Specie del Genere Nepenthes (Malesia 3, 1886, 1-15, t. 1-3).List; no key.

DANSER, B. H., The Nepenthaceae of the Netherlands Indies (Bull. Jard. Bot. Btzg III, 9, 1928, 249-438, fig. 1-36).-Complete revision of Mal. spp.; key.

Harms, H., Pflanzenfamilien ed. 2, 17 b (1936) 728765, fig. 453-472.

Korthals, P. W., in Temminck, Verh. Nat. Gesch. Bot. (1840) 1-44, t. 1-4, 13-15, 20-22.

MacFarlane, J. M., Pflanzenreich Heft 36 (1908) 1-92, fig. 1-19.-Monograph.

\section{Local}

Sumatra: DANSER, Bull. Jard. Bot. Btzg III, 13 (1935) 465-467.-New sp. S. Sumatra.

一, ibid. 16 (1940) 268-271.-New sp. N. Sumatra.

Miquel, Ill. Fl. Arch. Ind. 1 (1870) 1-8, t. 1-6.List; $n$. spp.

Borneo: DaNSER, Mitt. Inst. Allg. Bot. Hamburg 7 (1931) 217-221, 1 fig.-List 4 spp.

一, Bull. Jard. Bot. Btzg III, 13 (1935) 467469.-Crit. notes.

Hooker $f$., Trans. Linn. Soc. 22 (1859) 415-424, t. 69-74.-Synopsis; no key.

MACFARLANE, Kew Bull. (1925) 35-37.-New sp. (N. decurrens).

Philippines: MacFarlane, Contr. Bot. Lab. Univ. Penns. 3 (1911) 207-210, t. 1-2.-2 new spp.

-, Philip. J. Sc. 33 (1927) 127-140.-Revision; key.

New Guinea: MacFarlane, Nova Guinea 8 (1911) 339-341.

\section{NYCTAGINACEAE}

Hetmerl, A., Pflanzenfamilien ed. 2, 16c (1934) 86-134, 587.

SkotTsberG, C., Act. Hort. Gothob. 2 (1926) 231-232.-Replaces Calpidia by Ceodes.

StandLeY, P., The Nyctaginaceae of Northwestern South America (Publ. Field Mus. 11, 1931, 73-114).-Keys.

\section{Local}

New Guinea: Merrill \& Perry, J. Arn. Arb. 20 (1939) 327-329.-4 Ceodes.

Pulle, Nova Guinea 8 (1910) 353.

-, ibid. 8 (1912) 629-630.

VAleton, Bot. Jahrb. 52 (1915) 101-103.

Hawail: SkotTsberg, Svensk Bot. Tidskr. 30 (1936) 722-743, 6 fig.; ibid. 35 (1941) 364.Arbor. spp.

Polynesia: Heimerl, Occ. Pap. Bern. P. Bish. Mus. 13 (1937) 27-47.-List.

\section{Genera}

Boerhaavia: BACKER, Onkruidfl. Jav. Suiker. (1930) 233-237.-Key Jav. spp.

Bougainvillea: HeIMERL, Denkschr. Akad. Wiss. Wien 70 (1900) 98-124.-Key; descr.

Ceodes (Calpidia): HeImerL, Oester. Bot. Zeitschr. 63 (1913) 279-290.-List.

Pisonia: Bargagli-Petrucci, Nuovo Giom. Bot. Ital. new ser. 8 (1901) App. 603-625, t. 4.Rev. Mal. spp.; key.

AIRY Shaw, Kew Bull. (1952) 87-97, map.Syn.; distr.; ecol. $P$. grandis with crit. notes on other spp.

St John, Webbia 8 (1951) 225-228, 1 fig.Distr. $P$. grandis.

\section{NYMPHAEACEAE}

Caspary, R., Nymphaeaceae (in MiQ. Ann. Mus. Bot. Lugd.-Bat. 2, 1866, 241-256).-Also extra-Mal.; no key. 
Henkel, F., F. Rehnelt \& L. Ditmann, Das Buch der Nymphaeaceen. Darmstadt (1907) 1-158, plates and many fig. - Monograph; uses; cultivation methods; new species; keys.

Planchon, J. E., Étude sur les Nymphéacées (Ann. Sc. Nat. III, Bot. 19 (1853) 17-63.Census of genera \& spp.; no key.

-, Enumération succincte des espèces de la famille des Nymphéacées (Revue Hortic. Paris IV, 2, 1853, 62-68).-Conspectus.

\section{Local}

Java: BACKER, Schoolfi. Java (1911) 48-51.

New Guinea: Merrill \& Perry, J. Arn. Arb. 23 (1942) 388-390.

Pulle, Nova Guinea 8 (1910) 361.

\section{Genera}

Barclaya: MIQueL, Ill. Fl. Arch. Ind. (1870) 43-44. -2 spp.; no key.

Wallich, Trans. Linn. Soc. Lond. 15 (1827) 442-448, t. 18.-Type descr.

Brasenia: vaN DER MEer MoHR, Trop. Natuur 26 (1937) 33-34.

Cabomba: FassetT, Castanea 18 (1953) 116-128. -Monograph; key.

Nymphaea: Conard, Publ. Carnegie Inst. Wash. 4 (1905) 1-279, t. 1-30.-Monograph.

DIETRICH, Allg. Gartenzeit. 21 (1853) 353-355, $364,370-372,379-380,386-388,395-396$, 406-407.

\section{NYSSACEAE}

Wangerin, W., Pflanzenreich Heft 41 (2) (1910) 1-20, fig. 1-4.

WAsscher, J., Nyssa in the Netherlands Indies (Blumea 1, 1935, 343-350).-Revision.

_, Fl. Mal. I, 4 (1948) 29-31, fig. 1.

\section{OCHNACEAE}

BartelletT, V., Studio monografico intorno alle famiglia delle Ochnaceae e specialmente delle Specie Malesi (Malpighia 15, 1901 (an 1902?) 105-174, t. 5-11).-No keys.

GiLG, E., Beiträge zur Kenntnis der Ochnaceae, besonders im Hinblick auf die neueste Bearbeitung dieser Pflanzenfamilie durch VAN Tieghem (Festschrift Ascherson 1904, 97-118). -, Pflanzenfamilien ed. 2, 21 (1925) 53-87.

Planchon, J. E., Sur le genre Godoya et ses analogues, avec des observations sur les limites des Ochnacées et une revue des genres et especes de ce groupe (in Hook. Lond. J. Bot. 5, 1846, $584-600$, 644-656, pl. 19-22; ibid. 6, 1847, 1-31). -No keys.

Tieghem, P. VAN, Sur les Ochnacées (Ann. Sc. Nat. VIII, Bot. 16, 1902, 161-416).-Disc. of genera; no spp. mentioned; no keys.

-, Constitution nouvelle de la famille des Ochnacées (in MoroT, J. Bot. 16, 1902, 181-212).Delimitation of the family with a key to the genera.

\section{Local}

Borneo: Diels \& Hackenberg, Bot. Jahrb. 60 (1926) 310-311.-Euthemis, 2 records.
Ridley, Kew Bull. (1930) 76-77.-List records. AIRY Shaw, Kew Bull. (1940) 249-252.-List RICHARDS coll.; key to Neckia.

Philippines: Merrill \& Quisumbing, Philip. J. Sc. 82 (1953) 329-330, t. 2.-Neckia from Samar.

New Guinea: Pulle, Nova Guinea 8 (1911) 667.

Smith, J. Arn. Arb. 22 (1941) 524-528.-No key; Schuurmansia.

\section{Genera}

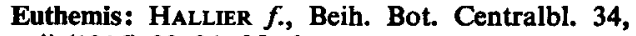
ii (1916) 30-34.-No key.

Neckia (?Gomphia): AIRY SHAw, Kew Bull. (1940) 252.-Key to spp.

Ouratea: Hallier $f$., Beih. Bot. Centralbl. 34, ii (1916) 34-35.-2 spp.; no key.

Schuurmansia: Hallier $f$., Rec. Trav. Bot. Néerl. 10 (1913) 345-351.-Rev.; no key.

Merrill, Philip. J. Sc. 11 (1916) Bot. 2, 19-20.Record \& new spp. from the Philippines.

MiQuel, Ill. Fl. Arch. Ind. (1870) 66-67.Moluccas, 1 sp.

Smith, J. Arn. Arb. 10 (1929) 524-528.-Descr. 4 Pap. spp.

Schuurmansiella: Hallier $f$, Rec. Trav. Bot. Néerl. 10 (1913) 344-345.-1 sp.

Ridley, Kew Bull. (1930) 77.

\section{OENOTHERACEAE See Onagraceae \\ OLACACEAE (incl. Erythropalaceae)}

Blume, C. L., Mus. Bot. Lugd.-Bat. 1 (1850) 245-251.-List, incl. also Icac., Opil.

Sleumer, H. O., Pflanzenfamilien ed. 2, 16b (1935) 5-32.

Valeton, Th., Critisch overzicht der Olacineae. Thesis, Groningen (1886) 1-280, t. 1-6.

\section{Local}

Java: BACKER, Schoolf. Java (1911) 220-230.Incl. also Icac., Opil.

Borneo: RIDLEY, Kew Bull. (1931) 33-35.-List; 3 new Olax spp.

Philippines: Elmer, Leafl. Philip. Bot. 10 (1939) 3770-3771.-New Strombosia.

New Guinea: Schellenberg, Bot. Jahrb. 58 (1923) 157-158.-Short list.

\section{Genera}

Erythropalum: Fagerlind, Svensk Bot. Tidskr. 40 (1946) 9-14.-Morph., embr.

QUISUMBING \& Merrill, Philip. J. Sc. 37 (1928) 143-144.-Aberrant Philip. sp.

Sleumer, Pflanzenfamilien ed. 2, 20b (1942) 401-403.-As a distinct family Erythropalaceae.

Fissipetalum = Erycibe (Convolvulaceae).

Phytocrene: MiQuel, Ann. Mus. Bot. Lugd.-Bat. 3 (1867) 247-248.-No key.

Schoepfia: ENGLER, Pflanzenfamilien, Nachtr. (1897) 145-146.-Sect. key.

VAN Steenis, Reinw. 1 (1952) 467-481, 2 fig.

Scorodocarpus: BeCCARI, Nuovo Giorn. Bot. Ital. 9 (1877) 100, 273-276, t. 11 (fig. 12-17).Descr. S. borneensis. 
Tropidopetalum $=$ Bouea (Anacardiaceae).

Ximenia: BecCari. Nuovo Giorn. Bot. Ital. 9 (1877) 276-279, t. 11 (fig. 1-11).-Record $X$. americana from New Guinea.

SARGENT, Trees \& Shrubs 2 (1913) 127-132, pl. 154.

\section{OLEACEAE}

DeCAISNE, J., Monographie des genres Ligustrum et Syringa (Nouv. Arch. Mus. Hist. Nat. Paris II, 2, 1879, 16-38, t. 1-3).-Descr.; no keys.

KNoblauch, E., Zur Kenntnis einiger OleaceenGenera (Bot. Centralbl. 61, 1894, 81-87, 129136).-Lists; no keys.

LINGELSHEIM, A., Oleaceae-Oleoideae-Fraxineae \& Syringeae (Pflanzenreich Heft 72, 1920, 1-125, fig. 1-11, 1-11, map).-Forsythia, Fraxinus, Schrebera, and Syringa.

\section{Local}

Siam: KerR, Kew Bull. (1938) 127-133.-New spp.

Sumatra: BAKHUIZEN VAN DEN BRINK Jr, Blumea 6 (1950) 383-384.-Jasm. multiflorum and its infraspecific taxa.

Merrill, Pap. Mich. Acad. Sc. 19 (1934) 186188.-2 new Jasminums, 1 new Linociera.

Merrill, Contr. Arn. Arb. 8 (1934) 136-137.Listed records.

Borneo: Fischer, Kew Bull. (1932) 294.-New Jasminum.

RidLeY, Kew Bull. (1934) 121-123.-3 new Jasminums; 1 Linociera.

AIRY SHAw, in Hook. Ic. Pl. 33 (1935) t. 3282.New Jasminum.

Philippines: Elmer, Leafl. Philip. Bot. 5 (1913) 1651-1657.-List Linociera.

—, ibid. 9 (1934) 3208-3209.-New Ligustrum.

Merrill, Philip. J. Sc. 27 (1925) 48-49.-New Linociera.

Quisumbing \& Merrill, Philip. J. Sc. 37 (1928) 187-190.-New Linociera, Jasminum, and Ligustrum.

Moluccas: Merrill, Philip. J. Sc. 11 (1916) Bot. 304-306.-2 new spp. from Ambon and Celebes.

New Guinea: KnoblaUCH, Notizbl. Berl.-Dahlem 11 (1934) 1029-1031.-New Linociera.

Kobusk1, J. Arn. Arb. 21 (1940) 328-335.List; descr.; no key.

LiNGELSHEM, Nova Guinea 14 (1927) 329-331.

- List.

-, Bot. Jahrb. 61 (1927) 1-22.-Keys.

WHITE, Proc. Linn. Soc. N.S.W. 51 (1926) 297.

-New Jasminum.

-, J. Arn. Arb. 10 (1929) 259.-3 spp. listed.

\section{Genera}

Fraxinus: Boerlage \& KoOrders, Nat. Tijd. Ned. Ind. 56 (1896) 185-190, t. 1-2.-E. Java.

LINGELSHEIM, Bot. Jahrb. 40 (1908) 185-223.Revision; no keys.

Wesmael, Bull. (Mém.) Soc. Bot. Belg. 31 (1892) 69-117.-Synopsis; no key.

Jasminum: KoBUskı, J. Arn. Arb. 13 (1932) 145-179.-Synops. Chin. spp.; keys.
Ligustrum: HöFKer, Mitt. Deut. Dendr. Ges. 24 (1915) 51-66, t. 1-6.-Revision; keys.

Mansfeld, Bot. Jahrb. 59 (1924) Beibl: 132, 19-75.-Precurs. notes to monogr.

Myxopyrum: Hill, Kew Bull. (1910) 37-44.Revision; key.

Schrebera: Kostermans, Reinwardtia 2 (1953) 360-362, fig. 2.-Borneo record; new sp.

Syringa: MCKelveY, S. D., The Lilac, a monograph. New York (1928) i-xiv, 1-581, t. 1-171.Complete monograph with keys to the spp.

\section{ONAGRACEAE \\ (Oenotheraceae)}

\section{Local}

Java: BACKer, Onkruidfl. Jav. Suiker. (1930) 467-472.-Key.

New Guinea: Diels, Bot. Jahrb. 62 (1929) 485 486.-2 new Epilobiums.

Pulle, Nova Guinea 8 (1910) 395.

一, ibid. 8 (1911) 681.

WARBURG, Bot. Jahrb. 16 (1892) 15, 23-24.Epilobium prostratum n. sp.

\section{Genera}

Epilobium: Haussknecht, Monographie der Gattung Epilobium. (1884) i-viii, 1-318, pl. 1-23.

LÉVEILlÉ, Iconographie du genre Epilobium. Le Mans (1910) 1-328, t. 1-272.-Unreliable work.

Fuchsia: Munz, Proc. Calif. Acad. Sc. IV, 25 (1943) 1-139, t. 1-16.-Revision; keys.

Jussieua: BACKER, Trop. Natuur 3 (1914) 55-63.Key Jav, spp.

Brenan, Fl. Trop. E. Afr. (Onagrac.) (1953) 6-20, fig. 3-4.-Key; critical treatment.

FAWCETT, J. Bot. 64 (1926) 10-13.-Interpr. Linnean $s p p$.

HARA, J. Jap. Bot. 28 (1953) 289-294.-Jussieua is reduced to Ludwigia; necessary recomb.

LEVEILLE, Bull. Soc. Bot. Fr. 54 (1907) 421-427.

-Revision; list; no key.

Livera, Ann. R. Bot. Gard. Perad. 9 (1924) 199-200.-Key to Ceylon spp.

Munz, Darwiniana 4 (1942) 179-284, t. 1-20, 2 fig., 9 maps.-Full monogr. of New World spp. RidLeY, J. Bot. 59 (1921) 257-260.-No key.

\section{OPILIACEAE}

Sleumer, H. O., Pflanzenfamilien ed. 2, 16b (1935) 33-43.

\section{Local}

Indo-China: GagnepaIn, Suppl. Fl. Gén. IndoChine (1946) 731-734.-Descr. \& key Lepionurus BL.

Philippines: MerriLl, Philip. J. Sc. 29 (1926) 477. -A new sp. of Melientha.

New Guinea: LAUTERBACH, Nova Guinea 8 (1912) 817-818.-Descr. Gjellerupia (= Lepionurus).

\section{Genera}

Lepionurus (Gjellerupia): Hatusima, Bot. Mag. Tokyo 65 (1952) 109-111.-Reduction. 


\section{ORCHIDACEAE}

(excl. Apostasiaceae)

Bentham, G., Notes on Orchideae (J. Linn. Soc. Lond. Bot. 18, 1881, 281-360).-System of the family with notes on genera; no descr. of spp.

Kraenzlin, F., Orchidacearum genera et species 1 (1901) i-viii, 1-986; 2, 1 (1904) 1-143, t. 1-16.With keys to spp. but not covering whole family. -, Pflanzenreich Heft 45 (1910) 1-382, fig. 1-35.Dendrobiinae: Dendrobium, Desmotrichum, Diplocaulobium, Sarcopodium.

-, ibid. Heft 50 (1) (1911) 1-182, fig. 1-35.Orchidaceae-Monandrae-Dendrobiinae: Dendrobium, Sarcopodium, Diplocaulobium, Desmotrichum, Eria, Trichotosia, Porpax, Chitonanthera. -, ibid. 50 (2) (1911) 1-46, fig. 1-5.-Orchidaceae, Monandrae-Thelasiinae: Phreatia, Thelasis.

LINDLEY, J., The genera and species of Orchidaceous plants $(1830-40) \mathrm{i}-\mathrm{xiii}, 1-553$.-Keys to the genera.

-, Folia Orchidacea. Enumeration of the known species of Orchids. 9 parts. London (1852-59) 1-300.-Keys to subgenera and sections, sometimes to spp.

Mansfeld, R., Ueber das System der Orchidaceae-Monandrae (Notizbl. Berl.-Dahlem 13, 1937, 666-676).-Key to tribes \& subtribes.

Pfitzer, E., Beiträge zur Systematik der Orchideen (Bot. Jahrb. 19, 1895, 1-42).

-, Pflanzenreich Heft 12 (1903) 9-132, fig. 1-41.Monogr. Paphiopedilum.

- \& Kraenzlin, F., Pflanzenreich Heft 32 (1907) 1-169, fig. 1-54.-Orchidaceae-Monandrae-Coelogyninae: Coelogyne (Ptychogyne), Dendrochilum, Gynoglottis, Hologyne, Crinonia, Chelinostele, Chelonanthera, Pholidota, Camelostalix.

Reichenbach, H. G., Orchides (in Walpers, Ann. Bot. Syst. 6, 1861, 167-933).-Enum. world's orchids; sometimes partial keys.

Schlechter, R., Monographie der Podochilinae (Mém. Herb. Boiss. no 21, 1900, 1-78). Podochilus (incl. Appendicula), Lobogyne, Thelasis, Oxyanthera. Keys!

-, Die Polychondreae (Neottiinae Pritz.) und ihre systematische Einteilung (Bot. Jahrb. 45, $1911,375-410$ ).-Key to subtribes; review of and key to the genera of the Gastrodiinae; keys to species of Epipogum, Didymoplexis, Nervilia, and Gastrodia.

-, Die Orchideen, ihre Beschreibung, Kultur und Züchtung, Handbuch für Orchideen Liebhaber, Züchter und Botaniker (1915) 1-836, fig. 1-230, t. 1-12. Ed. 2 (1927) xii + 959, 250 fig., 16 tab.Descr.; no keys.

- Das System der Orchidaceen Notizbl. Berl.Dahlem 9, 1926, 563-591).-Synopsis of names.

SMITH, J. J., Artificial key to the orchid genera of the Netherlands Indies, etc. (Blumea 1, 1934, 194-215).-Comprising the whole of Malaysia.

\section{Mixed contributions to Malaysia}

Ames, O., c.s., Orchidaceae, etc. North Easton, Mass. 1-7 (1905-1922).
Blume, Rumphia 1 (1836) 195-201, t. 67-70.Col. pl. \& descr. Vanilla, Galeola.

—, ibid. 4 (1849) 38-56, t. 192-200B.-Various orch. with plates.

-, Mus. Bot. Lugd.-Bat. 1 (1849) 29-32, 46-48, 60-64, 112.

-, ibid. 2 (1856) 172-190.-Lists of spp. of various genera.

-, Collection des Orchidées les plus remarquables de l'Archipel Indien et du Japon (1858) i-vi, $1-190$, t. 1-66.-Miscell. descr. This is a republication with minor changes of Blume's Flora Javae nova series vol. 1 (1858). See under Java.

-, Monographie des Anectochilus, Goodyera et genres voisins, etc. (Belg. Hort. 11, 1861, 369378 , t. 1 (fig. 1-7).-Condensed from the above; key to genera; descr. of a number of spp.

KING \& Pantling, Some new. Indo-Malayan Orchids (J. As. Soc. Beng. 66, 1897, 578-605).

LINDLEY, Sertum Orchidaceum: A wreath of the most beautiful orchidaceous flowers (1837-42) t. 1-49.-Descr. and plates; no keys.

ReICHENBACH, Orchidaceae in Walper's Annales 6 (1861) 167-933.-Synopsis.

-, Orchidiographische Beiträge (Linnaea 41, 1877, 17-98).-New descr. partly Malaysian.

-, Xenia Orchidacea. Beitr. zur Kenntnis der Orchideen 1 (1854-58) 1-245, t. 1-100; 2 (1862-74) 1-232, t. 101-200; 3 (by KRAENZLIN) (1900) 1-193, t. 200-300.

ReichenbaChIa, Orchids illustrated and described by F. SANDER. Abbildungen, Beschreibungen und Kulturanweisungen der schönsten Orchideen. Published by Sander. 2 series, in 4 vols ( 48 parts). London and Berlin (1886-1895) folio, with 192 col. pl.-Descr. \& plates; no keys.

Schlechter, Neue Orchidaceen der Flora des Monsun-Gebietes (Bull. Herb. Boiss. II, 6, 1906, 295-310, 453-472).-Mixed diagn.

-, Orchidaceae novae vel criticae (in FEDde, Rep. 3, 1906-07, 15-20, 45-51, 276-280, 316321 ; ibid. 9, 1910-11, 25-27, 162-165, 212-217, 284-287, 290-294, 337-342, 428-439; ibid. 10, 1911, 250-252; ibid. 11, 1912, 140-146).Mixed descr.

-, Notizbl. Berl.-Dahlem 8 (1921) 14-20.New spp. coll. BECCARI in Borneo \& Sumatra. -, in FEDde, Rep. Beih. 74 (1934) 85 tab.Fig. of Ind. \& Mal. spp.

SmitH, J. J., Bull. Inst. Bot. Btzg no 7 (1900) 1-5.List mixed descr.

-, Rec. Trav. Bot. Néerl. 1 (1904) 146-159.-List mixed descr.

-, Neue Orchideen des Malaiischen Archipels (Bull. Dép. Agric. Ind. Néerl. no 5, 1907, 1-36). -Descr.

-, ditto II (ibid. no 15, 1908, 1-29).-Descr.

-, ditto III (ibid. no 22, 1909, 1-51).-Descr.

-, ditto IV (ibid. no 45, 1911, 13-25).-Descr.

-, ditto V (Bull. Jard. Bot. Btzg II, no 3, 1912, 53-69).-Descr.

-, ditto VI (ibid. II, no 8, 1912, 38-47).-Descr. -, ditto VII (ibid. II, no 13, 1914, 1-52).-Descr. - ditto VIII (ibid. II, no 25, 1917, 1-103).Descr. 
-, ditto IX (ibid. III, 2, 1920, 15-127).-Descr.

-, ditto X (ibid. III, 5, 1922, 12-102).-Descr.

-, ditto XI (ibid. III, 8, 1926, 35-70).-Descr.

一, ditto XII (ibid. III, 9, 1927, 138-195).-Descr.

-, ditto XIII (ibid. III, 10, 1928, 25-84).-Descr.

一, ditto XIV (ibid. III, 12, 1932, 105-150).-Descr.

-, ditto XV (in FeDDE, Rep. 36, 1934, 110-119).Descr.

—, ditto XVI (Bull. Jard. Bot. Btzg III, 14, 1937, 160-168),-Descr.

一, ditto XVII (Blumea 5, 1943, 297-315).-Descr.

一, ditto XVIII (ibid. 5, 1945, 692-699, 6 fig.).Descr.

-, Blumea 5 (1945) 764-785.-Compl. \& emend. descr.

-, Icones Orchidacearum malayensium I (Bull. Jard. Bot. Btzg III, Suppl. 2, 1930, t. 1-100).Plates belonging to series above.

-, ditto II (ibid. Suppl. 3, 1938-1949, t. 101-176). -Ditto.

一, idem Additamenta t. 178-189 (Reinwardtia 1, 1950, 1-25).-Ditto.

-, Ic. Bog. 2 (1903) t. 51-75; 3 (1906) t. 204-225; 3 (1907) t. $240-243 ; 4$ (1910) t. 301-305.Descr. fig.

-, Bull. Jard. Bot. Btzg III, 8 (1927) 353-369.Notes from ReICHENBACH's Herb. I.

-, ibid. 9 (1928) 482-492.-Ditto II.

一, ibid. 11 (1930) 19-27.-Ditto III.

-, in Fedde, Rep. 31 (1932) 76-82.-3 new spp.

-, Gard. Bull. Str. Settl. 9 (1935) 89-92.-1 Oberonia, 2 Dendrobiums.

Van Steenis, Trop. Natuur 23 (1934) 53-57, fig. 18-24.-Key to saprophytic Mal. Orch. mostly Javan.

TeysmanN, Nat. Tijd. Ned. Ind. 5 (1853) 487-494. -Mixed diagn.

-, Ned. Kruidk. Arch. 3 (1854) 396-402. Mixed diagn. ex Hort. Bog.

- \& BinnendiJK, Nat. Tijd. Ned. Ind. 24 (1862) 305-326.-Mixed diagn.

-, ibid. 27 (1864) 16-22.-Ditto.

-, ibid. 29 (1867) 241-244.-Ditto.

DE VRIESE, Illustrations d'orchidées orientales néerlandaises, ou choix de plantes nouvelles ou peu connues de la famille des Orchidées. Avec texte explicatif et scientifique. La Haye (1854) 19 tab. (col.).-Descr. various spp.; no keys.

\section{Local}

India: HoOKer, A century of Indian Orchids (Ann. R. Bot. Gard. Calc. 5, 1895, 1-68, t. 1-101).-Descr.; pl.

King \& Pantling, The Orchids of the Sikkim Himalaya (Ann. R. Bot. Gard. Calc. 8, 1898, 1-342, t. 1-448).-Descr.; pl.

Lindley, J. \& Proc. Linn. Soc. Lond. 1 (1857) 170-190; ibid. 3 (1859) 1-63.-Enum.; descr.

Indo-China: Gagnepan \& Guillaumin, Fl. Gén. Indo-Chine 6 (1932-34) 142-647.

Sumatra: Ames, in Merrill, Contr. Arn. Arb. 8 (1934) 22-40.

ReICHENBACH, Bonplandia 5 (1857) 34-43, 5358.-List Zollinger.
RIDLeY, J. Fed. Mal. Stat. Mus. 8, 4 (1917) 87114.-Coll. Mt Kerintji; list.

-, J. Mal. Br. R. As. Soc. no 87 (1923) 94-98.List E. Coast coll. RIDLEY.

SCHLeChTER, Bot. Jahrb. (1911) Beibl. no 104, p. 1-61:-List SCHLeCHTER coll.

SmIth, in Fedde, Rep. 32 (1933) 129-386.Critical, complete list Orch. of Sumatra \& adjacent islands.

—- Blumea 5 (1943) 316-323; ibid. 5 (1945) 700-

708.-List Atjeh coll. van STEenIS.

Malay Peninsula: CARR, Some Malayan orchids (Gard. Bull. Str. Settl. 5, 1929, 1-50, pl. 1-18). -List; descr.

- , ditto II (ibid. 5, 1930, 124-160, pl. 1-4).Ditto.

-, ditto III (ibid. 7, 1932, 1-59, pl. 1-5).Ditto.

-, ditto IV (J. Mal. Br. R. As. Soc. 11, 1933, 66-109, pl. 1-13).-Ditto.

$\longrightarrow$, ditto V (Gard. Bull. Str. Settl. 8, 1935, 69129).-Ditto.

-, ibid. 7 (1932) 61-86, pl. 6-14.-Key Taeniophyllum.

Henderson, J. Mal. Br. R. As. Soc. 5 (1927) 268-272.-List Cameron Highlands.

-, J. Fed. Mal. Stat. Mus. 13, 4 (1927) 7-10.List Pahang.

-, ibid. 223-226.-List; 2 new spp.

-, Gard. Bull. Str. Settl. 5 (1930) 79-80.List P. Tioman.

Holtrum, Mal. Nat. J. 2 (1947) 1-7, 2 fig.Key \& descr. Paphiopedilum.

-, Gard. Bull. Str. Settl. 11 (1947) 275-292,

fig. 2-5.-New spp.

-, Mal. Nat. J. 4 (1949) 85-92, 4 fig.-Key Arachnis.

-, ibid. 13 (1951) 251-253.-New Vanilla.

-, Gard. Bull. Sing. 14 (1953) 4-7.-Descr. 3 new spp.

-, Orchids of Malaya. Revised Flora of Malaya 1 (1953) $v+753,234$ fig.-Keys; descr.

RIDley, J. Linn. Soc. Bot. 32 (1896) 213-415.List of spp. from Mal. Pen.

-, Mat. Fl. Mal. Pen. (Monocot.) 1 (1907) 7-231.-Keys.

Anambas \& Natuna Islands: SMTth, in FeDde, Rep. 30 (1932) 327-336.-List coll. 'VAN STEENIS.

Java: BACKER, Bekn. Fl. Java (em. ed.) vol. 12 Orchidaceae. 3 pts, 494 pp., 2 pl. (folio-mimeogr.). Leyden (Aug.-Sept. 1952).-In Dutch; keys, etc.

Blume, Tabellen en platen voor de Javaansche Orchideèn. Batavia (1825) text t. 1-5, fig. 1-78. -Generic key \& figures.-See for a bibliogr. note on this work MANSFELD, in Fedde, Rep. 39 (1936) 318.

-, Flora Javae, nov. ser. vol. 1 (1858) 1-162, t. 1-66.-Col. plates \& descr.

BredA, Genera et species Orchidearum et Asclepiadearum quas in itinere per insulam Java . . collegerunt G. Kuhl \& J. C. van Hasselt. 3 fasc. (1828-29). -15 col. pl. and descr.

LoOgen, Trop. Natuur 27 (1938) 113-115, 1 fig.-Disperis javanica. 
ReICHENBACH, Bonplandia 5 (1857) 34-43, 5358.-List ZOLLINGER.

SMITH, Die Orchideen von Java. Leiden (1905) viii + 672 pp.-Rev.; keys; descr.

-, ditto Atlas, 6 parts, 483 fig. (1908-1912).

- Die Orchideen von Java. Nachtrag I (Bull.

Dép. Agric. Ind. Néerl. no 13, 1907, 1-78,

2 tab.).-Lists add.; descr.

-, ditto II (ibid. no 43, 1910, 1-77).-Ditto.

-, ditto III (Bull. Jard. Bot. Btzg II, no 9, 1913, 1-130).-Ditto.

-, ditto IV (ibid. II no 14, 1914, I-56).-Ditto. -, ditto V (ibid. II no 26, 1918, 1-135).-Ditto with key to Luisia \& Taeniophyllum.

-, ditto VI (ibid. III, no 3, 1921, 227-333).Keys to Cryptostylis, Thrixspermum \& Dendrocolla, and to Agrostophyllum.

-, ditto VII (ibid. III, no 9, 1927, 23-66, Taf. 2-8).-List.

-, ditto VIII (ibid. III, no 16, 1939, 121-138).List.

-, Trop. Natuur 5 (1916) 179-183.-Key to Taeniophyllum, \& Chiloschista.

-, Tafeln Javanischer Orchideen I (Bull. Jard. Bot. Btzg III, 5, 1922, t. 18-37).

一, ditto II (ibid. III, 6, 1924, t. 1-25).

一, in FEDDE, Rep. 24 (1931) 248-252.-4 n. spp.

Borneo: Ames, in Merrill, En. Born. (1921) 134-204.-List.

- \& SChWEInfurth, in AMEs, Orchidaceae 6 (1920) xiv + 271 pp.-List \& descr. Orchid. Mt Kinabalu; no keys.

Carr, Gard. Bull. Str. Settl. 8 (1935) 165-240.List Clemens \& Carr coll. Kinabalu part 1.

Holtium, Sarawak Mus. J. 5 (1949) 172-173.New Arachnis.

-, ibid. 5 (1950) 389-390, pl. 20.-New Vanda.

RIDLEY, J. Linn. Soc. Lond. Bot. 31 (1896) 261306, t. 13-15.-Complete list.

Smith, J. Str. Br. R. As. Soc. no 63 (1912) 63-70. -Coll. Moulton Sarawak.

-, Not. R. Bot. Gard. Edinb. 13 (1921) 188.New Chelonistele; descr.

-, Rec. Trav. Bot. Néerl. 22 (1925) 262-267.Phalaenopsis denevei.

-, Inst. Allg. Bot. Hamburg 7 (1927) 9-76, 10 pl.-List Hans WinkLer coll.

—, Bull. Jard. Bot. Btzg III, 11 (1931) 83-160.Coll. ENDERT.

-, Brittonia 1 (1931) 105-111,-List Sarawak Clemens, coll.

Williams, Bot. Mus. Leafl. Harv. Univ. 6 (1938) 57-61.-3 new spp.

Philippines: AMES, Orchidaceae 1 (1905) 63-107.Descr. list Orch. Philip.

-, Proc. Biol. Soc. Wash. 19 (1906) 143-154.New Acoridiums.

_, Philip. J. Sc. 2 (1907) Bot. 311-337.-Orchids from Mt Halcon.

-, Orchidaceae 3 (1908) 17-257.-Mixed descr. -, Notes on Philippine Orchids with descriptions of new species I (Philip. J. Sc. 4, 1909, Bot. 593-600).

$\longrightarrow$, ditto II (ibid. 4 (1909) Bot. 663-676).

-, ditto III (ibid. 6 (1911) Bot. 35-56). ditto IV (ibid. 7 (1912) Bot. 1-27, 126-143). -, ditto V (ibid. 7 (1912) Bot. 125-143).-Bulbophyllum.

-, ditto VI (ibid. 8 (1913) Bot. 407-440).

-, ditto VII (ibid. 6 (1920) 273-318).

_, in Elmer, Leafl. Philip. Bot. 5 (1912) 1549 1588.-Mixed not. \& descr.

-, Orchidaceae 5 (1915) 3-271.-The genera and spp. of Philip. Orchids.

- Orchidaceae 7 (1922) 141-155.-Add. Orchid. Mountain Prov. Luzon.

-, Sched. Orchidaceae 5 (1923) 1-42.-List. -, in Merrill, En. Philip. Fl. Pl. 1 (1924) 252-458.-List.

-, Bot. Mus. Leafl. Harv. Univ. 2 (1934) 31-32, 39-40.-Mixed notes.

-, Blumea Suppl. 1 (1937) 66-77.-New Acoridiums.

-, Amer. Orch. Soc. Bull. 13 (1945) 320-326.Mixed notes.

- \& QUisumbing, New or noteworthy Philippine Orchids I (Philip. J. Sc. 44, 1931, 369-383). -, ditto II (ibid. 47, 1932, 197-220, 29 pl.).

-, ditto III (ibid. 49, 1932, 483-504, 28 pl.).

-, ditto IV (ibid. 52, 1933, 443-473, 17 pl.).

-, ditto V (ibid. 56, 1935, 453-469, pl. 1-10).

—, ditto VI (ibid. 59, 1936, 1-14, 9 pl.).

DAvis, Orch. Digest 11 (1947) 264-266.-Genus Phreatia.

-, Philip. Orch. Rev. 1, no 2 (1948) 10-15, 3 fig. -Dendrobium.

- \& SteIner, Philippine Orchids. New York (1952) 278 pp. 112 fig.-Popular.

Kraenzln, in Fedde, Rep. 7 (1910) 97-98.-2 new spp.

-, ibid. 17 (1921) 382-392.-Various diagn. coll. LOHER.

LeavitT, Philip. J. Sc. 4 (1909) Bot. 201-244, fig. 1-26.-Eria; census; no key.

Quisumbing, Philip. J. Sc. 52 (1933) 271-279, pl. 1-3.-Difference between Anota violacea \& Rhynchostylis retusa.

-, Philip. J. Sc. 72 (1940) 481-492, 8 pl.-Key to Philip. Cymbidiums.

-, ibid. 74 (1941) 175-187, 2 pl.-Studies in various Phalaenopsis spp.; no key.

-, ibid. 76 (1941) 81-98, 9 pl.-Phalaenopsis. Non vidi.

-, ibid. 77 (1947) 1-18, t. 1-5.-Phalaenopsis; key.

—, ibid. 66 (1948) 141-154, t. 1-7.-Various descr.

_, Philip. Orch. Rev, $1^{1}$ (1948) 4-9.-Spathoglottis; no key.

-, ibid. 1 (1948) 4-9, 1 fig.-Spathoglottis, descr.; no key.

—, ibid. $2^{1}$ (1949) 8-15, 4 fig.-Vanda; key.

-, ibid. 32 (1950) 3-11.-Renanthera; key; many fig.

一, ibid. 31 (1950) 9-11.-Angraecum.

-, ibid. 41 (1951) 2-5, t. 1-2.-New Dendrobium. -, ibid. $4^{1}$ (1951) 26-32.-Grammatophyllum; key.

—, ibid. $4^{2}$ (1951) 8-14, 2 photos, 1 fig.-Coelogyne; key. 
- ibid. $4^{3}$ (1951) 2-10, 3 pl.-Eulophia; key. -, ibid. $5^{1}$ (1953) 2-6.-Vanilla; no key.

REICHENBACH $f$., Bonplandia 3 (1855) 212-227. -Descr. Cuming coll.

一, Linnaea 41 (1877) 99-118.-Descr. coll. WALLIS.

RENDLE, J. Bot. 34 (1896) 357-358.-2 new spp. Schlechter, in Perkins, Fragm. Fl. Philip. pt 1 (1904) 43-44.-Small list.

SteIner, Philip. Orch. Rev. $2^{3}$ (1949) 3-8.Aerides; key.

一, ibid. $5^{3}$ (1953) 4-15, 2 pl.-Revision; key.

Williams, Philip. J. Sc. 65 (1938) 385-397.-

Rev. Trichoglottis; key.

-, Bot. Mus. Leafl. Harv. Univ. 6 (1938) 4648.-Syn. Plocoglottis.

-, ibid. 6 (1938) 53-57, tab.-Descr. Phragmorchis n.g.

—, ibid. 6 (1938) 77-95.-Rev. Thrixspermum; key.

-, ibid. 6 (1938) 96-111.-Mixed descr.

—, Philip. J. Sc. 71 (1940) 113-114.-Epiblastus;

1 new $s p$.

-, ibid. 80 (1952) 281-334.-Revision of Dendrochilum sect. Acoridium; key.

Celebes: Rolfe, Kew Bull. (1899) 126-133.New spp. Minahasa coll. KoORders.

SChleChter, in Fedde, Rep. 8 (1910) 500-512. -, ibid. 10 (1911) 1-40, 66-96, 177-213.-List SCHLECHTER coll.

一, ibid. 21 (1925) 113-212.-List.

SMITH, Nat. Tijd. Ned. Ind. 58 (1898) 358-363. -Descr. 3 n. spp.

-, Svensk Bot. Tidskr. 20 (1926) 470-482.-

List W. Kaudern coll.

-, Bull. Jard. Bot. Btzg III, 10 (1928) 1-24.List BÜNNEMEIJER coll.

-, Bot. Jahrb. 65 (1933) 449-508.-List coll. KJELLBERG.

-, Blumea 5 (1945) 563-566.-List coll. MoNOD DE FROIDEVILLE.

Lesser Sunda Islands: Kraenzln, Bot. Jahrb. 7 (1886) 435-443.-Some records of Timor.

ReichenbaCH, Bonplandia 5 (1857) 34-43, 5358.-List ZoLLINGER coll.

SMITH, Med. Rijksherb. Leiden no 53 (1925) 1-17.-List Elbert coll.

Moluccas.-

Ambon: Finet, Not. Syst. 2 (1911-13) 253-255.New Phalaenopsis.

Smith, Die Orchideen von Ambon. Batavia 1905. 125 pp.-Descr.

-, Philip. J. Sc. 12 (1917) 249-262.-List RoBinson coll.

Buru: SmIth, Bull. Jard. Bot. Btzg III, 9 (1928) 439-481.-List coll. Toxopeus, StresemanN.

Ceram : Smith, Bull. Jard. Bot. Btzg III, 10 (1928) 85-172.-List coll. Rutten, StresemanN.

Talaud \& Morotai: SMITH, Bull. Jard. Bot. Btzg III, 11 (1930) 67-81.-List coll. LAM.

New Guinea: BaILEY, Various small contributions, in Queensl. Agric. J. 3 (1898) 159.-Papua. —, ibid. 4 (1899) 48.-Dendrobium armittii. -, ibid. 5 (1899) 41.-Ornithochilus moretonii. -, ibid. 6 (1900) 287.-Papua.
-, ibid. 7 (1900) 349.-Papua.

-, ibid. 16 (1906) 410-412.-Dendrobium litoreum.

-, ibid. 19 (1907) 273-274.-Papua (leg.

Copland King).

一, ibid. 2 (1914) 75.-Eulophia papuana.

KrAENZLIN, Bot. Jahrb. 7 (1886) 435-443.Some new spp.

一, Oester. Bot. Z. 44 (1894) 161-164, 208-212, 253-257, 298-302, 333-338, 418-421, 459-462.

-Descr. new spp.

MANSFELD, Bot. Jahrb. 62 (1929) 462-474.KEYSSER coll. from Sarawaged.

Reichenbach, Bot. Centralbl. 28 (1886) 343346.-New spp. BECCARI coll.

RIDLEY, J. Bot. 24 (1886) 322-327.-Descr. new spp. For BES coll.

-, Trans. Linn. Soc. Lond. Bot. II, 9 (1916) 159-209.-List Boden KLoss coll. etc.

Rogers, Trans. R. Soc. South Austr. 49 (1925) 254-265.-List.

一, ibid. 54 (1930) 37-39.

— \& WhITE, ibid. 44 (1920) 110-119, pl. 5-8.Coll. C. T. White.

SCHLECHTER, Die Orchidaceen von DeutschNeu Guinea (in Fedde, Rep. Beih. 1, 1914, i-lxvi, 1-1079).-Descr.; no keys.

-, in FedDe, Rep. 16 (1919) 214-219.-New spp. coll. KeYsSER.

-, Bot. Jahrb. 58 (1923) 50-154.-List add.

Smith, in Fedde, Rep. 10 (1912) 487-488.-

4 n. spp.

-, Vorläufige Beschreibungen neuer Papuanischer Orchideen I (Bull. Dép. Agric. Ind. Néerl. no 19, 1908, 1-39).-Coll. VeRSTEEG.

-, ditto II (ibid. 39, 1910, 1-22).-Coll. VON RÖMER.

-, ditto III (ibid. 45, 1911, 1-12).-Coll. GJeLLERUP.

-, ditto IV (Bull. Jard. Bot. Btzg II, 2, 1911, 1-20).-Coll. A. C. DE Kock.

-, ditto V (ibid. II, 3, 1912, 70-79).-Coll. GJELLERUP.

-, ditto VI (in FeDDE, Rep. 11, 1912, 130-140). -Ditto.

-, ditto VII (ibid. 11, 1912, 274-280).-Ditto. -, ditto VIII (ibid. 11, 1913, 552-560).-Ditto \& others.

-, ditto IX (ibid. 12, 1913, 24-34),-Ditto.

-, ditto X (ibid. 12, 1913, 110-123).-Ditto.

一, ditto XI (ibid. 12, 1913, 394-406).-Ditto.

-, ditto XII (Bull. Jard. Bot. Btzg II, 13, 1914,

53-77).-Coll. Pulles

-, ditto XIII (Med. Rijksherb. no 23, 1915, 1-21).-Coll. JANOWSKI \& others.

-, Die Orchideen von Niederländisch NeuGuinea (Nova Guinea 8, 1909, 1-148, t. 1-46). -, ditto (ibid. 8, 1911, 521-611, t. 75-112).

一, ditto (ibid. 12, 1913, 1-108, t. 1-28).

-, ditto (ibid. 12, 1915, 173-477, t. 55-99).

-, ditto (ibid. 14, 1929, 337-516, t. 41-87).-List.

-, ditto (ibid. 18, 1934, 9-85, t. 3-16).

-, in Gibns, Contr. Fl. Phytog. Arfak Mts (1917) 105-127, 203-206.-List.

-, Neue Orchideen Papuasiens (Beitr. z. Fl. 
v. Papuasien XX) (Bot. Jahrb. 66, 1934, 161215).-Coll. Stein, MaYr; also some spp. from Japen \& Waigeou Isl.

Williams, Amer. Orch. Soc. Bull. 10 (1942) 373-375.-New Paphiopedilum.

一, ibid. 11 (1943) 297-298.-2 new Saccoglossum.

7 , Bot. Mus. Leafl. Harv. Univ. 12 (1946) 149-183, pl. 21-23.-Part BRAss coll.

Solomon Islands: CARR, Kew Bull. (1934) 375383.-Records coll. WATERHOUSE.

Micronesia: Schlechter, Bot. Jahrb. 56 (1920) 434-501.-Keys!

New Hebrides: Ames, J. Arn. Arb. 13 (1932) 127144.

一, ibid. 14 (1933) 101-112.

Australia: Fitzgerald, R. D., Australian Orchids (1875-1895) vol. 1 (7 parts), vol. 2 (5 parts).204 spp. represented on coloured plates; unfinished. Non vidi.

Nicholls, W. H., Orchids of Australia. Parts 1-2 (1 dated 1951), each with 24 col. pl.-Key to genera and $s p p$.

RUPP, H. M. R., The Orchids of New South Wales. Sydney (1943) 1-152, fig.-Keys.

\section{Genera}

Acriopsis: SchlechteR, Oester. Bot. Z. 50 (1900) 245-250, 286-290.-Revision; descr.; key.

Appendicula (Lobogyne, Chilopogon, Cyphochilus): Smith, Bull. Jard. Bot. Btzg II, no 8 (1912) 3037.-List.

Arachnis (Dimorphorchis): RolfE, Orch. Rev. 27 (1919) 149.

SMITH, Nat. Tijd. Ned. Ind. 72 (1912) 71-76.List.

Basigyne: SмIтH, Bull. Jard. Bot. Btzg II, no 25 (1924) 4-6.-Celebes, monotypic.

Bromheadia: RIDley, J. Linn. Soc. Bot. 28 (1891) 331-339.-Key.

Bulbophyllum § Cirrhopetalum: SmiтH, Bull. Jard. Bot. Btzg II, no 8 (1912) 19-29.-List spp.

Caladenia: RuPP, Proc. Linn. Soc. N.S.W. 71, 1946, 278-281.-Variab. C. carnea.

Camarotis: Smith, Nat. Tijd. Ned. Ind. 72 (1912) 96-98.-List.

Chiloschista: Diels \& Mansfeld, Notizbl. Berl.Dahlem 11 (1932) 491-498.-Rev.; key.

Chrysoglossum (Collabium): SMITH, Bull. Jard. Bot. Btzg II, no 8 (1912) 1-3.-Sect. divisions.

Corybas (Corysanthes): SChlechter, in Fedde, Rep. 19 (1923) 18-24.-Nomencl.; census of all spp.

Cymbidium: Schlechter, in Fedde, Rep. 20 (1924) 96-105.-List.

Cyperorchis (Iridorchis): Schlechter, in Fedde, Rep. 20 (1924) 105-110.-List.

Cystorchis: Smith, in Fedde, Rep. 30 (1932) 329-331.-Key to Mal. spp.

Dendrobium: Finet, Bull. Mus. Hist. Nat. Paris 9 (1903) 295-303.-Key to sections; enum. spp. represented at Paris.

Dendrobium \$ Cadetia (Cadetia); SMITH, Bull. Jard. Bot. Btzg II, no 8 (1912) 15-18.-List spp.
Dendrobium \& Rhopalanthe (partim): SMITH, Trop. Natuur 19 (1928) 23-26.

Dendrochilum (Platyclinis): AMEs, Orchidaceae 3 (1908) 3-20.-New sect.; 4 new spp.; observ.; later he accepted Acoridium for Philip. spp.; $c f$. under Philippines.

Smith, Rec. Trav. Bot. Néerl. 1 (1904) 52-80.Key!

Didymoplexis (Leucolaena): KuRz, J. Bot. 4 (1886) 40-41.-Discussion synonymy.

Schlechter, Bot. Jahrb. 45 (1911) 407-408.Key.

Smith, Bull. Jard. Bot. Btzg III, 2 (1920) 20.

Epipogium: SCHLECHTER, Bot. Jahrb. 45 (1911) 398-399.-Key.

Sprague \& Green, Kew Bull. (1937) 475-476.

Eria \& Aporodes: SMITH, Blumea 5 (1945) 735-739. - List.

Eria (Forbesina): Smith, in Fedde, Rep. 32 (1933) 244.

Gastrochilus: Schlechter, in Fedde, Rep. 12 (1913) 313-317.-Enum. spp.; segregate from Saccolabium.

Gastrodia: SchLECHTER, Bot. Jahrb. 45 (1911) 409 410.-Key.

Glomera (Giulianettia, Glossorrhyncha): SMIтH, Bull. Dép. Agric. Ind. Néerl. no 15 (1908) 2729.-List.

—, Bull. Jard. Bot. Btzg II, no 8 (1912) 7-14.Sect. division; list spp.

Goodyera (Salacistus): SmIr, Bull. Jard. Bot. Btzg III, 8 (1927) 353.

Grammatophyllum (Grammangis): SCHLECHTER, Orchis 9 (1915) 99-109, 115-122.-Census; descr.; no keys.

Gynoglottis: SmITh, Rec. Trav. Bot. Néerl. 1 (1904) 49-51.

Habenaria: Kraenzlin, Bot. Jahrb. 16 (1892) 52-223.-Rev.; keys!

Liparis: RIDLEY, J. Linn. Soc. Lond. Bot. 22 (1886) 244-297.-Monograph.

Malaxis (Microstylis): Ridley, J. Linn. Soc. Lond. Bot. 24 (1888) 308-351.

Nephelaphyllum: Gagnepan, Bull. Mus. Hist. Nat. Paris II, 4 (1932) 705-710.-Reduces the genus to Tainia.

Nervilia: SCHLECHTER, Bot. Jahrb. 45 (1911) 399405.-Key.

Sprague \& Fischer, Kew Bull. (1927) 363-365. -Generic syn. (nomencl.).

Paphiopedilum: Hallier $f$, , Ann. Jard. Bot. Btzg 14 (1897) 34-52, t. 4.-Conspectus.

Phalaenopsis (Doritis, Kingiella): RerchenbaCH, Hamb. Gartenzeit. 16 (1860) 114-117.-Census; clav. part.

Rolfe, Gard. Chron. 26 (1886) 168-170, 212, 276-277.-Revision; no key.

SmItH, Blumea 5 (1945) 755.-Synonymy genus.

Polystachya: Kraenzlin, in Fedde, Rep. Beih. 39 (1926) 1-136.

Pomatocalpa: Smith, Nat. Tijd. Ned. Ind. 72 (1912) 102-108.-List.

Pristiglottis (Cystopus BL.): SмITH, in Act. pro Fauna \& Flora Univ. II Bot. 1 no 14 (1934) 1-6; ibid. no 17 (1935) 4.-List of spp. 
Pterostylis: RuPP, Proc. Linn. Soc. N.S.W. 58 (1933) 421-428.

Rhynchostylis (Anota): Holtrum, Orchid J. 2 (1953) 123-125.-Anota reduced; key; references.

Robiquetia: Smrth, Nat. Tijd. Ned. Ind. 72 (1912) 112-115.-List.

Sarcanthus (Cleisostoma): SMITH, Nat. Tijd. Ned. Ind. 72 (1913) 79-96.-List.

Sarcochilus: RUPP, Proc. Linn. Soc. N.S.W. 76 (3-4) (1951) 49-56.-Rev. Australian spp.

Sarcopodium: Rolfe, Orch. Rev. 18 (1910) 237240.-Mostly incl. as a section of Dendrobium; census of 21 spp.

Schoenorchis: SmIth, Nat. Tijd. Ned. Ind. 72 (1912) 99-101.-List.

Spiranthes: VAN SteEnIs, c.s., Blumea 6 (1950) 361.-Syn. S. lancea.

Taeniophyllum (Geissanthera): WiLliams, Bot. Mus. Leafl. Harv. Univ. 5 (1938) 164-170.Some new comb. \& descr.

Tainia (Mitapetalum, Ascotainia, Mischobulbum): Gagnepain, Bull. Mus. Hist. Nat. Paris II, 4 (1932) 705-710.-Includes Nephelaphyllum in Tainia.

Smith, Bull. Jard. Bot. Btzg II, no 8 (1912) 3-6. -Sect. division.

Thrixspermum (Dendrocolla): SCHLECHTER, Orchis 5 (1911) 46-48, 54-58.-Census; concise descr.; no keys.

Tropidia (Schoenomorphus): MANSFeld, in Fedde, Rep. 36 (1934) 58-59.-Reduction.

Vanda (Papilionanthe): LINDley \& PAXToN, F1. Gard. 2 (1852) 20-22.

SCHLECHTER, Orchis $9^{4}$ (1915) 77-80.

SMITH, Teysmannia 9 (1898) 200-208.-Disc. \& char. Mal. spp.; in Dutch.

Vandopsis: SMITH, Nat. Tijd. Ned. Ind. 72 (1912) 76-78.-List.

Vanilla: RoLfe, J. Linn. Soc. Lond. Bot. 32 (1896) 439-478.-Revision; key.

Zeuxine (Monochilus): Ames, Bot. Mus. Leafl. Harv. Univ. 5 (1938) 100-101.-Nomencl. transfers.

SMITH, Bull. Jard. Bot. Btzg III, 8 (1927) 353.Generic syn.

\section{OROBANCHACEAE (Aeginetiaceae)}

Beck-von Mannagetta, G., Pflanzenreich Heft 96 (1930) 1-348, 24 fig.

LIVERA, E. J., Aeginetiaceae. A new natural family of flowering plants (Ann. R. Bot. Gard. Perad. 10, 1927, 145-159).-List.

\section{Genera}

Aeginetia: Baxhuizen Van Den Brink, Trop. Natuur 10 (1921) 154-158, 164-173, 11 fig.Key.

-, Bull. Jard. Bot. Btzg III, 13 (1933) 77-91, 5 fig.-Revision; key.

Christisonia: Worsdell, Ann. Bot. 9 (1895) 103-136, t. 5-6.-Anat.

\section{OXALIDACEAE}

KNUTH, R., Pflanzenreich Heft 95 (1930) 1-481, fig. 1-28.

-, Pflanzenfamilien ed. 2, 19a (1931) 11-42, fig. 2-19.

\section{Local}

New Guinea: LAUTERBACH, Bot. Jahrb. 62 (1929) 482.

Pulle, Nova Guinea 8 (1910) 389.

一, ibid. 8 (1911) 655 .

\section{Genera}

Biophytum: van SteEnis, Bull. Bot. Gard. Btzg III, 18 (1950) 449-455, 2 fig.-Revision; key. -, Reinwardtia 1 (1952) 467.-Addition.

Connaropsis (cf. sub Dapania): RIDLEY, Kew Bull. (1930) 75-76.-New sp. Borneo.

Dapania: Hallier, Beih. Bot. Centralbl. 34, ii (1916) 25-27.

KNUTH, Bot. Jahrb. 50 Suppl. (1914) 234. Connaropsis is reduced to Dapania (sic!).

Korthals, Ned. Kruidk. Arch. 3 (1853) 381384.-Type descr.

Oxalis: Calder, Rec. Bot. Surv. Ind. 6 (1919) 325-341, t. 1-7.-Key Indian spp. native \& introduced.

Petch, Ann. R. Bot. Gard. Perad. 5 (1914) 541. -In Ceylon.

Van STEEnIs, Bull. Jard. Bot. Btzg III, 13 (1934) 235.-List native spp.

Sarcotheca: HALLIER $f$., Med. Rijksherb. Leiden no 1 (1910) 1-2.-List.

-, Beih. Bot. Centralbl. 34, ii (1916) 27-30.List new spp.

Miquel, Ill. Fl. Arch. Ind. (1871) 69-71.Descr.

RidLeY, Kew Bull. (1930) 75-76.-As Connaropsis.

\section{PALMAE}

BecCArI, O., Reliquiae Schefferianae (Ann. Jard. Bot. Btzg 2, 1885, 77-171, t. 1-14).-Descr.; list of spp. of a number of genera.

-, Nuovi studi sulle Palme Asiatiche (Malesia 3, 1886, 58-149, pl. 6-11).

-, Nuove Palme Asiatiche (Malesia 3, 1889, 169-200).-Mixed diagn.

-, Asiatic Palms. Lepidocaryae and Corypheae (Ann. R. Bot. Gard. Calc. 11-13, 1908-1931).

- Recensio della palme del vecchio mondo appartenenti alla Tribù delle Corypheae con descrizione delle specie e varietà nuove che vi appartengono (Webbia 5, 1921, 1-70).

Blume, C. L., Rumphia 2 (1837/8) 37-176, t. 82137.-Descr. Licuala, Pericycla, Saribus, Corypha, Areca, Pinanga, Kentia, Oncosperma, Cyrtostachys, Calyptrocalyx, Iguanura, Wallichia, Orania, Ptychosperma, Saguerus, Caryota, Sagus, Salacca, Ceratolobus, Korthalsia. Extr. in Bull. Sc. Phys. Nat. Néerl. (1838) 61-67 and in Ann. Sc. Nat. II, 10 (1838) 369-377.

-, Rumphia 3 (1842/3) 1-90.-Descr. of Daemonorops, Calamus, Nypa, Plectocomia; notes on uses of palms. 
Burret, M., Beiträge zur Kenntnis der Palmen von Malesia, Papua, and der Südsee (in FEDDE, Rep. 24, 1928, 253-296).-Mixed notes, descr. \& lists.

- Palmae Gerontogeae IV (Notizbl. Berl.Dahlem 12, 1935, 590-602).

-, Palmae Gerontogeae V (Notizbl. Berl.Dahlem 13, 1936, 185-200).

-, Indomalayische Palmen (Notizbl. Bot. Gart. Berl.-Dahlem 15, 1940, 164-210).-New descr. of Calamus, Daemonorops, Caryota, and Pinanga, all from Sumatra except one, coll. BURRET.

-, Systematische Ubersicht über die Gruppen der Palmen (Mitt. Bot. Gart. \& Mus. Berl.Dahlem 1, 1953, 59-74).-Key to genera of palms (not finished).

Martelli, U., Generi, specie e varietà nuove di palme gerontogee della tribù Arecaceae lasciate inedite dalDr. Od. Beccari (Atti Soc. Tosc. Sc. Nat. 44, 1934, 114-176).-Descr. new spp.; new comb. $\&$ records of many genera from various sources of Indo-Malaysia; some of Pacific. In Ital.

Martius, K. F. P. von, Historia naturalis Palmarum ... (1823-50). Three volumes in two, pp. 1cxcviii, $1-350$, t. 1-445. Volume 2 and 3 Expositio Palmarum systematica, 350 pp., $180 \mathrm{pl}$.

Miquel, F. A. W., De Palmis Archipelagi Indici observationes novae (Verh. Kon. Akad. Wet. A'dam 115, 1868, 1-33, t. 1).-Descr. \& list new or noteworthy spp.

SCheffer, R. H. C. C., Sur quelques palmiers du groupe des Arécinées. I (Nat. Tijd. Ned. Ind. $32,1873,149-193)$.-Descr, and keys to spp. of genera of Arecineae.

-, ditto II (Ann. Jard. Bot. Btzg 1, 1876, 5254, 103-164, pl. 1-30).-List Papuan spp. of palms; disc. \& descr.; key to genera of Arecineae; spp. listed, exceptionally a key.

\section{Local}

India: BeCCARI \& HoOKer, in HoOK. $f$. Fl. Br. Ind. 6 (1892) 402-448; ibid. (1893) 449-483.

Blatter, The palms of British India \& Ceylon. London (1926) i-xxviii, 1-600. t, 1-106, fig. 127.-Descr.; keys to genera, a few to spp.

Indo-China: BecCARI, Webbia 3 (1910) 191.-List Indo-Chinese palms.

—, Bull. Mus. Hist. Nat. Paris. 17 (1911) 148160.-Concise key.

Gagnepain \& Conrard, Fl. Gén. Indo-Chine 6 (1937) 946-1056.

Sumatra: Miquel, in MiQuel, J. Bot. 1 (1861) 1-29. -List; some new spp.

Malay Peninsula: Burret, Notizbl. Bot. Gart. Berl.-Dahlem 15 (1941) 316-318.-Symphyogyne n. g., 2 spp., formerly Livistona rupicola. Furtado, Palmae Malesicae I (in Fedde, Rep. 35, 1934, 273-283).-Mostly Mal. Pen \& Bornean Pinanga.

—, ditto II (Gard. Bull. Str. Settl. 8, 1935, 159_ 163).-Nenga; nomencl., synon.

-, ditto III (ibid. 8, 1935, 241-262).-Descr. Calamus; also of Borneo.

一, ditto V (ibid. 8, 1935, 339-367).-Daemonorops; list.
-, ditto VI (ibid. 9, 1937, 152-181).-Some

spp. of Daemonorops $\$$ Cymbospathae.

- , ditto VIII (ibid. 11, 1940, 31-73, 9 fig.).Licuala; keys.

-, ditto IX (ibid. 11, 1941, 236-243, fig. 1-2).2 monotypic new gen. Liberbaileya and Maxburrettia (Coryphoideae).

-, ditto X (ibid. 12, 1949, 378-403).-Salacca; key.

-, ditto XI (ibid. 13, 1951, 300-324).-Korthalsia; key.

一, ditto XII (ibid. L. c. 325-338).-Plectocomiopsis; key.

一, ditto XIII (ibid. l. c. 339-344).-Myrialepis. -, ditto XIV (ibid. l.c. 345-350).-Plectocomia.

-, ditto XV (ibid. l.c. 351-359).-Ceratolobus.

- , ditto XVI (ibid. l.c. 360-365).-Calospatha; key.

-, ditto (ibid. 14, 1953, 49-147, 41 tab.).Rev. Daemonorops; plates; key!

Ridley, Mat. Fl. Mal. Pen. (Monocot.) 2 (1907) 133-221.-Keys.

Borneo: Beccari, Nuovo Giorn. Bot. Ital. 3 (1871) 11-30.-Some new descr. \& records in Caryota, Eugeissona, Metroxylon, and Teysmannia.

Merrill, Sarawak Mus. J. 3 (1928) 518.New Pinanga.

MiQuel, Anal. Bot. Ind. 1 (1850) 2-7; repr. Verh. Kon. Ned. Inst. le kl. 3e Reeks vol. 3.Enumeration; some new Calamus.

Philippines: BeccarI, in Perkins, Fragm. Fl. Philip. pt 1 (1904) 45-48.-List.

-, Webbia 1 (1905) 315-359.-List; descr.

-, Philip. J. Sc. 2 (1907) Bot. 219-240.-Descr. n. $s p p$.

-, Philip. J. Sc. 3 (1908) Bot. 339-342.-List spp. of Batanes and Babuyanes Isl.

- , in ElMER, Leafl. Philip. Bot. 36 (1909) 639_ 650.-List.

-, Philip. J. Sc. 4 (1909) Bot. 601-637, 2 pl.Descr. new spp.

-, Philip. J. Sc. 6 (1911) Bot. 229-230.-List Polillo Isl. spp.

-, in Elmer, Leafl. Philip. Bot. 8 (1919) 2997-3067.-Descr. of spp. coll. by ELMER; also key to genera.

-, Philip. J. Sc. 14 (1919) 295-363, t. 1-3.Revision with keys!

Furtado, Gard. Bull. Str. Settl. 8 (1935) 321338.-Verification ratans descr. by BLANCo.

Moluccas: BecCarI, Philip. J. Sc. 12 (1917) Bot. 81.-New Calamus (Ambon).

New Guinea: Beccari, Nuovo Giorn. Bot. Ital. 20 (1888) 177-180.-New spp.

-, Webbia 1 (1905) 281-313, fig. 1-8.-New gen. \& new spp.; descr. only.

-, in Nova Guinea 8 (1909) 203-220, t. 50-51. -Descr. new spp.

-, Bot. Jahrb. 52 (1914) 19-39.-List; new spp. -, in Grbss, Contr. Fl. Phytog. Arfak Mts (1917) 91-98, 200.-List.

-, Bot. Jahrb. 58 (1924) 441-462.-List new spp. Burret, J. Arn. Arb. 12 (1931) 264-269.Descr. new spp. 
—, Notizbl. Berl.-Dahlem 11 (1933) 704-713. -Descr. new spp.

—, ibid. 12 (1935) 309-348.-Descr. new spp.; records; no keys.

-, ibid. 13 (1936) 65-101.-Descr. new spp., many of Solomon Isl.

-, ibid. 13 (1936) 317-332.-Descr. new spp.

-, ibid. 13 (1937) 468-475.-Descr. new spp.

-, J. Arn. Arb. 20 (1939) 187-212.-Records

and descr. new spp.

Furtado, Gard. Bull. Str. Settl. 9 (1937) 182-186.-New Calamus.

Ridley, J. Bot. 24 (1886) 358-359.-2 new spp. FORBES coll.

Solomon Islands: Burret, Notizbl. Berl.-Dahlem 13 (1936) 65-101.-List new sp. \& n.g.

Micronesia: BeCCARI, Bot. Jahrb. 59 (1924) 11-16. -Gulubiopsis, Ponapea n.g.

\section{Genera}

Actinophloeus: BurReT, in FEDdE, Rep. 24 (1928) 271-273.-List.

-, Notizbl. Berl.-Dahlem 11 (1930) 205-207.2 new spp.

Adelonenga (Hydriastele): BurReT, Notizbl. Berl.Dahlem 13 (1936) 482-487.-List.

Areca (Mischophloeus): BurreT, Notizbl. Berl.Dahlem 13 (1935) 199-200.-New sp. Celebes.

Furtado, in Fedde, Rep. 33 (1933) 217-239.Delimitation; key to infrageneric divisions; list of spp.

Scheffer, Nat. Tijd. Ned. Ind. 32 (1873) 149170.-List.

Barkerwebbia: BecCARI, Webbia 1 (1905) 281289.-Monotypic.

Borassodendron: BeCCARI, Webbia 4 (1914) 359373.-New genus from Malaya.

Borassus: BeCCARI, Webbia 4 (1914) 293-359; 373-385.-Key.

Brassiophoenix: Burret, Notizbl. Berl.-Dahlem 12 (1935) 345-348.-Monotypic; New Guinea.

Calamus: Bartlett, Pap. Mich. Acad. Sc. 251 (1939) 3-14, pl. 1-4.-Descr. new Sumatran sp., type of new subgenus.

BeCCARI, Rec. Bot. Surv. Ind. 2 (1903) 197-218. -Compl. list; descr. new spp.

-, Ann. R. Bot. Gard. Calc. 11 (1908) 1-518, t. 1-231.-Complete revision.

-, ibid. (1913) Suppl., i-x, 1-142, t. 1-83.Records \& descr.

Burret, Notizbl. Berl.-Dahlem 15 (1943) 800819.-Some descr. new spp.

Ceratolobus: BecCARI, Ann. R. Bot. Gard. Calc. $12^{2}$ (1918) 18.

Cocos: Beccari, Malpighia 1 (1887) 343-354, 441-454, t. 9; ibid. 2 (1888) 85-95, 147-156.Segregation of Cocos.

-, Agric. Col. 10 (1916) 435 seq., extracted in Kew Bull. (1917) 331-335.-Key to Cocos and allied genera; list.

—, Philip. J. Sc. 12 (1917) Bot. 27-43.-Origin and distr. of $C$. nucifera.

Coleospadix: Burret, in Fedde, Rep. 24 (1928) 285-288.-List; descr.

Corypha: BeCCARI, Webbia 5 (1921) 6-10.
-, Ann. R. Bot. Gard. Calc. 13 (1931) 10-32.Key; descr.

Daemonorops: Beccari, Rec. Bot. Surv. Ind. 2 (1903) 218-230.-Compl. list; descr. new spp. -, Ann. R. Bot. Gard. Calc. $12^{1}$ (1911) 1-237, t. 1-109.

Burret, Notizbl. Berl.-Dahlem 15 (1943) 797-800.-Some new spp.

Eugeissona: BecCARI, Webbia 4, pt 1 (1913) 190202, fig. 8-11.-Genus transferred from the Lepidocaryeae to Cocoineae.

-, Ann. R. Bot. Gard. Calc. $12^{2}$ (1918) $196-$ 205.

Gronophyllum: Burret, Notizbl. Berl.-Dahlem 13 (1936) 200-205.-List.

Hydriastele: BurRet, in FEDDE, Rep. 24 (1928) 291-292.

—, Notizbl. Berl.-Dahlem 13 (1937) 482-487.

Iguanura: BeCCARI, Malesia 3 (1886) 100-107.

Kentia: BecCARI, Webbia 4 (1913) 143-156.-List.

Korthalsia: BecCARI, Ann. R. Bot. Gard. Calc. $12^{2}$ (1918) 104-155.-Key; descr.

Laccospadix: BurRet, in FeDde, Rep. 24 (1928) 289-291.

Licuala: BecCARI, Malesia 3 (1886) 70-90.-List. -, Webbia 5 (1921) 22-55.-Old World spp. key.

-, Ann. R. Bot. Gard. Calc. 13 (1931) 109-224. -Key; descr.

Burret, Notizbl. Berl.-Dahlem 15 (1941) 327336.-List; no key.

Linospadix: Burret, Notizbl. Berl.-Dahlem 12 (1935) 330-331.-List.

Livistona: BecCARI, Ann. R. Bot. Gard. Calc. 13 (1931) 43-108.-Key; descr.

Burret, Notizbl. Berl.-Dahlem 15 (1941) 319327.-List; no key.

Metroxylon: Beccari, Ann. R. Bot. Gard. Calc. $12^{2}$ (1918) 156-195.-Key; descr.

Nengella (Leptophoenix): Burret, Notizbl. Berl.Dahlem 13 (1936) 312-317.-List.

Paralinospadix: Burret, Notizbl. Berl.-Dahlem 12 (1935) 331-336.-List.

Phoenix: Beccari, Malesia 3 (1890) 345-416, pl. 43-44.-Key.

Gamble \& Fischer, Fl. Pres. Madras pt 9 (1931) 1558-1560.-Key.

Pholidocarpus: BECCARI, Malesia 3 (1886) 90-92. -Key.

-, Ann. R. Bot. Gard. Calc. 13 (1931) 227238.-Key; descr.

Pigafetta: Beccari, Ann. R. Bot. Gard. Calc. $12^{2}$ (1918) 99-103.-Key; descr.

Pinanga: BecCari, Malesia 3 (1886) 110-147.-Key. Burret, Notizbl. Berl.-Dahlem 13 (1935) 185-186.-New sp. Sumatra.

Plectocomia: Beccari, Ann. R. Bot. Gard. Calc. $12^{2}$ (1918) 19-45.-Key; descr.

Plectocomiopsis: Beccari, Ann. R. Bot. Gard. Calc. $12^{2}$ (1918) 46-66.-Key; descr.

Gagnepain, Not. Syst. 6 (1937) 149-160.Discussion affinities with Myrialepis and a new genus from Indo-China.

Pritchardia: Beccari, Malesia 3 (1889) 281-317, t. 37-38. 
-, Webbia 4 (1913) 202-240.-Key.

$\rightarrow$ Ann. R. Bot. Gard. Calc. 13 (1931) 308316.-Key; descr.

Pseudopinanga: Burret, Notizbl. Berl.-Dahlem 13 (1936) 188-198.-List; segregate from Pinanga.

Ptychococcus: Burret, in Fedde, Rep. 24 (1928) 261-262.-List.

Ptychosperma: BurRet, in FedDe, Rep. 24 (1928) 263-271.-List.

_, Notizbl. Berl.-Dahlem 12 (1935) 595-599.List.

一, ibid. 13 (1936) 384-385.

Rehderophoenix: Burret, Notizbl. Berl.-Dahlem 13 (1936) 86.

Rhopaloblaste: Burret, in Fedde, Rep. 24 (1928) 288-289.-Descr.

Roystonea (Oreodoxa): Balley, Gentes Herb. 3 (1935) 343-387.-Key.

-, ibid. 8 (1949) 114-134, fig. 24-40.-New revision; key.

Sabal: BAILEY, Gentes Herb. 3 (1934) 275-337.Key.

Salacca (Zalacca): BeCCARI, Malesia 3 (1886) 6368.-List.

-, Ann. R. Bot. Gard. Calc. $12^{2}$ (1918) 67-98. -Key; descr.

Siphokentia: Burret, Notizbl. Berl.-Dahlem 10 (1927) 198-201.-New genus; Moluccas.

Symphyogyne: BurReT, Notizbl. Berl.-Dahlem 15 (1941) 316-318.-2 spp.; Mal. Peninsula.

Teysmannia: BecCari, Ann. R. Bot. Gard. Calc. 13 (1931) 238-241.

Miquel, Ann. Mus. Bot. Lugd.-Bat. 4 (1868) 89-90.-Descr.

Palm \& Jochems, Trop. Natuur 13 (1924) 9-12, fig. 1-4.-Descr.; in Dutch.

\section{PANDANACEAE}

KURz, S., Revision of the Indian screwpines and their allies (J. Bot. 5, 1867, 93-106, 125-136, t. 62-65).-List; no keys; contains also the Mal. spp.

Martelli, U., Enumerazione delle 'Pandanaceae' (Webbia 3, 1910, 307-327).-Freycinetia; list.

一, ditto (ibid. 4, 1913, 5-105, t. 1-17).-Pandanus; list.

一, ditto (ibid. 4, 1914, 399-435, t. 18-43).-Descr. new spp. of Pandanus.

-, La distribuzione geographica delle Pandanaceae (Atti Soc. Toscana Sc. Nat. Pisa, Mem. 43, 1933, 190-209).-General; in Ital.

Solms-Laubach, H., Monographia Pandanacearum (Linnaea 42, 1878, 1-110).-No keys.

-, Ann. Jard. Bot. Btzg 3 (1883) 89-104, t. 16.New spp., coll. Beccari in Celebes, Aru, Key and New Guinea; list.

Warburg, O., Pflanzenreich Heft 3 (1900) 1-97, fig. 1-22.

\section{Local}

India: Beccari \& Hooker f., Fl. Br. Ind. 6 (1893) 483-488.

Kurz, J. As. Soc. Beng. 38, ii (1869) 145-151.Rev. Ind. Pandanus.
Indo-China: Martelli, Fl. Gén. Indo-Chine 6 (1937) 1056-1069.

Sumatra: KaneHIRA, J. Jap. Bot. Tokyo 16 (1940) 101-105.-New spp.; list of Sumatran Pandanus.

Malay Peninsula: KanehIra, J. Jap. Bot. Tokyo 14 (1938) 429-438.-Some records.

Ridley, Mat. Fl. Mal. Pen. (Monocot.) 2 (1907) 221-235.-Keys.

Borneo: Kanehira, J. Jap. Bot. 14 (1938) 429-438, 10 fig.-Some records.

Martelli, Univ. Calif. Publ. Bot. 12 (1930) 369-370, t. 48.-New sp.

Java: BACKeR, Handb. Fl. Java 1 (1925) 32-47.Key; also on sterile characters \& incl. some cult. spp.

Celebes: Fagerlind, Svensk Bot. Tidskr. 34, (1940) 101-116.-New sp.

-, Bot. Notiser (1941) 173-182.-Coll. KJELLBERG.

Philippines: Elmer, Leafl. Philip. Bot. 1 (1906) 74-77, 80-82.-New spp. Pandanus.

-, ibid. 1 (1907) 212-219.-New Freycinetia.

-, ibid. 10 (1938) 3601-3610.-Descr. new Freycinetia.

-, ibid. 10 (1939) 3771-3776.-New spp. of Pandanus \& Freycinetia.

-, ibid. 10 (1939) 3811-3818, t. 1-3.-Notes on Pandanus utilissimus ElM.

Martelli, Philip. J. Sc. 3 (1908) Bot. 59-72.List new spp.; no key.

-, Webbia 3 (1910) 5-35.-Key to Freycinetia. -, in Elmer, Leafl. Philip. Bot. 3 (1911) 11091132.-New spp. of Pandanus \& Freycinetia.

Merrill, Philip. J. Sc. 3 (1908) Bot. 307-315.Rev. of Freycinetia with key.

-, ibid. 26 (1925) 448-451.-4 new Pandanus from Busuanga.

-, ibid. 29 (1926) 476.-New Pandanus from Bohol.

Quisumbing \& MerRill, Philip. J. Sc. 37 (1928) 134-135.-2 new Pandanus from Luzon.

Warburg, in Perkins, Fragm. Fl. Philip. pt 1 (1904) 50.-New Pandanus.

Moluccas: FAGERLind, Svensk Bot. Tidskr. 34 (1940) 101-116.-New sp. Buru.

New Guinea: Kanehira, Bot. Mag. Tokyo 54 (1940) 249-260, 9 fig.-Compl. list; no key.

Kanehira \& Hatusima, Bot. Mag. Tokyo 55 (1941) 251-308.-List, with new spp.

MaRTelli, Bot. Jahrb. 49 (1913) 60-67.-List; no key.

-, J. Arn. Arb. 10 (1929) 137-142, t. 18.-New spp. Brass coll.

Merrill \& Perry, J. Arn. Arb. 20 (1939) 139 186, t. 1-2.-Descr., list Brass coll.; no keys. -, ibid. 21 (1940) 163-175.-Many new spp. Pandanus \& Freycinetia.

RIDLEY, J. Bot. 24 (1886) 359.-List ForBEs coll.; 2 new Freycinetia.

White, J. Arn. Arb. 10 (1929) 201-202.-List Brass coll.

Micronesia: KanehirA, J. Jap. Bot. 12 (1936) 495-501, 545-554, 21 fig.-List; no keys.

_, ibid. 12 (1936) 783-792, 6 fig.-Figures.

-, ibid. 13 (1937) 322-331, fig. 7-14.-Figures. 
Fiji: Martelli, Univ. Calif. Publ. Bot. 12 (1930) 325-335, t. 37-44.

PerRY, J. Arn. Arb. 31 (1950) 208-213.Freycinetia, revis.; key.

Queensland: Martelli, Proc. R. Soc. Queensl. 36 (1925) 129-130, t. 25.-New sp.

\section{Genera}

Freycinetia: Hemsley, Kew Bull. (1896) 164-167.Descr. new Freycinetias.

Martella, Webbia 3 (1910) 167-186.-New spp. from Borneo, Moluccas, Timor, and New Guinea.

Pandanus: Balfour, J. Linn. Soc. Lond. Bot. 17 (1878) 33-68.-Enumeration spp.

FAgerlind, Svensk Bot. Tidskr. 34 (1940) 101116. -4 new spp. cult. Bogor.

Kurz, in Miquel, Ann. Mus. Bot. Lugd.-Bat. 2 (1865) 52-54.-Descr.; no key.

-, J. As. Soc. Beng. 38, ii (1869) 145-151.Rectification of sections.

Martelli, Bull. Soc. Bot. Ital. (1904) 298-304.

-Brief descr. keyed out.

-, Nuovo Giorn. Bot. Ital. n.s. 36 (1929) 328-

337.-Nomencl. Pandanus tectorius \& odoratissimus.

Sararanga: Stapf \& Hemsley, J. Linn. Soc. Lond. Bot. 32 (1896) 479-489, t. 4-7.-Revision; morphol.

\section{PAPAVERACEAE}

Fedde, F., Pflanzenreich Heft 40 (1909) 1-430, fig. 1-43.-Revision of Hypecoideae \& Papaveroideae; some genera are: Papaver, Argemone, Eschscholzia, and Bocconia.

-, Pflanzenfamilien ed. 2, 17 b (1936) 5-145, fig. 1-76.

Hutchinson, J., The genera of Papaveraceae (Kew Bull. 1925, 161-168).-Key to genera.

Steenis, C. G. G. J. vaN, Fl. Mal. I, 5 (1954) 114-117, fig. 1-2.

\section{Local}

Java: BACKER, Schoolfl. Java (1911) 51-53. -, Onkruidfl. Jav. Suiker. pt 2 (1930) 249-250.

\section{Genera}

Argemone: PraIN, J. Bot. 33 (1895) 129-135. 176-178, 207-209, 307-312, 325-333, 363-371. -Revision; key!

Bocconia: Hutchinson, Kew Bull. (1920) 275281.-Revision; key!

Chelidonium: Prann, Bull. Herb. Boiss. 3 (1895) 570-587.

Meconopsis: TAYLOR \& Cox, An account of the genus Meconopsis. London (1934) 1-130.

\section{PASSIFLORACEAE}

Blume, C. L., Rumphia 1 (1836) 165-170.-No key.

Hallier $f$., H., Indonesische Leidensblumen (Meded. Rijksherb. Leiden no 42, 1922, 1-17). -Revision; list.

Harms, H., Pflanzenfamilien ed. 2, 21 (1925) $470-507$.
KIlliP, E. P., The American species of Passifloraceae (Field Mus. Nat. Hist. (bot. ser.) 19, 1938, 7-613).-Keys!

Masters, M. T., Contributions to the natural history of the Passifloraceae (Trans. Linn. Soc. $27,1871,593-645$, t. $64-65,1$ fig.).-With enumeration of genera and spp. of tribe Passifloreae with synonyms.

\section{Local}

India: Chakravarty, Bull. Bot. Soc. Bengal 3 (1949) 45-71, 2 pl.-Revision Ind. spp.; descr.; keys.

Sumatra: Merrill, Contr. Arn. Arb. 8 (1934) 110-111.-New Adenia.

Borneo: Ridley, Kew Bull. (1938) 112.-List of Adenia records.

Java: BACKER, Bekn. Fl. Java (em. ed.) 4a (1942) fam. 87.

Van Steenis, Bull. Bot. Gard. Btzg III, 17 (1948) 400-401.-P. capsularis.

New Guinea: Merrill \& Perry, J. Arn. Arb. 24 (1943) 210.-Record Hollrungia. 一, ibid. 29 (1948) 160.-Record Hollrungia. -, ibid. 30 (1949) 44.-List. Pulle, Nova Guinea 8 (1911) 673.-Record. White, J. Arn. Arb. 10 (1929) 244,-Record.

\section{Genera}

Hollrungia: Van SteEnIS, Reinwardtia 1 (1952) 480-481.-Revision; E. Malaysia.

\section{PEDALIACEAE}

(incl. Martyniaceae)

BACker, C. A., Fl. Mal. I, 4 (1951) 216-221, fig. 1-3.

BruCe, E. A., Notes on African Pedaliaceae (Kew Bull. 1953, 417-429).-Contains notes on Sesamum and Josephinia.

\section{PENTAPHRAGMATACEAE}

Poulson, V. A., Pentaphragma ellipticum sp. nov. Et Bidrag til Kundskab om Slaegten Penta-. phragma Wall. (Vidensk. Medd. Naturh. Før. Kjøbenhavn, 1903, 319-331, t. 4-5).-Descr. new sp. Mal. Pen.; anatomy; considerations on affinity.

Shaw, H. K. AIRY, The Pentaphragmataceae of the Oxford University Expedition to Sarawak (Kew Bull. 1941, 233-236).-Descr. family; discussion affinity.

-, Fl. Mal. I, 4 (1953) 517-528, fig. 1-3.

\section{Local}

Sumatra: Merrill, Pap. Mich. Ac. Sc. Arts \& Lett. 19 (1934) 201-203.-2 new spp.

RidLey, J. Fed. Mal. St. Mus. 8, pt 4 (1917) 56. -New sp.

Borneo: AIRY SHAw, Kew Bull. (1941) 235-236.Records RichaRds coll.

Philippines: Philip. J. Sc. 37 (1928) 211.-New sp. Moluccas: KuRz, Bot. Zeit. 30 (1872) 136.-New $s p$. from Batjan.

New Guinea: Pulle, Nova Guinea 8 (1910) 407, (1911) 691.-Records. 


\section{Genera}

Pentaphragma: AIRY SHAw, Kew Bull. (1953) 241249.-Descr. new spp.

\section{PENTAPHYLACACEAE}

Mattfeld, J. Pflanzenfamilien ed. 2, $20 \mathrm{~b}$ (1942) 13-21.

Tieghem, Ph. van, in Morot, J. Bot. 14 (1900) 189-193.-Morphol.

\section{Local}

Indo-China: Gagnepain, Suppl. Fl. Gén. IndoChine 1 (1943) 332-334.-Descr.; key.

Sumatra: van SteEnis, Bull. Bot. Gard. Btzg III, 17 (1948) 404.-First record.

Malay Peninsula: Ridlex, F1. Mal. Pen. 1 (1922) 205-206.

一, ibid. 5 (1925) 291.

\section{PERIPTERYGIACEAE}

See Icacinaceae

\section{PHILYDRACEAE}

Caruel, T., in DC. Monogr. Phan. 3 (1881) 1-6.No key.

Pilger, R., Pflanzenfamilien ed. 2, 15a (1930) 190-191.

SkottsBerg, C., Bemerkungen über die Philydraceen (Bot. Jahrb. 65, 1932, 253-274, 65 fig.)

-, The Philydraceae of Netherlands India (Bull. Jard. Bot. Btzg III, 13, 1933, 110-113).-Revision; key.

-, Fl. Mal. I, $4^{1}$ (1948) 5-7, fig. 1.

\section{PHYTOLACCACEAE}

Backer, C. A., Fl. Mal. I, 4 (1951) 228-232, fig. 1. Heimerl, A., Pflanzenfamilien ed. 2, 16c (1934) 135-164.

Walter, H., Pflanzenreich Heft 39 (1909) 1-154, fig. 1-42.

\section{PINACEAE}

See Coniferae

\section{PIPERACEAE}

Blume, C. L., Monographie der Oost-Indische pepersoorten (Verh. Bat. Gen. K. \& W. 11, 1826, 139-245),-Descr. many new spp. of Piper.

Candolle, C. De, in DC. Prod. 16, 1 (1869) 235-471.-Descr.; partial keys.

- Piperacearum clavis analytica (Candollea 1, 1923, 65-415).-Keys to Piper \& Peperomia.

Mrquel, F. A. W., Systema Piperacearum. Fasc. 1 (1843) 1-304, fasc. 2 (1844) 305-575.-Key to genera; keys or partial keys to spp.

-, Illustrationes Piperacearum (Nov. Act. Acad. Leop.-Carol. Nat. Cur. 21, Suppl. 1846, 1-87, t. 1-92).-Descr.; no keys.

- Animadversiones in Piperaceas Herbarii Hookeriani (Lond. J. Bot. 4, 1845, 410-470).Descr.

- Mantissa Piperacearum e speciminibus Musei Vindobonensis, Regii Monacensis et Martiana (Linnaea 20, 1847, 117-182).-Enum. and descr.
-, Piperaceae Reinwardtianae (ibid. 21, 1848 , 480-486).-Enum. and descr.

-, Ann. Mus. Bot. Lugd.-Bat. 1 (1863-64) 134 141.-List new spp.

\section{Local}

Malay Peninsula: de Candolle, Rec. Bot. Surv. Ind. 6 (1912) 1-29.-Descr. new spp.

Borneo: de Candolle, Ann. Cons. Jard. Bot. Genève 21 (1920) 279-295.-Descr.

Schwartz, Mitt. Allg. Inst. Bot. Hamburg 7 (1931) 231-234.-List; new spp.

Java: de CANDolle, Ann. Cons. Jard. Bot. Genève 15-16 (1912) 231-235.-Enum.; descr. coll. HochreUtiNer; some new spp.

-, Med. Rijksherb. Leiden 22 (1914) 1-7.Enum., descr.

-, Ann. Cons. Jard. Bot. Genève 21 (1920) 271-277.

Koorders, Verh. Kon. Ak. Wet. A'dam sect. 2, 14, no 4 (1908) repr. 1-75.-Revision; keys.

Lesser Sunda Islands: DE CANDOLle, Med. Rijksherb. Leiden 22 (1914) 1-7.-List, descr.

Celebes: de Candolle, Ann. Cons. Jard. Bot. Genève 21 (1920) 297-304.-Descr. new spp.

Philippines: de Candolle, in Perkins, Fragm. Fl. Philip. pt 1 (1905) 153-160.-Descr.

-, in Elmer, Leafl. Philip. Bot. 3 (1910) 759-789.-Descr.

—, Philip. J. Sc. 5 (1910) Bot. 405-463.-Revision; keys!

-, in Elmer, Leafl. Philip. Bot. 6 (1914) 2291-2294.-Descr. 6 new spp.

—, Philip. J. Sc. 11 (1916) Bot. 207-225.-New spp. and records of Piper.

Miquel ex Meyen in Nov. Act. Acad. Caes. Leop.-Car. 19 (1843) Suppl. 1, 483-494.Descr.

Quisumbing, Philip. J. Sc. 43 (1936) 1-246, fig. 1-124, t. 1-24.-Revision; descr. \& keys!

Moluccas: de Candolle, Philip. J. Sc. 11 (1916) Bot. 260-261.-Descr. new spp. Ambon. -, Med. Rijksherb. Leiden no 32 (1918) 1-2. -Descr. new spp. Key Isl.

New Guinea: DE CANDolle, Nova Guinea 8 (1910) 415-422; (1914) 1005-1010.-Descr.

-, in Grbes, Contr. Fl. Phytog. Arfak Mts (1917) 127-128, 207-208.

-, Bot. Jahrb. 55 (1918) 204-220.-Descr.; no keys.

-, Candollea 2 (1925) 216-222.-Descr. new spp.

Trelease, J. Arn. Arb. 9 (1928) 146-150.-Descr. new spp.

Fiji: SmIrh, J. Arn. Arb. 24 (1943) 347-361. -Crit. not. Pacific spp.; key to Fijian spp. of Piper.

Hawaii: Yuncker, Bern. P. Bish. Mus. Bull. 112 (1933) 1-131, fig. 1-38.-Rev. Peperomia; keys.

Polynesia: Yuncker, Bern. P. Bish. Mus. Bull. 143 (1937) 1-73, fig. 1-35.-Rev. Peperomia; keys.

\section{PIROLACEAE}

See Ericaceae 


\section{PITTOSPORACEAE}

Blume, C. L., Mus. Bot. Lugd.-Bat. 1 (1850) 158-162.-Descr.; \pm key.

Miquel, F. A. W., Ill. Fl. Arch. Ind. (1871) 7584, t. 34-37.-Descr.; \pm key.

Pritzel, E., Pflanzenfamilien ed. 2, 18a (1930) 265-286.

PutTerlick, A., Synopsis Pittosporearum (in NeEs, Gen. Pl. fasc. 22-24? Non vidi). Separate print Vienna (1839) 30 pp.-Descr. of spp.; partial keys to spp.; no key to genera.

\section{Local}

Java: BACKer, Schoolf. Java (1911) 73-75. KoORders \& VAleton, Ic. Bog. 2 (1904) t. 138, p. 1-2.-Descr.

New Guinea: Merrill \& Perry, J. Árn. Arb. 21 (1940) 175-179.-Descr.; no key.

Pritzel, Bot. Jahrb. 62 (1929) 338-340.-Descr.; no key.

Pulle, Nova Guinea 8 (1910) 365; (1911) 643. -Descr.

\section{Genera}

Citriobates: Boerlage \& Koorders, Ic. Bog. 1 (1901) t. 77, p. 1-2.-Descr. Jav. sp.

Hymenosporum: BAKHUIZEN VAN DEN BRINK \& VAN Steenis, Blumea 7 (1952) 292.-Record from New Guinea.

Pittosporum: Gagnepann, Bull. Soc. Bot. Fr. 55 (1908) 544-548.-Descr. SE. Asiat. spp.

GowdA, J. Arn. Arb. 32 (1951) 263-343.-Rev. Sino-Indian spp.; keys!

Sherf,, Field. Mus. Nat. Hist. bot. ser. 22 (1942) 467-566, 574-580.-Revision Hawaiian spp.; keys per island!

\section{PLANTAGINACEAE}

Pilger, R., Bot. Jahrb. 50, 1. Suppl. (1914) 61-71. -Distr. \& morph. of Sect. Plantaginella DECNE.

- Die Arten der Plantago major-Gruppe in Ostasien (Notizbl. Berl.-Dahlem 8, 1922, 104 116).-Key!

—, Pflanzenreich Heft 102 (1937) 1-466, fig. 1-45.

Local

Java: BACKer \& VAN SLoOten, Theeonkruiden Java (1924) 200.

New Guinea: Merrill \& Perry, J. Arn. Arb. 30 (1949) 52-55.-Descr. new spp.

PILGER, Bot. Jahrb. 67 (1935) 236.-Record.

\section{PLUMBAGINACEAE}

Steenis, C. G. G. J. Van, Fl. Mal. I, 4 (1949) 107-112, fig. 2.

\section{Local}

New Guinea: Pulle, Nova Guinea 8 (1910) 397. Genera

Plumbago: van Steenis, Trop. Natuur 26 (1937) 12-13.-Key.

\section{PODOCARPACEAE See Coniferae}

\section{PODOSTEMACEAE}

Engler, A., Pflanzenfamilien ed. 2, 18a (1930) 3-68.

Steenis, C. G. G. J. van, Bull. Jard. Bot. Btzg III, 13 (1936) 530-534.-Key.

-, Fl. Mal. I, 4 (1949) 65-68, fig.1-2; 503.

\section{Local}

New Guinea: van Steenis, J. Arn. Arb. 28 (1947) 421.

\section{Genera}

Cladopus: Mö́ler, Ann. Jard. Bot. Btzg 16 (1899) 115-132, t. 12-15. -Type descr.

Lemnopsis = Halophila \& Utricularia.

Torrenticola: van Steenis, Proc. R. Soc. Queensl. 62 (1952) 67-68, t. 2.

\section{POLEMONIACEAE}

BACKer, C. A., Fl. Mal. I, 4 (1951) 195-196, 1 fig. Brand, A., Pflanzenreich Heft 27 (1907) 1-202, fig. 1-39.

\section{Genera}

Cobaea: Standley, Contr. U. S. Nat. Herb. 17 (1914) 448-458, t. 26-31.-Revision; key.

Polemonium: Davidson, Univ. Cal. Publ. Bot. 23 (1950) 209-282, fig. 1-23.-Revision; keys.

\section{POLYGALACEAE \\ (incl. Xanthophyllaceae)}

Chodat, R., Monographia Polygalacearum (Mém. Soc. Phys. Hist. Nat. Genève Suppl. 1890 (1891) 1-143, t. 1-12; 31, 1893, 1-500, t. 13-35). -, Polygalaceae novae vel parum cognitae $\mathbf{V}$ (Bull. Herb. Boiss. II, 4, 1906, 233-237).-Some new spp.; no keys.

HasskarL, J. K., Polygalaceae, praesertim indicae (Ann. Mus. Bot. Lugd.-Bat. 1, 1863-64, 142196).-Semi-monogr.; some keys.

\section{Local}

Indo-China: Gagnepain, Suppl. Fl. Gén. IndoChine 1 (1939) 217-236; ibid. (1943) 237-238.Keys.

Sumatra: Chodat, Bull. Herb. Boiss. 4 (1896) 234.-Polyg. sumatrana.

Merrill, Contr. Arn. Arb. 8 (1934) 84-85.Crit. not. P. pulchra.

Ridley, J. Mal. Br. R. As. Soc. no 87 (1923) 53. -List 5 records.

Borneo: Chodat, in Merrill, Plant. Elm. Born. (1929) 133-137.-Rev. \& new spp. of Xanthophyllum; no key.

FISCHER, Kew Bull. (1932) 176-177.-New Xanthophyllum.

RidLEY, Kew Bull. (1938) 113-115.-New Xanthophyllums.

AIRY SHAw, Kew Bull. (1940) 252-253.-Records Oxf. Univ. Exp.

Philippines: Chodat, Bull. Herb. Boiss. 4 (1896) 233.

Elmer, Leafl. Philip. Bot. 5 (1913) 1671-1678.Descr. new Securidaca \& Xanthophyllums. 
-, ibid. 10 (1939) 3776-3777.-New Xanthophyllum.

MERRILL, Philip. J. Sc. 27 (1925) 29-30.-New Xanthophyllum.

New Guinea: Kassau, in Fedde, Rep. 35 (1934) 160.-Descr. 2 Securidacas.

SMITH, in FedDE, Rep. 10 (1912) 486.

-, Nova Guinea 8 (1912) 897-898, t. 158.Descr. Epirixanthes papuana.

WENT, Nova Guinea 8 (1909) 169.

\section{Genera}

Epirixanthes: Penzig, Ann. Jard. Bot. Btzg 17 (1901) 142-170, t. 20-26.-Anat.

Van Steenis, Trop. Natuur 23 (1934) 51.-Key. _, Bull. Bot. Gard. Btzg III, 17 (1948) 461. -Generic syn.

Eriandra: van RoYen \& van SteEnis, J. Arn. Arb. 33 (1952) 91-96, 1 fig.-End. new genus of New Guinea.

Semeiocardium. See Balsaminaceae.

Xanthophyllum: CHоDAт, Bull. Herb. Boiss. 4 (1896) 254-264.-Conspectus; new spp.; no key. Gagnepain, in Morot, J. de Bot. 21 (1908) 241-253.-Discuss. taxon.; key!

Miquel, Ann. Mus. Bot. Lugd.-Bat. 1 (1864) 271-277, 317-318.-Descr.

\section{POLYGONACEAE}

BruYn, A. J. DE, in MiQueL, Pl. Jungh. (1854) 302311.-No key.

DANSER, B. H., Die Polygonaceen NiederländischOstindiens (Bull. Jard. Bot. Btzg III, 8, 1927, 117-261, fig. 1-17).-Rev.; keys.

-, ibid. III, 12 (1932) 65-70, 3 fig.-Descr. some Rumex and Polygonum hybrids from Java.

HedrerG, O., Pollen morphology in the genus Polygonum L. sens. lat. and its taxonomic significance (Svensk Bot. Tidskr. 40, 1946, 371404, fig. 1-47).-Pollen types are typical for segregate genera; proposed segregation and key to these.

Meissner, C. F., in Miquel, Ann. Mus. Bot. Lugd.-Bat. 2 (1865) 55-65.-List; descr.; no key.

\section{Local}

Java: Danser, Med. Proefst. Thee no 98 (1926) 16 pp., 9 fig.-Descr.\& fig. Polyg. of tea plantauons; key.

-, in BACKer, Bekn. Fl. Java (em. ed.) 4A (1942) fam. 60.

New Guinea: DANSER, Nova Guinea 14 (1927) 333-336.

-, Bull. Jard. Bot. Btzg III, 13 (1935) 429-431. -List of records; no key.

Merrill \& Perry, J. Arn. Arb. 23 (1942) 384-385.-Records.

一, ibid. 29 (1948) 154.-New record $P$. attenuatum. Pulle, Nova Guinea 8 (1910) 359; (1911) 625-626.-Records; descr. Muehl. monticola.

\section{Genera}

Muehlenbeckia: Danser, Bull. Jard. Bot. Btzg 16 (1940) 324-328.-Descr. Pap. \& Queensl. spp.
Polygonum: Danser, Bull. Jard. Bot. Btzg III, 8 (1926) 25-31.-Syn. Reynoutria \& Truellum.

GAGE, Rec. Bot. Surv. Ind. 2 (1903) 371-452. -Enum. of Indian spp.; keys!

Steward, Contr. Gray Herb. Harv. Univ. 88 (1930) 3-129, t. 1-4.-Revision E. Asiat. spp.; keys.

Rumex: Rechinger, Beih. Bot. Centralbl. 49, ii (1932) 1-132.-Prelim. to monogr.; no keys. - , Bot. Notiser, Suppl. $3: 3$ (1954) 1-114.Monogr. African spp.

\section{PONTEDERIACEAE}

BACKeR, C. A., Fl. Mal. I, 4 (1951) 255-261, fig. 1-3.

Schwartz, O., Anatomische, morphologische und systematische Untersuchungen uber die Pontederiaceen (Beih. Bot. Centralbl. 42, i, 1926, 263-320).-No taxon.

-, Zur Systematik und Geographie der Pontederiaceen (Bot. Jahrb. 61, 1927, Beibl. 139, p. 28-50).-Syst. geogr. disc.; partial key.

-, Pflanzenareale II, 2 (1928) Karten 11-17.Geogr. maps.

-, Pflanzenfamilien ed. 2, 15a (1930) 181-188, fig. 67-71.

Solms-Laubach, H., in DC. Monogr. Phan. 4 (1883) 501-535.-Rev.; keys.

\section{Local}

Java: BACKer, Handb. Fl. Java 3 (1924) 39-42. -Key.

New Guinea : HalliER $f$, Nova Guinea 8(1913)909. -One record.

VAN Steenis, J. Arn. Arb. 28 (1947) 419.Record.

\section{PORTULACA CEAE}

Pax, F. \& K. Hoffmann, Pflanzenfamilien ed. 2, $16 \mathrm{c}$ (1934) 234-262, 587.

\section{Local}

Java: BACKer, Schoolfl. Java (1911) 84-85.-Key. New Guinea: Merrill \& Perry, J. Arn. Arb. 23 (1942) 386.-Montia recorded!

Pulle, Nova Guinea 8 (1910) 357.-P. oleracea.

\section{Genera}

Portulaca: von Poellnitz, in Fedde, Rep. 37 (1934) 240-320.-Precurs. to monogr.; keys!

Talinum: voN Poellnitz, Ber. Deut. Bot. Ges. 51 (1933) 112-127.

-, in Fedde, Rep. 35 (1934) 1-34.-Monogr.

\section{POTAMOGETONACEAE}

Ascherson. P., Nuovo Giorn. Bot. Ital. 3 (1871) 299-302.-List marine spp. of BECCARI coll.; em. descr. of Halophila with a new $s p$.

- \& P. Graebner, Pflanzenreich Heft 31 (1907) 1-184, fig. 1-36.

Steenis, C. G. G. J. van, Trop. Natuur 22 (1933) 43-46.-Key to marine representatives.

-, Webbia 8 (1952) 435.-List records of BECCARI coll. ditto. 


\section{Local}

Java: BACKeR, Handb. Fl. Java pt 1 (1924) 47-51. Philippines: Bennetr, Philip. J. Sc. 9 (1914) Bot. 339-344; ibid. 40 (1929) 183.-List; descr. new spp.

Australia: Ostenfeld, Dansk Bot. Ark. 2 (6) (1916) 1-44.-W. Australian seagrasses.

-, Proc. R. Soc. Victoria n.s. 42 (1929) 1-3.List Austr. spp. of marine representatives. 一, ibid. 43 (1930) 1 pp.-Additions.

\section{Genera}

Potamogeton: BenNeTt, J. Bot. 28 (1890) 297-302; ibid. 29 (1891) 121-122, 150-152, 154, 186.List; nomencl.; notes on $P$. javanicus.

-, J. Bot. 65 (1927) 113-116.-Various notes; also $P$. javanicus.

Hagström, Critical researches on the Potamogetons (Kungl. Svenska Vet.-Ak. Handl. 55 (1916) 1-281, fig. 1-119).-Revision; no key!

Miquel, III. Fl. Arch. Ind. pt 1 (1870) 44-48. -List.

Van Steenis, Arch. Hydrobiol. Suppl. 10 (1932) 268-271, fig. 1.-List; crit. notes.

Ruppia: Setchell, Proc. Calif. Acad. Sc. IV, 25 (1946) 469-478, t. 47-48).-Revision.

\section{PRIMULACEAE}

Pax, F. \& KNUth, R., Pflanzenreich Heft 22 (1905) 1-386, fig. 1-75, 2 maps.

Steenis, C. G. G. J. van, Bull. Jard. Bot. Btzg III, 13 (1934) 236-238.-List.

\section{Local}

Sumatra: Merrill, Not. Nat. Acad. Nat. Sc. Philad. no 47 (1940) 6-7.-Descr. new Primula.

SMITH, W. W., Trans. \& Proc. Bot. Soc. Edinb. 35 (1950) 181-182.-Discuss. Primula sumatrana.

Malay Peninsula: Henderson, J. Mal. Br. R. As. Soc. 17 (1939) 53.-Record of Lysimachia peduncularis.

Java: BACKer, Bekn. Fl. Java (em. ed.) 8 (1949) fam. 180, p. 1-4.-Keys; in Dutch.

New Guinea: Merrill \& Perry, J. Arn. Arb. 29 (1948) 162-163.-Record.

WENT Jr, Nova Guinea 14 (1924) 113.-List.

\section{Genera}

Lysimachia (Orescia): Handel-Mazzetti, Not. R. Bot. Gard. Edinb. 16 (1928) 51-123.-New system of genus; revision Chinese spp. with a key.

-, Pflanzenareale $2^{5}$ (1929) 39-41, Karten 44-49.-Distr.

Van Steenis, Bull. Bot. Gard. Btzg III, 17 (1948) 458.-Syn.

Primula: Ernst, Ann. Jard. Bot. Btzg 49 (1940) 99-161.-Flower morph. of $\boldsymbol{P}$. imperialis.

PaX, Bot. Jahrb. 10 (1889) 75-241.-Concise revision; keys.

Smith, W. W. \& Fletcher, Trans. \& Proc. Bot. Soc. Edinb. 33 (1941) 122-181.-Rev. $\S$ Candelabra; key.

— \& Forrest, J. R. Hort. Soc. Lond. 54 (1928)
4-49, fig. 1-70.-Definition \& keys to sections; synopsis of species.

\section{PROTEACEAE}

Sleumer, H., Proteaceae Americanae (Bot. Jahrb. 76, 1954, 139-211).-Keys!

Local

Malay Peninsula: Symington, Kew Bull. (1937) 319.-Syn. Helicia excelsa.

Borneo: Merrill, Plant. Elm. Born. (1929) 52-53. -Records.

Moluccas: Miquel, Ann. Mus. Bot. Lugd.-Bat. I (1864) 204.-Descr. spp. Ambon.

New Guinea: Diels, Bot. Jahrb. 50 (1913) 328334.-Keys!

—, ibid. 54 (1917) 198-206.-No keys.

-, Nova Guinea 14 (1923) 74.

LAUTERBACH, Nova Guinea 8 (1910) 285; (1912) 811.-Records.

VON MUELLER, Vict. Nat. 3 (1886) 63-64.-Descr. Sleumer, Bot. Jahrb. 70 (1939) 125-148.-Compl. revision; keys!

-, Bot. Jahrb. 76 (1954) 139-211.

WhITE, Proc. R. Soc. Queensl. 34 (1922) 24-27. -Records; descr.

-, J. Arn. Arb. 10 (1929) 209-210.-Records; a new Helicia.

\section{Genera}

Finschia: Sleumer, Bot. Jahrb. 70 (1939) 126.Red. to Grevillea.

WARBURG, Bot. Jahrb. 13 (1891) 297-298.

WhITE, Pac. Sc. 3 (1949) 187-194, 3 fig.-Revision; key.

Grevillea: Sleumer, Bot. Jahrb. 70 (1939) 126130.-Key.

Helicia: Blume, Ann. Sc. Nat. II, 1 (Bot.) (1834) 211-220.-Census.

Macadamia: Johnson, Proc. Linn. Soc. N. S. W. 79 (1954) 15-18.-Descr. new sp. allied to $M$. ternifolia.

Van Steenis, Reinwardtia 1 (1952) 467-481, 2 fig.-New sp. from Celebes.

\section{PUNICACEAE}

BACKer, C. A., Fl. Mal. I, 4 (1951) 226-227, fig. 1.

\section{Local}

Java: BACKer, Bekn. Fl. Java (em. ed.) 4a (1942) fam. 74.

\section{Genera}

Punica: CaIRns, J. Bomb. Nat. Hist. Soc. 42 (1940) 13-37.-P. granatum.

\section{RAFFLESIACEAE}

Harms, H., Pflanzenfamilien ed. 2, 16b (1935) 243-281.

KoORders, S. H., Botanisch overzicht der Rafflesiaceae van Nederlandsch-Indië (1918) vi, 1128, t. 1-18.-Revision; in Dutch; keys.

Solms-Laubach, H., Pflanzenreich Heft 5 (1901) 1-19, fig. 1-13. 


\section{Local}

Sumatra: Suringar in Boerlage, in Veth, Midden-Sumatra 4, 2 (1884) 26-30, 2 pl.Descr. Rafflesia hasseltii; discussion specific differences in the genus; in Dutch.

Java: Blume, Flora Javae fasc. 1-2 (1828) 1-26. VAN STEENIS, Trop. Natuur 23 (1934) 47-48.Key.

Borneo: BecCarI, Atti Soc. Ital. Sc. Nat. 11 (1868) repr. 1-2.-Descr. new Brugmansia and Rafflesia.

—, Nuovo Giorn. Bot. Ital. 1 (1869) repr. 20-27, t. 5.-Descr. new Brugmansia.

\section{Genera}

Mitrastemon: Hayata, Ic. Plant. Formos. 3 (1913) $156,199-213$.-Syst. position of genus. $C f$. also Bot. Jahrb. 51 (1913) 164-176.

Jochems, Rec. Trav. Bot. Néerl. 25a (1928) 203-207.-Descr. N. Sumatran new sp.

MatudA, Bull. Torrey Bot. Club 74 (1947) 133-141.-Key!

Palm, Medd. Göteb. Bot. Trädg. 9 (1934) 147152, 1 fig.-Record from Sumatra; descr.

WATANABE, J. Jap. Bot. 12 (1936) 603-618.Revision; key.

Yamamoto, Bot. Mag. Tokyo 50 (1936) 539541.-First record from Mexico.

Raffiesia: Mrouel, Analecta Bot. Ind. pt 3 (is 23-30, t. 2-3.-Obs. $R$. rochussenii.

Solms-Laubach, Ann. Jard. Bot. Btzg 9 (1891) 184-246, t. 25-28.-Descr.

-, Ann. Jard. Bot. Btzg Suppl. 3 (1910) 1-7.Descr. $R$. cantleyi from the Malay Peninsula.

Van Steenis, c.s., Bull. Brit. Mus. (Nat. Hist.) hist. ser. 1 (1954) 56,61, t. 13.-First collection ever made of Rafflesia by Deschamps.

Rhizanthes (Brugmansia BL., Mycetanthe): BLUME, in VAN HALL, Bijdr. Nat. Wet. 2 (1827) 422-423. FawCETt, Trans. Linn. Soc. Lond. II, Bot. 2 (1886) 244-245, t. 36.-Descr. B. lowii.

HARMS, in FedDE, Rep. 36 (1934) 286-287.Nomencl.

\section{RANUNCULACEAE}

Hutchinson, J., Kew Bull. (1923) 75-89.-Key to tribes and genera.

Korthals, P. W., Ned. Kruidk. Arch. 1 (1847) 207-211.-Census Indonesian representatives.

PrantL, K., Beiträge zur Morphologie und Systematik der Ranunculaceen (Bot. Jahrb. 9, 1887, 225-273).-Contr. morphol. \& taxon.; partial keys to spp.

Steenis, C. G. G. J. van, Bull. Jard. Bot. Btzg III, 13 (1934) 238-240.-List mountain spp.

\section{Local}

Sumatra: van Steenis, Bull. Bot. Gard. Btzg III, 17 (1948) 403-404.-Anemone rivularis.

Borneo: STAPF, Trans. Linn. Soc. Lond. II, Bot. 4 (1894) 127-128.-Descr.

Java: BACKER, Schoolfl. Java (1911) 5-8.-In Dutch; key.

New Guinea: Drels, Bot. Jahrb. 62 (1929) 479.Descr.
Merrill \& Perry, J. Arn. Arb. 24 (1943) 34

-41.-Descr.!

-, ibid. 29 (1948) 154-155.-Records.

Pulle, Nova Guinea 8 (1911) 631.

\section{Genera}

Aconitum: Stapf, Ann. Roy. Bot. Gard. Calc. 10 (1905) 115-197, t. 92-116.-Revision Asiat. spp.; key.

Anemone: Pritzel, Linnaea 15 (1842) 561-698, t. 1-6.-Revision, no key.

UlRRICH, Bot. Jahrb. 37 (1905) 172-334, 37 fig. -Infrageneric segr.; geogr. census of species; no key.

Aquilegia: BRÜHL, J. As. Soc. Beng. 61, ii (1892) 270-324, t. 3-6.-Rev. Ind. spp.; no key.

Munz, Gentes Herb. 7 (1946) 1-150, fig. 1-38.Monogr.; keys.

Clematis: Hallier $f$., Ann. Jard. Bot. Btzg 14 (1897) 248-276, t. 18-20.-No key.

Kuntze, Verh. Bot. Ver. Prov. Brandenburg 26 (1885) 83-202. Nachtr. in Verh. Zool.-Bot. Ges. Wien 37 (1887) 47-50.-Monogr.; keys.

Delphinium: Huth, Bot. Jahrb. 20 (1895) 322-499, t. 6-8.-World monogr.

Thalictrum: BoIvin, Rhodora 46 (1944) 337-377, 391-445, 453-487, fig. 1-114.-Revision Amer. spp. and their Old World allies; keys.

LeCOYer, Bull. Soc. Roy. Bot. Belg. 24 (1885) 78-325, t. 1-5.-Monogr.; key.

Von Regel, Bull. Soc. Nat. Moscou 34, 1 (1861) 14-63, 3 pl.-Key to spp. of Russia, Siberia, and N. China.

\section{RESEDACEAE}

Mueller, J. von, Monographie de la famille des Résédacées (Neue Denkschr. Allg. Schweiz. Ges. 16 (1) (1857) 1-239, t. 1-10.-Keys!

$$
\text { Lacal }
$$

Java: BACKeR, Schoolfl. Java (1911) 64.-Culta.

\section{RESTIONACEAE}

Masters, M. T., in DC. Monogr. Phan. 1 (1878) 218-398, t. 1-4.-Monogr.; no keys to spp.

Steudel, E. G., Synopsis plantarum Glumacearum pars II. Stuttgart (1855) 246-266.-Enum. descr. in Latin.

\section{Local}

Malay Peninsula: RidLey, Fl. Mal. Pen. 5 (1925) 136.-Descr. Leptocarpus disjunctus MAsT.

\section{R H A M N A C E A E}

Suessenguth, K., Pflanzenfamilien ed. 2, 20d (1953) 7-173, fig. 1-49.

\section{Local}

Sumatra: Merrill, Contr. Arn. Arb. 8 (1934) 96-97.-Critical notes.

Malay Peninsula: Symington, Mal. Br. R. As. Soc. 14 (1936) 350.

Borneo: RIDLEY, Kew Bull. (1931) 493-495.New spp. of Ventilago and Zizyphus; new records.

Suessenguth \& Overkott, in Fedde, Rep. 50 (1941) 330-331.-New Zizyphus. 
Java: BACKer, Schoolf. Java (1911) 239-244.

- \& BAKHUIZEN VAN DEN BRINK Jr, Blumea 5 (1945) 519-521.-List.

Philippines: Merrill, Philip. J. Sc. 27 (1925) 35.Record of Sageretia hamosa BRONGN.

—, Philip. J. Sc. 30 (1926) 408.-New Zizyphus.

New Guinea: LaUterbach, Bot. Jahrb. 57 (1922) 326-340.-Keys.

$\rightarrow$ Bot. Jahrb. 59 (1925) 536-537.-A new doubtful Rhamnus.

MANSFezd, Notizbl. Berl.-Dahlem 10 (1928) 222-224, fig. 2.-New Alphitonia.

Merrill \& Perry, J. Arn. Arb. 20 (1939) 337339.-Descr.

-, J. Am. Arb. 22 (1941) 263-264.-New Ventilago \& Colubrina.

WeNT Jr, Nova Guinea 14 (1924) 110-111.

\section{Genera}

Alphitonia: Braid, Kew Bull. (1925) 168-186.Revision; key!

Rhamnus: VAN STEenis, J. Bot. 72 (1934) 6-7.New spp. Borneo \& Sumatra.

-, Bull. Jard. Bot. Btzg III, 13 (1934) 240.List mountain spp.

Sageretia: Drummond \& Sprague, Kew Bull. (1914) 175.-Correction on next paper.

Schneider, in Sargent, Pl. Wils. 2 (1914) 226-231.-Revis. Asiat. spp.; key.

Smythea: SummerhaYes, Kew Bull. (1928) 389.S. lanceata (TulasNe) SUMmerhaYes correct name for $S$. pacifica.

Ventilago (Apteron): BenthaM, J. Linn. Soc. Bot. 5 (1861) 75-77.-Revision; no key.

Merrill, Philip. J. Sc. 11 (1916) Bot. 287.New sp. from Ambon.

Ridley, Fl. Mal. Pen. 5 (1925) 300.

Van Steenis, Blumea 7 (1954) 597.-Distr. $V$. oblongifolia.

Zizyphus: Santapau, J. Bombay Nat. Hist. Soc. 51 (1953) 801-804.-Remarks on nomencl. of genus \& spp.; distinction of 4 Deccan spp.; no key.

Van Steens, Blumea 7 (1954) 595-596.-Syn. \& distr. Z. grewioides.

\section{RHIZOPHORACEAE}

Bentham, G., Synopsis of the Legnotideae, a tribe of the Rhizophoraceae (J. Linn. Soc. Bot. 3, 1858, 65-80).-Synopsis.

Blume, C. L., Mus. Bot. Lugd.-Bat. 1 (1849-'50) 126-145.-Census; descr.

Miquel, F. A. W., Ann. Mus. Bot. Lugd.-Bat. 2 (1865) 66-67.-Only subfam. Legnotideae. List; no key.

Valeton, Th., Bull. Jard. Bot. Btzg III, 2 (1920) 346-348.-New Carallia; new var. in Pellacalyx.

\section{Local}

Malay Peninsula: KING, J. As. Soc. Beng. 66, ii (1897) 311-326.-Keys.

Bomeo: Ridley, Kew Bull. (1938) 282-283.Descr. new Carallias.

Java: BACkER, Schoolfl. Java (1911) 478-486.In Dutch; keys.
New Guinea: Pulle, Nova Guinea 8 (1910) 393394; (1912) 679.

White, Proc. R. Soc. Queensl. 34 (1922) 46.List.

\section{Genera}

Anisophyllea: Burkill, Gard. Bull. Str. Settl. 4 (1927) 426.-Syn. of $A$. grandis.

SANDWITH, Kew Bull. (1952) 303-306.-Record from America.

Bruguiera: Merrill, Trans. Am. Phil. Soc. Philad. n.s. $24^{2}$ (1935) 282.-Nomencl.

Combretocarpus: AIRY SHAw, in Hook. Ic. Pl. (1937) t. 3335.-Ample discussion of ecol. and morphol.

Pellacalyx (Craterianthus): KoRThals, Tijd. Nat. Gesch. \& Phys. 3 (1836) 20-22.-Descr.

SCORTEChINI, Nuovo Giorn. Bot. Ital. 17 (1885) 140-144.-P. saccardianus n. sp.

Van SteEnis, Bull. Bot. Gard. Btzg III, 17 (1948) 457.-Synonymy.

Rhizophora: Salvoza, Univ. Philip. Nat. \& Appl. Sc. Bull. 5 (1936) 179-237, t. 1-9.-Revision whole genus; key.

\section{ROSACEAE}

Blume, C. L., Melanges botaniques (Flora 41, 1858, 255-256).-Concise review of an unpublished paper of BLume by HasskaRL.

Korthals, P. W., Nat. Tijd. Ned. Ind. 7 (1854) 206-212.-Latin diagn.

-, Bijdrage tot de kennis der Chrysobalaneae van Nederlandsch Oost-Indië (Ned. Kruidk. Arch. 3, 1855, 384-388).-Copy of the above; descr. 3 new genera.

Miquel, F. A. W., Chrysobalaneae quaedam Indicae (Ann. Mus. Bot. Lugd.-Bat. 3, 1867, 236-237).-List.

Steenis, C. G. G. J. van, Bull. Jard. Bot. Btzg III, 13 (1934) 240-246.-List mountain spp.

\section{Local}

Sumatra: Merrill, Pap. Mich. Acad. Sc. Arts \& Lett. 19 (1934) 155-157.-Descr. new Photinia, 2 new Pygeums.

- Not. Nat. Acad. Nat. Sc. Philadelphia no 47 (1940) 3.-Record Potentilla.

Malay Peninsula: Henderson, Gard. Bull. Str. Settl. 7 (1933) 101-103.-2 new spp.

Borneo: Merrill, J. Str. Br. R. As. Soc. no 76 (1917) 81-83.-New records in Parinari, new spp. of Pygeum.

-, Plant. Elm. Born. (1929) 92-94.-New Parinaris.

Ridley, Kew Bull. (1938) 280-282.-Records of Rubus \& new Pygeum.

Java: BACKER, Schoolfl. Java (1911) 443-465. Docters van LeEuWEN, Trop. Natuur 18 (1929) 148-151, fig. 1-3.-Rubus calycinus in E. Java. Koorders, Bull. Jard. Bot. Btzg III, 1 (1918) 84-85, t. 5.-Key to Prunus; one new sp.

VAN STEENIS, Trop. Natuur 21 (1932) 103-104, fig. 3-4.-Potentilla mooniana.

- Trop. Natuur 24 (1935) 122, fig. 1.-Rubus calycinus in E. Java. 
Lesser Sunda Islands: FocKE, Med. Rijksherb. Leiden no 14 (1912) 39-40.-List Rubus coll. ELBERT.

Von MaLm, Notizbl. Berl.-Dahlem 11 (1932) 630.-New Parinari from Flores.

Philippines: Elmer, Leafl. Philip. Bot. 2 (1908) 445-462.-Revision of Philippine Rubus; key! 一, ibid. 5 (1913) 1621-1628.-Census descr. Philip. Pygeum.

—, ibid. 10 (1939) 3777-3778.-New Rubus.

— \& Focke, ibid. 5 (1913) 1617-1619.-Descr. 2 new spp. Rubus.

Merrull, Philip. J. Sc. 29 (1926) 480.-New Potentilla.

-, ibid. 30 (1928) 395-396.-New Pygeum.

New Guinea: BAKER, J. Bot. 61 (1923) Suppl. 13.New Angelesia.

Diels, Bot. Jahrb. 62 (1929) 480-482.-Notes on Acaena, Potentilla, and Rubus.

Focke, Abh. Naturw. Ver. Bremen 13 (1894) 161-166.-Records of Potentilla, Rubus; some new.

-, Bot. Jahrb. 54 (1916) 69-73.-List spp. NE. part, some new.

Merrill \& Perry, J. Arn. Arb. 21 (1940) 179_ 198.-New spp. in Potentilla, Pygeum, Parastemon, and Rubus; no key.

-, ibid. 22 (1941) 256.-Note on Parastemon.

Pulle, Nova Guinea 8 (1910) 367.

一, ibid. 8 (1912) 647.-New Rubus.

Ridley, Trans. Linn. Soc. Lond. II, Bot. 9 (1916) 35-37.-On Rubus \& Potentilla.

Whrte, J. Arn. Arb. 31 (1950) 86-88.-New spp. of Parinari.

\section{Genera}

Acaena: BitTer, Bibl. Bot. Heft 74 (1910-1911) 1336, t. 1-37.-Revision; key.

—, in FEDde, Rep. 10 (1912) 498-501.-Suppl. notes to revision.

Alchemilla : Rothmaler, in Fedde, Rep. 38 (1935) 33-43.

—, ibid. 40 (1936) 208-212.-Sect. div.

Angelesia (Trichocarya): BoERLAGE \& KoORders, Ic. Bog. 1 (1901) t. 96.

Eriobotrya: CARDot, Bull. Mus. Hist. Nat. Paris 25 (1919) 205-207.-Critical notes; census Paris sheets.

Geum: Bolle, in Fedde, Rep. Beih. 72 (1933) 119 pp.-Revision; with keys.

Inodaphnis = Microcos (Tiliaceae).

Parastemon (Diemenia): BOERLAGE \& KOORD., Ic. Bog. 1 (1901) t. 97.

Korthals, Nat. Tijd. Ned. Ind. 7 (1854) 212.

Parinari (Cyclandrophora, Parinarium): BLUME, Mus. Bot. Lugd.-Bat. 2 (1852) 94-99.-Descr.

KANEHIRA, J. Japan. Bot. 16 (1940) 471-475, 4 fig.

Van Steenis, Bull. Bot. Gard. Btzg III, 17 (1948) 461.-Synon.

Potentilla: WolfF, Bibl. Bot. Heft 71 (1908) 1-715, 20 tab., 2 maps.-Monogr.; keys. Obsolete for Malaysia.

Prunus: Koehne, in Sargent, PI. Wils. pt 2 (1912) 196-282.-Principally account of Chin. spp.; subdivision genus; keys to spp. in some subgen. $\&$ sect.

Pygeum: Koehne, Bot. Jahrb. 51 (1913) 177-224. -Unsatisfactory \& very incomplete now; geogr. key

-, ibid. 52 (1915) 334-345.-Suppl. notes; descr. new spp.

Miqued, Ann. Mus. Bot. Lugd.-Bat. 1 (1864) 212.-Descr.

Rosa: Boulenger, Bull. Jard. Bot. Brux. 13 (1935) 165-266.-Revision of some groups of Rosa $\S$ Eglanteriae in Asia.

ibid. 14 (1936) 115-221, (1937) 274-292.

一, ibid. 14 (1937) 241-273.-Introd. to taxon. of genus.

Rubus: Focke, Bot. Jahrb. 1 (1881) 87-103.General disc. taxon.

-, Bibl. Bot. Heft 72 (1910) 1-120, fig. 53; ibid. (1911) 121-223, fig. 54-87; ibid. Heft 83 (1914) 1-274, fig. 88-155.-Revision; keys.

\section{ROXBURGHIACEAE \\ See Stemonaceae}

\section{RUBIACEAE}

BAKhuizen VAN DEN BrINK Jr, R. C., Notes on Malaysian Rubiaceae (Blumea 7, 1953, 329338).-Critical notes; new descr.

Haviland, G. D., A revision of the tribe Naucleae (J. Linn. Soc. Lond. Bot., 33, 1897, 1-94, t. 1-4).-Monogr. of the tribe; keys!

Korthals, P. W., Over het geslacht Nauclea en eenige verwante geslachten (in TEMMINCK, Verhand. (Botanie), 1842, 150-174, pl. 32-35, 48.-Deals with the tribe Naucleae.

-, Overzigt der Rubiaceën van de NederlandschOostindische Koloniën (Ned. Kruidk. Arch. 22, 1851, 99-136, 145-269).-Disc. \& enum. Neth. East Indian spp. \& genera.

Miquel, F. A. W., in de VRIESe, Plant. Ind. Bat. Orient. (1856) 156-160.-Descr. of some spp. from herb. REINWARDT, mainly from Java.

-, De quibusdam Rubiaceis, Apocyneis et Asclepiadeis (Ann. Mus. Bot. Lugd.-Bat. 4, 1869, 128-137).-Descr. of various genera \& spp.

-, Ecloge Rubiacearum archipelagi Indici (ibid. 4, 1869, 179-213, 221-262).-Ditto; no keys.

RidLey, H. N., Notes on some Malayan Rubiaceae (Kew Bull. 1939, 493-513, sphalm. 593-613).Crit. notes on various genera \& spp.

RobIJNs, W., Tentamen monographiae Vangueriae generumque affinium (Bull. Jard. Bot. Brux. 11, 1928, 1-359, fig. 1-36).-Monogr. of Vangueria and segregates; keys.

Steenis, C. G. G. J. van, Bull. Jard. Bot. Btzg III, 13 (1934) 246-248.-List mountain spp.

Valeton, TH., in Ic. Bog. 2 (1903-1906) t. 141148, 183-184.-Descr. various records and spp. -, ibid. 3 (1906-1909) t. 221 (BACKER), 247-248, 267-273, 278-299.-Ditto.

-, ibid. 4 (1913) t. 355-366.-Ditto.

\section{Local}

Siam: RIdLEY, Kew Bull. (1931) 441-448.-Descr. - new spp. coll. KERR. 
Sumatra: Merrill, Contr. Arn. Arb. 8 (1934) 156-164.-List.

—, Pap. Mich. Acad. Sc. Arts \& Lett. 19 (1934) 194-199.-Descr. new spp.

-, Pap. Mich. Acad. Sc. 23 (1937) 1938, 191202.-Descr. new spp.

VALETON, Ic. Bog. 4 (1914) t. 390.-New sp. from Billiton.

Malay Peninsula: Corner, Gard. Bull. Str. Settl. 10 (1939) 46-55.-Critical notes.

Ridley, J. Bot. 66 (1932) 191-197, 219-226.Census of Urophyllum in Malaya; no key.

-, ibid. 72 (1934) 251-257, 271-274.-Descr. of new Ixora, Tarenna, Gardenia, Geocardia.

Scortechini, J. Bot. 22 (1884) 369-370.Descr. Creaghia (= Mussaendopsis).

Java: BACKER, Onkruidfl. Jav. Suiker. (1931) 685-722.

Borneo: Merrill, J. Str. Br. R. As. Soc. no 76 (1917) 117.-New Uncaria.

-, ibid. no 77 (1917) 238-245.-Descr. new Timonius from Borneo.

一, J. R. As. Soc. Mal. Br. 1 (1923) 37-44.Descr. new spp.

-, Sarawak Mus. J. 3 (1928) 534-537, 553-556. -, Mitt. Inst. Allg. Bot. Hamb. 7 (1937) 270 301.-Descr. \& new records.

Ridley, Sarawak Mus. J. 1 (1912) 30-32.Descr. of some new spp.

—, Kew Bull. (1934) 119-120.-Descr. 3 new spp.

STaPf, Trans. Linn. Soc. Lond. II, Bot. 4 (1894) 168-187.-List; descr. new spp.

Van Steenis, J. Bot. 72 (1934) 9.-Descr. Coptosapelta montana.

WERnhAM, J. Linn. Soc. Bot. 42 (1914) 87-97.Descr. \& records.

Celebes: BremEKAMP, Blumea 5 (1945) 587-591, fig. 11-12.-Records \& descr. new spp.

KoOrders, Suppl. Flora N.O. Celebes 2 (1922) t. 123-127; ibid. 3 (1922) 58-60.-Descr. new spp.

Moluccas: BremeKamp, Blumea 5 (1942) 243-251. -List Talaud \& Morotai.

Philippines: Elmer, Leafl. Philip. Bot. 1 (1906) 1-41.-Survey of fam.; keys to spp.

-, ibid. (1906) 63-73.-Addit. new spp.

一, ibid. 3 (1911) 971-1046.-New \& noteworthy spp.

-, ibid. 4 (1912) 1327-1362.-New spp. \& records from Palawan Island.

一, ibid. 5 (1913) 1855-1905.-New spp. \& records from Mt Urdaneta (Mindanao).

一, ibid. 9 (1934) 3209-3216.-New spp. from Mt Pinatubo (Luzon).

_, ibid. 9 (1937) 3242-3272.-Various new spp.

-, ibid. 10 (1939) 3778-3781.-Two new spp.

Merrill, Philip. J. Sc. 8 (1913) Bot. 31-62, t. 1.-Descr. new spp., records, critical notes on synon.

—, ibid. 10 (1915) Bot. 99-144.-Ditto.

-, ibid. 12 (1917) Bot. 159-176.-Descr. new spp.

—, ibid. 17 (1920) 425-485.-Descr. new spp.

_, ibid. 26 (1925) 492-496.-Descr. new spp.
—, ibid. 27 (1925) 55-59.-Descr. new spp.

—, ibid. 29 (1926) 489-495.-Descr. new gen. \& spp. from Bohol \& Mindanao.

-, ibid. 35 (1928) 7-9.-New comb. for Philip.

'Plectronia'.

Quisumbing, Philip. J. Sc. 41 (1930) 360-368.-

Descr. new spp.

— \& Merrill, Philip. J. Sc. 37 (1928) 200-211.

-Descr. new spp.

New Guinea: Diels, Bot. Jahrb. 62 (1929) 493-494. -New Galium.

Merrill \& Perry, J. Arn. Arb. 25 (1944) 183-205; ibid. 26 (1945) 1-36, 1 pl., 229-266; ibid. 27 (1946) 193-233.-Account BRAss coll.; no keys.

—, ibid. 29 (1948) 164-167.-Descr. new spp.

MoORE, J. Bot. 65 (1927) 241-247, 265-272.Descr. \& records coll. Brass.

-, ibid. 67 (1929) 49.-New Psychotria.

Valeton, Bull. Dép. Agr. Ind. Néerl. 10 (1907) 61-67.-List.

-, Nova Guinea 8 (1911) 437-519, (1912) 755-775.-Descr.

-, in Gibss, Contr. Fl. Phytog. Arfak Mts (1917) 180-182, 220-221.-Descr.

- Bot. Jahrb. 60 (1925) 1-104; 61 (1927) 32163.-Descr.; keys!

-, Nova Guinea 14 (1926) 229-274, t. 20-29. WernhaM, J. Bot. 56 (1918) 68-77, 129-135.List Forbes coll.; new records and descr. new spp.

White, Proc. R. Soc. Queensl. 34 (1922) 52-59. -List; descr. new spp.

一, Proc. Linn. Soc. N.S.W. 51 (1926) 296, t. 17.-New Plectronia.

New Caledonia: Guillaumin, in Viguier, Arch. Bot. 3 (1930) Mém. 5, p. 1-47.-Keys!

\section{General}

Acranthera (Psilobium, Gonyanera): BREMEKAMP, J. Arn. Arb. 28 (1947) 261-308.-Revision; key. Adina: Haviland, J. Linn. Soc. Lond. Bot. 33 (1897) 42-48.-Revision; key.

VerhoEF, Tectona 31 (1938) 802-814.-List.

Antherostele: Bremekamp, J. Arn. Arb. 21 (1940) 25-31.-Segregate of Urophyllum; $4 \mathrm{spp}$. in Philippines.

Anthocephalus: Haviland, J. Linn. Soc. Lond. Bot. 33 (1897) 22-24.-Revision; key.

Antirrhea: Valeton, Bull. Dép. Agr. Ind. Néerl. 26 (1909) 31-32.

White, J. Arn. Arb. 27 (1946) 121-122, t. 1.List 3 Australian spp.

Aphaenandra: Bremekamp, Blumea Suppl. 1 (1937) 116-122.

Jochems, Trop. Natuur 18 (1929) 153-154.Descr.; fig.

Van Steenis, Bull. Bot. Gard. Btzg III, 18 (1950) 457-458.

DE Voogd, Trop. Natuur 18 (1929) 110-111.Descr.; photogr.

Argostemma (Argostemmella): BAKHuIzEN VAN

(1) Not all segregate genera proposed by BREMEKAMP are mentioned here separately. 
DEN BRINK Jr, Blumea 7 (1953) 329-334.Sect. div.; descr. new spp.; critical notes. Ridley, J. Bot. 65 (1927) 25-42.-Census; no key.

Asemanthia (Mussaenda \& Asemanthia): RidLey, Kew Bull. (1939) 499-500.-Segregate; Mal. Pen. \& Borneo; list 4 spp.

Bikkia: Beumée, Trop. Natuur 15 (1926) 198-200. -Distr. \& descr. B. grandiflora.

Guillaumin, Not. Syst. 1 (1909) 111-112.Discuss. gen. syn. of New Caledon. Bikkia.

Valeton, Philip. J. Sc. 23 (1923) 573-574.New sp. from Cebu.

Boholia: Merrill, Philip. J. Sc. 29 (1926) 492494.-Descr.; Bohol Isl.

Borreria: BACKER, Onkruidfl. Jav. Suiker. (1931) 714-722.-In Dutch; Jav. spp.; key.

Canthium (Plectronia auct. non L.): BuRTT, Kew Bull. (1936) 463.-Recomb. in a Canthium.

Merrill, Philip. J. Sc. 35 (1928) 7-9.-New comb. for Philippine 'Plectronia'.

Carlemannia, See Caprifoliaceae; cf. Fl. Mal. I, 4 (1951) 192-194.

Cinchona: KunTze, O., Monographie der Gattung Cinchona. Leipzig (1878).-Non vidi.

Miquel, Ann. Mus. Bot. Lugd.-Bat. 4 (1869) 263-275.-Descr. spp. cult. in Java.

Coelospermum: Guillaumin, Arch. Bot. Caen 3 (1929) Mém. V, p. 15, 41.

Coffea: Chevalier, Rev. Int. Bot. Appl. \& Agr. Col. 26 (1946) 81-94, map.-Ecol. \& geogr. distr. of the species.

—, Les Caféiers du globe fasc. 3. Systématique (Encycl. Biol. 28, 1947, 117-263).-Revision; keys!

Froehner, Notizbl. Berl.-Dahlem 1 (1897) 230-238.-Revision; key.

一, Bot. Jahrb. 25 (1898) 233-295.-Revision; key.

VALEToN, Bull. Inst. Bot. Btzg no 8 (1901) 15-25. -Discussion; no key.

Coprosma: Oliver, Bern. P. Bish. Mus. Bull. 132 (1935) 1-207, t. 1-59, 67 fig.-Monogr.; key.

-, Rec. Dominion Mus. 1 (1942) 44-47, t. 14.Descr. 3 new spp., 2 from Papua, one from Hawaii.

Coptosapelta: Valeton, Versl. Kon. Akad. Wet. A'dam (Sept. 1908) 120-126.-Discussion; census. —, Rec. Trav. Bot. Néerl. 19 (1922) 281-292, t. 10-11.-Descr.; key!

_, Versl. Kon. Akad. Wet. A'dam 26 (1923) 423-440, fig. 1-14.-Descr.

Craterianthus = Pellacalyx (Rhizophoraceae); $c f$. Bull. Bot. Gard. Btzg III, 17 (1948) 457.

Dentella: AIRy Shaw, Kew Bull. (1932) 289-292.Descr. some extra-Mal. spp.

-, Kew Bull. (1934) 290-301.-Australian spp.; keys!

Didymoecium: Bremekamp, Bull. Jard. Bot. Btzg III, 13 (1935) 425-428, 1 fig.-Descr.; monotypic; Sumatra.

Dolianthus : BrEMEKAMP, Kew Bull. (1936) 103-105. -Formerly incl. in Logan. but closely allied to Amaracarpus. In my opinion at most a sect. of it (Ed.).
Galium: van Steenis, Trop. Natuur 25 (1936) 152-155.-In Dutch; key to Mal. spp.

Gardenia: Sprague, Kew Bull. (1929) 12-16.Syn. of G. jasminoides ElLIs.

Geocardia: (Geophila D. DON, non BergereT): STandley, Contr. U.S. Nat. Herb. 17 (1914) 444-445.-Census; no key.

Guettarda: BaIllon, Adansonia 12 (1879) 316319.-Delimitation of the genus.

Hedyotis (Oldenlandia, Pleiocraterium): BACKER, Onkruidfl. Jav. Suiker. (1931) 688-700.-Key Jav. spp.

Bremekamp, Rec. Trav. Bot. Néerl. 36 (1939) 438-445.-Rev. Pleiocraterium.

-, Verh. Kon. Akad. Wet. 2e reeks, 48, no 2 (1952) 297 pp., 13 tab.-Rev. African spp.; keys.

Fosberg, Bern. P. Bish. Mus. Bull. no 174 (1943) 102 pp., 9 fig., t. 1-4.-Revision Hawaiian spp.

Kanehira, Trans. Nat. Hist. Soc. Formosa 25 (1925) $2-7,35-39 .-$ List, descr. of records and new spp.

Merrill \& Metcalf, J. Arn. Arb. 23 (1942) 226-230, 1 pl.-Discussion generic concept and synonymy; descr.

RidLey, Kew Bull. (1939) 511-513.-New spp.; records.

Ixora: BaIlloN, Adansonia 12 (1878) 213-219.Delimitation of the genus.

BremeKamp, Bull. Jard. Bot. Btzg III, 14 (1937) 197-367.-Revision; keys!

—, ibid. 16 (1940) 276-299.-Add. \& emend. to revision.

-, J. Bot. 75 (1937) 108-111, 169-175, 260-266, 295-298, 318-326; ibid. 76 (1938) 330-336.Census Andaman Isl. and Burma; no key.

一, J. Arn. Arb. 20 (1939) 216-219.-New spp. from Solomons.

CORNER, Gard. Bull. Str. Settl. 11 (1941) 177235.-Key \& disc. Mal. Pen. spp.

Kajewskiella: Merrill \& PerRY, J. Arn. Arb. 28 (1947) 331-332, t. 1.-Descr.; Solomons.

Lachnastoma: VALETON, Bull. Inst. Bot. Btzg no 8 (1901) 25-33.-Key.

Lasianthus: Wight, Calcutta J. Nat. Hist. 6, p. 494-518.-Non vidi.

Lecariocalyx: BremEKAMP, Kew Bull. (1940) 192193.-Aff. Grumilea (Psychotrieae); Borneo.

Lerchea: BAKHUIzeN VAN DEN BRINK Jr, Blumea 7 (1953) 334-335.-New Jav. sp.

Mastixiodendron (Dorisia): MERRILL \& PERRY, J. Arn. Arb. 23 (1942) 416; ibid. 25 (1944) 205; ibid. 26 (1945) 254-256.

Meyna: Robisns, Bull. Jard. Bot. Brux. 11 (1928) 226-242.-The As.-Mal. complement of Vangueria and formerly included in that genus; key!

Mitragyne: Haviland, J. Linn. Soc. Lond. Bot. 33 (1897) 68-73.-Revision; key.

Morinda: Balllon, Bull. Soc. Linn. Paris 1 (1879) 205-206.-Delimitation of the genus; remarks on some BECCARI nos.

Mussaenda: RIDLEY, Kew Bull. (1929) 497-499.Descr. new spp.

SulIT, Philip. J. For. 2 (1939) 35-41, pl. 1-3.- 
Descr. \& key to spp. in Makiling National Park, Luzon.

Mussaendopsis: Bremekamp, Rec. Trav. Bot. Nérl. 36 (1939) 367-371.-Revision; key.

STAPF, in HoOK. Ic. Pl. 24 (1895) t. 2388.M. beccariana.

Mycetia (Adenosacme): SPRAgue \& Fischer, Kew Bull. (1928) 253.-Gen. nomencl.

VALETON, Ic. Bog. 3 (1908-1909) t. 270, 281-282.

-Fig., descr., syn. of 3 Jav. spp.

Myrmeconauclea: Merrill, Philip. J. Sc. 17 (1920) 375-376.-Monotypic; Mal.

Nauclea (Sarcocephalus): Haviland, J. Linn. Soc. Lond. Bot. 33 (1897) 24-33.-Revision; key.

Merrill, J. Wash. Acad. Sc. 5 (1915) 530-537. -Typification; new comb. under Nauclea.

Neonauclea (Nauclea auct.): HAvilAND, J. Linn. Soc. Lond. Bot. 33 (1897) 48-68.

Merrill, J. Wash. Acad. Sc. 5 (1915) 530-542. -Typification; new comb. under Neonauclea.

Pavetta: Bremekamp, in Fedde, Rep. 37 (1934) 1-208.-Monogr.; keys.

-, Blumea Suppl. 1 (1937) 112-116.-Notes on synon.

二, in Fedde, Rep. 47 (1939) 12-28, 81-98.Emend. to monograph.

Pentas: Verdcourt, Bull. Jard. Bot. Brux. 23 (1953) 237-371.-Revision; keys!

Perakanthus: RoBisss, Bull. Jard. Bot. Brux. 11 (1928) 330-331.-2 spp. Mal. Pen.; segregate of Canthium.

Pleiocarpidia: Bremekamp, Rec. Trav. Bot. Néerl. 37 (1940) 198-236.-Revision; key.

Polycycliska: RidLeY, Kew Bull. (1926) 67-68.Monotypic; Mentawai; said to be allied to Pomazota.

Pomazota (Coptophyllum KoRTH. non GARDN.): Bremekamp, J. Arn. Arb. 28 (1947) 186-203.Revision; key.

Porterandia (Randia \& Anisophyllea Hook. f.): RIDLEY, Kew Bull. (1939) 493-497.-Segregate; W. Malaysia; key.

Praravinia (Williamsia): Bremekamp, Rec. Trav. Bot. Nérl. 37 (1940) 237-278.-Revision of spp. from Celebes \& Borneo; key.

KOORDers, Nat. Tijd. Ned. Ind. 63 (1903) 7375.-Descr. 2 spp. from Borneo \& Celebes.

Korthals, in Temminck, Verh. Nat. Gesch. Botanie (1842) 189-191.

Prismatomeris: RIdLey, Kew Bull. (1939) 501-507. -Crit. remarks; list; records.

Valeton, Bull. Inst. Bot. Btzg no 8 (1901) 1-34.-Discussion.

Psilobium: KERR, in HoOK. Ic. Pl. V, 4 (1937) t. 3332.-Disc.; fig.

Psychotria (Gouldia): BremekAMP, J. Bot. 71 (1933) 271-280.-Descr. \& key to the bacteriophilous species of Psychotria.

Ernst, Arch. J. Klaus Stift. (Zürich) 7 (1932) 241-280, 9 fig.-Heterostyly.

St.John, Bern. P. Bish. Mus. Occ. Pap. 12 no 7 (1936) 1-4, t. 1.-Reduction of Gouldia.

Randia (Anomanthodia, Canthopsis, Gynopachys): Baillon, Bull. Soc. Linn. Paris 1 (1879) 206.Reduction of Canthopsis.
FAgerlind, Arkiv Bot. 30A, no 7 (1942) 1-57, 10 fig. -Survey of morphol.; precursory for a future revison \& segregation.

GAMBLE, Kew Bull. (1921) 312-313.-Critical notes on some spp. from India.

Valeton, Not. Syst. 3 (1914) 54-55.-New sp. from Rawak Isl.

Rennellia: RIDley, Kew Bull. (1929) 507-509.Key.

Rhadinopus: Moore, J. Arn. Arb. 11 (1930) 129131, fig. 1.-Monotopic; New Guinea; aff. Gardenia.

Siderobombyx: Bremekamp, J. Arn. Arb. 28 (1947) 204-206.-Monotypic; aff. Pomazota.

Spermacoce: RENDLE, J. Bot. 72 (1934) 329-333.Exam. Linnean specimens.

Steenisia (Neurocalyx p.p.): BAKHUIzEN VAN DEN Brink Jr, Webbia 8 (1952) 381-382.-Census of the genus.

AIRY SHaw, Kew Bull. (1937) 281-291, t. 9. Valeton, in Fedde, Rep. 12 (1913) 513-514.

Stichianthus: Valeton, Bull. Jard. Bot. Btzg III, 2 (1920) 349-350, t. 10.-Descr.

Winkler, Hans, Planta 13 (1931) 85-101, 5 fig. -Morph. inflor.

Streblosa: Bremekamp, J. Arn. Arb. 28 (1947) 145-185.-Revision; key!

Sulitia: MerRILL, Philip. J. Sc. 29 (1926) 494-495. -Descr.; Mindanao.

Timonius: Merrill, J. Arn. Arb. 18 (1937) 130132.-Some new comb.

—, ibid. 26 (1945) 235-254.-Numerous Pap. new spp.

Valeton, Bull. Dép. Agr. Ind. Nérl. no 26 (1909) 1-61.-Descr.; key.

Uncaria: Haviland, J. Linn. Soc. Lond. Bot. 33 (1897) 73-92.-Revision; key.

Urophyllum: BremeKAMP, J. Arn. Arb. 21 (1940) 32-47.-Revisions Pleiocarpidia, Praravinia, and Urophyllum; keys.

一, Rec. Trav. Bot. Néerl. 37 (1940) 171-197.Urophyllum split into a number of segregate genera.

Wendlandia: Cowan, Not. Roy. Bot. Gard. Edinb. 16 (1932) 233-316, t. 232-235; ibid. 18 (1934) 183-188.-Revision; keys.

-, Bull. Jard. Bot. Btzg III, 14 (1936) 8-46, fig. 1-26.-Rev. Mal. spp.; key.

Xanthophytum: BAKHUIZEN VAN DEN BRINK Jr, Blumea 7 (1953) 335-337.-Descr. new spp. from Borneo.

Zeuxanthe: (Morinda \& Dibrachya): RIDLEY, Kew Bull. (1939) 509-511.-Borneo; 3 spp.; key.

\section{RUTACEAE'}

ENGLER, A., Uber die geographische Verbreitung der Rutaceen im Verhältnis zu ihrer systematischen Gliederung (Abh. K. Preuss. Akad. Wiss. Phys. Abh. 1896 i, 1-27, 3 maps).

-, Pflanzenfamilien ed. 2, 19a (1931) 187-359, fig. 89-165.

(1) There are various opinions on generic delimitation in this family. Many data in this bibliography should be handled with great caution. 
Guillaumin, A., Espèces ou localités nouvelles pour les Rutacées d'Extrême-Orient (Not. Syst. 1, $1909,207-223 ; 1911,327-328)$.-List of records and notes, also several from Malaysia.

MiQuel, F. A. W., Aurantiaceae novae (Ann. Mus. Bot. Lugd.-Bat. 1, 1864, 211).-Diagn. 5 new species.

-, Rutacearum quarundam illustratio (Ann. Mus. Bot. Lugd.-Bat. 3, 1867, 242-246).-New diagn. in Evodia, Acronychia, and Zanthoxylum.

Oliver, D., The natural order Aurantiaceae, with a synopsis of the Indian species (J. Linn. Soc. Lond. Bot. 5, 1861, Suppl. 2, 1-44).-Synopsis; delimitation of genera; no keys!

Shaw, H. K. AIRY, Notes on the nomenclature of certain Rutaceae-Aurantioideae (Kew Bull. 1939, 290-293).-Straightening out nomencl. of Limonia-Atalantia-Feronia.

Swingle, W. T., A new taxonomic arrangement of the orange subfamily, Aurantioideae (J. Wash. Acad. Sc. 28, 1938, 530-533).-Concise concept of a new classification; some new comb.

-, New varieties and new combinations in the genera Clausena, Oxanthera, and Triphasia of the orange subfamily, Aurantioideae (J. Wash. Acad. Sc. 30, 1940, 79-83, 2 fig.).-New comb., mostly of varieties.

-, The Botany of Citrus and the wild relatives of the orange subfamily (in The Citrus Industry 1, 1943, 127-474).-Non vidi.

Tanaka, T., Revisio Aurantiacearum I (Bull. Soc. Bot. Fr. 75, 1928, 708-715).-Various notes, new comb. and descr. new spp.

-, Notes on the Dutch Indian species of RutaceaeAurantieae (Med. Rijksherb. Leiden no 69, 1931, 1-13).-List of records; some new spp. in Clausena, Glycosmis, Lavanga.

-, The taxonomy and nomenclature of RutaceaeAurantioideae (Blumea 2, 1936, 101-110).Considerations in general and on Citrus in particular.

\section{Local}

SE. Asia: Guillaumin, Not. Syst. 1 (1910) 207-224; (1911) 327-328.-List of records and notes.

Tanaka, Bull. Mus. Hist. Nat. II, 2 (1930) 157-164.-Compendium with several new spp. and comb.

-, Enumeration of Indian species of RutaceaeAurantioideae (J. Bot. 68, 1930, 225-236).List; syn.; some new spp.

-, J. Ind. Bot. Soc. 16 (1937) 227-240.-List of records with new spp. and comb.

Malay Peninsula: Burkill, Gard. Bull. Str. Settl. 5 (1931) 212-223.-List of Atalantia, Citrus, and Paramignya in the Peninsula.

Sumatra: Merrill, Pap. Mich. Acad. Sc. 23 (1938) 180-183.-New spp. of Evodia \& Clausena.

Borneo: Merrill, J. Str. Br. R. As. Soc. no 86 (1922) 315.-New Evodia.

RIDLEY, Kew Bull. (1930) 77-80.-Records and descr. of new spp.

Java: BACKER, Schoolfl. Java (1911) 174-189.-In Dutch; keys!
BAKHUIZEN VAN DEN BRINK Jr, Blumea 6 (1950) 365-367.-List; some new spp. \& new names.

Philippines: Elmer, Leafl. Philip. Bot. 9 (1934) 3216-3217.-A new Evodia.

一, ibid. 10 (1939) 3781-3783.-A new Clausena and Lunasia.

MerRILL, Philip. J. Sc. 26 (1925) 458-459.-

New spp. in Clausena and Triphasia.

一, ibid. 27 (1925) 26.-New spp. in Micromelum and Clausena.

—, ibid. 29 (1926) 481.-New Lunasia.

-, ibid. 30 (1926) 399-400.-Syn. of Merope ROEM.; new Glycosmis.

Quisumbing \& Merrill, Philip. J. Sc. 37 (1928) 154.-New Clausena.

New Guinea: BAILEY, Ann. Rep. Br. New Guinea (1901-02) app. p. 1, pl. (1902).-2 new Citrus.

Francis, Kew Bull. (1931) 188-195.-Synon. of an Evodia and an Acronychia.

Kurata, Bull. Tokyo Univ. For. no 36 (1948) 124-129, 2 fig.-2 new Evodias \& other records. LaUTERBACH, Nova Guinea 8 (1910) 291-293.Records \& descr. new spp.

-, ibid. 8 (1912) 823-825.-Ditto.

-, ibid. 14 (1924) 139-147.-Ditto.

-, Bot. Jahrb. 55 (1918) 221-265.-Revision; keys.

一, ibid. 59 (1925) 535-536.-New Terminthodia. 一, ibid. 61 (1927) 30-31.-New Evodia.

Merrill \& Perry, J. Arn. Arb. 20 (1939) 330-333.-List; 2 new Flindersias.

-, ibid. 22 (1941) 32-59.-List; many new spp. RIDley, Trans. Linn. Soc. Lond. II, Bot. 9 (1916) 24-25.-Descr. some new spp.

Swingle, J. Wash. Acad. Sc. 30 (1940) 79-83.

TanakA, J. Arn. Arb. 9 (1928) 137-141.Descr. of Monanthocitrus, Echinocitrus; new comb. in Atalantia, Wenzelia \& Clausena.

Valeton, Bull. Dép. Agr. Ind. Néerl. no 10 (1907) 23-24.-Records and a new Melicope.

White, Proc. R. Soc. Queensl. 34 (1922) 37-38. -List of records.

-, J. Arn. Arb. 7 (1926) 231-233.-New Paramignya; on spp. of Citrus and Lunasia.

- \& Francis, Proc. R. Soc. Queensl. 38 (1927) 232-234.-New Flindersia.

Melanesia: BurTt, Kew Bull. (1935) 300-301.New Fagara and Melicope from the Solomons.

New Caledonia: Guillaumin, Bull. Soc. Bot. Fr. 85 (1938) 294-305.-Keys.

\section{Genera}

Acronychia (Terme) : Hallier $f$., Rec. Trav. Bot. Néerl. 15 (1918) 65-67.-Generic syn.

Atalantia: Guillaumin, Not. Syst. 1 (1910) 175-184.-List and key for Asiat. spp.

AIRY SHAw, Kew Bull. (1939) 290-293,-Exact syn. of some spp.

SwIngle, Not. Syst. 2 (1911) 158-163.-Addit. notes to GuILlaumin's paper and reduction of one $s p$. to Glycosmis.

Bouchardatia: RIDLEY, Trans. Linn. Soc. II, Bot. 9 (1916) 25.-B. cyanosperma n.sp. from New Guinea. 
Burkillianthus: Swingle, J. Arn. Arb. 20 (1939) 255-258, t. 2-3.-Segregate from Citrus; monotypic; Mal. Pen.

Citrus: Alston, in Trimen, Handb. Fl. Ceyl. Suppl. (1931) 39.-Key for Citrus; new comb. for some other Rutaceae.

Bonavia, J. Linn. Soc. Lond. Bot. 22 (1887) 213-218.-Discussion on the probable wild source.

-, The cultivated oranges and lemons of India and Ceylon. 2 vols. London (1890) i-xix, 1-384, Atlas t. 1-158 with letterpress.-No keys.

Guillaumin, Bibl. Agric. Col. Paris (1917) 1-80.-Repr. from Agric. Pratique des Pays Chauds.-Revision; no keys.

Lushington, Ind. Forester 36 (1910) 323-353. -Key.

Marcovitch, Landbouw 2 (1926) 1-25, 1 pl.Key; both in Dutch \& English, translated from Russian.

Swingle, in The Citrus Industry (ed. by $\mathbf{H}$. J. WeBber and L. D. BACHELOR) 1 (1943) 129-474, fig.-Taxon. of Citrus and its wild relatives. Non vidi.

TANAKA, Bull. Miyazaki College Agric. \& For. no 1 (1929) 109-114.-Notes on origin \& delimitation of spp.; key to spp. of the looseskin oranges ( $C$. nobilis auct.).

De Vries, Trop. Natuur 17, 1928, 157-158.Key for some cult. spp. in Java; in Dutch.

Clymenia: Swnngle, J. Arn. Arb. 20 (1939) 251254, t. 1.-Bismarck Arch.; segreg. from Citrus; monotypic.

Echinocitrus: Tanaka, J. Arn. Arb. 9 (1928) 137138.-Monotypic; New Guinea.

Fagara: Reeder \& Cheo, J. Arn. Arb. 32 (1951) 67-71, t. 1-2.-Critical notes on Xanthoxylum and Fagara in China.

Feroniella: KoORders \& VAleton, Ic. Bog. 2 (1904) t. 149.-Descr. Feronia lucida SCHEFF. Swingle, Bull. Soc. Bot. Fr. 59 (1912) 774-781 (1913).

Flindersia: Francis, Queensl. Agric. J. 50 (1938) 456-458.-Notes; key to Australian rain-forest spp.

WhITE, Proc. Linn. Soc. N.S.W. 46 (1921) 324 329.-List of spp. with critical notes; no key.

Fortunella: Swingle, J. Wash. Ac. Sc. 5 (1915) 167-175.

Tanaka, Bull. Soc. Bot. Fr. 75 (1928) 714.New sp. from the Mal. Pen.

Geyera: WhITE, Queensl. Agric. J. (1925) 146-148, t. 27-28.-Descr. \& pl. of G. salicifolia.

Glycosmis: KURz, J. Bot. 14 (1876) 33-40, t. 174-175.-Discussion of some allied spp.; key. Narayanaswami, Rec. Bot. Surv. Ind. 14 (1941) 1-72, fig. 1-30.-Revision Indian spp.; keys.

Swingle, Not. Syst. 2 (1911) 158-163.-Some notes.

Hunsteinia $=$ Rapanea (Myrsinaceae).

Lamiofrutex = Vavaea (Meliaceae).

Lavanga (Luvunga): KURz, J. As. Soc. Beng. 39, ii (1870) 69.-Syn. of $L$. sarmentosa.

Limnocitrus: SwIngle, J. Arn. Arb. 21 (1940)
2-6, t. 1.-Monotypic, formerly in other genera. Cf. Valeton, Ic. Bog. 4 (1912) t. 348.

Limonia (Feronia): KoORders \& VAleton, Ic. Bog. 2 (1904) t. 149.-Descr. \& pl. of F. lucida SCHEFF.

AIRY SHaw, Kew Bull. (1939) 293. $F$. elephantum $=$ L. acidissima.

Swingle, J. Wash. Acad. Sc. 4 (1914) 325-328.

Lunasia (Androcephalium, Mytilicoccus): MIQUeL, Ann. Mus. Bot. Lugd.-Bat. 3 (1867) 89.Census.

White, Proc. R. Soc. Queensl. 34 (1922) 38.-Syn.

Merope: SwINGle, J. Wash. Acad. Sc. 5 (1915) 420-425.-Syn. \& distr. of $M$. angulata.

Microcitrus: Swingle, J. Wash. Acad. Sc. 5 (1915) 569-578.-A genus of Queensland; key!

Monanthocitrus: TanaKa, J. Arn. Arb. 9 (1928) 138-139.-Spp. 3; New Guinea.

Murraya (Chalcas): Tanaka, J. Soc. Trop. Agric. Formosa 1 (1929) 23-42.-List of spp.; key (under Chalcas).

Paramignya: VAleton, Ic. Bog. 4 (1912) t. 148.$P$. angulata.

Pleiospermium: Swingle, J. Wash. Acad. Sc. 6 (1916) 426-431.-Descr.; 2 spp.; key!

-, J. Arn. Arb. 20 (1939) 258-263, t. 3-6.Descr. new spp. from Sumatra and Borneo.

Skimmia: GAMBLE, Kew Bull. (1917) 301-303.Note on delimitation of some Sino-Himalayan spp.

Masumune, Bull. Soc. Bot. Fr. 84 (1937) 91.Descr. S. philippinensis n. sp.

Swinglea (Chaetospermum SwINGLE non SACCARDo): MERRILL, J. Arn. Arb. 8 (1927) 131-132.

SwINGLe, J. Wash. Acad. Sc. 3 (1913) 99-102.Descr.; Philip. genus.

Tetractomia (Terminthodia): MERrILL \& PerRy, J. Arn. Arb. 22 (1941) 53-55.-Reduction; census; no key.

Toddalia: VerdOORN, Kew Bull. (1926) 400-401.Account in Africa.

Xanthoxylum: ReEder \& Cheo, J. Arn. Arb. 32 (1951) 67-71, t. 1-2.-Critical notes on Chin. spp.

Wenzelia: Swingle, J. Arn. Arb. 26 (1940) 6-17, t. 2-3.

Tanaka, J. Arn. Arb. 9 (1928) 139-141.-Some new comb. Papuan spp.

\section{SABIACEAE}

Miquel, F. A. W., Revue des Sabiacées de l'Archipel Indien (MIQ. Ill. Fl. Arch. Ind. 1870, 71-75).-Descr.; no keys.

RENARD, A. LE, Recherches anatomiques sur la tige et la feuille des Sabiacées (in MOROT, J. de Bot. 21, 1908, 290-332).-Key to Sabia on anat. characters; descr, anat. Sabia \& Meliosma spp.

\section{Local}

Sumatra: Merrill, Contr. Arn. Arb. 8 (1934) 94-95.-Syn. some Meliosmas.

Malay Peninsula: Henderson, Gard. Bull. Str. Settl. 7 (1933) 96-97, t. 18.-New Meliosma.

Java: BACKER, Schoolfl, van Java (1911) 272-275. -Key, descr.; in Dutch. 
Borneo: Merrill, Plant. Elm. Born. (1929) 176177.-A new Meliosma.

Ridley, Kew Buil. (1933) 193.-New sp. and new name in Meliosma.

Philippines: Elmer, Leafi. Philip. Bot. 10 (1939) 3784-3788.-Descr. 3 new Meliosmas.

New Guinea: Merrill \& Perry, J. Arn. Arb. 22 (1941) 262-263.-New Meliosma.

\section{Genera}

Meliosma (Heteropithmos): BLume, Rumphia 3 (1847) 196-204, t. 168-169.--Descr.

Cufodontis, Oester. Bot. Z. 88 (1939) 246-268.

-Revision Chin. spp.; descr.; key!

Fedtschenko, Svensk Bot. Tidskr. 19 (1926) $493-494$.

Merrill \& Perry, J. Arn. Arb. 20 (1939) 356360.-Records \& descr. of a number of Mal. spp.

Urban, Ber. Deut. Bot. Ges. 13 (1895) 211-222, t. 19.-Morphol.

Sabia: Blume, Mus. Bot. Lugd.-Bat. 1 (1851) 368-370, t. 44.-Diagn.

Chen, Sargentia 3 (1943) 1-75.-Monogr.; key.

\section{SALICACEAE}

Andersson, N. J., Monographia Salicum (Kongl. Svenska Vet.-Ak. Handl. n.s. 6, 1, 1865, 1-181, t. 1-9).-Monogr.; no keys.

-, in DC. Prod. 16, 2 (1868) 190-331.-Census; descr.; no keys.

JACOBS, M., Fl. Mal. I, 5 (1954) 107-110, fig. 1-3.

Miquel, F. A. W., Salicinées (Ill. Fl. Arch. Ind. 1870, 11-14).-Synopsis.

\section{Local}

Sumatra: Jochems, Trop. Natuur 15 (1926) 200203, fig. 1 .

Merrill, Contr. Arn. Arb. 8 (1934) 41-42.Record.

\section{Genera}

Salix: SCHNeIDER, Oester. Bot. Z. 62 (1915) 273278.-Generic subdiv.

\section{SALVADORACEAE}

BACker, C. A., F1. Mal. I, 4 (1951) 224-225, fig. 1. Candolle, A. de, Prod. 17 (1873) 27-31.-Synopsis; in Latin.

Sleumer, H., Pflanzenfamilien ed. 2, 20 b (1942) 232-239.

\section{SAMYDACEAE \\ See Flacourtiaceae}

\section{SANTALACEAE}

Blume, C. L., Mus. Bot. Lugd.-Bat. 1 (1850) 180-182, 242-245.-Descr. in Latin.

DANSER, B. H., On some genera of Santalaceae Osyrideae from the Malay Archipelago, mainly from New Guinea (Nova Guinea n.s. 4, 1940, 133-149, t. 14).-Descr. of new genera and spp.

- Supplementary notes on the Santalaceous genera Dendromyza and Cladomyza (Nova
Guinea n.s. 6, 1955, 23 pp., 9 tab., inedit.).For both genera keys and descr. of spp.; many new spp.

Pilger, R., Pfianzenfamilien ed. 2, $16 \mathrm{~b}$ (1935) 52-91.

Planchon, J. E., Sur les affinités des genres Henslowia, Wall. (Crypteronia? Blume. Quilamum? Blanco), Raleighia, Gardn. et Alzatea, Ruiz. \& Pav. (Hook. Lond. J. Bot. 4, 1845, 474-478, t. 16B-D).-Discussion; list of some old and new spp. of Henslowia from Malaya and the Philippines.

Sprague, T. A. \& V. S. Summerhayes, Santalum, Eucarya, and Mida (Kew Bull. 1927, 193-202, 1 map).-Splitting of Santalum into 3 genera.

\section{Local}

Java: van SteEnis, Trop. Natuur 22 (1933) 97-99, 1 fig.-Distinction 2 spp.

Moluccas: Merrill, Philip. J. Sc. 11 (1916) Bot. 267-268.-New spp. from Ambon.

New Guinea: LaUTERBaCH, Nova Guinea 8 (1910) 287.-Record.

-, ibid. 8 (1912) 813.-New Henslowia.

Pilger, Bot. Jahrb. 59 (1924) 118-128.-Keys.

Summerhayes, Kew Bull. (1929) 125.-Descr.

Santalum papuanum.

\section{Genera}

Calyptrosepalum: BaILeY, \& Smith, J. Arn. Arb. 34 (1953) 52-64, t. 1-2.-2nd sp. from Fiji.

Moore, J. Bot. (1925) Suppl. 90-92, 1 fig.Descr. typ.

Phacellaria: Danser, Blumea 3 (1939) 212-235, t. 3-12.-Monogr.; key.

Santalum: FiscHER, Kew Bull. (1927) 193-202. —, J. Bomb. Nat. Hist. Soc. 40 (1938) 458-466. $-S$. album introd. in contin. Asia.

Skottsberg, Act. Hort. Gothob. 5 (1929) 135145.-Disc. geogr. distr. \& alliances Pacific spp.

\section{SAPINDACEAE}

Blume, C. L., De quibusdam Sapindaceis maxima parte Indiae orientali propriis (Rumphia 3, ?1849, 91-192, t. 166-167.)-Descr.

Herzog, Th., Pflanzenareale Reihe 4, Heft 4 (1936) 35-42, Karte 31-37b.-Distr. tribes and genera, based on RADLKOFER's monogr.

Miquel, F. A. W., Ann. Mus. Bot. Lugd.-Bat. 3 (1867) 88-89.

RAdlkofer, L., Ueber die Sapindaceen Holländisch Indiens (Act. Congr. Intern. Amsterdam 1877 (1879), 70-133).-Precursory; descr. new $s p p$. and genera; discussions; no keys.

-, Nachträge ibid. 216-254.-Ditto. The reprint of these 2 papers is paged $1-63,65-103$.

-, Ueber Capania und damit verwandte Pflanzen (Sitz.-Ber. Math.-Phys. K1. Akad. Wiss. München 9, 1879, 457-678).-Survey of trib. Cupanieae; key to the genera of this tribe; list of all names used in the tribe; additions to Arytera with new spp.; ditto of Cupania; key \& new spp. of Cupaniopsis; ditto of Elattostachys, Guioa, Rhysotoechia and some other small genera. - Ueber die Gliederung der Sapindaceen 
(Sitz.-Ber. Math.-Phys. K1. Bayer. Akad. Wiss. München 20,1890, 105-379).-Delimitation of Sapind. against other families with special use of the anatomical method; conspectus of tribes and genera of the family (non vidi); from extract in Bot. Jahrb. 12 (1890) Lit.-Ber. p. 78-80.

- Sapindaceae novae Indicae et Malaicae ex herbario Calcuttensi (Rec. Bot. Surv. Ind. 3, 1907, 341-355).-Descr.

-, Pflanzenreich Heft 98 (1931-34) 1-1539, fig. $1-46$.

\section{Local}

Indo-China: RADlKofer, Not. Syst. 1 (1910) 298306.-Descr. new spp.

Sumatra: Merrill, Pap. Mich. Acad. Sc. 23 (1937) 183-184 (1938).-New Allophylus.

Malay Peninsula: CoRner, Gard. Bull. Str. Settl. 10 (1939) 38-45, 263.-Disc. on specific delimitation in Allophylus and nomencl. in Mischocarpus.

Borneo: Riduey, Kew Bull. (1932) 178-179.Record; one new Otophora. - , ibid. (1933) 190-193.-New records and spp.

Java: ADELBERT, Blumea 6 (1948) 322-325.-Critical notes; no key.

BACKER, Schoolfl. Java (1911) 256-272.-Keys; in Dutch.

RADLKOFER, Flora 118-119 (1925) 399-401.Descr. 3 new spp.

Valeton, Bull. Inst. Bot. Btzg no 15 (1902) 1-12.-Some critical notes.

Celebes: Koorders, Suppl. Flora N. O. Celebes 2 (1922) t. 52-54; ibid. Suppl. 3 (1922) 26-27.Descr. \& pl. of two spp.

Lesser Sunda Islands: RADLxofer, Med. Rijksherb. Leiden no 22 (1914) 10-20.-List records coll. Elbert.

Philippines: MerriLl, Philip. J. Sc. 26 (1925) 468470.-Descr. of a new Allophylus.

Quisumbing, Philip. J. Sc. 76 (1944) 44-45.New Allophylus from Palawan.

— \& MerRILL, Philip. J. Sc. 37 (1928) 163.-New Otophora from Mindanao.

Radlkofer, in Perkins, Fragm. Fl. Philip. (1904) 56-66.-New records and spp.

-, in Elmer, Leafl. Philip. Bot. 1 (1907) 208-

211.-Descr. new spp.

一, ibid. 5 (1913) 1601-1616.-Ditto.

-, Philip. J. Sc. 6 (1911) Bot. 181-183.-Descr.

4 new spp. from Polillo Isl.

一, ibid. 8 (1913) Bot. 443-473.-List; descr. new spp.

-, ibid. 20 (1922) 657-662.-Descr. new spp.

Moluccas: Radlkofer, Philip. J. Sc. 12 (1917) 83. -A new Guioa from Ambon.

New Guinea: Kanehira \& Hatusima, Bot. Mag. Tokyo 57 (1943) 74-83.-New records and several new spp.

KOORDERS, Nova Guinea 8 (1909) 171-172.Some records.

MelChIOR, J. Arn. Arb. 14 (1933) 63-65.Suppl. records of Brass coll.

Merrill \& Perry, J. Arn. Arb. 21 (1940) 511527.-Descr. new spp. coll. Brass.
RAdLKOFER, Nova Guinea 8 (1911) 617-618.Some records and a new Harpullia.

-, Bot. Jahrb. 50 (1913) 73-80.-List records coll. SCHLECHTER.

-, ibid. 56 (1920) 251-316.-Revision; keys.

一, in FEDDE, Rep. 20 (1924) 33-42.-Descr. some new spp.

-, Nova Guinea 14 (1926) 181-185.-Some records.

WhItE, Proc. R. Soc. Queensl. 34 (1922) 42.Some records.

- \& Francis, ibid. 38 (1927) 238.-Some records coll. LANE-POOLE.

Melanesia: Melchior, Notizbl. Berl.-Dahlem 10 (1930) 227-281.-New Aphania and Harpullia from New Britain.

\section{Genera}

Allophylus: Corner, Gard. Bull. Str. Settl. 10 (1939) 38-45.-Severe critic on RADLKOFER's species concept in this genus; in Mal. only one species with some varieties.

RADlKofer, Sitz.-Ber. Math.-Phys. Kl. Kgl. Bayer. Akad. Wiss. München 38 (1909) 201240.-Keys to all spp. of the genus.

Cardiospermum: Alston, Handb. Fl. Ceyl. 6 Suppl. (1931) 54.-Key to 3 spp.

Dodonaea: Sherff, Field Mus. Nat. Hist. Publ. Bot. 23 (1947) 269-317.-Descr.

Mischocarpus: CoRNER, Gard. Bull. Str. Settl. 10 (1939) 263.-Disputes MerRILL's recombinations based on doubtful LOUREIRO descriptions.

Napaeodendron $=$ Walsura (Meliaceae), cf. $\mathrm{Kew}$ Bull. (1937) 319.

Paranephelium (Mildea): MrQuel, Ann. Mus. Bot. Lugd.-Bat. 3 (1867) 88-89.-Recombination.

\section{SAPOTACEAE}

\section{(excl. Boerlagellaceae and Sarcospermataceae)}

Baehni, Ch., Mémoires sur les Sapotacées. I. Système de classification (Candollea 7, 1938, 394508).-Crit. notes on morph.; system of the family; critical notes on the genera; key to the genera.

Boerlage, J. G., Sapotacées (Bull. Inst. Bot. Btzg no 5, 1900, 20-27).-List caoutchouc furnishing spp. coll. VAN ROMBURGH.

Burck, W., Sur les Sapotacées des Indes néerlandaises et les origines botaniques de la guttapercha (Ann. Jard. Bot. Btzg 5, 1886, 1-85, t. 1-10).-Descr.; no key.

Dubard, M., Les Sapotacées du groupe des Isonandrées (Rev. Gén. Bot. 21, 1909, 392-398, fig. 1-3).-General discussion; struct. of genera; no descr. of spp.; no keys.

-, ditto des Sideroxylinées (Ann. Mus. Col. Marseille (II, 10) 20, 1912, 1-90).-Key to genera; enum. spp.

-, ditto des Sideroxylinées - Mimusopées (ibid. (III, 3) 23, 1915, 1-62, fig. 1-27).-Census; no keys.

ENGLER, A., Beiträge zur Kenntnis der Sapotaceen (Bot. Jahrb. 12, 1890, 496-525).-Discussion delimitation genera.

HASSKARL, J. K., Ueber einige neue Gattungen 
der Sapotaceae welche Getah-pertjah liefern (Flora 38, 1855, 577-579).-Descr. 2 new gen. and a new sp.

LAM, H. J., The Sapotaceae, Sarcospermaceae, and Boerlagellaceae of the Dutch East Indies and surrounding countries (Bull. Jard. Bot. Btzg III, 7, 1925, 1-289).-Revision; keys.

- Further studies on Malayan Sapotaceae I (ibid. III, 8, 1927, 381-493).-Addition to the revision; no keys.

-, On the system of the Sapotaceae, with some remarks on taxonomical methods (Rec. Trav. Bot. Néerl. 36, 1939, 509-525).-Discussion of BAEHNI's work; scheme of a new system of the family.

_, Note on the Sapotaceae-Mimusopoideae in general and the Far-Eastern Manilkara-allies in particular (Blumea 4, 1941, 323-358)--Mimusops p. p. and Northia auct. reduced to Manilkara; key to Far-Eastern spp. of Manilkara.

- \& B. J. D. Meeuse, Monograph of the genus Nesoluma (Sapotaceae). A primitive Polynesian endemic of supposed Antarctic origin (Occas. Pap. Bern. P. Bish. Mus. 14, 1938, 127-165, t. 1-5).-Contains new system of the family; a key to Planchonellidae of the Pacific; monograph of Nesoluma (Pac.).

Pierre, L., Notes botaniques, Sapotacées (189091) 1-68 (not finished).-Critical notes on genera and their circumscription.

Valeton, Th., Bull. Inst. Bot. Btzg no 15 (1902) 13-16, 2 fig.-New Payena.

VRIESE, W. H. DE, Plant. Ind. Batav. Orient. (1856) 57-63.-List of misc. records and descr.

\section{Local}

Malay Peninsula: Gamble, Kew Bull. (1907) 109121.-Discussion on getah-pertja trees.

LAM, Gard. Bull. Str. Settl. 9 (1935) 105-106, t. 7. - Madhuca ridleyi $n$. sp.

Borneo: LAM, Kew Bull. (1936) 17-19; ibid. (1938) 306-307.-Descr. new Palaquium and some records.

- , in Hook. Ic. P1. 34 (1938) t. 3360, 2 pp.Descr. new Aulandra.

PierRe, Arch. Néerl. Sc. Nat. 19 (1884) 104-106, t. 4.-Descr. Diploknema sebifera n.g. n.sp.

Ridley, Kew Bull. (1934) 121.-Descr. new Madhuca.

Celebes: KoOrders, Suppl. F1. N.O. Celebes 2 (1922) t. 104; ibid. 3 (1922) 51.-Sideroxylon koordersii PIERRE.

Philippines: Dubard, Bull. Mus. Hist. Nat. Paris 15 (1909) 379-385.-Records \& descr. of new spp. of Palaquium.

ElMER, Leafl. Philip. Bot. 3 (1910) 867-874.-

Records \& new spp. from Sibuyan Isl.

一, ibid. 10 (1939) 3788-3790.-2 new Palaquiums from Sorsogon Isl.

Merril., Philip. J. Sc. 27 (1925) 46-48.-2 new Palaquiums from Luzon.

Moluccas: Holthuis \& LAM, Blumea 5 (1942) 226-228.-List records Talaud \& Morotai.

New Guinea: Burck, Nova Guinea 8 (1910) 435. -Unimportant record.
KRause, Bot. Jahrb. 58 (1924) 463-487.-Revision; keys!

LaM, Nova Guinea 14 (1931) 549-570, t. 94 129.-Enum; keys!

-, Boissiera 7 (1943) 91-99, 6 fig.-3 new spp. of Planchonella; new comb. in Krausella.

White \& Francis, Proc. R. Soc. Queensl. 38 (1927) 252-255, fig. 15-16.-Descr. new spp. in Sideroxylon and Achradotypus.

Pacific: Lam, Proc. 6th Pac. Sc. Congr. 4 (1940) 673-683, 4 maps.-Geogr. distr. Sapot. in Pacific; some new comb. in Philip. Planchonella.

-, Blumea 5 (1942) 1-46.-Revision Melanesian-

Polynesian spp.; keys; New Caledonia excluded.

\section{Genera}

Aulandra: LAM, in Hook. Ic. Pl. 34 (1938) t. 3360, pp. 1-2.-Second sp. from Borneo quite distinct from the other one.

Burckella: LAM \& VAN ROYEN, Blumea 6 (1952) 580-593, 3 fig.-Revision; key.

Ganua: VAN DEN Assem, Blumea 7 (1953) 364-400. -Revision; key.

-, Blumea 7 (1954) 481-483.-Additional note.

Isonandra: JEUKEN, Blumea 6 (1952) 547-579, 4 fig.

Manilkara (Northiopsis, Achras): LAM, Blumea 4 (1941) 323-343.-Key to Mal.-Pac. spp.

VAN ROYEN, Blumea 7 (1953)401-412.-Revision; key.

Mimusops: van RoYen, Blumea 6(1952) 594-595. -Only $M$. elengi.

Palaquium: Dubard, Mémoire 16, Soc. Bot. France (Déc. 1909) 1-24.-Revision; species listed; several Bornean spp. distinguished by Pierre.

Planchonella: Dubard, C. R. Acad. Sc. Paris 152 (1911) 392-393.-Delimitation of the genus.

Pouteria: BAEHNI, Candollea 9 (1942) 147-476.Revision; some recombinations of Planchonellas. LAM, Blumea 5 (1943) 336-338.-Some transfers from Planchonella.

\section{SARCOSPERMATACEAE}

LAM, H. J., Bull. Jard. Bot. Btzg III, 7 (1925) 248250.-One Mal. sp.

- Concise revision of the Sarcospermaceae (Bull. Jard. Bot. Btzg III, 8, 1926, 18-24, 2 fig.). -Key to all spp.

-, Fl. Mal. I, 4 (1948) 32-34, fig. 1.

- \& P. VAN Royen, Concise revision of the Sarcospermataceae (Blumea 7, 1952, 148-153, map).-Key to all spp.

- \& W. W. Varossieau, Revision of the Sarcospermataceae (Blumea 3, 1938, 183-200).Key to all spp.

-, Additional notes on Sarcosperma (Blumea 3, 1939, 261-262).-Notes \& records.

\section{Local}

Indo-China: Merrill, J. Arn. Arb. 20 (1939)351.Syn. \& disc. of S. kachinense.

Philippines: LAM, Philip. J. Sc. 19 (1932) 143-146. -Reduction of Apoia to Sarcosperma.

New Guinea: LAM, Blumea 4 (1941) 322.-Record. 


\section{SAURAUIACEAE \\ See Actinidiaceae}

\section{SAURURACEAE}

Groff, G. W., in Piperales of Kwantung China (Lingn. Sc. J. 11, 1932, 81-86).-Key to Saururus and Houttuynia; descr. of species.

P'eI, ChIEN, The Saururaceae and Chloranthaceae of Eastern China (Bot. Bull. Acad. Sinica 1, no $2,1947,111-117)$.- Keys to genera and species.

SteEnis, C. G. G. J. VAN, Blumea 6 (1948) 244 245.-Key to genera.

-, Fl. Mal. I, 4 (1949) 47-48, fig. 1.

\section{Local}

Java: VAN STEENIS, Blumea Suppl. 1 (1937) 137139.-Note on Houttuynia.

\section{SAXIFRAGACEAE}

Engler, A., Pflanzenfamilien ed. 2, 18a (1930) 74-226, fig. 64-132.

Hutchinson, J., The genera of Hydrangeaceae and J. E. DANDY ditto of Saxifragaceae (Kew Bull. 1927, 101-118, 6 maps).-Keys!

Maximowicz, C. J., Revision Hydrangeearum Asiae Orientalis (Mém. Acad. Imp. Sc. St.Pétersb. VII, $10^{16}, 1867,1-48$, t. 1-3).-Incl. also key to spp. of Hydrangea and Deutzia.

Steenis, C. G. G. J. van, Bull. Jard. Bot. Btzg III, 13 (1934) 249-250.-List spp. of mountain genera.

\section{Local}

Sumatra: Merrill, Contr. Arn. Arb. 8 (1934) 6667.-Small list; one new Polyosma.

-, Pap. Mich. Acad. Sc. Arts \& Lett. 20 (1935) 97-98.-Records of some Polyosma.

-, Not. Nat. Acad. Nat. Sc. Philad. no 47 (1940) 2.-Record of Parnassia.

Malay Peninsula: KING, J. As. Soc. Beng. 66, ii (1897) 297-305.-Keys!

Borneo: Merrill, Sarawak Mus. J. 3 (1928) 523524.-New Polyosma.

Java: BACKER, Schoolfi. Java (1911) 466-472.Key; in Dutch.

Philippines: Elmer, Leaff. Philip. Bot. 2 (1908) 527-529.-Key of 2 Philip. spp.

-, ibid. 5 (1913) 1633-1639.-Records \& new spp. of Polyosma.

Merrill, En. Philip. Fl. Pl. 2 (1923) 218-221.List.

Moluccas: Merrull, Philip. J. Sc. 11 (1916) Bot. 273-274.-New Polyosma.

New Guinea: Kanehira \& Hatusima, Bot. Mag. Tokyo 56 (1942) 304-308.-New Polyosmas. LAUTERbach, Nova Guinea 8 (1912) 821.-New Polyosma.

MatTfELd, Bot. Jahrb. 69 (1938) 273-275.-Two new Polyosmas.

—, ibid. 70 (1940) 469-470.-New Quintinia.

ReEder, J. Arn. Arb. 27 (1946) 275-288.-New spp. in Quintinia, Carpodetus, and Polyosma.

SCHLECHTER, Nova Guinea 12 (1912) 487-490.Descr. new spp. in Astilbe, Dichroa, Quintinia, and Carpodetus.
-, Bot. Jahrb. 52 (1914) 118-138.-Important; keys!

New Caledonia: Guillaumin, Bull. Soc. Bot. Fr. 86 (1939) 275-278.-Keys.

\section{Genera}

Astilbe: HeNRY, Gard. Chron. III, 32 (1902) 95, 154-156, 171.-Revision; species listed; new Philip. sp.

KNOLL, Sitz.-Ber. Math.-Nat. Kl. Akad. Wiss. Wien 118, Abt. 1 (1909) 45-88, 11 fig., t. 1-4.Key \& descr.; nomencl. antedated.

Deutxia: LemoIne, J. Soc. Nat. Hort. Fr. IV, 3 (1902) 298-314, fig. 8-9.-Hortic. monograph; no keys.

AIRY Shaw, Kew Bull. (1934) 177-182.-Key to the spp. of the Asiat. $\$$ Mesodeutzia.

Dichroa: Hallier $f$, Meded. Rijksherb. Leiden no 37 (1918) 11-12.-Small note.

Wu, Bot. Jahrb. 71 (1941) 180.-Key to Chinese spp.

Heuchera: Rosendahl, Butters \& LAKela, Minnesota Stud. P1. Sc. 2 (1936) 1-180, 5 fig.-Monogr. with descr. \& keys.

Itea: Wu, Bot. Jahrb. 71 (1941) 178.-Key to Chinese spp.

Yamamoto, Acta Phytotax. \& Geobot. 6 (1937) 245-250, fig. 1-3.-Key \& descr. Formosan spp.

Parnassia: Franchet, Bull. Soc. Bot. Fr. 44 (1897) 244-263.-Synopsis with partial key!

Handel-Mazzetti, Oester. Bot. Z. 90 (1941) 127-136.-Key to Chinese spp.

KERR, J. Siam Soc., Nat. Hist. Suppl. 9 (1934) 327-328, 1 fig.-Occurrence in Siam.

Merrill, Not. Nat. Ac. Nat. Sc. Philad. no 47 (1940) 2.-Record from Sumatra.

Polyosma: Blume, Mus. Bot. Lugd.-Bat. 1 (1850) 258-261.-List; descr.

Saxifraga: Engler \& IRMSCHER, Pflanzenreich Heft 67 (1916) 1-448; Heft 69 (1919) 449-709, pars generalis $1-47$, fig. 101-124.

\section{SCHEUCHZERIACEAE See Juncaginaceae}

\section{SCHISANDRACEAE}

Blume, C. L., Flora Javae fasc. 33 (1830) 18 pp., 5 tab.

Smith, A. C., The families Illiciaceae and Schisandraceae (Sargentia 7, 1947, 79-224).-Monograph; keys! Comprises Kadsura and Schizandra.

\section{SCROPHULARIACEAE}

Bentham, G.; Scrophularineae Indicae. A synopsis of the East Indian Scrophularineae (1835) 1-57.-No key to genera; short descr.; partial keys to spp.

- Synopsis of the Buchnereae, a tribe of the Scrophulariaceae (in Hook. Comp. Bot. Mag. 1, 1836, 356-384).-Conspectus incl. Striga and Buchnera.

-, Synopsis of the Gerardieae, a tribe of Scro- 
phulariaceae (in Hook. Comp. Bot. Mag. 1, 1836, 192-212, t. 11).-Synopsis; no key.

一, in DC. Prod. 10 (1846) 186-586, 589-598.

Pennell, F. W., The Scrophulariaceae of the Southeastern United States (Proc. Ac. Nat. Sc. Philad. 71, 1920, 224-291).-Revision; keys.

-, Scrophulariaceae of Cuba (ibid. 75, 1923, 1-21).

-, Genotypes of the Scrophulariaceae in the first edition of Linne's 'Species Plantarum' (Proc. Acad. Nat. Sc. Philad. 82 (1931) 9-26).Typification.

- The Scrophulariaceae of Eastern temperate North America (Acad. Nat. Sc. Philad. Monogr. 1, 1935, 1-650).-Revision; keys to tribes, genera and spp.

- The Scrophulariaceae of the Western Himalayas (Acad. Nat. Sc. Philad. Monogr. 5, 1943, i-vii, 1-163, t. 1-25).

PraIN, D., Some additional Scrophulariaceae (J. As. Soc. Beng. 72, 1904, 11-23).-A number of additions to Fl. Br. Ind. incl. Malay Peninsula; critical notes on synon.; no keys.

SteENIS, C. G. G. J. van, Bull. Jard. Bot. Btzg III, 13 (1934) 250-253.-List of spp. of mountain genera in Mal.

\section{Local}

East Asia: Yamazaki, J. Jap. Bot. 25 (1950) 209217, 3 fig.; ibid. 27 (1952) 61-68.-Misc. notes; some new spp. and comb.

India: Santapau, J. Bomb. Nat. Hist. Soc. 49 (1950) 25-49.-Keys, synonymy, and notes on Bombay spp., based on PenNell's work.

Indo-China: Bonat1, Not. Syst. 1 (1911) 331-339. -Descr. new spp.

-, Bull. Soc. Bot. Fr. 71 (1924) 1091-1100.Descr. new spp. No key.

Formosa: LI, Quart. J. Taiwan Mus. 3 (1950) 55-80.-Critical review; no key.

Sumatra: Merrill, Pap. Mich. Acad. Sc. 23 (1937) (1938) 189-191.-Records.

Borneo: Merrill, Sarawak Mus. J. 3 (1928) 549.Record of a Lindernia.

Java: BACKER, Onkruidfl. Jav. Suiker. (1931) 598629.-In Dutch; key.

Philippines: Quisumbing, Philip. J. Sc. 41 (1930) 345.-Record of Veronica javanica in Luzon.

— \& Merrill, Philip. J. Sc. 37 (1928) 198-199.New record of a Torenia from Luzon.

Du RIETZ, Svensk Bot. Tidskr. 25 (1931) 500542, 11 fig.-Descr. 2 new Philip. Euphrasias and their plant-geogr. significance.

Moluccas: MerriLL, Philip. J. Sc. 11 (1916) Bot. 312.-Ambon records; 2 new comb.

New Guinea: DiELs, Bot. Jahrb. 62 (1929) 490492.-Records and Detzneria n.g.

- \& Lanjouw, Nova Guinea 14 (1928) 534539.-List; one new Euphrasia.

Pennell, Brittonia 2 (1936) 177-188.-Enum. Pap. spp. coll. Brass.

-, J. Arn. Arb. 24 (1943) 243-275, t. 1-5.Second revision; keys!

Du RiETz, Svensk Bot. Tidskr. 25 (1931) 526539.-Some new names (nom. nud.) of proposed
New Guinean Euphrasias are mentioned in a discussion on plant geography.

SCHLEChTER, Bot. Jahrb. 59 (1924) 99-117.Rev. Pap. spp.; keys!

\section{Genera}

Alectra: Handel-Mazzetti, Symb. Sin. 7 (1936) 843. Cf. Yamazaki, J. Jap. Bot. 25 (1950) 213.A. arvensis reduced to Melasma arvense. Melchior, Notizbl. Berl.-Dahlem 15 (1941) 423-447.-Revision of the genus; keys.

Bacopa (Herpestis): Pennell, Proc. Acad. Nat. Sc. Philad. 98 (1946) 83-98.-Redefinition of the genus; complete revision; keys!

Brookea: Hallier $f$., Bull. Herb. Boiss. II, 3 (1903) $194,206 .-H$. assumes this genus to belong to the Gesneriaceae.

Calceolaria: Kraenzlin, Pflanzenreich Heft 28 (1907) 21-128.-Monograph; keys.

Centranthera (Razumovia SPR. 1807, non SPR. 1805): Merrill, Bull. Torr. Bot. Club 64 (1937) 589-598.-Spp. under Razumovia; key!

-, 150th Anniv. vol. Ann. R. Bot. Gard. Calcutta (1942) 53-58.-Revision; key.

YAMAZAKI, J. Jap. Bot. 25 (1950) 209.-New comb. for $C$, tranquebarica.

Ellisiophyllum: BaIllon, Bull. Soc. Linn. Paris 103 (1890) 817-818.-Transfers the genus from $\boldsymbol{H y}$ drophyllaceae to Scrophulariaceae.

Makino, Tokyo Bot. Mag. 20 (1906) 91-92, t. 5.-Syn.; new comb.

YAMAZAKI, J. Jap. Bot. 27 (1952) 61, fig. 1.Descr. new variety from Japan.

Euphrasia: LI, Not. Nat. Ac. Sc. Philad. no 254 (1953) 1-6.-Key to Chinese spp.

VoN WetTstein, Oester. Bot. Z. 43 (1893) 77-83, 126-133, 193-202, 238-241, 305-310, t. 6; ibid. 44 (1894) 5-11, 53-60, 92-97, 132-138, 169$173,244-249,288-294,328-333,374-381,405$ 410 , 448-455; ibid. 45 (1895) 14-17, 111-112, t. 3.-Revision; descr. spp.; key to Austrian spp. (1895).

-, Monographie der Gattung Euphrasia. Leipzig (1896) 316 pp., 18 Taf.-Key to spp.; no Mal. spp. included.

Hemiphragma: Yamazaki, J. Jap. Bot. 25 (1950) 212.-Philip. sheets represent a variety.

Limnophila: AIRY SHAw, Kew Bull. (1951) (1952) 370.-L. viscosa RIDL. appears to be Staurogyne glauca.

Lindernia (Ilysanthes): HARA, J. Jap. Bot. 19 (1943) 203-209.-Spp. of Japan; several new comb. of Mal. spp. and their syn.

Mukerjee, J. Ind. Bot. Soc. 24 (1945) 127-134. -Revision Indo-Burmese Lindernias; key to 28 spp.

Urban, Ber. Deut. Bot. Ges. 2 (1884) 429-442. -Discussion of alliance Ilysanthes, Bonnaya, Lindernia, Vandellia. Survey of Ilysanthes spp.

Mazus: Bonati, Bull. Herb. Boiss. II, 8 (1908) 525-539.-Revision; key.

LI, Brittonia 8 (1954) 29-38.-Revision; key.

Mimulus: Grant, Ann. Missouri Bot. Gard. 11 (1924) 99-388, t. 3-10, fig. 1-3.-Monogr.; keys! 
VAN Steenis, Reinwardtia 1 (1952) 472-474.First record from Sumatra.

Pentstemon: KrautTer, Contr. Bot. Lab. Univ. Philad. 3 (1908) 93-206.-Monogr.; key.

Picria (Curanga): Merrill, Trans. Am. Phil. Soc. n. s. 24 (2) (1935) 353.-Nomencl.

Russelia: Bor \& RAIZADA, J. Bomb. Nat. Hist. Soc. 46 (1946) $411-413$, t. 26, 1-4.-Key to 2 cult. spp.

Holtrum, M. A. H. A. Mag. 11 (1941) 73-75, $1 \mathrm{fig} .-S p p$. in Malaya.

Robinson, Proc. Amer. Ac. Arts \& Sc. 35 (1900) 319-321 (repr. in Contr. Gray Herb. new ser. 18).-Revision; key.

STANDley, Contr. U. S. Nat. Herb. 23 (1924) 1308-1310.-Key Mexic. spp.

Scoparia: Chodat, Bull. Herb. Boiss. II, 8 (1908) 1-16.-Revision; key!

FrIEs, Arkiv Bot. 6 (no 9) (1907) 1-31, t. 1-8.Revision; key.

—, Bull. Herb. Boiss. II, 8 (1908) 934-940.Some remarks on ChODAT's revision.

Striga: Barnes, J. Ind. Bot. Soc. 15 (1936) 125130 , t. 11.-Key to 4 spp.

BURNs, ibid. I (1920) 212-216, 4 fig.-Variation in flower colour of Bombay spp.

Veronica : LeHMANN, Ann. Jard. Bot. Btzg 25 (1912) 189-201.-Precurs. study on syn. \& distr. of $V$. javanica.

RIEK, in Fedde, 'Rep. Beih. 79 (1935) 1-68.Syst. notes on \$ Chamaedrys; no keys!

RöMPP, in FEDDE, Rep. Beih. 50 (1928) 1-172.Precursory to monograph; keys!

Wightia: Hallier $f$., Bull. Herb. Boiss. II, 3 (1903) 194-196, 206.-H. assumes this genus to belong to the Bignoniaceae.

Van Steenis, Bull. Bot. Gard. Btzg III, 18 (1949) 213-227.-Revision; key.

\section{SCYPHOSTEGIACEAE}

BaEHNI, CH., L'inflorescence mâle du Scyphostegia borneensis (C. R. Sc. Soc. Phys. Hist. Nat. Genève 54, 1937, 91-92).-Extract of next paper.

- Note sur les inflorescences mâles et femelles du Scyphostegia borneensis Stapf (Bull. Soc. Bot. Suisse 48, 1938, 22-28, 3 fig.).-Descr. of flowers \& discussion of taxon. position.

StapF, O., Trans. Linn. Soc. Lond. II, Bot. 4 (1893) 217-219, t. 17.

\section{SIMAROUBACEAE \\ (incl. Irvingiaceae)}

BoAs, F., Beiträge zur Anatomie und Systematik der Simarubaceae (Beih. Bot. Centralbl. 29, ii (1913) 303-356, fig. 1-7).-Non vidi.

Cronquist, A., Studies in the Simaroubaceae IV. Resume of the American genera (Brittonia 5, 1944, 128-147).-Keys to genera \& spp.; descr.

- Additional notes on the Simaroubaceae (Brittonia 5, 1945, 469-470).-Revised key to the genera.

Engler, A., Pflanzenfamilien ed. 2, 19a (1931) 359-405, fig. 166-190.

JADIN, F., Contribution à l'étude des Simaruba- cées (Ann. Sc. Nat. Bot. VIII, 13, 1901, 201304).-Morph. \& anat. of genera.

Planchon, J. E., Revue de la famille des Simaroubacées (Hook. Lond. J. Bot. 5, 1846, 560-584).-List of genera and spp.

Tieghem, PH. von, Sur les Irvingiacées (Ann. Sc. Nat. IX, 1, 1905, 247-320).-Treatment of Irvingia and Irvingella as belonging to a separate family.

\section{Local}

Indo-China \& China: LeComte, Not. Syst. 1 (1909) 101-105.-List.

Sumatra: Merrill, Contr. Arn. Arb. 8 (1934) 8081. -2 records.

Borneo: Merrill, Philip. J. Sc. 30 (1926) 82.First record of Harrisonia.

Java: BACKER, Schoolf. Java (1911) 189-194.Keys; in Dutch.

Philippines: Radrkofer, Philip. J. Sc. 6 (1911) Bot. 365-367.-Descr. Hebonga n.g. with 2 new spp.

New Guinea: LAUTERBACH, Bot. Jahrb. 56 (1921) 341-344.-Census.

WhITE, J. Arn. Arb. 31 (1950) 91.-Records of Ailanthus.

Melanesia: Melchior, Notizbl. Berl.-Dahlem 10 (1930) 893-896.-New Ailanthus from Bismarck Arch.

New Caledonia: Gulllaumin, Bull. Soc. Bot. Fr. 85 (1938) 19-20.-Genera Suriana and Soulamea; with keys.

\section{SIPHONODONTACEAE See Celastraceae}

\section{SOLANA CEAE}

BaEhNi, Ch., L'ouverture du bouton chez les Solanées (Candollea 10, 1946, 399-492).-New classification of tribes and genera based on the opening of the bud.

BITTER, G., Weitere Untersuchungen über das Vorkommen von Steinzellkonkretionen im Fruchtfleisch beerentragender Solanaceen (Abh. Naturw. Ver. Bremen 23, 1915, 114-163).Anat.

Dunal, F., in DC. Prod. 13, 1 (1852) 1-690.

Morton, C. V., in Studies of tropical American plants (Contr. U. S. Nat. Herb. 29, 1944, 54-55). -Reduces Lycianthes to a section of Solanum.

\section{Local}

India: NeEs von Esenbeck, Trans. Linn. Soc. 17 (1837) 37-82.-Monogr. East Indian Solaneae; no keys.

Santapau, J. Bombay Nat. Hist. Soc. 47 (1948) 652-662.-Revision spp. Bombay Pres.; keys.

Sumatra: WrTASEK, Oester. Bot. Z. 80 (1931) 162167.-Enum. coll. SchiffNer.

Java: ADELBERT, Blumea 6 (1948) 327-333.Critical notes; one new sp.

WrTaseK, Oester. Bot. Z. 80 (1931) 162-167.List coll. SchIFFNER:

Philippines: Elmer, Leafl. Philip. Bot. 2 (1910) 729-734.-Account of Solanums from Mt Apo; some new spp. 
New Guinea: BITTER, Bot. Jahrb. 55 (1917) 59113.-Solanum spp.; key!

Merrill \& Perry, J. Arn. Arb. 30 (1949) 45-52. -New spp. and records.

White, Proc. R. Soc. Queensl. 34 (1922) 51-52. -Some records.

\section{Genera}

Brugmansia (Pseudodatura): LAGerheIM, Bot. Jahrb. 20 (1895) 655-668, t. 11.-Monogr. Ecuador spp.; key.

SAFFord, J. Wash. Acad. Sc. 11 (1921) 182-189. -Treated as Datura $\S$; key!

Van Steenis, Bull. Jard. Bot. Btzg III, 11 (1930) 15-18.-Syn. Mal. spp.

-, Trop. Natuur 30 (1941) 33-37, fig. 1-2.-Key 2 Mal. spp.; in Dutch.

VAN ZIJP, Nat. Tijd. Ned. Ind. 80 (1920) 24-28.

Capsicum: Birter, in FEDde, Rep. 15 (1917) 9698.-Crit. notes on a Philip. sp.

Irish, 9th Ann. Rep. Missouri Bot. Gard. (1898) $53-110$, t. 8-28.-Key! With special regard to garden varieties.

Paul, Trop. Agric. Ceylon 94 (1940) 10-18, 198-213, 332-354.-With keys to the varieties of the spp.

Cestrum: Francey, Candollea 6 (1935) 46-398; ibid. 7 (1936) 1-132, 5 pl.-Monograph; key!

PitTier, J. Wash. Acad. Sc. 22 (1932) 25-37.Venezuelan spp., several new; key!

Cyphomandra: SENDTNER, Flora 28 (1845) 161176, t. 1-9.-Monograph Brazilian spp.; key; differences with other genera.

Datura: SAFford, J. Wash. Acad. Sc. 11 (1921) 173-189, fig. 1-3.-Revision; key! Includes Brugmansia.

Van SteEnIS, Trop. Natuur 30 (1941) 35-38.Key 2 Mal. spp.; in Dutch.

Lycianthes: AMSHOFF, Act. Bot. Neerl. 3 (1954) 419-420.-Suggests that Parascopolia is the oldest name for this segregate.

BitTer, Abh. Naturw. Ges. Bremen 24 (1919) 292-520.-Precursory revision; no key; segregate of Solanum.

一, in FEDDE, Rep. 18 (1922) 314-321.-Addition to the above.

Lycium: Hrtchcock, Ann. Missouri Bot. Gard. 19 (1932) 179-374.-Monograph of New World spp.

Nicandra: BrtTer, Beibl. Bot. Centralbl. 14 (1903) 145-176, Taf. 9-14.-Races of $N$. physaloides.

Nicotiana: Comes, Atti Reale Istituto d'Incorrag. Napoli V, 1 (1899) 1-80, t. 1-7 (fig. 1-14).Monogr.; infrageneric subdivis.; enum. spp.; no keys.

- Monographie du genre Nicotiana, etc. Naples (1899), 1-80, t. 1-7.-French translation of the above. Non vidi.

Goodspeed, Univ. Cal. Publ. Bot. 18 (1945) 345-368, 7 fig.-Sect. div.; no keys.

Wheeler, Univ. Calif. Publ. Bot. 18 (1935) 4568.-Revision of Australasian spp.; key!

Petunia: Fries, Kungl. Svenska Vet.-Ak. Handl. 46, no 5 (1911) 1-72, t. 1-5.-Monograph; key.

Salpichroa: BenoIsT, Bull. Soc. Bot. Fr. 85 (1938)
408-410.-S. American; some new spp.; provisional key!

Solanum: BITTER, in FEDdE, Rep. 10 (1912) 529-565; ibid. 11 (1912) 1-18, 202-237, 241-260, 349-394, 431-473, 481-491, 561-562; ibid. 12 (1913) 1-10, $49-90,136-162,433-467,542-555$; ibid. 13 (1914) 88-103, 169-173; ibid. 15 (1917) 93-98; ibid. 16 (1919) 10-15, 79-103; (1920) 389-409.Descr. and notes on new and noteworthy spp. all over the world, mostly the New World. -, Bot. Jahrb. 49 (1913) 560-569; ibid. 54 (1917) 416-506; ibid. 57 (1921) 248-286.-Revision, keys; descr., and notes on new and noteworthy spp. from Africa.

-, in Fedde, Rep. Beih. 16 (1923) 1-320.Ditto.

Correll, U. S. Dep. Agric., Agr. Monogr. no 11 (1952) 1-243, fig. 1-168.-Revision sect. Tuberarium of N. \& Central America; keys.

VAN SteENIS, Bull. Jard. Bot. Btzg III, 13 (1934) 253.-Some records of mountain spp.

\section{SONNERATIACEAE}

BACKer, C. A. \& C. G. G. J. VAN STeENIS, Fl. Mal. I, 4 (1951) 280-289, fig. 1-6.-Complete revisions of the genera Sonneratia and Duabanga.

\section{Local}

New Guinea: Merrill \& Perry, J. Arn. Arb. 22 (1941) 269.-2 Records.

Pulle, Nova Guinea 8 (1911) 677.-One record. White, Proc. R. Soc. Queensl. 34 (1922) 45-46. -Records.

\section{Genera}

Sonneratia: Backer, Bull. Jard. Bot. Btzg III, 2 (1920) 329-330.-Excellent key to 3 spp.; one new. Parker, Ind. For. 51 (1925) 505-510.-Revision.

\section{SPARGANIACEAE}

BaCker, C. A., Fl. Mal. I, 4 (1951) 233-234, fig. 1. Graebner, P., Pflanzenreich Heft $2^{2}$ (1900) 1-26.

\section{SPHENOCLEACEAT}

Shaw, H. K. AIRY, Fl. Mal. I, 4 (1948) 27-28, fig. 1.

\section{STACKHOUSIACEAE}

BrouWer, F. I., Fl. Mal. I, 4 (1948) 35-36, fig. 1-2.

Mattfeld, J., Pflanzenfamilien ed. 2, 20b (1942) 240-254.

Pampanini, R. \& G. Bargagli-Petrucci, Monografia della famiglia delle Stackhousiacee (Bull. Herb. Boiss. II, 5, 1905, 901-916, 1045-1060, $1145-1160$, t. 10-15; ibid. 6, 1906, 39-44).

\section{Local}

New Guinea: van Steenis, Nova Guinea 14 (1927) 307.-New variety.

-, J. Arn. Arb. 28 (1947) 422.-Record.

\section{Genera}

Stackhousia: Brouwer, Blumea 3 (1938) 174-178. -Revision.

\section{STAPHYLEACEAE}

KraUse, J., Pflanzenfamilien ed. 2, $20 \mathrm{~b}$ (1942) 255-321. 


\section{Local}

Philippines: Elmer, Leafl. Philip. Bot. 9 (1934) 3217-3218.-Descr. new Turpinia.

Merrill, Philip. J. Sc. 27 (1925) 33-35.-Descr. 2 new Philip. spp., one 1-foliolate.

\section{Genera}

Turpinia: Merrill \& Perry, J. Arn. Arb. 22 (1941) 543-555.

\section{STEMONACEAE \\ (Roxburghiaceae)}

Krause, K, Pflanzenfamilien ed. 2, 15a (1930) 224-227.

PranN, D., Notes on the Roxburghiaceae, etc. (J. As. Soc. Beng. 73, 1904, 39-44).-Key to spp.

\section{Local}

Indo-China: Gagnepain, Bull. Soc. Bot. Fr. 81 (1934) 146-148.-Descr. 5 new spp. from Siam \& Indo-China.

Java: BACKER, Handb. Fl. Java 3 (1924) 44-45.One $s p$.

New Guinea: SchlechteR, Bot. Jahrb. 59 (1924) 541-543.-Descr. 2 new spp.; key!

SMITH, Nova Guinea 8 (1909) 198.-Record. 一, ibid. 14 (1926) 208-209.-Descr.

\section{Genera}

Stemona: Krause, Notizbl. Berl.-Dahlem 10 (1928) 289-290.-Notes on synon. \& distr. of some spp. Prain, J. As. Soc. Beng. 73 (1904) 39-43.-Key! Schlechter, Notizbl. Berl.-Dahlem 9 (1924) 190-196, fig. 7.-Infrageneric division; key to sections; enumeration of all spp.; descr. 2 new Chin. spp.

SMITH, Ic. Bog. 3 (1907) 244-246.-Discussion St. javanica and St. moluccana.

—, Bull. Jard. Bot. Btzg III, 6 (1924) 73-78.Descr. new spp.

WRIGHT, J. Linn. Soc. Bot. 32 (1896) 490-496.Revision; key!

\section{STERCULIACEAE}

Bentham, G., Notes on Malvaceae and Sterculiaceae (J. \& Proc. Linn. Soc. Lond. 6, 1862, 110-122).-Generic distinction.

Hochreutiner, B. P. G., Ann. Jard. Bot. Btzg Suppl. 3 (1910) 821-831.-Descr. spp. cult. in Hort. Bog.

Korthals, P. W., Overzigt der Sterculiaceae en Buttneriaceae van de Nederlandsche OostIndische Bezittingen (Ned. Kruidk. Arch. 1, 1848, 301-313).-In Dutch; list of Neth.-Ind. spp., incl. also Bombacaceae and Tiliaceae; descr. Covilhamia and Schoutenia.

\section{Local}

Sumatra: Merrill, Contr. Arn. Arb. 8 (1934) 103-104.-Records; synon.

-, Pap. Mich. Acad. Sc. Arts \& Lett. 19 (1934) 169-171.-3 new spp. of Sterculia.
Malay Peninsula: BurkiLl, Kew Bull. (1935) 317. -New comb. in Firmiana.

Corner, Gard. Buili. Str. Settl. 10 (1939) 262.Record of Pterospermum acerifolium.

Symington, Kew Bull. (1937) 319.-Peniculifera reduced to Trigonopleura.

Borneo: MerRILL, Philip. J. Sc. 21 (1922) 524-529. -Descr. 5 new Sterculias.

-, ibid. 29 (1926) 394-395.-Descr. 2 Sterculias. -, ibid. 30 (1926) 84.-Record of a Pterospermum.

Ridley, Kew Bull. (1938) 222-226.-List; descr. new spp. in Sterculia, Tarrietia and Buettneria. AIRY Shaw, Kew Bull. (1949) 160-162.-List Oxford Exp.; one new Sterculia.

WARBURG, in FEDDE, Rep. 18 (1922) 330.-Descr. new Buettneria.

Java: ADELBERT, Blumea 5 (1945) 505-507.Critical notes; a new Melhania and Sterculia. BACKER, Schoolfl. Java (1911) 133-146.-Keys! In Dutch.

Celebes: Koorders, Suppl. Flora N. O. Celebes 2 (1922) t. 66-68; ibid. 3 (1922) 32-34.-Pl. of 3 spp.

Philippines: Elmer, Leafi. Philip. Bot. 5 (1913) 1641-1644.-List Philip. Trichospermums; descr. 2 new spp.

Merrill, Philip. J. Sc. 30 (1926) 409-411.Descr. new Sterculia.

New Guinea: Kanehira \& Hatusima, Bot. Mag. Tokyo 55 (1941) 389.-Some records.

Merrill \& Perry, J. Arn. Arb. 30 (1949) 4044.-Additions; some new spp. of Sterculia.

MildBRAeD, Bot. Jahrb. 62 (1929) 347-367.Revision; keys!

VoN MUELLER, Vict. Nat. 3 (1886) 45-52.-Some new Pterygota, Sterculias, and Brachychiton.

Pulle, Nova Guinea 8 (1911) 665-666.-Some records.

White, J. Arn. Arb. 10 (1929) 239-240.-Misc. records.

Melanesia: Mildbraed, Notizbl. Berl.-Dahlem 10 (1930) 281-282.-Descr. new Sterculia.

VoN MUeller, Australas. J. Pharm. 2 (1887) 43-45.-Descr. 2 new spp. New Britain.

\section{Genera}

Abroma: Miquel, Ill. Fl. Arch. Ind. (1871) 105.Notes.

Buettneria: MiQueL, Ill. Fl. Arch. Ind. (1870) 9092.-List; descr.

Commersonia: Gagnepain, Not. Syst. 1 (1909) 96-97.-Varieties of C. echinata.

Covilhamia $=$ Stixis (Capparidaceae).

Erythropsis: Burkill, Gard. Bull. Str. Settl. 5 (1932) 231.-Discussion; one new comb.

RIDLEY, Kew Bull. (1934) 215-216.-List of Mal. spp.; one new sp. from India.

Firmiana: RidLeY, Kew Bull. (1934) 214-215.List of spp.

Hildegardia: Kostermans, J. Sc. Res. Indon. 2 (1953) 11.-Revision; one new sp. in Mal.

Melochia (Aleurodendron): GARCKE, Bot. Jahrb. 12 (1890) Beibl. no 27, 29-32.-Discussion synon. and confusion with Waltheria and other genera.

\section{CXXXII}


Leptonychia: BURreT, Notizbl. Berl.-Dahlem 9 (1926) 727-731.-Several spp. of Grewia appear to belong to $L$.

Peniculifera $=$ Trigonopleura (Euphorbiaceae), $c f$. Symington, Kew Bull. (1937) 319.

Pterocymbium: Kostermans, Reinwardtia 1 (1950) 41-50.-Revision Mal. spp.; key!; descr.; one new comb. in Sterculia.

一, ibid. 2 (1953) 363-365, fig. 3.-New sp.; revised key.

Pterospermum: MiQuel, Ill. Fl. Arch. Ind. (1870) 84-89.-List; descr.

Pterygota: Kostermans, Reinwardtia 2 (1953) 365-366, fig. 4.-New comb.; Tetradia incorporated in Pterygota.

Reevesia: Anthony, Not. R. Bot. Gard. Edinb. 15 (1926) 121-129, 2 fig.-Revision of the genus; enum. and key!

—, ibid. 15 (1927) 237-238.-Revision of specimens from Java.

Scaphium: Kostermans, J. Sc. Res. Indon. 2 (1953) 2-11, fig. 1-6, tab.-Revision Mal. spp.; key; remarks on other genera.

Sterculia: SchumanN, Bot. Jahrb. 24 (1898) Beibl. 58, 14-21.-BECCARI collection.

Tarrietia: SpRague, Kew Bull. (1916) 85-86.Some critical notes concerning distinction Heritiera, Tarrietia, \& Argyrodendron.

Theobroma: BerNoulli, Neue Denkschr. Allg. Schweiz. Ges. Naturwiss. Zürich 24 (1871) 1-15, t. 1-7.-Revision; no key.

Chevalier, Rev. Bot. Appl. \& Agric. Trop. 26 (1946) 265-285, t. 5-6.-Revision; key; based on the coll. of the Paris Herbarium.

Triumfetta: Sprague \& Hutchinson, J. Linn. Soc. Bot. 39 (1909) 231-276, t. 17.-Revision African spp.; key!

\section{STYLIDIACEAE}

Good, R. D., New Phytol. 24 (1925) 225-240, 3 maps.-Discuss. of geogr. distr.

Mildbraed, J., Pflanzenreich Heft 35 (1908) 1-98, fig. 1-26.

Slooten, D. F. van, Bull. Jard. Bot. Btzg III, 6 (1924) 65-67.-Revision; key!

_, ibid. III, 14 (1937) 169-174.-New revision; key!

-, Fl. Mal. I, 4 (1954) 529-532, fig. 1-5.

\section{Local}

New Guinea: Van Slooten, Nova Guinea 14 (1926) 195.-Record.

\section{STYRACACEAE}

Perkins, J., Pflanzenreich Heft 30 (1907) 1-111, fig. 1-18.

-, Uebersicht über die Gattungen der Styracaceae, etc. Leipzig (1928) 1-36, fig. 1-19.-Separately published account of the genera intended for 2nd ed. of the Pfianzenfamilien.

Steenis, C. G. G. J. van, Bull. Jard. Bot Btzg III, 12 (1932) 212-272.-Revision; keys!

-, Fl. Mal. I, 4 (1949) 49-56, fig. 1-12.

\section{Local}

Sumatra: SMrтH, J. J., Tectona 10 (1917) 204-206; Bull. Jard. Bot. Btzg III, 4 (1922) 238-240, t. 10.-Descr. new sp.

De VRIEse, Pl. Nov. Ind. Bat. Orient. (1845) 9-10, t. 3.-Descr. new spp.

Philippines: Merrill \& Quisumbing, Philip. J. Sc. 56 (1935) 313-317, t. 1. -Descr. new sp.

New Guinea: van Steenis, J. Arn. Arb. 28 (1947) 422-423.-Ident. Pap. specim.

Melanesia: Van Steenis, J. Arn. Arb. 20 (1939) 220-221.-Record of Styrax in the Solomons.

\section{SYMPHOREMACEAE See Verbenaceae}

\section{SYMPLOCACEAE}

Brand, A., Pflanzenreich Heft 6 (1901) 1-100, fig. 1-9.

- Ueber einige Symplocaceen des Herbier Delessert (Ann. Cons. Jard. Bot. Genève 7-8, 1904, 277-287).-Addit. to monogr.; list; no keys.

- Additamenta nova, etc. (Bull. Herb. Boiss. II, 6, 1906, 747-750).-Some new spp. and comb. from W. Malaysia.

-, Decas specierum novarum (in Fedde, Rep. 14, 1916, 323-326).-Some new spp. from the Philippines, one from Malaya.

\section{Local}

China: Handel-Mazzetti \& Peter-Stibal, Beih. Bot. Centralbl. 62 B (1943) 1-42.-Revision; key! Indo-China: Gulllaumin, Bull. Soc. Bot. Fr. 71 (1924) 273-288.-Descr.; key!

Merrill, Univ. Calif. Publ. Bot. 13 (1926) 140-141. - Descr. new. sp.

Sumatra \& Java: RidLeY, J. Fed. Mal. St. Mus. 8, part 4 (1917) 60-61.-New sp. from Mt Kerintji. De VRIESE, Pl. Nov. Ind. Bat. Or. (1845) 11-12. -Descr. 3 new spp. from Sumatra and Java.

Borneo: MerRill, Sarawak Mus. J. 3 (1928) 545546.-Descr. 2 new spp.

—, Pl. Elm. Born. (1929) 248-249.-New records. AIry Shaw, Kew Bull. (1939) 508-509.-2 records.

STAPF, Trans. Linn. Soc. II, Bot. 4 (1894) 204 206.-Descr. 5 good new spp.

Philippines: Brand, in Perkins, Fragm. Fl. Philip. (1904) 36.-Records; descr. new sp.

-, Philip. J. Sc. 3 (1908) Bot. 1-10.-Revision; key!

一, ibid. 4 (1909) 107-110.-Descr. new spp.; key!

—, ibid. 7 (1912) 29-36.-Addit. new spp.; new key!

Elmer, Leafl. Philip. Bot. 2 (1908) 508-511.Descr. 3 new spp.

-, ibid. 7 (1914) 2319-2324.-Descr. 4 new spp. 一, ibid. 10 (1939) 3792-3794.-Descr. 2 new spp. Merrill, Philip. J. Sc. 35 (1928) 7.-Nomencl. note; nom. nov.

Perknns, Fragm. F1. Philip. (1904) 37.-Syn. S. polyandra. 
New Guinea: Brand, Bot. Jahrb. 54 (1916) 212225.-Descr. new spp.; key!

-, Nova Guinea 14 (1924) 186-189.-Descr. 9 new spp.

一, in FEDDE, Rep. 26 (1929) 172.-S. oranjeensis n. sp.

Kanehira \& Hatusima, Bot. Mag. Tokyo 56 (1942) 485-489.-Descr. some new spp.

Lauterbach, Nova Guinea 8 (1912) 857.Record.

White \& Francis, Proc. R. Soc. Queensl. 38 (1927) 256-257, fig. 17.-Descr. new sp.

Fiji : Turrill, J. Linn. Soc. Bot. 43 (1915) 30-31.Descr. new sp.

New Caledonia: GuIllaumin, Bull. Soc. Bot. Fr. 71 (1924) 939-946.-Census; key!

\section{Genera}

Symplocos: Brand, Bull. Herb. Boiss. II, 6 (1906) 750.-Revision sect. Cordyloblaste.

VAN SteEnis, Bull. Bot. Gard. Btzg III, 17 (1948) 429-446, 2 fig. - Ditto.

\section{TACCACEAE}

LmPricht, W., Beitrag zur Kenntnis der Taccaceae. Inaug. Diss. Bot. Gart. Univ. Breslau (1902) 1-59.-Monogr.; keys.

-. Pfianzenreich Heft 92 (1928) 1-32, fig. 1-5. -Practically a copy of the thesis.

Pax, F., Pflanzenfamilien ed. 2, $15 a$ (1930) 434 437, fig. 192-193.

\section{Local}

Malay Peninsula: RIdlex, Mat. Fl. Mal. Pen. (Monocot.) 2 (1907) 75-78.-Key.

Borneo: Merrill, Philip. J. Sc. 29 (1926) 356357.-Descr. new $s p$.

Java: BACKer, Handb. Fl. Java pt 3 (1924) 106108.-Key; in Dutch.

Celebes: Smith, Bull. Jard. Bot. Btzg III, 6 (1924) 79-80.-Descr. new sp.

Philippines: Elmer, Leaff. Philip. Bot. 10 (1939) 3794-3796.-Descr. new sp.

Krause, in Elmer, Leafl. Philip. Bot. 6 (1914) 2283-2284.-Descr. new sp.

Quisumbing \& Merrill, Philip. J. Sc. 37 (1928)

137-138.-Crit. notes; synon.

\section{Genera}

Tacca: Merrill, J. Arn. Arb. 26 (1945) 85-92, 2 pl.-Syn. T. leontopetaloides; morph. rhizome.

\section{TAMARICACEAE}

Niedenzu, Fr., Pflanzenfamilien ed. 2, 21 (1925) 282-289.

\section{TAXACEA E \\ See Coniferae}

\section{THEACEAE}

(Ternstroemiaceae, incl. Tetrameristaceae) (excl. Pentaphylacaceae)

Chorsy, J., Mémoire sur les familles des Ternstroemiacées et Camelliacées (Mém. Soc. Phys. Hist. Nat. Genève 14, 1854, 91-186, t. 1-3). -Generic descr. and delimitation.
Korthals, P. W., Bijdrage tot de kennis der. Ternstroemiaceae (in Temminck, Verh. Nat. Gesch. Bot. 1841-42, 93-149, t. 25-31).-Descr. in Latin of spp. and genera.

MelchioR, H., Pflanzenfamilien ed. 2, 21 (1925) 109-154, fig. 59-67.

- Ueber die systematische Gliederung und Phylogenie der Theaceen (in FEDDE, Rep. Beih. $56,1929,232-235)$.-Validation of morphology for the system.

-, Notizbl. Berl.-Dahlem 11 (1934) 1093-1100. -Survey of the few Afr. representatives.

Miquel, F. A. W., Adnotationes de Ternstroemiaceis (Ann. Mus. Bot. Lugd.-Bat. 4, 1869, 103$105,112-114)$.-Various notes; list; many new spp.

Shaw, H. K. AIRY, The classification of the Camellioideae (Kew Bull. 1936, 498-499).Modified version of MELCHIOR's treatment excluding several genera which are assigned to other families.

\section{Local}

Formosa: KENG, Taiwania 1 (1950) 223-268.Revision; key.

Sumgtra: Merrill, Contr. Arn. Arb. 8 (1934) 106107.-List misc. records.

Borneo: Merrill, J. Str. Br. R. As. Soc. no 86 (1922) 331.-New sp. \& new comb. in Gordonia. RiDleY, Kew Bull. (1938) 173-175.-List; new records in Ternstroemia; new sp. of Adinandra \& Ternstroemia.

AIRy Shaw, in Hook. Ic. Pl. (1936) t. 3309.New Schima.

_, Kew Bull. (1939) 404-408 (sphalm. 504-508). -List RichaRDs coll.; new records and spp. of Adinandra \& Ternstroemia.

一, in Hook. Ic. Pl. V, 5 (1940) t. 3408.-Descr. \& pl. of Ternstroemia magnifica.

Warburg, in Fedde, Rep. 18 (1922) 329.-New Schima.

Java: BACKER, Schoolf. Java (1911) 95-105.Key; in Dutch.

Philippines: Burkall, Philip. J. Sc. 15 (1919) 475478.-Revision; key!; descr.

New Guinea: Diels, Bot. Jahrb. 57 (1923) $431-$ 435.-Keys.

-, ibid. 62 (1929) 483.-Descr. new Eurya.

Hatusima, J. Jap. Bot. 26 (1951) 371.-New comb. in Eurya.

Kobuskı, J. Arn. Arb. 21 (1940) 134-162.Census; no key; descr. new spp.

-, Bot. Mag. Tokyo 55 (1941) 504-507, fig. 2. -Records; one new Ternstroemia.

Kurata, Bull. Tokyo Univ. For. no 36 (1948) 132-133, 1 fig.-Descr. new Ternstroemia.

White, J. Arn. Arb. 10 (1929) 241.-Record of a Philippine Ternstroemia.

\section{Genera}

Adinandra: KoBuski, J. Arn. Arb. 28 (1947) 1-98. -Revision; keys (geogr.)!

-, J. Arn. Arb. 34 (1953) 125-134.-Descr. misc. new spp.

Anneslea: Kobuski, J. Arn. Arb. 33 (1952) 79-90. -Revision; key. 
Camellia (Thea): Cohen Stuart, Bull. Jard. Bot. Btzg III, 1 (1919) 193-320, 3 maps, pl. 21-31.Monogr.; key.

Kochs, Bot. Jahrb. 27 (1900) 577-635, t. 8.Monograph; key; varieties of cult. spp.; anat. ReHDER, J. Arn. Arb. 5 (1924) 238-239.-R. accepts Thea as the correct name on page priority basis which is, according to the Rules, nomenclaturally inadmissable.

Sealy, Kew Bull. (1949) 215-222.-Descr. new spp. \& new comb. of SE. Asia, precursory to a revision of the genus.

SeemanN, Trans. Linn. Soc. Lond. 22 (1859) 337-352, t. 60-61.-Camellia \& Thea kept distinct; synopsis of spp.; no keys.

Cleyera: Kobuski, J. Arn. Arb. 18 (1937) 118-129. -Asia \& Japan.

-, ibid. 28 (1947) 34, 44.-New var. from Luzon, originally descr. in Adinandra.

Eurya: KobUSki, Ann. Missouri Bot. Gard. 25 (1938) 299-359.-Revision of subg. Euryodes and Pentaneurya; keys!

—, J. Arn. Arb. 20 (1939) 361-374.-Misc. notes and descr. new spp.

Vesque, Bull. Soc. Bot. Fr. 42 (1895) 151-161. -Revision; key.

DE WIT, Bull. Bot. Gard. Btzg III, 17 (1947) 329-375, 5 fig.-Revision; keys!; spp. of the Philippines and the Malay Peninsula are not treated.

Gordonia: Burkill, J. Str. Br. R. As. Soc. no 76 (1917) 133-159, fig. 1-15.-Enum. of spp.

Laplacea: Sprague, J. Bot. 61 (1923) 17-19, 8385.-Proposed to be conserved against Lindleya; nomencl.

Pentaphylax. See Pentaphylacaceae.

Ploiarium (Archytaea auct.): HALLIER $f$., Beih. Bot. Centralbl. 34, ii (1916) 34, note 1.-Short note on the genus.

Kовuski, J. Arn. Arb. 31 (1950) 201-207.Revision; key.

Schima: BLOEMBERGEN, Reinwardtia 2 (1952) 133183, fig. A-J.-The genus is reduced to one sp. with a number of subsp.; key!

Airy Shaw, Kew Bull. (1936) 496-497.-Various critical notes.

Ternstroemia (Cyclandra): HatusimA, Bot. Mag. Tokyo 65 (1952) 109-111.-Cyclandra (formerly Guttiferae) is reduced to Ternstroemia; 1 new comb.

Tetramerista: Hallier $f$, Beih. Bot. Centralbl. 34, ii (1916) 35-40.-Revision.

Trematanthera = Saurauia (Actinidiaceae).

\section{THEOPHRASTACEAE}

\section{See Myrsinaceae}

\section{THYMELAEACEAE}

(incl. also Gonystylaceae)

BaIllon, H., Nouvelles observations sur les Aquilariées (Adansonia 11, 1875, 313-322).-General discussion.

- Sur les Aquilariées des herbiers de la Hollande et sur une affinité peu connue de ce groupe
(Adansonia 11, 1875, 326-329).-General discussion.

DeCaIsne, J., Description des genres Drymispermum, Pseudaîs et Gyrinopsis du groupe des Aquilariées (Ann. Sc. Nat. II, 19, 1843, 35-42, t. 1).- Descr. n. g. \& n. spp.; list of Phaleria (under Drymispermum), Pseudals and Gyrinopsis.

DOMKe, W., Zur Kenntnis einiger Thymelaeaceae (Notizbl. Berl.-Dahlem 11, 1932, 348-362).For Malaysia only new Aquilaria, new sp. \& comb. in Gyrinops.

- Untersuchungen über die systematische und geographische Gliederung der Thymelaeaceen nebst einer Neubeschreibung ihrer Gattungen (Bibl. Bot. Heft 111, 1934, 1-151, 1 fig., t. 1-7, 5 maps).-Subdiv. of fam.; keys to and descr. of genera.

HaLlier $f$., H., Beiträge zur Kenntnis der Thymelaeaceen und ihrer natürlichen Umgrenzung (Med. Rijksherb. Leiden no 44, 1922, 1-31).Discussion on affinities within the family. List of Malayan representatives with critical syn.; descr. new spp.; no keys.

MEISNER, K. F., Ueber die Ostindischen Thymeläen (Denkschr. Bot. Ges. Regensb. 3, 1841, 271294,t.6-7).-Taxonomy, tribes; mostly Asiatic spp.

MiQuel, F. A. W., Thymelaeacearum genera nova (Ann. Mus. Bot. Lugd.-Bat. 1, 1863, 132-133, t. 4).-Descr. Lachnolepis and Gonystylus.

Quisumbing, E., A critical study of Philippine species of the tribe Aquilarieae (J. Arn. Arb. 27, 1946, 401-407).-Various new comb. of Mal. spp. in this tribe.

Shaw, H. K. AIRY, Thymelaeaceae-Gonystyloideae (Fl. Mal. I, 4, 1953, 349-365, 6 fig.).

SteEnis, C. G. G. J. vaN, Bull. Jard. Bot. Btzg III, 13 (1934) 254.-List of Daphne and Kelleria \& Drapetes records.

\section{Local}

Sumatra: MERrill, Contr. Arn. Arb. 8 (1934) 111 -Syn. of Eriosolena.

Borneo: Domke \& VAN STEenis, Notizbl. Berl.Dahlem 12 (1934) 233-235.-Descr. new Gony. stylus.

AIRY SHAw, Kew Bull. (1940) 261-262.-Some records.

一, in Hook. Ic. P1. V, 5 (1947) t. 3474-3475.

Java: BACKER, Bekn. Fl. Java (em. ed.) 4A (1942) fam. 77, p. 1-5.

-, Blumea 5 (1945) 494-495.-New Phaleria and a new name in Wikstroemia.

Celebes: Koorders, Suppl. Flora N. O. Celebes 2 (1922) t. 98; ibid. 3, p. 48.-Phaleria urens.

Philippines: Elmer, Leafl. Philip. Bot. 5 (1913) 1629-1632.-Survey of Gyrinopsis; 3 new spp. Quisumbing, J. Arn. Arb. 27 (1946) 401-407.Crit. study of Aquilarieae; various new comb. and new spp.

Warburg, in Perkins, Fragm. Fl. Philip. (1905) 171-172.-New Wikstroemia.

Moluccas: MerriLl, Philip. J. Sc. 11 (1916) Bot. 294-295.-New Phaleria from Ambon.

-, J. Arn. Arb. 31 (1950) 283.-New comb. in Aquilaria. 
New Guinea: Domke, Bot. Jahrb. 62 (1929) 484485.-New Kelleria (Drapetes).

Gilg, Nova Guinea 8 (1910) 411-412.-New Phaleria and Brachythalamus.

Merrill \& Perry, J. Arn. Arb. 22 (1941) 264 267.-New spp. and records in Phaleria, Wikstroemia, and Kelleria.

VAN Steenis, Nova Guinea 14 (1927) 306.Record Gonystylus.

\section{Genera}

Aetoxylon: AIry Shaw, Kew Bull. (1950) 145. 一, Fl. Mal. I, 4 (1952) 364-365, fig. 6.

Amyxa: AIRy SHAw, in Hook. Ic. Pl. V, 5 (1947) t. 3475 .

-, Fl. Mal. I, 4 (1952) 362-363, fig. 5.

Aquilaria: GiLG, Bot. Jahrb. 28 (1901) 145-146.Enum. spp.; key!; new sp. from New Guinea.

Gonystylus: AIRY SHAw, Kew Bull. (1947) 9-17.Key; 3 sections; some new spp.

一, in Hook. Ic. Pl. V, 5 (1947) t. 3474.- \& Auxanthus; descr. G. augescens.

-, Kew Bull. (1950) 138-147.-New or noteworthy spp. of Gonystylus and allied genera. -, Fl. Mal. I, 4 (1952) 350-361.

Gyrinops (Brachythalamus): Domke, Notizbl. Berl.-Dahlem 11 (1932) 349-350.

Gilg, Bot. Jahrb. 28 (1901) 146-147.-Descr. 2 Papuan spp.

Phaleria: VAleton, Ic. Bog. 4 (1913) t. 368-371.Critical notes \& fig. of several spp.

Pimelea: Engler, Bot. Jahrb. 7 (1886) 459-460.Nomencl. Timor $s p$.

FAWCETT, in Forbes, Wanderings ed. 2 (1885) 516.-Descr. new sp. from Timor.

\section{TILIACEAE}

Bentham, G., Notes on Tiliaceae (J. Proc. Linn. Soc. 2nd Suppl. to vol. 5, 1861, 52-62).-Census of the family with keys to gen. \& spp.; many new spp.

BURReT, M., Beiträge zur Kenntnis der Tiliaceen (Notizbl. Bot. Gart. Berl.-Dahlem 9, 1926, 592-880).-Important precursory to a revision; subdivision of the family; see also under genera.

-, ditto II (ibid. 1927, 1161-1174).-Additions to paper above.

-, ditto III (ibid. 12, 1934, 160-167).-Ditto.

-, ditto IV (ibid. 12, 1935, 602-605).-Ditto.

-, ditto V (ibid. 13, 1936, 252-255).-Descr. new Brownlowia (Borneo), Grewia and Trichospermum (Celebes).

Hochreutiner, B. P. G., Notes sur les Tiliacées (Ann. Cons. Jard. Bot. Genève 18/19, 1914, 68-128).-New subdiv. of family; descr. of spp., practically none Malaysian.

\section{Local}

Sumatra: RIDley, Kew Bull. (1924) 262.-New Grewia from Mal. Pen. \& Sumatra.

Borneo: Merrill, Philip. J. Sc. 30 (1926) 82-83.Record of and new Pentace.

-, Pl. Elm. Born. (1929) 184-188.-Records; new spp. in Pentace, Brownlowia and Microcos. Ridley, Kew Bull. (1938) 226-229.-List re- cords; new spp. of Brownlowia, Pentace and Microcos.

AIRY Shaw, Kew Bull. (1949) 159-160.-Record and new spp. of Microcos.

Warburg, in Fedde, Rep. 18 (1922) 330.-New spp. of Pentace.

Java: ADELBERT, Blumea 5 (1945) 503-505.-Critical notes on delimitation.

BACKER, Schoolfl. Java (1911) 147-157.-Keys!; in Dutch.

-, Bekn. Fl. Java (em. ed.) 4B (1944) fam. 105, p. 1-25.-Keys!

Philippines: Elmer, Leafl. Philip. Bot. 2 (1909) 631-632.-New Grewia from Negros.

-, ibid. 5 (1913) 1641-1644.-List of Philip. Trichospermum; 2 new spp.; no key.

Quisumbing \& Merrill, Philip. J. Sc. 37 (1928) 167-170.-Verif. Philip. nomencl. with BURRET's work.

New Guinea: Burret, Notizbl. Berl.-Dahlem 11 (1932) 511-512.-2 new spp. of Trichospermum. Merrill \& Perry, J. Arn. Arb. 20 (1939) 339345.-New spp. in Berrya, Trichospermum, Microcos, and Colona.

Pulle, Nova Guinea 8 (1911) 663-664.-Misc. records; new Grewia.

White, Proc. R. Soc. Queensl. 34 (1922) 43.Misc. records.

—, J. Arn. Arb. 10 (1929) 238.-Records Brass coll.

—, Proc. R. Soc. Queensl. 47 (1936) 54.-Record of a Pap. Grewia in Queensland.

- \& Francis, Proc. R. Soc. Queensl. 38 (1927) 240-241.-Descr. new Columbia.

\section{Genera}

Althoffia: Burret, Notizbl. Berl.-Dahlem 9 (1926) 857-861.-Revision; key.

White, Proc. R. Soc. Queensl. 34 (1922) 43.Crit. note on 2 Pap. spp. of Althoffia.

Berrya: BurReT, Notizbl. Berl.-Dahlem 9 (1926) 605-607.-Revision; key!

KoORders \& Valeton, Ic. Bog. 1 (1897) 19-20, t. 5.-New Berrya from Java.

Brownlowia: Burret, Notizbl. Berl.-Dahlem 9 (1926) 613-619, 869.-Revision; key!

-, ibid. 9 (1927) 1163-1165.-New comb.; additions.

—, ibid. 12 (1934) 160-161.-Correction; identical with 9 (1927) 1163-1164 (sic).

Colona (Diplophractum): BurReT, Notizbl. Berl.Dahlem 9 (1926) 796.-Revision; key!

—, ibid. 12 (1934) 161-162.-Some notes; new sp. from Celebes.

Diplodiscus: Burret, Notizbl. Berl.-Dahlem 9 (1926) 618-619.-Enum.

-, ibid. 12 (1934) 162.-New comb. from Borneo.

Eleutherostylis: BuRRET, Notizbl. Berl.-Dahlem 9 (1926) 629-632.-Monotypic; New Guinea!

Grewia: Burret, Notizbl. Berl.-Dahlem 9 (1926) 632-736; ibid. (1927) 1165-1171.-Key to sections; Microcos split off as separate genus; revision \& key to non-African spp.!

-, ibid. 12 (1935) 604-605.-New Papuan spp.

Corner, Gard. Bull. Str. Settl. 10 (1939) 262.- 
Criticism on generic distinction of Grewia \& Microcos; new name in Grewia.

Drummond, J. Bot. 49 (1911) 329-337, 357-363. -Critical notes on RoxBURGH's spp.

GagnePaIN, Not. Syst. 1 (1909) 119-132.Revision; enum. \& key of spp.

GARCKE, Bonplandia 5 (1857) 257-258.-Critical notes on 2 wrongly placed Philip. Grewias by WALPERS.

- Bot. Zeit. 22 (1864) 337-339, 345-347.Miscell. notes on Grewia.

Narayanaswami \& Rao, J. Ind. Bot. Soc. 29 (1950) 177-190.-Notes Indo-Burm. spp.

Microcos (Omphacarpus, Inodaphnis): BURRET, Notizbl. Berl.-Dahlem 9 (1926) 728-736, 756795.-Revision \& key to Malaysian spp.!; not all spp. are keyed out.

-, ibid. (1927) 1171-1173.-Some additions.

-, ibid. 12 (1934) 162-165.-New spp. from Borneo and New Guinea.

-, ibid. 12 (1935) 602-604.-2 new spp. from Borneo \& New Guinea.

Korthals, Kruidkunde in Temminck, Verh. Nat. Gesch. (1842) 192-194, t. 42.-Descr. 2 new spp.

KURz, Flora 55 (1872) 397-398.-Reduction.

Pentace: BurreT, Notizbl. Berl.-Dahlem 9 (1926) 619-621, 814.-Notes; new comb.

Schoutenia (Actinophora, Chartacalyx): BuRRET, Notizbl. Berl.-Dahlem 9 (1926) 625-628.-Revision; key!

Korthals, Ned. Kruidk. Arch. 1 (1846) 312313.-Type descr.

Trichospermum (Bixagrewia): BURRET, Notizbl. Berl.-Dahlem 9 (1926) 846-855.-Revision; key! 一, ibid. 11 (1932) 511-512.-2 new Pap. spp.

KURZ, J. Bot. 13 (1875) 325, 373.

Merrill, Philip. J. Sc. 11 (1916) Bot. 288-290. -New sp. from Ambon.

Triumfetta: BACKER, Trop. Natuur 8 (1919) 6-7, fig. 12.-Distinction $T$. repens and procumbens.

Gagnepain, Not. Syst. 1 (1910) 170-174.-Crit. notes on 3 spp. allied to $T$. procumbens; key to these spp.

HemSLeY, J. Bot. 28 (1890) 1-2, t. 293.-Critical note on $T$. procumbens.

LAy, Ann. Missouri Bot. Gard. 37 (1950)

315-395.-Revision Amer. spp.; keys!

\section{TRAPACEAE \\ See Hydrocaryaceae}

TRIGONIACEAE

Miquel, F. A. W., Fl. Ind. Bat. Suppl. 1 (1860) 394-395.-Descr. Trigoniastrum.

Steenis, C. G. G. J. YAN, Fl. Mal. I, 4 (1949) $58-60,1$ fig.

\section{Local}

Borneo: AIRy Shaw, Kew Bull. (1940) 253-254. -Record; 2 new varieties.

\section{TRIURIDACEAE}

Beccari, O., Le Triuridaceae della Malesia (Malesia 3, 1890, 318-344, t. 39-42).-Revision; key!
Giesen, H., Pflanzenreich Heft 104 (1938) 1-84, fig. 1-18, 3 maps.

\section{Local}

Java: BACKeR, Handb. FI. Java 1 (1925) 64-66.Key!; in Dutch.

Moluccas: Smith, Bull. Jard. Bot. Btzg III, 16 (1939) 111-112, fig. 1.-Descr. new sp. from Buru.

New Guinea: Schlechter, Bot. Jahrb. 49 (1913) 70-89, 3 fig.-Descr. new gen. \& spp.; no key. Smith, Nova Guinea 14 (1927) 323-327, t. 35-36.-Records \& descr. new spp.

Went Sr, Nova Guinea 8 (1909) 165-167, t. 47. -Ditto.

Micronesia: TuYama, Bot. Mag. Tokyo 52 (1938) 61-65, fig. 1-4.-New Andruris; key to Andruris.

\section{TROPAEOLACEAE}

Buchenau, F., Pflanzenreich Heft 10 (1902) 1-36, fig. 1-14.

Farenholtz, H., Pflanzenfamilien ed. 2, 19a (1931) 67-82, fig. 35-42.

\section{Genera}

Tropaeolum: Buchenau, Bot. Jahrb. 15 (1893) 180-259.-Revision; key! -, ibid. 22 (1895) 157-183.-Additions.

\section{TURNERACEAE}

BACKer, C. A., Fl. Mal. I, 4 (1951) 235-238, fig. 1-2.

GiLG, E., Pflanzenfamilien ed. 2, 21 (1925) 459466.

URBAN, I., Monographie der Familie der Turneraceen (Jahrb. Bot. Gart. Berlin 2, 1883, 1-152, t. 1-11),-Monograph with keys!

\section{Genera}

Turnera: van Steenis, Trop. Natuur 20 (1931) 227-231, fig. 1-3.-Distinction Mal. spp.

\section{TYPHACEAE}

BaCker, C. A., Fl. Mal. I, 4 (1951) 242-244, fig. 1. Graebner, P., Pflanzenreich Heft 2, pt 1 (1900) 1-18, fig. $1-4$.

\section{Local}

Java: Backer, Handb. Fl. Java 1 (1925) 31.-In Dutch.

New Guinea: Hallier $f$, Nova Guinea 8 (1913) 911-914.-New revision of the genus!

\section{Genera}

Typha: Géze, Bull. Soc. Bot. Fr. 58 (1911) 457461.-Critical remarks on the syn. of some spp. -, Etudes botaniques et agronomiques sur les Typha, etc. Villefranche-de-Bouergue (1912) 1-174, t. 1-7.-Monograph; keys!

Kronfeld, Verh. K. K. Zool.-Bot. Ges. Wien 39 (1889) 89-192, t. 4-5.-Monograph; key!

\section{ULMACEAE}

Bernard, Ch., Sur la distribution géographique des Ulmacées (Bull. Herb. Boiss. II, 5, 1905, 1098-1112; ibid. II, 6, 1906, 23-38, pl. 1-7).Geogr. distr. of the family. 
Planchon, J. E., Sur les Ulmacées (Ulmacées et Celtidées de quelques auteurs) considérées comme tribu de la famille des Urticées (Ann. Sc. Nat. Bot. III, 10, 1848, 244-341).-Census; no keys!

\section{Local}

East Asia: P'er, Bot. Bull. Acad. Sin. 1 (1947) 283-297.-Revision; keys!

Sumatra: Merrill, Contr. Arn. Arb. 8 (1934) 44. -Record of Ulmus!

Java: Smith, in KOORD. \& Val. Bijdr. Booms. Java 13 (1910) 638-671.

Philippines: Elmer, Leafl. Philip. Bot. 9 (1934) 3218-3219.-New Trema.

一, ibid. 10 (1939) 3796-3797.-New Celtis.

Warburg, in Perkns, Fragm. Fl. Philip. (1905) 164-165.-Descr. new Celtis.

New Guinea: Lauterbach, Bot. Jahrb. 50 (1913) 308-327.-Keys!

Merrill \& Perry, J. Arn. Arb. 20 (1939)

324-327.-New Parasponia and Gironnieras.

-, ibid. 22 (1941) 253-256.-2 new Celtis and 2 new Parasponias.

Smrrh, Nova Guinea 8 (1912) 891-892, t. 158. -New Parasponia, and new var. in Gironniera.

\section{Genera}

Aphananthe: Leroy, Bull. Mus. Hist. Nat. Paris 18 (1946) 118-123, 180-184.-Discussion; no key; 4 spp. distinguished.

Gironniera: KURZ, Flora 55 (1872) 446-448.-Subdivision of the Celtideae; some notes on spp.

Trema: Lauterbach, Bot. Jahrb. 50 (1913) 312325.-Revision Indo-Mal. spp.

DE WIT, Bull. Bot. Gard. Btzg III, 18 (1949) 184-192.-Revision Mal. spp.; key.

Ulmus: SCHNEIDER, Oester. Bot. Z. 66 (1916) 2134, 65-82.-Revision of genera \& spp.; keys!; 2nd part treats nomencl. of Europ. spp.

\section{UMBELLIFERAE}

Buwalda, P., The Umbelliferae of the Netherlands Indies (Blumea 2, 1936, 119-220, 6 fig.).Revision; key.

- (\& STEenis, C. G. G. J. VAN): Fl. Mal. 4 (1949) 113-140, fig. 1-11; ibid. (1954) 595-597, fig. 5a.

Miquel, F. A. W., Ombellifères, in Ill. Fl. Arch. Ind. (1870) 36-43.-Census; no key.

Molkenboer, J. H., in Miquel, Plant. Jungh. (1851) 89-98.-List; descr. new spp.

WolfF, H., Pflanzenreich Heft 43 (1910) 1-214, fig. 1-24.-Apioideae-Ammineae heteroclitae. $\mathrm{Ge}-$ nus Bupleurum, etc.

-, ibid. Heft 61 (1913) 1-305, fig. 1-42.-Saniculoideae. Genera Sanicula, Eryngium.

-, ibid. Heft 90 (1927) 1-398, fig. 1-26.-Apioideae-Ammineae-Carinae, etc. Genera Apium, Cryptotaenia, Cuminum, Petroselinum, Pimpinella, Trachyspermum, etc.

\section{Local}

Java: Van SteEnis, Trop. Natuur 19 (1930) 78. -Reduction of Pimpinella leeuwenii.

WolfF, in FEDDE, Rep. 20 (1924) 159-160.Descr. new Pimpinella from Java.
Borneo: Merrill, Amer. J. Bot. 5 (1918) 514-515, t. 36.-Descr. new Oreomyrrhis.

Lesser Sunda Islands: NormaN, J. Bot. 69 (1931) 287.-New Trachymene from Timor.

Celebes: Hemsley, Kew Bull. (1896) 37-38.New Trachymene.

WolfF, in Fedde, Rep. 17 (1921) new Didiscus from Celebes.

Philippines: Elmer, Leafl. Philip. Bot. 2 (1909) 627-629.-Key!; 2 new spp.

Merrill, Philip. J. Sc. 29 (1926) 482-483.Descr. new Pimpinella from Luzon.

-, ibid. 30 (1926) 418-419.-Record of Peucedanum from Batan Isl.

— \& QuIsUMBING, ibid. 82 (1953) 332.-Record of Apium leptophyllum.

Moluccas: van Steenis, Fl. Mal. I, 4 (1954) 595597, fig. 5a.-Eryngium from Ceram.

New Guinea: Buwalda, J. Arn. Arb. 32 (1951) 59-66, 1 fig.-List BRAss records; descr. new spp. DANSER, Brittonia 2 (1936) 135-136, t. 2.-New Trachymene.

HeMsLeY, in Hook. Ic. P1. 26 (1899) t. 2590.Descr. \& pl. of Oreomyrrhis linearis.

\section{Genera}

Centella: Domin, Bot. Jahrb. 41 (1908) 148-169. -Monograph; no key.

Petroselinum: AIRy Shaw, Kew Bull. (1938) 256-258.-Nomencl. $P$. crispum $=$ vulgare.

Sanicula: Shan \& Constance, Univ. Calif. Publ. Bot. 25 (1951) 1-78.-World revision; keys!

Trachymene (Didiscus); BURTT, J. Bot. 79 (1941) 44-46.-New comb.

Domin, Bull. Acad. Intern. Géogr. Bot. 18 (1908) 481-494.-Revision Trachymene sens. str.; key; Australian spp.

一, Sitz.-Ber. Kön. Böhm. Ges. Wiss. Prag. II. Classe (1908) no 10, p. 1-77, t. 1-4.-Monogr.; key!

NoRman, J. Bot. 69 (1931) 287-288.-Notes on congenericy of Trachymene and Didiscus.

\section{URTICACEAE}

Bigalke, H., Die Blattspodogramme der Urticaceae und ihre Verwendbarkeit für die Systematik (in Corn's Beitr. z. Biol. der Pfi. 21, 1933, 1-58, t. 1-5).-Anat. of the cystoliths examined in all genera found useful for taxon. aims. Keys to the spp. on the basis of the crystal configuration which represents a constant specific character.

Blume, C. L., Mus. Bot. Lugd.-Bat. 2 (1852) 43-57; ibid. (1855) 137-160; ibid. (1856) 161170, 193-256, fig. 12-24, 48-49, 54-58.-List gen. \& spp. in Herb. Lugd.

Miquel, F. A. W., Plantae Junghuhnianae (1851) 18-41.-List.

-, Observationes de Urticeis quibusdam et de Fatoua (Ann. Mus. Lugd.-Bat. 4, 1869, 301307).-Enum.

UNRUH, M., Anisophyllie und Wechselbeziehungen des Wachstums und Spross bei Urticaceen (Ber. Deut. Bot. Ges. 58, 1940, 484-488). -Morph. 
VRIESE, W. H. DE, Pl. Ind. Bat. Or. (1856) 143148.-List; records copied from WEDDELL.

Weddell, H. A., Revue de la famille des Urticées (Ann. Sc. Nat. Bot. IV, 1, 1854, 173-212).Preliminary census; no keys!

- Monographie de la famille des Urticées (Arch. Mus. Hist. Nat. Paris 9, 1856-57, 1-592, t. 1-20).-Monograph! With keys to tribes and genera.

- Considérations générales sur la famille des Urticées suivie de la description des tribus et des genres (Ann. Sc. Nat. IV, 7, 1857, 307-396). -Extr. from monogr. above.

一, in DC. Prod. 16, 1 (1869) 32-235. 64_Conspectus tribes, genera, and species; partial keys.

\section{Local}

Sumatra: Merrill, Contr. Arn. Arb. 8 (1934) 52-53.-Some misc. records. —, Pap. Mich. Acad. Sc. 23 (1937) (1938) 177.New comb. in Pseudopipturus.

Borneo: Hallier $f$., Ann. Jard. Bot. Btzg 13 (1896) $300-316$, t. 25-27.-Descr. some new spp.; many recomb. under Elatostema.

-, Bull. Herb. Boiss. 6 (1898) 352-356.Descr. new Elatostemas.

-, Meded. Rijksherb. Leiden no 26 (1915) 1-4.

-Records and descr. new spp.

Robinson, Philip. J. Sc. 6 (1911) Bot. 291-314. -Misc. records coll. Sarawak Mus.

SCHRÖTER \& HuB. WINKLER, Mitt. Inst. Allg. Bot. Hamburg 7 (1937) 263-268.-Elatostemas coll. HaNs WinkLER; some new spp.

Java: Amshoff, Blumea 5 (1945) 515-517. -Critical notes \& records.

BAKHUIZEN VAN DEN BRINK $f$., Blumea 6 (1950) 363-365.-Addit. critical notes \& records.

SMITH, in KoORd. \& VAL. Bijdr. Booms. Java 12 (1910) 672-753.-Only arboreous spp.; excellent treatment; keys!

Van Steenis, Trop. Natuur 30 (1941) 154-155. -Distinction 2 Pileas: peploides \& microphylla. -, Bull. Bot. Gard. Btzg III, 17 (1948) 400.Record of Parietaria.

Celebes: AmshofF, Blumea 5 (1945) 593-594. -Record of a Chamabainia and an Elatostema.

Philippines: Elmer, Leafl. Philip. Bot. 3 (1910) 875-901.-List spp. from Mt Apo, Mindanao; many new spp.

—, Leafl. Philip. Bot. 9 (1934) 3219-3222, 32273241.-New Elatostemas and a Pipturus.

Merrill, Philip. J. Sc. 27 (1925) 23.-New Pilea from Luzon.

Quisumbing, Philip. J. Sc. 41 (1930) 317-322, fig. 1-3.-Descr. new Laportea and 2 Elatostemas. -, in Fedde, Rep. 36 (1934) 284-285, t. 178.New Elatostema.

- \& Merrill, Philip. J. Sc. 37 (1928) 142-143. -Descr. new Elatostema from Bohol.

Robinson, Philip. J. Sc. 5 (1910) Bot. 465-543; ibid. 6 (1911) Bot. 1-31, t. 1-3.-Revision; keys! -, ibid. 6 (1911) Bot. 299-314.-Additions to revision; new spp.

Warburg, in Perkins, Fragm. Fl. Philip. (1905) 168.-3 records of Laportea.
Moluccas: van Steenis, Bull. Bot. Gard. Btzg III, 18 (1950) 458.-Record of Poikilospermum.

New Guinea: Diels, J. Arn. Arb. 10 (1929) 75-76. -New Elatostema and Cypholophus.

Gibis (\& Rendle), in Gibis, Contr. Fl. Phytog. Arfak Mts (1917) 128-132, 209-210, fig. 8.-List records and descr. new gen. \& spp.

Perry, J. Arn. Arb. 32 (1951) 369-389.-List records and new spp. coll. BRAss.

Winkler, HuB., in Fedde, Rep. 15 (1919) 418419.-3 new Pileas.

-, ibid. 16 (1919) 175-176.-3 new Pipturus.

-, ibid. 18 (1922) 238.-New Elatostema.

-, Bot. Jahrb. 57 (1922) 501-608, fig. 1-15. -Revision; keys!

-, Nova Guinea 14 (1924) 119-134.-Records and descr. new spp.

\section{Genera}

Boehmeria: Warburg, Notizbl. Berl.-Dahlem 7, no 68 (1920) 345-351 (sphalm. 1-7).-Discussion on various names of fibre yielding $s p p$.

Elatostema (Pellionia, Weddellia, Elatostemoides): Schröter \& HUb. WinkLER, in FEdde, Rep. Beih. $83^{1}$ (1935) 1-56; ibid. $83^{2}$ (1936) 1-174, t. 9-40.-Monogr.; keys!

Fatoua: Miquel, Ann. Mus. Bot. Lugd.-Bat. 4 (1869) 306-307.-List; no key.

Leucosyke: UNRUH, Bot. Jahrb. 73 (1943) 191-258. -Monograph; key!

Oreocnide (Villebrunea): SPRAGUE, Kew Bull. (1928) 395-397.-Generic nomencl.

Pipturus: Skottsberg, Medd. Göteb. Bot. Trädg. 7 (1932) 43-63, 49 fig.-Differential studies between $P$. argenteus and $P$. incanus. ㄷ. ibid. 8 (1933) 111-117, 13 fig.-On the complex $P$. albidus; Pseudopipturus n.g. proposed. -, Bull. Bern. P. Bish. Mus. no 117 (1934) 38-77, fig. 13-32, t. 16-38.-Revision Hawaiian spp.; key!

Procris: Schröter, in Fedde, Rep. 45 (1938) 179-192, 257-300.-Monograph; key!

Pseudopipturus. See under Pipturus.

\section{VACCINIACEAE See Ericaceae}

\section{VALERIANACEAE}

BaCker, C. A., Fl. Mal. I, 4 (1951) 253-254, 1 fig. Graebner, P., Die Gattungen der natürlichen Familie der Valerianaceae (Bot. Jahrb. 37, $1906,464-480$ ).-Genera of the family.

Höck, F., Beiträge zur Morphologie, Gruppierung und geographischen Verbreitung der Valerianaceen (Bot. Jahrb. 3, 1882, 1-73).-Comparative morphology; geogr. distr.

\section{VERBENACEAE}

\section{(incl. Avicenniaceae \& Symphoremaceae)}

Hallier $f$., H., Verbenaceae (Med. Rijksherb. Leiden no 37, 1918, 17-91).-Properly a paper on Elbert's Lesser Sunda Islands coll., but with numerous other data on the family; descr. new spp. and critical notes. 
JUNELL, S., Zur Gynäceummorphologie und Systematik der Verbenaceen und Labiaten, etc. (Symb. Bot. Uppsala 1, no 4, 1934, 1-219, fig. 1-257).-Delimitation of the families on the basis of $\%$ morph.; result very deviating from current usage.

LAM, H. J., The Verbenaceae of the Malayan Archipelago, etc. Thesis Utrecht. Groningen (1919) 1-371, t. 1-3.-Revision of Mal. spp. based on materials at Leyden, Utrecht, and Berlin; keys!

- \& Bakhuizen VAN den BRINK, R. C., Revision of Verbenaceae of the Dutch East-Indies and surrounding countries (Bull. Jard. Bot. Btzg III, 3, 1921, 1-116, iii).-New revision; only descr. of new taxa; keys!

Moldenke, H. N., An alphabetic list of invalid and incorrect scientific names proposed in the Verbenaceae and Avicenniaceae (New York, 1942, 59 pp.).

-, ditto. Suppl. 1 (New York, 1947, 30 pp.).

- The known geographic distribution of the members of the Verbenaceae and Avicenniaceae (New York, 1942, 104 pp.).-List of names arranged by countries.

-, ditto. Suppl. 1 (New York, 1943, 4 pp.).

-, ditto. Suppl. 2 (Bot. Gaz. 106, 1944, 158-164).

-, ditto. Suppl. 3 (Castanea 10, 1945, 35-46).

—, ditto. Suppl. 4 (Amer. J. Bot. 32, 1945, 609612).

-, Many other Supplements in Phytologia.-Non vidi.

- The known geographic distribution of the members of the Verbenaceae, Avicenniaceae, Stilbaceae, Symphoremaceae, and Eriocaulaceae (New York, 1949, 215 pp.).

-, Phytologia 4 (1953) 173-184, 285-295.-Some transfers and new var. of Mal. Verb.

\section{Local}

China: P'eI, Mem. Sc. Soc. China 1, no 3 (1932) 1-193, t. 1-33.-Revision Chin. Verben.; keys!

Siam: Fletcher, Kew Bull. (1938) 199-209.Descr. new spp.

-, Kew Bull. (1938) 401-445.-Important revision with syn., distr. and full keys!

Malaysia (general): KING \& GaMBLE, Kew Bull. (1908) 105-114.-Descr, various new spp.

Sumatra: Merrill, Contr. Arn. Arb. 8 (1934) 146-148.-Records; crit. syn. of some spp.

Moldenke, Phytologia 4 (1953) 286-287, 294. -New Callicarpa and Vitex.

Malay Peninsula: Henderson, J. Mal. Br. Roy. As. Soc. 5 (1927) 262-263.-New Vitex.

-, Gard. Bull. Str. Settl. 7 (1933) 118-119, t. 29.-New Clerodendron.

Java: BACKeR, Trop. Natuur 5 (1916) 87-94.-Key to spp. in Java; in Dutch.

MeEuse, Blumea 5 (1942) 66-80.-Critical notes. -, in BACKER, Bekn. Fl. Java (em. ed.) 9 (1949) fam. 200, p. 1-45.-Keys; in Dutch.

Borneo: Moldenke, Phytologia 4 (1953) 285-286, 368.-New Callicarpa and Sphenodesme.

Ridley, Kew Bull. (1929) 260-262.-New spp.
Lesser Sunda Islands: HALLIER $f$., Med. Rijksherb. Leiden no 37 (1918) 17-91.-List ELBERT coll.

Philippines: Elmer, Leafl. Philip. Bot. 3 (1910) 861-866.-Callicarpas of Mt Apo, Mindanao. -, ibid. 9 (1934) 3222-3225.-New Callicarpa and Clerodendron.

-, ibid. 10 (1939) 3797-3799.-New Vitex and Callicarpa.

Merrill, Philip. J. Sc. 30 (1926) 424-426.Descr. new Clerodendron and Callicarpa from Sulu Arch.

-, Phytologia 2 (1941) 5-6.-New Premna. Non vidi.

Perkins, Fragm. Fl. Philip. (1904) 1-3.-Syn. of Symphorema.

Quisumbing, Philip. J. Sc. 41 (1930) 343-345, fig. 15.-Descr. new Clerodendron.

— \& Merrill, Philip. J. Sc. 37 (1928) 195-198. -Descr. 2 new Callicarpas and a Clerodendron.

Moluccas: Moldenke, Amer. J. Bot. 32 (1945) 612.-New comb. in Geunsia, new Clerodendron from Manipa Isl.

New Guinea: Bakhuizen van den Brink, J. Arn. Arb. 10 (1929) 69-75, t. 16, 17.-List Brass coll. East N. Guinea; one new Gmelina.

- \& LAM, Nova Guinea 14 (1924) 167-172. -List; no key.

BEeR \& LAM, Blumea 2 (1936) 221-228, 3 fig.List Brass coll.; some new spp. in Premna and Clerodendron.

Kurata, Bull. Tokyo Univ. For. no 35, art. 17 (1947) 201-207, 1 fig.-List coll. INOKUMA \& HARA; one new Teysmanniodendron.

LAM, Bot. Jahrb. 59 (1924) 87-98.-List records. -, Blumea 7 (1954) 553-556, fig. 1-2.-New Premna.

Pulle, Nova Guinea 8 (1910) 401-403; 8 (1911) 685-687.-Lists.

White, Proc. R. Soc. Queensl. 34 (1922) 50.Small list.

- \& Francis, Proc. R. Soc. Queensl. 38 (1927) 257-259, fig. 18.-One new Gmelina and some records coll. LANE-POOLE.

Solomon Isl.: BAKHUIZEN VAN DEN BRINK, J. Arn. Arb. 16 (1935) $70-75$, t. 120-122.-List records some new comb. and varieties; one new Gmelina.

Micronesia: LAM, Bot. Jahrb. 59 (1924) 24-29.List of records.

\section{Genera}

Archboldia: BeER \& LAM, Blumea 2 (1936) 31-33, 1 fig.-Monotypic; New Guinea.

Avicennia: BaKhuizen VAN DEN BRINK, Bull. Jard. Bot. Btzg III, 3 (1921) 199-226, t. 14-22.Revision; key!

Biswas, Notes R. Bot. Gard. Edinb. 18 (1934) 159-166, t. 243-246.-Revision; key to Indian spp.

Wyatт-Smith, Mal. For. 16 (1953) 156-161, fig. 1-5.-Key to 5 spp. of the Mal. Pen.

Callicarpa: Merrill, Frans. Am. Phil. Soc. n.s. $24^{2}$ (1935) 332-333.-Synon. of a Callicarpa.

Moldenke, in Fedde, Rep. 39 (1936) 288-317; ibid. 40 (1936) 38-131.-Monogr. of cultivated and American spp.; keys. 
Faradaya: LAM \& MeEuse, Blumea 3 (1938) 201.F. chrysoclada is a Bignon.

SeEmanN, J. Bot. 3 (1865) 256-258.-Revision; no key.

Garrettia: FleTcher, Kew Bull. (1937) 70-74, fig. 1.-Type descr.; Siam.

Lam \& Meeuse, Blumea 3 (1939) 248-254, fig. 1.-The Vitex described proved later to be a Garrettia, $c \hat{f}$. next reference.

Meeuse, Blumea 5 (1942) 67-68.-A second $s p$. occurs in Mal.

Lantana: BACKER, Trop. Natuur 2 (1913) 27-31, 33-36, 4 fig.-Key.

Petraea: Moldenke, in Fedde, Rep. 43 (1938) 1-48, 161-221.-Monograph; key!

Phyla (Lippia): Greene, Pittonia 4 (1899) 45-48. -Lippia versus Phyla.

Premna (Pygmaeopremna, Tatea): DoP, Bull. Soc. Bot. Fr. 70 (1923) 437-446, 829-836.-Descr. \& records from Indo-China.

FleTCHeR, Not. R. Bot. Gard. Edinb. 19 (1936) 177-178. $-P$. integrifolia $=P$. corymbosa.

MeEuse, Blumea 5 (1942) 71.

Merrill, J. Arn. Arb. 32 (1951) 73-78.Reduction of Tatea and Pygmaeopremna to Premna.

Priva: Kobuski, Ann. Missouri Bot. Gard. 13 (1926) 1-34, t. 1-5.-Revision; key!

Moldenke, in Fedde, Rep. 41 (1936) 1-76.Revision; key!

Stachytarpheta: Danser, Ann. Jard. Bot. Btzg 40 (1929) 1-43, t. 1-10.-Revision Mal. spp.; key!; hybrids!

Teysmanniodendron (Xerocarpa): KooRders, Ann. J. Bot. Btzg 19 (1904) 19-31, t. 2-3.-Orig. descr.

Kostermans, Reinwardtia 1 (1951) 75-106.Monograph; key!

Vitex: LAM, Bull. Jard. Bot. Btzg III, 5 (1922) 175-178.-Additions and records; one new sp.

\section{VIOLACEAE}

Burgersdijk, L. A. J., in Miquel, Plant. Jungh. (1852) 118-122.-List records; several new spp. in Java \& Sumatra.

Korthals, P. W., Aanteekeningen op de familie der Violarieae van den Indischen Archipel (Ned. Kruidk. Arch. 1, 1848, 356-363, t. 1).Survey of the family; no keys; 2 new Rinoreas.

Melchior, H. \& W. Becker, Pflanzenfamilien ed. 2, 21 (1925) 329-377, fig. 148-162.

Miquel, F. A. W., Violacearum quarundam recensio (Ann. Mus. Bot. Lugd.-Bat. 4, 1869, 214-218).-Survey of fam. in Mal.

Oudemans, C. A. J. A., in Miquel, Ann. Mus. Bot. Lugd.-Bat. 3 (1867) 67-78.-Revision; keys!

\section{Local}

Sumatra: Merrill, Pap. Mich. Acad. Sc. Arts \& Lett. 19 (1934) 171-174.-New records and spp. of Rinorea.

Borneo: Merrill, Philip. J. Sc. 29 (1926) 400.Some records in Rinorea.

Celebes: Melchior, Notizbl. Berl.-Dahlem 12
(1934) 205-207.-Records KJELLBERG coll.; one new $s p$.

VaN Ooststroom, Blumea 5 (1945) 594-595.Misc. records of Viola.

Philippines: Becker, Philip. J. Sc. 19 (1921) 707 722.-Revision; key!

Merrill, Philip. J. Sc. 30 (1926) 413.-Records of a Bornean sp. in Sulu.

Moluccas: Merrill, Philip. J. Sc. 11 (1916) Bot. 292-293.-New Rinorea from Ambon.

New Guinea: Kanehira \& Hatusima, Bot. Mag. Tokyo 55 (1941) 390-391.-Some records.

MelChioR, Bot. Jahrb. 62 (1929) 368-375.Revision; keys!

Merrill \& Perry, J. Arn. Arb. 24 (1943) 208-210.-Descr. \& records; new Agatea from the Solomons.

Pulle, Nova Guinea 8 (1911) 669-670.-Descr. of a new Viola and Rinorea.

\section{Genera}

Indovethia: BoerlaGe, in Feestbundel Taal-, Letter-, Geschied.- en Aardrijkskundige Bijdragen 80sten geboortedag P. J. VETH (1894) p. 89-90, 1 pl.; repr. in Ic. Bog. 1 (1897) t. 1.

Hybanthus (Ionidium): BACKER, Onkruidfl. Jav. Suiker. (1930) 454 455.-Descr.; in Dutch.

Schulze, Bot. Jahrb. 67 (1936) 437-492.Revision; keys!

Viola: Becker, Beih. Bot. Centralbl. 34, ii (1916) 208-266; ibid. 34, ii (1917) 373-433; ibid. 36, ii (1918) 15-59; ibid. 40, ii (1923) 20-171.Misc. notes and descr. on spp. from Asia, Malaysia, Australia, and the Pacific; no keys. —, Bot. Jahrb. 54 (1917) Beibl. 120, p. 156-189. - Ditto.

Boissieu \& Capitaine, Bull. Soc. Bot. Fr. 57 (1910) 337-344, pl. 11-13.-Account of violets of Herb. Bog.; 1 new sp., 1 new var.

LIN, Taiwania 1 (1950) 269-283.-Key to Formosan spp.

Ridley, J. Bot. 73 (1935) 13-19.-Critical notes and new spp. and var. from West Malaysia.

VAN Steenis, Bull. Jard. Bot. Btzg III, 13 (1934) 258-260'.-List.

\section{VITACEAE \\ (Ampelidaceae, incl. Leeaceae)}

Gagnepain, F., Révision des Ampélidacées Asiatiques et Malaises (Bull. Soc. Nat. Autun 24, 1911, 1-41).-Key to genera \& spp.; no descr.; enum. coll.; several transfers but no new spp.

KING, G., Notes on the Indian species of Vitis (J. As. Soc. Beng. 65, ii, 1896, 108-114).Critical notes; no key.

Miquel, F. A. W., Ampelideae novae (Ann. Mus. Bot. Lugd.-Bat. 1, 1863, 72-101).-Survey Mal. spp.; no keys.

Planchon, J. E., Monographie des Ampélidées

(1) Dr BECKER determined all sheets of Herb. Bog. before he died; unfortunately no MS was left. The list is made according to his identifications. 
vraies (in DC. Mon. Phan. 5, 1887, 305-654).Monogr. without Leea.

Suessenguth, K., Pflanzenfamilien ed. 2, $20 \mathrm{~d}$ (1953) 174-398, fig. 50-104.

\section{Local}

Sumatra: Merrill, Contr. Arn. Arb. 8 (1934) 97-99.-List.

-, Pap. Mich. Acad. Sc. Arts \& Lett. 19 (1934) 167-168, t. 30.-New Pterisanthes.

$\rightarrow$ ibid. 23 (1938) 184-185.-2 records of Ampelocissus.

Java: BACKER, Schoolfl. Java (1911) 244-256.-In Dutch; key.

Borneo: Winkler, HuB., Bot. Jahrb. 44 (1910) 534-537.-Records; new spp. of Ampelocissus.

Celebes: SuessenguTH, in Fedde, Rep. 49 (1940) 13-14.-New Ampelopsis and Ampelocissus leg. RIEDEL.

Philippines: Elmer, Leafl. Philip. Bot. 10 (1939) 3799-3803.-New sp. of Leea, Cayratia, and Tetrastigma.

MerRill, Philip. J. Sc. 11 (1916) Bot. 125-145. -Records and new spp. in Ampelocissus, Cissus, Columella, Tetrastigma, and Leea.

Quisumbrng, Philip. J. Sc. 76 (1944) 45-47.Recombinations of Philip. Cayratias; new Leea. — \& Merrill, Philip. J. Sc. 37 (1928) 164-166. -Descr. 2 new Ampelocissus and one new Leea.

New Guinea: Lauterbach, Nova Guinea 8 (1910) 301-303; (1912) 831-833; 14 (1924) 136-138. -, Bot. Jahrb. 59 (1925) 505-534; ibid. 61 (1927) 31.-Revision; keys.

Merrill \& PerRy, J. Arn. Arb. 22 (1941) 375-383.-Records; new spp. of Leea, also from Solomons.

VAletoN, IC. Bog. 3 (1908) t. 258.-Leea macropus from New Guinea.

\section{Genera}

Cayratia (Columellá): Gagnepan,, Not. Syst. 1 (1911) 339-362.-Segreg. of the genus; revision; key for Mal. \& As. spp.

Cissus: Gagnepann, Not. Syst. 1 (1911) 339-362.Key to 23 spp.

Leea: BurTT, Kew Bull. (1935) 304-305.-Descr. 4-merous sp. from Solomons.

Clarke, J. Bot. 19 (1881) 100-106, 135-142, 163-167.-Revision of Indian spp., including several Malaysian ones.

CorNer, Gard. Bull. Str. Settl. 10 (1939) 45-46.Critical note on $L$. gigantea.

GagnepaIN, Not. Syst. 1 (1910) 229-231.-Some new Leeas from Indo-China.

-, Bull. Soc. Bot. Fr. 57 (1910) 331-336.-Revision Asiatic spp.; key.

Hallier $f$., Ann. Jard. Bot. Btzg 14 (1897) 241247.-Descr. L. amabilis from Borneo; anat. \& ecol. of this sp. with water-containing buds.

Parthenocissus (Landukia): Merrill, Philip. J. Sc. 11 (1916) Bot. 129.-Nomencl.

Pterisanthes: BACKER, Trop. Natuur 6 (1917) 12-16, 2 fig. - Key \& descr. of 2 Jav. spp.

Tetrastigma: GAGNEPAIN, Not. Syst. 1 (1910) 261-271, 306-326; ibid. (1911) 376-379.-New spp. from Indo-China, China, and Mal.; revision; key!

\section{WINTERACEAE}

Hutchinson, J., The family Winteraceae (Kew Bull. 1921, 185-191).-Key 'to the genera; incl. also Illicium.

Smith, A. C., Taxonomic notes on the Old World species of Winteraceae (J. Arn. Arb. 24, 1943, 119-164, 6 fig.).-Precursory to monograph; keys to genera and species.

-, Geographical distribution of the Winteraceae (J. Arn. Arb. 26, 1945, 48-59).

\section{Local}

New Guinea: BuRT, in Hook. Ic. Pl. V, 4 (1936) t. 3315.-Note on Bubbias; many Drimys and Belliolum reduced.

一, Kew Bull. (1938) 458-460.-Reduction of Tetrathalamus to Bubbia.

DANDY, J. Bot. 72 (1934) 41-42.-Transfers from Drimys to Bubbia, mostly New Cal.

Diels, Bot. Jahrb. 54 (1916) 240-245.-Key to Drimys!

Smith, J. Arn. Arb. 23 (1942) 417-441.-Descr. \& records; no key.

\section{XYRIDACEAE}

Malme, G. O., De nonnullis speciebus Xyridis praecipue Australisiensibus et Asiaticis (Svensk Bot. Tidskr. 21, 1927, 381-396).-Key to Austral. and Asiat. spp.

-, Xyridaceae Indiae Batavae (Bull. Jard. Bot. Btzg III, 10, 1929, 385-392).-Incomplete revision Mal. spp.; key.

- Pflanzenfamilien ed. 2, 15a (1930) 35-38, fig. 10-15.

Nilson, A., Studien uber die Xyrideen (Kongl. Svenska Vet. Akad. Handl. 24, no 14, 1892, 1-75, pl. 1-6).-Descr. of two Xyris from Malaya \& Sum.; further on Amer. Abolboda.

Rendle, A. B., Notes on Xyris (J. Bot. 37, 1899, 497-509).-Notes \& descr.; no key.

ROYEN, P. VAN, Fl. Mal. I, 4 (1953) 366-376, fig. 1-10; 598-599.

—, Blumea 7 (1953) 307-309.-Descr. new spp. \& var.

STEUdel, E. G., Enumeratio plantarum Glumacearum pars II. Stuttgart (1855) 283-290.Enum. descr.; in Latin.

\section{Local}

Indo-China: MALme, Bull. Mus. Hist. Nat. Paris II, 2 (1930) 684-685.-2 new spp.

Java: BACKER, Handb. Fl. Java 3(1924)4-5.-Key!; in Dutch.

Beumée, Trop. Natuur 6 (1917) 155-159, fig. 1-10.-Ecol. \& anat. $X$. indica L.; in Dutch.

New Guinea: Hallier $f$., Nova Guinea 8 (1913) 903.-Record.

\section{ZINGIBERACEAE}

Holtrum, R. E. See under Malay Peninsula.

Horaninow, P., Prodromus monographiae Sci- 
taminarum, etc. (1862) 1-45, t. 1-4.-Survey; no keys.

LOESENER, TH., Zingiberaceae novae vel minus cognitae (Notizbl. Berl.-Dahlem 10, 1927, 6268).-Critical notes on Achasma, Geanthus, and Costus.

-, ditto (ibid. 10, 1929, 706-716).

-, Pflanzenfamilien ed. 2, 15a (1930) 541-640, fig. 236-289.

Miquel, F. A. W., Observationes de Zingiberaceis (Ann. Mus. Bot. Lugd.-Bat. 4, 1869, 99-102).-Notes \& descr. of Hedychium and Tapeinocheilos.

SchumanN, K., Monographie der Zingiberaceae von Malaisien und Papuasien (Bot. Jahrb. 27, $1899,259-350$, t. 2-6).-Keys to genera and spp. -, Pflanzenreich Heft 20 (1904) 1-458, fig. 1-52.

VAleton, TH., Ic. Bog. 2 (1904-1906) t. 154-175, 188-200; ibid. 3 (1906-1909) t. 202-203, 249, 275, 300; ibid. 4 (1913-1914) t. 350, 372-378. -Descr. various new or noteworthy spp.

-, Bull. Jard. Bot. Btzg II, no 27 (1919) 1-167, pl. 1-30.-New notes on Zing. of Java and Mal. Arch.; no keys!

\section{Local}

Indo-China: GagnePAIn, Bull. Soc. Bot. Fr. (1906) 132-150.-Descr. new spp. Herb. Pier Re.

Sumatra: BurKILL, Gard. Bull. Str. Settl. 3 (1923) 18-19.- New Haplochorema.

RIDley, J. Fed. Mal. Stat. Mus. 8, 4 (1917) 114 115.-List of records.

_, J. Mal. Br. R. As. Soc. no 87 (1923) 98-100. -Descr. several new spp. Sum. East Coast.

VALEToN, Bull. Jard. Bot. Btzg III, 3 (1921) 148149, t. 9.-Descr. new Elettariopsis.

Malay Peninsula: Holtrum, Gard. Bull. Sing. 13 (1950) 1-249, 33 fig.-Complete revision; of great value for a future monograph of Mal. spp.; keys!

RIDley, J. Str. Br. Roy. As. Soc. no 32 (1899) 87-175.-Descr. revision; no keys.

Ridley, Mat. Fl. Mal. Pen. (Monocot.) 2 (1907) 1-55.-Keys!

Borneo: Merrill, Sarawak Mus. J. 3 (1928) 519. Ridley, J. Str. Br. R. As. Soc. no 46 (1906) 229-245.-Records \& descr. new spp. -, J. Str. Br. R. As. Soc. no 63 (1912) 62.

-, J. Bot. 51 (1913) 247.-Descr. new Alpinia.

-, J. Linn. Soc. Bot. 42 (1914) 162-164.-List; descr. 2 new Globbas and 2 new Alpinias.

- \& Hub. Winkler, Bot. Jahrb. 44 (1910) 529-532.-List; several new spp.

Java: Valeton, Bull. Inst. Bot. Btzg I, no 20 (1904) 1-99.-Descr. new and noteworthy spp.; keys!

—, Bull. Jard. Bot. Btzg II, no 27 (1919) 1-167, t. 1-30.-New notes on Jav. Zing.; no keys.

VAN ZiJP, Rec. Trav. Bot. Néerl. 12 (1915) $340-347$, t. 2-3.-Descr. new Curcuma \& notes on a Zingiber.

— \& VAleron, ibid. 14 (1917) 127-142, t. 11-13. -Further notes on Curcuma with descr. new spp.

Celebes: Valeton, Bull. Jard. Bot. Btzg III, 5 (1923) 343-344.-Key to 5 spp.
Philippines: Elmer, Leafl. Philip. Bot. 8 (1915) 2885-2919.-Descr. many new spp.

一, ibid. 8 (1919) 2963-2995.-Descr. new spp. Sorsogon Peninsula.

一, ibid. 9 (1934) 3225-3226.-New Alpinia.

-, ibid. 10 (1939) 3803-3806.-Descr. 2 new spp. Merrill, Philip. J. Sc. 30 (1926) 390-391.Record of Amomum and new Boesenbergia from Sulu Arch.

Quisumbing \& Merrill, Philip. J. Sc. 37 (1928) 138-141.-Descr. 2 new Alpinias (Languas).

Ridley, Govt Lab. Publ. Philip. no 35 (1905) 83-87.-List records with descr. new spp.

一, Philip. J. Sc. 4 (1909) Bot. 155-193.-Survey; descr. new spp.; keys!

-, in Elmer, Leafl. Philip. Bot. 2 (1909) 569572.-Records and descr. new genera (Elmeria) and new spp.

-, ibid. 2 (1909) 603-607.-Records and new spp. from S. Negros; Adelmeria new name for Elmeria.

Moluccas: Valeton, Bull. Jard. Bot. Btzg III, 5 (1923) 343-345, t. 39.-Descr. new spp.

New Guinea: RIDLEY, J. Bot. 24 (1886) 356-358. -New spp. coll. Forbes.

-, Trans. Linn. Soc. Lond. II, Bot. 9 (1916) 209-226.-List; several new genera, many new spp.

-, Proc. R. Soc. Queensl. 34 (1922) 19-21.Records; 2 new Riedelias, 1 new Eriolopha.

Valeton, Nova Guinea 8 (1913) 923-988, t. 162-179.-List; many new spp.; subdivision of Riedelia.

-, Bot. Jahrb. 52 (1914) 40-100, 11 fig.-List and descr. new spp.; keys!; only German New Guinea.

-, in Gibes, Contr. Fl. Phytog. Arfak Mts (1917) 102-103.-List; one new Riedelia.

\section{Genera}

Amomum: Valeton, Bull. Jard. Bot. Btzg III, 2 (1920) 354-356.-Records and descr. new spp.

Burbidgea: Ridley, J. Bot. 75 (1937) 202-203.-2 new spp. Borneo.

Elettariopsis: Valeton, Bull. Jard. Bot. Btzg III, 3 (1921) 148-149, t. 9.-Descr. new sp. from Sumatra.

Eriolopha: RIDLEY, Trans. Linn. Soc. II, Bot. 9 (1916) 217-222.-Descr, new sp.; key!

Geanthus: Valeton, Bull. Jard. Bot. Btzg III, 3 (1921) 143-146, t. 7-8.-Descr. 2 new spp. from Java \& Sumatra.

Geostachys: Valeton, Bull. Jard. Bot. Btzg III, 3 (1921) 146-147.-Descr. new sp. from Sumatra.

Globba: Gagnepan, Bull. Soc. Bot. Fr. 48 (1901) 201-216, t. 4-8.-Survey of Globbas preserved at Paris; no key!

Valeton, Ann. Jard. Bot. Btzg 31 (1921) 18-25, t. 5-6.-Descr. 3 new spp., from Sumatra (2) and Borneo (1).

Hedychium: TuRriL., Kew Bull. (1914) 368-372, $2 \mathrm{pl}, 8 \mathrm{fig}$.-Key to $8 \mathrm{spp}$. aff. $H$. coronarium.

Hornstedtia (subg. of Amomum): VAleton, Bull. Jard. Bot. Btzg III, 3 (1921) 150-179, t. 9-13.Reconsideration of generic limits; key! 
Phaeomeria (Nicolaia): VAleton, Bull. Jard. Bot. Btzg III, 3 (1921) 128-140, t. 1-6.-Descr. misc. spp.

Rhynchanthus: Valeton, Bull. Jard. Bot. Btzg III, 3 (1921) 141-142, t. 7.-Descr. new sp. from Sumatra.

Riedelia: Valeton, Nova Guinea 8 (1913) 959961.-Infrageneric subdivision.

\section{ZYGOPHYLLACEAE}

ENGLER, A., Geographische Verbreitung der Zygophyllaceen in Verhältnis zu ihrer systema- tischen Gliederung (Abh. K. Preuss. Akad.Wiss., Phys. Abh. 1896 ii, 1-36, 1 map).

-, Pflanzenfamilien ed. 2, 19a (1931) 144-184, fig. 68-87.

Steenis, C. G. G. J. van, Bull. Jard. Bot. Btzg III, 3 (1934) 104-105.-Revision.

-, Fl. Mal. I, 4 (1949) 64, 2 fig.

\section{Local}

New Guinea: Merrill \& Perry, J. Arn. Arb. 21 (1940) 511.-Record.

C. G. G. J. van STEEnis 